University of Oviedo

Faculty of Sciences $\widehat{\underline{\underline{\text { IIIIIII }}}}$

GHENT

UNIVERSITY

\section{Ghent University}

Faculty of Bioscience Engineering

\title{
MONOTONICITY-BASED CONSENSUS \\ STATES FOR THE MONOMETRIC \\ RATIONALISATION OF RANKING RULES \\ WITH APPLICATION IN DECISION MAKING
}

Raúl Pérez-Fernández

Thesis submitted in fulfillment of the requirements for the degree of

Doctor by the University of Oviedo (Doctoral Program of Mathematics and Statistics)

Doctor of Applied Biological Sciences by Ghent University

Academic year 2016-2017

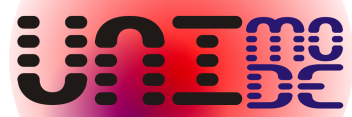

Fo FACULTY OF

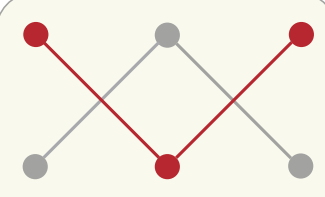

KERMIT 


\section{Supervisors:}

Prof. dr. Bernard De Baets

Department of Mathematical Modelling, Statistics and Bioinformatics Ghent University, Belgium

Prof. dr. Irene Díaz

Department of Computer Science

University of Oviedo, Spain

Prof. dr. Susana Montes

Department of Statistics and O.R.

University of Oviedo, Spain

\section{Examination board:}

Prof. dr. Pedro Alonso

Prof. dr. Bernard De Baets

Prof. dr. José Luis García-Lapresta

Prof. dr. Ignacio Montes

Prof. dr. ir. Wim Verbeke

Dean Faculty of Sciences (University of Oviedo):

Prof. dr. José Manuel Noriega

Rector University of Oviedo:

Prof. dr. Santiago García-Granda

Dean Faculty of Bioscience Engineering (Ghent University):

Prof. dr. ir. Marc Van Meirvenne

Rector Ghent University:

Prof. dr. Anne De Paepe 
Raúl Pérez-Fernández

\section{MONOTONICITY-BASED CONSENSUS STATES FOR THE MONOMETRIC RATIONALISATION OF RANKING RULES WITH APPLICATION IN DECISION MAKING}

Thesis submitted in fulfillment of the requirements for the degree of

Doctor by the University of Oviedo (Doctoral Program of Mathematics and Statistics) Doctor of Applied Biological Sciences by Ghent University

Academic year 2016-2017 


\section{Spanish translation of the title:}

Estados de consenso basados en la propiedad de monotonìa para la racionalización monométrica de las reglas de ordenación con aplicación en la toma de decisiones

\section{Dutch translation of the title:}

Monotoniteit-gebaseerde consensustoestanden voor de monometrische rationalisatie van rankschikkingsregels met toepassing in besluitvorming

\section{Please refer to this work as follows:}

Raúl Pérez-Fernández (2017). Monotonicity-based consensus states for the monometric rationalisation of ranking rules with application in decision making, $\mathrm{PhD}$ Thesis. University of Oviedo, Oviedo, Spain. KERMIT, Department of Mathematical Modelling, Statistics and Bioinformatics, Ghent University, Ghent, Belgium.

This research has been partially supported by the Campus of International Excellence of the University of Oviedo, by the BOF grant with code 01DI2816 and by the project IWT-SBO130036: CHECKPACK - Integrated optical sensors in food packaging to simultaneously detect early-spoilage and check package integrity.

The author and the supervisors give the authorisation to consult and to copy parts of this work for personal use only. Every other use is subject to the copyright laws. Permission to reproduce any material contained in this work should be obtained from the author. 


\section{Agradecimientos}

A todos los que me acompañaron en estos años de altos y bajos, de caricias y hachazos. A todos los que injustamente olvidaré en estos míseros párrafos. A todos los que, de una manera u otra, me han ayudado a llegar hasta aquí. Gracias por ayudarme a entender que la vida consiste en ser feliz.

A Samu, Sabi, Aitor, Enol, Mikel, Victor, Andrés, Christian y resto de amigos del cachopo y la caleya. A Pelayo, cachopero, caleyero y hermano. A Julia, Kike y Jandro, eternos compañeros de indecisiones, escapes y sopas agripicantes. A Coru, que aparece y desaparece, que siempre está presente en mi corazón. To Marcelo and Marcela, with whom I still have lots of saideiras to share. Gracias por todas esas innumerables noches de filosofía y cerveza.

To Michael, my partner in crime, my vriendo. To Andreia and Niels, my very best friends in Ghent. To Marc (and Carlotta), my foal(s), who taught me that life tastes better mixed with ginger jenever. To Aisling, my favourite Irish-Canadian, my favourite Irish, my favourite Canadian. To David, who shared my fever, who will always be my favoured coffee partner. To Giacomo, who deserves all the love of Ramón. To Youri, the only beard I feel envy for. To (bad) Wouter, my co-organiser, who managed to create a magic potion from the sewer. To Peter, the unique and only scout-judoist. To Michiel, with whom I wandered the last paths of this PhD life. To José, el pollo loco, the spicy chili. To Christina, who suffered my face all day in front. To Guillermo, who enlivened this department even more than he can imagine. To Bram, the golden boy. To Dorien, who should never stop smiling. To Hassane, who was as sweet to me as his dates. To Tim, Gang, Stijn, Timpe, André Puré, and rest of biomatchers, partners of victories and cheers, of defeats and tears, of fun and beers. To Giulio, Bac, Yuanyuan, Laura, Carlos, Dailé, Shuyun, Wenwen, Omar and Gisele, who meant so much in a ridiculously short amount 
of time. To Nacho, Davide, Quique, Susana D., and rest of members of the research unit UNIMODE. To Chaïm, Tinne, Marlies, Hilde, Timothy, Ivo, Sophie, Jenna, Jim, Yohannis, and rest of members of this bw10 family, which I hold dear. Thanks for making me feel at home during these amasing $\mathrm{PhD}$ years.

To Michaël, who helped this little duck take his first steps, who called my attention to the field of social choice, who is close to being the best man I have ever met. Thanks for your help, time and friendship.

A Pedro, Irene y Susana, que me iniciaron en la investigación, que guiaron mis pasos, que, desgraciadamente, vieron demasiadas veces mi lado más gris. To Bernard, who has supported me in every single sense, who believed in me when not even I did. Thanks for carrying me here.

A mi padre, mi héroe, mi futuro ser. A mi madre, la persona con la que más injusto soy, a la que no digo suficiente lo mucho que quiero. A mi hermana, que me ha sufrido más de 21 años y, aún así, sigue a mi lado. A mi abuelo, que me mima con locura. A Juanjo, Ana, Dani y Alba, que vienen de Oriente por Navidad. A Carmen y Alberto, mi familia adoptiva. Al resto de familia repartida por el mundo: Sanse, Málaga, Alcalá, París... A aquellos que, desafortunadamente, ya no están. A los que no hay palabras suficientes para agradecer lo mucho que debo. Gracias por ayudarme a ser quien quiero ser. Gracias por ayudarme a querer ser quien soy.

A la sonrisa que alumbra mis mañanas. A los labios que soplan mis noches en vela. A la mano que, con mimo, mece mi vida. A mi cita con la felicidad. A mi compañera de viaje. A ti, siempre a ti, Laura. Gracias por estos inolvidables ocho años juntos.

Happiness only real when shared.

Christopher J. McCandless 


\section{Contents}

\begin{tabular}{|ll}
\hline Agradecimientos & i \\
\hline
\end{tabular}

Contents $\quad$ ix

List of symbols $\quad$ xi

\begin{tabular}{lll}
\hline 1 & Prologue & 1
\end{tabular}

1.1 Preface . . . . . . . . . . . . . . . . . . . . . . 1

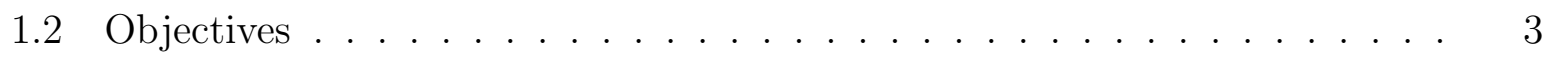

1.3 Outline of this dissertation . . . . . . . . . . . . . . . . . . 3

\begin{tabular}{lll}
\hline The state of the art & 5
\end{tabular}

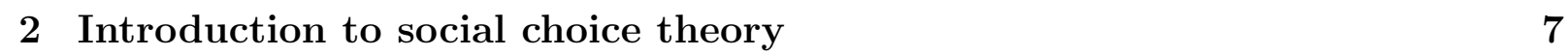

2.1 Social choice theory $\ldots \ldots \ldots \ldots \ldots$. . . . . . . . . . . . . . . . . .

2.2 Best-known ranking rules in social choice theory . . . . . . . . . . . . . . . 9

$2.2 .1 \quad$ Plurality (first-past-the-post) $\ldots \ldots \ldots$. . . . . . . . . . . 9 
$2.2 .2 \quad$ Borda count $\ldots \ldots \ldots \ldots \ldots$

$2.2 .3 \quad$ Scoring ranking rules $\ldots \ldots \ldots \ldots \ldots$

2.2 .4 Majority $\ldots \ldots \ldots \ldots \ldots \ldots$

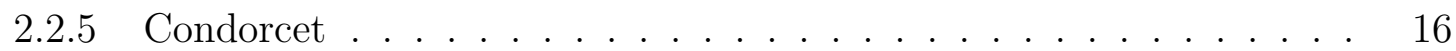

2.2 .6 Kemeny (Kemeny-Young) $\ldots \ldots \ldots$

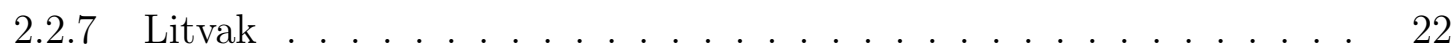

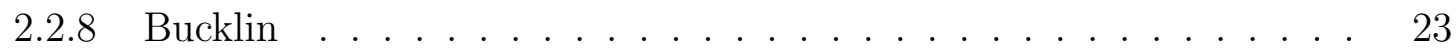

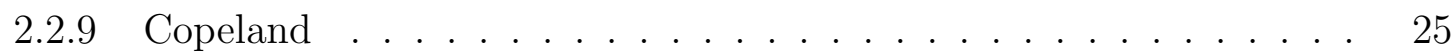

2.2 .10 Simpson (Simpson-Kramer, Minimax, Maximin) . . . . . . . . 26

2.2 .11 Tideman (ranked pairs) $\ldots \ldots \ldots \ldots \ldots$. . . . . . . . 27

2.2 .12 Schulze. . . . . . . . . . . . . . . . . . . . . . . . . . . . . 29

$2.2 .13 \mathrm{WVM}$ and EWVM $\ldots \ldots \ldots \ldots \ldots$

2.2 .14 Elimination methods . . . . . . . . . . . . . . . . . . . . 33

$2.3 \quad$ A ranking rule based on monotonicity $\ldots \ldots \ldots \ldots \ldots \ldots$

\begin{tabular}{lll}
\hline II & The methodology & 39
\end{tabular}

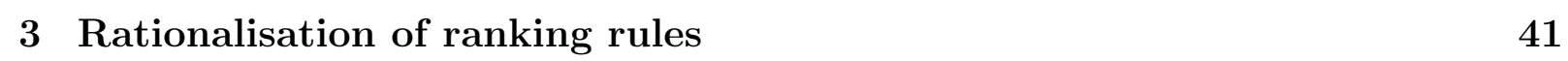

$3.1 \quad$ Metric rationalisation of ranking rules $\ldots \ldots \ldots \ldots$. . . . . . . . . . . . 41

3.2 Monometrics. . . . . . . . . . . . . . . . . . . . . . . . . . . . . . . . . 44

3.2 .1 General case . . . . . . . . . . . . . . . . . . . . . . . 44

$3.2 .2 \quad$ Monometrics on $\mathcal{L}(\mathscr{C}) \quad \ldots \ldots \ldots \ldots \ldots \ldots$ 
$3.2 .3 \quad$ Monometrics on $\mathcal{L}(\mathscr{C})^{r} \ldots \ldots \ldots \ldots \ldots 5$

3.2 .4 Monometrics on $\mathcal{M}_{r}(\mathcal{L}(\mathscr{C})) \ldots \ldots \ldots \ldots$

$3.3 \quad$ Cost calculation as an optimization problem . . . . . . . . . . . . . . . 68

3.3.1 Calculating the cost of changing an anonymised profile of rankings into another one . . . . . . . . . . . . . . . 6 68

3.3 .2 Searching the 'closest' (anonymised) profile of rankings . . . . . . . 69

3.3 .3 The special case of the zero-one monometric . . . . . . . . . . . . 71

3.4 Hierarchical combination of monometrics . . . . . . . . . . . . . . . . . . 72

3.5 Monometric rationalisation of ranking rules . . . . . . . . . . . . . . . . . . 74

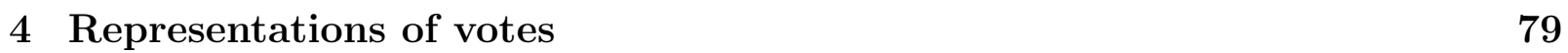

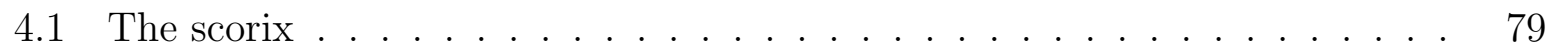

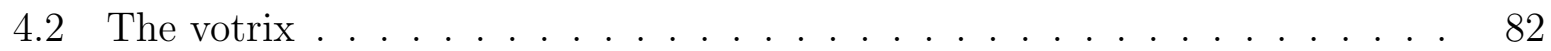

4.3 The votex $\ldots \ldots \ldots \ldots$

4.4 The beatpath matrix $\ldots \ldots \ldots \ldots$. . . . . . . . . . . . . . . . 95

\begin{tabular}{|lll}
5 & Monotonicity of a representation of votes & 97
\end{tabular}

5.1 Monotonicity of the scorix $\ldots \ldots \ldots$. . . . . . . . . . . . 97

5.2 Recursive monotonicity of the scorix $\ldots \ldots \ldots$. . . . . . . . . 100

5.3 Monotonicity of the votrix . . . . . . . . . . . . . 106

5.4 Monotonicity of the votex . . . . . . . . . . . . . . . 108

5.5 Monotonicity of the beatpath matrix . . . . . . . . . . . . . . . . 111

5.6 Monotonicity of the (anonymised) profile . . . . . . . . . . . . . . . 113 
5.7 Acclamation . . . . . . . . . . . . . . . . . . . . . 117

\begin{tabular}{|cc|}
6 & The search for monotonicity as an optimization problem \\
\hline
\end{tabular}

$6.1 \quad$ Changes in the representation of votes $\ldots \ldots \ldots \ldots$

6.1 .1 Search for a monotone scorix . . . . . . . . . . . . . . . . 126

6.1 .2 Search for a monotone (quasi)votrix . . . . . . . . . . . . . 131

6.1 .3 Search for a monotone (quasi) votex . . . . . . . . . . . . 136

6.2 Changes in the profile of rankings $\ldots \ldots \ldots \ldots \ldots$

\begin{tabular}{lll}
\hline 7 & Monotonicity and axiomatic social choice theory & 147
\end{tabular}

$7.1 \quad$ Axiomatic social choice theory $\ldots \ldots \ldots \ldots$. . . . . . . . . . . 147

7.2 Independence of all proposed ranking rules $\ldots \ldots \ldots \ldots \ldots$

7.2.1 The search for monotonicity of the scorix and the search for recursive monotonicity of the scorix . . . . . . . . . . . . . . . . 149

7.2.2 The search for (recursive) monotonicity of the scorix and all scoring ranking rules $\ldots \ldots \ldots \ldots \ldots$

7.2.3 The search for monotonicity of the votrix and the search for monotonicity of the votex . . . . . . . . . . . . . . . . . . 151

7.2.4 The search for monotonicity of the votrix and the search for a Condorcet ranking and unanimity . . . . . . . . . . . . . . . . 152

7.2.5 The search for monotonicity of the profile and the search for all other consensus states . . . . . . . . . . . . . . . . . . . . . 153

7.2.6 The search for monotonicity of the scorix and the search for monotonicity of the votrix . . . . . . . . . . . . . . . . . 153 
7.2.7 The search for acclamation and the search for a Condorcet ranking and unanimity . . . . . . . . . . . . . . . . . . . 154

7.2.8 The search for a Condorcet ranking and the search for unanimity . 154

7.2 .9 Summary . . . . . . . . . . . . . . . . . . . . 155

$\begin{array}{lll}8 & \text { Impact of ties on the representations of votes } & 157\end{array}$

8.1 The three-way setting . . . . . . . . . . . . . . . . . . . 157

8.2 The weak-scorix $\ldots \ldots \ldots \ldots$. . . . . . . . . . . . . . . . . . . . . . . . . . 158

8.3 The weak-votrix . . . . . . . . . . . . . . . . . . . . . . . 161

8.4 The weak-votex $\ldots \ldots \ldots \ldots$. . . . . . . . . . . . . . . . . . . 162

8.5 The profile of rankings with ties . . . . . . . . . . . . . . . . . . 171

$8.6 \quad$ Search for monotonicity of representations of votes affected by ties . . . . . 183

8.7 Relation with the antisymmetric case $\ldots \ldots$. . . . . . . . . . . . . 186

\begin{tabular}{lll}
\hline 9 & Winning candidates & 191
\end{tabular}

$9.1 \quad$ On being better than another candidate . . . . . . . . . . . . . . 192

9.1.1 On being quasipositionally better than another candidate . . . . . . 192

9.1 .2 On being positionally better than another candidate. . . . . . . . . 195

9.1.3 On being pairwisely better than another candidate . . . . . . . . . 197

9.2 On being the best candidate . . . . . . . . . . . . . . . . . . 202

9.2.1 Some existing notions of best candidate . . . . . . . . . . . . 202

$9.2 .2 \quad$ On being the quasipositionally best candidate . . . . . . . . . . . 204

$9.2 .3 \quad$ On being the positionally best candidate . . . . . . . . . . . . 208 
9.2 .4 On being the pairwisely best candidate . . . . . . . . . . . . . . 213

9.2.5 Relation between the (quasi)positional and the pairwise winners . . 217

$9.3 \quad$ On being a potential best candidate . . . . . . . . . . . . . . . . . 219

9.3 .1 The quasipositionally undominated set . . . . . . . . . . . . 220

9.3 .2 The positionally undominated set . . . . . . . . . . . . . 221

9.3 .3 The pairwisely undominated set . . . . . . . . . . . . . . . 222

$9.4 \quad$ On being the best ranking $\ldots \ldots \ldots \ldots \ldots \ldots$

9.4 .1 On being the quasipositionally best ranking . . . . . . . . . . . . 223

$9.4 .2 \quad$ On being the positionally best ranking . . . . . . . . . . . . 225

$9.4 .3 \quad$ On being the pairwisely best ranking . . . . . . . . . . . . 226

9.5 A new social choice function . . . . . . . . . . . . . . . . . . . . . 228

III The application $\quad 239$

\begin{tabular}{ll}
\hline 10 Case studies & 241
\end{tabular}

10.1 Lar rangeland in Iran . . . . . . . . . . . . . . . . . . . . . . . . 242

10.1 .1 The wildlife diversity criterion . . . . . . . . . . . . . . . 242

10.1 .2 Allowing for ties . . . . . . . . . . . . . . 253

10.2 Orange harvest in Argentina . . . . . . . . . . . . . . . . . . 257

10.3 Food spoilage in Belgium . . . . . . . . . . . . . . . . . . 265 
$\begin{array}{ll}\text { IV Epilogue } & 273\end{array}$

\begin{tabular}{ll}
\hline 11 Conclusions and perspectives & 275
\end{tabular}

\begin{tabular}{ll}
\hline Bibliography & 281
\end{tabular}

\begin{tabular}{ll}
\hline Summaries & 295
\end{tabular}

11.1 English summary . . . . . . . . . . . . . . . . . . . . . . 295

11.2 Spanish summary . . . . . . . . . . . . . . . . . . . 299

11.3 Dutch summary . . . . . . . . . . . . . . . . . . . . 302

\begin{tabular}{ll}
\hline Curriculum vitae & 307
\end{tabular} 



\section{List of symbols}

List of symbols in order of appearence.

$\mathbb{N} \quad$ Set of natural numbers

$\mathbb{Z} \quad$ Set of integer numbers

$\mathbb{R} \quad$ Set of real numbers

$\mathbb{R}^{+} \quad$ Set of positive real numbers

$\mathscr{C} \quad$ Set of candidates

$\mathscr{C}_{\neq}^{2} \quad$ Set of couples of different candidates

$k \quad$ Number of candidates

$\mathscr{R} \quad$ Profile of rankings

$r \quad$ Number of voters

$\mathscr{R}_{\sigma} \quad$ Profile of rankings obtained by permuting the order of the candidates

$\sigma(\mathscr{R}) \quad$ Profile of rankings obtained by permuting the order of the voters

$\succ \quad$ Ranking (with ties)

$\succeq \quad$ Total order relation

$\sim \quad$ Indifference relation

$\mathcal{L}(\mathscr{C}) \quad$ Set of all rankings on $\mathscr{C}$

$\mathcal{L}(\mathscr{C})^{r} \quad$ Set of all profiles of $r$ rankings on $\mathscr{C}$

$P_{\succ}(a) \quad$ Position of the candidate $a$ in the ranking $\succ$

$P_{j}(a) \quad$ Position of the candidate $a$ in the ranking $\succ_{j}$

$\left(\alpha_{i}\right)_{i=1}^{n} \quad$ Vector of scores (sums up to one)

$s(a) \quad$ Score of the candidate $a$

$K \quad$ Kendall (Kemeny in the case of rankings with ties) distance function

$a_{i} \rightarrow a_{j}$ Path from candidate $a_{i}$ to candidate $a_{j}$

$B \quad$ Beatpath matrix 


\begin{tabular}{|c|c|}
\hline $\mathcal{P}(X)$ & Power set of the set $X$ \\
\hline$\sigma$ & Permutation \\
\hline$\Pi(X)$ & Set of permutations of $X$ \\
\hline$\Pi_{n}$ & Set of permutations of $\{1, \ldots, n\}$ \\
\hline$d$ & Distance function \\
\hline$R$ & Relation (either binary or ternary) \\
\hline$m, M, \mathbb{M}$ & Monometric \\
\hline$w, W, \mathbb{W}$ & Betweenness relation \\
\hline$[x, y, z]$ & $y$ is in between $x$ and $z$ \\
\hline$\left\{x_{1}, \ldots, x_{n}\right\}$ & Set of objects \\
\hline$\left(x_{1}, \ldots, x_{n}\right)$ & List of objects \\
\hline$\left\{\left\{x_{1}, \ldots, x_{n}\right\}\right\}$ & Multi-set of objects \\
\hline$\delta$ & Zero-one distance function \\
\hline $\mathbb{1}_{X}, \mathbb{1}(X)$ & Indicator function of $X$ \\
\hline $\mathscr{Y}$ & Anonymised profile \\
\hline $\mathcal{M}_{r}(\mathcal{L}(\mathscr{C}))$ & Set of all anonymised profiles of $r$ rankings on $\mathscr{C}$ \\
\hline$\sigma(\mathscr{Y})$ & Anonymised profile obtained by permuting the order of the voters \\
\hline$\Theta_{r}^{M}\left(\mathscr{Y}_{1}, \mathscr{Y}_{2}, \mathscr{Y}_{3}\right)$ & Set of all optimal permutation triplets \\
\hline$C$ & Matrix of costs \\
\hline$C_{i j}$ & Element at the $i$-th row and $j$-th column of the matrix $C$ \\
\hline$S$ & Scorix \\
\hline $\mathcal{S}_{r}(\mathscr{C})$ & Set of all scorices on $\mathscr{C}$ \\
\hline$S_{i}$ & Vector of positions of candidate $a_{i}$ \\
\hline$s\left(a_{i}, a_{j}\right)$ & Strength of support of candidate $a_{i}$ over candidate $a_{j}$ \\
\hline$V$ & Votrix \\
\hline $\mathcal{V}_{r}(\mathscr{C})$ & Set of all votrices on $\mathscr{C}$ \\
\hline $\mathcal{V}_{r}^{\mathcal{Q}}(\mathscr{C})$ & Set of all quasivotrices on $\mathscr{C}$ \\
\hline$W$ & Votex \\
\hline $\mathcal{W}_{r}(\mathscr{C})$ & Set of all votices on $\mathscr{C}$ \\
\hline $\mathcal{W}_{r}^{\mathcal{Q}}(\mathscr{C})$ & Set of all quasivotices on $\mathscr{C}$ \\
\hline $\mathcal{K}^{+}$ & Shorthand for $\{1, \ldots, k-1\}$ \\
\hline $\mathcal{K}^{-}$ & Shorthand for $\{1-k, \ldots,-1\}$ \\
\hline $\mathcal{K}$ & Shorthand for $\{1-k, \ldots,-1,1, \ldots, k-1\}$ \\
\hline$n_{i}\left(a_{i_{1}}, a_{i_{2}}\right)$ & Number of voters considering that $a_{i_{1}}$ is $i$ positions before $a_{i_{2}}$ \\
\hline$N_{i}\left(a_{i_{1}}, a_{i_{2}}\right)$ & Number of voters considering that $a_{i_{1}}$ is at most $i$ positions before $a_{i}$ \\
\hline$\unrhd_{1}$ & First-order dominance relation \\
\hline
\end{tabular}


$\unrhd_{2} \quad$ Second-order dominance relation

$\Subset \quad$ Relation 'is a sub-scorix of'

$\sqsupset \quad$ Strict order relation between couples of candidates

$\sqsupset^{+} \quad$ Upper part of the strict order relation between couples of candidates

$\unrhd \quad$ Dominance relation

$\unrhd \quad$ Order relation between rankings (with ties)

$\mathscr{R}^{*} \quad$ Profile of rankings with ties

$\mathcal{L}^{*}(\mathscr{C}) \quad$ Set of all rankings with ties on $\mathscr{C}$

$\mathcal{L}^{*}(\mathscr{C})^{r} \quad$ Set of all profiles of $r$ rankings with ties on $\mathscr{C}$

$\mathcal{P}_{k} \quad$ Set of all interval positions for a set of $k$ candidates

$\leq_{\mathcal{P}_{k}} \quad$ Order relation on $\mathcal{P}_{k}$

$S^{*} \quad$ Weak-scorix

$\mathcal{S}_{r}^{*}(\mathscr{C}) \quad$ Set of all weak-scorices on $\mathscr{C}$

$V^{*} \quad$ Weak-votrix

$\mathcal{V}_{r}^{*}(\mathscr{C}) \quad$ Set of all weak-votrices on $\mathscr{C}$

$\mathcal{B}_{k} \quad$ Set of all positional triplets for a set of $k$ candidates

$\leq_{\mathcal{B}_{k}} \quad$ Order relation on $\mathcal{B}_{k}$

$W^{*} \quad$ Weak-votex

$\mathcal{W}_{r}^{*}(\mathscr{C}) \quad$ Set of all weak-votices on $\mathscr{C}$

$\mathscr{S} \quad$ Signature

$\mathbb{S}(\mathscr{C}) \quad$ Set of all signatures on $\mathscr{C}$

$\mathscr{O} \quad$ Ordered signature

$\mathbb{O}(\mathscr{C}) \quad$ Set of all ordered signatures on $\mathscr{C}$

$\mathscr{S} \rightsquigarrow \mathscr{O}$ The signature $\mathscr{S}$ leads to the ordered signature $\mathscr{O}$

$\gg^{\mathbb{S}} \quad$ Covering relation on $\mathbb{S}(\mathscr{C})$

$\geq^{\mathbb{S}} \quad$ Order relation on $\mathbb{S}(\mathscr{C})$

$\geq 0 \quad$ Order relation on $\mathbb{O}(\mathscr{C})$

$\gtrsim_{B} \quad$ Relation 'is positionally as good as'

$>_{B} \quad$ Relation 'is positionally better than'

$\geq_{B} \quad$ Reflexive closure of the relation 'is positionally better than'

$\gtrsim_{C} \quad$ Relation 'is pairwisely as good as'

$>_{C} \quad$ Relation 'is pairwisely better than'

$\geq_{C} \quad$ Reflexive closure of the relation 'is pairwisely better than' 


\section{CHAPTER 1}

\section{Prologue}

\subsection{Preface}

A genuine leader is not a searcher for consensus but a molder of consensus.

Martin Luther King Jr.

From social to mathematical sciences, passing by computer, economical and biological sciences, collective decision making is a problem setting that has been addressed systematically by the research community, covering a varied spectrum of scientific disciplines. When a decision among several candidates concerns a large number of people, it is desirable to choose the option that best benefits the group as a whole. This well-being of the entire group is usually referred to as social welfare. As this social welfare is hardly measurable, a voting procedure is commonly invoked to determine the best option to be taken. This best option may either be a single candidate, a set of candidates or a ranking (with ties) of the different candidates. In this dissertation, we mainly focus on the search for the best option of the latter type.

The choice of this best ranking of the candidates is a difficult problem in itself, let aside the fact that the voting procedure may easily be manipulated by strategic votes of some dishonest voters. In general, there is no absolute truth and we can say that no method 
is truly best. In fact, as Arrow stated in his well-known impossibility theorem, among all methods based on the search for the best ranking of the candidates, only dictatorship satisfies the following two natural properties at the same time:

- If a candidate is preferred to another candidate by all the voters, then it should be preferred to this other candidate in the best ranking.

- The relative position of two candidates in the best ranking should not depend on the other candidates.

The consequences of Arrow's impossibility theorem are despairing. Even worse, it is known that all prominent methods may yield different winners given the same set of votes. One then wonders if the best ranking is just the truly best in case all the power of decision is given to an individual. Should we forget about democracy and surrender to an all powerful leader? No, we just need to accept that a truly best candidate might not exist, that the only truth is that we may need to compromise. The notion of consensus turns then out to be a crucial concept that leads to the study of conditions under which the existence of a truly best candidate can be assured. In this dissertation, we analyse different types of consensus (states) and, turning a deaf ear to Martin Luther King Jr. and his introductory quote to this chapter, we search for consensus in order to identify the best ranking of the candidates. All these notions of consensus are centred on the property of monotonicity, which is a common desired property in mathematical modelling exercises.

The property of monotonicity is an old acquaintance for scholars of social choice theory that can easily be traced back to the early 1970s. This concept of monotonicity is different from the one considered throughout this dissertation. Monotonicity, understood in this classical sense, means that a candidate remains being the winning candidate in case he/she is raised on some of the voters' rankings. Although the consequences of the absence of this property may seem paradoxical, several existing methods do not fulfill monotonicity. Indeed, social choice theory is a field full of paradoxes. Among all the existing paradoxes of voting, the one pointed out by Condorcet has managed to become known as 'the' voting paradox. This relevant paradox states that the transitivity of the voters' rankings does not imply the transitivity of the majority rule (a candidate is said to defeat another candidate by simple majority if the number of voters who prefer the first candidate to the second candidate is greater than the number of voters who prefer the second candidate to the first candidate). However, this majority rule disregards a considerable part of the information provided by 
the voters. The transitivity of the voters' rankings is surprisingly ignored. Monotonicity, now understood in the sense of this dissertation, will be again proved to be a key property for social choice, this time by helping to avoid the inconvenient voting paradox.

\subsection{Objectives}

Motivated by the numerous potential applications in many fields of interest such as ecosystem management, sustainability assessment and consumer preference analysis, this research studies many aspects concerning the theory of social choice. Mainly, this dissertation focuses on the following objectives:

- In real-life problems, where voters rarely unanimously agree, the analysis of conditions under which a consensus can be easily reached becomes of utmost importance. In particular, we delve into the role of the monotonicity property in the definition of natural consensus states, leading to an obvious consensus decision.

- Mainly due to the confrontation of several groups with opposing opinions or to the usual presence of noise in real-life data, these newly-introduced monotonicity-based consensus states may still not hold. The proposal of a methodology for facilitating the decision making based on the search for these monotonicity-based consensus states is the ultimate goal of this research.

\subsection{Outline of this dissertation}

The rest of this dissertation is divided in four clearly differentiated parts.

Part I consists of one chapter (Chapter 2) and is devoted to an exhaustive study of the most prominent ranking rules in the theory of social choice. A knowledgeable reader, aware of the current state of the art of the field, could skip this first part and go directly to Part II.

In Part II, which consists of seven chapters, we explain in detail all the theoretical development addressed during this $\mathrm{PhD}$ research. The first chapter (Chapter 3) of this part is devoted to the (mono)metric rationalisation of ranking rules, a branch of social choice 
theory that amounts to explaining ranking rules by the minimisation of the distance to a consensus state for some appropriate (mono)metric. In Chapter 4, we analyse four different representations of votes used in the field of social choice theory. The property of monotonicity of these representations of votes is studied in Chapter 5, leading to the introduction of natural consensus states for the (mono)metric rationalisation of ranking rules. The search for these consensus states is formulated as an optimization problem in Chapter 6. In Chapter 7, we discuss some properties of the ranking rules based on the search for a monotonicity-based consensus state and we prove that all proposed ranking rules are independent with respect to each other. The more general setting where voters express a ranking with ties instead of a ranking is analysed in Chapter 8 . In the last chapter (Chapter 9) of this part, we restrict our attention to the setting where the goal is to choose the best candidate rather than the best ranking.

In Part III, we show some examples of application of this research to real-life problems. We highlight three particular problems: an ecosystem management problem in the Lar rangeland in Iran; an environmental decision making problem in the Argentinian province of Corrientes; and a consumer preference analysis problem at the Laboratory of Food Microbiology and Food Preservation at Ghent University, Belgium. All is here applied.

Finally, we end this dissertation with the closing Part IV] where a discussion on some conclusions and open problems of this research is addressed. 


\section{PART I}

THE STATE OF THE ART 



\section{CHAPTER 2}

\section{Introduction to social choice theory}

In this chapter, we introduce the concepts of social choice theory that are necessary for a correct understanding of this dissertation, together with a brief review of the most prominent ranking rules. A reader aware of the current state of the art in the theory of social choice may direct to Section 2.3 for an explanation of the ranking rule that was the starting point of this dissertation.

\subsection{Social choice theory}

Social choice theory concerns the study of the conclusions that can be drawn from the preferences expressed by several voters over a set of candidates. Throughout this dissertation, we consider the problem of ranking candidates, meaning we are dealing with the social choice subdiscipline of ranking rules. The simplest and oldest ranking rule is the plurality rule [153], which can be traced back to times of the Ancient Greece. This ranking rule simply ranks candidates according to the number of voters who considered them as their preferred choice. Although it is a really simple and naive proposal, it is still the most frequently used ranking rule, for example, in national elections. Many ranking rules have been proposed since the eighteenth century, when the works of Rousseau [139], Borda [18] and Condorcet [35] laid the foundations of social choice theory. Already in the twentieth century, the contributions of Arrow [3] and Fishburn [57, 58, 59] were fundamental in the settling of social choice theory as a relevant research field. In the last fifty years, researchers have focused on the analysis of strategies and strategy-proof ranking rules [68, 178, 79, 146]. 
Several researchers, concerned about how, in some extreme cases, every ranking can be elected winner when considering an appropriate ranking rule, called attention to the fact that the choice of ranking rule is linked to a notion of consensus (or consensus state). In this direction, Nitzan [106], Lerer and Nitzan [88] and Campbell and Nitzan [27] characterized social choice rules (where, instead of a ranking, the winner is a subset of the set of candidates) by means of a consensus state and a distance function. This characterization is known as metric rationalisation of social choice rules. In general, a profile of rankings is said to be in a consensus state when determining a winning ranking is obvious. A trivial consensus state is that of unanimity [80, where each voter expresses the exact same ranking on the set of candidates. Another slightly more involved one is that of the existence of a Condorcet ranking [35]. Meskanen and Nurmi [99] demonstrated that most ranking rules can be characterized as minimizing the distance to a consensus state for some appropriate distance function. A well-known method of this type is the method of Kemeny [80, where the Kendall distance function [82] between rankings and the consensus state of unanimity are considered. Other relevant proposals are due to Bogart [16, 17] or Cook and Seiford [37, 38], where different distance functions are considered; or due to Meskanen and Nurmi [99, 109], Rademaker and De Baets [133] or Pérez-Fernández et al. [116, 121, 122, 124, 125], where different consensus states are considered.

There exist many ranking rules in social choice theory and, in some extreme cases, every ranking can be elected winner considering an appropriate ranking rule. Unfortunately, the choice of a best ranking rule is not an absolute truth as Arrow proved in his applauded work "A difficulty in the concept of social welfare" [3]. Arrow's impossibility theorem states that there is no ranking rule satisfying, at the same time, three properties that can be considered natural or desirable: non-dictatorship (there is no voter whose ranking always is elected the winning ranking), unanimity (if a candidate is preferred to another candidate by all the voters it should be ranked at a better position than this candidate in the winning ranking) and independence of irrelevant alternatives (the order of two candidates in the winning ranking does not depend on the rest of candidates). Arrow's impossibility theorem leads to the conclusion that the choice of an 'appropriate' ranking rule depends on the nature of the problem and that, unfortunately, a truly best choice does not always exist.

In social choice theory, two different settings are normally considered when choosing a winner. On the one hand, when the goal is to look for the winning candidate(s), one talks about a social choice rule. On the other hand, when the goal is to look for the winning ranking, one talks about a ranking rule. When the distinction between a social choice 
rule and a ranking rule does not matter, one talks about a voting rule. Note that a social choice rule can be seen as a special case of ranking rule where all candidates following the first-ranked one are not taken into account. Due to the nature of this dissertation, which is centered on ranking rules and not on social choice rules, we present here an overview of the most important ranking rules ${ }^{1}$ in social choice theory. A more exptensive review on voting rules can be found in [6, 14].

\subsection{Best-known ranking rules in social choice theory}

Throughout this dissertation, we denote by $\mathscr{C}=\left\{a_{1}, \ldots, a_{k}\right\}$ the considered set of $k$ candidates. Each of $r$ voters expresses his/her preferences on the set of candidates in the form of a strict linear order relation or ranking $\succ_{j}$ on $\mathscr{C}$, i.e., the asymmetric part of a total order relation $\succeq_{j}$ on $\mathscr{C}$. This list of $r$ rankings is called a profile of rankings and is denoted by $\mathscr{R}=\left(\succ_{j}\right)_{j=1}^{r}$. The set of all rankings on $\mathscr{C}$ is denoted by $\mathcal{L}(\mathscr{C})$. The position of a candidate $a \in \mathscr{C}$ in a ranking $\succ$ is called the position or rank of the candidate $a$ and is denoted by $P_{\succ}(a)$ (for short, $P_{\succ_{j}}(a)$ is directly denoted by $P_{j}(a)$ ).

\subsubsection{Plurality (first-past-the-post)}

The simplest and most ancient ranking rule in social choice theory is plurality [153], which is proved to be used at the latest in one of the city states of the Ancient Greece: Sparta. When taking into account this ranking rule, candidates are ranked according to the number of voters that considered this candidate to be their preferred option. Although it is a really simple and naive proposal, it is still the most frequently used ranking rule, for example, in national elections.

Plurality leads to a ranking rule where only the main choice of each voter is taken into account and voters are not asked to provide a ranking on the set of candidates. This is one of the reasons why, although it does not accurately represent the preferences of the voters, it is still the most used ranking rule in national elections.

\footnotetext{
${ }^{1}$ Some of these ranking rules provide a ranking with ties instead of a ranking on the set of candidates.
} 
Example 2.1 Let us consider a set of four candidates $\mathscr{C}=\{a, b, c, d\}$ and the profile $\mathscr{R}=$ $\left(\succ_{i}\right)_{i=1}^{14}$ of fourteen rankings given by the voters shown in Table 2.1.

\begin{tabular}{|c|c|}
\hline$\# \succ_{i}$ & Ranking on $\mathscr{C}$ \\
\hline 6 & $c \succ b \succ a \succ d$ \\
5 & $a \succ d \succ b \succ c$ \\
3 & $b \succ a \succ d \succ c$ \\
\hline
\end{tabular}

Table 2.1: Profile of rankings on $\mathscr{C}$ given by fourteen voters.

First-place votes are the only votes taken into account when considering the plurality ranking rule. Candidate $c$ is the first choice six times, candidate a is the first choice five times, candidate $b$ is the first choice three times and candidate $d$ is not the first choice for any of the voters. Therefore, considering the plurality ranking rule, the winning ranking is:

$$
c \succ a \succ b \succ d \text {. }
$$

Unfortunately, plurality is really sensitive to manipulation, as can be understood by reading the following extract of a paper by Tideman where he discusses how he manipulated a treasurer election [157]: "When I was 12 years old I was nominated to be treasurer of my class at school. A girl named Michelle was also nominated. I relished the prospect of being treasurer, so I made a quick calculation and nominated Michelle's best friend, Charlotte. In the ensuing election I received 13 votes, Michelle received 12, and Charlotte received 11 , so I became treasurer".

\subsubsection{Borda count}

In 1770, Jean-Charles de Borda [18] proposed to the French Academy of Sciences his ideas for reforming the election procedures in the academy itself [170]. He called attention to the fact that the plurality rule can easily elect a candidate that is supported by only a small minority of the electorate and suggested that each voter should provide his/her preferences in the form of a ranking on the set of candidates. In that way, each candidate is rewarded a point every time that another candidate is ranked at a worse position than him/her in the profile of rankings given by the voters. Candidates are then sorted based on the number of points obtained. 
Although his method only lasted about two decades as the academy official election procedure, the Borda count undoubtedly is one of the best-known ranking rules in social choice theory [96]. For more details concerning the history of the Borda count and preceding similar procedures, we refer to [50].

Example 2.2 Let us consider a set of four candidates $\mathscr{C}=\{a, b, c, d\}$ and the profile $\mathscr{R}=$ $\left(\succ_{i}\right)_{i=1}^{14}$ of fourteen rankings given by the voters shown in Table 2.1 .

Considering the Borda count, the respective score for each candidate is:

$$
\begin{aligned}
& B(a)=5 \cdot 3+3 \cdot 2+6 \cdot 1+0 \cdot 0=27, \\
& B(b)=3 \cdot 3+6 \cdot 2+5 \cdot 1+0 \cdot 0=26, \\
& B(c)=6 \cdot 3+0 \cdot 2+0 \cdot 1+8 \cdot 0=18, \\
& B(d)=0 \cdot 3+5 \cdot 2+3 \cdot 1+6 \cdot 0=13 .
\end{aligned}
$$

A winning ranking on the set of candidates is defined by sorting the candidates according to their respective score. Therefore, considering the Borda count, the winning ranking is:

$$
a \succ b \succ c \succ d
$$

The Borda count also is really sensitive to manipulation. In fact, it was already criticized in the eighteenth century by one of Borda's contemporaries: Marquis de Condorcet. When confronted with this criticism, Borda was merely moved to comment: "My scheme is only intended for honorable men" [140].

\subsubsection{Scoring ranking rules}

Scoring ranking rules ${ }^{2}$ [32, 57, 67, 102, 104, 169] constitute a large family of ranking rules based on positional information, with as most prominent examples plurality [153], the Borda count [18, veto [7, 141] (also known as anti-plurality) and best-worst voting systems [66]. A scoring ranking rule assigns a score to each of the $k$ candidates based on the positions at which the candidate is ranked by each voter. Each scoring ranking rule

\footnotetext{
${ }^{2}$ In case we are considering social choice rules instead of ranking rules, scoring ranking rules are just referred to as 'scoring rules'.
} 
has an associated vector of scores ${ }^{3}\left(\alpha_{\ell}\right)_{\ell=1}^{k}$, where $\alpha_{\ell} \geq \alpha_{\ell+1} \geq 0$ for any $\ell \in\{1, \ldots, k-1\}$ and $\alpha_{1}>\alpha_{k}$. For instance, the vector of scores corresponding to plurality is $\alpha_{1}=1$ and, for any $\ell \in\{2, \ldots, k\}, \alpha_{\ell}=0$; the vector of scores corresponding to the Borda count is $\alpha_{\ell}=k-\ell$, for any $\ell \in\{1, \ldots, k\}$; the vector of scores corresponding to veto is $\alpha_{k}=0$ and, for any $\ell \in\{1, \ldots, k-1\}, \alpha_{\ell}=1$; the vector of scores corresponding to best-worst voting systems is $\alpha_{1}=1, \alpha_{k}=0$ and, for any $\left.\ell \in\{2, \ldots, k-1\}, \alpha_{\ell}=\alpha \in\right] 0,1[$.

Every candidate is assigned a score of $\alpha_{\ell}$ every time that he/she is ranked at the $\ell$-th position in one of the rankings in the profile. The total score $s\left(a_{i}\right)$ of each candidate $a_{i} \in \mathscr{C}$, usually obtained as the sum of the received scores, can be used to define a weak order relation ${ }^{4}$ on the set of candidates by sorting these total scores in decreasing order. The asymmetric part of a weak order relation is referred to as a ranking with ties. As a scoring ranking rule may define a ranking with ties, the notion of linear extension (also known as refinement) of a ranking with ties must be introduced. A ranking $\succ$ linearly extends (is a linear extension of) a ranking with ties $\succ^{\prime}$ if $\succ^{\prime} \subseteq \succ$.

A relevant subfamily of scoring ranking rules, known as $t$-approva $5^{5}$ scoring ranking rules [93], contains all scoring ranking rules where the first $t \in\{1, \ldots, k-1\}$ scores $\alpha_{1}, \ldots, \alpha_{t}$ are equal to one and the last $k-t$ scores $\alpha_{t+1}, \ldots, \alpha_{k}$ are equal to zero. $t$-approval scoring ranking rules allow voters to divide the set of candidates in two subsets: the $t$ candidates that they approve and the $k-t$ candidates that they refuse. Note that plurality and veto belong to the family of $t$-approval scoring ranking rules, but the Borda count does not belong to this family.

Another relevant subfamily of scoring ranking rules, known as convex scoring ranking rules [154], contains all scoring ranking rules such that $\alpha_{\ell}-\alpha_{\ell+1} \geq \alpha_{\ell+1}-\alpha_{\ell+2}$ for any $\ell \in\{1, \ldots, k-2\}$. Convex scoring ranking rules reward first place votes over second place votes more than second place votes over third place votes; second place votes over third place votes more than third place votes over fourth place votes; etc. Note that plurality and the Borda count are convex scoring ranking rules, but veto (and any $t$-approval scoring ranking rule different from plurality) is not a convex scoring ranking rule.

\footnotetext{
${ }^{3}$ This vector of scores is unique up to a positive multiplicative constant.

${ }^{4} \mathrm{~A}$ weak order relation is a complete and transitive relation.

${ }^{5}$ Not to be confused with approval voting [20] where voters are able to freely choose the number of candidates that they approve.
} 
Example 2.3 Let us consider a set of four candidates $\mathscr{C}=\{a, b, c, d\}$ and the profile $\mathscr{R}=$ $\left(\succ_{i}\right)_{i=1}^{14}$ of fourteen rankings (with ties) given by the voters shown in Table 2.2.

\begin{tabular}{|c|c|}
\hline$\# \succ_{i}$ & Ranking on $\mathscr{C}$ \\
\hline 6 & $a \sim b \sim c \succ d$ \\
5 & $a \sim b \sim d \succ c$ \\
3 & $a \sim c \sim d \succ b$ \\
\hline
\end{tabular}

Table 2.2: Profile of rankings (with ties) on $\mathscr{C}$ given by fourteen voters.

Note that, in the given profile of rankings (with ties), six voters have vetoed (ranked last) candidate d, five voters have vetoed candidate $c$ and three voters have vetoed candidate $b$. Considering the veto ranking rule ((k-1)-approval), the respective score for each candidate is:

$$
\begin{aligned}
& s(a)=14-0=14, \\
& s(b)=14-3=11, \\
& s(c)=14-5=9, \\
& s(d)=14-6=8 .
\end{aligned}
$$

A winning ranking on the set of candidates is defined by sorting the candidates according to their respective score. Therefore, considering the veto ranking rule, the winning ranking is:

$$
a \succ b \succ c \succ d
$$

\subsubsection{Majority}

The simple majority rule [54, 76, 95, 149] is one of the most widespread and studied concepts in social choice theory. A candidate is said to defeat another candidate by simple majority if the number of voters who prefer the first candidate to the second candidate is greater than the number of voters who prefer the second candidate to the first candidate. The simple majority rule has been proved [95] to be the unique solid and meaningful voting rule when we are restricted to a set of two candidates.

\footnotetext{
${ }^{6}$ May [95] proved that majority is the only social choice rule that is decisive (it leads to a unique choice for an odd number of votes), egalitarian (it is anonymous), neutral (it does not favour any alternative)
} 
A closely related concept is that of the absolute majority, where a candidate is said to defeat another candidate by absolute majority if the number of voters who prefer the first candidate to the second candidate is greater than half of the number of voters. As in the setting of this dissertation each voter provides his/her preferences in the form of a ranking on the set of candidates, both simple and absolute majority coincide.

The strongest type of majority is that of the unanimous majority. A candidate is said to defeat another candidate by unanimous majority if every voter prefers the first candidate to the second candidate. Nevertheless, unanimous majority obviously is too strong a condition that almost never holds in an election.

Another type of majority that lies in between the notions of absolute majority and unanimous majority are qualified majorities [51]. Qualified majorities require the number of voters who prefer the first candidate to the second candidate to be greater than or equal to a certain quota $\alpha r$ (where $r$ equals the number of voters and $\alpha \in[0.5,1]$ ), fixed before the election. Note that $\alpha=0.5$ corresponds to weak absolute majority and $\alpha=1$ corresponds to unanimous majority. In the eighteenth century, Rousseau [139] already encouraged the use of qualified majorities for important decisions: "The more the deliberations are important and serious, the more the opinion that carries should approach unanimity."

In the same way that qualified majorities lie in between absolute majority and unanimous majority, there exists another type of majorities (majorities based on differences of votes) that lie in between simple majority and unanimous majority. Qualified majorities and majorities based on differences of votes become equivalent in the setting of this dissertation, where each voter provides his/her preferences in the form of a ranking on the set of candidates. For more details on all these types of majorities, we refer to [112].

Unfortunately, all these majority rules (except unanimous majority) might lead to the well-known voting paradox [35] (also known as Condorcet's paradox), where a candidate defeats a second candidate, this second candidate defeats a third different candidate, which, at the same time, defeats the first candidate. Furthermore, the given relation on the set of candidates might not be complete. Therefore, majority rules are not considered ranking rules.

and positively responsive (if a candidate is the winner of the election, then it should still be the winner if a single voter changes its preference in a way favourable to this candidate) for a set of two candidates. 
Example 2.4 Let us consider a set of four candidates $\mathscr{C}=\{a, b, c, d\}$ and the profile $\mathscr{R}=$ $\left(\succ_{i}\right)_{i=1}^{14}$ of fourteen rankings given by the voters shown in Table 2.1. Candidate $b$ is preferred to candidates $a, c$ and $d$ by more than half of the number of voters. At the same time, candidate $a$ is preferred to candidates $c$ and $d$ by more than half of the number of voters and candidate $d$ is preferred to candidate $c$ by more than half of the number of voters. Therefore, considering the simple majority rule, the winning ranking is:

$$
b \succ a \succ d \succ c .
$$

Unfortunately, the simple majority rule does not always lead to a ranking on the set of candidates. Consider that, instead of the profile of rankings in Table 2.1, the profile of rankings listed in Table 2.3 is provided.

\begin{tabular}{|c|c|}
\hline$\# \succ_{i}$ & Ranking on $\mathscr{C}$ \\
\hline 6 & $c \succ b \succ a \succ d$ \\
5 & $a \succ d \succ c \succ b$ \\
3 & $b \succ a \succ d \succ c$ \\
\hline
\end{tabular}

Table 2.3: Profile of rankings on $\mathscr{C}$ given by fourteen voters.

Candidate a is preferred to candidate $c$ by more than half of the number of voters, candidate $c$ is preferred to candidate $b$ by more than half of the number of voters but, at the same time, candidate $b$ is preferred to candidate a by more than half of the number of voters. This inconvenience is referred to as the voting paradox. Therefore, the simple majority rule defines no ranking on the set of candidate for the profile of rankings given in Table 2.3.

For improving the performance of the simple majority rule, several authors have encouraged the use of degrees of preferences in social choice theory [64, 123, 131]; quoting a well-known phrase by Sen [150], “... the method of majority decision takes no account of intensities of preference, and it is certainly arguable that what matters is not merely the number who prefer $x$ to $y$ and the number who prefer $y$ to $x$, but also by how much each prefers one alternative to the other". 


\subsubsection{Condorcet}

In 1785, Marie Jean Antoine Nicolas Caritat, mostly known as Marquis de Condorcet, followed the direction started by Rousseau in his remarkable work 'Du Contrat Social' [139] where he discusses about the 'general will': "When a law is proposed in the people's assembly, what is asked of them is not precisely whether they approve of the proposition or reject it, but whether it is in conformity with the general will [...]". In that way, Condorcet [35] stated that it is not a compromise ranking what is searched, but an unknown truth that remains hidden due to the fact that "voters sometimes make mistakes in their judgements". In order to identify this unknown truth, Condorcet proposed a probabilistic model [170] for finding the ranking that is the "most likely to be best".

In this same direction, Arrow [4] stated a couple of centuries later the following: "[...] each individual has two orderings, one which governs him in his everyday actions, and one which would be relevant under some ideal conditions and which is in some sense truer than the first ordering. It is the latter which is considered relevant to social choice, and it is assumed that there is complete unanimity with regard to the truer individual ordering".

Nevertheless, Condorcet's most important contribution to social choice theory is the notion nowadays known as the Condorcet winner. He stated that if a candidate is preferred by more than half of the voters to all the other candidates, then it is presumptively the best and must be elected the winning candidate 7 . This candidate is commonly known as the Condorcet winner. Analogously, a candidate such that every other candidate is preferred to him/her by more than half of the voters is known as the Condorcet loser. Another term related to Condorcet is that of the Condorcet ranking, which is a ranking such that every candidate is preferred by more than half of the voters to all the candidates ranked at a worse position than him/her.

One may note that a Condorcet winner/loser/ranking might not exist, but the uniqueness (in case of existence) is always assured. When both a Condorcet winner (resp. loser) and a Condorcet ranking exist, the first (resp. last) candidate of the Condorcet ranking and the Condorcet winner (resp. loser) coincide. Any ranking (resp. social choice) rule

\footnotetext{
${ }^{7}$ Some authors consider that a Condorcet winner is one that satisfies that, the support of this candidate over any other candidate is greater than the support of this other candidate over him/her. Note that both definitions are equivalent in the setting considered in this dissertation where each voter expresses his/her preferences in the form of a ranking on the set of candidates.
} 
always electing a Condorcet ranking (resp. winner), if it exists, as the winning ranking (resp. candidate) is called a Condorcet ranking rule (resp. Condorcet method). A wellknown Condorcet method is the social choice rule introduced by Black [14], where, if there is a Condorcet winner, then he/she should be chosen the winner and, otherwise, the Borda winner should be considered.

A weaker definition of the Condorcet winner/loser/ranking can be found in the literature when instead of 'being preferred by more than half of the voters' one requires 'being preferred by at least half of the voters'. In such a case, one talks about a weak Condorcet winner/loser/ranking. The Condorcet winner/loser/ranking always is a weak Condorcet winner/loser/ranking, but the converse is only true when the number of voters is odd. Both the existence and the uniqueness of a weak Condorcet winner/loser/ranking are not assured.

The fact that a Condorcet winner/ranking might not exist has led to the introduction of many different ranking rules where the winning candidate/ranking is the one that is the closest to being a Condorcet winner/ranking. Several ways of measuring such closeness have been proposed. Here, we restrict our attention to the two most prominent ones: Dodgson's method [46] and Condorcet's least-reversal method [99]. The former one addresses the search for the closest Condorcet winner by means of the Kendall distance function between rankings [82]. The latter one addresses the search for the closest Condorcet winner by counting the minimum number of reversals in the voting matrix. For more details, we refer to 99 .

Example 2.5 Let us consider a set of four candidates $\mathscr{C}=\{a, b, c, d\}$ and the profile $\mathscr{R}=$ $\left(\succ_{i}\right)_{i=1}^{14}$ of fourteen rankings given by the voters shown in Table 2.1.

Every profile of rankings is represented by a voting matrix (from now on referred to as the votrix $\left.\right|^{8} V$. This votrix is formed by the pairwise comparisons between each couple of candidates. In that way, $V_{i j}$ denotes the number of voters that prefer the $i$-th candidate to the $j$-th candidate (by convention, all diagonal elements are equal to zero).

$$
V=\left(\begin{array}{cccc}
0 & 5 & 8 & 14 \\
9 & 0 & 8 & 9 \\
6 & 6 & 0 & 6 \\
0 & 5 & 8 & 0
\end{array}\right)
$$

\footnotetext{
${ }^{8}$ Later on in this dissertation, we will provide a more formal definition of the votrix.
} 
Candidate $b$ is preferred to all the other candidates by more than half of the voters: nine voters prefer candidate $b$ to candidate $a$, eight voters prefer candidate $b$ to candidate $c$ and nine voters prefer candidate $b$ to candidate $d$. Therefore, candidate $b$ is the Condorcet winner. In addition, candidate a is preferred to candidate $c$ by eight voters and to candidate $d$ by fourteen voters and candidate $d$ is preferred to candidate $c$ by eight voters. We conclude that candidate $c$ is the Condorcet loser and that the Condorcet ranking is:

$$
b \succ a \succ d \succ c .
$$

However, the existence of the Condorcet ranking is not assured for every profile of rankings.

In the following, we will consider the profile of rankings given in Table 2.4 in order to illustrate the method of Dodgson [46].

\begin{tabular}{|c|c|}
\hline$\# \succ_{i}$ & Ranking on $\mathscr{C}$ \\
\hline 6 & $a \succ b \succ c \succ d$ \\
5 & $b \succ c \succ a \succ d$ \\
3 & $c \succ d \succ a \succ b$ \\
\hline
\end{tabular}

Table 2.4: Profile of rankings on $\mathscr{C}$ given by fourteen voters.

The votrix $V$ corresponding to this profile of rankings is:

$$
V=\left(\begin{array}{cccc}
0 & 9 & 6 & 11 \\
5 & 0 & 11 & 11 \\
8 & 3 & 0 & 14 \\
3 & 3 & 0 & 0
\end{array}\right)
$$

There is no Condorcet winner (and therefore no Condorcet ranking) because candidate a is preferred to candidate $b$ by more than half of the voters (nine), candidate $b$ is preferred to candidate $c$ by more than half of the voters (eleven) and candidate $c$ is preferred to candidate a by more than half of the voters (eight).

Note that we can reverse the preference of two voters w.r.t. candidates a and $c$ and obtain the profile of rankings in Table 2.5. 


\begin{tabular}{|c|c|}
\hline$\# \succ_{i}$ & Ranking on $\mathscr{C}$ \\
\hline 6 & $a \succ b \succ c \succ d$ \\
3 & $b \succ c \succ a \succ d$ \\
3 & $c \succ d \succ a \succ b$ \\
2 & $b \succ a \succ c \succ d$ \\
\hline
\end{tabular}

Table 2.5: Profile of rankings on $\mathscr{C}$ given by fourteen voters.

The votrix $V^{\prime}$ corresponding to this profile of rankings is:

$$
V^{\prime}=\left(\begin{array}{cccc}
0 & 9 & 8 & 11 \\
5 & 0 & 11 & 11 \\
6 & 3 & 0 & 14 \\
3 & 3 & 0 & 0
\end{array}\right)
$$

Now, the Condorcet winner, $a$, and the Condorcet ranking, $a \succ b \succ c \succ d$, exist. In Table 2.6, the number of reversals needed to turn each possible ranking on $\mathscr{C}$ into a Condorcet ranking is shown.

\begin{tabular}{|c|c||c|c||c|c||c|c|}
\hline Ranking & Rev. & Ranking & Rev. & Ranking & Rev. & Ranking & Rev. \\
\hline$a \succ b \succ c \succ d$ & $\mathbf{2}$ & $b \succ a \succ c \succ d$ & 5 & $c \succ a \succ b \succ d$ & 5 & $d \succ a \succ b \succ c$ & 20 \\
$a \succ b \succ d \succ c$ & 10 & $b \succ a \succ d \succ c$ & 13 & $c \succ a \succ d \succ b$ & 10 & $d \succ a \succ c \succ b$ & 25 \\
$a \succ c \succ b \succ d$ & 7 & $b \succ c \succ a \succ d$ & 3 & $c \succ b \succ a \succ d$ & 8 & $d \succ b \succ a \succ c$ & 23 \\
$a \succ c \succ d \succ b$ & 12 & $b \succ c \succ d \succ a$ & 8 & $c \succ b \succ d \succ a$ & 13 & $d \succ b \succ c \succ a$ & 21 \\
$a \succ d \succ b \succ c$ & 15 & $b \succ d \succ a \succ c$ & 18 & $c \succ d \succ a \succ b$ & 15 & $d \succ c \succ a \succ b$ & 23 \\
$a \succ d \succ c \succ b$ & 20 & $b \succ d \succ c \succ a$ & 16 & $c \succ d \succ b \succ a$ & 18 & $d \succ c \succ b \succ a$ & 26 \\
\hline
\end{tabular}

Table 2.6: Number of reversals needed to make each ranking the Condorcet ranking.

Therefore, considering the method of Dodgson, the winning candidate is a. Extending the method of Dodgson to the search for the Condorcet ranking we obtain the following winning ranking:

$$
a \succ b \succ c \succ d .
$$

The work of Condorcet [35] undoubtedly is one of the most important contributions to social choice theory. Condorcet's proposal has suffered criticism among the research community, especially due to the fact that it is easy to find profiles of rankings for which 
the Condorcet winner is a more than questionable winner [120]. As an example of such received criticism, we refer to a brief reflection by Saari [142]: "the combination of the pairwise vote with the Condorcet terms loses the crucial fact that voters have transitive preferences. [...] An equally surprising assertion is that rather than being the standard, the Condorcet winner must be held suspect".

\subsubsection{Kemeny (Kemeny-Young)}

The method of Kemeny [80] is a ranking rule where the winning ranking is the one that minimizes the sum of the Kendall distances [82 to the given profile of rankings. The Kendall distance function between two rankings $\succ_{1}$ and $\succ_{2}$ is defined as:

$$
K\left(\succ_{1}, \succ_{2}\right)=\#\left\{(a, b) \in \mathscr{C}^{2} \mid a \neq b \wedge a \succ_{1} b \wedge b \succ_{2} a\right\}
$$

Intuitively, the winning ranking according to the method of Kemeny is the one that can become unanimous in the profile of rankings with the least number of reversals.

Under the assumption that: (i) each voter will choose the best candidate with some fixed probability $p$, where $0.5<p<1$ and $p$ is the same for all voters; (ii) every voter's judgement on every pair of candidates is independent on every other pair; (iii) each voter's judgement is independent of the other voters' judgements; Young [171] proved that the ranking that has the maximum likelihood coincides with the winning ranking according to the method of Kemeny. Due to this result, the method of Kemeny is also known as the maximum likelihood method.

A common criticism against the method of Kemeny concerns the fact that it is NP-hard to compute its winning ranking [9]. Some researchers have focused on the search for approximate methods that can be executed in polynomial time. Nevertheless, quoting Conitzer et al. [36], "an approximation algorithm for a voting rule is, in effect, a different voting rule; and in real-world elections, voters may feel deceived if a different voting rule is used than the one that was promised to them".

Example 2.6 Let us consider a set of four candidates $\mathscr{C}=\{a, b, c, d\}$ and the profile $\mathscr{R}=$ $\left(\succ_{i}\right)_{i=1}^{14}$ of fourteen rankings given by the voters shown in Table 2.4.

The Kendall distance between two rankings is the minimum number of reversals we need to do in one of them in order to obtain the other one. For instance, $K(b \succ c \succ a \succ d, a \succ$ 
$b \succ c \succ d)=2$, as we can reverse $c \succ a$ to $a \succ c$ in the ranking $b \succ c \succ a \succ d$ and we will obtain $b \succ a \succ c \succ d$. Then we can reverse $b \succ a$ to $a \succ b$ and we will obtain the ranking $a \succ b \succ c \succ d$. The Kendall distances of all the rankings in the profile to the ranking $a \succ b \succ c \succ d$ are the following:

$$
\begin{aligned}
& K(a \succ b \succ c \succ d, a \succ b \succ c \succ d)=0, \\
& K(b \succ c \succ a \succ d, a \succ b \succ c \succ d)=2, \\
& K(c \succ d \succ a \succ b, a \succ b \succ c \succ d)=4 .
\end{aligned}
$$

Therefore, the Kendall distance between the profile of rankings in Table 2.4 and the profile of rankings where all voters have expressed the ranking $a \succ b \succ c \succ d$ equals

$$
\sum_{j=1}^{14} K\left(\succ_{j}, a \succ b \succ c \succ d\right)=6 \cdot 0+5 \cdot 2+3 \cdot 4=22 .
$$

In Table 2.7, the number of reversals needed to make each ranking the unanimous ranking (measured my the Kendall distance function) is shown.

\begin{tabular}{|c|c||c|c||c|c||c|c|}
\hline Ranking & Rev. & Ranking & Rev. & Ranking & Rev. & Ranking & Rev. \\
\hline$a \succ b \succ c \succ d$ & $\mathbf{2 2}$ & $b \succ a \succ c \succ d$ & 26 & $c \succ a \succ b \succ d$ & 28 & $d \succ a \succ b \succ c$ & 52 \\
$a \succ b \succ d \succ c$ & 36 & $b \succ a \succ d \succ c$ & 40 & $c \succ a \succ d \succ b$ & 36 & $d \succ a \succ c \succ b$ & 60 \\
$a \succ c \succ b \succ d$ & 30 & $b \succ c \succ a \succ d$ & 24 & $c \succ b \succ a \succ d$ & 32 & $d \succ b \succ a \succ c$ & 56 \\
$a \succ c \succ d \succ b$ & 38 & $b \succ c \succ d \succ a$ & 32 & $c \succ b \succ d \succ a$ & 40 & $d \succ b \succ c \succ a$ & 54 \\
$a \succ d \succ b \succ c$ & 44 & $b \succ d \succ a \succ c$ & 48 & $c \succ d \succ a \succ b$ & 44 & $d \succ c \succ a \succ b$ & 58 \\
$a \succ d \succ c \succ b$ & 52 & $b \succ d \succ c \succ a$ & 46 & $c \succ d \succ b \succ a$ & 48 & $d \succ c \succ b \succ a$ & 62 \\
\hline
\end{tabular}

Table 2.7: Number of reversals needed to make each ranking the unanimous ranking.

Therefore, considering the method of Kemeny, the winning ranking, which minimizes the costs in Table 2.7, is:

$$
a \succ b \succ c \succ d
$$

As previously discussed, Condorcet [35] proposed a probabilistic approach [170] for finding the ranking that is the "most likely to be best". Actually, Condorcet formalized the probabilistic approach for the case where the set of candidates only consists of three candidates 
and what he meant to do for a set of candidates of bigger cardinality is unclear. Quoting Nanson [14, 105, 170]: "the general rules for the case of any number of candidates as given by Condorcet are stated so briefly as to be hardly intelligible [...] and as no examples are given it is quite hopeless to find out what Condorcet meant".

Young declares in [170] that the method of Kemeny is what Condorcet 35] really had in mind: "Condorcet's overall intention is quite clear. It is to find the most probable combination of opinions. In describing his method he seems to have used the term 'successively deletes' rather loosely as a heuristic for computing the solution. (He may also have believed, mistakenly, that successive deletion always does give the most probable combination.) But there appears to be little doubt about his objective. [...] It seems reasonably likely that this is what Condorcet meant to say. In any case, it is the only interpretation that is consistent with his goal of finding the ranking that is most likely to be correct".

\subsubsection{Litvak}

The method of Litvak [108] follows similar principles as the method of Kemeny [80]. Instead of selecting the ranking that minimizes the sum of the Kendall distances [82] to the given profile of rankings, the method of Litvak selects the ranking that minimizes the Litvak sum. Given the profile $\mathscr{R}=\left(\succ_{i}\right)_{i=1}^{r}$, the Litvak sum of a ranking $\succ$ is defined as:

$$
\sum_{i=1}^{k} s\left(a_{i}\right)=\sum_{i=1}^{k} \sum_{j=1}^{r}\left|P_{\succ}\left(a_{i}\right)-P_{j}\left(a_{i}\right)\right| .
$$

The winning ranking according to the method of Litvak is sometimes referred to as the Litvak median.

Example 2.7 Let us consider a set of four candidates $\mathscr{C}=\{a, b, c, d\}$ and the profile $\mathscr{R}=$ $\left(\succ_{i}\right)_{i=1}^{14}$ of fourteen rankings given by the voters shown in Table 2.4.

For the ranking $a \succ b \succ c \succ d$, we see that candidate $a$ is ranked at the first position, which leads to a coincidence with the ranking $a \succ b \succ c \succ d$ and to a difference of two positions with respect to the rankings $b \succ c \succ a \succ d$ and $c \succ d \succ a \succ b$. This leads to a score for candidate $a$ of

$$
s(a)=\sum_{j=1}^{r}\left|P_{\succ}(a)-P_{j}(a)\right|=6 \times 0+5 \times 2+3 \times 2=16 .
$$


Analogously, the scores for the other candidates are given by:

$$
\begin{aligned}
& s(b)=\sum_{j=1}^{r}\left|P_{\succ}(b)-P_{j}(b)\right|=6 \times 0+5 \times 1+3 \times 2=11, \\
& s(c)=\sum_{j=1}^{r}\left|P_{\succ}(c)-P_{j}(c)\right|=6 \times 0+5 \times 1+3 \times 2=11, \\
& s(d)=\sum_{j=1}^{r}\left|P_{\succ}(d)-P_{j}(d)\right|=6 \times 0+5 \times 0+3 \times 2=6 .
\end{aligned}
$$

Therefore, the Litvak sum of the ranking $a \succ b \succ c \succ d$ equals 44. In Table 2.8, the Litvak sum of each the rankings is shown.

\begin{tabular}{|c|c||c|c||c|c||c|c|}
\hline Ranking & Rev. & Ranking & Rev. & Ranking & Rev. & Ranking & Rev. \\
\hline$a \succ b \succ c \succ d$ & 44 & $b \succ a \succ c \succ d$ & 46 & $c \succ a \succ b \succ d$ & 56 & $d \succ a \succ b \succ c$ & 94 \\
$a \succ b \succ d \succ c$ & 66 & $b \succ a \succ d \succ c$ & 68 & $c \succ a \succ d \succ b$ & 72 & $d \succ a \succ c \succ b$ & 88 \\
$a \succ c \succ b \succ d$ & 50 & $b \succ c \succ a \succ d$ & $\mathbf{4 2}$ & $c \succ b \succ a \succ d$ & 46 & $d \succ b \succ a \succ c$ & 84 \\
$a \succ c \succ d \succ b$ & 66 & $b \succ c \succ d \succ a$ & 64 & $c \succ b \succ d \succ a$ & 68 & $d \succ b \succ c \succ a$ & 84 \\
$a \succ d \succ b \succ c$ & 82 & $b \succ d \succ a \succ c$ & 74 & $c \succ d \succ a \succ b$ & 78 & $d \succ c \succ a \succ b$ & 84 \\
$a \succ d \succ c \succ b$ & 76 & $b \succ d \succ c \succ a$ & 74 & $c \succ d \succ b \succ a$ & 84 & $d \succ c \succ b \succ a$ & 90 \\
\hline
\end{tabular}

Table 2.8: Litvak sum of each ranking.

Therefore, considering the method of Litvak, the winning ranking, which leads to a Litvak sum of 42 , is:

$$
b \succ c \succ a \succ d
$$

\subsubsection{Bucklin}

The term Bucklin voting refers to the process of considering the comparisons in the profile of rankings that are above some threshold and then adjusting that threshold down until a majority is reached. In particular, Bucklin [23] introduced a ranking rule that consists of looking for a majority of more than half of the voters supporting some candidate starting from the first-place choices. If there is no candidate with a majority of first-place choices, then second-place choices are included and the search for a majority is addressed considering both first-place and second-place choices. The procedure continues until the smallest 
$n \in \mathbb{N}$ is reached such that one or more candidates are supported by more than half of the voters taking the $n$-first-place choices into account 5 . All candidates obtaining a majority considering the $n$-first-place choices are then ordered according to the number of times that they are considered in the $n$-first-place choices by a voter ${ }^{10}$. In case of tie, Bucklin specified that first-place choices are used, but no further tie-breaking rule is explicitly mentioned in [23].

Example 2.8 Let us consider a set of four candidates $\mathscr{C}=\{a, b, c, d\}$ and the profile $\mathscr{R}=$ $\left(\succ_{i}\right)_{i=1}^{14}$ of fourteen rankings given by the voters shown in Table 2.1 .

First-place choices are firstly taken into account when considering the method of Bucklin. Candidate $c$ appears six times, candidate a appears five times, candidate $b$ appears three times and candidate $d$ does not appear any time. As there is no candidate with at least seven appearances as a first-place choice, the consideration of second-place choices needs to be addressed.

Considering both first-place and second-place choices, candidate b appears nine times, candidate a appears eight times, candidate $c$ appears six times and candidate d appears five times. Now, both candidate $a$ and candidate $b$ reach more than half of the first and secondplace choices. Then, as candidate b appears more times as a first or second-place choice than candidate $a$, candidate $b$ is elected the Bucklin winner.

In order to obtain a winning ranking on the set of candidates, we continue with the procedure considering also third-place votes. Note that candidate d appears eight times as a first-place, second-place or third-place choice, so it reaches a majority. However, candidate c appears only six times and it will not reach a majority without additionally considering the fourthplace choices. Therefore, considering the method of Bucklin, the winning ranking is:

$$
b \succ a \succ d \succ c .
$$

\footnotetext{
${ }^{9}$ Although he mentions the variation of omitting "the scheme of dropping the lowest candidate", Bucklin [23] actually states that the candidate with the lowest score should be dropped after each step.

${ }^{10}$ Bucklin actually proposed his method as a social choice rule and how to order the candidates that do not reach a majority in the $n$-th step is not explicitly addressed. We propose to continue with his procedure until every candidate reaches a majority.
} 


\subsubsection{Copeland}

Copeland [40] is a Condorcet ranking rule measuring the number of victories, ties and defeats of each candidate with respect to the other candidates. There are many equivalent definitions of this ranking rule considering different score assessments. In this dissertation, we are going to consider the following score assessment:

(i) 1 point per victory.

(ii) $\frac{1}{2}$ point per tie.

(iii) 0 points per defeat.

The candidate with the highest score is considered the winner and the winning ranking is given by decreasingly sorting these scores.

Example 2.9 Let us consider a set of four candidates $\mathscr{C}=\{a, b, c, d\}$ and the profile $\mathscr{R}=$ $\left(\succ_{i}\right)_{i=1}^{14}$ of fourteen rankings given by the voters shown in Table 2.9.

\begin{tabular}{|c|l|}
\hline$\# \succ_{i}$ & Ranking on $\mathscr{C}$ \\
\hline 6 & $a \succ b \succ c \succ d$ \\
4 & $b \succ c \succ a \succ d$ \\
3 & $c \succ d \succ a \succ b$ \\
1 & $a \succ c \succ d \succ b$ \\
\hline
\end{tabular}

Table 2.9: Profile of rankings on $\mathscr{C}$ given by fourteen voters.

When considering the method of Copeland, we need to calculate the pairwise confrontation between each two candidates. Candidate a beats candidate $b$ (10 voters out of 14 prefer candidate a to candidate b) and candidate $d(11 / 14)$ and ties with candidate $c$ (7/14), candidate $b$ beats candidate $c(10 / 14)$ and candidate $d(10 / 14)$ and, finally, candidate $d$ 
beats candidate $c(14 / 14)$. The scores corresponding to each candidate are then given by:

$$
\begin{aligned}
& s(a)=2 \cdot 1+1 \cdot \frac{1}{2}+0 \cdot 0=\frac{5}{2}, \\
& s(b)=2 \cdot 1+0 \cdot \frac{1}{2}+1 \cdot 0=2, \\
& s(c)=0 \cdot 1+1 \cdot \frac{1}{2}+2 \cdot 0=\frac{1}{2}, \\
& s(d)=1 \cdot 1+0 \cdot \frac{1}{2}+2 \cdot 0=1 .
\end{aligned}
$$

Therefore, considering the method of Copeland, the winning ranking is:

$$
a \succ b \succ d \succ c .
$$

\subsubsection{Simpson (Simpson-Kramer, Minimax, Maximin)}

The method of Simpson [85, 151] is a Condorcet method ${ }^{11}$ that ranks candidates according to their maximum defeat. In that way, the best ranked candidates are the ones that are not clearly defeated by any other candidate. A relevant (and unintuitive) property of this method is that, although it is a Condorcet method (if there is a Condorcet winner it is elected winner), a Condorcet loser might be elected winner.

Example 2.10 Let us consider a set of four candidates $\mathscr{C}=\{a, b, c, d\}$ and the profile $\mathscr{R}=$ $\left(\succ_{i}\right)_{i=1}^{14}$ of fourteen rankings given by the voters shown in Table 2.10.

\begin{tabular}{|c|l|}
\hline$\# \succ_{i}$ & Ranking on $\mathscr{C}$ \\
\hline 4 & $a \succ d \succ b \succ c$ \\
4 & $c \succ a \succ b \succ d$ \\
4 & $b \succ c \succ d \succ a$ \\
1 & $d \succ a \succ b \succ c$ \\
1 & $d \succ c \succ a \succ b$ \\
\hline
\end{tabular}

Table 2.10: Profile of rankings on $\mathscr{C}$ given by fourteen voters.

\footnotetext{
${ }^{11}$ The method of Simpson is not a Condorcet ranking rule.
} 
When considering the method of Simpson, we need to obtain the biggest defeat of each candidate. Firstly, we obtain the votrix $V$ corresponding to the given profile:

$$
V=\left(\begin{array}{cccc}
0 & 10 & 5 & 8 \\
4 & 0 & 9 & 8 \\
9 & 5 & 0 & 8 \\
6 & 6 & 6 & 0
\end{array}\right)
$$

The biggest defeat of candidate $a$ is against candidate $c$ (five against nine), the biggest defeat of candidate $b$ is against candidate a (four against ten), the biggest defeat of candidate $c$ is against candidate $b$ (five against nine) and the biggest defeat of candidate $d$ is against either candidate $a$, candidate $b$ or candidate $c$ (six against eight). Note that candidate $d$ is a Condorcet loser, but it is the winner considering the method of Simpson due to the fact that all his/her defeats are by a small margin. Therefore, considering the method of Simpson, the winning ranking is:

$$
d \succ a \sim c \succ b
$$

\subsubsection{Tideman (ranked pairs)}

The method of Tideman [157] is a Condorcet ranking rule that consists of two steps. Firstly, all couples of candidates are sorted from stronger to weaker. Secondly, these couples of candidates are locked by order (ignoring those that contradict stronger victories) until an order on the set of candidates is decided. Although in social choice theory normally there are a huge number of voters and the probability of a tie is low, a tie breaking rule needs to be defined when two or more couples of candidates have the same strength. The most adopted tie breaking rule [172] consists of randomly ${ }^{12}$ selecting a voter and use his/her personal ranking to break the tie: ${ }^{13}$.

Example 2.11 Let us consider a set of four candidates $\mathscr{C}=\{a, b, c, d\}$ and the profile $\mathscr{R}=$ $\left(\succ_{i}\right)_{i=1}^{14}$ of fourteen rankings given by the voters shown in Table 2.10.

\footnotetext{
${ }^{12}$ This selection is usually made by means of a discrete uniform distribution on the set of voters.

${ }^{13}$ The order on the set of couples of candidates induced by a ranking on the set of candidates is given by firstly ordering by the best ranked candidate of the couple, secondly ordering by the worst ranked candidate of the couple and, finally, the two couples of candidates involving the same two candidates are ranked according to the candidate that appears the first in the couple.
} 
When considering the method of Tideman, we need to rank all the couples of candidates. $A$ random ranking ${ }^{14} b \succ c \succ d \succ a$ given by a voter was selected in order to act as a tie-breaking rule. We firstly recall that the votrix $V$ corresponding to this profile is the following:

$$
V=\left(\begin{array}{cccc}
0 & 10 & 5 & 8 \\
4 & 0 & 9 & 8 \\
9 & 5 & 0 & 8 \\
6 & 6 & 6 & 0
\end{array}\right) .
$$

The couples of candidates are then sorted in the following way:

1. $a \succ b(10)$,

2. $b \succ c(9), c \succ a(9)$,

4. $a \succ d(8), b \succ d(8), c \succ d(8)$,

7. $d \succ a(6), d \succ b(6), d \succ c(6)$,

10. $c \succ b(5), a \succ c(5)$,

12. $b \succ a(4)$.

We should apply the tie-breaking rule in each group. For instance, between $b \succ c$ and $c \succ a$, as the randomly selected ballot was $b \succ c \succ d \succ a$, the couple $b \succ c$ should be ranked first as it involves the first candidate of $b \succ c \succ d \succ a$. In that way, the resulting order on the preferences is:
1. $a \succ b(10)$,
7. $d \succ b(6)$,
2. $b \succ c(9)$,
8. $d \succ c(6)$,
3. $c \succ a(9)$,
9. $d \succ a(6)$,
4. $b \succ d(8)$,
10. $c \succ b(5)$,
5. $c \succ d(8)$,
11. $a \succ c(5)$,
6. $a \succ d(8)$,
12. $b \succ a(4)$.

\footnotetext{
${ }^{14}$ This random ranking should be changed after each comparison but, in order to ease the illustration of the method, we will work with this first ranking all the time.
} 
We will proceed to construct the winning ranking locking these preferences and ignoring the contradictory ones until a ranking is established. Firstly, we lock $a \succ b$. Secondly, $b \succ c$ is locked providing $a \succ b \succ c$. Thirdly, $c \succ a$ is ignored as it contradicts the current order $a \succ b \succ c$. Fourthly, $b \succ d$ is introduced so the winning ranking will either be $a \succ b \succ c \succ d$ or $a \succ b \succ d \succ c$. Finally, $c \succ d$ is locked and the winning ranking, considering the method of Tideman, is:

$$
a \succ b \succ c \succ d
$$

The method of Tideman, sometimes referred to as the ranked pairs rule, has been proved to be one of the few ranking rules that is independent of clones. Two candidates are said to be clones if they are ranked at consecutive positions by every voter. Intuitively, a ranking rule is said to be independent of clones if adding one (or more) candidate to the election that is a clone of an existing candidate does not affect the outcome of the election.

The study of the notion of clone is not exclusive of the field of social choice theory. For instance, De Baets et al. [42] analysed the clone relation of a (partial) order relation and Bouremel et al. [19] extended this notion to any binary relation.

\subsubsection{Schulze}

The method of Schulze was developed in 1997 and it was first discussed in public mailing lists. Due to the increasing success of the method, which has been used by several private organizations, such as the Wikimedia Foundation, The Pirate Party of Sweden and the Pirate Party of Germany, Schulze decided to publish his method in a well-known academic journal in 2011 [147].

The method of Schulze is based on the notion of a beatpath. A path from a candidate $a_{i_{1}}$ to a candidate $a_{i_{2}}$, denoted by $a_{i_{1}} \rightarrow a_{i_{2}}$, is a list of couples $\left(\left(a^{i}, a^{i+1}\right)\right)_{i=1}^{n}$ such that $a^{1}=a_{i_{1}}, a^{n+1}=a_{i_{2}}$ and $\left(a^{1}, \ldots, a^{n+1}\right)$ are pairwisely different. The weakest link of a path $\left(\left(a^{i}, a^{i+1}\right)\right)_{i=1}^{n}$ from $a_{i_{1}}$ to $a_{i_{2}}$ is the couple $\left(a^{i}, a^{i+1}\right)$ such that the number of voters preferring $a^{i}$ to $a^{i+1}$ (called the strength of the weakest link) is the minimum among all the couples in $\left(\left(a^{i}, a^{i+1}\right)\right)_{i=1}^{n}$. A path from $a_{i_{1}}$ to $a_{i_{2}}$ that maximizes the strength of the weakest link is called a beatpath from $a_{i_{1}}$ to $a_{i_{2}}$. 
Finally, the strength of all the beatpaths are gathered in a beatpath matrix $B$, where the element $B_{i j}$ at the $i$-th row and $j$-th column equals the strength of the beatpath from the $i$-th candidate to the $j$-th candidate. The relation defined by $a_{i} R a_{j}$ if $B_{i j} \geq B_{j i}$ or $i=j$ turns out to be a weak order relation on the set of candidates.

Example 2.12 Let us consider a set of four candidates $\mathscr{C}=\{a, b, c, d\}$ and the profile $\mathscr{R}=$ $\left(\succ_{i}\right)_{i=1}^{14}$ of fourteen rankings given by the voters shown in Table 2.1.

In a set of four candidates, there are five different paths from a candidate $a_{i_{1}}$ to a candidate $a_{i_{2}}$. For instance, for going from candidate a to candidate $b$, there are the following five possible paths:

$$
\begin{array}{r}
((a, b)) \text { leading to } \min (5)=5, \\
((a, c),(c, b)) \text { leading to } \min (8,6)=6, \\
((a, d),(d, b)) \text { leading to } \min (14,5)=5, \\
((a, c),(c, d),(d, b)) \text { leading to } \min (8,6,5)=5, \\
((a, d),(d, c),(c, b)) \text { leading to } \min (14,8,6)=6 .
\end{array}
$$

The paths maximizing the strength of the weakest link, leading to a value of six, are $((a, c),(c, b))$ and $((a, d),(d, c),(c, b))$. Therefore, the element of the beatpath matrix corresponding to $(a, b)$ equals six. The beatpath matrix is the following:

$$
B=\left(\begin{array}{cccc}
- & 6 & 8 & 14 \\
9 & - & 8 & 9 \\
6 & 6 & - & 6 \\
6 & 6 & 8 & -
\end{array}\right)
$$

Therefore, considering the method of Schulze, the winning ranking is:

$$
b \succ a \succ d \succ c .
$$

\subsubsection{WVM and EWVM}

The Weighted Voting Method (WVM) [74] is a natural voting procedure that considers the proportion of victories/defeats of each candidate for each couple of candidates and it assigns to each candidate a score based on the aggregation of all the proportions of victories 
and ranks the candidates according to this score. It must be remarked that this method comes from the field of group decision making [33].

A natural extension of this Weighted Voting Method, known as the Extended Weighted Voting Method (EWVM) [113, 114, 115], introduces a parameter $\alpha$ allowing to model the importance of 'beating other candidates' or 'not being defeated by other candidates'.

We consider the matrix $P$ of proportions of victories/defeats where $P_{i j}$ is the proportion of times that candidate $a_{i}$ is preferred to candidate $a_{j}$, for any $i \neq j$, and $P_{i i}=0.5$. The victories and the defeats are separated in two matrices $P^{+}$and $P^{-}$:

$$
\begin{aligned}
& P_{i j}^{+}=\max \left\{0, p_{i j}-0.5\right\}, \\
& P_{i j}^{-}=\min \left\{0, p_{i j}-0.5\right\} .
\end{aligned}
$$

The weighted matrix $P^{\alpha}$ is constructed as

$$
P^{\alpha}=\alpha \cdot P^{+}+(1-\alpha) \cdot P^{-} .
$$

Therefore, for each candidate $a_{i} \in \mathscr{C}$, we calculate the score:

$$
s\left(a_{i}\right)=A\left(P_{i 1}^{\alpha}, \ldots, P_{i k}^{\alpha}\right),
$$

where $A$ is an aggregation function [11, 71], usually the arithmetic mean. Finally, candidates are ranked according to this score.

Note that the EWVM coincides with the WVM in case $\alpha=\frac{1}{2}$. Different combinations of aggregation functions and different values of the parameter $\alpha$ may lead to well-known ranking rules. For instance, when the aggregation function is the arithmetic mean and the considered value of the parameter $\alpha$ is $\frac{1}{2}$, the EWVM coincides with the Borda count. On the other hand, when the aggregation function is the minimum and the considered value of the parameter $\alpha$ is 0 , the EWVM coincides with the method of Simpson.

Example 2.13 Let us consider a set of four candidates $\mathscr{C}=\{a, b, c, d\}$ and the profile $\mathscr{R}=$ $\left(\succ_{i}\right)_{i=1}^{14}$ of fourteen rankings given by the voters shown in Table 2.11 .

The votrix $V$ corresponding to this profile of rankings is the following:

$$
V=\left(\begin{array}{cccc}
0 & 7 & 8 & 8 \\
7 & 0 & 5 & 11 \\
6 & 9 & 0 & 9 \\
6 & 3 & 5 & 0
\end{array}\right)
$$




\begin{tabular}{|c|l|}
\hline$\# \succ_{i}$ & Ranking on $\mathscr{C}$ \\
\hline 5 & $a \succ c \succ b \succ d$ \\
3 & $b \succ d \succ a \succ c$ \\
2 & $c \succ a \succ b \succ d$ \\
2 & $d \succ b \succ c \succ a$ \\
1 & $c \succ b \succ a \succ d$ \\
1 & $c \succ d \succ b \succ a$ \\
\hline
\end{tabular}

Table 2.11: Profile of rankings on $\mathscr{C}$ given by fourteen voters.

The matrix $P$ of proportions of victories/defeats:

$$
P=\left(\begin{array}{cccc}
\frac{1}{2} & \frac{1}{2} & \frac{4}{7} & \frac{4}{7} \\
\frac{1}{2} & \frac{1}{2} & \frac{5}{14} & \frac{11}{14} \\
\frac{3}{7} & \frac{9}{14} & \frac{1}{2} & \frac{9}{14} \\
\frac{3}{7} & \frac{3}{14} & \frac{5}{14} & \frac{1}{2}
\end{array}\right)
$$

When considering the Weighted Voting Method, the score for each candidate (considering the arithmetic mean) is:

$$
\begin{aligned}
& s(a)=\frac{1}{4}\left(\frac{1}{2}+\frac{1}{2}+\frac{4}{7}+\frac{4}{7}\right)=\frac{30}{48}, \\
& s(b)=\frac{1}{4}\left(\frac{1}{2}+\frac{1}{2}+\frac{5}{14}+\frac{11}{14}\right)=\frac{30}{48}, \\
& s(c)=\frac{1}{4}\left(\frac{3}{7}+\frac{9}{14}+\frac{1}{2}+\frac{9}{14}\right)=\frac{31}{48}, \\
& s(d)=\frac{1}{4}\left(\frac{3}{7}+\frac{3}{14}+\frac{5}{14}+\frac{1}{2}\right)=\frac{21}{48} .
\end{aligned}
$$

Therefore, considering the Weighted Voting Method, the winning ranking is

$$
c \succ a \sim b \succ d .
$$

If we consider the Extended Weighted Voting Method, then we need to define the matrices $P^{+}$and $P^{-}$:

$$
P^{+}=\left(\begin{array}{cccc}
0 & 0 & \frac{1}{14} & \frac{1}{14} \\
0 & 0 & 0 & \frac{2}{7} \\
0 & \frac{1}{7} & 0 & \frac{1}{7} \\
0 & 0 & 0 & 0
\end{array}\right), \quad P^{-}=\left(\begin{array}{cccc}
0 & 0 & 0 & 0 \\
0 & 0 & \frac{-1}{7} & 0 \\
\frac{-1}{14} & 0 & 0 & 0 \\
\frac{-1}{14} & \frac{-2}{7} & \frac{-1}{7} & 0
\end{array}\right)
$$


Therefore, for any $\alpha \in[0,1], P^{\alpha}$ is given by:

$$
P^{\alpha}=\left(\begin{array}{cccc}
0 & 0 & \frac{\alpha}{14} & \frac{\alpha}{14} \\
0 & 0 & \frac{\alpha-1}{7} & \frac{2 \alpha}{7} \\
\frac{\alpha-1}{14} & \frac{\alpha}{7} & 0 & \frac{\alpha}{7} \\
\frac{\alpha-1}{14} & \frac{2 \alpha-2}{7} & \frac{\alpha-1}{7} & 0
\end{array}\right) .
$$

When considering the Weighted Voting Method, the score for each candidate (considering the arithmetic mean) is:

$$
\begin{aligned}
& s(a)=\frac{1}{4}\left(0+0+\frac{\alpha}{14}+\frac{\alpha}{14}\right)=\frac{2 \alpha}{48}, \\
& s(b)=\frac{1}{4}\left(0+0+\frac{\alpha-1}{7}+\frac{2 \alpha}{7}\right)=\frac{6 \alpha-2}{48}, \\
& s(c)=\frac{1}{4}\left(\frac{\alpha-1}{14}+\frac{\alpha}{7}+0+\frac{\alpha}{7}\right)=\frac{5 \alpha-1}{48}, \\
& s(d)=\frac{1}{4}\left(\frac{\alpha-1}{14}+\frac{2 \alpha-2}{7}+\frac{\alpha-1}{7}+0\right)=\frac{7 \alpha-7}{48} .
\end{aligned}
$$

Therefore, considering the Extended Weighted Voting Method, the winning ranking is

$$
\begin{cases}a \succ c \succ b \succ d & , \text { if } \alpha \in\left[0, \frac{1}{3}[,\right. \\ a \sim c \succ b \succ d & , \text { if } \alpha=\frac{1}{3}, \\ c \succ a \succ b \succ d & , \text { if } \alpha \in] \frac{1}{3}, \frac{1}{2}[, \\ c \succ a \sim b \succ d & , \text { if } \alpha=\frac{1}{2}, \\ c \succ b \succ a \succ d & , \text { if } \alpha \in] \frac{1}{2}, 1[, \\ c \sim b \succ a \succ d & , \text { if } \alpha=1 .\end{cases}
$$

Note that, as expected, the winning ranking according to the WVM coincides with the winning ranking according to the EWVM when $\alpha=\frac{1}{2}$.

\subsubsection{Elimination methods}

Although an elimination method is not a ranking rule by itself, it can be combined with any ranking rule to define a new ranking rule. Elimination methods [5, 59, 137, 152] consist of a multi-stage voting procedure based on iteratively eliminating the worst candidates until the winning candidate is obtained. An important family of elimination methods contains the 
ones with only two steps where the two best candidates (according to the chosen ranking rule) are finally compared and the one that is preferred by the highest number of voters is elected the winning candidate. Here, we are interested in elimination methods based on a scoring ranking rule [60]. Relevant elimination methods based on a scoring ranking rule are plurality with run-off [137], the method of Nanson [105] or the method of Coombs [39].

Example 2.14 Let us consider a set of four candidates $\mathscr{C}=\{a, b, c, d\}$ and the profile $\mathscr{R}=$ $\left(\succ_{i}\right)_{i=1}^{14}$ of fourteen rankings given by the voters shown in Table 2.12. In the following, we will analyse how different elimination methods may change the winning ranking considering the same ranking rule. For instance, we will consider plurality combined with different elimination procedures.

\begin{tabular}{|c|l|}
\hline$\# \succ_{i}$ & Ranking on $\mathscr{C}$ \\
\hline 5 & $a \succ c \succ b \succ d$ \\
4 & $b \succ c \succ a \succ d$ \\
3 & $c \succ d \succ b \succ a$ \\
2 & $d \succ c \succ b \succ a$ \\
\hline
\end{tabular}

Table 2.12: Profile of rankings on $\mathscr{C}$ given by fourteen voters.

When considering the plurality ranking rule, first-place votes are the only votes taken into account. Only considering these votes, candidate a is the main choice five times, candidate $b$ is the main choice four times, candidate $c$ is the main choice three times and candidate $d$ is the main choice two times. Therefore, considering the plurality ranking rule, the winning ranking is:

$$
a \succ b \succ c \succ d .
$$

Nevertheless, if we consider a three-step elimination method, then we will firstly eliminate candidate $d$ as it is the one which is the main choice for the least number of voters. Only taking candidates $a, b$ and $c$ into account, candidate $a$ is the main choice five times, candidate $b$ is the main choice four times and candidate $c$ now is the main choice five times (the original three and the two cases where candidate $d$ was the main choice). Therefore, candidate $b$ is eliminated and candidates $a$ and $c$ are the only two candidates taken into account in the last step. Finally, candidate $a$ is the main choice five times and candidate $c$ is the main choice nine times. Therefore, considering a three-step elimination procedure, the winning ranking is:

$$
c \succ a \succ b \succ d .
$$


Finally, if we consider plurality with run-off, then we will eliminate candidates $c$ and $d$ as they are the ones that are the main choice for the least number of voters. Only taking into account candidates $a$ and $b$, candidate $a$ is the main choice five times and candidate $b$ is the main choice nine times. Therefore, considering plurality with run-off, the winning ranking is:

$$
b \succ a \succ c \succ d
$$

Note that the same ranking rule combined with three different elimination methods leads to three totally different winning rankings.

\subsection{A ranking rule based on monotonicity}

Monotonicity is a common desired property in mathematical modelling exercises, and its importance has been acknowledged in several disciplines, e.g. in machine learning [12, 28, 90] and fuzzy modelling [128, 155, 161]. However, real-life data is often imperfect and does not fully comply with the monotonicity hypothesis. One option then is to (minimally) adjust the data set restoring the monotonicity [132, 134, 135]. This is particularly important as, for instance, in machine learning, some algorithms cannot be trained with non-monotone datasets [134].

Returning to the field of social choice theory, several ranking rules centered on this monotonicity property have already been proposed. Rademaker and De Baets [133] advocated that, for a ranking $a \succ b \succ c$, monotonicity implies that the number of voters preferring $a$ to $c$ should not be less than both the number of voters preferring $a$ to $b$ and the number of voters preferring $b$ to 15 .

More formally, each ranking $\succ$ on the set of candidates defines a strict order relation $\sqsupset \succ$ between couples of candidates $\left(a_{i_{1}}, a_{j_{1}}\right),\left(a_{i_{2}}, a_{j_{2}}\right) \in \mathscr{C}^{2}$ :

$$
\left(a_{i_{1}} \succeq a_{j_{1}}\right) \wedge\left(a_{j_{2}} \succeq a_{i_{2}}\right) \wedge\left(a_{i_{1}} \succ a_{j_{1}} \vee a_{j_{2}} \succ a_{i_{2}}\right)
$$

This relation is represented in Figure 2.1 for an example of a set of four candidates.

According to the proposal of Rademaker and De Baets, if the strength of support (number of times that the first candidate of the couple is preferred to the second candidate of

\footnotetext{
${ }^{15}$ This intuitive notion is closely related to the property of strong stochastic transitivity [138].
} 


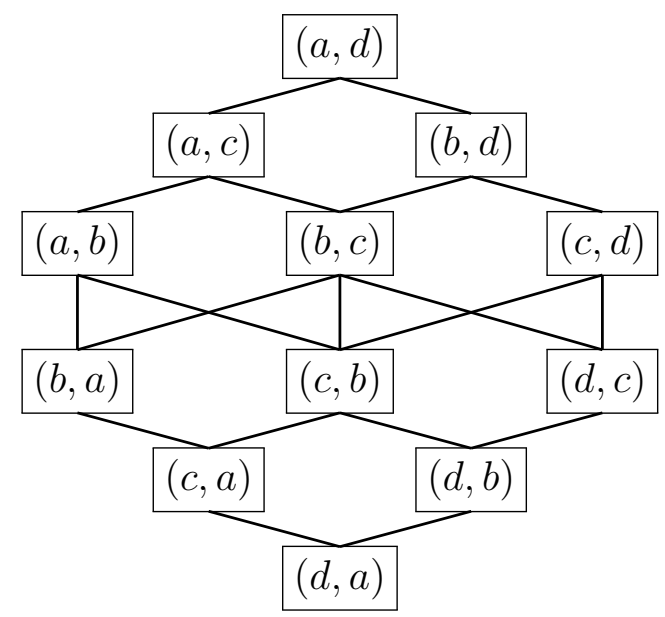

Figure 2.1: Hasse diagram of the order relation $\sqsupset \succ$ for the ranking $a \succ b \succ$ $c \succ d$.

the couple) is monotone decreasing on the Hasse diagram of the order relation $\sqsupset_{\succ}$ for a ranking $\succ$, then $\succ$ should be elected the winning ranking. If there is no such ranking, then the costs of imposing monotonicity w.r.t. each possible ranking are obtained. These costs are measured in the same way that they are measured in Condorcet's least reversals method [99], by counting the minimum number of reversals of pairwise comparisons.

Example 2.15 Let us consider a set of four candidates $\mathscr{C}=\{a, b, c, d\}$ and the profile $\mathscr{R}=$ $\left(\succ_{i}\right)_{i=1}^{14}$ of fourteen rankings given by the voters shown in Table 2.1. This profile of rankings is not monotone w.r.t. any ranking on $\mathscr{C}$. However, we can see in Figure 2.2 that, with a small number of reversals (three), we can impose monotonicity w.r.t. the ranking $a \succ b \succ$ $c \succ d$.

Of course, the number of changes needed in order to impose monotonicity totally depends on the chosen ranking. For instance, the cost of imposing monotonicity w.r.t. the ranking $d \succ c \succ b \succ a$ (fourteen) is much larger than the cost of imposing monotonicity w.r.t. to the ranking $a \succ b \succ c \succ d$ (three). The number of reversals needed in order to impose monotonicity w.r.t. the ranking $d \succ c \succ b \succ a$ can be seen in Figure 2.3.

In Table 2.13, the number of changes needed to impose monotonicity w.r.t. each possible ranking is shown. 


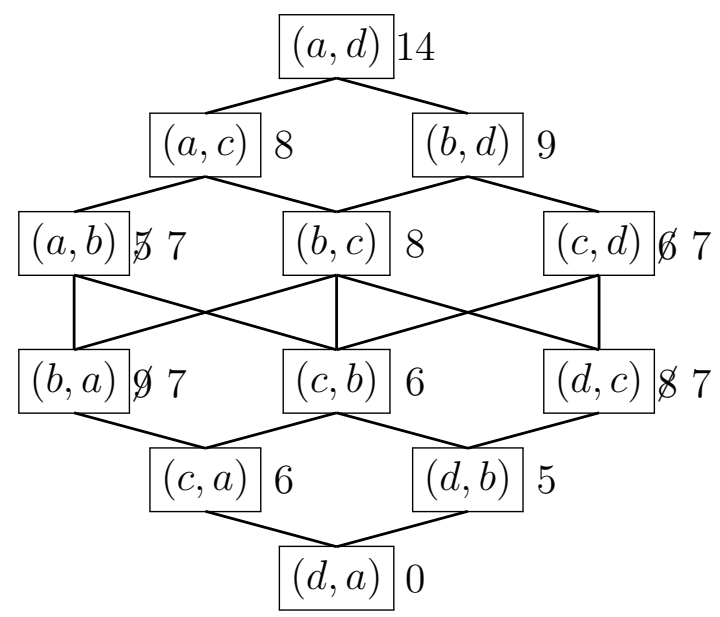

Figure 2.2: Minimum number of reversals needed for imposing monotonicity w.r.t. the ranking $a \succ b \succ c \succ d$.

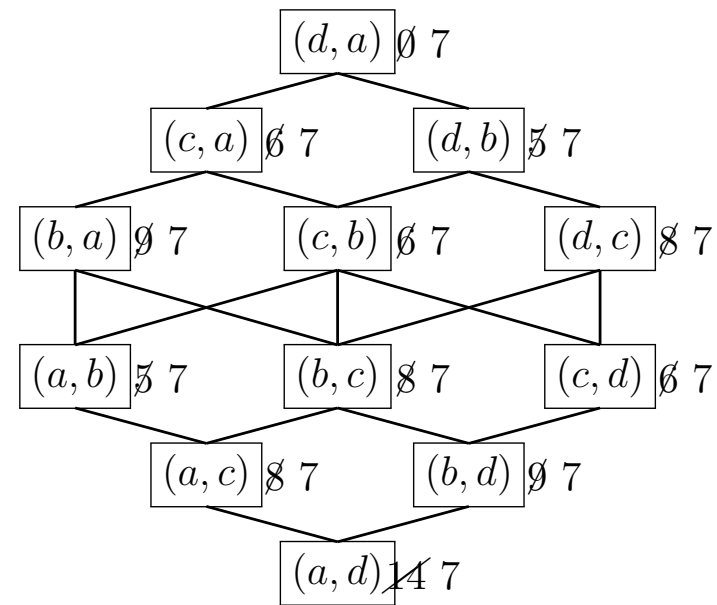

Figure 2.3: Minimum number of reversals needed for imposing monotonicity w.r.t. the ranking $d \succ c \succ b \succ a$.

Therefore, considering the ranking rule proposed by Rademaker and De Baets, the winning ranking is:

$$
a \succ b \succ c \succ d
$$

This simple and intuitive ranking rule was the starting point of this research. The natural property of monotonicity turns out to be a cornerstone of social choice theory, and it is only in case this property is not fulfilled that the need of making a decision arises. Therefore, 


\begin{tabular}{|c|c||c|c||c|c||c|c|}
\hline Ranking & Rev. & Ranking & Rev. & Ranking & Rev. & Ranking & Rev. \\
\hline$a \succ b \succ c \succ d$ & $\mathbf{3}$ & $b \succ a \succ c \succ d$ & 7 & $c \succ a \succ b \succ d$ & 12 & $d \succ a \succ b \succ c$ & 11 \\
$a \succ b \succ d \succ c$ & 9 & $b \succ a \succ d \succ c$ & 7 & $c \succ a \succ d \succ b$ & 14 & $d \succ a \succ c \succ b$ & 14 \\
$a \succ c \succ b \succ d$ & 7 & $b \succ c \succ a \succ d$ & 9 & $c \succ b \succ a \succ d$ & 12 & $d \succ b \succ a \succ c$ & 11 \\
$a \succ c \succ d \succ b$ & 13 & $b \succ c \succ d \succ a$ & 9 & $c \succ b \succ d \succ a$ & 14 & $d \succ b \succ c \succ a$ & 12 \\
$a \succ d \succ b \succ c$ & 11 & $b \succ d \succ a \succ c$ & 8 & $c \succ d \succ a \succ b$ & 14 & $d \succ c \succ a \succ b$ & 14 \\
$a \succ d \succ c \succ b$ & 13 & $b \succ d \succ c \succ a$ & 10 & $c \succ d \succ b \succ a$ & 14 & $d \succ c \succ b \succ a$ & 14 \\
\hline
\end{tabular}

Table 2.13: Cost imposing monotonicity w.r.t. each ranking on $\mathscr{C}$.

we advocate for exploiting the search for this monotonicity property for the introduction of new ranking rules. 


\section{PART II}

THE METHODOLOGY 



\section{CHAPTER 3}

\section{Rationalisation of ranking rules}

\subsection{Metric rationalisation of ranking rules}

One of the most well-known methods for the aggregation of rankings is that of Kemeny [80]. According to Kemeny, we select as the winning ranking the one that minimizes the sum of the Kendall distances to the profile of rankings given by the voters. Note that this ranking rule can be seen as the search for the 'closest' unanimous profile of rankings in the sense of minimizing the sum of the Kendall distances, i.e., considering the following distance function between profiles of rankings:

$$
d\left(\mathscr{R}^{1}, \mathscr{R}^{2}\right)=\sum_{i=1}^{r} K\left(\succ_{i}^{1}, \succ_{i}^{2}\right) .
$$

However, requiring a profile of rankings to be unanimous is a very restrictive property. Therefore, in social choice theory, another less restrictive property is commonly accepted. According to Condorcet [35], if it exists, the Condorcet ranking should be the winning ranking.

In the same way that a profile of rankings does not need to be unanimous, a Condorcet ranking might not exist either. The method of Dodgson [46] and Condorcet's least reversals method [109] look for the minimum number of reversals that need to be made in order to find a Condorcet ranking. 
Rademaker and De Baets [133] proposed a ranking rule based on the search for monotonicity in the strength of support. According to their proposal, for a ranking $a \succ b \succ c$, monotonicity implies that the strength with which $a \succ c$ is supported (number of rankings where $a$ is ranked before $c$ ) should not be less than both the strength with which $a \succ b$ and the strength with which $b \succ c$ are supported. They advocated that the winning ranking should be the one that is the 'closest' to satisfying this assumption. This 'closeness' was measured in terms of the minimum number of reversals.

There is a common pattern between these methods. In each of these ranking rules, a condition is imposed on the profile of rankings in order to decide which ranking is the winner. As they allow to undoubtedly decide the winning ranking, these important conditions are called consensus states. A consensus state satisfies the following three properties: anonymity (reassigning the rankings over the voters does not affect the belonging to the consensus state), neutrality (if some permutation of candidates is applied to each voter's ranking, the same permutation should be observed in the consensus state) and unanimity (if every voter provides the same ranking, then it belongs to the consensus state).

In the following definition, we consider the following notations. For any set $X, \mathcal{P}(X)$ denotes the power set of $A$. For any permutation $\sigma$ of $\{1, \ldots, r\}, \sigma(\mathscr{R})$ represents the profile $\left(\succ_{j}\right)_{j=\sigma(1)}^{\sigma(r)}$ obtained by permuting the order of the voters. For any permutation $\sigma$ of $\{1, \ldots, k\}, \mathscr{R}_{\sigma}$ represents the profile $\left(\sigma\left(\succ_{j}\right)\right)_{j=1}^{r}$ obtained by permuting the order of the candidates.

Definition 3.1 Let $\mathscr{C}$ be a set of $k$ candidates and $r$ be the number of voters. A consensus state is a couple $(\mathscr{X}, \mathscr{F})$ where $\mathscr{X} \subseteq \mathcal{L}(\mathscr{C})^{r}$ is a set of profiles of r rankings and $\mathscr{F}: \mathscr{X} \rightarrow$ $\mathcal{P}(\mathcal{L}(\mathscr{C}))$ is a function satisfying the following three properties:

(i) Anonymity: for any $\mathscr{R} \in \mathcal{L}(\mathscr{C})^{r}$, any $\left(a_{i_{1}}, a_{i_{2}}\right) \in \mathscr{C}^{2}$ such that $a_{i_{1}} \neq a_{i_{2}}$ and any permutation $\sigma$ of $\{1, \ldots, r\}$, it holds that

$$
(\mathscr{R} \in \mathscr{X} \Leftrightarrow \sigma(\mathscr{R}) \in \mathscr{X}) \wedge(\mathscr{F}(\mathscr{R})=\mathscr{F}(\sigma(\mathscr{R})))
$$

(ii) Neutrality: for any $\mathscr{R} \in \mathcal{L}(\mathscr{C})^{r}$, any $\left(a_{i_{1}}, a_{i_{2}}\right) \in \mathscr{C}^{2}$ such that $a_{i_{1}} \neq a_{i_{2}}$ and any permutation $\sigma$ of $\{1, \ldots, k\}$, it holds that

$$
\left(\mathscr{R} \in \mathscr{X} \Leftrightarrow \mathscr{R}_{\sigma} \in \mathscr{X}\right) \wedge\left(\sigma(\mathscr{F}(\mathscr{R}))=\mathscr{F}\left(\mathscr{R}_{\sigma}\right)\right)
$$


(iii) Unanimity: for any $\succ \in \mathcal{L}(\mathscr{C})$, it holds that

$$
\left(\succ^{r} \in \mathscr{X}\right) \wedge\left(\mathscr{F}\left(\succ^{r}\right)=\succ\right)
$$

The two most common consensus states in the rationalisation of ranking rules are unanimity, which holds when every voter expresses the same ranking on the set of candidates, and presence of a (weak) Condorcet ranking, which holds when there exists a ranking such that every candidate is preferred by more than (resp. at least) half of the number voters to all candidates ranked at a worse position.

Note that a consensus state is usually quite restrictive and it is not satisfied by every profile of rankings. Therefore, when aggregating a profile of rankings, the search for the 'closest' profile of rankings in the chosen consensus state needs to be addressed.

Several authors, such as Nitzan [106], Lerer and Nitzan [88, Campbell and Nitzan [27], Meskanen and Nurmi [99, 100], Andjiga et al. 2] and Elkind et al. [49], have advocated that (most) ranking rules can be characterized as minimizing the distance to a consensus state for some appropriate distance function. This characterization is known as metric rationalisation of ranking rules.

In that way, all these ranking rules based on a consensus state can be seen as a two-step procedure:

(i) Search for $\mathscr{R}^{\prime}$, the 'closest' profile of rankings in the chosen consensus state by means of a distance function or metric.

(ii) Analysis of the consensus state to obtain the winning ranking $\succ$.

In Figure 3.1, this two-step procedure is illustrated for the method of Kemeny.

Many ranking rules, such as plurality [6] or the Borda count [18], apparently do not require any specific consensus state. However, as they determine the winning ranking by ordering based on the total score awarded to each candidate, all scoring ranking rules [57, 102, 104, 169] (in particular plurality or the Borda count) can be characterized by the distance to the set of profiles of rankings where the first-ranked candidate coincides for all rankings [99, 100 . 


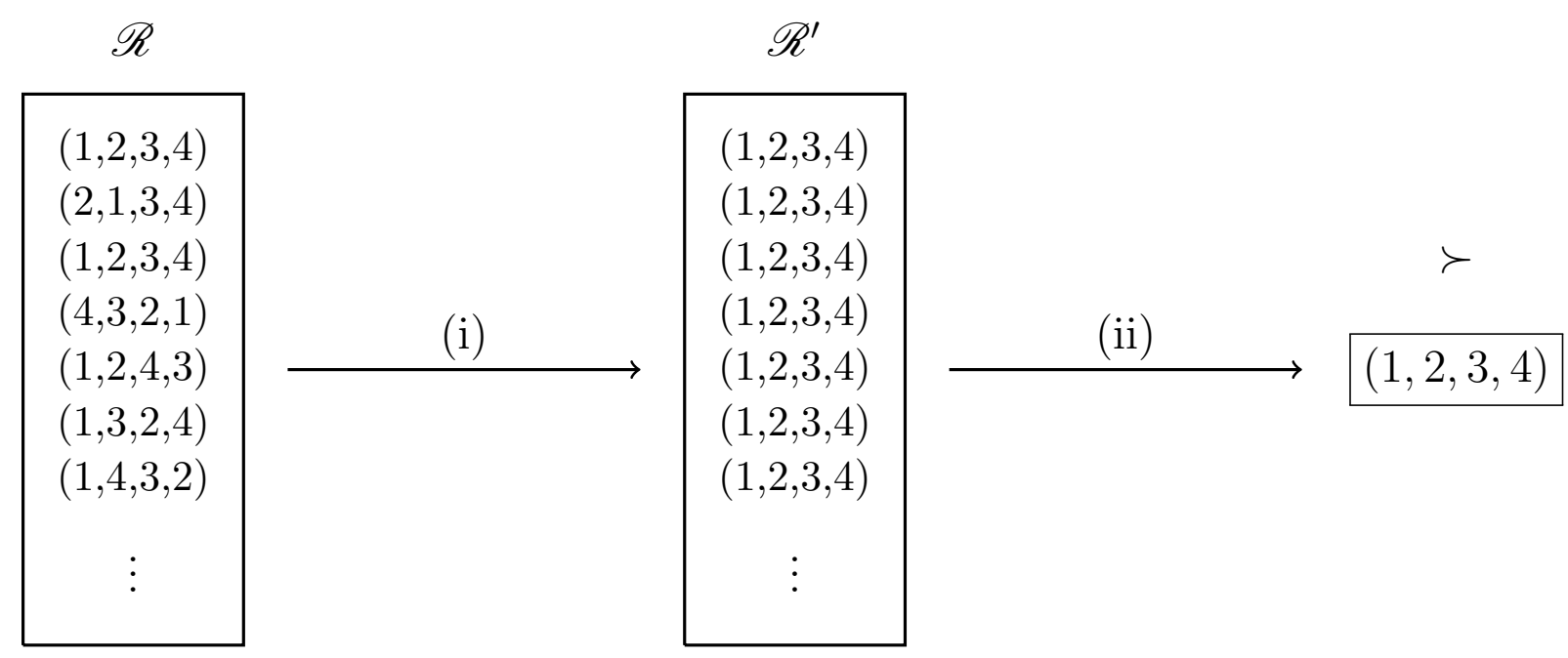

Figure 3.1: Diagram of the two-step procedure.

\subsection{Monometrics}

In this section, we introduce the concept of a monometric [126], which is closely related to that of a distance function or metric. Like a distance function, a monometric satisfies the axioms of non-negativity and coincidence, but a monometric requires compatibility with a given betweenness relation [111] and does not impose symmetry nor the triangle inequality.

\subsubsection{General case}

The notion of a distance function or metric is a well-known concept in mathematics.

Definition 3.2 A function $d: A \times A \rightarrow \mathbb{R}$ is called a distance function (on the set $A$ ) if it satisfies the following four properties:

(i) Non-negativity: for any $a, b \in A$, it holds that

$$
d(a, b) \geq 0
$$

(ii) Coincidence: for any $a, b \in A$, it holds that

$$
d(a, b)=0 \Leftrightarrow a=b .
$$


(iii) Symmetry: for any $a, b \in A$, it holds that

$$
d(a, b)=d(b, a)
$$

(iv) Triangle inequality: for any $a, b, c \in A$, it holds that

$$
d(a, c) \leq d(a, b)+d(b, c) .
$$

A betweenness relation is a ternary relation, introduced by Pasch [111] and further developed by Huntington and Kline [75], that describes when an element is in between two other ones. In what follows, we adhere to the formal relaxed definition given by Pitcher and Smiley [130], requiring a minimal set of axioms. Actually, they also proposed additional axioms concerning transitivity. Further additional axioms have been proposed in literature [55, 75, 111].

Definition 3.3 A ternary relation $R$ on a set $A$ is called a betweenness relation if it satisfies the following two properties:

(i) Symmetry in the end points: for any $a, b, c \in A$, it holds that

$$
(a, b, c) \in R \quad \Leftrightarrow \quad(c, b, a) \in R
$$

(ii) Closure: for any $a, b, c \in A$, it holds that

$$
((a, b, c) \in R \wedge(a, c, b) \in R) \quad \Leftrightarrow \quad b=c .
$$

Remark 3.4 Note that, for any $a, b \in A,(a, b, a) \in R$ implies that $a=b$. It suffices to see that it always holds that $(b, a, a) \in R$ due to the closure axiom and, therefore, $(a, a, b) \in R$ due to the symmetry in the end points. As it always holds that $(a, a, b) \in R$, due to the closure axiom we conclude that, if it holds that $(a, b, a) \in R$, then $a=b$.

The formula ' $(a, b, c) \in R$ ' is read as ' $b$ is in between $a$ and $c$ ' and is denoted as $[a, b, c]$ when no confusion is possible.

Although no transitivity axioms are required here, they are necessary conditions in order to guarantee the existence of an order relation $\leq$ that agrees with $R$, i.e., for which it holds 
that $(a, b, c) \in R$ if and only if $a=b$ or $b=c$ or $a \leq b \leq c$ or $c \leq b \leq a$. For further details about the relationship between order relations and betweenness relations, we refer to [55].

After fixing a betweenness relation, monometrics can be introduced, which are functions satisfying the non-negativity and coincidence axioms of a distance function, while preserving the given betweenness relation.

Definition 3.5 Let $A$ and $B$ be two sets such that $A \subseteq B$ and let $R$ be a betweenness relation on $B$. A function $M: A \times B \rightarrow \mathbb{R}$ is called a monometric (w.r.t. $R$ ) if it satisfies the following three properties:

(i) Non-negativity: for any $a \in A$ and any $b \in B$, it holds that

$$
M(a, b) \geq 0 .
$$

(ii) Coincidence: for any $a \in A$ and any $b \in B$, it holds that

$$
M(a, b)=0 \Leftrightarrow a=b .
$$

(iii) Compatibility: for any $a \in A$ and any $b, c \in B$ such that $[a, b, c]$, it holds that

$$
M(a, b) \leq M(a, c)
$$

In case the sets $A$ and $B$ coincide, we say that $M$ is a monometric on $A$.

For any $a \in A$ and any $b \in B, M(a, b)$ is called the cost of changing $a$ into $b$. The set $A$ is called the set of observable elements and the set $B$ is called the set of reachable elements.

Note that, by considering an appropriate betweenness relation, every distance function can be considered a monometric. In the following example, three generic betweenness relations are proposed w.r.t. which every distance function is a monometric.

Proposition 3.6 $A$ distance function $d: A \times A \rightarrow \mathbb{R}$ (on the set $A$ ) is a monometric w.r.t. the three following betweenness relations:

(i) $R_{1}=\left\{(a, b, c) \in A^{3} \mid a=b \vee b=c\right\}$; 
(ii) $R_{2}=\left\{(a, b, c) \in A^{3} \mid d(a, c)=d(a, b)+d(b, c)\right\}$;

(iii) $R_{3}=R_{1} \cup\left\{(a, b, c) \in A^{3} \mid d(a, b)<d(a, c) \wedge d(c, b)<d(c, a)\right\}$.

Proof: We first prove that $R_{1}, R_{2}$ and $R_{3}$ satisfy the two axioms of a betweenness relation (on $A$ ).

Symmetry in the end points: for any $a, b, c \in A$, it holds that

$$
\begin{aligned}
(a, b, c) \in R_{1} & \Leftrightarrow \quad(a=b) \vee(b=c) \\
& \Leftrightarrow \quad(c=b) \vee(b=a) \\
& \Leftrightarrow \quad(c, b, a) \in R_{1},
\end{aligned}
$$

and

$$
\begin{aligned}
(a, b, c) \in R_{2} & \Leftrightarrow d(a, c)=d(a, b)+d(b, c) \\
& \Leftrightarrow \quad d(c, a)=d(b, a)+d(c, b) \\
& \Leftrightarrow \quad(c, b, a) \in R_{2},
\end{aligned}
$$

due to the symmetry of $d$, and

$$
\begin{aligned}
(a, b, c) \in R_{3} & \Leftrightarrow \quad(d(a, b)<d(a, c) \wedge d(c, b)<d(c, a)) \vee(a=b \vee b=c) \\
& \Leftrightarrow \quad(d(c, b)<d(c, a) \wedge d(a, b)<d(a, c)) \vee(c=b \vee b=a) \\
& \Leftrightarrow \quad(c, b, a) \in R_{3} .
\end{aligned}
$$

Closure: for any $a, b, c \in A$, it holds that

$$
\begin{aligned}
& \left((a, b, c) \in R_{1}\right) \wedge\left((a, c, b) \in R_{1}\right) \\
& \Leftrightarrow \quad(a=b \vee b=c) \wedge(a=c \vee b=c) \\
& \Leftrightarrow \quad b=c,
\end{aligned}
$$

and

$$
\begin{aligned}
& \left((a, b, c) \in R_{2}\right) \wedge\left((a, c, b) \in R_{2}\right) \\
& \Leftrightarrow \quad d(a, c)=d(a, b)+d(b, c) \wedge d(a, b)=d(a, c)+d(c, b) \\
& \Leftrightarrow \quad d(b, c)=0 \\
& \Leftrightarrow \quad b=c,
\end{aligned}
$$


and

$$
\begin{aligned}
& \left((a, b, c) \in R_{3}\right) \wedge\left((a, c, b) \in R_{3}\right) \\
& \Leftrightarrow((d(a, b)<d(a, c) \wedge d(c, b)<d(c, a)) \vee(a=b \vee b=c)) \\
& \quad \wedge((d(a, c)<d(a, b) \wedge d(b, c)<d(b, a)) \vee(a=c \vee c=b)) \\
& \Leftrightarrow(d(a, b)<d(a, c) \wedge d(c, b)<d(c, a) \wedge d(a, c)<d(a, b) \wedge d(b, c)<d(b, a)) \\
& \quad \vee(d(a, b)<d(a, c) \wedge d(c, b)<d(c, a) \wedge(a=c \vee c=b)) \\
& \quad \vee((a=b \vee b=c) \wedge d(a, c)<d(a, b) \wedge d(b, c)<d(b, a)) \\
& \quad \vee((a=b \vee b=c) \wedge(a=c \vee c=b)) \\
& \Leftrightarrow(a=b \vee b=c) \wedge(a=c \vee c=b) \\
& \Leftrightarrow b=c .
\end{aligned}
$$

Next, we prove that $d$ satisfies the three axioms of a monometric (on $A$ ) w.r.t. $R_{1}, R_{2}$ and $R_{3}$. The non-negativity and coincidence axioms are trivially satisfied since $d$ is a distance function. We only need to prove the compatibility axiom.

Firstly, we prove it for $R_{1}$. Let $a, b, c \in A$ be such that $(a, b, c) \in R_{1}$, then $a=b$ or $b=c$. Therefore, $d(a, b)=0$ or $d(a, b)=d(a, c)$. Hence,

$$
d(a, b) \leq \max (0, d(a, c))=d(a, c) .
$$

Secondly, we prove it for $R_{2}$. Let $a, b, c \in A$ be such that $(a, b, c) \in R_{2}$, then $d(a, c)=$ $d(a, b)+d(b, c)$. Therefore,

$$
d(a, b)=d(a, c)-d(b, c) \leq d(a, c) .
$$

Thirdly, we prove it for $R_{3}$. Let $a, b, c \in A$ be such that $(a, b, c) \in R_{3}$, then $d(a, b)<d(a, c)$ or $a=b$ or $b=c$. Therefore,

$$
d(a, b) \leq d(a, c)
$$

Thus, $d$ is a monometric (on $A$ ) w.r.t. $R_{1}, R_{2}$ and $R_{3}$.

Note that the introduction of monometrics does not imply a rejection of the symmetry property or the triangle inequality, but a call for attention to the fact that both properties are not indispensable in the aggregation of rankings (although these properties are satisfied by the most widespread monometrics used in the aggregation of rankings). Furthermore, although any distance function can be seen as a monometric due to Propositon 3.6, it 
must be noted that requiring compatibility with a betweenness relation fixes a notion of 'closeness' that needs to be preserved. After fixing a betweenness relation, not every distance function can be seen as a monometric w.r.t. the fixed betweenness relation.

Different transformations of distance functions have called the attention of the scientific community [44. Here, we consider a natural transformation that takes the value of the original function whenever this value does not exceed a fixed lower bound, but is penalized and set to a fixed upper bound when it does.

Definition 3.7 For any function $f: A \times B \rightarrow \mathbb{R}$ and any two constants $0<l \leq u$, the function $f_{l, u}: A \times B \rightarrow \mathbb{R}$, defined as

$$
f_{l, u}(a, b)= \begin{cases}f(a, b) & , \text { if } f(a, b) \leq l, \\ u & , \text { otherwise }\end{cases}
$$

is called the $(l, u)$-penalized version of $f$.

Truncation [44] is a common transformation of a distance function, resulting in a so-called truncated distance function, which can be been as an $(l, l)$-penalized distance function.

We are interested in fixing a maximum admissible disagreement. Therefore, we measure the distance between elements, penalizing this measurement if this maximum admissible disagreement is reached. In order to do so, we set the lower bound $l$ and the upper bound $u$ such that $l \leq \sup _{a \in A, b \in B} d(a, b) \leq u$. This penalized distance function might not satisfy the triangle inequality, but still is a monometric if the original distance function was. In general, we prove in the following proposition that any $(l, u)$-penalized version of a monometric still is a monometric.

Proposition 3.8 Let $A$ and $B$ be two sets such that $A \subseteq B$ and let $R$ be a betweenness relation on $B$. For any monometric $M: A \times B \rightarrow \mathbb{R}$ (w.r.t. $R$ ) and any two constants $0<l \leq u$, the (l,u)-penalized version of $M_{l, u}: A \times B \rightarrow \mathbb{R}$ of $M$ is a monometric (w.r.t. $R$ ).

Proof: We prove that $M_{l, u}$ satisfies the three axioms of a monometric w.r.t. $R$.

Non-negativity: for any $a \in A$ and any $b \in B$, it holds that $M_{l, u}(a, b) \in\{M(a, b), u\}$ and, therefore, $M_{l, u}(a, b) \geq 0$. 
Coincidence: for any $a \in A$ and any $b \in B$, it holds that $M_{l, u}(a, b)=0$ if and only if $M(a, b)=0$. Therefore, it holds that $M_{l, u}(a, b)=0$ if and only if $a=b$.

Compatibility: for any $a \in A$ and any $b, c \in B$ such that $(a, b, c) \in R$, it holds that $M(a, b) \leq M(a, c)$. We distinguish four cases:

(i) $M_{l, u}(a, b)=M(a, b)$ and $M_{l, u}(a, c)=M(a, c)$. It holds that $M_{l, u}(a, b)=M(a, b) \leq$ $M(a, c)=M_{l, u}(a, c)$.

(ii) $M_{l, u}(a, b)=M(a, b)$ and $M_{l, u}(a, c)=u$. By definition, it holds that $M_{l, u}(a, b)=$ $M(a, b) \leq l \leq u=M_{l, u}(a, c)$.

(iii) $M_{l, u}(a, b)=u$ and $M_{l, u}(a, c)=M(a, c)$. Note that this implies that $(M(a, b)>l$ or $M(a, b)=l=u)$ and $M(a, c) \leq l$. Note that $(M(a, b)>l$ and $M(a, c) \leq l)$ leads to an impossible case $(M(a, b) \leq M(a, c) \leq l<M(a, b)$, a contraction) and $(M(a, b)=l=u$ and $M(a, c) \leq l)$ trivially implies that $M_{l, u}(a, b) \leq M_{l, u}(a, c)$.

(iv) $M_{l, u}(a, b)=u$ and $M_{l, u}(a, c)=u$. It trivially holds that $M_{l, u}(a, b) \leq M_{l, u}(a, c)$.

Thus, $M_{l, u}$ is a monometric w.r.t. $R$.

\subsubsection{Monometrics on $\mathcal{L}(\mathscr{C})$}

First of all, we clarify the notation that we will use from now on. Curly brackets \{\} are used for representing a set of elements without considering a specific order among them and parentheses () are used for representing an ordered list. Multi-sets, which are sets of elements where duplicated elements are allowed, are denoted with double curly brackets $\{\{\}\}$.

Distance functions on a set of rankings have been deeply studied in literature. Some of the most well-known ones are the Kendall distance [82], the Hamming distance [73] and the Cayley distance [29]. For the aggregation of rankings, these distance functions have been used in order to look for the 'closest' profile of rankings satisfying some given properties. However, distance functions are not the most appropriate type of functions for this problem. Rather, our newly introduced concept of monometric turns out to be better suited to represent the cost of changing a ranking into another one, as will be thoroughly discussed in Section 3.5 . 
Betweenness relations on $\mathcal{L}(\mathscr{C})$ have been deeply studied [37, 80, 81]. In practical problems, especially in social choice theory, Kemeny's proposal [80], which is based on the Kendall distance function between rankings, is the one that best preserves the preferences given by the voters. Basically, the Kendall distance between two rankings $\succ_{1}$ and $\succ_{2}$ counts the minimum number of consecutive candidates that need to be swapped in order to obtain $\succ_{2}$ starting from $\succ_{1}$ (or vice versa). The swap of two consecutive candidates in a ranking is called a reversal.

Definition 3.9 Let $\mathscr{C}$ be a set of $k$ candidates. The number of pairwise discordances between two rankings $\succ, \succ^{\prime} \in \mathcal{L}(\mathscr{C})$ is measured by means of the Kendall distance function $K$ :

$$
K\left(\succ, \succ^{\prime}\right)=\#\left\{\left(a_{i_{1}}, a_{i_{2}}\right) \in \mathscr{C}^{2} \mid a_{i_{1}} \neq a_{i_{2}} \wedge P_{\succ}\left(a_{i_{1}}\right)<P_{\succ}\left(a_{i_{2}}\right) \wedge P_{\succ^{\prime}}\left(a_{i_{1}}\right)>P_{\succ^{\prime}}\left(a_{i_{2}}\right)\right\} .
$$

Kemeny [80] proposed a related betweenness relation on $\mathcal{L}(\mathscr{C})$. Basically, it says that a ranking $\succ_{2}$ is in between two rankings $\succ_{1}$ and $\succ_{3}$ if there exists a way of going from $\succ_{1}$ to $\succ_{3}$ with the fewest number of reversals that passes by $\succ_{2}$.

Proposition 3.10 Let $\mathscr{C}$ be a set of $k$ candidates. The ternary relation $w$ on $\mathcal{L}(\mathscr{C})$ defined as

$$
w=\left\{\left(\succ_{1}, \succ_{2}, \succ_{3}\right) \in \mathcal{L}(\mathscr{C})^{3} \mid K\left(\succ_{1}, \succ_{3}\right)=K\left(\succ_{1}, \succ_{2}\right)+K\left(\succ_{2}, \succ_{3}\right)\right\}
$$

is a betweenness relation.

Proving that $w$ is a betweenness relation is straightforward as the Kendall distance function is a distance function and, therefore, it is symmetric and satisfies that two rankings are at Kendall distance zero if and only if they coincide. A more formal proof can be found in Proposition 3.6. For this particular betweenness relation, additional axioms of transitivity are satisfied [55].

Figure 3.2 illustrates the elements that are in between the rankings $a \succ b \succ c \succ d$ and $d \succ b \succ a \succ c$ according to the betweenness relation $w$. These rankings are $a \succ b \succ d \succ c$, $a \succ d \succ b \succ c, b \succ a \succ c \succ d, b \succ a \succ d \succ c, b \succ d \succ a \succ c$ and $d \succ a \succ b \succ c$.

Fixing a betweenness relation determines how 'closeness' is measured. After fixing a betweenness relation on $\mathcal{L}(\mathscr{C})$, if a ranking $\succ_{2}$ is in between two rankings $\succ_{1}$ and $\succ_{3}$, then the cost of changing $\succ_{1}$ into $\succ_{2}$ should not be larger than the cost of changing $\succ_{1}$ into $\succ_{3}$. The cost of changing these rankings is measured by means of a monometric on $\mathcal{L}(\mathscr{C})$. 


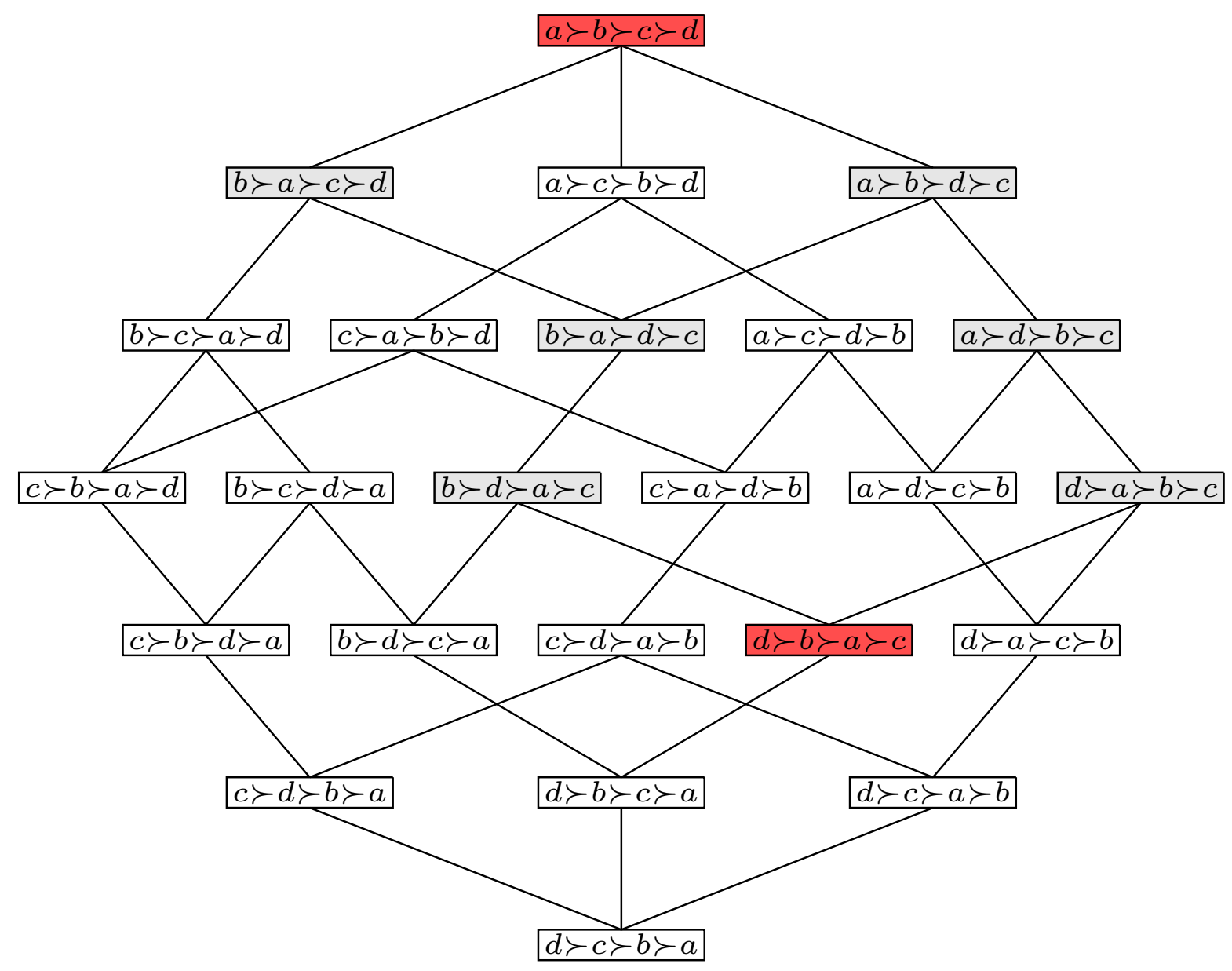

Figure 3.2: Graphical representation of $w$.

We have seen in Proposition 3.6 that, when considering an appropriate betweenness relation, every distance function can be seen as a monometric. The goal is to choose a meaningful betweenness relation determining our notion of 'closeness'.

Any monometric defined w.r.t. Kemeny's betweenness relation will be called a reversalbased monometric. These monometrics are the most interesting ones in social choice theory as they prevent from changing distant candidates in a ranking without changing close candidates first. Some relevant reversal-based monometrics are:

(i) $m_{1}\left(\succ_{1}, \succ_{2}\right)=K\left(\succ_{1}, \succ_{2}\right)$. The monometric $m_{1}$ counts the number of reversals needed in order to go from a ranking to another one. 


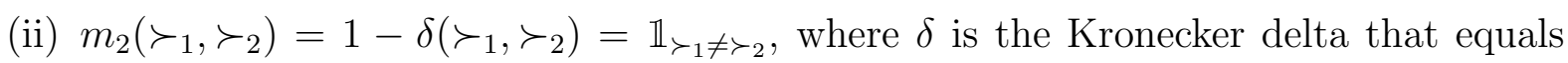
one in case the variables are equal and zero otherwise. The monometric $m_{2}$ is the common zero-one distance function that equals zero if and only if both elements coincide.

(iii) $m_{3}\left(\succ_{1}, \succ_{2}\right)=2^{-p}$ (with $m_{3}(\succ, \succ)=0$ for any ranking $\succ$ ), where $p$ is the first position at which both rankings differ. The monometric $m_{3}$ counts the number of high-ranked candidates that need to be changed in order to go from a ranking to another one.

(iv) $m_{4}\left(\succ_{1}, \succ_{2}\right)=K\left(\succ_{1}, \succ_{2}\right)^{2}$. The monometric $m_{4}$ computes the squared number of reversals.

(v) $m_{5}\left(\succ_{1}, \succ_{2}\right)=K_{l, \frac{k(k-1)}{2}}\left(\succ_{1}, \succ_{2}\right)$, where $l \in\left\{1, \ldots, \frac{k(k-1)}{2}\right\}$. The monometric $m_{5}$ fixes a maximum admissible disagreement between rankings and counts the number of reversals needed in order to go from a ranking to another one, penalizing this count if this maximum admissible disagreement is reached.

Remark 3.11 Although $m_{1}, m_{2}$ and $m_{3}$ are distance functions $\left(m_{3}\right.$ is even an ultrametri $\left.q^{1}\right)$, not every monometric is a distance function. For instance, $m_{4}$ and $m_{5}$ are two examples of a monometric that are not a distance function because they do not satisfy the triangle inequality.

Two interesting examples of monometrics that are not reversal-based are the Hamming distance function [73] and the Cayley distance function [29].

In the following example, monometrics on $\mathcal{L}(\mathscr{C})$ are illustrated.

Example 3.12 Let $\succ_{1}: a \succ b \succ c \succ d$ and $\succ_{2}: b \succ a \succ d \succ c$ be two rankings on $\mathscr{C}=\{a, b, c, d\}$. Candidates $a$ and $b$ lead to a pairwise discordance between both rankings; so do $c$ and d. Hence,

$$
\left\{(u, v) \in \mathscr{C}^{2} \mid u \neq v \wedge P_{\succ_{1}}(u)<P_{\succ_{1}}(v) \wedge P_{\succ_{2}}(u)>P_{\succ_{2}}(v)\right\}=\{(a, b),(c, d)\} .
$$

Therefore,

$$
K\left(\succ_{1}, \succ_{2}\right)=\#\{(a, b),(c, d)\}=2 .
$$

\footnotetext{
${ }^{1}$ An ultrametric $d$ (on a set $A$ ) is a distance function (on $A$ ) that satisfies the additional axiom $d(a, c) \leq$ $\max (d(a, b), d(b, c))$, for any $a, b, c \in A$.
} 
Hence, the minimum number of reversals needed in order to go from $\succ_{1}$ to $\succ_{2}$ is two.

Let us denote by $\succ_{3}: b \succ a \succ c \succ d$ and $\succ_{4}: a \succ b \succ d \succ c$. There are two possible ways of going from $\succ_{1}$ to $\succ_{2}$ with the least number of reversals (two):

$$
\begin{aligned}
& C_{1}: \succ_{1} \rightarrow \succ_{3} \rightarrow \succ_{2}, \\
& C_{2}: \succ_{1} \rightarrow \succ_{4} \rightarrow \succ_{2},
\end{aligned}
$$

where $\succ \rightarrow \succ^{\prime}$ represents that $\succ$ is changed into $\succ^{\prime}$ by means of a unique reversal $(K(\succ$ ,$\left.\succ^{\prime}\right)=1$ ).

It holds that $\left[\succ_{1}, \succ_{1}, \succ_{2}\right],\left[\succ_{1}, \succ_{2}, \succ_{2}\right],\left[\succ_{1}, \succ_{3}, \succ_{2}\right]$ and $\left[\succ_{1}, \succ_{4}, \succ_{2}\right]$ considering Kemeny's betweenness relation. On the other hand, there exists no $\succ \in \mathcal{L}(\mathscr{C})$ such that $\left[\succ_{1}, \succ, \succ_{2}\right]$ and, at the same time, $\succ \notin\left\{\succ_{1}, \succ_{2}, \succ_{3}, \succ_{4}\right\}$.

Note that $m_{1}\left(\succ_{1}, \succ_{2}\right)=K\left(\succ_{1}, \succ_{2}\right)=2$ is the cost of changing $\succ_{1}$ into $\succ_{2}$ in terms of the number of reversals.

Analogously, $m_{2}\left(\succ_{1}, \succ_{2}\right)=1-\delta\left(\succ_{1}, \succ_{2}\right)=1$ represents the cost of changing $\succ_{1}$ into $\succ_{2}$ in terms of the number of rankings changed. This monometric will be of interest when minimizing the number of rankings that need to be changed between two profiles of rankings.

As $\succ_{1}$ and $\succ_{2}$ do not coincide in their first ranked candidate, it holds that $m_{3}\left(\succ_{1}, \succ_{2}\right)$ $=2^{-p}=2^{-1}$. This monometric is useful when the goal is to minimize the number of high-ranked candidates that need to be changed in order to go from a ranking to another one.

The cost of changing $\succ_{1}$ into $\succ_{2}$ in terms of the squared number of reversals equals $m_{4}\left(\succ_{1}\right.$ ,$\left.\succ_{2}\right)=K\left(\succ_{1}, \succ_{2}\right)^{2}=4$.

Finally, $m_{5}$ counts the number of reversals needed in order to go from a ranking to another one, penalizing this count if a maximum admissible disagreement is reached. For $l \leq 2$, it holds that $m_{5}\left(\succ_{1}, \succ_{2}\right)=K\left(\succ_{1}, \succ_{2}\right)=2$ and, for $l>2$, it holds that $m_{5}\left(\succ_{1}, \succ_{2}\right)=\frac{k(k-1)}{2}=$ 6. 


\subsubsection{Monometrics on $\mathcal{L}(\mathscr{C})^{r}$}

When dealing with the aggregation of rankings, it is common to work with profiles of rankings instead of single rankings. A profile is denoted by $\mathscr{R}$ and, when $n \in \mathbb{N}$ profiles of rankings of the same size $r \in \mathbb{N}$ are considered, they are denoted by $\mathscr{R}_{1}, \ldots, \mathscr{R}_{n}$. For any $j \in\{1, \ldots, n\}$, we will denote by $\left(\succ_{i}^{j}\right)_{i=1}^{r}$ the corresponding $r$ rankings in $\mathscr{R}_{j}$.

Some ranking rules require the given profile of rankings to satisfy some specific properties. However, these properties are not always satisfied and the search for the 'closest' profile of rankings satisfying these properties is addressed. This 'closeness' is measured by means of monometrics on $\mathcal{L}(\mathscr{C})^{r}$. Note that any monometric on $\mathcal{L}(\mathscr{C})$ obviously is a monometric on $\mathcal{L}(\mathscr{C})^{r}$ with $r=1$.

Extending a betweenness relation on $\mathcal{L}(\mathscr{C})$ to a betweenness relation on $\mathcal{L}(\mathscr{C})^{r}$ can be realised by comparing the elements of both profiles one to one.

Proposition 3.13 Let $\mathscr{C}$ be a set of $k$ candidates, $r$ be the number of voters and $w$ be a betweenness relation on $\mathcal{L}(\mathscr{C})$. The ternary relation $W$ on $\mathcal{L}(\mathscr{C})^{r}$ defined as

$$
W=\left\{\left(\mathscr{R}_{1}, \mathscr{R}_{2}, \mathscr{R}_{3}\right) \in \mathcal{L}(\mathscr{C})^{r} \mid(\forall i \in\{1, \ldots, r\})\left(\left(\succ_{i}^{1}, \succ_{i}^{2}, \succ_{i}^{3}\right) \in w\right)\right\}
$$

is a betweenness relation.

Proof: We prove that $W$ satisfies the two axioms of a betweenness relation on $\mathcal{L}(\mathscr{C})^{r}$.

Symmetry in the end points: for any $\mathscr{R}_{1}, \mathscr{R}_{2}, \mathscr{R}_{3} \in \mathcal{L}(\mathscr{C})^{r}$, it holds that

$$
\begin{aligned}
\left(\mathscr{R}_{1}, \mathscr{R}_{2}, \mathscr{R}_{3}\right) \in W \quad & \quad(\forall i \in\{1, \ldots, r\})\left(\left(\succ_{i}^{1}, \succ_{i}^{2}, \succ_{i}^{3}\right) \in w\right) \\
\Leftrightarrow & (\forall i \in\{1, \ldots, r\})\left(\left(\succ_{i}^{3}, \succ_{i}^{2}, \succ_{i}^{1}\right) \in w\right) \\
\Leftrightarrow & \left(\mathscr{R}_{3}, \mathscr{R}_{2}, \mathscr{R}_{1}\right) \in W .
\end{aligned}
$$

Closure: for any $\mathscr{R}_{1}, \mathscr{R}_{2}, \mathscr{R}_{3} \in \mathcal{L}(\mathscr{C})^{r}$, it holds that

$$
\begin{aligned}
& \left(\left(\mathscr{R}_{1}, \mathscr{R}_{2}, \mathscr{R}_{3}\right) \in W\right) \wedge\left(\left(\mathscr{R}_{1}, \mathscr{R}_{3}, \mathscr{R}_{2}\right) \in W\right) \\
& \Leftrightarrow \quad(\forall i \in\{1, \ldots, r\})\left(\left(\left(\succ_{i}^{1}, \succ_{i}^{2}, \succ_{i}^{3}\right) \in w\right) \wedge\left(\left(\succ_{i}^{1}, \succ_{i}^{3}, \succ_{i}^{2}\right) \in w\right)\right) \\
& \Leftrightarrow \quad(\forall i \in\{1, \ldots, r\})\left(\succ_{i}^{2}=\succ_{i}^{3}\right) \\
& \Leftrightarrow \quad \mathscr{R}_{2}=\mathscr{R}_{3} .
\end{aligned}
$$


Thus, $W$ is a betweenness relation on $\mathcal{L}(\mathscr{C})^{r}$.

$W$ is called a 'betweenness relation on $\mathcal{L}(\mathscr{C})^{r}$ induced by $w$ '. The formula ' $\left(\mathscr{R}_{1}, \mathscr{R}_{2}, \mathscr{R}_{3}\right) \in$ $W$ ' is read ' $\mathscr{R}_{2}$ is in between $\mathscr{R}_{1}$ and $\mathscr{R}_{3}$ ' and is denoted as $\left[\mathscr{R}_{1}, \mathscr{R}_{2}, \mathscr{R}_{3}\right]$.

Since a ranking is a profile with just one element and both definitions coincide for $r=1$, using the same notation for a betweenness relation on $\mathcal{L}(\mathscr{C})^{r}$ and a betweenness relation on $\mathcal{L}(\mathscr{C})$ is justified.

Given a monometric on $\mathcal{L}(\mathscr{C})$, a monometric on $\mathcal{L}(\mathscr{C})^{r}$ can be constructed through the use of an 'aggregation' function.

Proposition 3.14 Let $\mathscr{C}$ be a set of $k$ candidates, $r$ be the number of voters, $m: \mathcal{L}(\mathscr{C}) \times$ $\mathcal{L}(\mathscr{C}) \rightarrow \mathbb{R}$ be a monometric on $\mathcal{L}(\mathscr{C})$ (w.r.t. a betweenness relation $w$ on $\mathcal{L}(\mathscr{C})$ ) and $W$ be the betweenness relation on $\mathcal{L}(\mathscr{C})^{r}$ induced by $w$. For any $r$-ary strictly increasing function $A:\left(\mathbb{R}^{+}\right)^{r} \rightarrow \mathbb{R}^{+}$such that $A(0, \ldots, 0)=0$, it holds that the function $M: \mathcal{L}(\mathscr{C})^{r} \times \mathcal{L}(\mathscr{C})^{r} \rightarrow$ $\mathbb{R}$ defined by

$$
M\left(\mathscr{R}_{1}, \mathscr{R}_{2}\right)=\underset{i=1}{\operatorname{A}}\left(m\left(\succ_{i}^{1}, \succ_{i}^{2}\right)\right)
$$

where $\underset{i=1}{\operatorname{A}}\left(x_{i}\right)$ is a shorthand for $A\left(x_{1}, \ldots, x_{r}\right)$, is a monometric on $\mathcal{L}(\mathscr{C})^{r}$ w.r.t. $W$

Proof: We prove that $M$ satisfies the three axioms of a monometric on $\mathcal{L}(\mathscr{C})^{r}$ w.r.t. $W$. Non-negativity: immediate.

Coincidence: for any $\mathscr{R}_{1}, \mathscr{R}_{2} \in \mathcal{L}(\mathscr{C})^{r}$, as $A\left(x_{1}, \ldots, x_{r}\right)=0$ if and only if $(\forall i \in\{1, \ldots, r\})$ $\left(x_{i}=0\right)$ and $m$ is a monometric on $\mathcal{L}(\mathscr{C})$, it holds that

$$
\begin{aligned}
& M\left(\mathscr{R}_{1}, \mathscr{R}_{2}\right):=\stackrel{A}{A}_{i=1}^{r}\left(m\left(\succ_{i}^{1}, \succ_{i}^{2}\right)\right)=0 \\
& \Leftrightarrow \quad(\forall i \in\{1, \ldots, r\})\left(m\left(\succ_{i}^{1}, \succ_{i}^{2}\right)=0\right) \\
& \Leftrightarrow \quad(\forall i \in\{1, \ldots, r\})\left(\succ_{i}^{1}=\succ_{i}^{2}\right) \\
& \Leftrightarrow \quad \mathscr{R}_{1}=\mathscr{R}_{2} .
\end{aligned}
$$

Compatibility: for any $\mathscr{R}_{1}, \mathscr{R}_{2}, \mathscr{R}_{3} \in \mathcal{L}(\mathscr{C})^{r}$ such that $\left[\mathscr{R}_{1}, \mathscr{R}_{2}, \mathscr{R}_{3}\right]$, as $A$ is increasing and $m$ is a monometric on $\mathcal{L}(\mathscr{C})$, it holds that

$$
M\left(\mathscr{R}_{1}, \mathscr{R}_{2}\right)=\underset{i=1}{A}\left(m\left(\succ_{i}^{1}, \succ_{i}^{2}\right)\right) \leq \underset{i=1}{A}\left(m\left(\succ_{i}^{1}, \succ_{i}^{3}\right)\right)=M\left(\mathscr{R}_{1}, \mathscr{R}_{3}\right) .
$$


Thus, $M$ is a monometric on $\mathcal{L}(\mathscr{C})^{r}$ w.r.t. $W$.

In particular, the addition defines a monometric on $\mathcal{L}(\mathscr{C})^{r}$.

Corollary 3.15 Let $\mathscr{C}$ be a set of $k$ candidates, $r$ be the number of voters, $m: \mathcal{L}(\mathscr{C}) \times$ $\mathcal{L}(\mathscr{C}) \rightarrow \mathbb{R}$ be a monometric on $\mathcal{L}(\mathscr{C})$ (w.r.t. a betweenness relation $w$ on $\mathcal{L}(\mathscr{C})$ ) and $W$ be the betweenness relation on $\mathcal{L}(\mathscr{C})^{r}$ induced by $w$. The function $M: \mathcal{L}(\mathscr{C})^{r} \times \mathcal{L}(\mathscr{C})^{r} \rightarrow \mathbb{R}$ defined as

$$
M\left(\mathscr{R}_{1}, \mathscr{R}_{2}\right)=\sum_{i=1}^{r} m\left(\succ_{i}^{1}, \succ_{i}^{2}\right)
$$

is a monometric on $\mathcal{L}(\mathscr{C})^{r}$ w.r.t. $W$.

Considering the monometrics on $\mathcal{L}(\mathscr{C})$ introduced in Subsection 3.2 .2 , we obtain the following monometrics on $\mathcal{L}(\mathscr{C})^{r}$ :

(i) $M_{1}\left(\mathscr{R}_{1}, \mathscr{R}_{2}\right)=\sum_{i=1}^{r} K\left(\succ_{i}^{1}, \succ_{i}^{2}\right)$. The monometric $M_{1}$ counts the number of reversals needed in order to go from a profile of rankings to another one.

(ii) $M_{2}\left(\mathscr{R}_{1}, \mathscr{R}_{2}\right)=\sum_{i=1}^{r} 1-\delta\left(\succ_{i}^{1}, \succ_{i}^{2}\right)$. The monometric $M_{2}$ counts the number of rankings that differ from a profile of rankings to another one.

(iii) $M_{3}\left(\mathscr{R}_{1}, \mathscr{R}_{2}\right)=\sum_{i=1}^{r} m_{3}\left(\succ_{i}^{1}, \succ_{i}^{2}\right)$. The monometric $M_{3}$ counts the number of highranked candidates that need to be changed in order to go from a profile of rankings to another one.

(iv) $M_{4}\left(\mathscr{R}_{1}, \mathscr{R}_{2}\right)=\sum_{i=1}^{r} K\left(\succ_{i}^{1}, \succ_{i}^{2}\right)^{2}$. The monometric $M_{4}$ computes the squared number of reversals.

(v) $M_{5}\left(\mathscr{R}_{1}, \mathscr{R}_{2}\right)=\sum_{i=1}^{r} K_{l, \frac{k(k-1)}{2}}\left(\succ_{i}^{1}, \succ_{i}^{2}\right)$ (with $\left.l \in\left\{1, \ldots, \frac{k(k-1)}{2}\right\}\right)$. The monometric $M_{5}$ fixes a maximum admissible disagreement between rankings and counts the number of reversals needed in order to go from a profile of rankings to another one, penalizing this count each time that this maximum admissible disagreement is reached. 
(vi) $M_{6}\left(\mathscr{R}_{1}, \mathscr{R}_{2}\right)=1-\delta\left(\mathscr{R}_{1}, \mathscr{R}_{2}\right)$. The monometric $M_{6}$ only takes into account whether or not two profiles of rankings differ. It must be noted that $M_{6}$ is a monometric on $\mathcal{L}(\mathscr{C})^{r}$ that is not defined by means of a monometric on $\mathcal{L}(\mathscr{C})$.

In the following example, these monometrics on $\mathcal{L}(\mathscr{C})^{r}$ are illustrated.

Example 3.16 Let $\mathscr{R}_{1}, \mathscr{R}_{2}$ and $\mathscr{R}_{3}$ be the following profiles of rankings on $\mathscr{C}=\{a, b, c, d\}$ :

$$
\begin{aligned}
& \mathscr{R}_{1}=\left(\succ_{1}^{1}, \succ_{2}^{1}, \succ_{3}^{1}, \succ_{4}^{1}\right)=(a \succ b \succ c \succ d, a \succ b \succ d \succ c, d \succ c \succ a \succ b, d \succ c \succ b \succ a), \\
& \mathscr{R}_{2}=\left(\succ_{1}^{2}, \succ_{2}^{2}, \succ_{3}^{2}, \succ_{4}^{2}\right)=(a \succ b \succ d \succ c, d \succ c \succ a \succ b, d \succ c \succ b \succ a, d \succ c \succ b \succ a), \\
& \mathscr{R}_{3}=\left(\succ_{1}^{3}, \succ_{2}^{3}, \succ_{3}^{3}, \succ_{4}^{3}\right)=(a \succ b \succ d \succ c, d \succ c \succ b \succ a, d \succ c \succ b \succ a, a \succ b \succ c \succ d) .
\end{aligned}
$$

It is easy to see that, considering Kemeny's betweenness relation on $\mathcal{L}(\mathscr{C}),\left[\succ_{i}^{1}, \succ_{i}^{2}, \succ_{i}^{3}\right]$ for any $i \in\{1, \ldots, 4\}$. Therefore, it holds that $\left[\mathscr{R}_{1}, \mathscr{R}_{2}, \mathscr{R}_{3}\right]$ considering the betweenness relation on $\mathcal{L}(\mathscr{C})^{r}$ induced by Kemeny's betweenness relation on $\mathcal{L}(\mathscr{C})$.

Furthermore,

$$
\begin{aligned}
& M_{1}\left(\mathscr{R}_{1}, \mathscr{R}_{2}\right)=\sum_{i=1}^{4} K\left(\succ_{i}^{1}, \succ_{i}^{2}\right)=1+4+1+0=6, \\
& M_{1}\left(\mathscr{R}_{1}, \mathscr{R}_{3}\right)=\sum_{i=1}^{4} K\left(\succ_{i}^{1}, \succ_{i}^{3}\right)=1+5+1+6=13 .
\end{aligned}
$$

Note that it indeed holds that

$$
M_{1}\left(\mathscr{R}_{1}, \mathscr{R}_{2}\right)=6 \leq 13=M_{1}\left(\mathscr{R}_{1}, \mathscr{R}_{3}\right)
$$


Other possible monometrics lead to:

$$
\begin{aligned}
& M_{2}\left(\mathscr{R}_{1}, \mathscr{R}_{2}\right)=\sum_{i=1}^{4} 1-\delta\left(\succ_{i}^{1}, \succ_{i}^{2}\right)=3, \\
& M_{2}\left(\mathscr{R}_{1}, \mathscr{R}_{3}\right)=\sum_{i=1}^{4} 1-\delta\left(\succ_{i}^{1}, \succ_{i}^{3}\right)=4, \\
& M_{3}\left(\mathscr{R}_{1}, \mathscr{R}_{2}\right)=\sum_{i=1}^{4} m_{3}\left(\succ_{i}^{1}, \succ_{i}^{2}\right)=\frac{3}{4}, \\
& M_{3}\left(\mathscr{R}_{1}, \mathscr{R}_{3}\right)=\sum_{i=1}^{4} m_{3}\left(\succ_{i}^{1}, \succ_{i}^{3}\right)=\frac{5}{4}, \\
& M_{4}\left(\mathscr{R}_{1}, \mathscr{R}_{2}\right)=\sum_{i=1}^{4} K\left(\succ_{i}^{1}, \succ_{i}^{2}\right)^{2}=18, \\
& M_{4}\left(\mathscr{R}_{1}, \mathscr{R}_{3}\right)=\sum_{i=1}^{4} K\left(\succ_{i}^{1}, \succ_{i}^{3}\right)^{2}=63, \\
& M_{5}\left(\mathscr{R}_{1}, \mathscr{R}_{2}\right)=\sum_{i=1}^{4} K_{3,6}\left(\succ_{i}^{1}, \succ_{i}^{2}\right)=8, \\
& M_{5}\left(\mathscr{R}_{1}, \mathscr{R}_{3}\right)=\sum_{i=1}^{4} K_{3,6}\left(\succ_{i}^{1}, \succ_{i}^{3}\right)=14, \\
& M_{6}\left(\mathscr{R}_{1}, \mathscr{R}_{2}\right)=1-\delta\left(\mathscr{R}_{1}, \mathscr{R}_{2}\right)=1, \\
& M_{6}\left(\mathscr{R}_{1}, \mathscr{R}_{3}\right)=1-\delta\left(\mathscr{R}_{1}, \mathscr{R}_{3}\right)=1 .
\end{aligned}
$$

The relationship between the costs of changing $\mathscr{R}_{1}$ into $\mathscr{R}_{2}$ and $\mathscr{R}_{1}$ into $\mathscr{R}_{3}$ are, of course, still satisfied:

$$
\begin{gathered}
M_{2}\left(\mathscr{R}_{1}, \mathscr{R}_{2}\right)=3 \leq 4=M_{2}\left(\mathscr{R}_{1}, \mathscr{R}_{3}\right), \\
M_{3}\left(\mathscr{R}_{1}, \mathscr{R}_{2}\right)=\frac{3}{4} \leq \frac{5}{4}=M_{3}\left(\mathscr{R}_{1}, \mathscr{R}_{3}\right), \\
M_{4}\left(\mathscr{R}_{1}, \mathscr{R}_{2}\right)=18 \leq 63=M_{4}\left(\mathscr{R}_{1}, \mathscr{R}_{3}\right), \\
M_{5}\left(\mathscr{R}_{1}, \mathscr{R}_{2}\right)=8 \leq 14=M_{5}\left(\mathscr{R}_{1}, \mathscr{R}_{3}\right), \\
M_{6}\left(\mathscr{R}_{1}, \mathscr{R}_{2}\right)=1 \leq 1=M_{6}\left(\mathscr{R}_{1}, \mathscr{R}_{3}\right) .
\end{gathered}
$$

\footnotetext{
${ }^{2}$ For the monometric $M_{5}$, the maximum admissible disagreement is set to $l=3$.
} 


\subsubsection{Monometrics on $\mathcal{M}_{r}(\mathcal{L}(\mathscr{C}))$}

In some cases, a profile of rankings is given in the form of a multi-set where voters cannot be related with their respective votes in order to preserve their privacy. These multisets of rankings are called anonymised profiles of rankings. An anonymised profile is denoted by $\mathscr{Y}$ and, when $n \in \mathbb{N}$ anonymised profiles of rankings of the same size $r \in \mathbb{N}$ are considered, they are denoted by $\mathscr{Y}_{1}, \ldots, \mathscr{Y}_{n}$. The set of all anonymised profiles of $r$ rankings on $\mathscr{C}$ is denoted by $\mathcal{M}_{r}(\mathcal{L}(\mathscr{C}))$. Although no order is defined between the rankings in an anonymised profile of rankings, we will assign a label to each ranking in order to ease notations. For any $j \in\{1, \ldots, n\}$, we will denote by $\left\{\left\{\succ_{i}^{j}\right\}_{i=1}^{r}\right.$ the corresponding $r$ rankings in $\mathscr{Y}_{j}$. For a permutation $\sigma \in \Pi_{r}$, where $\Pi_{r}=\mathcal{L}(\{1, \ldots, r\})$ denotes the set of permutations of $\{1, \ldots, r\}$, we will denote by $\sigma(\mathscr{Y})$ the profile of rankings where each ranking of the anonymised profile of rankings $\mathscr{Y}$ is in the position given by $\sigma$.

After choosing a monometric, the notion of an optimal permutation triplet can be defined. Intuitively, a permutation triplet is optimal if there are no other ways of re-ordering the anonymised profiles leading to a lower cost considering the chosen monometric on $\mathcal{L}(\mathscr{C})^{r}$. More formally, we introduce optimal permutation triplets in the following definition.

Definition 3.17 Let $\mathscr{C}$ be a set of $k$ candidates, $r$ be the number of voters, $\mathscr{Y}_{1}, \mathscr{Y}_{2}, \mathscr{Y}_{3} \in$ $\mathcal{M}_{r}(\mathcal{L}(\mathscr{C}))$ be three anonymised profiles and $M$ be a monometric on $\mathcal{L}(\mathscr{C})^{r}$ (w.r.t. a betweenness relation on $\left.\mathcal{L}(\mathscr{C})^{r}\right)$. A permutation triplet $\left(\sigma_{1}, \sigma_{2}, \sigma_{3}\right) \in \Pi_{r}^{3}$ is called optimal if

$$
\left\{\begin{array}{l}
M\left(\sigma_{1}\left(\mathscr{Y}_{1}\right), \sigma_{2}\left(\mathscr{Y}_{2}\right)\right)=\min _{\sigma, \sigma^{\prime} \in \Pi_{r}} M\left(\sigma\left(\mathscr{Y}_{1}\right), \sigma^{\prime}\left(\mathscr{Y}_{2}\right)\right), \\
M\left(\sigma_{1}\left(\mathscr{Y}_{1}\right), \sigma_{3}\left(\mathscr{Y}_{3}\right)\right)=\min _{\sigma, \sigma^{\prime} \in \Pi_{r}} M\left(\sigma\left(\mathscr{Y}_{1}\right), \sigma^{\prime}\left(\mathscr{Y}_{3}\right)\right), \\
M\left(\sigma_{2}\left(\mathscr{Y}_{2}\right), \sigma_{3}\left(\mathscr{Y}_{3}\right)\right)=\min _{\sigma, \sigma^{\prime} \in \Pi_{r}} M\left(\sigma\left(\mathscr{Y}_{2}\right), \sigma^{\prime}\left(\mathscr{Y}_{3}\right)\right) .
\end{array}\right.
$$

The set of all optimal permutation triplets is denoted by $\Theta_{r}^{M}\left(\mathscr{Y}_{1}, \mathscr{Y}_{2}, \mathscr{Y}_{3}\right)$.

Although it may be non-intuitive at first sight, a betweenness relation on $\mathcal{M}_{r}(\mathcal{L}(\mathscr{C}))$ depends on a monometric on $\mathcal{L}(\mathscr{C})^{r}$. In the following example, the need of introducing optimal permutation triplets for constructing a betweenness relation on $\mathcal{M}_{r}(\mathcal{L}(\mathscr{C}))$ is illustrated. 
Example 3.18 Let $\mathscr{Y}_{1}, \mathscr{Y}_{2}$ and $\mathscr{Y}_{3}$ be the following anonymised profiles of rankings on $\mathscr{C}=\{a, b, c, d\}$ :

$$
\begin{aligned}
& \mathscr{Y}_{1}=\left\{\left\{\succ_{1}^{1}, \succ_{2}^{1}\right\}\right\}=\{\{a \succ b \succ c \succ d, d \succ c \succ b \succ a\}, \\
& \mathscr{Y}_{2}=\left\{\left\{\succ_{1}^{2}, \succ_{2}^{2}\right\}\right\}=\{\{a \succ c \succ b \succ d, a \succ c \succ b \succ d\}, \\
& \mathscr{Y}_{3}=\left\{\left\{\succ_{1}^{3}, \succ_{2}^{3}\right\}\right\}=\{\{a \succ b \succ c \succ d, d \succ c \succ b \succ a\}\} .
\end{aligned}
$$

In case $r=2$, it holds that $\Pi_{r}=\left\{\sigma_{I}, \sigma_{R}\right\}$, with $\sigma_{I}=(1,2)$ and $\sigma_{R}=(2,1)$. We identify a triplet of permutations $\left(\sigma_{I}, \sigma_{I}, \sigma_{R}\right) \in \Pi_{r}^{3}$ such that $\left(\sigma_{I}\left(\mathscr{Y}_{1}\right), \sigma_{I}\left(\mathscr{Y}_{2}\right), \sigma_{R}\left(\mathscr{Y}_{1}\right)\right) \in W$, where $W$ is the betweenness relation on $\mathcal{L}(\mathscr{C})^{r}$ induced by Kemeny's betweenness relation on $\mathcal{L}(\mathscr{C})$.

This would contradict the closure axiom of a betweenness relation on $\mathcal{M}_{r}(\mathcal{L}(\mathscr{C}))$. One may note that this non-intuitive result is due to the fact that $\left(\sigma_{I}, \sigma_{I}, \sigma_{R}\right)$ may not be an optimal permutation triplet (due to the coincidence axiom of a monometric).

Therefore, extending a betweenness relation on $\mathcal{L}(\mathscr{C})^{r}$ to a betweenness relation on $\mathcal{M}_{r}$ $(\mathcal{L}(\mathscr{C}))$ depends on the chosen monometric on $\mathcal{L}(\mathscr{C})^{r}$. In particular, we are interested in a particular kind of monometrics on $\mathcal{L}(\mathscr{C})^{r}$.

Definition 3.19 Let $\mathscr{C}$ be a set of $k$ candidates and $r$ be the number of voters. A monometric $M$ on $\mathcal{L}(\mathscr{C})^{r}$ (w.r.t. a betweenness relation on $\left.\mathcal{L}(\mathscr{C})^{r}\right)$ is called order-invariant if, for any three anonymised profiles $\mathscr{Y}_{1}, \mathscr{Y}_{2}, \mathscr{Y}_{3} \in \mathcal{M}_{r}(\mathcal{L}(\mathscr{C}))$, the following two statements are equivalent:

(i) $\left(\sigma_{1}, \sigma_{2}, \sigma_{3}\right) \in \Theta_{r}^{M}\left(\mathscr{Y}_{1}, \mathscr{Y}_{2}, \mathscr{Y}_{3}\right)$;

(ii) $\left(\forall \varepsilon \in \Pi_{3}\right)\left(\left(\sigma_{\varepsilon(1)}, \sigma_{\varepsilon(2)}, \sigma_{\varepsilon(3)}\right) \in \Theta_{r}^{M}\left(\mathscr{Y}_{\varepsilon(1)}, \mathscr{Y}_{\varepsilon(2)}, \mathscr{Y}_{\varepsilon(3)}\right)\right)$.

Remark 3.20 In order to prove that a monometric is order invariant, it is sufficient to prove that the following statements are equivalent:

(i) $\left(\sigma_{1}, \sigma_{2}, \sigma_{3}\right) \in \Theta_{r}^{M}\left(\mathscr{Y}_{1}, \mathscr{Y}_{2}, \mathscr{Y}_{3}\right)$;

(ii) $\left(\sigma_{2}, \sigma_{3}, \sigma_{1}\right) \in \Theta_{r}^{M}\left(\mathscr{Y}_{2}, \mathscr{Y}_{3}, \mathscr{Y}_{1}\right)$;

(iii) $\left(\sigma_{3}, \sigma_{1}, \sigma_{2}\right) \in \Theta_{r}^{M}\left(\mathscr{Y}_{3}, \mathscr{Y}_{1}, \mathscr{Y}_{2}\right)$. 
Remark 3.21 Any symmetric monometric is, by definition of optimal permutation triplet, order invariant. In particular, all the monometrics introduced in Subsection 3.2.3 are order invariant.

The notion of an optimal permutation triplet defined by an order-invariant monometric on $\mathcal{L}(\mathscr{C})^{r}$ allows us to introduce a betweenness relation on $\mathcal{M}_{r}(\mathcal{L}(\mathscr{C}))$. Obviously, different monometrics on $\mathcal{L}(\mathscr{C})^{r}$ may yield different betweenness relations on $\mathcal{M}_{r}(\mathcal{L}(\mathscr{C}))$.

Proposition 3.22 Let $\mathscr{C}$ be a set of $k$ candidates, $r$ be the number of voters, $W$ be a betweenness relation on $\mathcal{L}(\mathscr{C})^{r}$ and $M$ be an order-invariant monometric on $\mathcal{L}(\mathscr{C})^{r}$ w.r.t. W. The ternary relation $\mathbb{W}$ on $\mathcal{M}_{r}(\mathcal{L}(\mathscr{C}))$ defined as

$$
\mathbb{W}=\left\{\begin{array}{l|l}
\left(\mathscr{Y}_{1}, \mathscr{Y}_{2}, \mathscr{Y}_{3}\right) \in \mathcal{M}_{r}(\mathcal{L}(\mathscr{C}))^{3} & \begin{array}{c}
\Theta_{r}^{M}\left(\mathscr{Y}_{1}, \mathscr{Y}_{2}, \mathscr{Y}_{3}\right) \neq \emptyset \\
\wedge\left(\forall\left(\sigma_{1}, \sigma_{2}, \sigma_{3}\right) \in \Theta_{r}^{M}\left(\mathscr{Y}_{1}, \mathscr{Y}_{2}, \mathscr{Y}_{3}\right)\right) \\
\left(\left(\sigma_{1}\left(\mathscr{Y}_{1}\right), \sigma_{2}\left(\mathscr{Y}_{2}\right), \sigma_{3}\left(\mathscr{Y}_{3}\right)\right) \in W\right)
\end{array}
\end{array}\right\}
$$

is a betweenness relation.

Proof: We prove that $\mathbb{W}$ satisfies the two axioms of a betweenness relation on $\mathcal{M}_{r}(\mathcal{L}(\mathscr{C}))$.

Symmetry in the end points: for any $\mathscr{Y}_{1}, \mathscr{Y}_{2}, \mathscr{Y}_{3} \in \mathcal{M}_{r}(\mathcal{L}(\mathscr{C}))$, it holds that

$$
\begin{aligned}
& \left(\mathscr{Y}_{1}, \mathscr{Y}_{2}, \mathscr{Y}_{3}\right) \in \mathbb{W} \\
& \Leftrightarrow\left\{\begin{array}{l}
\Theta_{r}^{M}\left(\mathscr{Y}_{1}, \mathscr{Y}_{2}, \mathscr{Y}_{3}\right) \neq \emptyset \\
\left(\forall\left(\sigma_{1}, \sigma_{2}, \sigma_{3}\right) \in \Theta_{r}^{M}\left(\mathscr{Y}_{1}, \mathscr{Y}_{2}, \mathscr{Y}_{3}\right)\right)\left(\left(\sigma_{1}\left(\mathscr{Y}_{1}\right), \sigma_{2}\left(\mathscr{Y}_{2}\right), \sigma_{3}\left(\mathscr{Y}_{3}\right)\right) \in W\right)
\end{array}\right. \\
& \Leftrightarrow\left\{\begin{array}{l}
\Theta_{r}^{M}\left(\mathscr{Y}_{1}, \mathscr{Y}_{2}, \mathscr{Y}_{3}\right) \neq \emptyset \\
\left(\forall\left(\sigma_{1}, \sigma_{2}, \sigma_{3}\right) \in \Theta_{r}^{M}\left(\mathscr{Y}_{1}, \mathscr{Y}_{2}, \mathscr{Y}_{3}\right)\right)\left(\left(\sigma_{3}\left(\mathscr{Y}_{3}\right), \sigma_{2}\left(\mathscr{Y}_{2}\right), \sigma_{1}\left(\mathscr{Y}_{1}\right)\right) \in W\right)
\end{array}\right. \\
& \underset{(*)}{\Leftrightarrow}\left\{\begin{array}{l}
\Theta_{r}^{M}\left(\mathscr{Y}_{1}, \mathscr{Y}_{2}, \mathscr{Y}_{3}\right) \neq \emptyset \\
\left(\forall\left(\sigma_{3}, \sigma_{2}, \sigma_{1}\right) \in \Theta_{r}^{M}\left(\mathscr{Y}_{3}, \mathscr{Y}_{2}, \mathscr{Y}_{1}\right)\right)\left(\left(\sigma_{3}\left(\mathscr{Y}_{3}\right), \sigma_{2}\left(\mathscr{Y}_{2}\right), \sigma_{1}\left(\mathscr{Y}_{1}\right)\right) \in W\right)
\end{array}\right. \\
& \Leftrightarrow \quad\left(\mathscr{Y}_{3}, \mathscr{Y}_{2}, \mathscr{Y}_{1}\right) \in \mathbb{W},
\end{aligned}
$$

(*) as $M$ is order invariant. 
Closure: for any $\mathscr{Y}_{1}, \mathscr{Y}_{2}, \mathscr{Y}_{3} \in \mathcal{M}_{r}(\mathcal{L}(\mathscr{C}))$, it holds that

$$
\begin{aligned}
& \left(\left(\mathscr{Y}_{1}, \mathscr{Y}_{2}, \mathscr{Y}_{3}\right) \in \mathbb{W}\right) \wedge\left(\left(\mathscr{Y}_{1}, \mathscr{Y}_{3}, \mathscr{Y}_{2}\right) \in \mathbb{W}\right)
\end{aligned}
$$

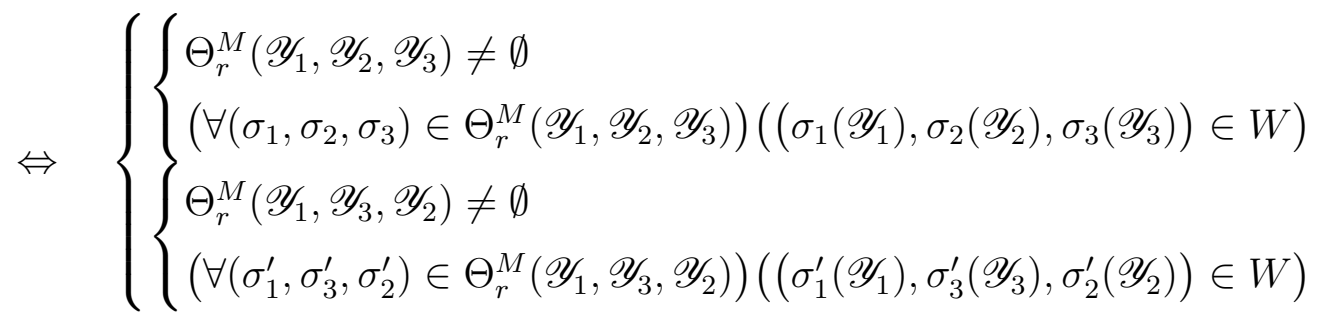

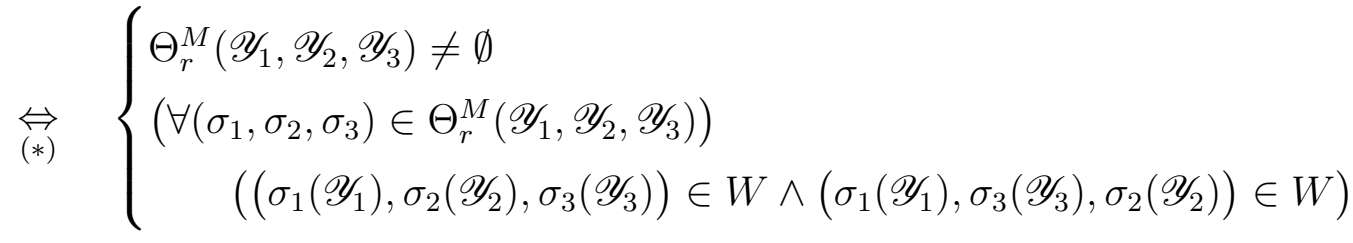

$$
\begin{aligned}
& \Leftrightarrow\left\{\begin{array}{l}
\Theta_{r}^{M}\left(\mathscr{Y}_{1}, \mathscr{Y}_{2}, \mathscr{Y}_{3}\right) \neq \emptyset \\
\left(\forall\left(\sigma_{1}, \sigma_{2}, \sigma_{3}\right) \in \Theta_{r}^{M}\left(\mathscr{Y}_{1}, \mathscr{Y}_{2}, \mathscr{Y}_{3}\right)\right)\left(\sigma_{2}\left(\mathscr{Y}_{2}\right)=\sigma_{3}\left(\mathscr{Y}_{3}\right)\right)
\end{array}\right. \\
& \Leftrightarrow \quad \mathscr{Y}_{2}=\mathscr{Y}_{3} \text {, }
\end{aligned}
$$

$(*)$ as $M$ is order invariant.

Thus, $\mathbb{W}$ is a betweenness relation on $\mathcal{M}_{r}(\mathcal{L}(\mathscr{C}))$.

$\mathbb{W}$ is called a 'betweenness relation on $\mathcal{M}_{r}(\mathcal{L}(\mathscr{C}))$ induced by $W$ and $M$ '. The formula ' $\left(\mathscr{Y}_{1}, \mathscr{Y}_{2}, \mathscr{Y}_{3}\right) \in \mathbb{W}$ ' is read ' $\mathscr{Y}_{2}$ is in between $\mathscr{Y}_{1}$ and $\mathscr{Y}_{3}$ ' and is denoted as $\left[\mathscr{Y}_{1}, \mathscr{Y}_{2}, \mathscr{Y}_{3}\right]$.

Given a monometric on $\mathcal{L}(\mathscr{C})^{r}$, we can construct a monometric on $\mathcal{M}_{r}(\mathcal{L}(\mathscr{C}))$.

Proposition 3.23 Let $\mathscr{C}$ be a set of $k$ candidates, $r$ be the number of voters, $W$ be a betweenness relation on $\mathcal{L}(\mathscr{C})^{r}$ and $M: \mathcal{L}(\mathscr{C})^{r} \times \mathcal{L}(\mathscr{C})^{r} \rightarrow \mathbb{R}$ be an order-invariant monometric on $\mathcal{L}(\mathscr{C})^{r}$ w.r.t. $W$. The function $\mathbb{M}: \mathcal{M}_{r}(\mathcal{L}(\mathscr{C})) \times \mathcal{M}_{r}(\mathcal{L}(\mathscr{C})) \rightarrow \mathbb{R}$ defined by

$$
\mathbb{M}\left(\mathscr{Y}_{1}, \mathscr{Y}_{2}\right)=\min _{\sigma, \sigma^{\prime} \in \Pi_{r}} M\left(\sigma\left(\mathscr{Y}_{1}\right), \sigma^{\prime}\left(\mathscr{Y}_{2}\right)\right)
$$

is a monometric on $\mathcal{M}_{r}(\mathcal{L}(\mathscr{C}))$ w.r.t. $\mathbb{W}$, the betweenness relation on $\mathcal{M}_{r}(\mathcal{L}(\mathscr{C}))$ induced by $W$ and $M$.

Proof: We prove that $\mathbb{M}$ satisfies the three axioms of a monometric on $\mathcal{M}_{r}(\mathcal{L}(\mathscr{C}))$ w.r.t. $\mathbb{W}$. 
Non-negativity: immediate.

Coincidence: for any $\mathscr{Y}_{1}, \mathscr{Y}_{2} \in \mathcal{M}_{r}(\mathcal{L}(\mathscr{C}))$, it holds that

$$
\begin{aligned}
& \mathbb{M}\left(\mathscr{Y}_{1}, \mathscr{Y}_{2}\right):=\min _{\sigma, \sigma^{\prime} \in \Pi_{r}} M\left(\sigma\left(\mathscr{Y}_{1}\right), \sigma^{\prime}\left(\mathscr{Y}_{2}\right)\right)=0 \\
& \Leftrightarrow \quad\left(\exists \sigma_{1}, \sigma_{2} \in \Pi_{r}\right)\left(M\left(\sigma_{1}\left(\mathscr{Y}_{1}\right), \sigma_{2}\left(\mathscr{Y}_{2}\right)\right)=0\right) \\
& \Leftrightarrow \quad\left(\exists \sigma_{1}, \sigma_{2} \in \Pi_{r}\right)\left(\sigma_{1}\left(\mathscr{Y}_{1}\right)=\sigma_{2}\left(\mathscr{Y}_{2}\right)\right) \\
& \Leftrightarrow \quad \mathscr{Y}_{1}=\mathscr{Y}_{2} .
\end{aligned}
$$

Compatibility: for any $\mathscr{Y}_{1}, \mathscr{Y}_{2}, \mathscr{Y}_{3} \in \mathcal{M}_{r}(\mathcal{L}(\mathscr{C}))$ such that $\left(\mathscr{Y}_{1}, \mathscr{Y}_{2}, \mathscr{Y}_{3}\right) \in \mathbb{W}$, it holds by definition for any $\left(\sigma_{1}, \sigma_{2}, \sigma_{3}\right) \in \Theta_{r}^{M}\left(\mathscr{Y}_{1}, \mathscr{Y}_{2}, \mathscr{Y}_{3}\right)$ that

$$
\left(\sigma_{1}\left(\mathscr{Y}_{1}\right), \sigma_{2}\left(\mathscr{Y}_{2}\right), \sigma_{3}\left(\mathscr{Y}_{3}\right)\right) \in \mathbb{W} .
$$

Hence,

$$
\begin{aligned}
\mathbb{M}\left(\mathscr{Y}_{1}, \mathscr{Y}_{2}\right) & =\min _{\sigma, \sigma^{\prime} \in \Pi_{r}} M\left(\sigma\left(\mathscr{Y}_{1}\right), \sigma^{\prime}\left(\mathscr{Y}_{2}\right)\right)=M\left(\sigma_{1}\left(\mathscr{Y}_{1}\right), \sigma_{2}\left(\mathscr{Y}_{2}\right)\right) \\
& \leq M\left(\sigma_{1}\left(\mathscr{Y}_{1}\right), \sigma_{3}\left(\mathscr{Y}_{3}\right)\right)=\min _{\sigma, \sigma^{\prime} \in \Pi_{r}} M\left(\sigma\left(\mathscr{Y}_{1}\right), \sigma^{\prime}\left(\mathscr{Y}_{3}\right)\right)=\mathbb{M}\left(\mathscr{Y}_{1}, \mathscr{Y}_{3}\right) .
\end{aligned}
$$

Thus, $\mathbb{M}$ is a monometric on $\mathcal{M}_{r}(\mathcal{L}(\mathscr{C}))$ w.r.t. $\mathbb{W}$.

The following corollary shows how any monometric on $\mathcal{L}(\mathscr{C})$ naturally leads to a monometric on $\mathcal{M}_{r}(\mathcal{L}(\mathscr{C}))$.

Corollary 3.24 Let $\mathscr{C}$ be a set of $k$ candidates, $r$ be the number of voters, $w$ be a betweenness relation on $\mathcal{L}(\mathscr{C})$ and $m: \mathcal{L}(\mathscr{C}) \times \mathcal{L}(\mathscr{C}) \rightarrow \mathbb{R}$ be an order-invariant monometric on $\mathcal{L}(\mathscr{C})$ w.r.t. $w$. The function $\mathbb{M}: \mathcal{M}_{r}(\mathcal{L}(\mathscr{C})) \times \mathcal{M}_{r}(\mathcal{L}(\mathscr{C})) \rightarrow \mathbb{R}$ defined by

$$
\mathbb{M}\left(\mathscr{Y}_{1}, \mathscr{Y}_{2}\right)=\min _{\sigma \in \Pi_{r}} \sum_{i=1}^{r} m\left(\succ_{i}^{1}, \succ_{\sigma(i)}^{2}\right)
$$

is a monometric on $\mathcal{M}_{r}(\mathcal{L}(\mathscr{C}))$ w.r.t. $\mathbb{W}$, the betweenness relation on $\mathcal{M}_{r}(\mathcal{L}(\mathscr{C}))$ induced by $W$ (the betweenness relation on $\mathcal{L}(\mathscr{C})$ induced by $w$ ) and $M$ (the monometric on $\mathcal{L}(\mathscr{C})$ induced by the addition).

Considering the monometrics on $\mathcal{L}(\mathscr{C})$ introduced in Subsection 3.2.2, we obtain the following monometrics on $\mathcal{M}_{r}(\mathcal{L}(\mathscr{C}))$ : 
(i) $\mathbb{M}_{1}\left(\mathscr{Y}_{1}, \mathscr{Y}_{2}\right)=\min _{\sigma \in \Pi_{r}} \sum_{i=1}^{r} K\left(\succ_{i}^{1}, \succ_{\sigma(i)}^{2}\right)$. The monometric $\mathbb{M}_{1}$ counts the number of reversals needed in order to go from an anonymised profile of rankings to another one.

(ii) $\mathbb{M}_{2}\left(\mathscr{Y}_{1}, \mathscr{Y}_{2}\right)=\min _{\sigma \in \Pi_{r}} \sum_{i=1}^{r} 1-\delta\left(\succ_{i}^{1}, \succ_{\sigma(i)}^{2}\right)$. The monometric $\mathbb{M}_{2}$ counts the number of rankings that differ from an anonymised profile of rankings to another one.

(iii) $\mathbb{M}_{3}\left(\mathscr{Y}_{1}, \mathscr{Y}_{2}\right)=\min _{\sigma \in \Pi_{r}} \sum_{i=1}^{r} m_{3}\left(\succ_{i}^{1}, \succ_{\sigma(i)}^{2}\right)$. The monometric $\mathbb{M}_{3}$ counts the number of high-ranked candidates that need to be changed in order to go from an anonymised profile of rankings to another one.

(iv) $\mathbb{M}_{4}\left(\mathscr{Y}_{1}, \mathscr{Y}_{2}\right)=\min _{\sigma \in \Pi_{r}} \sum_{i=1}^{r} K\left(\succ_{i}^{1}, \succ_{\sigma(i)}^{2}\right)^{2}$. The monometric $\mathbb{M}_{4}$ computes the the squared number of reversals needed in order to go from an anonymised profile of rankings to another one.

(v) $\mathbb{M}_{5}\left(\mathscr{Y}_{1}, \mathscr{Y}_{2}\right)=\min _{\sigma \in \Pi_{r}} \sum_{i=1}^{r} K_{l, \frac{k(k-1)}{2}}\left(\succ_{i}^{1}, \succ_{\sigma(i)}^{2}\right)$ (with $\left.l \in\left\{1, \ldots, \frac{k(k-1)}{2}\right\}\right)$. The monometric $\mathbb{M}_{5}$ fixes a maximum admissible disagreement between rankings and counts the number of reversals needed in order to go from an anonymised profile of rankings to another one, penalizing this count each time that this maximum admissible disagreement is reached.

(vi) $\mathbb{M}_{6}\left(\mathscr{Y}_{1}, \mathscr{Y}_{2}\right)=1-\delta\left(\mathscr{Y}_{1}, \mathscr{Y}_{2}\right)$. The monometric $\mathbb{M}_{6}$ only takes into account whether or not two anonymised profiles of rankings differ. It must be noted that $\mathbb{M}_{6}$ is a monometric on $\mathcal{M}_{r}(\mathcal{L}(\mathscr{C}))$ that is not defined by means of a monometric on $\mathcal{L}(\mathscr{C})$.

Remark 3.25 As the addition is commutative, permutations of the first anonymised profile are not needed.

In the following example, these concepts are illustrated. 
Example 3.26 Let $\mathscr{Y}_{1}, \mathscr{Y}_{2}$ and $\mathscr{Y}_{3}$ be the following anonymised profiles of rankings on $\mathscr{C}=\{a, b, c, d\}$ :

$$
\begin{aligned}
& \mathscr{Y}_{1}=\left\{\left\{\succ_{1}^{1}, \succ_{2}^{1}\right\}\right\}=\{\{a \succ b \succ c \succ d, d \succ c \succ b \succ a\}, \\
& \mathscr{Y}_{2}=\left\{\left\{\succ_{1}^{2}, \succ_{2}^{2}\right\}\right\}=\{\{a \succ d \succ b \succ c, d \succ c \succ b \succ a\}, \\
& \mathscr{Y}_{3}=\left\{\left\{\succ_{1}^{3}, \succ_{2}^{3}\right\}\right\}=\{\{d \succ a \succ b \succ c, c \succ d \succ b \succ a\}\} .
\end{aligned}
$$

In this example, we will consider $W$ to be the betweenness relation on $\mathcal{L}(\mathscr{C})^{r}$ induced by Kemeny's betweenness relation $w$ on $\mathcal{L}(\mathscr{C})$.

As was stated in Proposition 3.22, in case the monometric $M_{1}$ (w.r.t. $W$ ) on $\mathcal{L}(\mathscr{C})^{r}$ given by the sum of the Kendall distances is considered, a betweenness relation $\mathbb{W}_{1}$ on $\mathcal{M}_{r}(\mathcal{L}(\mathscr{C}))$ is induced by $W$ and $M_{1}$.

In case $r=2$, it holds that $\Pi_{r}=\left\{\sigma_{I}, \sigma_{R}\right\}$, with $\sigma_{I}=(1,2)$ and $\sigma_{R}=(2,1)$. Considering $M_{1}$, only $\left(\sigma_{I}, \sigma_{I}\right)$ and $\left(\sigma_{R}, \sigma_{R}\right)$ minimize $M_{1}\left(\sigma\left(\mathscr{Y}_{1}\right), \sigma^{\prime}\left(\mathscr{Y}_{2}\right)\right)$. Analogously, only $\left(\sigma_{I}, \sigma_{I}\right)$ and $\left(\sigma_{R}, \sigma_{R}\right)$ minimize $M_{1}\left(\sigma\left(\mathscr{Y}_{1}\right), \sigma^{\prime}\left(\mathscr{Y}_{3}\right)\right)$ and only $\left(\sigma_{I}, \sigma_{I}\right)$ and $\left(\sigma_{R}, \sigma_{R}\right)$ minimize $M_{1}\left(\sigma\left(\mathscr{Y}_{2}\right), \sigma^{\prime}\left(\mathscr{Y}_{3}\right)\right)$. Therefore, there are only two optimal permutation triplets $\left(\sigma_{1}, \sigma_{2}, \sigma_{3}\right) \in$ $\Theta_{r}^{M}\left(\mathscr{Y}_{1}, \mathscr{Y}_{2}, \mathscr{Y}_{3}\right):\left(\sigma_{I}, \sigma_{I}, \sigma_{I}\right)$ and $\left(\sigma_{R}, \sigma_{R}, \sigma_{R}\right)$.

Firstly, considering $\left(\sigma_{1}, \sigma_{2}, \sigma_{3}\right)=\left(\sigma_{I}, \sigma_{I}, \sigma_{I}\right)$, it holds that

$$
\begin{aligned}
& \left(\succ_{\sigma_{I}(1)}^{1}, \succ_{\sigma_{I}(1)}^{2}, \succ_{\sigma_{I}(1)}^{3}\right)=\left(\succ_{1}^{1}, \succ_{1}^{2}, \succ_{1}^{3}\right)=(a \succ b \succ c \succ d, a \succ d \succ b \succ c, d \succ a \succ b \succ c) \in w \\
& \left(\succ_{\sigma_{I}(2)}^{1}, \succ_{\sigma_{I}(2)}^{2}, \succ_{\sigma_{I}(2)}^{3}\right)=\left(\succ_{2}^{1}, \succ_{2}^{2}, \succ_{2}^{3}\right)=(d \succ c \succ a \succ b, d \succ c \succ b \succ a, c \succ d \succ b \succ a) \in w
\end{aligned}
$$

and, therefore, $\left(\sigma_{I}\left(\mathscr{Y}_{1}\right), \sigma_{I}\left(\mathscr{Y}_{2}\right), \sigma_{I}\left(\mathscr{Y}_{3}\right)\right) \in W$. Analogously, considering $\left(\sigma_{1}, \sigma_{2}, \sigma_{3}\right)=$ $\left(\sigma_{R}, \sigma_{R}, \sigma_{R}\right)$, it holds that

$$
\begin{aligned}
& \left(\succ_{\sigma_{R}(1)}^{1}, \succ_{\sigma_{R}(1)}^{2}, \succ_{\sigma_{R}(1)}^{3}\right)=\left(\succ_{2}^{1}, \succ_{2}^{2}, \succ_{2}^{3}\right)=(d \succ c \succ a \succ b, d \succ c \succ b \succ a, c \succ d \succ b \succ a) \in w \\
& \left(\succ_{\sigma_{R}(2)}^{1}, \succ_{\sigma_{R}(2)}^{2}, \succ_{\sigma_{R}(2)}^{3}\right)=\left(\succ_{1}^{1}, \succ_{1}^{2}, \succ_{1}^{3}\right)=(a \succ b \succ c \succ d, a \succ d \succ b \succ c, d \succ a \succ b \succ c) \in w
\end{aligned}
$$

Therefore, $\left(\sigma_{R}\left(\mathscr{Y}_{1}\right), \sigma_{R}\left(\mathscr{Y}_{2}\right), \sigma_{R}\left(\mathscr{Y}_{3}\right)\right) \in W$. Finally, as every optimal permutation triplet belongs to $W$, it holds that $\left(\mathscr{Y}_{1}, \mathscr{Y}_{2}, \mathscr{Y}_{3}\right) \in \mathbb{W}_{1}$.

Note that, in case the monometric $\mathbb{M}_{1}$ on $\mathcal{M}_{r}\left(\mathcal{L}(\mathscr{C})\right.$ ) (w.r.t. $\left.\mathbb{W}_{1}\right)$ defined as

$$
\mathbb{M}_{1}\left(\mathscr{Y}, \mathscr{Y}^{\prime}\right)=\min _{\sigma, \sigma^{\prime} \in \Pi_{r}} M_{1}\left(\sigma(\mathscr{Y}), \sigma^{\prime}\left(\mathscr{Y}^{\prime}\right)\right)
$$


is considered, it holds that

$$
\begin{aligned}
\mathbb{M}_{1}\left(\mathscr{Y}_{1}, \mathscr{Y}_{2}\right)= & \min _{\sigma, \sigma^{\prime} \in \Pi_{r}} M_{1}\left(\sigma\left(\mathscr{Y}_{1}\right), \sigma^{\prime}\left(\mathscr{Y}_{2}\right)\right) \\
= & \min \left(M_{1}\left(\sigma_{I}\left(\mathscr{Y}_{1}\right), \sigma_{I}\left(\mathscr{Y}_{2}\right)\right), M_{1}\left(\sigma_{I}\left(\mathscr{Y}_{1}\right), \sigma_{R}\left(\mathscr{Y}_{2}\right)\right),\right. \\
& \left.M_{1}\left(\sigma_{R}\left(\mathscr{Y}_{1}\right), \sigma_{R}\left(\mathscr{Y}_{2}\right)\right), M_{1}\left(\sigma_{R}\left(\mathscr{Y}_{1}\right), \sigma_{I}\left(\mathscr{Y}_{2}\right)\right)\right) \\
= & \min (3,9,3,9)=3 .
\end{aligned}
$$

Analogously,

$$
\begin{aligned}
\mathbb{M}_{1}\left(\mathscr{Y}_{1}, \mathscr{Y}_{3}\right)= & \min _{\sigma, \sigma^{\prime} \in \Pi_{r}} M_{1}\left(\sigma\left(\mathscr{Y}_{1}\right), \sigma^{\prime}\left(\mathscr{Y}_{3}\right)\right) \\
= & \min \left(M_{1}\left(\sigma_{I}\left(\mathscr{Y}_{1}\right), \sigma_{I}\left(\mathscr{Y}_{3}\right)\right), M_{1}\left(\sigma_{I}\left(\mathscr{Y}_{1}\right), \sigma_{R}\left(\mathscr{Y}_{3}\right)\right),\right. \\
& \left.M_{1}\left(\sigma_{R}\left(\mathscr{Y}_{1}\right), \sigma_{R}\left(\mathscr{Y}_{3}\right)\right), M_{1}\left(\sigma_{R}\left(\mathscr{Y}_{1}\right), \sigma_{I}\left(\mathscr{Y}_{3}\right)\right)\right) \\
= & \min (5,7,5,7)=5 .
\end{aligned}
$$

Therefore, as expected due to $\left(\mathscr{Y}_{1}, \mathscr{Y}_{2}, \mathscr{Y}_{3}\right) \in \mathbb{W}_{1}$, it holds that

$$
\mathbb{M}_{1}\left(\mathscr{Y}_{1}, \mathscr{Y}_{2}\right)=3 \leq 5=\mathbb{M}_{1}\left(\mathscr{Y}_{1}, \mathscr{Y}_{3}\right)
$$

On the other hand, in case the monometric $M_{2}$ (w.r.t. $W$ ) on $\mathcal{L}(\mathscr{C})^{r}$ given by the sum of the zero-one comparisons between rankings is considered, a betweenness relation $\mathbb{W}_{2}$ on $\mathcal{M}_{r}(\mathcal{L}(\mathscr{C}))$, is also induced by $W$ and $M_{2}$, as was stated in Proposition 3.22.

Now, considering $M_{2}$, any couple of permutations minimizes $M_{2}\left(\sigma\left(\mathscr{Y}_{1}\right), \sigma^{\prime}\left(\mathscr{Y}_{2}\right)\right)$. Analogously, any couple of permutations minimizes $M_{2}\left(\sigma\left(\mathscr{Y}_{1}\right), \sigma^{\prime}\left(\mathscr{Y}_{3}\right)\right)$ and $M_{2}\left(\sigma\left(\mathscr{Y}_{2}\right), \sigma^{\prime}\left(\mathscr{Y}_{3}\right)\right)$. Therefore, considering, for instance, $\left(\sigma_{I}, \sigma_{R}, \sigma_{I}\right)$, it holds that

$$
\left\{\begin{array}{l}
M_{2}\left(\sigma_{I}\left(\mathscr{Y}_{1}\right), \sigma_{R}\left(\mathscr{Y}_{2}\right)\right)=\min _{\sigma, \sigma^{\prime} \in \Pi_{r}} M_{2}\left(\sigma\left(\mathscr{Y}_{1}\right), \sigma^{\prime}\left(\mathscr{Y}_{2}\right)\right), \\
M_{2}\left(\sigma_{I}\left(\mathscr{Y}_{1}\right), \sigma_{I}\left(\mathscr{Y}_{3}\right)\right)=\min _{\sigma, \sigma^{\prime} \in \Pi_{r}} M_{2}\left(\sigma\left(\mathscr{Y}_{1}\right), \sigma^{\prime}\left(\mathscr{Y}_{3}\right)\right), \\
M_{2}\left(\sigma_{R}\left(\mathscr{Y}_{2}\right), \sigma_{I}\left(\mathscr{Y}_{3}\right)\right)=\min _{\sigma, \sigma^{\prime} \in \Pi_{r}} M_{2}\left(\sigma\left(\mathscr{Y}_{2}\right), \sigma^{\prime}\left(\mathscr{Y}_{3}\right)\right),
\end{array}\right.
$$

i.e., it is an optimal permutation triplet. However, as

$$
\begin{aligned}
& \left(\succ_{\sigma_{I}(1)}^{1}, \succ_{\sigma_{R}(1)}^{2}, \succ_{\sigma_{I}(1)}^{3}\right)=\left(\succ_{1}^{1}, \succ_{2}^{2}, \succ_{1}^{3}\right)=(a \succ b \succ c \succ d, d \succ c \succ b \succ a, d \succ a \succ b \succ c) \notin w, \\
& \left(\succ_{\sigma_{I}(2)}^{1}, \succ_{\sigma_{R}(2)}^{2}, \succ_{\sigma_{I}(2)}^{3}\right)=\left(\succ_{2}^{1}, \succ_{1}^{2}, \succ_{2}^{3}\right)=(d \succ c \succ a \succ b, a \succ d \succ b \succ c, c \succ d \succ b \succ a) \notin w,
\end{aligned}
$$

it holds that $\left(\sigma_{I}\left(\mathscr{Y}_{1}\right), \sigma_{R}\left(\mathscr{Y}_{2}\right), \sigma_{I}\left(\mathscr{Y}_{3}\right)\right) \notin W$.

Thus, $\left(\mathscr{Y}_{1}, \mathscr{Y}_{2}, \mathscr{Y}_{3}\right) \notin \mathbb{W}_{2}$ (even though $\left.\left(\mathscr{Y}_{1}, \mathscr{Y}_{2}, \mathscr{Y}_{3}\right) \in \mathbb{W}_{1}\right)$. 


\subsection{Cost calculation as an optimization problem}

Many problems related with monometrics can be formulated as optimization problems. In this section, two particular problems are analysed: the calculation of the cost of changing an anonymised profile of rankings into another one and the search for a 'closest' (anonymised) profile of rankings satisfying a certain property.

\subsubsection{Calculating the cost of changing an anonymised profile of rankings into another one}

Calculating the cost of changing a profile into another one considering a monometric on $\mathcal{L}(\mathscr{C})^{r}$ of the form

$$
M\left(\mathscr{R}_{1}, \mathscr{R}_{2}\right)=\sum_{i=1}^{r} m\left(\succ_{i}^{1}, \succ_{i}^{2}\right),
$$

where $m$ is a monometric on $\mathcal{L}(\mathscr{C})$, is a trivial problem.

However, calculating the cost of changing an anonymised profile into another one considering a monometric on $\mathcal{M}_{r}(\mathcal{L}(\mathscr{C}))$ of the form

$$
\mathbb{M}\left(\mathscr{Y}_{1}, \mathscr{Y}_{2}\right)=\min _{\sigma} \sum_{i=1}^{r} m\left(\succ_{i}^{1}, \succ_{\sigma(i)}^{2}\right),
$$

where $m$ is a monometric on $\mathcal{L}(\mathscr{C})$, is not trivial as the best matching needs to be found. The search for this best matching can be seen as an assignment problem [110], which is a particular case of Integer Linear Programming (ILP).

In an assignment problem, there are a number of agents and the same number of tasks. Each agent has an associated cost for performing each of the tasks. The goal of an assignment problem is to search for a distribution of tasks with the minimum possible total cost such that every agent performs one and exactly one task.

In our particular setting, we have two anonymised profiles $\mathscr{Y}_{1}$ and $\mathscr{Y}_{2}$ consisting of $r$ rankings. For solving the problem, we will define $r^{2}$ variables $x_{i j}$ taking values in $\{0,1\}$. For any $i, j \in\{1, \ldots, r\}, x_{i j}=1$ means that the $i$-th ranking in $\mathscr{Y}_{1}$ is assigned to the $j$-th ranking in $\mathscr{Y}_{2}$. Analogously, $x_{i j}=0$ means that the $i$-th ranking in $\mathscr{Y}_{1}$ is not assigned to the $j$-th ranking in $\mathscr{Y}_{2}$. As the assignment needs to be a bijection, the sums of the $x_{i j}$ for 
either $i$ 's or $j$ 's needs to be equal to one. Also a matrix of costs $C$ needs to be defined, where the element $C_{i j}$ represents the cost of changing the $i$-th ranking in $\mathscr{Y}_{1}$ into the $j$-th ranking in $\mathscr{Y}_{2}$.

The mathematical formulation of the problem is:

$$
\begin{array}{cl}
\text { Minimize } & \sum_{i=1}^{r} \sum_{j=1}^{r} C_{i j} x_{i j} \text { w.r.t. }\left\{x_{i j}\right\}_{i, j=1}^{r} \\
\text { s.t. } & \sum_{i=1}^{r} x_{i j}=1, \text { for any } j \in\{1, \ldots, r\}, \\
& \sum_{j=1}^{r} x_{i j}=1, \text { for any } i \in\{1, \ldots, r\}, \\
& x_{i j} \geq 0, \text { for any } i, j \in\{1, \ldots, r\}, \\
& x_{i j} \in \mathbb{Z}, \text { for any } i, j \in\{1, \ldots, r\} .
\end{array}
$$

Assignment problems are one of the fundamental problems in combinatorial optimization and have been deeply studied. There exist several methods to solve this problem in polynomial time, for instance, the Hungarian method [86].

\subsubsection{Searching the 'closest' (anonymised) profile of rankings}

In some cases, we are not interested in obtaining the cost of changing a profile of rankings into another one, but in the 'closest' profile of rankings satisfying a certain property given a profile of rankings $\mathscr{R}$. In this case, we will no longer have an assignment problem, as the 'tasks' are unknown. However, a transportation problem can be defined [101].

In a transportation problem, we have a number of supply points and a number of demand points. At each supply point certain product is produced and it needs to be transported to the demand points satisfying the required demands at each demand point. Transporting a unit of product from a supply point to a demand point has an associated cost. An optimal transportation distribution needs to be defined such that the demand is satisfied.

Note that in our particular setting, each ranking in $\mathcal{L}(\mathscr{C})$ is both a supply point and a demand point. The quantity of product produced at each supply point is given by $\mathscr{R}$ and, as the number of rankings needs to be preserved, all the produced units need to be transported to a demand point. There are no demands at each demand point. Formalizing the problem is similar to the assignment problem where $(k !)^{2}$ variables $x_{i j}$ taking values 
in $\mathbb{N} \cup\{0\}$ are defined, $k$ being the number of candidates in $\mathscr{C}$. For any $i, j \in\{1, \ldots, k !\}$, $x_{i j}=n$ means that $n$ units of the $i$-th ranking in $\mathcal{L}(\mathscr{C})$ are assigned to $n$ units of the $j$-th rankings in $\mathcal{L}(\mathscr{C})$. In this case, we have an initial profile of rankings where each ranking $\succ_{i}$ appears $s_{i}$ times. These $s_{i}$ can be seen as the number of units of product that are produced at each supply point. The goal is to distribute these products satisfying the required global demand and a certain property $P$ that can be defined as a linear condition on the profile of rankings. The constraints used in the assignment problem need to be modified, leading to the following problem:

$$
\begin{aligned}
\text { Minimize } & \sum_{i=1}^{k !} \sum_{j=1}^{k !} C_{i j} x_{i j} \text { w.r.t. }\left\{x_{i j}\right\}_{i, j=1}^{k !} \\
\text { s.t. } & \sum_{j=1}^{k !} x_{i j}=s_{i}, \text { for any } i \in\{1, \ldots, k !\} \\
& x_{i j} \geq 0, \text { for any } i, j \in\{1, \ldots, k !\}, \\
& x_{i j} \in \mathbb{Z}, \text { for any } i, j \in\{1, \ldots, k !\} \\
& P, \text { the property to be satisfied by the profile of rankings } .
\end{aligned}
$$

Transportation problems with additional constraints can also be solved in polynomial time [84]. Note that, on the one hand, in the calculation of the cost of changing a profile of rankings into another one, this polynomial time is in terms of $r$, the number of rankings in the profiles. On the other hand, in the calculation of the 'closest' profile of rankings satisfying a fixed property, this polynomial time is in terms of $k$ !, which turns out to be an euphemism for non-polynomial time. This is an obvious computational drawback, even though in some fields of application of the aggregation of rankings, for instance in social choice theory, the number of candidates in $\mathscr{C}$ is typically quite small. The simplest case where the property $P$ requires all the rankings in the profile to be the same (Kemeny [80]) has called the attention of several researchers. Although to compute its winning ranking is proved to be an NP-hard problem [9], several algorithms improving the performance of the method have been proposed [1, 13, 77]. Further research on the computational aspects of the search for the 'closest' profile of rankings still needs to be addressed in the general case.

Note that in case the required property does not take the order of the voters into account, every profile of rankings given by a permutation of an anonymised profile of rankings satisfies the same property. In that case, the search for the 'closest' profile of rankings satisfying certain property is equivalent to the search for the 'closest' anonymised profile of rankings. 


\subsubsection{The special case of the zero-one monometric}

The complexity of the problem of finding the 'closest' profile of rankings may depend on the chosen monometric. For instance, a less complex problem can be solved in case the zero-one monometric on $\mathcal{L}(\mathscr{C})^{r}$ is considered.

$$
M_{2}\left(\mathscr{R}_{1}, \mathscr{R}_{2}\right)=\sum_{i=1}^{r} 1-\delta\left(\succ_{i}^{1}, \succ_{i}^{2}\right) .
$$

In this case, as every change is equally costly, looking for the assignment that will lead to the lowest cost is not necessary. Therefore, an easier Integer Linear Programming problem can be defined.

Firstly, we will define $k$ ! positive integer variables that will be denoted by $x_{i}$. Each $x_{i}$ will represent the number of times the $i$-th ranking in $\mathcal{L}(\mathscr{C})$ appears in the closest profile of rankings. Analogously, each $o_{i}$ will represent the number of times the $i$-th ranking in $\mathcal{L}(\mathscr{C})$ appears in the given profile of rankings.

We will minimize the zero-one monometric $M_{2}\left(\mathscr{R}_{1}, \mathscr{R}_{2}\right)$, which can be written in terms of our variables as:

$$
\frac{1}{2} \sum_{i=1}^{k !}\left|x_{i}-o_{i}\right|
$$

Note that, due to the absolute values, we have an Integer Non-Linear Programming problem (INLP) instead of an Integer Linear Programming problem (ILP). Fortunately, an absolute value constraint can be turned into two linear constraints by adding an additional positive variable. In particular, minimizing Eq. (3.1) is equivalent to minimizing:

$$
\frac{1}{2} \sum_{i=1}^{k !} y_{i}
$$

under the additional constraints, for any $i \in\{1, \ldots, k !\}$,

$$
\begin{aligned}
& x_{i}-o_{i} \leq y_{i}, \\
& o_{i}-x_{i} \leq y_{i} .
\end{aligned}
$$


Therefore, the problem to be optimized is the following:

$$
\begin{aligned}
\text { Minimize } & \frac{1}{2} \sum_{i=1}^{k !} y_{i} \text { w.r.t. }\left\{y_{i}\right\}_{i=1}^{k !} \\
\text { s.t. } & \sum_{i=1}^{k !} x_{i}=r \\
& x_{i}-o_{i} \leq y_{i}, \\
& o_{i}-x_{i} \leq y_{i}, \\
& x_{i}, y_{i} \geq 0, \text { for any } i \in\{1, \ldots, k !\} \\
& x_{i}, y_{i} \in \mathbb{Z}, \text { for any } i \in\{1, \ldots, k !\} \\
& P, \text { the property to be satisfied by the profile of rankings } .
\end{aligned}
$$

Although the computational cost of the algorithm is still polynomial in terms of $k$ !, a significant reduction in the number of variables was addressed $\left(2 k\right.$ ! instead of $\left.(k !)^{2}\right)$ with respect to the general case.

\subsection{Hierarchical combination of monometrics}

There exist many different monometrics defined according to different criteria: number of reversals made, number of rankings changed, etc. Moreover, in general, the uniqueness of a 'closest' (anonymised) profile of rankings is not assured. An intuitive idea to reduce the set of 'closest' (anonymised) profiles of rankings satisfying a fixed property is to hierarchically combine monometrics.

In the following proposition, we will see that any convex combination of a finite number of monometrics is a monometric.

Proposition 3.27 Let $A$ and $B$ be two sets such that $A \subseteq B$, let $R$ be a betweenness relation on $B$ and $M_{1}, \ldots, M_{n}: A \times B \rightarrow \mathbb{R}$ be $n$ monometrics (w.r.t. $R$ ). For any $\left(\alpha_{1}, \ldots, \alpha_{n}\right) \in[0,1]^{n}$ such that $\sum_{i=1}^{n} \alpha_{i}=1$, the function $M: A \times B \rightarrow \mathbb{R}$ defined by

$$
M(a, b)=\sum_{i=1}^{n} \alpha_{i} M_{i}(a, b)
$$

is a monometric w.r.t. $R$. 
Proof: We prove that $M$ satisfies the three axioms of a monometric w.r.t. $R$.

Non-negativity: immediate.

Coincidence: for any $a \in A$, any $b \in B$ and any $\left(\alpha_{1}, \ldots, \alpha_{n}\right) \in[0,1]^{n}$, it holds that

$$
\begin{aligned}
& M(a, b):=\sum_{i=1}^{n} \alpha_{i} M_{i}(a, b)=0 \\
& \Leftrightarrow \quad(\forall i \in\{1, \ldots, n\})\left(\alpha_{i}=0 \vee M_{i}(a, b)=0\right) \\
& \quad \wedge(\exists i \in\{1, \ldots, n\})\left(\alpha_{i} \neq 0 \wedge M_{i}(a, b)=0\right) \\
& \Leftrightarrow a=b .
\end{aligned}
$$

Compatibility: for any $a \in A$ and any $b, c \in B$ such that $[a, b, c]$ and any $\left(\alpha_{1}, \ldots, \alpha_{n}\right) \in$ $[0,1]^{n}$, it holds that

$$
\begin{aligned}
M(a, b) & =\sum_{i=1}^{n} \alpha_{i} M_{i}(a, b) \\
& \leq \sum_{i=1}^{n} \alpha_{i} M_{i}(a, c) \\
& =M(a, c) .
\end{aligned}
$$

Thus, $M$ is a monometric w.r.t. $R$.

Remark 3.28 It must be noted that in the case of anonymised profiles of rankings, betweenness relations induced by monometrics on $\mathcal{L}(\mathscr{C})^{r}$ are commonly used. The result of Proposition 3.27 does not hold when considering, instead of a fixed betweenness relation, a different betweenness relation for each monometric. For instance, let us consider a betweenness relation $W$ on $\mathcal{L}(\mathscr{C})^{r}$. The function $\mathbb{M}: \mathcal{M}_{r}(\mathcal{L}(\mathscr{C})) \times \mathcal{M}_{r}(\mathcal{L}(\mathscr{C})) \rightarrow \mathbb{R}$ defined by

$$
\mathbb{M}\left(\mathscr{Y}_{1}, \mathscr{Y}_{2}\right)=\sum_{i=1}^{n} \alpha_{i} \mathbb{M}_{i}\left(\mathscr{Y}_{1}, \mathscr{Y}_{2}\right)
$$

where $\mathbb{M}_{1}, \ldots, \mathbb{M}_{n}$ are $n$ monometrics on $\mathcal{M}_{r}(\mathcal{L}(\mathscr{C}))$ w.r.t. $\mathbb{W}_{1}, \ldots, \mathbb{W}_{n}$ (the $n$ betweenness relations on $\mathcal{M}_{r}(\mathcal{L}(\mathscr{C}))$ induced by $\left.M_{1}, \ldots, M_{n}\right)$ respectively, is not guaranteed to satisfy compatibility w.r.t. $\mathbb{W}$ (the betweenness relation on $\mathcal{M}_{r}(\mathcal{L}(\mathscr{C})$ ) induced by $M$ ). This is because $\cap_{i=1}^{n} \mathbb{W}_{i}$ is not necessarily included in $\mathbb{W}$. 
According to these results, a hierarchical combination of two monometrics where the second monometric is used as a tie-breaker method between all the 'closest' anonymised profiles of rankings can be proposed.

Let us consider two monometrics on $\mathcal{L}(\mathscr{C})^{r}$ bounded from above by an upper bound $B_{u}$ and such that the cost of changing any profile of rankings into a different one is bounded from below by a lower bound $B_{l}>0$. If we consider the function

$$
M\left(\mathscr{R}_{1}, \mathscr{R}_{2}\right)=\alpha M_{1}\left(\mathscr{R}_{1}, \mathscr{R}_{2}\right)+(1-\alpha) M_{2}\left(\mathscr{R}_{1}, \mathscr{R}_{2}\right),
$$

then we can express $M_{2}$ as a tie-breaker of $M_{1}$ considering any $\left.\alpha \in\right] \frac{B_{u}}{B_{u}+B_{l}}, 1[$. Analogously, we can express $M_{1}$ as a tie-breaker of $M_{2}$ considering any $\left.\alpha \in\right] 0, \frac{B_{l}}{B_{u}+B_{l}}[$.

These results can be trivially extended to monometrics on $\mathcal{M}_{r}(\mathcal{L}(\mathscr{C}))$.

\begin{tabular}{|c|c|c|c|}
\hline$i$ & $M_{i} / \mathbb{M}_{i}$ & $B_{l}$ & $B_{u}$ \\
\hline \hline 1 & $M_{1} / \mathbb{M}_{1}$ & 1 & $\frac{r k(k-1)}{2}$ \\
\hline 2 & $M_{2} / \mathbb{M}_{2}$ & 1 & $r$ \\
\hline 3 & $M_{3} / \mathbb{M}_{3}$ & $2^{1-k}$ & $\frac{r}{2}$ \\
\hline 4 & $M_{4} / \mathbb{M}_{4}$ & 1 & $\left(\frac{r k(k-1)}{2}\right)^{2}$ \\
\hline 5 & $M_{5} / \mathbb{M}_{5}$ & 1 & $\frac{r k(k-1)}{2}$ \\
\hline 6 & $M_{6} / \mathbb{M}_{6}$ & 1 & 1 \\
\hline
\end{tabular}

Table 3.1: Lower and upper bounds of the monometrics on $\mathcal{L}(\mathscr{C})^{r}$ defined in Subsection 3.2 .3 (left) and of the monometrics on $\mathcal{M}_{r}(\mathcal{L}(\mathscr{C}))$ defined in Subsection 3.2 .4 (right).

Note that, as can be seen in Table 3.1, the monometrics on $\mathcal{L}(\mathscr{C})^{r}$ defined in Subsection 3.2 .3 and the monometrics on $\mathcal{M}_{r}(\mathcal{L}(\mathscr{C}))$ defined in Subsection 3.2 .4 are bounded in this way.

\subsection{Monometric rationalisation of ranking rules}

Under the name of metric rationalisation of ranking rules [2, 27, 49, 88, 100, 106], several authors have discussed how most ranking rules can be characterized as minimizing the 
distance to a consensus state for some appropriate distance function. In this section, we advocate for the use of a monometric instead, leading to the monometric rationalisation of ranking rules.

However, when we are not dealing with a notion of closeness in the most geometrical sense, the symmetry axiom of a distance function might not be necessary. Quoting Tversky and Gati on the study of similarity measures [158, 159], one can understand that the term closeness is not always interpreted as a symmetric term: "The poet writes "my love is as deep as the ocean', not 'the ocean is as deep as my love', because the ocean epitomizes depth".

Even more importantly, the triangle inequality of a distance function may not always be linked to a notion of closeness. Quoting again Tversky and Gati, one may think about how close two countries are: "Consider the similarity between countries: Jamaica is similar to Cuba (because of geographical proximity); Cuba is similar to Russia (because of their political affinity); but Jamaica and Russia are not similar at all. This example [...] suggests that the perceived distance of Jamaica to Russia exceeds the perceived distance of Jamaica to Cuba, plus that of Cuba to Russia - contrary to the triangle inequality."

As another example, when thinking of a human, a centaur and a horse, the term closeness is neither related with the triangle inequality (the perceived distance of a human to a horse exceeds the perceived distance of a human to a centaur, plus that of a centaur to a horse). Nevertheless, there is a clear betweenness relation: A centaur is between a human and a horse and, therefore, a human should always be closer to a centaur than to a horse.

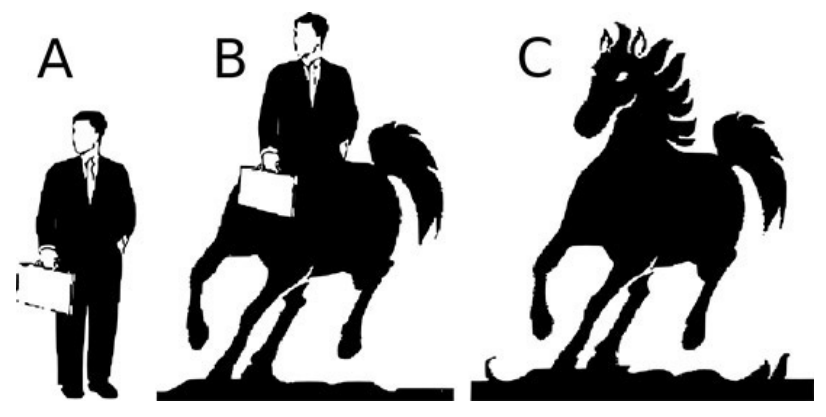

Figure 3.3: Images of a human (A), a centaur (B) and a horse (C).

Closeness is a vague term here. From a geometrical point of view, symmetry and the triangle inequality are needed. Nevertheless, in the rationalisation of ranking rules, closeness is not defined by a geometrical concept. Here, this closeness is related to the notion of 
(local) penalty function used in the aggregation of real numbers [25], where the axioms of symmetry and the triangle inequality are no longer required, but an additional axiom of quasi-convexity providing the penalty with a well-founded semantic basis is required. In this work, this well-founded semantic basis is captured by requiring the preservation of a natural betweenness relation. Therefore, closeness is no longer measured by a metric, but by a monometric.

In that way, monometrics (instead of distance functions) should be considered in the rationalisation of ranking rules. Of course, a meaningful betweenness relation needs to be fixed according to the nature of the considered problem. Although it may seem natural to consider the one defined by Kemeny, this does not need to be an imposition.

The two-step procedure of the rationalisation of ranking rules is now as follows:

(i) Search for the 'closest' profile of rankings in the chosen consensus state by means of a monometric.

(ii) Analysis of the consensus state to obtain the winning ranking.

Note that most consensus states do not take the order of the voters into account. Therefore, every profile of rankings given by a permutation of an anonymised profile of rankings is in the same consensus state of the anonymised profile as was explained in Subsection 3.3.2. In case the order of the rankings is not relevant, the search for the 'closest' profile of rankings in some consensus state is equivalent to the search for the 'closest' anonymised profile of rankings in the same consensus state. This result allows us to systematically work with anonymised profiles of rankings instead of profiles and to assure the privacy of the voters. Conversely, we can, and will, directly work with profiles of rankings in order to ease the understanding of this dissertation. Of course, when a consensus state takes the order of the rankings into account (such as dictatorship or other weighted voting systems), this anonymisation cannot be addressed.

We conclude that the use of a monometric better fits the spirit of the rationalisation of ranking rules than the use of a distance function. Indeed, the (mono)metric rationalisation of ranking rules can be understood as a penalty-based approach for the aggregation of rankings. Most of the times, as Yager described in his "general theory of information aggregation" back in 1993 [166], aggregation outside the real line is understood as a process of minimizing some notion of penalty that measures the deviation from a consensus ele- 
ment [62, 63]. Obviously, the ideas of Yager have long time been surpassed. However, over the years, the use of penalty functions has shifted towards the aggregation of values in a closed interval of the real line [24, 25, 26, 165, 167]. In this setting, the considered penalty is more similar to the notion of monometric than to the notion of a distance function, enforcing the monometric rationalisation of ranking rules over the metric rationalisation of ranking rules. 


\section{CHAPTER 4}

\section{Representations of votes}

In the field of social choice theory, the preferences of the voters are usually compressed into representations of votes gathering the most significant information. For instance, the Borda count [18] reduces the preferences given by the voters to the scorix [125], which is a matrix where the element at the $i$-th row and $j$-th column represents the number of times that the $i$-th candidate is ranked at the $j$-th position in the profile of rankings given by the voters. Besides the Borda count, all other scoring ranking rules [57, 102, 104, 169] also reduce the profile of rankings given by the voters to the scoring matrix. Based on the ideas of Condorcet [35], another representation of votes gathering a completely different type of information is commonly used in social choice theory: the votrix [124]. The votrix is a matrix where the element at the $i$-th row and $j$-th column represents the number of times that the $i$-th candidate is preferred to the $j$-th candidate in the preferences given by the voters. This type of representation of votes where candidates are head-to-head compared with each other is said to be based on pairwise information. Many other representations of votes based on pairwise information, such as the beatpath matrix [147] and the votex [124, have been used in social choice theory.

\subsection{The scorix}

Let us recall that the position of candidate $a_{i}$ in ranking $\succ_{j}$ is denoted by $P_{j}\left(a_{i}\right)$ (considering position 1 is the best). Each profile of rankings defines a matrix, henceforth called 
a scorix ${ }^{1}[125]$, where each row represents a candidate in $\mathscr{C}$ and each column represents a position $\ell \in\{1, \ldots, k\}$. In that way, the element at the $i$-th row and $\ell$-th column equals the number of times that the $i$-th candidate is ranked at the $\ell$-th position.

Definition 4.1 Let $\mathscr{C}$ be a set of $k$ candidates and $r$ be the number of voters. A matrix $S \in\{0,1, \ldots, r\}^{k \times k}$ is called a scorix (plural scorices) on $\mathscr{C}$ if there exists a profile $\mathscr{R}$ of $r$ rankings on $\mathscr{C}$ such that, for any $a_{i} \in \mathscr{C}$ and any $\ell \in\{1, \ldots, k\}$, it holds that

$$
S_{i \ell}=\#\left\{j \in\{1, \ldots, r\} \mid P_{j}\left(a_{i}\right)=\ell\right\} .
$$

The set of all scorices on $\mathscr{C}$ induced by any profile of $r$ rankings on $\mathscr{C}$ is denoted by $\mathcal{S}_{r}(\mathscr{C})$.

Remark 4.2 The notion of a scorix is well known [52, 53, 57, 58, 92, 93, 144, 154, 169] in social choice theory, usually considered either in the form of a matrix or in the form of an ensemble of vectors corresponding to the different rows of the scorix.

For any scorix $S \in \mathcal{S}_{r}(\mathscr{C})$, the $i$-th row $(i \in\{1, \ldots, k\})$ is called the vector of positions of candidate $a_{i}$ and is denoted by $S_{i}$. Note that, for any $i \in\{1, \ldots, k\}$, it holds that $S_{i} \in\{0,1, \ldots, r\}^{k}$.

In order to illustrate how the scorix induced by a profile of rankings can be obtained, we consider the following example.

Example 4.3 Consider a set of four candidates $\mathscr{C}=\{a, b, c, d\}$ and a profile of rankings $\mathscr{R}=\left(\succ_{i}\right)_{i=1}^{3}$ given by three voters. These rankings are shown in Table 4.1.

\begin{tabular}{|c|c|}
\hline$\# \succ_{i}$ & Rankings on $\mathscr{C}$ \\
\hline 1 & $a \succ b \succ c \succ d$ \\
1 & $a \succ d \succ b \succ c$ \\
1 & $d \succ a \succ c \succ b$ \\
\hline
\end{tabular}

Table 4.1: Frequency of the rankings on $\mathscr{C}$ expressed by three voters.

Note that candidate a is ranked twice at the first position and once at the second position and it is not ranked at the third or fourth position in any of the rankings. Therefore, the

\footnotetext{
${ }^{1}$ Scorix is a contraction of the term 'scoring matrix'.
} 
vector of positions of candidate a is $(2,1,0,0)$. In the same way, the vector of positions of candidate $b$ is $(0,1,1,1)$, the vector of positions of candidate $c$ is $(0,0,2,1)$ and the vector of positions of candidate $d$ is $(1,1,0,1)$. The scorix is then given by:

$$
S=\left(\begin{array}{llll}
2 & 1 & 0 & 0 \\
0 & 1 & 1 & 1 \\
0 & 0 & 2 & 1 \\
1 & 1 & 0 & 1
\end{array}\right)
$$

As the number of candidates and the number of candidates ranked at each position are constant, it is straightforward to see that a scorix always satisfies the properties listed in the following proposition.

Proposition 4.4 Let $\mathscr{C}$ be a set of $k$ candidates and $r$ be the number of voters. For any scorix $S \in \mathcal{S}_{r}(\mathscr{C})$, the following properties are fulfilled:

(i) Completeness in candidates: for any $i \in\{1, \ldots, k\}$, it holds that

$$
\sum_{\ell=1}^{k} S_{i \ell}=r .
$$

(ii) Completeness in positions: for any $\ell \in\{1, \ldots, k\}$, it holds that

$$
\sum_{i=1}^{k} S_{i \ell}=r .
$$

Proof: Completeness in candidates: for any $i \in\{1, \ldots, k\}, \sum_{\ell=1}^{k} S_{i \ell}$ represents the total number of times that the $i$-th candidate is ranked at any position; as every voter provides a ranking on the set of candidates, it obviously equals $r$.

Completeness in positions: for any $\ell \in\{1, \ldots, k\}, \sum_{i=1}^{k} S_{i \ell}$ represents the total number of times that any candidate is ranked at the $\ell$-th position; as every voter provides a ranking on the set of candidates, it obviously equals $r$.

The following result, due to Fine and Fine [52], expresses that any matrix satisfying the properties listed in Proposition 4.4 is a scorix, i.e., there exists a profile of rankings on the set of candidates such that the element at the $i$-th row and $\ell$-th column of the matrix equals the number of times that the $i$-th candidate is ranked at the $\ell$-th position. 
Proposition 4.5 Let $\mathscr{C}$ be a set of $k$ candidates and $r$ be the number of voters. Any matrix $S \in\{0,1, \ldots, r\}^{k \times k}$ satisfying the properties listed in Proposition 4.4 is a scorix.

\subsection{The votrix}

The strength of support of candidate $a_{i_{1}}$ over candidate $a_{i_{2}}$ is then defined as the number of times that candidate $a_{i_{1}}$ is preferred to candidate $a_{i_{2}}$ :

$$
s\left(a_{i_{1}}, a_{i_{2}}\right)=\#\left\{j \in\{1, \ldots, r\} \mid a_{i_{1}} \succ_{j} a_{i_{2}}\right\} .
$$

This strength of support defines a matrix $V$, henceforth called a votrix ${ }^{2}$ [124], where the element at the $i$-th row and the $j$-th column is the strength of support of the $i$-th candidate over the $j$-th candidate.

Definition 4.6 Let $\mathscr{C}$ be a set of $k$ candidates and $r$ be the number of voters. A matrix $V \in\{0,1, \ldots, r\}^{k \times k}$ is called a votrix (plural votrices) on $\mathscr{C}$ if there exists a profile $\mathscr{R}$ of $r$ rankings on $\mathscr{C}$ such that, for any $a_{i}, a_{j} \in \mathscr{C}$, it holds that

$$
V_{i j}=s\left(a_{i}, a_{j}\right)
$$

The set of all votrices on $\mathscr{C}$ induced by any profile of $r$ rankings on $\mathscr{C}$ is denoted by $\mathcal{V}_{r}(\mathscr{C})$.

Remark 4.7 The notion of a votrix is well known [35, 170] in social choice theory.

In order to illustrate how the votrix induced by a profile of rankings can be obtained, we consider the following example.

Example 4.8 Consider a set of four candidates $\mathscr{C}=\{a, b, c, d\}$ and a profile of rankings $\mathscr{R}=\left(\succ_{i}\right)_{i=1}^{3}$ given by three voters provided in Table 4.1 .

Note that the strength of support of candidate a over candidate $b$ equals three, as $a$ is preferred to $b$ in all three rankings. Analogously, the strength of support of candidate b over

\footnotetext{
${ }^{2}$ Votrix is a contraction of the term 'voting matrix'.
} 


\begin{tabular}{|c|c|}
\hline$\# \succ_{i}$ & Rankings on $\mathscr{C}$ \\
\hline 1 & $a \succ b \succ c \succ d$ \\
1 & $a \succ d \succ b \succ c$ \\
1 & $d \succ a \succ c \succ b$ \\
\hline
\end{tabular}

Table 4.2: Frequency of the rankings on $\mathscr{C}$ expressed by three voters.

candidate a equals zero, as there is no ranking where $b$ is preferred to $a$. The votrix is then given by:

$$
V=\left(\begin{array}{llll}
0 & 3 & 3 & 2 \\
0 & 0 & 2 & 1 \\
0 & 1 & 0 & 1 \\
1 & 2 & 2 & 0
\end{array}\right)
$$

Compressing a profile of rankings into a votrix is a common tool in social choice theory [170]. Unfortunately, recovering a profile of rankings from a given votrix is not possible. Moreover, necessary and sufficient conditions that a matrix needs to satisfy in order to be a representation of a profile of rankings are also unknown for a number of candidates greater than or equal to six [61, 94]. In the following proposition, some properties of votrices are listed.

Proposition 4.9 Let $\mathscr{C}$ be a set of $k$ candidates and $r$ be the number of voters. For any votrix $V \in \mathcal{V}_{r}(\mathscr{C})$, the following properties are fulfilled:

(i) Strictness: for any $a_{i_{1}} \in \mathscr{C}$, it holds that

$$
V_{i_{1} i_{1}}=0
$$

(ii) Reciprocity: for any two different $a_{i_{1}}, a_{i_{2}} \in \mathscr{C}$, it holds that

$$
V_{i_{1} i_{2}}+V_{i_{2} i_{1}}=r
$$

(iii) Triangle inequality: for any $a_{i_{1}}, a_{i_{2}}, a_{i_{3}} \in \mathscr{C}$, it holds that

$$
V_{i_{1} i_{2}}+V_{i_{2} i_{3}} \geq V_{i_{1} i_{3}}
$$


Proof: Strictness: evident.

Reciprocity: for any two different $a_{i_{1}}, a_{i_{2}} \in \mathscr{C}$, it holds that

$$
\begin{aligned}
V_{i_{1} i_{2}}+V_{i_{2} i_{1}}= & s\left(a_{i_{1}}, a_{i_{2}}\right)+s\left(a_{i_{2}}, a_{i_{1}}\right) \\
= & \#\left\{j \in\{1, \ldots, r\} \mid a_{i_{1}} \succ_{j} a_{i_{2}}\right\} \\
& +\#\left\{j \in\{1, \ldots, r\} \mid a_{i_{2}} \succ_{j} a_{i_{1}}\right\} \\
= & \#\left\{j \in\{1, \ldots, r\} \mid a_{i_{1}} \succ_{j} a_{i_{2}} \vee a_{i_{2}} \succ_{j} a_{i_{1}}\right\}=r,
\end{aligned}
$$

as $a_{i_{1}} \succ_{j} a_{i_{2}}$ and $a_{i_{2}} \succ_{j} a_{i_{1}}$ are disjoint events.

Triangle inequality: for any $a_{i_{1}}, a_{i_{2}}, a_{i_{3}} \in \mathscr{C}$, it holds that

$$
\begin{aligned}
V_{i_{1} i_{2}}+V_{i_{2} i_{3}}= & s\left(a_{i_{1}}, a_{i_{2}}\right)+s\left(a_{i_{2}}, a_{i_{3}}\right) \\
= & \#\left\{j \in\{1, \ldots, r\} \mid a_{i_{1}} \succ_{j} a_{i_{2}}\right\} \\
& +\#\left\{j \in\{1, \ldots, r\} \mid a_{i_{2}} \succ_{j} a_{i_{3}}\right\} \\
\geq & \#\left\{j \in\{1, \ldots, r\} \mid a_{i_{1}} \succ_{j} a_{i_{3}}\right\} \\
& =s\left(a_{i_{1}}, a_{i_{3}}\right)=V_{i_{1} i_{3}} .
\end{aligned}
$$

Note that these properties are the necessary and sufficient conditions that a matrix needs to satisfy in order to be a representation of a profile of rankings for a set of at most five candidates [61]. Although the sufficiency does not hold, they obviously remain necessary conditions for a set of six or more candidates. Even though a characterization of votrices is not known, we can identify a set of matrices that are 'close' to being votrices, by imposing the necessary conditions listed in Proposition 4.9 .

Definition 4.10 Let $\mathscr{C}$ be a set of $k$ candidates and $r$ be the number of voters. A matrix $V \in\{0,1, \ldots, r\}^{k \times k}$ is called a quasivotrix (plural quasivotrices) on $\mathscr{C}$ if it satisfies the properties listed in Proposition 4.9. The set of all quasivotrices on $\mathscr{C}$ with $r$ voters is denoted by $\mathcal{V}_{r}^{\mathcal{Q}}(\mathscr{C})$.

Of course, any votrix is a quasivotrix, i.e., $\mathcal{V}_{r}(\mathscr{C}) \subseteq \mathcal{V}_{r}^{\mathcal{Q}}(\mathscr{C})$

We recall that a common notion in social choice theory related to the votrix is that of the Condorcet ranking, which is a ranking such that every candidate is preferred by more 
than half of the voters to all the candidates ranked at a worse position than him/her. Analogously, a weak Condorcet ranking is a ranking such that every candidate is preferred by not less than half of the voters to all the candidates ranked at a worse position than him/her.

Definition 4.11 Let $\mathscr{C}$ be a set of $k$ candidates, $\mathscr{R}$ be the profile of $r$ rankings on $\mathscr{C}$ given by the voters and $V \in \mathcal{V}_{r}(\mathscr{C})$ be the votrix induced by $\mathscr{R}$.

(i) A ranking $\succ$ on $\mathscr{C}$ is called the Condorcet ranking if, for any $a_{i}, a_{j} \in \mathscr{C}$ such that $a_{i} \succ a_{j}$, it holds that $V_{i j}>V_{j i}$.

(ii) A ranking $\succ$ on $\mathscr{C}$ is called a weak Condorcet ranking if, for any $a_{i}, a_{j} \in \mathscr{C}$ such that $a_{i} \succ a_{j}$, it holds that $V_{i j} \geq V_{j i}$.

The Condorcet ranking is clearly unique, but there might exist several weak Condorcet rankings. The existence of the Condorcet ranking or the existence of at least one weak Condorcet ranking are not assured.

\subsection{The votex}

When a voter is providing a ranking $a \succ b \succ c$, he is actually declaring that he supports $a$ over $c$ stronger than both $a$ over $b$ and $b$ over $c$. This is not explicitly taken into account in the votrix and a new tool gathering this additional information is introduced here. To that end, we will provide a few notations that will be used throughout this paper. Let $\mathscr{C}_{\neq}^{2}=\left\{\left(a_{i_{1}}, a_{i_{2}}\right) \in \mathscr{C}^{2} \mid a_{i_{1}} \neq a_{i_{2}}\right\}, \mathcal{K}^{+}=\{1, \ldots, k-1\}, \mathcal{K}^{-}=\{1-k, \ldots,-1\}$ and $\mathcal{K}=\mathcal{K}^{-} \cup \mathcal{K}^{+}$.

Now, we will consider the 'relative position' of a candidate $a_{i_{1}}$ w.r.t. a different candidate $a_{i_{2}}$. This relative position of $a_{i_{1}}$ w.r.t. $a_{i_{2}}$ can be obtained as $P_{j}\left(a_{i_{2}}\right)-P_{j}\left(a_{i_{1}}\right)$ for each possible $j \in\{1, \ldots, r\}$. This difference can take any value in $\mathcal{K}$. Collecting the information given by all the voters, we denote by $n_{i}\left(a_{i_{1}}, a_{i_{2}}\right)$ the absolute frequency for the value $i$ of the relative position of $a_{i_{1}}$ w.r.t. $a_{i_{2}}$, that is, the number of voters considering that $a_{i_{1}}$ is $i$ positions before $a_{i_{2}}$ (or after when considering negative values of $i$ ):

$$
n_{i}\left(a_{i_{1}}, a_{i_{2}}\right)=\#\left\{j \in\{1, \ldots, r\} \mid P_{j}\left(a_{i_{2}}\right)-P_{j}\left(a_{i_{1}}\right)=i\right\} .
$$


Note that $n_{i}\left(a_{i_{1}}, a_{i_{2}}\right)=n_{-i}\left(a_{i_{2}}, a_{i_{1}}\right)$. Of course, these frequencies of relative positions can be obtained for any couple of candidates in $\mathscr{C}_{\neq}^{2}$.

In order to illustrate how the frequencies of relative positions given by a profile of rankings can be obtained, we consider the following example.

Example 4.12 Consider a set of four candidates $\mathscr{C}=\{a, b, c, d\}$ and a profile of rankings $\mathscr{R}=\left(\succ_{i}\right)_{i=1}^{3}$ given by three voters provided in Table 4.1 .

\begin{tabular}{|c|c|}
\hline$\# \succ_{i}$ & Rankings on $\mathscr{C}$ \\
\hline 1 & $a \succ b \succ c \succ d$ \\
1 & $a \succ d \succ b \succ c$ \\
1 & $d \succ a \succ c \succ b$ \\
\hline
\end{tabular}

Table 4.3: Frequency of the rankings on $\mathscr{C}$ expressed by three voters.

It holds that $n_{3}(a, d)=1, n_{-1}(a, d)=1, n_{1}(a, d)=1$ and $n_{i}(a, d)=0$ for any $i \in$ $\mathcal{K} \backslash\{-1,1,3\}$, since in the first ranking a is preferred to $d$ and there are two candidates in between them, in the second ranking a is preferred to $d$ and there are no candidates in between them and in the third ranking $d$ is preferred to $a$ and there are no candidates in between them. Thus, the frequency distribution of relative positions for the couple $(a, d)$ is given by

\begin{tabular}{|c|rrrrrr|}
\hline$i$ & -3 & -2 & -1 & 1 & 2 & 3 \\
\hline$n_{i}(a, d)$ & 0 & 0 & 1 & 1 & 0 & 1 \\
\hline
\end{tabular}

Relative positions will always be listed from $1-k$ to $k-1$ (not including 0 ). Hence, this frequency distribution can be denoted just by $(0,0,1,1,0,1)$.

The frequency distributions for all couples of candidates are given by:

$$
\begin{array}{rlr}
(a, b) & \rightarrow(0,0,0,1,2,0) & (c, a) \rightarrow(1,1,1,0,0,0) \\
(a, c) \rightarrow(0,0,0,1,1,1) & (c, b) \rightarrow(0,0,2,1,0,0) \\
(a, d) \rightarrow(0,0,1,1,0,1) & (c, d) \rightarrow(0,2,0,1,0,0) \\
(b, a) \rightarrow(0,2,1,0,0,0) & (d, a) \rightarrow(1,0,1,1,0,0) \\
(b, c) \rightarrow(0,0,1,2,0,0) & (d, b) \rightarrow(0,1,0,1,0,1) \\
(b, d) \rightarrow(1,0,1,0,1,0) & (d, c) \rightarrow(0,0,1,0,2,0)
\end{array}
$$


Note that, as can be seen in Example 4.12, any profile of rankings $\mathscr{R}$ defines a function of the form $W: \mathscr{C}_{\neq}^{2} \rightarrow\{0,1, \ldots, r\}^{2 k-2}$. This function will be henceforth called the votex ${ }^{3}[124$ induced by the profile $\mathscr{R}$.

Definition 4.13 Let $\mathscr{C}$ be a set of $k$ candidates and $r$ be the number of voters. A function $W: \mathscr{C}_{\neq}^{2} \rightarrow\{0,1, \ldots, r\}^{2 k-2}$ is called a votex (plural votices) on $\mathscr{C}$ if there exists a profile $\mathscr{R}$ of $r$ rankings on $\mathscr{C}$ such that, for any $\left(a_{i_{1}}, a_{i_{2}}\right) \in \mathscr{C}_{\neq}^{2}$ and any $i \in \mathcal{K}$, it holds that

$$
W\left(a_{i_{1}}, a_{i_{2}}\right)(i)=n_{i}\left(a_{i_{1}}, a_{i_{2}}\right) .
$$

The set of all votices on $\mathscr{C}$ induced by any profile of $r$ rankings on $\mathscr{C}$ is denoted by $\mathcal{W}_{r}(\mathscr{C})$.

Given a votex it is always possible to obtain the corresponding votrix. Unfortunately, the converse is not possible.

Proposition 4.14 Let $\mathscr{C}$ be a set of $k$ candidates, $\mathscr{R}$ be the profile of $r$ rankings on $\mathscr{C}$ given by the voters and $W: \mathscr{C}_{\neq}^{2} \rightarrow\{0,1, \ldots, r\}^{2 k-2}$ be the votex induced by $\mathscr{R}$. Then the votrix induced by $\mathscr{R}$ can be obtained from the votex by means of the formula:

$$
V_{i_{1} i_{2}}=\sum_{i \in \mathcal{K}^{+}} W\left(a_{i_{1}}, a_{i_{2}}\right)(i)
$$

for any $\left(a_{i_{1}}, a_{i_{2}}\right) \in \mathscr{C}_{\neq}^{2}$. Obviously, $V_{i_{1} i_{1}}=0$ for any $a_{i_{1}} \in \mathscr{C}$.

\section{Proof:}

The result follows from the fact that $\bigcup_{i \in \mathcal{K}^{+}}\left\{P_{j}\left(a_{i_{2}}\right)-P_{j}\left(a_{i_{1}}\right)=i\right\}=\left\{a_{i_{1}} \succ_{j} a_{i_{2}}\right\}$.

As in the case of the votrix, necessary and sufficient conditions that a function $W: \mathscr{C}_{\neq}^{2} \rightarrow$ $\{0,1, \ldots, r\}^{2 k-2}$ needs to satisfy in order to be the votex of a profile of rankings are unknown.

In the following proposition, some properties of votices are listed. They can be seen as necessary conditions that a function $W: \mathscr{C}_{\neq}^{2} \rightarrow\{0,1, \ldots, r\}^{2 k-2}$ needs to satisfy in order

\footnotetext{
${ }^{3}$ Votex is a contraction of the words 'voting' and 'vertex'. Note that a votex is the set of all frequency distributions induced by a voting profile on the vertices of the Hasse diagram of the order relation $\sqsupset$ (see Definition 5.16p.
} 
to be a votex. These properties are completeness (for each couple of candidates the sum of the frequencies should be equal to the number of voters), reciprocity (the distribution of frequencies of any couple $\left(a_{i_{1}}, a_{i_{2}}\right)$ should be opposite to the distribution of frequencies of the couple $\left(a_{i_{2}}, a_{i_{1}}\right)$ ), regularity (the total number of couples of candidates at each relative position is fixed), peakedness (if two candidates are not consecutive there should be a candidate in between them), the triangle inequality (if $a_{i_{1}}$ is preferred to $a_{i_{3}}$ by more than half of the number of voters, then for any other candidate $a_{i_{2}}$ it is not possible that $a_{i_{3}}$ is preferred to $a_{i_{2}}$ by more than half of the number of voters and, at the same time, $a_{i_{2}}$ is preferred to $a_{i_{1}}$ by more than half of the number of voters) and maximality (if two candidates $a_{i_{1}}$ and $a_{i_{2}}$ are at maximum distance, then only $a_{i_{1}}$ and $a_{i_{2}}$ can be ranked first or last).

Proposition 4.15 Let $\mathscr{C}$ be a set of $k$ candidates and $r$ be the number of voters. For any votex $W \in \mathcal{W}_{r}(\mathscr{C})$, the following properties are fulfilled:

(i) Completeness: for any $\left(a_{i_{1}}, a_{i_{2}}\right) \in \mathscr{C}_{\neq}^{2}$ :

$$
\sum_{i \in \mathcal{K}} n_{i}\left(a_{i_{1}}, a_{i_{2}}\right)=r .
$$

(ii) Reciprocity: for any $i \in \mathcal{K}$ and any $\left(a_{i_{1}}, a_{i_{2}}\right) \in \mathscr{C}_{\neq}^{2}$ :

$$
n_{i}\left(a_{i_{1}}, a_{i_{2}}\right)=n_{-i}\left(a_{i_{2}}, a_{i_{1}}\right) \text {. }
$$

(iii) Regularity: for any $i \in \mathcal{K}$ :

$$
\sum_{(u, v) \in \mathscr{C}_{\neq}^{2}} n_{i}(u, v)=r(k-|i|) .
$$

(iv) Peakedness: for any $a_{i_{1}} \in \mathscr{C}$ and any $i \in \mathcal{K}^{+} \backslash\{1\}$ :

$$
\begin{aligned}
\sum_{u \in \mathscr{C} \backslash\left\{a_{i_{1}}\right\}} n_{i}\left(a_{i_{1}}, u\right) & \leq \sum_{u \in \mathscr{C} \backslash\left\{a_{i_{1}}\right\}} n_{i-1}\left(a_{i_{1}}, u\right), \\
\sum_{u \in \mathscr{C} \backslash\left\{a_{i_{1}}\right\}} n_{i}\left(u, a_{i_{1}}\right) \leq & \sum_{u \in \mathscr{C} \backslash\left\{a_{i_{1}}\right\}} n_{i-1}\left(u, a_{i_{1}}\right),
\end{aligned}
$$

for any $a_{i_{1}} \in \mathscr{C}$ and any $i \in \mathcal{K}^{-} \backslash\{-1\}$ :

$$
\sum_{u \in \mathscr{C} \backslash\left\{a_{i_{1}}\right\}} n_{i}\left(a_{i_{1}}, u\right) \leq \sum_{u \in \mathscr{C} \backslash\left\{a_{i_{1}}\right\}} n_{i+1}\left(a_{i_{1}}, u\right),
$$




$$
\sum_{u \in \mathscr{C} \backslash\left\{a_{i_{1}}\right\}} n_{i}\left(u, a_{i_{1}}\right) \leq \sum_{u \in \mathscr{C} \backslash\left\{a_{i_{1}}\right\}} n_{i+1}\left(u, a_{i_{1}}\right) .
$$

(v) Triangle inequality: for any three different $a_{i_{1}}, a_{i_{2}}, a_{i_{3}} \in \mathscr{C}$ :

$$
\begin{aligned}
& \sum_{i \in \mathcal{K}^{+}} n_{i}\left(a_{i_{1}}, a_{i_{2}}\right)+\sum_{i \in \mathcal{K}^{+}} n_{i}\left(a_{i_{2}}, a_{i_{3}}\right) \geq \sum_{i \in \mathcal{K}^{+}} n_{i}\left(a_{i_{1}}, a_{i_{3}}\right), \\
& \sum_{i \in \mathcal{K}^{-}} n_{i}\left(a_{i_{1}}, a_{i_{2}}\right)+\sum_{i \in \mathcal{K}^{-}} n_{i}\left(a_{i_{2}}, a_{i_{3}}\right) \geq \sum_{i \in \mathcal{K}^{-}} n_{i}\left(a_{i_{1}}, a_{i_{3}}\right) .
\end{aligned}
$$

(vi) Maximality: for any $\left(a_{i_{1}}, a_{i_{2}}\right) \in \mathscr{C}_{\neq}^{2}$ :

$$
\begin{gathered}
\sum_{u \in \mathscr{C} \backslash\left\{a_{i_{1}}, a_{i_{2}}\right\}} n_{k-1}\left(a_{i_{1}}, u\right)+\sum_{v \in \mathscr{C} \backslash\left\{a_{i_{1}}, a_{i_{2}}\right\}} n_{k-1}\left(v, a_{i_{2}}\right) \leq \sum_{i \in \mathcal{K}^{+} \backslash\{k-1\}} n_{i}\left(a_{i_{1}}, a_{i_{2}}\right), \\
\sum_{u \in \mathscr{C} \backslash\left\{a_{i_{1}}, a_{i_{2}}\right\}} n_{1-k}\left(a_{i_{1}}, u\right)+\sum_{v \in \mathscr{C} \backslash\left\{a_{i_{1}}, a_{i_{2}}\right\}} n_{1-k}\left(v, a_{i_{2}}\right) \leq \sum_{i \in \mathcal{K}^{-} \backslash\{1-k\}} n_{i}\left(a_{i_{1}}, a_{i_{2}}\right) .
\end{gathered}
$$

Proof: Completeness: for any $\left(a_{i_{1}}, a_{i_{2}}\right) \in \mathscr{C}_{\neq}^{2}, \sum_{i \in \mathcal{K}} n_{i}\left(a_{i_{1}}, a_{i_{2}}\right)$ is the number of voters.

Reciprocity: for any $i \in \mathcal{K}^{+}$and any $\left(a_{i_{1}}, a_{i_{2}}\right) \in \mathscr{C}_{\neq}^{2}$, if $a_{i_{1}}$ is ranked $i$ positions before $a_{i_{2}}$, then $a_{i_{2}}$ is ranked $i$ positions after $a_{i_{1}}$. Similarly, for any $i \in \mathcal{K}^{-}$and any $\left(a_{i_{1}}, a_{i_{2}}\right) \in \mathscr{C}_{\neq}^{2}$, if $a_{i_{1}}$ is ranked $|i|$ positions after $a_{i_{2}}$, then $a_{i_{2}}$ is ranked $|i|$ positions before $a_{i_{1}}$. Therefore, $n_{i}\left(a_{i_{1}}, a_{i_{2}}\right)=n_{-i}\left(a_{i_{2}}, a_{i_{1}}\right)$.

Regularity: consider the notation

$$
\mathbb{1}(x=i)= \begin{cases}1, & \text { if } x=i \\ 0, & \text { if } x \neq i\end{cases}
$$

We have that

$$
\begin{aligned}
\sum_{(u, v) \in \mathscr{C}_{\neq}^{2}} n_{i}(u, v) & =\sum_{(u, v) \in \mathscr{C}_{\neq}^{2}} \sum_{j=1}^{r} \mathbb{1}\left(P_{j}(v)-P_{j}(u)=i\right) \\
& =\sum_{j=1}^{r} \sum_{(u, v) \in \mathscr{C}_{\neq}^{2}} \mathbb{1}\left(P_{j}(v)-P_{j}(u)=i\right) \\
& =\sum_{j=1}^{r}(k-|i|)=r(k-|i|),
\end{aligned}
$$


since there are exactly $k-|i|$ couples of candidates with relative position $i$.

Peakedness: for any $a_{i_{1}} \in \mathscr{C}$ and any $i \in \mathcal{K}^{+} \backslash\{1\}$ :

$$
\begin{aligned}
\sum_{u \in \mathscr{C} \backslash\left\{a_{i_{1}}\right\}} n_{i}\left(a_{i_{1}}, u\right) & =\sum_{u \in \mathscr{C} \backslash\left\{a_{i_{1}}\right\}} \sum_{j=1}^{r} \mathbb{1}\left(P_{j}(u)-P_{j}\left(a_{i_{1}}\right)=i\right) \\
& =\sum_{j=1}^{r} \sum_{u \in \mathscr{C} \backslash\left\{a_{i_{1}}\right\}} \mathbb{1}\left(P_{j}(u)-P_{j}\left(a_{i_{1}}\right)=i\right) \\
& =\sum_{j=1}^{r} \mathbb{1}\left(k-P_{j}\left(a_{i_{1}}\right) \geq i\right) \\
& \leq \sum_{j=1}^{r} \mathbb{1}\left(k-P_{j}\left(a_{i_{1}}\right) \geq i-1\right) \\
& =\sum_{j=1}^{r} \sum_{u \in \mathscr{C} \backslash\left\{a_{i_{1}}\right\}} \mathbb{1}\left(P_{j}(u)-P_{j}\left(a_{i_{1}}\right)=(i-1)\right) \\
& =\sum_{u \in \mathscr{C} \backslash\left\{a_{i_{1}}\right\}} n_{i-1}\left(a_{i_{1}}, u\right) .
\end{aligned}
$$

The other three peakedness formulas can be proved analogously.

Triangle inequality: for any $(u, v) \in \mathscr{C}_{\neq}^{2}$, it is immediate that $\sum_{i \in \mathcal{K}^{+}} n_{i}(u, v)$ is equal to the number of voters that preferred $u$ to $v$, that is,

$$
\sum_{i \in \mathcal{K}^{+}} n_{i}(u, v)=\sum_{j=1}^{r} \mathbb{1}\left(u \succ_{j} v\right) .
$$

Thus, for any three different $a_{i_{1}}, a_{i_{2}}, a_{i_{3}} \in \mathscr{C}$, we have that

$$
\begin{aligned}
\sum_{i \in \mathcal{K}^{+}} n_{i}\left(a_{i_{1}}, a_{i_{2}}\right)+\sum_{i \in \mathcal{K}^{+}} n_{i}\left(a_{i_{2}}, a_{i_{3}}\right) & =\sum_{j=1}^{r}\left(\mathbb{1}\left(a_{i_{1}} \succ_{j} a_{i_{2}}\right)+\mathbb{1}\left(a_{i_{2}} \succ_{j} a_{i_{3}}\right)\right) \\
& \geq \sum_{j=1}^{r} \mathbb{1}\left(a_{i_{1}} \succ_{j} a_{i_{3}}\right) \\
& =\sum_{i \in \mathcal{K}^{+}} n_{i}\left(a_{i_{1}}, a_{i_{3}}\right)
\end{aligned}
$$

due to the transitivity of the rankings $\succ_{j}$.

The other triangle inequality formula can be proved analogously. 
Maximality: for any $\left(a_{i_{1}}, a_{i_{2}}\right) \in \mathscr{C}_{\neq}^{2}$ :

$$
\begin{aligned}
& \sum_{u \in \mathscr{C} \backslash\left\{a_{i_{1}}, a_{i_{2}}\right\}} n_{k-1}\left(a_{i_{1}}, u\right)+\sum_{v \in \mathscr{C} \backslash\left\{a_{i_{1}}, a_{i_{2}}\right\}} n_{k-1}\left(v, a_{i_{2}}\right) \\
& =\sum_{j=1}^{r}\left(\sum_{u \in \mathscr{C} \backslash\left\{a_{i_{1}}, a_{i_{2}}\right\}} \mathbb{1}\left(P_{j}(u)-P_{j}\left(a_{i_{1}}\right)=k-1\right)\right. \\
& \left.+\sum_{v \in \mathscr{C} \backslash\left\{a_{i_{1}}, a_{i_{2}}\right\}} \mathbb{1}\left(P_{j}\left(a_{i_{2}}\right)-P_{j}(v)=k-1\right)\right) \\
& =\sum_{j=1}^{r}\left(\sum_{u \in \mathscr{C} \backslash\left\{a_{i_{1}}, a_{i_{2}}\right\}} \mathbb{1}\left(P_{j}\left(a_{i_{1}}\right)=1, P_{j}(u)=k\right)\right. \\
& \left.+\sum_{v \in \mathscr{C} \backslash\left\{a_{i_{1}}, a_{i_{2}}\right\}} \mathbb{1}\left(P_{j}(v)=1, P_{j}\left(a_{i_{2}}\right)=k\right)\right) \\
& =\sum_{j=1}^{r}\left(\sum_{u \in \mathscr{C} \backslash\left\{a_{i_{1}}, a_{i_{2}}\right\}} \mathbb{1}\left(P_{j}\left(a_{i_{1}}\right)=1,1<P_{j}\left(a_{i_{2}}\right)<k, P_{j}(u)=k\right)\right. \\
& \left.+\sum_{v \in \mathscr{C} \backslash\left\{a_{i_{1}}, a_{i_{2}}\right\}} \mathbb{1}\left(P_{j}(v)=1,1<P_{j}\left(a_{i_{1}}\right)<k, P_{j}\left(a_{i_{2}}\right)=k\right)\right) \\
& =\sum_{j=1}^{r}\left(\mathbb{1}\left(P_{j}\left(a_{i_{1}}\right)=1,1<P_{j}\left(a_{i_{2}}\right)<k\right)\right. \\
& \left.+\mathbb{1}\left(1<P_{j}\left(a_{i_{1}}\right)<k, P_{j}\left(a_{i_{2}}\right)=k\right)\right) \\
& \leq \sum_{j=1}^{r} \mathbb{1}\left(1 \leq P_{j}\left(a_{i_{2}}\right)-P_{j}\left(a_{i_{1}}\right)<k-1\right)=\sum_{\mathcal{K}^{+} \backslash\{k-1\}} n_{i}\left(a_{i_{1}}, a_{i_{2}}\right) \text {. }
\end{aligned}
$$

The other maximality formula can be proved analogously.

Remark 4.16 Assuming reciprocity is fulfilled, the regularity, peakedness, triangle inequality and maximality properties can be reduced to:

Regularity: for any $i \in \mathcal{K}^{+}$:

$$
\sum_{(u, v) \in \mathscr{C}_{\neq}^{2}} n_{i}(u, v)=r(k-i)
$$


Peakedness: for any $a_{i_{1}} \in \mathscr{C}$ and any $i \in \mathcal{K}^{+} \backslash\{1\}$ :

$$
\sum_{u \in \mathscr{C} \backslash\left\{a_{i_{1}}\right\}} n_{i}\left(a_{i_{1}}, u\right) \leq \sum_{u \in \mathscr{C} \backslash\left\{a_{i_{1}}\right\}} n_{i-1}\left(a_{i_{1}}, u\right),
$$

for any $a_{i_{1}} \in \mathscr{C}$ and any $i \in \mathcal{K}^{-} \backslash\{-1\}$ :

$$
\sum_{u \in \mathscr{C} \backslash\left\{a_{i_{1}}\right\}} n_{i}\left(a_{i_{1}}, u\right) \leq \sum_{u \in \mathscr{C} \backslash\left\{a_{i_{1}}\right\}} n_{i+1}\left(a_{i_{1}}, u\right) .
$$

Triangle inequality: for any three different $a_{i_{1}}, a_{i_{2}}, a_{i_{3}} \in \mathscr{C}$ :

$$
\sum_{i \in \mathcal{K}^{+}} n_{i}\left(a_{i_{1}}, a_{i_{2}}\right)+\sum_{i \in \mathcal{K}^{+}} n_{i}\left(a_{i_{2}}, a_{i_{3}}\right) \geq \sum_{i \in \mathcal{K}^{+}} n_{i}\left(a_{i_{1}}, a_{i_{3}}\right) .
$$

Maximality: for any $\left(a_{i_{1}}, a_{i_{2}}\right) \in \mathscr{C}_{\neq}^{2}$ :

$$
\sum_{u \in \mathscr{C} \backslash\left\{a_{i_{1}}, a_{i_{2}}\right\}} n_{k-1}\left(a_{i_{1}}, u\right)+\sum_{v \in \mathscr{C} \backslash\left\{a_{i_{1}}, a_{i_{2}}\right\}} n_{k-1}\left(v, a_{i_{2}}\right) \leq \sum_{i \in \mathcal{K}^{+} \backslash\{k-1\}} n_{i}\left(a_{i_{1}}, a_{i_{2}}\right) .
$$

A set of necessary properties that a function $W: \mathscr{C}_{\neq}^{2} \rightarrow\{0,1, \ldots, r\}^{2 k-2}$ needs to satisfy in order to be a votex has been given in Proposition 4.15. It is important to analyse the independence of these properties proving that none of them can be eliminated. An illustrative example showing that all the properties listed in Proposition 4.15 are indeed independent and that none of them is irrelevant is shown right after.

Example 4.17 Consider a set $\mathscr{C}=\{a, b, c, d\}$ of four candidates and three voters. In this example, we consider functions $W: \mathscr{C}_{\neq}^{2} \rightarrow\{0,1, \ldots, r\}^{2 k-2}$ fulfilling all the properties listed in Proposition 4.15 except for one. Reciprocity will be assumed in all cases, except in the one concerning reciprocity, in order to ease notations. Of course, none of the following functions is a votex as, as stated in Proposition 4.15, they must satisfy all the properties.

\section{Completeness:}

$$
\begin{array}{rl|rl}
W: \mathscr{C}_{\neq}^{2} & \longrightarrow\{0,1,2,3\}^{6} & W: \mathscr{C}_{\neq}^{2} & \longrightarrow\{0,1,2,3\}^{6} \\
W(a, b) & =(0,0,3,0,0,0) & W(b, c) & = \\
W(a, c) & =(0,2,1,0,0,0) & W(b, d) & =(0,2,1,0,0,0) \\
W(a, d) & =(3,1,0,0,0,0) & W(c, d) & =(0,1,2,0,0,0)
\end{array}
$$


Completeness is the only property that is not satisfied:

$$
\sum_{i \in \mathcal{K}} n_{i}(a, d)=4 \neq 3=r .
$$

\section{Reciprocity:}

$$
\begin{aligned}
& W: \quad \mathscr{C}_{\neq}^{2} \quad \longrightarrow \quad\{0,1,2,3\}^{6} \\
& W(a, b)=(1,1,0,1,0,0) \\
& W(a, c)=(0,2,1,0,0,0) \\
& W(a, d)=(1,0,2,0,0,0) \\
& W(b, a)=(0,1,0,0,1,1) \\
& W(b, c)=(0,0,2,1,0,0) \\
& W(b, d)=(1,1,0,1,0,0) \\
& \begin{aligned}
W: \mathscr{C}_{\neq}^{2} & \longrightarrow\{0,1,2,3\}^{6} \\
W(c, a) & =(0,0,1,0,1,1) \\
W(c, b) & =(0,0,1,1,1,0) \\
W(c, d) & =(0,1,1,1,0,0) \\
W(d, a) & =(0,0,0,3,0,0) \\
W(d, b) & =(0,0,1,0,1,1) \\
W(d, c) & =(0,0,0,1,2,0)
\end{aligned}
\end{aligned}
$$

Reciprocity is not satisfied as:

$$
n_{-2}(a, c)=2 \neq 1=n_{2}(c, a) .
$$

\section{Regularity:}

$$
\begin{aligned}
W: \mathscr{C}_{\neq}^{2} & \longrightarrow\{0,1,2,3\}^{6} \\
W(a, b) & =(0,1,2,0,0,0) \\
W(a, c) & =(0,2,1,0,0,0) \\
W(a, d) & =(2,0,1,0,0,0)
\end{aligned}
$$

$$
\begin{aligned}
W: \mathscr{C}_{\neq}^{2} & \longrightarrow\{0,1,2,3\}^{6} \\
W(b, c) & =(0,1,2,0,0,0) \\
W(b, d) & =(0,2,1,0,0,0) \\
W(c, d) & =(0,1,2,0,0,0)
\end{aligned}
$$

Regularity is not satisfied as:

$$
\sum_{(u, v) \in \mathscr{C}_{\neq}^{2}} n_{3}(u, v)=2 \neq 3=r(k-|i|) .
$$

\section{Peakedness:}

$$
\begin{aligned}
W: \mathscr{C}_{\neq}^{2} & \longrightarrow\{0,1,2,3\}^{6} \\
W(a, b) & =(0,2,0,0,0,1) \\
W(a, c) & =(0,2,0,1,0,0) \\
W(a, d) & =(2,0,0,1,0,0)
\end{aligned}
$$

$$
\begin{aligned}
W: \mathscr{C}_{\neq}^{2} & \longrightarrow\{0,1,2,3\}^{6} \\
W(b, c) & =(0,1,2,0,0,0) \\
W(b, d) & =(0,0,3,0,0,0) \\
W(c, d) & =(0,1,2,0,0,0)
\end{aligned}
$$

Peakedness is not satisfied as:

$$
\sum_{u \in \mathscr{C} \backslash\{a\}} n_{3}(a, u)=1 \not \leq 0=\sum_{u \in \mathscr{C} \backslash\{a\}} n_{i-1}(a, u) .
$$




\section{Maximality:}

$$
\begin{aligned}
W: \mathscr{C}_{\neq}^{2} & \longrightarrow\{0,1,2,3\}^{6} \\
W(a, b) & =(0,2,1,0,0,0) \\
W(a, c) & =(2,0,1,0,0,0) \\
W(a, d) & =(0,1,1,1,0,0)
\end{aligned}
$$

$$
\begin{aligned}
W: \mathscr{C}_{\neq}^{2} & \longrightarrow\{0,1,2,3\}^{6} \\
W(b, c) & =(0,2,1,0,0,0) \\
W(b, d) & =(1,0,1,1,0,0) \\
W(c, d) & =(0,1,1,1,0,0)
\end{aligned}
$$

Maximality is not satisfied as:

$$
\sum_{u \in \mathscr{C} \backslash\{b\}} n_{k-1}(a, u)+\sum_{v \in \mathscr{C} \backslash\{a\}} n_{k-1}(v, b)=1 \not \leq 0=\sum_{i \in \mathcal{K}^{+} \backslash\{k-1\}} n_{i}(a, b) .
$$

\section{Triangle inequality:}

It must be remarked that we could not identify an example of a function $W: \mathscr{C}_{\neq}^{2} \rightarrow$ $\{0,1, \ldots, r\}^{2 k-2}$ fulfilling all the properties listed in Proposition 4.15 except for the triangle inequality with four candidates. Therefore, we provide an example for a set of five candidates $\mathscr{C}=\{a, b, c, d, e\}$ and corresponding $\mathscr{C}_{\neq}^{2}$.

$$
\begin{aligned}
& W: \quad \mathscr{C}_{\neq}^{2} \quad \longrightarrow \quad\{0,1,2,3\}^{8} \\
& W(a, b)=(0,0,0,2,1,0,0,0) \\
& W(a, c)=(0,1,1,1,0,0,0,0) \\
& W(a, d)=(1,0,2,0,0,0,0,0) \\
& W(a, e)=(1,2,0,0,0,0,0,0) \\
& W(b, c)=(0,0,0,0,1,2,0,0) \\
& \begin{aligned}
W: \mathscr{C}_{\neq}^{2} & \longrightarrow\{0,1,2,3\}^{8} \\
W(b, d) & =(0,2,1,0,0,0,0,0) \\
W(b, e) & =(0,0,1,1,0,1,0,0) \\
W(c, d) & =(0,0,0,3,0,0,0,0) \\
W(c, e) & =(0,0,1,1,1,0,0,0) \\
W(d, e) & =(0,0,0,1,0,0,1,1)
\end{aligned}
\end{aligned}
$$

The triangle inequality is not satisfied as:

$$
\sum_{i \in \mathcal{K}^{+}} n_{i}(c, b)+\sum_{i \in \mathcal{K}^{+}} n_{i}(b, a)=2 \nsupseteq 3=\sum_{i \in \mathcal{K}^{+}} n_{i}(c, a) .
$$

As necessary and sufficient conditions that a function $W: \mathscr{C}_{\neq}^{2} \rightarrow\{0,1, \ldots, r\}^{2 k-2}$ needs to satisfy in order to be a votex are unkown, a family of characterizable functions will be defined. A function $W: \mathscr{C}_{\neq}^{2} \rightarrow\{0,1, \ldots, r\}^{2 k-2}$ will be called a quasivotex if it satisfies the necessary conditions listed in Proposition 4.15 .

Definition 4.18 Let $\mathscr{C}$ be a set of $k$ candidates and $r$ be the number of voters. A function $W: \mathscr{C}_{\neq}^{2} \rightarrow\{0,1, \ldots, r\}^{2 k-2}$ is called a quasivotex (plural quasivotices) on $\mathscr{C}$ if it satisfies the properties listed in Proposition 4.15. The set of all quasivotices on $\mathscr{C}$ with $r$ voters is denoted by $\mathcal{W}_{r}^{\mathcal{Q}}(\mathscr{C})$. 
Of course, any votex is a quasivotex, i.e., $\mathcal{W}_{r}(\mathscr{C}) \subseteq \mathcal{W}_{r}^{\mathcal{Q}}(\mathscr{C})$.

\subsection{The beatpath matrix}

Due to the close relation of the method of Schulze and the beatpath matrix, the content of this section is closely related to the content of Subsection 2.2.12. A reader aware of the content of Subsection 2.2 .12 could only glance through this section.

In a recent paper [147], Schulze formalized the ranking rule that he first discussed in public mailing lists in 1997. This ranking rule is based on the use of beatpaths, which lead to another natural representation of votes: the beatpath matrix. Recall that a path from a candidate $a_{i_{1}}$ to a candidate $a_{i_{2}}$, denoted by $a_{i_{1}} \rightarrow a_{i_{2}}$, is a list of couples $\left(\left(a^{i}, a^{i+1}\right)\right)_{i=1}^{n}$ such that $a^{1}=a_{i_{1}}, a^{n+1}=a_{i_{2}}$ and $\left(a^{1}, \ldots, a^{n+1}\right)$ are pairwisely different. The set of all paths from a candidate $a_{i_{1}}$ to a candidate $a_{i_{2}}$ is denoted by $\mathscr{P}\left(a_{i_{1}}, a_{i_{2}}\right)$.

The weakest link of a path $\left(\left(a^{i}, a^{i+1}\right)\right)_{i=1}^{n}$ from $a_{i_{1}}$ to $a_{i_{2}}$ is the couple $\left(a^{i}, a^{i+1}\right)$ such that the number of voters preferring $a^{i}$ to $a^{i+1}$ (called the strength of the weakest link ${ }^{4}$ ) is the minimum among all the couples in $\left(\left(a^{i}, a^{i+1}\right)\right)_{i=1}^{n}$. A path from $a_{i_{1}}$ to $a_{i_{2}}$ that maximizes the strength of the weakest link is called a beatpath ${ }^{5}$ from $a_{i_{1}}$ to $a_{i_{2}}$.

Definition 4.19 Let $\mathscr{C}$ be a set of $k$ candidates and $r$ be the number of voters. A matrix $B \in\{0,1, \ldots, r\}^{k \times k}$ is called a beatpath matrix on $\mathscr{C}$ if there exists a profile $\mathscr{R}$ of $r$ rankings on $\mathscr{C}$ such that, for any $a_{i_{1}}, a_{i_{2}} \in \mathscr{C}$, it holds that

$$
B_{i_{1} i_{2}}=\max _{\rightarrow \in \mathscr{P}\left(a_{i_{1}}, a_{i_{2}}\right)} \min _{\left(a^{i}, a^{i+1}\right) \in \rightarrow} \#\left\{j \in\{1, \ldots, r\} \mid a^{i} \succ_{j} a^{i+1}\right\} .
$$

Remark 4.20 In Schulze's original proposal [147], the strength of the weakest link is given by the couple formed by the number of voters preferring $a_{i_{1}}$ to $a_{i_{2}}$ and the number of voters preferring $a_{i_{2}}$ to $a_{i_{1}}$. However, as in the setting of this paper each voter provides his/her

\footnotetext{
${ }^{4}$ In Schulze's original proposal [147, the strength of the weakest link is given by the couple formed by the number of voters preferring $a_{i_{1}}$ to $a_{i_{2}}$ and the number of voters preferring $a_{i_{2}}$ to $a_{i_{1}}$. Nevertheless, as in the setting of this dissertation each voter provides his/her preferences in the form of a ranking on the set of candidates, the first element of the couple determines the second one.

${ }^{5}$ Schulze [147] actually allows candidates to appear several times in the path. Nevertheless, both approaches are equivalent when maximizing the strength of the weakest link.
} 
preferences in the form of a ranking on the set of candidates, the first element of the couple determines the second one.

In order to illustrate how the beatpath matrix induced by a profile of rankings can be obtained, we consider the following example.

Example 4.21 Consider a set of four candidates $\mathscr{C}=\{a, b, c, d\}$ and a profile of rankings $\mathscr{R}=\left(\succ_{i}\right)_{i=1}^{14}$ given by fourteen voters. These rankings are shown in Table 4.4 .

\begin{tabular}{|c|c|}
\hline$\# \succ_{i}$ & Rankings on $\mathscr{C}$ \\
\hline 6 & $c \succ b \succ a \succ d$ \\
5 & $a \succ d \succ b \succ c$ \\
3 & $b \succ a \succ d \succ c$ \\
\hline
\end{tabular}

Table 4.4: Frequency of the rankings on $\mathscr{C}$ expressed by 14 voters.

There are five different paths from a candidate $a_{i_{1}}$ to a candidate $a_{i_{2}}$. For instance, for going from a to $b$, there are the following five possible paths:

$$
\begin{array}{r}
((a, b)) \text { leading to } \min (5)=5, \\
((a, c),(c, b)) \text { leading to } \min (8,6)=6, \\
((a, d),(d, b)) \text { leading to } \min (14,5)=5, \\
((a, c),(c, d),(d, b)) \text { leading to } \min (8,6,5)=5, \\
((a, d),(d, c),(c, b)) \text { leading to } \min (14,8,6)=6 .
\end{array}
$$

The paths maximizing the strength of the weakest link, leading to a value of six, are $((a, c),(c, b))$ and $((a, d),(d, c),(c, b))$. Therefore, the element of the beatpath matrix corresponding to $(a, b)$, which is the element at the first row and second column, equals six. The beatpath matrix induced by the profile $\mathscr{R}$ is

$$
B_{\mathscr{R}}=\left(\begin{array}{cccc}
- & 6 & 8 & 14 \\
9 & - & 8 & 9 \\
6 & 6 & - & 6 \\
6 & 6 & 8 & -
\end{array}\right) .
$$




\section{CHAPTER 5}

\section{Monotonicity of a representation of votes}

Monotonicity is a common desired property in mathematical modelling exercises, and its importance has been acknowledged in several disciplines, e.g. in machine learning [12, 28, 43, 90] and fuzzy modelling [128, 155, 156, 160, 161. However, real-life data is often imperfect and does not fully comply with the monotonicity hypothesis. One option then is to (minimally) adjust the data set restoring the monotonicity [132, 134, 135]. This is particularly important as, for instance, in machine learning, some algorithms cannot be trained with non-monotone datasets [134].

\subsection{Monotonicity of the scorix}

Stochastic dominance is a popular tool for the comparison of probability distributions [89]. It has been shown to have applications in decision theory [89], economics [10, 72], machine learning [132, 163, 164] and social choice theory [124, 154]. Stochastic dominance establishes a relation between probability distributions that is based on the pairwise comparison of the corresponding cumulative probability distributions. A probability distribution is said to (first-order) stochastically dominate another probability distribution if the cumulative probability distribution of the first one is smaller than or equal to the cumulative probability distribution of the second one at any point. Similarly, a probability distribution is said to second-order stochastically dominate another probability distribution if the cumulative of the cumulative probability distribution of the first one is smaller than or equal to the cumulative of the cumulative probability distribution of the second one at any point. It 
is known that (first-order) dominance implies second-order dominance [89]. In a similar way, two dominance relations (first-order dominance and second-order dominance) between vectors of positions are defined.

Definition 5.1 Let $\mathscr{C}$ be a set of $k$ candidates, $r$ be the number of voters and $A=$ $\left(A_{1}, \ldots, A_{k}\right), B=\left(B_{1}, \ldots, B_{k}\right) \in\{0,1, \ldots, r\}^{k}$ be the vectors of positions of two candidates.

(i) $A$ is said to (first-order) dominate $B$, denoted by $A \unrhd_{1} B$, if, for any $j \in\{1, \ldots, k\}$, it holds that

$$
\sum_{i=1}^{j} A_{i} \geq \sum_{i=1}^{j} B_{i}
$$

(ii) $A$ is said to second-order dominate $B$, denoted by $A \unrhd_{2} B$, if, for any $\ell \in\{1, \ldots, k\}$, it holds that

$$
\sum_{j=1}^{\ell} \sum_{i=1}^{j} A_{i} \geq \sum_{j=1}^{\ell} \sum_{i=1}^{j} B_{i}
$$

These dominance relations have already been analysed by several authors in the context of scoring ranking rules. Fine and Fine [52] introduced the positional rule, which is a pre-order relation on the set of candidates that assigns candidate $a_{i_{1}}$ a better ranking than candidate $a_{i_{2}}$ if the vector of positions of candidate $a_{i_{1}}$ dominates the vector of positions of candidate $a_{i_{2}}$. Stein et al. [154] proved that the vector of positions of candidate $a_{i_{1}}$ strictly dominates ${ }^{1}$ the vector of positions of another candidate $a_{i_{2}}$ if and only if there is no scoring ranking rule ranking candidate $a_{i_{2}}$ at a better position than candidate $a_{i_{1}}$. They also proved that if the dominance is of second order, then this result holds for convex scoring ranking rules. Llamazares and Peña [92] used this dominance relation to interpret a well-known result by Saari [142, 144] (a candidate is a first ranked candidate for every scoring ranking rule if and only if it is a first ranked candidate for every $t$-approval scoring ranking rule) from a new point of view: the vector of positions of a candidate dominates the vector of positions of all the other candidates if and only if it is a first ranked candidate for every scoring ranking rule. The notion of (strict) dominance between vectors of positions is a weaker version of the notions of positional dominance [60] and permutation dominance [56] introduced by Fishburn.

\footnotetext{
${ }^{1} A$ is said to strictly dominate $B$, denoted by $A \triangleright_{1} B$, if $A \unrhd B$ and $A \neq B$.
} 
As can be seen, this dominance relation has called the attention of several researchers. Here, we propose to consider this dominance relation for introducing a consensus state [100, 126]. In that way, a ranking is considered a winning ranking when this dominance relation holds for all couples in the ranking. Note that we write 1-monotonicity in case of first-order dominance and, analogously, we write 2-monotonicty in case of second-order dominance.

Definition 5.2 Let $\mathscr{C}$ be a set of $k$ candidates and $r$ be the number of voters. A scorix $S \in \mathcal{S}_{r}(\mathscr{C})$ is said to be

(i) 1-monotone (or just monotone) w.r.t. a ranking $\succ$ on $\mathscr{C}$ if, for any $a_{i_{1}}, a_{i_{2}} \in \mathscr{C}$ such that $a_{i_{1}} \succ a_{i_{2}}$, it holds that

$$
S_{i_{1}} \unrhd_{1} S_{i_{2}}
$$

(ii) 2-monotone w.r.t. a ranking $\succ$ on $\mathscr{C}$ if, for any $a_{i_{1}}, a_{i_{2}} \in \mathscr{C}$ such that $a_{i_{1}} \succ a_{i_{2}}$, it holds that

$$
S_{i_{1}} \unrhd_{2} S_{i_{2}}
$$

Obviously, the scorix induced by most profiles of rankings is not monotone w.r.t. any ranking. The set of all profiles of rankings with a monotone scorix can be understood as a consensus state for the rationalisation of ranking rules. An interesting study subject is the relation between different notions of consensus states. In particular, monotonicity and 2-monotonicity of the scorix are two concepts that are obviously weaker than unanimity, but that respectively assure that all scoring ranking rules and all convex scoring ranking rule coincide.

Theorem 5.3 Let $\mathscr{C}$ be a set of $k$ candidates, $\mathscr{R}$ be the profile of $r$ rankings on $\mathscr{C}$ given by the voters, $S \in \mathcal{S}_{r}(\mathscr{C})$ be the scorix induced by $\mathscr{R}$ and $\succ$ be a ranking on $\mathscr{C}$. The following statements hold:

(i) If $\mathscr{R}$ is the unanimous profile where every voter expresses $\succ$, then $S$ is monotone w.r.t. $\succ$.

(ii) If $S$ is monotone w.r.t. $\succ$, then $S$ is 2-monotone w.r.t. $\succ$.

(iii) If $S$ is monotone w.r.t. $\succ$, then every scoring ranking rule defines a ranking with ties on the set of candidates that is linearly extended by $\succ$. 
(iv) If $S$ is 2-monotone w.r.t. $\succ$, then every convex scoring ranking rule defines a ranking with ties on the set of candidates that is linearly extended by $\succ$.

Proof: Statement (i). If $\mathscr{R}$ is the unanimous profile where every voter expresses $\succ$, then $S$ is the matrix such that, for any $i \in\{1, \ldots, k\}$, the $i$-th row is a vector where the $j$-th component equals $r$ if $j$ is the position at which the $i$-th candidate is ranked in $\succ$ and zero otherwise. Therefore, $S$ is monotone w.r.t. $\succ$.

Statement (ii) is straightforward due to the fact that (first-order) dominance implies second-order dominance.

Statement (iii) is a direct consequence of Theorem 1(a) $)^{2}$ in [154], when applied to all the couples in $\succ$.

Statement (iv) is a direct consequence of Theorem $1(b)^{2}$ in [154], when applied to all the couples in $\succ$.

\subsection{Recursive monotonicity of the scorix}

In social choice theory, there are two large families of ranking rules: ranking rules based on positional information [144] and ranking rules based on pairwise information [143]. Connecting both positional and pairwise election outcomes is a relevant topic in social choice theory [145]. As a result of Theorem 5.3, we know that all ranking rules belonging to the most prominent family of ranking rules based on positional information - scoring ranking rules - lead to the same outcome in case the scorix is monotone. However, a relation with the methods based on pairwise comparisons has not yet been established. In order to describe such relation, the notion of sub-scorix needs to be introduced. As a sub-scorix is related to the concept of restriction of a profile to a subset of the set of candidates, the latter notion is introduced first.

Definition 5.4 Let $\mathscr{C}$ be a set of $k$ candidates and $\mathscr{R}$ be the profile of $r$ rankings on $\mathscr{C}$ given by the voters. For any non-empty $\mathscr{C}^{\prime} \subseteq \mathscr{C}$, the restriction of $\mathscr{R}$ to $\mathscr{C}^{\prime}$ is the profile

\footnotetext{
${ }^{2}$ Note that Stein et al. [154] actually considered strict dominance. However, the proof is analogous for the case where dominance instead of strict dominance is considered.
} 
$\mathscr{R}^{\prime}=\left(\succ_{j}^{\prime}\right)_{j=1}^{r}$ of $r$ rankings on $\mathscr{C}^{\prime}$ such that, for any $j \in\{1, \ldots, r\}$ and any $a_{i_{1}}, a_{i_{2}} \in \mathscr{C}^{\prime}$, it holds that $a_{i_{1}} \succ_{j}^{\prime} a_{i_{2}}$ if $a_{i_{1}} \succ_{j} a_{i_{2}}$.

A matrix is said to be a sub-scorix of a scorix if it is the scorix associated with the restriction of the given profile of rankings to a subset of the set of candidates.

Definition 5.5 Let $\mathscr{C}$ be a set of $k$ candidates, $\mathscr{R}$ be the profile of $r$ rankings on $\mathscr{C}$ given by the voters and $S \in \mathcal{S}_{r}(\mathscr{C})$ be the scorix induced by $\mathscr{R}$. For any non-empty subset $\mathscr{C}^{\prime} \subseteq \mathscr{C}$, a matrix $S^{\prime} \in\{0,1, \ldots, r\}^{k^{\prime} \times k^{\prime}}$ (where $k^{\prime}=\left|\mathscr{C}^{\prime}\right|$ ) is called the sub-scorix (plural sub-scorices) of $S$ on $\mathscr{C}^{\prime}$ if $S^{\prime}$ is the scorix on $\mathscr{C}^{\prime}$ induced by the restriction of $\mathscr{R}$ to $\mathscr{C}^{\prime}$.

The fact that $S^{\prime}$ is the sub-scorix of $S$ on a non-empty subset $\mathscr{C}^{\prime} \subseteq \mathscr{C}$ is denoted by $S^{\prime} \subseteq S$.

Remark 5.6 A scorix has $2^{k}-1$ sub-scorices.

Remark 5.7 Any sub-scorix of a scorix on a singleton is a scalar expressing the number of voters.

These concepts are illustrated in the following example.

Example 5.8 Let $\mathscr{C}=\{a, b, c, d\}$ be a set of candidates and $\mathscr{R}=\left\{\succ_{1}, \succ_{2}, \succ_{3}\right\}$ be the profile from Example 4.3. We recall that $\succ_{1}: a \succ b \succ c \succ d, \succ_{2}: d \succ a \succ c \succ b$, $\succ_{3}: a \succ d \succ b \succ c$ and the scorix induced by $\mathscr{R}$ is given by:

$$
S=\left(\begin{array}{llll}
2 & 1 & 0 & 0 \\
0 & 1 & 1 & 1 \\
0 & 0 & 2 & 1 \\
1 & 1 & 0 & 1
\end{array}\right)
$$

Consider $\mathscr{C}^{\prime}=\{a, b, c\} \subseteq \mathscr{C}$. The restriction of $\mathscr{R}$ to $\mathscr{C}^{\prime}$ is the profile $\mathscr{R}^{\prime}=\left\{\succ_{1}^{\prime}, \succ_{2}^{\prime}, \succ_{3}^{\prime}\right\}$ with $\succ_{1}^{\prime}: a \succ b \succ c, \succ_{2}^{\prime}: a \succ c \succ b$ and $\succ_{3}^{\prime}: a \succ b \succ c$. The scorix induced by $\mathscr{R}^{\prime}$ is given by:

$$
S^{\prime}=\left(\begin{array}{lll}
3 & 0 & 0 \\
0 & 2 & 1 \\
0 & 1 & 2
\end{array}\right) .
$$

Thus, $S^{\prime}$ is the sub-scorix of $S$ on $\mathscr{C}^{\prime}$ and, therefore, it holds that $S^{\prime} \underline{\subseteq}$. 
In the same way a scorix can be monotone, the respective sub-scorices can also be monotone. The monotonicity of all the sub-scorices of a scorix leads to a stronger type of monotonicity: recursive monotonicity.

Definition 5.9 Let $\mathscr{C}$ be a set of $k$ candidates and $r$ be the number of voters.

(i) A scorix $S \in \mathcal{S}_{r}(\mathscr{C})$ is said to be recursively monotone w.r.t. a ranking $\succ$ on $\mathscr{C}$ if, for any non-empty subset $\mathscr{C}^{\prime} \subseteq \mathscr{C}$, the sub-scorix $S^{\prime}$ of $S$ on $\mathscr{C}^{\prime}$ is monotone w.r.t. the restriction ${ }^{3}$ of $\succ$ to $\mathscr{C}^{\prime}$.

(ii) A scorix $S \in \mathcal{S}_{r}(\mathscr{C})$ is said to be strictly recursively monotone w.r.t. a ranking $\succ$ on $\mathscr{C}$ if, for any non-empty subset $\mathscr{C}^{\prime} \subseteq \mathscr{C}$, the sub-scorix $S^{\prime}$ of $S$ on $\mathscr{C}^{\prime}$ is stricly monotone w.r.t. the restriction of $\succ$ to $\mathscr{C}^{\prime}$.

Again, the scorix induced by most profiles of rankings is not recursively monotone w.r.t. any ranking.

Remark 5.10 Recursive monotonicity of the scorix actually is a property of the profile of rankings and not of its scorix. This means that, given a scorix, it is not possible to identify whether or not it is recursively monotone without knowing the profile of rankings. For instance, consider the two profiles of rankings listed in Table 5.1.

\begin{tabular}{|c||c|}
\hline $\mathscr{R}_{1}$ & $\mathscr{R}_{2}$ \\
\hline$a \succ b \succ c$ & $a \succ c \succ b$ \\
$a \succ c \succ b$ & $a \succ c \succ b$ \\
$b \succ a \succ c$ & $b \succ a \succ c$ \\
$b \succ c \succ a$ & $b \succ a \succ c$ \\
$c \succ a \succ b$ & $c \succ b \succ a$ \\
\hline
\end{tabular}

Table 5.1: Profiles $\mathscr{R}_{1}$ and $\mathscr{R}_{2}$ of five rankings.

The scorices $S_{1}$ and $S_{2}$ induced by $\mathscr{R}_{1}$ and $\mathscr{R}_{2}$ coincide:

$$
S_{1}=S_{2}=\left(\begin{array}{lll}
2 & 2 & 1 \\
2 & 1 & 2 \\
1 & 2 & 2
\end{array}\right) .
$$

\footnotetext{
${ }^{3}$ As a ranking is a special case of a profile with just one ranking, the restriction of a ranking to a subset of the set of candidates follows from Definition 5.4 .
} 
In case we consider $\mathscr{C}^{\prime}=\{b, c\} \subseteq \mathscr{C}$, the corresponding restrictions $\mathscr{R}_{1}^{\prime}$ and $\mathscr{R}_{2}^{\prime}$ of $\mathscr{R}_{1}$ and $\mathscr{R}_{2}$ to $\mathscr{C}^{\prime}$ are listed in Table 5.2.

\begin{tabular}{|c||c|}
\hline $\mathscr{R}_{1}^{\prime}$ & $\mathscr{R}_{2}^{\prime}$ \\
\hline$b \succ c$ & $c \succ b$ \\
$c \succ b$ & $c \succ b$ \\
$b \succ c$ & $b \succ c$ \\
$b \succ c$ & $b \succ c$ \\
$c \succ b$ & $c \succ b$ \\
\hline
\end{tabular}

Table 5.2: Profiles $\mathscr{R}_{1}^{\prime}$ (left) and $\mathscr{R}_{2}^{\prime}$ (right) of five rankings.

The scorices $S_{1}^{\prime}$ and $S_{2}^{\prime}$ induced by $\mathscr{R}_{1}^{\prime}$ and $\mathscr{R}_{1}^{\prime}$ are given by:

$$
S_{1}^{\prime}=\left(\begin{array}{ll}
3 & 2 \\
2 & 3
\end{array}\right), \quad S_{2}^{\prime}=\left(\begin{array}{ll}
2 & 3 \\
3 & 2
\end{array}\right) .
$$

Note that, in contrast to $S_{1}^{\prime}, S_{2}^{\prime}$ is not monotone w.r.t. $b \succ c$. Therefore, $S_{2}$ is not recursively monotone w.r.t. $a \succ b \succ c$ (while $S_{1}$ is).

We conclude that indeed recursive monotonicity is a property of the profile of rankings and not of its scorix. Nevertheless, in order to ease the way of referring to this property, the term recursive monotonicity of the scorix will be used.

Due to the definition of sub-scorix, the following lemma is straightforward.

Lemma 5.11 Let $\mathscr{C}$ be a set of $k$ candidates, $\mathscr{R}$ be the profile of $r$ rankings on $\mathscr{C}$ given by the voters and $S \in \mathcal{S}_{r}(\mathscr{C})$ be the scorix induced by $\mathscr{R}$. The following statements hold:

(i) A ranking $\succ$ on $\mathscr{C}$ is a weak Condorcet ranking if and only if any sub-scorix $S^{\prime}$ of $S$ on a subset $\mathscr{C}^{\prime}$ of $\mathscr{C}$ of cardinality two is monotone w.r.t. the restriction of $\succ$ to $\mathscr{C}^{\prime}$.

(ii) A ranking $\succ$ on $\mathscr{C}$ is the Condorcet ranking if and only if any sub-scorix $S^{\prime}$ of $S$ on a subset $\mathscr{C}^{\prime}$ of $\mathscr{C}$ of cardinality two is strictly monotone w.r.t. the restriction of $\succ$ to $\mathscr{C}^{\prime}$.

The former lemma shows that, in case the set consists of three candidates, recursive monotonicity of the scorix is nothing else but monotonicity of the scorix plus presence of a (weak) Condorcet ranking. 
Corollary 5.12 Let $\mathscr{C}$ be a set of three candidates, $\mathscr{R}$ be the profile of $r$ rankings on $\mathscr{C}$ given by the voters, $S \in \mathcal{S}_{r}(\mathscr{C})$ be the scorix induced by $\mathscr{R}$ and $\succ$ be a ranking on $\mathscr{C}$. The following statements hold:

(i) $S$ is recursively monotone w.r.t. $\succ$ if and only if $S$ is monotone w.r.t. $\succ$ and $\succ$ is a weak Condorcet ranking.

(ii) $S$ is strictly recursively monotone w.r.t. $\succ$ if and only if $S$ is strictly monotone w.r.t. $\succ$ and $\succ$ is the Condorcet ranking.

Recursive monotonicity of the scorix obviously is a stronger property than monotonicity of the scorix and a weaker property than unanimity. Furthermore, as we prove in the following theorem, it guarantees the existence of a weak Condorcet ranking.

Theorem 5.13 Let $\mathscr{C}$ be a set of $k$ candidates, $\mathscr{R}$ be the profile of $r$ rankings on $\mathscr{C}$ given by the voters, $S \in \mathcal{S}_{r}(\mathscr{C})$ be the scorix induced by $\mathscr{R}$ and $\succ$ be a ranking on $\mathscr{C}$. The following statements hold:

(i) If $\mathscr{R}$ is the unanimous profile where every voter expresses $\succ$, then $S$ is recursively monotone w.r.t. $\succ$.

(ii) If $S$ is recursively monotone w.r.t. $\succ$, then $S$ is monotone w.r.t. $\succ$.

(iii) If $S$ is recursively monotone w.r.t. $\succ$, then the first ranked candidate according to $\succ$ is a weak Condorcet winner and $\succ$ is a weak Condorcet ranking.

(iv) If $S$ is recursively monotone w.r.t. $\succ$, then every elimination method based on a scoring ranking rule defines a ranking with ties on the set of candidates that is linearly extended by $\succ$.

Proof: Statement (i). If $\mathscr{R}$ is the unanimous profile where every voter expresses $\succ$, then $S$ is the matrix where, for any $i \in\{1, \ldots, k\}$, the $i$-th row is a vector where the $j$-th component equals $r$ if $j$ is the position at which the $i$-th candidate is ranked in $\succ$ and to zero otherwise. Furthermore, any sub-scorix $S^{\prime} \subseteq S$ will be of the same form considering the restriction of $\succ$ to the corresponding non-empty subset $\mathscr{C}^{\prime} \subseteq \mathscr{C}$. Therefore, $S$ is recursively monotone w.r.t. $\succ$. 
Statement (ii). By definition of recursive monotonicity, any sub-scorix $S^{\prime} \underline{\subseteq} S$ is monotone w.r.t. the corresponding restriction of $\succ$. As $S \Subset S$, it trivially follows that $S$ is monotone w.r.t. $\succ$.

Statement (iii). Direct result of Lemma 5.11.

Statement (iv). As $S$ is monotone w.r.t. $\succ$, the first set of $\ell$ eliminated candidates $\left\{a_{i_{1}}, \ldots, a_{i_{\ell}}\right\}$ corresponds to the last $\ell$ ranked candidates in $\succ$. Now, the current set of candidates is $\mathscr{C}^{\prime}=\mathscr{C} \backslash\left\{a_{i_{1}}, \ldots, a_{i_{\ell}}\right\}$ and the sub-scorix $S^{\prime}$ of $S$ on $\mathscr{C}^{\prime}$ is, due to the recursive monotonicity of $S$ w.r.t. $\succ$, monotone w.r.t. the restriction of $\succ$ to $\mathscr{C}^{\prime}$. Therefore, proceeding analogously as above, we prove that every elimination method based on a scoring ranking rule defines a ranking with ties on the set of candidates that is linearly extended by $\succ$.

The following result concerning the relation between a Condorcet ranking and a Borda ranking follows from Theorem 5.13 .

Corollary 5.14 Let $\mathscr{C}$ be a set of $k$ candidates and $\mathscr{R}$ be the profile of $r$ rankings on $\mathscr{C}$ given by the voters. If the scorix $S \in \mathcal{S}_{r}(\mathscr{C})$ induced by $\mathscr{R}$ is recursively monotone w.r.t. a ranking $\succ$ on $\mathscr{C}$, then $\succ$ is a weak Condorcet ranking and linearly extends the Borda ranking (with ties).

Corollary 5.15 Let $\mathscr{C}$ be a set of $k$ candidates and $\mathscr{R}$ be the profile of $r$ rankings on $\mathscr{C}$ given by the voters. If the scorix $S \in \mathcal{S}_{r}(\mathscr{C})$ induced by $\mathscr{R}$ is strictly recursively monotone w.r.t. a ranking $\succ$ on $\mathscr{C}$, then $\succ$ is the Condorcet ranking and the Borda ranking.

From the previous corollary, we conclude that (strict) recursive monotonicity is a property that links the works of Borda [18] and Condorcet [35], leading to the same social outcome. Therefore, recursive monotonicity of the scorix can be understood as a necessary condition for Condorcet and Borda to finally agree. 


\subsection{Monotonicity of the votrix}

In the context of social choice theory, there are many references to monotonicity (also known as positive association of social and individual values) as a desirable property for ranking rules [3, 60, 152]. In 2014, Rademaker and De Baets [133] proposed a new application of monotonicity in social choice theory. According to their proposal, for a ranking $a \succ b \succ c$, monotonicity means that the strength with which $a \succ c$ is supported should not be less than both the strength with which $a \succ b$ and the strength with which $b \succ c$ are supported. They advocated that the ranking that best reflects the preferences of the voters should be the one that is the closest to satisfying this assumption.

More formally, given a ranking $\succ$ on $\mathscr{C}$, a strict partial order relation between couples of candidates can be defined.

Definition 5.16 Let $\mathscr{C}$ be a set of $k$ candidates. A ranking $\succ$ on $\mathscr{C}$ induces a strict partial order relation $\sqsupset$ on $\mathscr{C}_{\neq}^{2}$ such that, for any two different $\left(a_{i_{1}}, a_{j_{1}}\right),\left(a_{i_{2}}, a_{j_{2}}\right) \in \mathscr{C}_{\neq}^{2}$, it holds that

$$
\left(a_{i_{1}}, a_{j_{1}}\right) \sqsupset\left(a_{i_{2}}, a_{j_{2}}\right) \text { if }\left(a_{i_{1}} \succeq a_{j_{1}}\right) \wedge\left(a_{j_{2}} \succeq a_{i_{2}}\right) \wedge\left(a_{i_{1}} \succ a_{j_{1}} \vee a_{j_{2}} \succ a_{i_{2}}\right)
$$

Figure 5.1 displays the Hasse diagram of the order relation $\sqsupset$ for the ranking $a \succ b \succ c \succ d$.

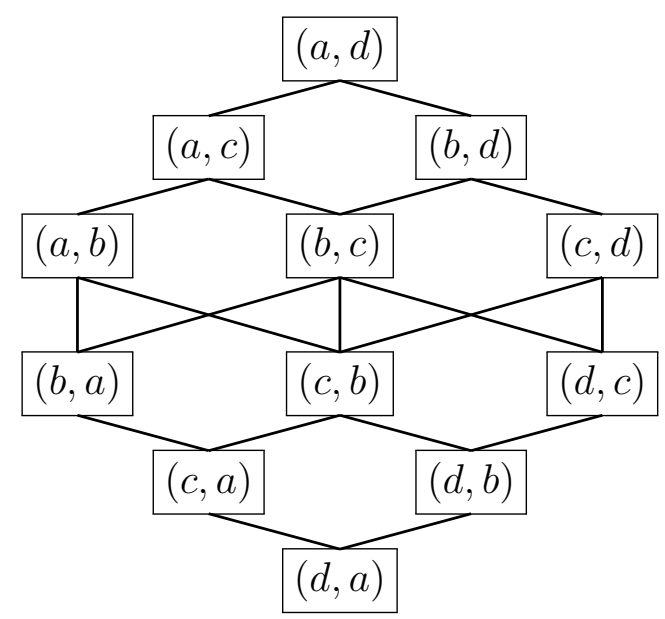

Figure 5.1: Hasse diagram of the order relation $\sqsupset$ for the ranking $a \succ b \succ$ $c \succ d$. 
According to Rademaker and De Baets, if the strength of support is decreasing on $\sqsupset$, then the strength of support is said to be monotone. Note that this property of the strength of support can be understood as a property of the votrix itself. Therefore, from now on, if the strength of support is decreasing on $\sqsupset$, then the votrix is called monotone w.r.t. $\succ$. The definition is naturally extended to any quasivotrix.

Definition 5.17 Let $\mathscr{C}$ be a set of $k$ candidates and $r$ be the number of voters. A quasivotrix $V \in \mathcal{V}_{r}^{\mathcal{Q}}(\mathscr{C})$ is said to be monotone w.r.t. a ranking $\succ$ on $\mathscr{C}$ (with corresponding $\sqsupset$ ) if, for any $\left(a_{i_{1}}, a_{j_{1}}\right),\left(a_{i_{2}}, a_{j_{2}}\right) \in \mathscr{C}_{\neq}^{2}$ such that $\left(a_{i_{1}}, a_{j_{1}}\right) \sqsupset\left(a_{i_{2}}, a_{j_{2}}\right)$, it holds that

$$
V_{i_{1} j_{1}} \geq V_{i_{2} j_{2}} .
$$

Obviously, the votrix induced by most profiles of rankings is not monotone w.r.t. any ranking. The set of all profiles of rankings with a monotone votrix can be understood as a consensus state for the rationalisation of ranking rules for which most methods based on pairwise information lead to the same social outcome, as will be discussed in Section 5.7 .

Remark 5.18 The fact that there exists a ranking w.r.t. which the votrix is monotone implies the property of strong stochastic transitivity [138]. A weaker property, usually referred to as weak stochastic transitivity, has also called the attention of researchers [136].

As we will work with votrices and quasivotrices and they fulfill the reciprocity property mentioned in Proposition 4.9, the lower half of the diagram defined by $\sqsupset$ does not actually provide additional information beyond that provided by the upper half. To exploit this knowledge, we may use the strict partial order relation $\sqsupset^{+}$instead of $\sqsupset$.

Definition 5.19 Let $\mathscr{C}$ be a set of $k$ candidates. A ranking $\succ$ on $\mathscr{C}$ induces a strict partial order relation $\sqsupset^{+}$on $\mathscr{C}_{\neq}^{2}$ such that, for any two different $\left(a_{i_{1}}, a_{j_{1}}\right),\left(a_{i_{2}}, a_{j_{2}}\right) \in \mathscr{C}_{\neq}^{2}$, it holds that

$$
\left(a_{i_{1}}, a_{j_{1}}\right) \sqsupset^{+}\left(a_{i_{2}}, a_{j_{2}}\right) \text { if } a_{i_{1}} \succeq a_{i_{2}} \succ a_{j_{2}} \succeq a_{j_{1}}
$$

Figure 5.2 displays the Hasse diagram of the order relation $\sqsupset^{+}$for the ranking $a \succ b \succ$ $c \succ d$.

Evidently, if the strength of support is decreasing on $\sqsupset^{+}$and, at the same time, equals at least half of the number of voters, then the votrix is monotone w.r.t. $\succ$. 


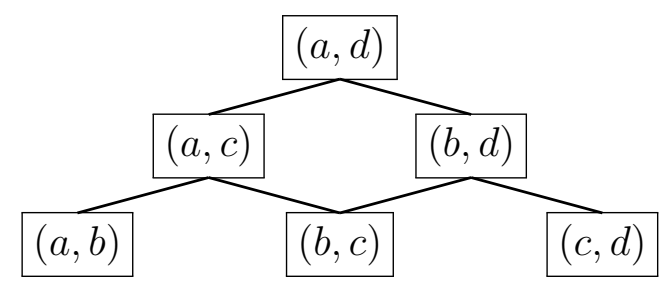

Figure 5.2: Hasse diagram of the order relation $\sqsupset^{+}$for the ranking $a \succ b \succ$ $c \succ d$.

Proposition 5.20 Let $\mathscr{C}$ be a set of $k$ candidates, $r$ be the number of voters, $V \in \mathcal{V}_{r}^{\mathcal{Q}}(\mathscr{C})$ be a quasivotrix and $\succ$ be a ranking on $\mathscr{C}$ (with corresponding $\sqsupset$ and $\sqsupset^{+}$). The following three statements are equivalent:

(i) A quasivotrix $V \in \mathcal{V}_{r}^{\mathcal{Q}}(\mathscr{C})$ is monotone w.r.t. $\succ$.

(ii) For any $\left(a_{i_{1}}, a_{j_{1}}\right),\left(a_{i_{2}}, a_{j_{2}}\right) \in \mathscr{C}_{\neq}^{2}$ such that $\left(a_{i_{1}}, a_{j_{1}}\right) コ^{+}\left(a_{i_{2}}, a_{j_{2}}\right)$, it holds that $V_{i_{1} j_{1}} \geq$ $V_{i_{2} j_{2}}$ and, for any $a_{i}, a_{j} \in \mathscr{C}$ such that $a_{i} \succ a_{j}$, it holds that $V_{i j} \geq \frac{r}{2}$.

(iii) For any $\left(a_{i_{1}}, a_{j_{1}}\right),\left(a_{i_{2}}, a_{j_{2}}\right) \in \mathscr{C}_{\neq}^{2}$ such that $\left(a_{i_{1}}, a_{j_{1}}\right) コ^{+}\left(a_{i_{2}}, a_{j_{2}}\right)$, it holds that $V_{i_{1} j_{1}} \geq$ $V_{i_{2} j_{2}}$ and, for any $a_{i}, a_{j} \in \mathscr{C}$ such that $a_{i} \succ a_{j}$ and $\nexists a_{\ell} \in \mathscr{C}$ satisfying $a_{i} \succ a_{\ell} \succ a_{j}$, it holds that $V_{i j} \geq \frac{r}{2}$.

\subsection{Monotonicity of the votex}

With the votex representation, monotonicity can no longer be represented as the decrease of the strength of support. Nevertheless, similarly to stochastic dominance, we introduce a way to compare frequency distributions of couples of candidates based on their cumulative frequency distributions.

Definition 5.21 Let $\mathscr{C}$ be a set of $k$ candidates and $r$ be the number of voters. For any quasivotex $W \in \mathcal{W}_{r}^{\mathcal{Q}}(\mathscr{C})$ and any $\left(a_{i_{1}}, a_{j_{1}}\right),\left(a_{i_{2}}, a_{j_{2}}\right) \in \mathscr{C}_{\neq}^{2}$, we say that $W\left(a_{i_{1}}, a_{j_{1}}\right)$ dominates $W\left(a_{i_{2}}, a_{j_{2}}\right)$, denoted as $W\left(a_{i_{1}}, a_{j_{1}}\right) \unrhd W\left(a_{i_{2}}, a_{j_{2}}\right)$, if, for any $j \in \mathcal{K}$, it holds that

$$
N_{j}\left(a_{i_{1}}, a_{j_{1}}\right) \leq N_{j}\left(a_{i_{2}}, a_{j_{2}}\right)
$$


where $N_{j}$ denotes the cumulative absolute frequency, i.e., $N_{j}(u, v)=\sum_{\substack{i \in \mathcal{K} \\ i \leq j}} n_{i}(u, v)$.

For any possible ranking $\succ$ on $\mathscr{C}$, we have an associated strict partial order relation $\sqsupset$ between couples of candidates (see Definition 5.16). Among all these rankings, we would like to consider the one that is the closest to imposing monotonicity w.r.t. $\succ$ on the provided votex.

Definition 5.22 Let $\mathscr{C}$ be a set of $k$ candidates and $r$ be the number of voters. A quasivotex $W \in \mathcal{W}_{r}^{\mathcal{Q}}(\mathscr{C})$ is said to be monotone w.r.t. a ranking $\succ$ on $\mathscr{C}$ (with corresponding $\sqsupset$ ) if, for any $\left(a_{i_{1}}, a_{j_{1}}\right),\left(a_{i_{2}}, a_{j_{2}}\right) \in \mathscr{C}_{\neq}^{2}$ such that $\left(a_{i_{1}}, a_{j_{1}}\right) \sqsupset\left(a_{i_{2}}, a_{j_{2}}\right)$, it holds that

$$
W\left(a_{i_{1}}, a_{j_{1}}\right) \unrhd W\left(a_{i_{2}}, a_{j_{2}}\right) .
$$

Obviously, the votex induced by most profiles of rankings is not monotone w.r.t. any ranking. The set of all profiles of rankings with a monotone votex can be understood as a consensus state for the rationalisation of ranking rules for which most methods based on pairwise information lead to the same social outcome and that (unlike most methods based on pairwise information) also exploits the given positional information.

An interesting study subject is the relation between different notions of consensus states. For instance, if a profile of rankings is the unanimous profile where every voter expresses the ranking $\succ$, then the corresponding votrix and votex are monotone w.r.t. $\succ$. In the following theorem, we establish a relation between four different notions of consensus: unanimity, monotonicity of the votex, monotonicity of the votrix and presence of a weak Condorcet ranking.

Theorem 5.23 Let $\mathscr{C}$ be a set of $k$ candidates, $\mathscr{R}$ be the profile of $r$ rankings on $\mathscr{C}$ given by the voters, $V \in \mathcal{V}_{r}(\mathscr{C})$ be the votrix induced by $\mathscr{R}, W \in \mathcal{W}_{r}(\mathscr{C})$ be the votex induced by $\mathscr{R}$ and $\succ$ be a ranking on $\mathscr{C}$. The following statements hold:

(i) If $\mathscr{R}$ is the unanimous profile where every voter expresses $\succ$, then $W$ is monotone w.r.t. $\succ$.

(ii) If $W$ is monotone w.r.t. $\succ$, then $V$ is monotone w.r.t. $\succ$. 
(iii) If $V$ is monotone w.r.t. $\succ$, then the first ranked candidate according to $\succ$ is a weak Condorcet winner and $\succ$ is a weak Condorcet ranking.

(iv) If $V$ is monotone w.r.t. $\succ$ and $r$ is an odd number, then the first ranked candidate according to $\succ$ is the Condorcet winner and $\succ$ is the Condorcet ranking.

Proof: Let $\succ: a_{1} \succ \ldots \succ a_{k}$ be the considered ranking.

Statement (i). If $\mathscr{R}$ is the unanimous profile where every voter expresses $\succ$ then, for any $\left(a_{i_{1}}, a_{i_{2}}\right) \in \mathscr{C}_{\neq}^{2}, W\left(a_{i_{1}}, a_{i_{2}}\right)$ is the vector where $n_{i_{2}-i_{1}}\left(a_{i_{1}}, a_{i_{2}}\right)=r$ and $n_{i}\left(a_{i_{1}}, a_{i_{2}}\right)=0$ for any $i \in \mathcal{K} \backslash\left\{i_{2}-i_{1}\right\}$. It is immediate to see that $W$ is monotone w.r.t. $\succ$.

Statement (ii). If $W$ is monotone w.r.t. $\succ$ then, for any $\left(a_{i_{1}}, a_{j_{1}}\right),\left(a_{i_{2}}, a_{j_{2}}\right) \in \mathscr{C}_{\neq}^{2}$ such that $\left(a_{i_{1}}, a_{j_{1}}\right) \sqsupset\left(a_{i_{2}}, a_{j_{2}}\right)$, it holds that $W\left(a_{i_{1}}, a_{j_{1}}\right) \unrhd W\left(a_{i_{2}}, a_{j_{2}}\right)$.

As a result of Proposition 4.14, we know that, for any $\left(a_{\ell}, a_{\ell^{\prime}}\right) \in \mathscr{C}_{\neq}^{2}$, it holds that

$$
V_{\ell, \ell^{\prime}}=\sum_{i \in \mathcal{K}^{+}} W\left(a_{\ell}, a_{\ell^{\prime}}\right)(i)
$$

Therefore, for any $\left(a_{i_{1}}, a_{j_{1}}\right),\left(a_{i_{2}}, a_{j_{2}}\right) \in \mathscr{C}_{\neq}^{2}$ such that $\left(a_{i_{1}}, a_{j_{1}}\right) \sqsupset\left(a_{i_{2}}, a_{j_{2}}\right)$, it holds that $V_{i_{1}, j_{1}} \geq V_{i_{2}, j_{2}}$. Thus, $V$ is monotone w.r.t. $\succ$.

Statement (iii). If $V$ is monotone w.r.t. $\succ$ then, for any $i \in\{2, \ldots, k\}$, it holds that

$$
V_{1, i} \geq V_{i, 1}
$$

In words, the number of voters preferring $a_{1}$ to any $a_{i}$ is not smaller than the number of voters preferring $a_{i}$ to $a_{1}$, i.e., $a_{1}$ is a weak Condorcet winner.

We now remove $a_{1}$ and consider $\mathscr{C}^{\prime}=\mathscr{C} \backslash\left\{a_{1}\right\}$. Then the restricted votrix on $\mathscr{C}$ will be monotone w.r.t. $\succ^{\prime}: a_{2} \succ^{\prime} \ldots \succ^{\prime} a_{k}$ and we proceed analogously as above. Proceeding iteratively, it is straightforward to prove that $\succ$ is a weak Condorcet ranking.

Statement (iv) is a consequence of statement (iii) and of the fact that a weak Condorcer winner (resp. ranking) always is the Condorcet winner (resp. ranking) in case the number of voters is odd.

Monotonicity of the votrix and monotonicity of the votex are two properties lying in between unanimity and the presence of a weak Condorcet ranking. Other properties such as simple majority [95], absolute majority [139] or majorities based on difference of votes [65] have no immediate relation with monotonicity of the votrix or the votex. 


\subsection{Monotonicity of the beatpath matrix}

The strict partial order relation between couples of candidates given in Definition 5.16 is also used for defining monotonicity of the beatpath matrix [122].

Definition 5.24 Let $\mathscr{C}$ be a set of $k$ candidates and $r$ be the number of voters. A beatpath matrix $B$ is said to be monotone w.r.t. a ranking $\succ$ on $\mathscr{C}$ if, for any $\left(a_{i_{1}}, a_{j_{1}}\right),\left(a_{i_{2}}, a_{j_{2}}\right) \in \mathscr{C}_{\neq}^{2}$ such that $\left(a_{i_{1}}, a_{j_{1}}\right) \sqsupset\left(a_{i_{2}}, a_{j_{2}}\right)$, it holds that

$$
B_{i_{1} j_{1}} \geq B_{i_{2} j_{2}}
$$

Again, the beatpath matrix induced by most profiles of rankings is not monotone w.r.t. any ranking.

Note that monotonicity of the beatpath matrix is a weaker property than monotonicity of the votrix and, therefore, than monotonicity of the votex and unanimity.

Theorem 5.25 Let $\mathscr{C}$ be a set of $k$ candidates, $\mathscr{R}$ be the profile of $r$ rankings on $\mathscr{C}$ given by the voters, $V \in \mathcal{V}_{r}(\mathscr{C})$ be the votrix induced by $\mathscr{R}, B$ be the beatpath matrix induced by $\mathscr{R}$ and $\succ$ be a ranking on $\mathscr{C}$. If $V$ is monotone w.r.t. $\succ$, then $B$ is monotone w.r.t. $\succ$.

Proof: In order to ease the understanding, and just throughout this proof, we consider the notation $r_{\succ}(i)$ to refer to the label corresponding to the candidate ranked at the $i$-th position in $\succ$. We first prove that, if $V$ is monotone w.r.t. $\succ$, then the beatpath from a candidate $a_{i_{1}}$ to a different candidate $a_{i_{2}}$ is the couple $\left(a_{i_{1}}, a_{i_{2}}\right)$ in case $a_{i_{1}} \succ a_{i_{2}}$ and the list of couples $\left(\left(a_{\ell}, a_{\ell+1}\right)\right)_{\ell=r_{\succ}\left(i_{1}\right)}^{r_{\succ}\left(i_{2}\right)-1}$ in case $a_{i_{2}} \succ a_{i_{1}}$. This is illustrated in Figure 5.3 .

1. The case $a_{i_{1}} \succ a_{i_{2}}$. Suppose that there is a path from $a_{i_{1}}$ to $a_{i_{2}}$ such that the strength of its weakest link is greater than the strength of support of $a_{i_{1}}$ over $a_{i_{2}}$. We distinguish three cases:

(a) There is a candidate $a_{i_{3}}$ in the path such that $a_{i_{3}} \succ a_{i_{1}} \succ a_{i_{2}}$. Therefore, one of the couples in the path is such that the first element is preferred to the second one by less than half of the number of voters, a contradiction with the fact that the strength of the weakest link is greater than the strength of support of $a_{i_{1}}$ over $a_{i_{2}}$. 


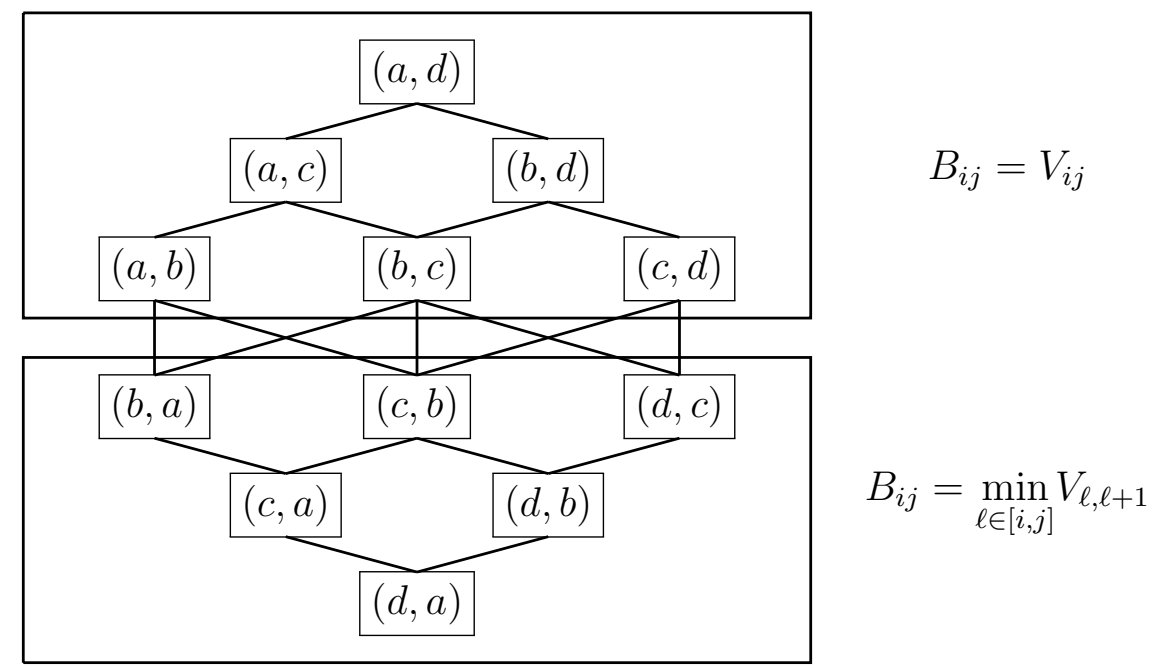

Figure 5.3: Characterization of the beatpath matrix $B$ in case the votrix $V$ is monotone.

(b) There is a candidate $a_{i_{3}}$ in the path such that $a_{i_{1}} \succ a_{i_{2}} \succ a_{i_{3}}$. Therefore, one of the couples in the path is such that the first element is preferred to the second one by less than half of the number of voters, a contradiction with the fact that the strength of the weakest link is greater than the strength of support of $a_{i_{1}}$ over $a_{i_{2}}$.

(c) Any candidate $a_{i_{3}}$ in the path is such that $a_{i_{1}} \succ a_{i_{3}} \succ a_{i_{2}}$. Therefore, as $V$ is monotone w.r.t. $\succ$, the strength of support of $a_{i_{1}}$ over $a_{i_{2}}$ is greater than or equal to the strength of all the links in the path, a contradiction with the fact that the strength of the weakest link is greater than the strength of support of $a_{i_{1}}$ over $a_{i_{2}}$.

2. The case $a_{i_{2}} \succ a_{i_{1}}$. Suppose that there is a path from $a_{i_{2}}$ to $a_{i_{1}}$ such that the strength of its weakest link is greater than the lowest strength of support of $a_{\ell}$ over $a_{\ell+1}$, for $\ell \in\left\{r_{\succ}\left(i_{1}\right), \ldots, r_{\succ}\left(i_{2}\right)-1\right\}$. We prove that, for any $\left(a_{\ell}, a_{\ell+1}\right)$ with $\ell \in$ $\left\{r_{\succ}\left(i_{1}\right), \ldots, r_{\succ}\left(i_{2}\right)-1\right\}$, there is a couple $\left(a_{j_{1}}, a_{j_{2}}\right)$ in the path such that $\left(a_{\ell}, a_{\ell+1}\right) \sqsupset$ $\left(a_{j_{1}}, a_{j_{2}}\right)$ or $\left(a_{\ell}, a_{\ell+1}\right)=\left(a_{j_{1}}, a_{j_{2}}\right)$. Let us suppose that, for a fixed $\ell \in\left\{r_{\succ}\left(i_{1}\right), \ldots\right.$, $\left.r_{\succ}\left(i_{2}\right)-1\right\}$, there is no $\left(a_{j_{1}}, a_{j_{2}}\right)$ in the path such that $\left(a_{\ell}, a_{\ell+1}\right) \sqsupset\left(a_{j_{1}}, a_{j_{2}}\right)$ or $\left(a_{\ell}, a_{\ell+1}\right)=\left(a_{j_{1}}, a_{j_{2}}\right)$. It follows that there is no $\left(a_{j_{1}}, a_{j_{2}}\right)$ in the path such that $a_{\ell} \succeq$ $a_{j_{1}}$ or that $a_{j_{2}} \succeq a_{\ell+1}$ or that $\left(a_{\ell}, a_{\ell+1}\right)=\left(a_{j_{1}}, a_{j_{2}}\right)$. However, as $a_{i_{2}} \succeq a_{\ell+1} \succ a_{\ell} \succeq a_{i_{1}}$, there needs to be a couple $\left(a_{j_{1}}, a_{j_{2}}\right)$ in the path such that $a_{j_{2}} \succeq a_{\ell+1} \succ a_{\ell} \succeq a_{j_{1}}$ due to the transitivity of $\succ$, the definition of path and the fact that $a_{\ell}$ and $a_{\ell+1}$ are 
consecutive candidates in $\succ$. We conclude that $a_{\ell} \succeq a_{j_{1}}$ or that $a_{j_{2}} \succeq a_{\ell+1}$ or that $\left(a_{\ell}, a_{\ell+1}\right)=\left(a_{j_{1}}, a_{j_{2}}\right)$, a contradiction.

Finally, as $V$ is monotone w.r.t. $\succ$, the strength of the weakest link in the path is smaller than or equal to the strength of the lowest strength of support of $a_{\ell}$ over $a_{\ell+1}$, for $\ell \in\left\{r_{\succ}\left(i_{1}\right), \ldots, r_{\succ}\left(i_{2}\right)-1\right\}$, a contradiction.

We conclude that, in case $V$ is monotone w.r.t. the ranking $\succ$, the beatpath matrix $B$ equals $V$ for couples in the upper half of the diagram of $\sqsupset$ and that for any couple in the lower half of the diagram it equals the minimum of the values of $V$ in the upper row of the lower half of the diagram that dominate the chosen couple. Thus, as $V$ is monotone w.r.t. $\succ, B$ obviously is decreasing on $\sqsupset$ and, therefore, monotone w.r.t. $\succ$.

Unfortunately, the search for monotonicity of the beatpath matrix is not easily characterizable as a transportation problem (see Chapter 6 for a discussion on how all other searches can be addressed by solving a transportation problem). For this reason, monotonicity of the beatpath matrix will no longer be considered in the computational studies of this dissertation.

\subsection{Monotonicity of the (anonymised) profile}

Any (anonymised) profile is determined by the number of times that each ranking is voted We denote by $\mathbf{n}_{\mathscr{R}} \in\{0,1, \ldots, r\}^{k !}$ the vector of absolute frequencies of $\mathscr{R}$, where $\mathbf{n}_{\mathscr{R}}(i)$ is the absolute frequency of the $i$-th ranking in $\mathcal{L}(\mathscr{C})$, i.e., the number of voters that have expressed the $i$-th ranking in $\mathcal{L}(\mathscr{C})$ in the profile $\mathscr{R}$. Analogously, we denote by $\mathbf{f}_{\mathscr{R}} \in\left\{0, \frac{1}{r}, \ldots, 1\right\}^{k !}$ the vector of relative frequencies of $\mathscr{R}$, where $\mathbf{f}_{\mathscr{R}}(i)$ is the relative frequency of the $i$-th ranking in $\mathcal{L}(\mathscr{C})$ in the profile $\mathscr{R}$. For any $i \in\{1, \ldots, k !\}$, it obviously holds that

$$
r \cdot \mathbf{f}_{\mathscr{R}}(i)=\mathbf{n}_{\mathscr{R}}(i)
$$

In addition, it holds that

$$
\sum_{i=1}^{k !} \mathbf{n}_{\mathscr{R}}(i)=r \quad \wedge \quad \sum_{i=1}^{k !} \mathbf{f}_{\mathscr{R}}(i)=1 .
$$

\footnotetext{
${ }^{4}$ Here, we do not take the order of the voters into account.
} 
The set of all possible vectors $\mathbf{f} \in\left\{0, \frac{1}{r}, \ldots, 1\right\}^{k !}$ that can be seen as the vector of relative frequencies of a profile $\mathscr{R}$ of $r$ rankings on $\mathscr{C}$ is denoted by $\mathcal{R}_{r}(\mathscr{C})$.

Each ranking $\succ$ on $\mathscr{C}$ defines an order relation $\geqq$ on $\mathcal{L}(\mathscr{C})$ according to how far two rankings in $\mathcal{L}(\mathscr{C})$ are from $\succ$ in terms of reversals. For any $\succ_{i}, \succ_{j} \in \mathcal{L}(\mathscr{C})$, the fact that $\left(\succ_{i}, \succ_{j}\right) \in \geqq$ is denoted by $\succ_{i} \geqq \succ_{j}$.

Proposition 5.26 Let $\mathscr{C}$ be a set of $k$ candidates and $\succ$ be a ranking on $\mathscr{C}$. The relation $\geqq$ defined as

$$
\Xi=\left\{\left(\succ_{i}, \succ_{j}\right) \in \mathcal{L}(\mathscr{C})^{2} \mid\left(\forall\left(a_{i_{1}}, a_{i_{2}}\right) \in \mathscr{C}^{2}\right)\left(\left(\left(a_{i_{1}} \succ a_{i_{2}}\right) \wedge\left(a_{i_{1}} \succ_{j} a_{i_{2}}\right)\right) \Rightarrow\left(a_{i_{1}} \succ_{i} a_{i_{2}}\right)\right)\right\}
$$

is an order relation on $\mathcal{L}(\mathscr{C})$.

Proof: We prove that $\geqq$ satisfies reflexivity, antisymmetry and transitivity.

Reflexivity: holds trivially.

Antisymmetry: for any $\succ_{i}, \succ_{j} \in \mathcal{L}(\mathscr{C})$, if $\succ_{i} \succ_{j}$ and $\succ_{j} \unlhd_{i}$, then it holds that:

$$
\begin{aligned}
& \left(\forall\left(a_{i_{1}}, a_{i_{2}}\right) \in \mathscr{C}^{2}\right)\left(\left(\left(a_{i_{1}} \succ a_{i_{2}}\right) \wedge\left(a_{i_{1}} \succ_{j} a_{i_{2}}\right)\right) \Rightarrow\left(a_{i_{1}} \succ_{i} a_{i_{2}}\right)\right), \\
& \left(\forall\left(a_{i_{1}}, a_{i_{2}}\right) \in \mathscr{C}^{2}\right)\left(\left(\left(a_{i_{1}} \succ a_{i_{2}}\right) \wedge\left(a_{i_{1}} \succ_{i} a_{i_{2}}\right)\right) \Rightarrow\left(a_{i_{1}} \succ_{j} a_{i_{2}}\right)\right) .
\end{aligned}
$$

Therefore, for any $a_{i_{1}}, a_{i_{2}} \in \mathscr{C}$ such that $a_{i_{1}} \succ a_{i_{2}}$, it holds that

$$
\left(a_{i_{1}} \succ_{i} a_{i_{2}}\right) \Leftrightarrow\left(a_{i_{1}} \succ_{j} a_{i_{2}}\right)
$$

As $\succeq$ is complete, if $a_{i_{1}} \nsucc a_{i_{2}}$, then it holds that $a_{i_{1}}=a_{i_{2}}$ or $a_{i_{2}} \succ a_{i_{1}}$. If $a_{i_{1}}=a_{i_{2}}$, then, as $\succ_{i}$ and $\succ_{j}$ are irreflexive by definition of ranking, it is trivial to see that $\left(a_{i_{1}} \succ_{i} a_{i_{2}}\right) \Leftrightarrow$ $\left(a_{i_{1}} \succ_{j} a_{i_{2}}\right)$. If $a_{i_{2}} \succ a_{i_{1}}$, then it holds that $\left(a_{i_{2}} \succ_{i} a_{i_{1}}\right) \Leftrightarrow\left(a_{i_{2}} \succ_{j} a_{i_{1}}\right)$, or, equivalently, $\left(\begin{array}{lll}a_{i_{2}} & \nsucc_{i} & a_{i_{1}}\end{array}\right) \Leftrightarrow\left(\begin{array}{lll}a_{i_{2}} & \nsucc_{j} & a_{i_{1}}\end{array}\right)$. As $\succeq_{i}$ and $\succeq_{j}$ are complete and $a_{i_{2}} \succ a_{i_{1}}$, it holds that $\left(a_{i_{1}} \succ_{i} a_{i_{2}}\right) \Leftrightarrow\left(a_{i_{1}} \succ_{j} a_{i_{2}}\right)$.

Hence, for any $a_{i_{1}}, a_{i_{2}} \in \mathscr{C}$, it holds that

$$
\left(a_{i_{1}} \succ_{i} a_{i_{2}}\right) \Leftrightarrow\left(a_{i_{1}} \succ_{j} a_{i_{2}}\right)
$$

i.e., it holds that $\succ_{i}=\succ_{j}$. 
Transitivity: for any $\succ_{i}, \succ_{j}, \succ_{\ell} \in \mathcal{L}(\mathscr{C})$, if $\succ_{i} \geqq \succ_{j}$ and $\succ_{j} \geqq \succ_{\ell}$, then it holds that

$$
\begin{aligned}
& \left(\forall\left(a_{i_{1}}, a_{i_{2}}\right) \in \mathscr{C}^{2}\right)\left(\left(\left(a_{i_{1}} \succ a_{i_{2}}\right) \wedge\left(a_{i_{1}} \succ_{j} a_{i_{2}}\right)\right) \Rightarrow\left(a_{i_{1}} \succ_{i} a_{i_{2}}\right)\right), \\
& \left(\forall\left(a_{i_{1}}, a_{i_{2}}\right) \in \mathscr{C}^{2}\right)\left(\left(\left(a_{i_{1}} \succ a_{i_{2}}\right) \wedge\left(a_{i_{1}} \succ_{\ell} a_{i_{2}}\right)\right) \Rightarrow\left(a_{i_{1}} \succ_{j} a_{i_{2}}\right)\right) .
\end{aligned}
$$

Hence, for any $a_{i_{1}}, a_{i_{2}} \in \mathscr{C}$ such that $a_{i_{1}} \succ a_{i_{2}}$, it holds that

$$
\left(a_{i_{1}} \succ_{\ell} a_{i_{2}}\right) \Rightarrow\left(a_{i_{1}} \succ_{j} a_{i_{2}}\right) \Rightarrow\left(a_{i_{1}} \succ_{i} a_{i_{2}}\right)
$$

i.e., it holds that $\succ_{i} \geqq \succ_{\ell}$.

Thus, $\geqq$ is an order relation on $\mathcal{L}(\mathscr{C})$.

Figure 5.4 displays the Hasse diagram of the order relation $\geqq$, where $a \succ b \succ c \succ d$ is the ranking assumed to be the true ranking on a set of four candidates $\mathscr{C}=\{a, b, c, d\}$. Clearly, every ranking $\succ_{i}$ is closer (in terms of reversals) to $\succ$ than $\succ_{j}$ if it holds that $\succ_{i} \geqq \succ_{j}$.

Under the assumption that there exists a true ranking $\succ$ on $\mathscr{C}$, it seems natural that the vector of frequencies of the given profile of rankings should be decreasing on the Hasse diagram of the order relation $\geqq$. A profile of rankings satisfying this property is said to be monotone w.r.t. the ranking $\succ$.

Definition 5.27 Let $\mathscr{C}$ be a set of $k$ candidates, $r$ be the number of voters and $\succ$ be a ranking on $\mathscr{C}$.

(i) A vector of relative frequencies $\mathbf{f} \in \mathcal{R}_{r}(\mathscr{C})$ is said to be monotone w.r.t. $\succ$ if, for any $\succ_{i}, \succ_{j} \in \mathcal{L}(\mathscr{C})$, it holds that

$$
\left(\succ_{i} \succ_{j}\right) \Rightarrow(\mathbf{f}(i) \geq \mathbf{f}(j))
$$

(ii) A vector of absolute frequencies $\mathbf{n}$ is said to be monotone w.r.t. $\succ$ if the corresponding vector of relative frequencies $\mathbf{f}=\frac{\mathbf{n}}{r}$ is monotone w.r.t. $\succ$.

(iii) A profile $\mathscr{R}$ of rankings on $\mathscr{C}$ is said to be monotone w.r.t. $\succ$ if the corresponding vector of relative frequencies of $\mathscr{R}$ is monotone w.r.t. $\succ$.

Obviously, not all profiles of rankings are monotone w.r.t. a at least one ranking. 


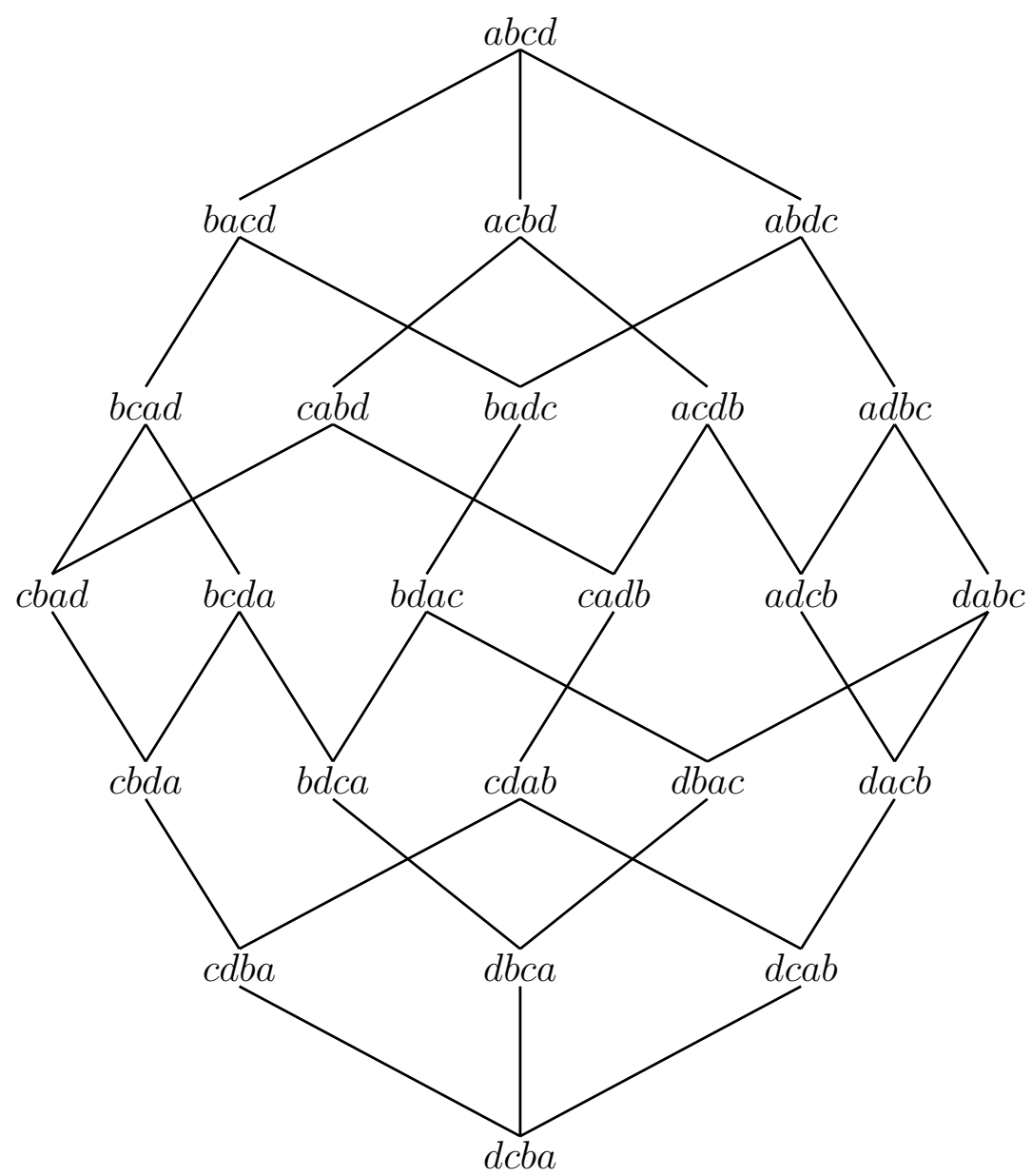

Figure 5.4: Hasse diagram of the order relation $\geqq$ for the ranking $a \succ b \succ$ $c \succ d$, where $x y z t$ is a shorthand for $x \succ y \succ z \succ t$.

Remark 5.28 If the profile of rankings is monotone w.r.t. a ranking $\succ$ on the set of candidates, then $\succ$ is a most frequent ranking in the profile of rankings.

Monotonicity of the profile of rankings implies the existence of a true ranking on the set of candidates instead of a compromise ranking.

Obviously, monotonicity of the profile of rankings is a weaker condition than unanimity.

Theorem 5.29 Let $\mathscr{C}$ be a set of $k$ candidates and $\mathscr{R}$ be the profile of $r$ rankings on $\mathscr{C}$ given by the voters. If $\mathscr{R}$ is the unanimous profile where every voter expresses $\succ$, then $\mathscr{R}$ is monotone w.r.t. $\succ$. 
Proof: The result follows immediately from the fact that, in case $\mathscr{R}$ is the unanimous profile where every voter expresses $\succ$, it holds that $\succ$ is the ranking at the top of the Hasse diagram of the order relation $\geqq$.

\subsection{Acclamation}

The main aim of this dissertation is to analyse the conditions under which determining the winning ranking on the set of candidates is obvious. Unanimity obviously is one of this situations and, unfortunately, it is the only situation under which the winning ranking on the set of candidates is indisputably determined. Fortunately, as discussed in the previous sections, monotonicity of different representations of votes can be understood as a cornerstone of social choice theory where almost all ranking rules lead to the same social outcome.

First, recursive monotonicity of the scorix assures that all ranking rules based on positional information lead to the same ranking on the set of candidates. Second, monotonicity of the votrix assures that all ranking rules based on pairwise information lead to the same ranking on the set of candidates. Note that monotonicity of the profile is not linked to an agreement of a family of ranking rules. Rather, as discussed in the upcoming Chapter 7 , monotonicity of the profile may lead to winning rankings that significantly differ from the rest of the ranking rules. Rather, as discussed in Section 5.6, monotonicity of the profile is linked to the real existence of a true ranking on the set of candidates, i.e., the philosophy advocated by Rousseau [139] and Condorcet [35] where personal preferences are not considered, to identify the 'general will'. This phylosophy is clearly described by Arrow [4]: "each individual has two orderings, one which governs him in his everyday actions, and one which would be relevant under some ideal conditions and which is in some sense truer than the first ordering. It is the latter which is considered relevant to social choice, and it is assumed that there is complete unanimity with regard to the truer individual ordering".

In this section, we propose to jointly consider these three types of monotonicity in order to define a weaker condition than unanimity, but that still leads to an obvious social outcome. From now on, a ranking w.r.t. which the scorix is recursively monotone, the votrix is monotone and the profile is monotone, is referred to as an acclaimed ranking [119]. 
Definition 5.30 Let $\mathscr{C}$ be a set of $k$ candidates, $\mathscr{R}$ be the profile of $r$ rankings on $\mathscr{C}$ given by the voters, $S \in \mathcal{S}_{r}(\mathscr{C})$ be the scorix induced by $\mathscr{R}$ and $V \in \mathcal{V}_{r}(\mathscr{C})$ be the votrix induced by $\mathscr{R}$. A ranking $\succ$ on $\mathscr{C}$ is called the acclaimed ranking for $\mathscr{R}$ if the following three statements hold:

(i) $\mathscr{R}$ is monotone w.r.t. $\succ$.

(ii) $S$ is recursively monotone w.r.t. $\succ$.

(iii) $V$ is monotone w.r.t. $\succ$.

Remark 5.31 The term acclamation historically refers to a voting system used in Ancient Greece, where the winning candidate was decided by the (loudest) shouts of the people [69].

In case there exists an acclaimed ranking for a given profile of rankings, we say that the profile belongs to the acclamation consensus state.

By definition, acclamation is obviously a weaker consensus state than unanimity and a stronger consensus state than (recursive) monotonicity of the scorix, monotonicity of the votrix and monotonicity of the profile.

Theorem 5.32 Let $\mathscr{C}$ be a set of $k$ candidates, $\mathscr{R}$ be the profile of $r$ rankings on $\mathscr{C}$ given by the voters, $V \in \mathcal{V}_{r}(\mathscr{C})$ be the votrix induced by $\mathscr{R}, W \in \mathcal{W}_{r}(\mathscr{C})$ be the votex induced by $\mathscr{R}$ and $\succ$ be a ranking on $\mathscr{C}$. The following statements hold:

(i) If $\mathscr{R}$ is the unanimous profile where every voter expresses $\succ$, then $\succ$ is the acclaimed ranking for $\mathscr{R}$.

(ii) If $\succ$ is the acclaimed ranking for $\mathscr{R}$, then $\mathscr{R}$ is monotone w.r.t. $\succ$.

(iii) If $\succ$ is the acclaimed ranking for $\mathscr{R}$, then $S$ is (recursively) monotone w.r.t. $\succ$.

(iv) If $\succ$ is the acclaimed ranking for $\mathscr{R}$, then $V$ is monotone w.r.t. $\succ$.

(v) If $\succ$ is the acclaimed ranking for $\mathscr{R}$, then the first ranked candidate according to $\succ$ is a weak Condorcet winner and $\succ$ is a weak Condorcet ranking. 
(vi) If $\succ$ is the acclaimed ranking for $\mathscr{R}$ and $r$ is an odd number, then the first ranked candidate according to $\succ$ is the Condorcet winner and $\succ$ is the Condorcet ranking.

\section{Proof:}

Statement (i) is a consequence of Theorems 5.3, 5.23 and 5.29 .

Statements (ii), (iii) and (iv) are straightforward due to the definition of an acclaimed ranking.

Statements (v) and (vi) are a consequence of Theorem 5.23 .

We recall here that the introduction of consensus states broader than unanimity, but that still lead to an obvious ranking on the set of candidates, represents a valuable topic in the field of social choice theory. This is due to the fact that the broader the consensus state, the less the ranking rule based on the search for this consensus state depends on the chosen monometric. Unfortunately, it also holds that the broader the consensus state, the weaker the support for the winning ranking associated with the consensus state. For this reason, we advocate for the use of acclamation, which is the broadest consensus state for which we could not identify a prominent ranking rule disagreeing with the associated winning ranking.

As illustrated in Table 5.3 , in case $\succ$ is the acclaimed ranking for a given profile of rankings, the ranking $\succ$ is a winning ranking and/or the first ranked candidate in $\succ$ is a winning candidate for the most prominent voting rules. In Table 5.3, a symbol $\checkmark$ (resp. -) in the column WC means that the first ranked candidate in the acclaimed ranking is (resp. does not need to be) a Winning Candidate for the method corresponding to the row; a symbol $\checkmark$ (resp. - ) in the column UWC means that the first ranked candidate in the acclaimed ranking is (resp. does not need to be) the Unique Winning Candidate for the method corresponding to the row; a symbol $\checkmark$ (resp. - ) in the column WR means that the acclaimed ranking is (resp. does not need to be) a Winning Ranking for the method corresponding to the row; and a symbol $\checkmark$ (resp. - ) in the column UWR means that the acclaimed ranking is (resp. does not need to be) the Unique Winning Ranking for the method corresponding to the row. The symbol $*$ means that the method corresponding to the row is not explicitly defined for identifying a winning ranking. 


\begin{tabular}{|l|c|c|c|c|}
\hline Method & WC & UWC & WR & UWR \\
\hline \hline Plurality [153] & $\checkmark$ & - & $\checkmark$ & - \\
\hline Borda count [18] & $\checkmark$ & $\checkmark$ & $\checkmark$ & $\checkmark$ \\
\hline Veto [141] & $\checkmark$ & - & $\checkmark$ & - \\
\hline Best-worst voting systems [66] & $\checkmark$ & - & $\checkmark$ & - \\
\hline Scoring (ranking) rules [169] & $\checkmark$ & - & $\checkmark$ & - \\
\hline Elimination methods based on a & $\checkmark$ & - & $\checkmark$ & - \\
scoring (ranking) rule [121] & & & & \\
\hline Simple majority rule [95] & $\checkmark$ & $\checkmark$ & $\checkmark$ & $\checkmark$ \\
\hline Dodgson [46] & $\checkmark$ & $\checkmark$ & $*$ & $*$ \\
\hline Condorcet's least-reversals [99] & $\checkmark$ & $\checkmark$ & $*$ & $*$ \\
\hline Kemeny [80] & $\checkmark$ & $\checkmark$ & $\checkmark$ & $\checkmark$ \\
\hline Copeland [40] & $\checkmark$ & $\checkmark$ & $\checkmark$ & $\checkmark$ \\
\hline Tideman [157] & $\checkmark$ & $\checkmark$ & $\checkmark$ & $\checkmark$ \\
\hline Schulze [147] & $\checkmark$ & $\checkmark$ & $\checkmark$ & $\checkmark$ \\
\hline Simpson [85, 151] & $\checkmark$ & $\checkmark$ & $\checkmark$ & - \\
\hline Bucklin [23] & $\checkmark$ & - & $*$ & $*$ \\
\hline
\end{tabular}

Table 5.3: Concordance with the notion of acclamation by the most prominent methods in social choice theory.

For plurality, the Borda count, veto, best-worst voting systems, scoring (ranking) rules and elimination methods based on a scoring (ranking) rule, the facts that the first ranked candidate in the acclaimed ranking is a winning candidate and that the acclaimed ranking is a winning ranking are a direct result from Theorem 5.13. This theorem also implies that the first ranked candidate in the acclaimed ranking is the unique winning candidate for the Borda count and that the acclaimed ranking is the unique winning ranking for the Borda count. We prove that the uniqueness is not assured for the remaining ranking rules mentioned in this paragraph by providing a counterexample.

Consider the set $\mathscr{C}=\{a, b, c, d\}$ of $k=4$ candidates and the profile $\mathscr{R}$ of $r=28$ rankings given in Table 5.4 . 


\begin{tabular}{|c|c||c|c||c|c||c|c|}
\hline Ranking & Freq. & Ranking & Freq. & Ranking & Freq. & Ranking & Freq. \\
\hline$a \succ b \succ c \succ d$ & 4 & $b \succ a \succ c \succ d$ & 3 & $c \succ a \succ b \succ d$ & 1 & $d \succ a \succ b \succ c$ & 1 \\
$a \succ b \succ d \succ c$ & 3 & $b \succ a \succ d \succ c$ & 2 & $c \succ a \succ d \succ b$ & 1 & $d \succ a \succ c \succ b$ & 1 \\
$a \succ c \succ b \succ d$ & 1 & $b \succ c \succ a \succ d$ & 2 & $c \succ b \succ a \succ d$ & 1 & $d \succ b \succ a \succ c$ & 1 \\
$a \succ c \succ d \succ b$ & 1 & $b \succ c \succ d \succ a$ & 1 & $c \succ b \succ d \succ a$ & 0 & $d \succ b \succ c \succ a$ & 0 \\
$a \succ d \succ b \succ c$ & 1 & $b \succ d \succ a \succ c$ & 2 & $c \succ d \succ a \succ b$ & 0 & $d \succ c \succ a \succ b$ & 0 \\
$a \succ d \succ c \succ b$ & 1 & $b \succ d \succ c \succ a$ & 1 & $c \succ d \succ b \succ a$ & 0 & $d \succ c \succ b \succ a$ & 0 \\
\hline
\end{tabular}

Table 5.4: Profile $\mathscr{R}$ of $r=28$ rankings on $\mathscr{C}=\{a, b, c, d\}$.

The scorix induced by $\mathscr{R}$ is:

$$
S=\left(\begin{array}{cccc}
11 & 9 & 6 & 2 \\
11 & 9 & 4 & 4 \\
3 & 5 & 10 & 10 \\
3 & 5 & 8 & 12
\end{array}\right)
$$

Although $a \succ b \succ c \succ d$ is the acclaimed ranking for $\mathscr{R}$, all rankings $a \succ b \succ c \succ d$, $a \succ b \succ d \succ c, b \succ a \succ c \succ d$ and $b \succ a \succ d \succ c$ are winning rankings for the plurality rule. Analogously, in case we reverse the order of the candidates in all rankings in the profile, we obtain a profile $\mathscr{R}^{\prime}$ where, although $d \succ c \succ b \succ a$ is the acclaimed ranking for $\mathscr{R}^{\prime}$, all rankings $c \succ d \succ a \succ b, c \succ d \succ b \succ a, d \succ c \succ a \succ b$ and $d \succ c \succ b \succ a$ are winning rankings for the veto rule. As best-worst voting systems, scoring (ranking) rules and elimination methods based on a scoring (ranking) rule have both the plurality and the veto rules as a particular case, we conclude that the first ranked candidate in the acclaimed ranking does not need to be the unique winner, and the acclaimed ranking does not need to be the unique winning ranking for any of the aforementioned ranking rules.

By definition of the simple majority rule, in case of existence of the Condorcet ranking - which is assured to coincide with the acclaimed ranking in case the latter exists - the (unique) winning candidate coincides with the first ranked candidate in the Condorcet ranking and the (unique) winning ranking coincides with the Condorcet ranking. Similarly, as both the method of Dodgson and Condorcet's least-reversals methods are based on the search for the candidate that is the closest to becoming the Condorcet winner, in case of existence of the Condorcet winner, the (unique) winning candidate for both methods coincides with the first ranked candidate in the Condorcet ranking. 
The methods of Kemeny, Copeland, Tideman, Schulze and Simpson are Condorcet methods, i.e., in case of existence of a Condorcet winner, they select this Condorcet winner as the unique winning candidate. Moreover, the first four are additionally Condorcet ranking methods, i.e., in case of existence of a Condorcet ranking, they select this Condorcet ranking as the unique winning ranking. Due to the property of monotonicity of the votrix, the method of Simpson, which ranks the candidates according to their greatest pairwise defeat, is trivially assured to select the acclaimed ranking as the winning ranking. However, the uniqueness is not assured in this case.

Consider the set $\mathscr{C}=\{a, b, c, d\}$ of $k=4$ candidates and the profile $\mathscr{R}$ of $r=11$ rankings given in Table 5.5 .

\begin{tabular}{|c|c|}
\hline Freq. & Rankings on $\mathscr{C}$ \\
\hline 6 & $a \succ b \succ c \succ d$ \\
5 & $b \succ a \succ c \succ d$ \\
\hline
\end{tabular}

Table 5.5: Profile $\mathscr{R}$ of $r=11$ rankings on $\mathscr{C}=\{a, b, c, d\}$.

The votrix induced by $\mathscr{R}$ is:

$$
S=\left(\begin{array}{cccc}
0 & 6 & 11 & 11 \\
5 & 0 & 11 & 11 \\
0 & 0 & 0 & 11 \\
0 & 0 & 0 & 0
\end{array}\right)
$$

Note that candidates $c$ and $d$ have the same greatest pairwise defeat. Therefore, although $a \succ b \succ c \succ d$ is the acclaimed ranking for $\mathscr{R}$, both rankings $a \succ b \succ c \succ d$ and $a \succ b \succ d \succ c$ are winning rankings for the method of Simpson.

As the vector of positions of candidate $a$ (strictly) dominates the vectors of positions of all other candidates, we conclude that the first ranked candidate in the acclaimed ranking is a winning candidate for the method of Bucklin. For proving that the uniqueness does not hold, we consider again the profile $\mathscr{R}$ of $r=28$ rankings given in Table 5.4. Note that there is no candidate that is ranked at the first position by more than half of the number of voters. Therefore, according to the method of Bucklin, we need to consider also the number of times that each candidate is ranked at the second position. Now, candidates $a$ and $b$ are ranked at the first or second position by 20 voters, which is more than half of the number of voters. Therefore, candidates $a$ and $b$ are winning candidates for the method of Bucklin, while candidate $b$ is not the first ranked candidate in the acclaimed ranking. 
We conclude this chapter with Figure 5.5 illustrating the relations between the different types of consensus states discussed here. In this figure, an arrow indicates that the consensus state from which the arrow starts implies the consensus state to which the arrow points.

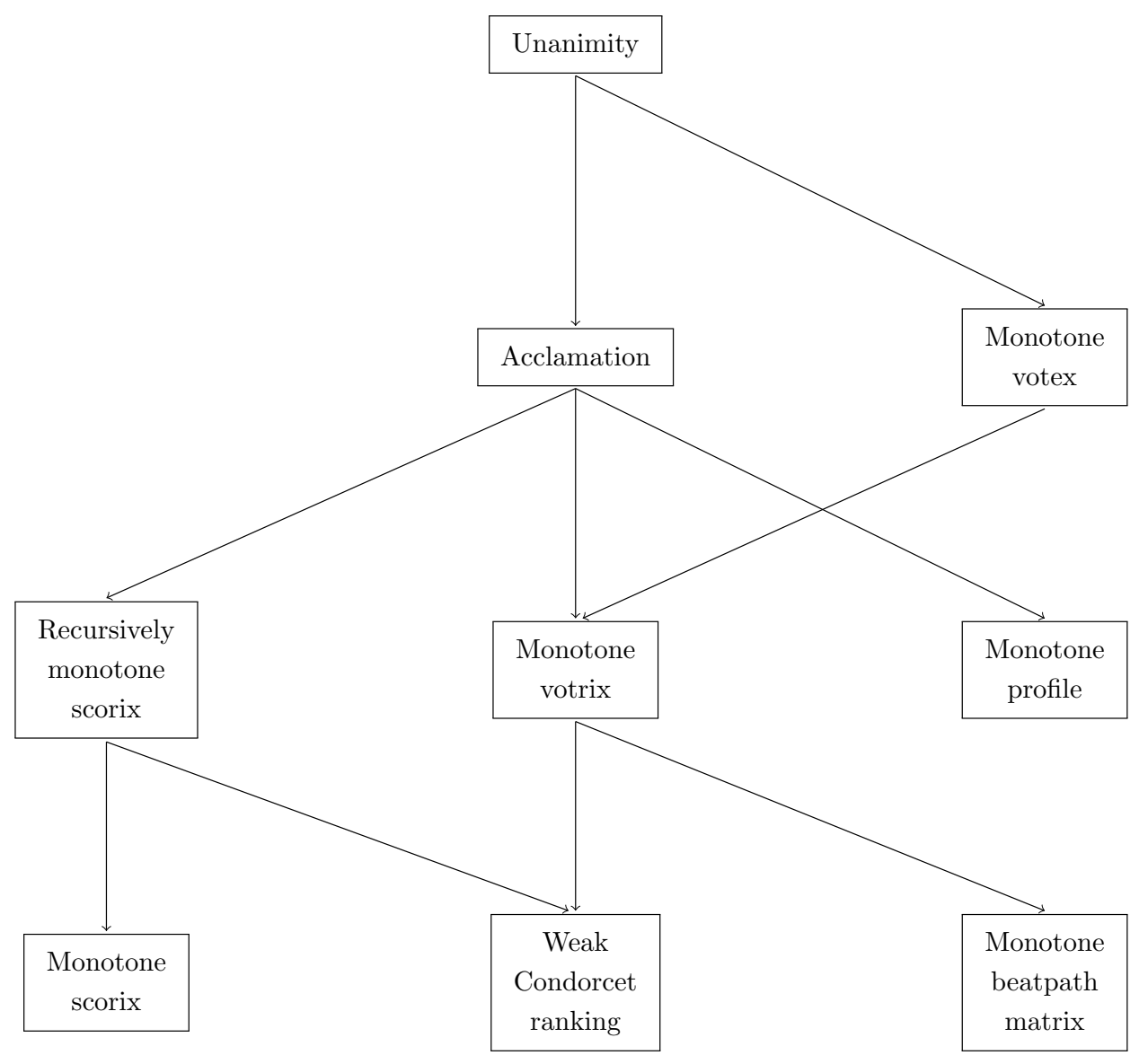

Figure 5.5: Relation between the different consensus states. 


\section{CHAPTER 6}

\section{The search for monotonicity as an optimization problem}

In this chapter, the search for all different types of monotonicity is addressed as an optimization problem. This chapter is divided into two different sections. First, the search for monotonicity is addressed by making changes in the representation of votes. Second, the search for monotonicity is addressed by making changes in the profile of rankings. Although both approaches are described here, we will only consider the latter after the conclusion of this chapter. This is due to the fact that the search for monotonicity by means of changes in the profile of rankings, unlike the search for monotonicity by means of changes in the representation of votes, does not disregard all the information that is lost when contracting the profile of rankings into a representation of votes. Moreover, in case we count changes in the profile of rankings, the closeness to any consensus states becomes comparable with the closeness to any other consensus states. The search for the same property by means of a distance function at the representation level and of a distance function at the profile level is a common topic in the field of social choice theory. For instance, the search for a Condorcet winner [35] is addressed by means of a distance function at the votrix level and of a distance function at the profile level by two well-known methods: Condorcet's least-reversal method [99] and Dodgson's method [46]. 


\subsection{Changes in the representation of votes}

In this section, we discuss the search for a monotone representation of votes. Note that, unlike in the case of the scorix, the search for a monotone votrix (resp. votex) does not assure the obtained construct to be a votrix (resp. votex), but a quasivotrix (resp. quasivotex).

\subsubsection{Search for a monotone scorix}

As discussed in [125], a natural ranking rule can be defined by searching for a construct that satisfies the properties mentioned in Proposition 4.4 and that at the same time is monotone w.r.t. a ranking on the set of candidates. Fortunately, as Fine and Fine proved in [52], scorices are characterizable by means of the properties listed in Proposition 4.4, thus the search for the closest scorix satisfying monotonicity can be addressed. This closest scorix has an associated cost that is calculated by means of a monometric on $\mathcal{S}_{r}(\mathscr{C})$.

Definition 6.1 Let $\mathscr{C}$ be a set of $k$ candidates and $r$ be the number of voters. A function $M: \mathcal{S}_{r}(\mathscr{C}) \times \mathcal{S}_{r}(\mathscr{C}) \rightarrow \mathbb{R}$ is called a monometric on $\mathcal{S}_{r}(\mathscr{C})$ if it satisfies the following three properties:

(i) Non-negativity: for any $S, S^{\prime} \in \mathcal{S}_{r}(\mathscr{C})$, it holds that

$$
M\left(S, S^{\prime}\right) \geq 0
$$

(ii) Coincidence: for any $S, S^{\prime} \in \mathcal{S}_{r}(\mathscr{C})$, it holds that

$$
M\left(S, S^{\prime}\right)=0 \Leftrightarrow S=S^{\prime}
$$

(iii) Compatibility: for any $S, S^{\prime}, S^{\prime \prime} \in \mathcal{S}_{r}(\mathscr{C})$ such that, for any $i \in\{1, \ldots, k\}$,

$$
\left(S_{i} \unrhd_{1} S_{i}^{\prime} \unrhd_{1} S_{i}^{\prime \prime}\right) \vee\left(S_{i}^{\prime \prime} \unrhd_{1} S_{i}^{\prime} \unrhd_{1} S_{i}\right),
$$

it holds that

$$
M\left(S, S^{\prime}\right) \leq M\left(S, S^{\prime \prime}\right)
$$

For any two scorices $S, S^{\prime} \in \mathcal{S}_{r}(\mathscr{C}), M\left(S, S^{\prime}\right)$ is referred to as the cost of changing $S$ into $S^{\prime}$. 
Definition 6.2 Let $\mathscr{C}$ be a set of $k$ candidates, $r$ be the number of voters, $\succ$ be a ranking on $\mathscr{C}, S \in \mathcal{S}_{r}(\mathscr{C})$ be a scorix and $M: \mathcal{S}_{r}(\mathscr{C}) \times \mathcal{S}_{r}(\mathscr{C}) \rightarrow \mathbb{R}$ be a monometric on $\mathcal{S}_{r}(\mathscr{C})$. A closest monotone scorix $S^{\prime} \in \mathcal{S}_{r}(\mathscr{C})$ to $S$ (w.r.t. $\succ$ ) is a scorix that is monotone w.r.t. $\succ$ and such that there exists no scorix $S^{\prime \prime} \in \mathcal{S}_{r}(\mathscr{C})$ that is monotone w.r.t. $\succ$ and, at the same time,

$$
M\left(S, S^{\prime \prime}\right)<M\left(S, S^{\prime}\right)
$$

Thus, for each ranking $\succ$, we have the corresponding cost associated with a closest monotone scorix (measured by means of the chosen monometric on $\mathcal{S}_{r}(\mathscr{C})$ ). An optimal ranking should be one whose corresponding closest monotone scorix is the closest to the scorix given by the voters.

Definition 6.3 Let $\mathscr{C}$ be a set of $k$ candidates, $\mathscr{R}$ be the profile of $r$ rankings on $\mathscr{C}$ given by the voters, $S_{\mathscr{R}} \in \mathcal{S}_{r}(\mathscr{C})$ be the scorix induced by $\mathscr{R}$ and $M: \mathcal{S}_{r}(\mathscr{C}) \times \mathcal{S}_{r}(\mathscr{C}) \rightarrow \mathbb{R}$ be a monometric on $\mathcal{S}_{r}(\mathscr{C})$. An optimal ranking $\succ$ (with a corresponding closest monotone scorix $S \in \mathcal{S}_{r}(\mathscr{C})$ ) is a ranking for which it holds that there exists no ranking $\succ^{\prime}$ (with a corresponding closest monotone scorix $\left.S^{\prime} \in \mathcal{S}_{r}(\mathscr{C})\right)$ such that

$$
M\left(S_{\mathscr{R}}, S^{\prime}\right)<M\left(S_{\mathscr{R}}, S\right) .
$$

Note that the search for a closest 2-monotone scorix can be addressed in an analogous way.

Definition 6.4 Let $\mathscr{C}$ be a set of $k$ candidates, $r$ be the number of voters, $\succ$ be a ranking on $\mathscr{C}, S \in \mathcal{S}_{r}(\mathscr{C})$ be a scorix and $M: \mathcal{S}_{r}(\mathscr{C}) \times \mathcal{S}_{r}(\mathscr{C}) \rightarrow \mathbb{R}$ be a monometric on $\mathcal{S}_{r}(\mathscr{C})$. A closest 2-monotone scorix $S^{\prime} \in \mathcal{S}_{r}(\mathscr{C})$ to $S$ (w.r.t. $\succ$ ) is a scorix that is 2-monotone w.r.t. $\succ$ and such that there exists no scorix $S^{\prime \prime} \in \mathcal{S}_{r}(\mathscr{C})$ that is 2-monotone w.r.t. $\succ$ and, at the same time,

$$
M\left(S, S^{\prime \prime}\right)<M\left(S, S^{\prime}\right)
$$

The resolution of the problem is addressed for an interesting monometric on $\mathcal{S}_{r}(\mathscr{C})$ based on the total amount of changes in the vectors of positions of all the candidates. This leads to the resolution of an Integer Linear Programming problem (ILP problem). The proposed monometric on $\mathcal{S}_{r}(\mathscr{C})$ is given by the sum of the absolute differences of the cumulative vectors of positions of all candidates:

$$
M\left(S, S^{\prime}\right)=\sum_{i=1}^{k} \sum_{\ell=1}^{k}\left|\sum_{j=1}^{\ell}\left(S_{i j}-S_{i j}^{\prime}\right)\right| .
$$


Obviously, in case the given scorix is not monotone w.r.t. any ranking on the set of candidates, different monometrics on $\mathcal{S}_{r}(\mathscr{C})$ might lead to different optimal rankings.

We will now prove that Eq. (6.1) defines a monometrid ${ }^{1}$ on $\mathcal{S}_{r}(\mathscr{C})$.

Proposition 6.5 Let $\mathscr{C}$ be a set of $k$ candidates, $r$ be the number of voters and $M$ : $\mathcal{S}_{r}(\mathscr{C}) \times \mathcal{S}_{r}(\mathscr{C}) \rightarrow \mathbb{R}$ be defined as in Eq. 6.1). Then $M$ is a monometric on $\mathcal{S}_{r}(\mathscr{C})$.

Proof: We will prove that $M$ satisfies the three axioms of a monometric on $\mathcal{S}_{r}(\mathscr{C})$.

Non-negativity. Immediate.

Coincidence. As it is a sum of absolute values, for any $S, S^{\prime} \in \mathcal{S}_{r}(\mathscr{C}), M\left(S, S^{\prime}\right)=0$ if and only if $\sum_{j=1}^{\ell} S_{i j}=\sum_{j=1}^{\ell} S_{i j}^{\prime}$, for any $i, \ell \in\{1, \ldots, k\}$. This is equivalent to saying that $S=S^{\prime}$.

Compatibility. For any $S, S^{\prime}, S^{\prime \prime} \in \mathcal{S}_{r}(\mathscr{C})$ satisfying that, for any $i, \ell \in\{1, \ldots, k\}$,

$$
\left(\sum_{j=1}^{\ell} S_{i j} \geq \sum_{j=1}^{\ell} S_{i j}^{\prime} \geq \sum_{j=1}^{\ell} S_{i j}^{\prime \prime}\right) \vee\left(\sum_{j=1}^{\ell} S_{i j} \leq \sum_{j=1}^{\ell} S_{i j}^{\prime} \leq \sum_{j=1}^{\ell} S_{i j}^{\prime \prime}\right),
$$

the following is fulfilled:

$$
\begin{aligned}
M\left(S, S^{\prime}\right) & =\sum_{i=1}^{k} \sum_{\ell=1}^{k}\left|\sum_{j=1}^{\ell}\left(S_{i j}-S_{i j}^{\prime}\right)\right| \\
& \leq \sum_{i=1}^{k} \sum_{\ell=1}^{k}\left|\sum_{j=1}^{\ell}\left(S_{i j}-S_{i j}^{\prime \prime}\right)\right|=M\left(S, S^{\prime \prime}\right) .
\end{aligned}
$$

Thus, $M$ is a monometric on $\mathcal{S}_{r}(\mathscr{C})$.

In order to search for an optimal ranking, we will need to solve an Integer Linear Programming problem for each possible ranking. The number of unknown variables (elements of the scorix) and the number of constraints (given by the properties of Proposition 4.4) in the optimization problem will depend on the number of candidates $k$ :

(i) Number of (positive integer) variables: $k^{2}$.

\footnotetext{
${ }^{1}$ As it is symmetric and satisfies the triangle inequality, Eq. 6.1 actually defines a distance function that satisfies the additional axiom of compatibility.
} 
(ii) Completeness in candidates constraints (equalities): $k$.

(iii) Completeness in positions constraints (equalities): $k$.

(iv) Monotonicity constraints (inequalities): $(k-1)^{2}$.

More specifically, the Integer Linear Programming problem to be resolved is then encoded in the following way. Firstly, we will define $k^{2}$ integer variables that will be denoted by $x_{i \ell}\left(x_{i \ell} \in\{0,1, \ldots, r\}\right)$, where $i, \ell \in\{1, \ldots, k\}$. Note that $x_{i \ell}$ denotes the number of times that the $i$-th candidate is ranked at the $\ell$-th position in the monotone scorix that we are looking for.

We intend to minimize $M\left(S, S^{\prime}\right)$, which can be written as

$$
\sum_{i=1}^{k} \sum_{\ell=1}^{k}\left|\sum_{j=1}^{\ell}\left(x_{i j}-o_{i j}\right)\right|,
$$

where the values $o_{i \ell}$ represent the number of times that the $i$-th candidate is ranked at the $\ell$-th position in the given profile of rankings.

It must be noted that Eq. (6.2) is a sum of absolute values and will lead to an Integer Non-Linear Programming problem instead of an ILP problem. Fortunately, we can define a family of auxiliary variables $y_{i \ell}($ where $i, \ell \in\{1, \ldots, k\})$ such that minimizing Eq. 6.2 is equivalent to minimizing:

$$
\sum_{i=1}^{k} \sum_{\ell=1}^{k} y_{i \ell}
$$

under the additional constraints, for any $i, \ell \in\{1, \ldots, k\}$ :

$$
\begin{array}{r}
\sum_{j=1}^{\ell}\left(x_{i j}-o_{i j}\right) \leq y_{i \ell}, \\
\sum_{j=1}^{\ell}\left(o_{i j}-x_{i j}\right) \leq y_{i \ell}, \\
y_{i \ell} \geq 0 .
\end{array}
$$

As we are looking for a closest monotone scorix, we need to require the properties listed in Proposition 4.4. These properties are encoded as follows. 
Completeness in candidates: for any $i \in\{1, \ldots, k\}$ :

$$
\sum_{\ell=1}^{k} x_{i \ell}=r .
$$

Completeness in positions: for any $\ell \in\{1, \ldots, k\}$ :

$$
\sum_{i=1}^{k} x_{i \ell}=r .
$$

Additionally, we require the new scorix to be monotone. As $\unrhd_{1}$ defines a transitive relation on the set of vectors of positions, in order to reduce the number of constraints, the dominance relation can be required for consecutive elements in the ranking, i.e., monotonicity can be required w.r.t. the covering relation $\gg$ associated with the ranking $\succ$. More formally, the covering relation is defined as:

$$
\gg=\left\{\left(a_{i_{1}}, a_{i_{2}}\right) \in \succ \mid\left(\nexists i_{3} \in\{1, \ldots, k\}\right)\left(a_{i_{1}} \succ a_{i_{3}} \succ a_{i_{2}}\right)\right\}
$$

Monotonicity: for any $\ell \in\{1, \ldots, k-1\}$ and any $i_{1}, i_{2} \in\{1, \ldots, k\}$ such that $a_{i_{1}} \gg a_{i_{2}}$ :

$$
\sum_{j=1}^{\ell} x_{i_{1} j} \geq \sum_{j=1}^{\ell} x_{i_{2 j}} .
$$

Therefore, the cost of a closest monotone scorix w.r.t. $\succ$ is the minimum value of Eq. 6.3 under the constraints of Eqs. (6.4)- 6.9).

Remark 6.6 The cost of a closest 2-monotone scorix w.r.t. $\succ$ is the minimum value of Eq. (6.3) under the constraints of Eqs. (6.4)- 6.8) and additionally requiring the following condition: for any $\ell \in\{1, \ldots, k\}$ and any $i_{1}, i_{2} \in\{1, \ldots, k\}$ such that $a_{i_{1}} \gg a_{i_{2}}$ :

$$
\sum_{j=1}^{\ell} \sum_{i=1}^{j} x_{i_{1} j} \geq \sum_{i=1}^{j} \sum_{j=1}^{\ell} x_{i_{2} j} .
$$

Proposition 6.7 There exists a solution of the Integer Linear Programming problem of minimizing Eq. (6.3) under the constraints of Eqs. (6.4)-(6.9).

Proof: There exists a finite number of possible scorices with $k$ candidates and $r$ voters. Each scorix has an associated cost of changing the scorix given by the voters into this 
new scorix defined by Eq. 6.2). As we are minimizing the function over a finite set, if the feasible region has at least one element, then there is an optimal solution of the optimization problem minimizing Eq. (6.2) under the constraints of Eqs. (6.7)-(6.9). Let us prove the existence of this optimal solution by showing that there exists at least one solution satisfying all the constraints.

Consider the profile of rankings where all $r$ rankings are identical to the ranking $\succ$ w.r.t. which the monotonicity is required. The scorix $S \in \mathcal{S}_{r}(\mathscr{C})$ induced by this particular profile is obviously monotone w.r.t. $\succ$. Furthermore, as it is a scorix, it will also fulfill all the properties listed in Proposition 4.4. Thus, $S$ is a solution of the optimization problem and, therefore, there exists an optimal solution.

Finally, we know that minimizing the function defined in Eq. (6.2) under the constraints of Eqs. 6.7)-6.9 is equivalent to minimizing the function defined in Eq. (6.3) under the constraints of Eqs. (6.4)- 6.9 .

Remark 6.8 The existence of a solution of the Integer Linear Programming problem of minimizing Eq. (6.3) under the constraints of Eqs. (6.4)-(6.8) and (6.10) is analogously proved.

\subsubsection{Search for a monotone (quasi)votrix}

If the votrix induced by a profile of rankings is monotone w.r.t. a ranking $\succ$, then $\succ$ is called an optimal ranking. If there is no such ranking, then the cost of imposing monotonicity w.r.t. each possible ranking is computed. This cost is measured by means of a monometric on $\mathcal{V}_{r}^{\mathcal{Q}}(\mathscr{C})$

Definition 6.9 Let $\mathscr{C}$ be a set of $k$ candidates and $r$ be the number of voters. A function $M: \mathcal{V}_{r}^{\mathcal{Q}}(\mathscr{C}) \times \mathcal{V}_{r}^{\mathcal{Q}}(\mathscr{C}) \rightarrow \mathbb{R}$ is called a monometric on $\mathcal{V}_{r}^{\mathcal{Q}}(\mathscr{C})$ if it satisfies the following three properties:

(i) Non-negativity: for any $V, V^{\prime} \in \mathcal{V}_{r}^{\mathcal{Q}}(\mathscr{C})$, it holds that

$$
M\left(V, V^{\prime}\right) \geq 0
$$


(ii) Coincidence: for any $V, V^{\prime} \in \mathcal{V}_{r}^{\mathcal{Q}}(\mathscr{C})$, it holds that

$$
M\left(V, V^{\prime}\right)=0 \Leftrightarrow V=V^{\prime}
$$

(iii) Compatibility: for any $V, V^{\prime}, V^{\prime \prime} \in \mathcal{V}_{r}^{\mathcal{Q}}(\mathscr{C})$ such that, for any two $i, j \in\{1, \ldots, k\}$,

$$
\left(V_{i j} \geq V_{i j}^{\prime} \geq V_{i j}^{\prime \prime}\right) \vee\left(V_{i j} \leq V_{i j}^{\prime} \leq V_{i j}^{\prime \prime}\right)
$$

it holds that

$$
M\left(V, V^{\prime}\right) \leq M\left(V, V^{\prime \prime}\right)
$$

For any two (quasi)votrices $V, V^{\prime} \in \mathcal{V}_{r}^{\mathcal{Q}}(\mathscr{C}), M\left(V, V^{\prime}\right)$ is referred to as the cost of changing $V$ into $V^{\prime}$.

Definition 6.10 Let $\mathscr{C}$ be a set of $k$ candidates, $r$ be the number of voters, $\succ$ be a ranking on $\mathscr{C}, V \in \mathcal{V}_{r}(\mathscr{C})$ be a votrix and $M: \mathcal{V}_{r}^{\mathcal{Q}}(\mathscr{C}) \times \mathcal{V}_{r}^{\mathcal{Q}}(\mathscr{C}) \rightarrow \mathbb{R}$ be a monometric on $\mathcal{V}_{r}^{\mathcal{Q}}(\mathscr{C})$. A closest monotone quasivotrix $V^{\prime} \in \mathcal{V}_{r}^{\mathcal{Q}}(\mathscr{C})$ to $V$ is a quasivotrix that is monotone w.r.t. $\succ$ and such that there exists no quasivotrix $V^{\prime \prime} \in \mathcal{V}_{r}^{\mathcal{Q}}(\mathscr{C})$ that is monotone w.r.t. $\succ$ and, at the same time,

$$
M\left(V, V^{\prime \prime}\right)<M\left(V, V^{\prime}\right)
$$

Thus, for each ranking $\succ$, we have the corresponding cost associated with a closest monotone quasivotrix (measured by means of the chosen monometric on $\mathcal{V}_{r}^{\mathcal{Q}}(\mathscr{C})$ ). An optimal ranking should be one whose corresponding closest monotone quasivotrix is the closest to the original votrix.

Definition 6.11 Let $\mathscr{C}$ be a set of $k$ candidates, $\mathscr{R}$ be the profile of $r$ rankings on $\mathscr{C}$ given by the voters, $V_{\mathscr{R}} \in \mathcal{V}_{r}(\mathscr{C})$ be the votrix induced by $\mathscr{R}$ and $M: \mathcal{V}_{r}^{\mathcal{Q}}(\mathscr{C}) \times \mathcal{V}_{r}^{\mathcal{Q}}(\mathscr{C}) \rightarrow \mathbb{R}$ be a monometric on $\mathcal{V}_{r}^{\mathcal{Q}}(\mathscr{C})$. An optimal ranking $\succ$ (with a corresponding closest monotone quasivotrix $V \in \mathcal{V}_{r}^{\mathcal{Q}}(\mathscr{C})$ ) is a ranking for which it holds that there exists no ranking $\succ^{\prime}$ (with a corresponding closest monotone quasivotrix $V^{\prime} \in \mathcal{V}_{r}^{\mathcal{Q}}(\mathscr{C})$ ) such that

$$
M\left(V_{\mathscr{R}}, V^{\prime}\right)<M\left(V_{\mathscr{R}}, V\right) .
$$


The cost of imposing monotonicity w.r.t. a given ranking on $\mathscr{C}$ was calculated in [133] as the sum of absolute differences between two quasivotrices. More formally, for any two quasivotrices $V, V^{\prime} \in \mathcal{V}_{r}^{\mathcal{Q}}(\mathscr{C})$, the following monometric on $\mathcal{V}_{r}^{\mathcal{Q}}(\mathscr{C})$ was considered:

$$
M\left(V, V^{\prime}\right)=\frac{1}{2} \sum_{i, j=1}^{k}\left|V_{i j}-V_{i j}^{\prime}\right| .
$$

Although in [133] only reciprocity was taken into account, we propose to extend that proposal by looking for a closest monotone quasivotrix to the given votrix.

We will now prove that Eq. 6.11) defines a monometric on $\mathcal{V}_{r}^{\mathcal{Q}}(\mathscr{C})$.

Proposition 6.12 Let $\mathscr{C}$ be a set of $k$ candidates, $r$ be the number of voters and $M$ : $\mathcal{V}_{r}^{\mathcal{Q}}(\mathscr{C}) \times \mathcal{V}_{r}^{\mathcal{Q}}(\mathscr{C}) \rightarrow \mathbb{R}$ be defined as in Eq. 6.11). Then $M$ is a monometric on $\mathcal{V}_{r}^{\mathcal{Q}}(\mathscr{C})$

Proof: We will prove that $M$ satisfies the three axioms of a monometric on $\mathcal{V}_{r}^{\mathcal{Q}}(\mathscr{C})$.

Non-negativity. Immediate.

Coincidence. As it is a sum of absolute values, for any $V, V^{\prime} \in \mathcal{V}_{r}^{\mathcal{Q}}, M\left(V, V^{\prime}\right)=0$ if and only if $V_{i j}=V_{i j}^{\prime}$, for any $i, j \in\{1, \ldots, k\}$. This is equivalent to saying that $V=V^{\prime}$.

Compatibility. For any $V, V^{\prime}, V^{\prime \prime} \in \mathcal{V}_{r}^{\mathcal{Q}}(\mathscr{C})$ such that, for any two $i, j \in\{1, \ldots, k\}$,

$$
\left(V_{i j} \geq V_{i j}^{\prime} \geq V_{i j}^{\prime \prime}\right) \vee\left(V_{i j} \leq V_{i j}^{\prime} \leq V_{i j}^{\prime \prime}\right),
$$

the following is fulfilled:

$$
\begin{aligned}
M\left(V, V^{\prime}\right) & =\frac{1}{2} \sum_{i, j=1}^{k}\left|V_{i j}-V_{i j}^{\prime}\right| \\
& \leq \frac{1}{2} \sum_{i, j=1}^{k}\left|V_{i j}-V_{i j}^{\prime \prime}\right|=M\left(V, V^{\prime \prime}\right) .
\end{aligned}
$$

Thus, $M$ is a monometric on $\mathcal{V}_{r}^{\mathcal{Q}}(\mathscr{C})$.

In their work, Rademaker and De Baets were able to calculate the cost of a closest monotone matrix satisfying reciprocity by solving a flow network problem [132, 133, 134, 135]. However, we propose to consider an Integer Linear Programming (ILP) problem instead, in order to consider new properties such as the triangle inequality. 
The number of unknown variables (elements of the matrix) and the number of constraints (given by the properties of Proposition 4.9 in the ILP problem depend on the number of candidates $k$. Note that strictness does not need to be considered in the ILP problem and only elements that are not on the diagonal are taken into account. In addition, due to reciprocity, half of these non-diagonal values become superfluous. Therefore, only the triangle inequality and monotonicity need to be considered. This yields the following numbers of constraints:

(i) Number of (positive integer) variables: $\frac{k(k-1)}{2}$.

(ii) Triangle inequality constraints (inequalities): $k(k-1)(k-2)$.

(iii) Monotonicity constraints (inequalities): $(k-1)^{2}$.

More specifically, the Integer Linear Programming problem to be resolved is then encoded in the following way. Firstly, we will define $\frac{k(k-1)}{2}$ integer variables that will be denoted by $x_{i}\left(x_{i} \in\{0,1, \ldots, r\}\right)$, where $i \in\left\{1, \ldots, \frac{k(k-1)}{2}\right\}$. Note that $x_{i}$ denotes the strength of support of the $i$-th couple in $\mathscr{C}_{\neq}^{2}$ (where, for simplicity, the elements in $\mathscr{C}_{\neq}^{2}$ are listed starting from top to bottom and in each level from left to right in the diagram of $\sqsupset^{+}$). We will denote by $i_{(u, v)}$ the index corresponding to a couple $(u, v) \in \mathscr{C}_{\neq}^{2}$ and by $i_{\left(a_{i_{1}}, a_{j_{1}}\right)} \rtimes i_{\left(a_{i_{2}}, a_{j_{2}}\right)}$ the fact that $\left(a_{i_{1}}, a_{j_{1}}\right) \sqsupset^{+}\left(a_{i_{2}}, a_{j_{2}}\right)$ for a given ranking $\succ$.

We now aim to minimize the cost $M\left(V, V^{\prime}\right)$ given by Eq. (6.11). In terms of the variables of the ILP problem, this cost is given by

$$
\sum_{i=1}^{\frac{k(k-1)}{2}}\left|x_{i}-o_{i}\right|,
$$

where the values $o_{i}$ represent the corresponding values of the votrix induced by the given profile of rankings.

It must be noted that Eq. 6.12 is a sum of absolute values and will lead to an Integer Non-Linear Programming (INLP) problem instead of an ILP problem. Fortunately, such absolute values can be easily handled. A well-known result in optimization theory [110] is that we can define a family of auxiliary variables $\left(y_{i}\right)_{i=1}^{\frac{k(k-1)}{2}}$ such that minimizing Eq. 6.12 
is equivalent to minimizing:

$$
\sum_{i=1}^{\frac{k(k-1)}{2}} y_{i}
$$

under the additional constraints, for any $i \in\left\{1, \ldots, \frac{k(k-1)}{2}\right\}$,

$$
\begin{aligned}
x_{i}-o_{i} & \leq y_{i}, \\
o_{i}-x_{i} & \leq y_{i}, \\
y_{i} & \geq 0 .
\end{aligned}
$$

The constraints associated with the triangle inequality and monotonicity can be given in the following way:

Triangle inequality: for any three different $a_{i_{1}}, a_{i_{2}}, a_{i_{3}} \in \mathscr{C}$ :

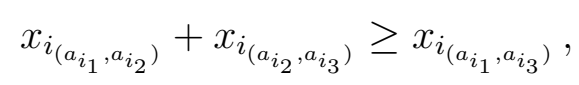

where $x_{i_{(v, u)}}$ equals $x_{i_{(u, v)}}$ when $u \succ v$.

Monotonicity: for any $i_{1}, i_{2} \in\left\{1, \ldots, \frac{k(k-1)}{2}\right\}$ such that $i_{1} \rtimes i_{2}$ :

$$
x_{i_{1}} \leq x_{i_{2}}
$$

For any $i \in\left\{\frac{(k-1)(k-2)}{2}+1, \ldots, \frac{k(k-1)}{2}\right\}$ :

$$
x_{i} \geq \frac{r}{2}
$$

Therefore, the cost of a closest monotone quasivotrix w.r.t. $\succ$ is the minimum value of Eq. 6.13) under the constraints of Eqs. 6.14 -6.19).

When dealing with an optimization problem, the existence of a solution and its uniqueness are relevant matters of study. In our case, the uniqueness is not necessary as we are interested in the cost of changing a votrix to a closest monotone quasivotrix and not in the closest monotone quasivotrix in itself. However, the existence of a solution is indeed an important question to be studied.

Proposition 6.13 There exists a solution of the Integer Linear Programming problem of minimizing Eq. (6.13) under the constraints of Eqs. 6.14 -6.19. 
Proof: There exists a finite number of possible quasivotrices with $k$ candidates and $r$ voters. Each quasivotrix has an associated cost w.r.t. the original votrix defined by Eq. 6.12). This cost takes a value in $\mathbb{N} \cup\{0\}$ and is bounded from below by 0 . As we are minimizing the function over a finite set, if the feasible region has at least one element, then there is an optimal solution of the optimization problem minimizing Eq. (6.12) under the constraints of Eqs. 6.17)-(6.19). Let us prove the existence of this optimal solution by showing that there exists at least one solution satisfying all the constraints.

Consider the profile of rankings where all $r$ rankings are identical to the ranking $\succ$ w.r.t. which the monotonicity is required. The votrix $V \in \mathcal{V}_{r}(\mathscr{C})$ induced by this particular profile is obviously monotone w.r.t. $\succ$. Furthermore, as it is a votrix, it will also fulfill all the properties listed in Proposition 4.9. Thus, $V$ is a solution of the optimization problem and, therefore, there exists an optimal solution.

Finally, we know that minimizing the function defined in Eq. 6.12) under the constraints of Eqs. 6.17) 6.19) is equivalent to minimizing the function defined in Eq. 6.13) under the constraints of Eqs. 6.14 -6.19).

\subsubsection{Search for a monotone (quasi)votex}

Of course, it is possible that a votex is not monotone w.r.t. a ranking on the set of candidates. Unfortunately, it can be even the case that it is not monotone w.r.t. any ranking. In that case, the goal would be to look for a new votex such that it is monotone w.r.t. at least one ranking. Obviously, among all the possible monotone votices, we want the closest to the observed one. Unfortunately, as we have discussed in the previous chapter, votices are not characterizable and, whenever a change in a votex is made, we can no longer assure that it still continues being a votex. Therefore, we will need to look for the closest monotone quasivotex as it can easily be characterized. The cost of imposing monotonicity on a votex can be measured by means of a monometric on $\mathcal{W}_{r}^{\mathcal{Q}}(\mathscr{C})$.

Definition 6.14 Let $\mathscr{C}$ be a set of $k$ candidates and $r$ be the number of voters. A function $M: \mathcal{W}_{r}^{\mathcal{Q}}(\mathscr{C}) \times \mathcal{W}_{r}^{\mathcal{Q}}(\mathscr{C}) \rightarrow \mathbb{R}$ is called a monometric on $\mathcal{W}_{r}^{\mathcal{Q}}(\mathscr{C})$ if it satisfies the following three properties: 
(i) Non-negativity: for any $W, W^{\prime} \in \mathcal{W}_{r}^{\mathcal{Q}}(\mathscr{C})$, it holds that

$$
M\left(W, W^{\prime}\right) \geq 0 \text {. }
$$

(ii) Coincidence: for any $W, W^{\prime} \in \mathcal{W}_{r}^{\mathcal{Q}}(\mathscr{C})$, it holds that

$$
M\left(W, W^{\prime}\right)=0 \Leftrightarrow W=W^{\prime} .
$$

(iii) Compatibility: for any $W, W^{\prime}, W^{\prime \prime} \in \mathcal{W}_{r}^{\mathcal{Q}}(\mathscr{C})$ such that, for any $\left(a_{i_{1}}, a_{i_{2}}\right) \in \mathscr{C}_{\neq}^{2}$, $\left(W\left(a_{i_{1}}, a_{i_{2}}\right) \unrhd W^{\prime}\left(a_{i_{1}}, a_{i_{2}}\right) \unrhd W^{\prime \prime}\left(a_{i_{1}}, a_{i_{2}}\right)\right) \vee\left(W^{\prime \prime}\left(a_{i_{1}}, a_{i_{2}}\right) \unrhd W^{\prime}\left(a_{i_{1}}, a_{i_{2}}\right) \unrhd W\left(a_{i_{1}}, a_{i_{2}}\right)\right)$, it holds that

$$
M\left(W, W^{\prime}\right) \leq M\left(W, W^{\prime \prime}\right)
$$

For any two (quasi)votices $W, W^{\prime} \in \mathcal{W}_{r}^{\mathcal{Q}}(\mathscr{C}), M\left(W, W^{\prime}\right)$ is referred to as the cost of changing $W$ into $W^{\prime}$.

After fixing a monometric on $\mathcal{W}_{r}^{\mathcal{Q}}(\mathscr{C})$, we look for the closest quasivotex to the original one such that it is monotone w.r.t. $\succ$.

Definition 6.15 Let $\mathscr{C}$ be a set of $k$ candidates, $r$ be the number of voters, $\succ$ be a ranking on $\mathscr{C}, W \in \mathcal{W}_{r}(\mathscr{C})$ be a votex and $M: \mathcal{W}_{r}^{\mathcal{Q}}(\mathscr{C}) \times \mathcal{W}_{r}^{\mathcal{Q}}(\mathscr{C}) \rightarrow \mathbb{R}$ be a monometric on $\mathcal{W}_{r}^{\mathcal{Q}}(\mathscr{C})$. A closest monotone quasivotex $W^{\prime} \in \mathcal{W}_{r}^{\mathcal{Q}}(\mathscr{C})$ to $W$ is a quasivotex that is monotone w.r.t. $\succ$ and such that there exists no quasivotex $W^{\prime \prime} \in \mathcal{W}_{r}^{\mathcal{Q}}(\mathscr{C})$ that is monotone w.r.t. $\succ$ and, at the same time,

$$
M\left(W, W^{\prime \prime}\right)<M\left(W, W^{\prime}\right)
$$

Thus, for each ranking $\succ$, we have the corresponding cost associated with a closest monotone quasivotex. An optimal ranking should be one whose corresponding closest monotone quasivotex is the closest to the original votex.

Definition 6.16 Let $\mathscr{C}$ be a set of $k$ candidates, $\mathscr{R}$ be the profile of $r$ rankings on $\mathscr{C}$ given by the voters, $W_{\mathscr{R}} \in \mathcal{W}_{r}(\mathscr{C})$ be the votex induced by $\mathscr{R}$ and $M: \mathcal{W}_{r}^{\mathcal{Q}}(\mathscr{C}) \times \mathcal{W}_{r}^{\mathcal{Q}}(\mathscr{C}) \rightarrow \mathbb{R}$ be a monometric on $\mathcal{W}_{r}^{\mathcal{Q}}(\mathscr{C})$. An optimal ranking $\succ$ (with a corresponding closest monotone quasivotex $\left.W \in \mathcal{W}_{r}^{\mathcal{Q}}(\mathscr{C})\right)$ is a ranking for which it holds that there exists no ranking $\succ^{\prime}$ (with a corresponding closest monotone quasivotex $W^{\prime} \in \mathcal{W}_{r}^{\mathcal{Q}}(\mathscr{C})$ ) such that

$$
M\left(W_{\mathscr{R}}, W^{\prime}\right)<M\left(W_{\mathscr{R}}, W\right) .
$$


For each possible ranking $\succ$ on $\mathscr{C}$, a closest monotone quasivotex needs to be identified. All the rankings whose corresponding closest monotone quasivotex has a minimal cost are declared optimal rankings.

Note that the existence of both a closest monotone quasivotex and an optimal ranking is assured. However, the uniqueness of neither of them can be assured.

Now that the problem setting has been established, the resolution of the problem can be addressed. We have introduced an optimization problem whose characteristics depend on the nature of the monometric on $\mathcal{W}_{r}^{\mathcal{Q}}(\mathscr{C})$. In the following, we will analyse the particular case of a natural and intuitive monometric on $\mathcal{W}_{r}^{\mathcal{Q}}(\mathscr{C})$ based on the total amount of changes in the cumulative frequencies. This leads to the resolution of an Integer Linear Programming problem (ILP problem).

Our proposal is based on the changes in the cumulative frequencies, where we can see reflected the changes between distant positions in the frequency distributions. It must be remarked that these changes will be made in the votex and not in the profile of rankings. The proposed monometric on $\mathcal{W}_{r}^{\mathcal{Q}}(\mathscr{C})$ is given by:

$$
M\left(W, W^{\prime}\right)=\sum_{(u, v) \in \mathscr{C}_{\neq}^{2}} m\left(W(u, v), W^{\prime}(u, v)\right)
$$

where, for any $\left(a_{i_{1}}, a_{i_{2}}\right) \in \mathscr{C}_{\neq}^{2}$,

$$
m\left(W\left(a_{i_{1}}, a_{i_{2}}\right), W^{\prime}\left(a_{i_{1}}, a_{i_{2}}\right)\right)=\sum_{j \in \mathcal{K}}\left|N_{i}\left(a_{i_{1}}, a_{i_{2}}\right)-N_{i}^{\prime}\left(a_{i_{1}}, a_{i_{2}}\right)\right| .
$$

We will now prove that Eq. 6.20 defines a monometric on $\mathcal{W}_{r}^{\mathcal{Q}}(\mathscr{C})$.

Proposition 6.17 Let $\mathscr{C}$ be a set of $k$ candidates, $r$ be the number of voters and $M$ : $\mathcal{W}_{r}^{\mathcal{Q}}(\mathscr{C}) \times \mathcal{W}_{r}^{\mathcal{Q}}(\mathscr{C}) \rightarrow \mathbb{R}$ be defined as in Eq. (6.20). Then $M$ is a monometric on $\mathcal{W}_{r}^{\mathcal{Q}}(\mathscr{C})$.

Proof: We will prove that $M$ satisfies the three axioms of a monometric on $\mathcal{W}_{r}^{\mathcal{Q}}(\mathscr{C})$.

Non-negativity. Immediate.

Coincidence. As it is a sum of absolute values, for any $W, W^{\prime} \in \mathcal{W}_{r}^{\mathcal{Q}}(\mathscr{C}), M\left(W, W^{\prime}\right)=0$ if and only if $N_{i}\left(a_{i_{1}}, a_{i_{2}}\right)=N_{i}^{\prime}\left(a_{i_{1}}, a_{i_{2}}\right)$, for any $j \in \mathcal{K}$ and any $\left(a_{i_{1}}, a_{i_{2}}\right) \in \mathscr{C}_{\neq}^{2}$. This is equivalent to saying that $W=W^{\prime}$. 
Compatibility. For any $W, W^{\prime}, W^{\prime \prime} \in \mathcal{W}_{r}^{\mathcal{Q}}(\mathscr{C})$ satisfying, for any $\left(a_{i_{1}}, a_{i_{2}}\right) \in \mathscr{C}_{\neq}^{2}$,

$$
\left(W\left(a_{i_{1}}, a_{i_{2}}\right) \unrhd W^{\prime}\left(a_{i_{1}}, a_{i_{2}}\right) \unrhd W^{\prime \prime}\left(a_{i_{1}}, a_{i_{2}}\right)\right) \vee\left(W^{\prime \prime}\left(a_{i_{1}}, a_{i_{2}}\right) \unrhd W^{\prime}\left(a_{i_{1}}, a_{i_{2}}\right) \unrhd W\left(a_{i_{1}}, a_{i_{2}}\right)\right),
$$

the following is fulfilled by definition of $\unrhd$ :

$$
\begin{aligned}
M\left(W, W^{\prime}\right) & =\sum_{(u, v) \in \mathscr{C}_{\neq}^{2}} \sum_{i \in \mathcal{K}}\left|N_{i}(u, v)-N_{i}^{\prime}(u, v)\right| \\
& \leq \sum_{(u, v) \in \mathscr{C}_{\neq}^{2}} \sum_{i \in \mathcal{K}}\left|N_{i}(u, v)-N_{i}^{\prime \prime}(u, v)\right|=M\left(W, W^{\prime \prime}\right) .
\end{aligned}
$$

Thus, $M$ is a monometric on $\mathcal{W}_{r}^{\mathcal{Q}}(\mathscr{C})$.

Once a monometric on $\mathcal{W}_{r}^{\mathcal{Q}}(\mathscr{C})$ has been fixed, the search for an optimal ranking can be addressed. Analogously to the votrix case, we will need to solve an Integer Linear Programming problem for each possible ranking. The number of unknown variables (frequencies of the votex) and the number of constraints (given by the properties of Proposition 4.15) in the ILP problem will depend on the number of candidates $k$. Due to reciprocity, some of these constraints become superfluous as the frequency distribution of each couple $\left(a_{i_{1}}, a_{i_{2}}\right) \in \mathscr{C}_{\neq}^{2}$ characterizes the frequency distribution of the couple $\left(a_{i_{2}}, a_{i_{1}}\right)$ (and vice versa). Nevertheless, for defining the monotonicity constraints, we cannot focus on $\sqsupset^{+}$instead of $\sqsupset$ as in the votrix case. This yields the following numbers of constraints:

(i) Number of (positive integer) variables: $k(k-1)^{2}$.

(ii) Completeness constraints (equalities): $\frac{k(k-1)}{2}$.

(iii) Regularity constraints (equalities): $(k-1)$.

(iv) Peakedness constraints (inequalities): $2 k(k-2)$.

(v) Triangle inequality constraints (inequalities): $k(k-1)(k-2)$.

(vi) Maximality constraints (inequalities): $k(k-1)$.

(vii) Monotonicity constraints (inequalities): $4(k-1)^{2}(k-2)+(k-1)^{2}$. 
More specifically, the Integer Linear Programming problem to be resolved is then encoded in the following way. Firstly, we will define $k(k-1)^{2}$ integer variables that will be denoted by $x_{i, j}\left(x_{i, j} \in\{0,1, \ldots, r\}\right)$, where $i \in\left\{1, \ldots, \frac{k(k-1)}{2}\right\}$ and $j \in\{1, \ldots, 2(k-1)\}$. Note that $x_{i, j}$ denotes the $j$-th component of the frequency distribution of the $i$-th couple in $\mathscr{C}_{\neq}^{2}$ (where, for simplicity, the elements in $\mathscr{C}_{\neq}^{2}$ are listed starting from top to bottom and in each level from left to right in the Hasse diagram of the order relation $\sqsupset$ ). We will denote by $i_{(u, v)}$ the index corresponding to a couple $(u, v) \in \mathscr{C}_{\neq}^{2}$ and by $i_{\left(a_{i_{1}}, a_{j_{1}}\right)} \rtimes i_{\left(a_{i_{2}}, a_{j_{2}}\right)}$ the fact that $\left(a_{i_{1}}, a_{j_{1}}\right) \sqsupset\left(a_{i_{2}}, a_{j_{2}}\right)$ for a given ranking $\succ$.

We intend to minimize $M\left(W, W^{\prime}\right)$, which can be written as

$$
\sum_{i=1}^{\frac{k(k-1)}{2}} \sum_{j=1}^{2 k-1}\left|\sum_{k=1}^{j}\left(x_{i, k}-o_{i, k}\right)\right|+\sum_{i=1}^{\frac{k(k-1)}{2}} \sum_{j=1}^{2 k-1}\left|\sum_{k=j}^{2 k-1}\left(x_{i, k}-o_{i, k}\right)\right|,
$$

where the values $o_{i, j}$ represent the values of the frequency distributions of the votex induced by the given profile of rankings, or, equivalently,

$$
2 \sum_{i=1}^{\frac{k(k-1)}{2}} \sum_{j=1}^{2 k-1}\left|\sum_{k=1}^{j}\left(x_{i, k}-o_{i, k}\right)\right| \text {. }
$$

It must be noted that Eq. (6.21) is a sum of absolute values and we will have an INLP problem instead of an ILP problem. We can define a family of auxiliary variables $y_{i, j}$ where $i \in\left\{1, \ldots, \frac{k(k-1)}{2}\right\}$ and $j \in\{1, \ldots, 2 k-1\}$ such that minimizing Eq. 6.21 is equivalent to minimizing:

$$
2 \sum_{i=1}^{\frac{k(k-1)}{2}} \sum_{j=1}^{2 k-1} y_{i, j}
$$

under the additional constraints, for any $i \in\left\{1, \ldots, \frac{k(k-1)}{2}\right\}$ and any $j \in\{1, \ldots, 2 k-1\}$,

$$
\begin{array}{r}
\sum_{k=1}^{j}\left(x_{i, k}-o_{i, k}\right) \leq y_{i, j}, \\
\sum_{k=1}^{j}\left(o_{i, k}-x_{i, k}\right) \leq y_{i, j}, \\
y_{i, j} \geq 0 .
\end{array}
$$

As we are looking for a closest monotone quasivotex, we will need to require the properties listed in Proposition 4.15. These properties are encoded as follows. 
Completeness: for any $i \in\left\{1, \ldots, \frac{k(k-1)}{2}\right\}$ :

$$
\sum_{j=1}^{2 k-2} x_{i, j}=r
$$

Regularity: for any $j \in\{1, \ldots, k-1\}$ :

$$
\sum_{i=1}^{\frac{k(k-1)}{2}} x_{i, j}+\sum_{i=1}^{\frac{k(k-1)}{2}} x_{i, 2 k-1-j}=j r
$$

Peakedness: for any $a_{i_{1}} \in \mathscr{C}$ and any $j \in\{2, \ldots, k-1\}$ :

$$
\sum_{u \in \mathscr{C} \backslash\left\{a_{i_{1}}\right\}} x_{i_{\left(a_{i_{1}}, u\right)}, j-1}+\sum_{u \in \mathscr{C} \backslash\left\{a_{i_{1}}\right\}} x_{i_{\left(u, a_{i_{1}}\right)}, 2 k-j} \leq \sum_{u \in \mathscr{C} \backslash\left\{a_{i_{1}}\right\}} x_{i_{\left(a_{i_{1}}, u\right)}, j}+\sum_{u \in \mathscr{C} \backslash\left\{a_{i_{1}}\right\}} x_{i_{\left(u, a_{i_{1}}\right), 2 k-1-j} .} .
$$

for any $a_{i_{1}} \in \mathscr{C}$ and any $j \in\{k, \ldots, 2 k-3\}$ :

$$
\sum_{u \in \mathscr{C} \backslash\left\{a_{i_{1}}\right\}} x_{i_{\left(a_{i_{1}}, u\right)}, j+1}+\sum_{u \in \mathscr{C} \backslash\left\{a_{i_{1}}\right\}} x_{i_{\left(u, a_{i_{1}}\right)}, 2 k-2-j} \leq \sum_{u \in \mathscr{C} \backslash\left\{a_{i_{1}}\right\}} x_{i_{\left(a_{i_{1}}, u\right)}, j}+\sum_{u \in \mathscr{C} \backslash\left\{a_{i_{1}}\right\}} x_{i_{\left(u, a_{i_{1}}\right)}, 2 k-1-j} .
$$

Triangle inequality: for any three different $a_{i_{1}}, a_{i_{2}}, a_{i_{3}} \in \mathscr{C}$ :

$$
\sum_{j=k}^{2 k-2} x_{i_{\left(a_{i_{1}}, a_{i_{2}}\right), j}}+\sum_{j=k}^{2 k-2} x_{i_{\left(a_{i_{2}}, a_{i_{3}}\right), j}} \geq \sum_{j=k}^{2 k-2} x_{i_{\left(a_{i_{1}}, a_{i_{3}}\right), j}},
$$

where $x_{i_{(v, u)}, j}$ equals $x_{i_{(u, v)}, 2 k-j-1}$ when $u \succ v$.

Maximality: for any $\left(a_{i_{1}}, a_{i_{2}}\right) \in \mathscr{C}_{\neq}^{2}$ :

$$
\sum_{\substack{i_{\left(a_{i_{1}}, u\right)} \\ v \in \mathscr{C} \backslash\left\{a_{i_{1}}, a_{i_{2}}\right\}}} x_{i_{\left(a_{i_{1}}, u\right)}, 2 k-2}+\sum_{\substack{i_{\left(v, a_{i_{2}}\right)} \\ v \in \mathscr{C} \backslash\left\{a_{i_{1}}, a_{i_{2}}\right\}}} x_{i_{\left(v, a_{i_{2}}\right)}, 2 k-2} \leq \sum_{j=k}^{2 k-1} x_{i_{\left(a_{i_{1}}, a_{i_{2}}\right)}, j},
$$

where $x_{i_{(v, u)}, j}$ equals $x_{i_{(u, v)}, 2 k-j-1}$ when $u \succ v$.

Additionally, we want to require the new quasivotex to be monotone.

Monotonicity: for any $j \in\{1, \ldots, 2(k-1)\}$ and any $i_{1}, i_{2} \in\left\{1, \ldots, \frac{k(k-1)}{2}\right\}$ such that $i_{1} \rtimes i_{2}$ :

$$
\sum_{\ell=1}^{j} x_{i_{1}, \ell} \leq \sum_{\ell=1}^{j} x_{i_{2}, \ell} .
$$


For any $j \in\{1, \ldots, k-1\}$ and any $i \in\left\{\frac{(k-1)(k-2)}{2}+1, \ldots, \frac{k(k-1)}{2}\right\}$ :

$$
\sum_{\ell=1}^{j} x_{i, \ell} \leq \sum_{\ell=2 k-1-j}^{2(k-1)} x_{i, \ell} .
$$

Therefore, the cost of a closest monotone quasivotex w.r.t. $\succ$ is the minimum value of Eq. 6.22 under the constraints of Eqs 6.23)-6.33).

Proposition 6.18 There exists a solution of the Integer Linear Programming problem of minimizing Eq. (6.22) under the constraints of Eqs. (6.23)-6.33).

Proof: There exists a finite number of possible quasivotices with $k$ candidates and $r$ voters. Each quasivotex has an associated cost w.r.t. the original votex defined by Eq. 6.21. As we are minimizing the function over a finite set, if the feasible region has at least one element, then there is an optimal solution of the optimization problem minimizing Eq. 6.21) under the constraints of Eqs. 6.26-6.33. Let us prove the existence of this optimal solution by showing that there exists at least one solution satisfying all the constraints.

Consider the profile of rankings where all $r$ rankings are identical to the ranking $\succ$ w.r.t. which the monotonicity is required. The votex $W \in \mathcal{W}_{r}(\mathscr{C})$ induced by this particular profile is obviously monotone w.r.t. $\succ$. Furthermore, as it is a votex, it will also fulfill all the properties listed in Proposition 4.15. Thus, $W$ is a solution of the optimization problem and, therefore, there exists an optimal solution.

Finally, we know that minimizing the function defined in Eq. 6.21 under the constraints of Eqs. 6.26) 6.33) is equivalent to minimizing the function defined in Eq. 6.22) under the constraints of Eqs. 6.23 -6.33.

The search for monotonicity by making changes in the representation of votes is computationally friendlier than the search for monotonicity by making changes in the profile. However, letting aside the fact that the obtained construct is not assured to be a votrix/votex, it is not possible to compare the cost of imposing monotonicity on different representations of votes. Therefore, in the following section we will address the search for monotonicity by making changes in the profile of rankings. 


\subsection{Changes in the profile of rankings}

Since the early 2000s, the field of social choice is facing a computational turn. The subfield of computational social choice is calling the attention of the scientific community to the fact that not only the normative properties of ranking rules are to be studied, but that also the execution time of these ranking rules needs to be taken into account [21]. One of the most prominent examples of ranking rules that are computationally unfriendly is the method of Kemeny [80, which has been extensively analysed from a computational point of view [9]. The Kemeny score problem (given a profile and a nonnegative integer $k$, is there a ranking that has score at most $k$ ?) is proved to be NP-complete, while the Kemeny winner problem (given a profile and a candidate $a_{i}$, is there a ranking that has minimum score and ranks $a_{i}$ first?), the Kemeny ranking problem (given a profile and two candidates $a_{i_{1}}$ and $a_{i_{2}}$, is there a ranking that has minimum score and ranks $a_{i_{1}}$ at a better position than $a_{i_{2}}$ ?) and the Kemeny rank aggregation problem (given a profile, find a ranking that has minimum score) are proved to be NP-hard (see Chapter 4 of [21]). Obviously, the corresponding adaptation of these problems to the search for monotonicity of a representation of votes is computationally harder. In [126], we proposed an algorithm for identifying the optimal ranking running in factorial time. We recall this algorithm throughout this section.

Although many betweenness relations on $\mathcal{L}(\mathscr{C})^{r}$ may be considered, as discussed in [126], the most interesting betweenness relation in the field of social choice is the one given by Kemeny [80]. A profile of rankings $\mathscr{R}^{\prime}=\left(\succ_{j}^{\prime}\right)_{j=1}^{r}$ is said to be in between two other profiles of rankings $\mathscr{R}=\left(\succ_{j}\right)_{j=1}^{r}$ and $\mathscr{R}^{\prime \prime}=\left(\succ_{j}^{\prime \prime}\right)_{j=1}^{r}$, denoted by $\left[\mathscr{R}, \mathscr{R}^{\prime}, \mathscr{R}^{\prime \prime}\right]$, if it holds that

$$
\sum_{j=1}^{r} K\left(\succ_{j}, \succ_{j}^{\prime \prime}\right)=\sum_{j=1}^{r} K\left(\succ_{j}, \succ_{j}^{\prime}\right)+\sum_{j=1}^{r} K\left(\succ_{j}^{\prime}, \succ_{j}^{\prime \prime}\right),
$$

where $K$ denotes the Kendall distance function [82] defined, for any two rankings $\succ_{1}$ and $\succ_{2}$, as $K\left(\succ_{1}, \succ_{2}\right)=\#\left\{\left(a_{i_{1}}, a_{i_{2}}\right) \in \mathscr{C}_{\neq}^{2} \mid a_{i_{1}} \succ_{1} a_{i_{2}} \wedge a_{i_{2}} \succ_{2} a_{i_{1}}\right\}$.

Definition 6.19 Let $\mathscr{C}$ be a set of $k$ candidates and $r$ be the number of voters. A function $M: \mathcal{L}(\mathscr{C})^{r} \times \mathcal{L}(\mathscr{C})^{r} \rightarrow \mathbb{R}$ is called a monometric if it satisfies the following three properties:

(i) Non-negativity: for any $\mathscr{R}, \mathscr{R}^{\prime} \in \mathcal{L}(\mathscr{C})^{r}$, it holds that $M\left(\mathscr{R}, \mathscr{R}^{\prime}\right) \geq 0$.

(ii) Coincidence: for any $\mathscr{R}, \mathscr{R}^{\prime} \in \mathcal{L}(\mathscr{C})^{r}$, it holds that $M\left(\mathscr{R}, \mathscr{R}^{\prime}\right)=0 \Leftrightarrow \mathscr{R}=\mathscr{R}^{\prime}$. 
(iii) Compatibility: for any $\mathscr{R}, \mathscr{R}^{\prime}, \mathscr{R}^{\prime \prime} \in \mathcal{L}(\mathscr{C})^{r}$ such that $\left[\mathscr{R}, \mathscr{R}^{\prime}, \mathscr{R}^{\prime \prime}\right]$, it holds that $M\left(\mathscr{R}, \mathscr{R}^{\prime}\right) \leq M\left(\mathscr{R}, \mathscr{R}^{\prime \prime}\right)$.

The ranking that is the closest to imposing monotonicity on the representation of votes should then be considered the optimal ranking. Of course, this optimal ranking depends on the chosen representation of votes.

Definition 6.20 Let $\mathscr{C}$ be a set of $k$ candidates and $\mathscr{R}$ be the profile of $r$ rankings on $\mathscr{C}$ given by the voters. Let $M: \mathcal{L}(\mathscr{C})^{r} \times \mathcal{L}(\mathscr{C})^{r} \rightarrow \mathbb{R}$ be a monometric and consider a fixed representation of votes.

(i) A closest profile of rankings with a monotone representation of votes is a profile of rankings $\mathscr{R}^{\prime}$ such that there exists a ranking $\succ$ w.r.t. which the representation of votes induced by $\mathscr{R}^{\prime}$ is monotone and for which it holds that there exists no profile of rankings $\mathscr{R}^{\prime \prime}$ such that there exists a ranking $\succ$ w.r.t. which the representation of votes induced by $\mathscr{R}^{\prime \prime}$ is monotone, while $M\left(\mathscr{R}, \mathscr{R}^{\prime \prime}\right)<M\left(\mathscr{R}, \mathscr{R}^{\prime}\right)$.

(ii) An optimal ranking $\succ$ is a ranking imposing monotonicity on at least one closest profile of rankings with a monotone representation of votes.

As there exists a finite number of profiles of $r$ rankings on $\mathscr{C}$, the existence of both a closest profile of rankings with a monotone scorix/votrix/votex and an optimal ranking is assured ${ }^{2}$. Unfortunately, the uniqueness cannot be assured.

As discussed by Pérez-Fernández et al. [126] for a more general problem setting, the search for a closest profile of rankings satisfying some desired property can be addressed as a transportation problem [101] in case the property can be expressed as a constraint of an Integer Linear Programming problem. Indeed, monotonicity of all previously discussed representations of votes can be expressed as a transportation problem. In a transportation problem, we have a number of supply points and a number of demand points. At each supply point certain product is produced and it needs to be transported to the demand points satisfying the required demands at each demand point. Transporting a unit of

\footnotetext{
${ }^{2}$ Unlike the setting of [124] where changes are considered in the votrix/votex leading to the introduction of the notion of quasivotrix/quasivotex, here changes are considered in the profile of rankings. Therefore, by definition, the votrix/votex associated to the new profile can always be obtained.
} 
product from a supply point to a demand point has an associated cost. An optimal transportation distribution needs to be defined such that the demand is satisfied.

In our setting, each ranking in $\mathcal{L}(\mathscr{C})$ is both a supply point and a demand point. The production in the supply point corresponding to a ranking $\succ$ on $\mathscr{C}$ represents the frequency of this ranking in the profile of rankings $\mathscr{R}$ given by the voters. The demand in the demand point corresponding to a ranking $\succ$ on $\mathscr{C}$ represents the frequency of this ranking in the closest profile of rankings $\mathscr{R}^{\prime}$ with a monotone representation of votes.

A general demand needs to be satisfied (the number of rankings in $\mathscr{R}^{\prime}$ needs to be equal to the number of rankings in $\mathscr{R})$. Formalizing the problem, $(k !)^{2}$ variables $x_{i j}$ taking values in $\mathbb{N} \cup\{0\}$ are defined, $k$ being the number of candidates in $\mathscr{C}$. For any $i, j \in\{1, \ldots, k !\}$, $x_{i j}=n$ means that $n$ units of the $i$-th ranking in $\mathcal{L}(\mathscr{C})$ are assigned to the $j$-th ranking in $\mathcal{L}(\mathscr{C})$. In this case, we have an initial profile of rankings where each ranking $\succ_{i}$ appears $s_{i}$ times. These $s_{i}$ 's can be seen as the number of units of product that are produced at each supply point. We are then dealing with the following optimization problem:

$$
\begin{array}{cl}
\text { Minimize } & \sum_{i=1}^{k !} \sum_{j=1}^{k !} C_{i j} x_{i j} \text { w.r.t. }\left\{x_{i j}\right\}_{i, j=1}^{k !} \\
\text { s.t. } & \sum_{j=1}^{k !} x_{i j}=s_{i}, \text { for any } i \in\{1, \ldots, k !\}, \\
& x_{i j} \geq 0, \text { for any } i, j \in\{1, \ldots, k !\}, \\
& x_{i j} \in \mathbb{Z}, \text { for any } i, j \in\{1, \ldots, k !\},
\end{array}
$$

under the additional constraint of monotonicity of the representation of votes w.r.t. the ranking $\succ \in \mathcal{L}(\mathscr{C})$.

A transportation problem can be solved in polynomial time [84]. Unfortunately, this polynomial time is in terms of the number of variables, which equals $k$ ! in our setting. This is an obvious computational drawback, even though in social choice theory, the number of candidates in $\mathscr{C}$ is typically quite small. The development of a more efficient $t^{3}$ method for searching for a closest profile of rankings with a recursively monotone scorix remains an open problem.

\footnotetext{
${ }^{3}$ Note that two similar ranking rules, such as the methods of Dodgson [46] and Kemeny [80, are proved to be NP-hard problems [9].
} 


\section{CHAPTER 7}

\section{Monotonicity and axiomatic social choice theory}

\subsection{Axiomatic social choice theory}

The study of properties that a ranking rule may or may not satisfy is a relevant matter of study in social choice theory [3, 60, 133, 152]. Unfortunately, as Arrow stated in [3], there is no ranking rule simultaneously satisfying all the properties that can be considered desirable. In the following, we will briefly introduce the most common ones:

(i) Non-dictatorship: there is no voter whose ranking is always elected as the winning ranking.

(ii) Anonymity: reassigning the rankings over the voters does not change the outcome.

(iii) Neutrality: if some permutation of candidates is applied to each voter's ranking, the same permutation should be observed in the winning ranking.

(iv) Non-imposition: for any ranking on the set of candidates there exists a profile of rankings such that this ranking is the winning ranking.

(v) Unanimity (Pareto efficiency): if every voter prefers a candidate over another one, then this must also be the case in the winning ranking. 
(vi) Independence of irrelevant alternatives: the order between two candidates depends only on the individual preferences between these two candidates.

The properties fulfilled by the ranking rules proposed in this dissertation may depend on the chosen monometric. It is immediate to see that, when considering the Kendall distance function and considering changes in the profile of rankings, the first four properties are trivially satisfied. Unanimity can also be proved to be satisfied (it suffices to see that, if every voter prefers $a_{i_{1}}$ over $a_{i_{2}}$, then it trivially holds that the cost of the closest profile of rankings in the chosen consensus state where the winning ranking $\succ$ satisfies that $a_{i_{1}} \succ a_{i_{2}}$ is lower than or equal to the cost of the closest profile of rankings in the chosen consensus state where the winning ranking is the result of permuting candidates $a_{i_{1}}$ and $a_{i_{2}}$ in $\succ$ ).

On the other hand, independence of irrelevant alternatives is not satisfied as monotonicity is a global property and does not depend only on the individual preferences between two candidates. Arrow's impossibility theorem [3] states that there is no ranking rule satisfying, at the same time, non-dictatorship, unanimity and independence of irrelevant alternatives. Therefore, as non-dictatorship and unanimity are satisfied, independence of irrelevant alternatives cannot be satisfied.

Finding axiomatic characterizations of ranking rules is an important aspect in the field of social choice theory. As pointed out by Merlin [97], Arrow's theorem [3] can be understood as an axiomatization of dictatorship. Characterizations of the simple majority rule [95], scoring functions [169], best-worst voting systems [66] and other voting rules have been proposed. Characterizing the search for monotonicity is still an open problem that will be addressed in the near future.

\subsection{Independence of all proposed ranking rules}

In this section, we prove the independence of the ranking rules proposed in this dissertation w.r.t. each other. The most important comparisons are detailed in an illustrative subsection. Note that, throughout this section, the Kendall distance function will be considered as monometric. 


\subsubsection{The search for monotonicity of the scorix and the search for recursive monotonicity of the scorix}

In this subsection, we prove that the search for monotonicity of the scorix and the search for recursive monotonicity of the scorix are independent w.r.t. each other by providing a profile of rankings for which both methods lead to a different winning ranking. Consider the profile of $r=20$ rankings listed in Table 7.1 .

\begin{tabular}{|c|c|}
\hline$\# \succ_{i}$ & Rankings on $\mathscr{C}$ \\
\hline 5 & $b \succ c \succ d \succ a$ \\
4 & $b \succ c \succ a \succ d$ \\
3 & $a \succ d \succ c \succ b$ \\
2 & $c \succ a \succ d \succ b$ \\
2 & $d \succ a \succ c \succ b$ \\
1 & $a \succ c \succ b \succ d$ \\
1 & $a \succ c \succ d \succ b$ \\
1 & $b \succ a \succ c \succ d$ \\
1 & $c \succ d \succ a \succ b$ \\
\hline
\end{tabular}

Table 7.1: Expressed rankings and their frequency.

On the one hand, in case we address the search for monotonicity of the scorix, the optimal ranking is $c \succ a \succ b \succ d$ with a cost of six, while the ranking $c \succ b \succ a \succ d$ leads to a cost of seven. On the other hand, in case we address the search for recursive monotonicity of the scorix, the optimal ranking is $c \succ b \succ a \succ d$ with a cost of seven, while the ranking $c \succ a \succ b \succ d$ leads to a cost of eight. Therefore, the search for monotonicity of the scorix and the search for recursive monotonicity of the scorix are independent w.r.t. each other.

\subsubsection{The search for (recursive) monotonicity of the scorix and all scoring rank- ing rules}

In this subsection, we prove that the search for monotonicity and the search for recursive monotonicity of the scorix are independent w.r.t. all scoring ranking rules by considering two examples that lead to contradictory conditions on the weights of the considered scoring ranking rule. 
On the one hand, we consider the profile of $r=20$ rankings listed in Table 7.2 .

\begin{tabular}{|c|c|}
\hline$\# \succ_{i}$ & Rankings on $\mathscr{C}$ \\
\hline 5 & $b \succ d \succ a \succ c$ \\
5 & $b \succ d \succ c \succ a$ \\
5 & $d \succ a \succ c \succ b$ \\
4 & $a \succ c \succ d \succ b$ \\
1 & $a \succ d \succ c \succ b$ \\
\hline
\end{tabular}

Table 7.2: Expressed rankings and their frequency.

The scorix induced by the profile of rankings listed in Table 7.2 is:

$$
S=\left(\begin{array}{cccc}
5 & 5 & 5 & 5 \\
10 & 0 & 0 & 10 \\
0 & 4 & 11 & 5 \\
5 & 11 & 4 & 0
\end{array}\right)
$$

Applying the search for monotonicity of the scorix, the optimal ranking is $d \succ b \succ a \succ c$ with a cost of eight. Analogously, applying the search for recursive monotonicity of the scorix, the optimal ranking is also $d \succ b \succ a \succ c$ with a cost of eight. As $b \succ a$ in the optimal ranking, any scoring ranking rule coinciding with both methods will satisfy that the score $s(b)$ of candidate $b$ is greater than the score $s(a)$ of candidate $a$. In particular, it holds that

$$
s(b)=10 \alpha_{1}+10 \alpha_{4}>5 \alpha_{1}+5 \alpha_{2}+5 \alpha_{3}+5 \alpha_{4}=s(a) .
$$

On the other hand, we consider the profile of $r=20$ rankings listed in Table 7.3 .

The scorix induced by the profile of rankings listed in Table 7.3 is:

$$
S=\left(\begin{array}{cccc}
5 & 5 & 5 & 5 \\
10 & 0 & 1 & 9 \\
0 & 6 & 11 & 3 \\
5 & 9 & 3 & 3
\end{array}\right)
$$

Applying the search for monotonicity of the scorix, the optimal ranking is $d \succ a \succ b \succ c$ with a cost of six. Analogously, applying the search for recursive monotonicity of the scorix, the optimal ranking is also $d \succ a \succ b \succ c$ with a cost of eight. As $a \succ b$ in the optimal ranking, any scoring ranking rule coinciding with both methods will satisfy that 


\begin{tabular}{|c|c|}
\hline$\# \succ_{i}$ & Rankings on $\mathscr{C}$ \\
\hline 4 & $a \succ d \succ c \succ b$ \\
4 & $b \succ d \succ c \succ a$ \\
3 & $b \succ c \succ a \succ d$ \\
3 & $d \succ a \succ c \succ b$ \\
2 & $b \succ a \succ d \succ c$ \\
2 & $d \succ c \succ a \succ b$ \\
1 & $a \succ d \succ b \succ c$ \\
1 & $b \succ c \succ d \succ a$ \\
\hline
\end{tabular}

Table 7.3: Expressed rankings and their frequency.

the score $s(a)$ of candidate $a$ is greater than the score $s(b)$ of candidate $b$. In particular, it holds that

$$
s(a)=5 \alpha_{1}+5 \alpha_{2}+5 \alpha_{3}+5 \alpha_{4}>10 \alpha_{1}+\alpha_{3}+9 \alpha_{4}=s(b) .
$$

As the intersection of Eqs. (7.1) and (7.2) with the constraints on the weights of a scoring ranking rule $\left(\alpha_{1} \geq \alpha_{2} \geq \alpha_{3} \geq \alpha_{4} \geq 0\right.$ and $\left.\alpha_{1}>\alpha_{4}\right)$ is empty, we conclude that there exists no scoring ranking rule satisfying at the same time both constraints. Therefore, the search for (recursive) monotonicity of the scorix is proved to be independent w.r.t. all scoring ranking rules.

\subsubsection{The search for monotonicity of the votrix and the search for monotonicity of the votex}

In this subsection, we prove that the search for monotonicity of the votrix and the search for monotonicity of the votex are independent w.r.t. each other by providing a profile of rankings for which both methods lead to a different winning ranking. Consider the profile of $r=20$ rankings listed in Table 7.4 .

On the one hand, in case we address the search for monotonicity of the votrix, the optimal ranking is $c \succ b \succ a \succ d$ with a cost of one, while the ranking $b \succ c \succ a \succ d$ leads to a cost of six. On the other hand, in case we address the search for monotonicity of the votex, the optimal ranking is $b \succ c \succ a \succ d$ with a cost of 13, while the ranking $c \succ b \succ a \succ d$ leads to a cost of 14. Therefore, the search for monotonicity of the votrix and the search for monotonicity of the votex are independent w.r.t. each other. 


\begin{tabular}{|c|c|}
\hline$\# \succ_{i}$ & Rankings on $\mathscr{C}$ \\
\hline 5 & $b \succ c \succ d \succ a$ \\
4 & $b \succ c \succ a \succ d$ \\
3 & $a \succ d \succ c \succ b$ \\
2 & $c \succ a \succ d \succ b$ \\
2 & $d \succ a \succ c \succ b$ \\
1 & $a \succ c \succ b \succ d$ \\
1 & $a \succ c \succ d \succ b$ \\
1 & $b \succ a \succ c \succ d$ \\
1 & $c \succ d \succ a \succ b$ \\
\hline
\end{tabular}

Table 7.4: Expressed rankings and their frequency.

\subsubsection{The search for monotonicity of the votrix and the search for a Condorcet ranking and unanimity}

In this subsection, we prove that the search for monotonicity of the votrix is independent w.r.t. the search for a Condorcet ranking and unanimity by providing a profile of rankings for which the first method leads to a different winning ranking than the other two methods. We consider the profile of $r=20$ rankings listed in Table 7.5.

\begin{tabular}{|c|c|}
\hline$\# \succ_{i}$ & Rankings on $\mathscr{C}$ \\
\hline 11 & $a \succ b \succ c \succ d$ \\
9 & $b \succ c \succ d \succ a$ \\
\hline
\end{tabular}

Table 7.5: Expressed rankings and their frequency.

When searching for monotonicity of the votrix, the optimal ranking is $b \succ a \succ c \succ d$ with a cost of ten. Note that the cost associated with the Condorcet ranking, $a \succ b \succ c \succ d$, equals 18. Therefore, the search for monotonicity of the votrix is independent w.r.t. the search for a Condorcet ranking. As in case a Condorcet ranking exists it always is the Kemeny winner, the independence w.r.t. the search for unanimity also holds. 


\subsubsection{The search for monotonicity of the profile and the search for all other con- sensus states}

In this subsection, we prove that the search for monotonicity of the profile is independent w.r.t. the search for all other consensus states by providing a profile of rankings for which the first method leads to a different winning ranking than all the other methods. We consider the profile of $r=20$ rankings listed in Table 7.6.

\begin{tabular}{|c|c|}
\hline$\# \succ_{i}$ & Rankings on $\mathscr{C}$ \\
\hline 9 & $a \succ b \succ c \succ d$ \\
6 & $b \succ a \succ c \succ d$ \\
3 & $b \succ c \succ a \succ d$ \\
2 & $b \succ c \succ d \succ a$ \\
\hline
\end{tabular}

Table 7.6: Expressed rankings and their frequency.

Note that the profile of rankings is monotone w.r.t. the ranking $a \succ b \succ c \succ d$. Therefore, it is the optimal ranking and trivially leads to a cost of zero. Note that this optimal ranking differs from the Condorcet ranking, $b \succ a \succ c \succ d$, which leads to a cost of two. Therefore, the search for monotonicity of the profile is independent w.r.t. the search for the Condorcet ranking and w.r.t. all Condorcet-consistent ranking rules. In addition, the optimal ranking according to the search for all other consensus states discussed in this dissertation is also $b \succ a \succ c \succ d$. Therefore, the search for monotonicity of the profile is proved to be independent w.r.t. the search for all other consensus states here discussed.

\subsubsection{The search for monotonicity of the scorix and the search for monotonicity of the votrix}

In this subsection, we prove that the search for monotonicity of the scorix and the search for monotonicity of the votrix are independent w.r.t. each other by providing a profile of rankings for which both methods lead to a different winning ranking. Consider the profile of $r=20$ rankings listed in Table 7.7 .

On the one hand, in case we address the search for monotonicity of the scorix, the optimal ranking is $b \succ a \succ c \succ d$ with a cost of seven, while the ranking $b \succ a \succ d \succ c$ leads 


\begin{tabular}{|c|c|}
\hline$\# \succ_{i}$ & Rankings on $\mathscr{C}$ \\
\hline 8 & $b \succ c \succ d \succ a$ \\
7 & $a \succ b \succ c \succ d$ \\
4 & $b \succ d \succ c \succ a$ \\
1 & $a \succ d \succ b \succ c$ \\
\hline
\end{tabular}

Table 7.7: Expressed rankings and their frequency.

to a cost of eight. On the other hand, in case we address the search for monotonicity of the votrix, the optimal ranking is $b \succ a \succ d \succ c$ with a cost of nine, while the ranking $b \succ a \succ c \succ d$ leads to a cost of ten. Therefore, the search for monotonicity of the scorix and the search for monotonicity of the votrix are independent w.r.t. each other.

\subsubsection{The search for acclamation and the search for a Condorcet ranking and unanimity}

In this subsection, we prove that the search for monotonicity of the votrix is independent w.r.t. the search for a Condorcet ranking and unanimity by providing a profile of rankings for which the first method leads to a different winning ranking than the other two methods. We consider the profile of $r=20$ rankings listed in Table 7.5.

As previously discussed, the ranking $a \succ b \succ c \succ d$ is the Condorcet ranking of the profile of rankings. Therefore, it is the optimal ranking according to the search for a Condorcet ranking and for unanimity. Nevertheless, when searching for acclamation, it leads to a cost of 18, which is lower than 16, the cost associated with the ranking $b \succ a \succ c \succ d$. Therefore, the search for acclamation is independent w.r.t. the search for a Condorcet ranking and unanimity.

\subsubsection{The search for a Condorcet ranking and the search for unanimity}

In this subsection, we prove that the search for a Condorcet ranking and the search for unanimity are independent w.r.t. each other by providing a profile of rankings for which both methods lead to a different winning ranking. This is a well-known result in social 
choice theory and we refer to an example profile given in [83]. This profile of $r=34$ rankings is listed in Table 7.8 .

\begin{tabular}{|c|c|}
\hline$\# \succ_{i}$ & Rankings on $\mathscr{C}$ \\
\hline 9 & $a \succ b \succ c \succ d$ \\
5 & $a \succ c \succ b \succ d$ \\
5 & $b \succ d \succ c \succ a$ \\
5 & $c \succ d \succ b \succ a$ \\
5 & $d \succ b \succ a \succ c$ \\
5 & $d \succ c \succ a \succ b$ \\
\hline
\end{tabular}

Table 7.8: Expressed rankings and their frequency.

On the one hand, in case we address the search for a Condorcet ranking, the optimal rankings are $b \succ c \succ d \succ a, b \succ d \succ a \succ c$ and $d \succ a \succ b \succ c$ with a cost of six, while the ranking $a \succ b \succ c \succ d$ leads to a cost of eight. On the other hand, in case we address the search for unanimity, the unique optimal ranking is $a \succ b \succ c \succ d$ with a cost of 95, while the costs associated with the other three rankings equals 97 . Therefore, the search for a Condorcet ranking and the search for unanimity are independent w.r.t. each other.

\subsubsection{Summary}

In this subsection, we provide a summary (see Table 7.9) of the set of optimal rankings in the search for all different consensus states mentioned in this dissertation for different profiles of rankings.

The reader may notice that the five considered profiles lead to an exhaustive comparison! 1 of the independence of the search for the eight different consensus states analysed in this dissertation w.r.t. each other. It suffices to check that, for any two consensus states, there is at least one profile of rankings for which the intersection of both sets of optimal rankings is empty.

\footnotetext{
${ }^{1}$ The search for a Condorcet ranking and the search for unanimity are not proved to be independent w.r.t. each other in Table 7.9 , but in the profile of rankings listed in Table 7.8 .
} 


\begin{tabular}{|c|c|c|c|c|c|}
\hline Search for & Table 7.1 & Table 7.2 & Table 7.5 & Table 7.6 & Table 7.7 \\
\hline $\begin{array}{l}\text { Monotonicity } \\
\text { of the scorix }\end{array}$ & $c \succ a \succ b \succ d$ & $d \succ b \succ a \succ c$ & $b \succ a \succ c \succ d$ & $b \succ a \succ c \succ d$ & $b \succ a \succ c \succ d$ \\
\hline $\begin{array}{l}\text { Recursive monotonicity } \\
\text { of the scorix }\end{array}$ & $c \succ b \succ a \succ d$ & $d \succ b \succ a \succ c$ & $b \succ a \succ c \succ d$ & $b \succ a \succ c \succ d$ & $b \succ a \succ c \succ d$ \\
\hline $\begin{array}{l}\text { Monotonicity } \\
\text { of the votrix }\end{array}$ & $c \succ b \succ a \succ d$ & $d \succ b \succ a \succ c$ & $b \succ a \succ c \succ d$ & $b \succ a \succ c \succ d$ & $b \succ a \succ d \succ c$ \\
\hline $\begin{array}{l}\text { Monotonicity } \\
\text { of the votex }\end{array}$ & $b \succ c \succ a \succ d$ & $\begin{array}{l}b \succ d \succ a \succ c \\
d \succ b \succ a \succ c\end{array}$ & $b \succ a \succ c \succ d$ & $b \succ a \succ c \succ d$ & $\begin{array}{l}b \succ a \succ c \succ d \\
b \succ c \succ a \succ d \\
b \succ c \succ d \succ a\end{array}$ \\
\hline $\begin{array}{l}\text { Monotonicity } \\
\text { of the profile }\end{array}$ & $c \succ a \succ b \succ d$ & $d \succ b \succ a \succ c$ & $a \succ b \succ c \succ d$ & $a \succ b \succ c \succ d$ & $\begin{array}{l}b \succ c \succ a \succ d \\
b \succ c \succ d \succ a\end{array}$ \\
\hline Condorcet ranking & $\begin{array}{l}b \succ c \succ a \succ d \\
c \succ a \succ b \succ d \\
c \succ b \succ a \succ d\end{array}$ & $b \succ d \succ a \succ c$ & $a \succ b \succ c \succ d$ & $b \succ a \succ c \succ d$ & $b \succ c \succ d \succ a$ \\
\hline Acclamation & $c \succ a \succ b \succ d$ & $\begin{array}{l}d \succ a \succ b \succ c \\
d \succ b \succ a \succ c\end{array}$ & $b \succ a \succ c \succ d$ & $b \succ a \succ c \succ d$ & $\begin{array}{l}b \succ c \succ a \succ d \\
b \succ c \succ d \succ a\end{array}$ \\
\hline Unanimity & $\begin{array}{l}b \succ c \succ a \succ d \\
c \succ a \succ b \succ d \\
c \succ b \succ a \succ d\end{array}$ & $b \succ d \succ a \succ c$ & $a \succ b \succ c \succ d$ & $b \succ a \succ c \succ d$ & $b \succ c \succ d \succ a$ \\
\hline
\end{tabular}

Table 7.9: Search for all consensus states and corresponding sets of optimal rankings. 


\section{CHAPTER 8}

\section{Impact of ties on the representations of votes}

\subsection{The three-way setting}

In the previous chapters, we have discussed several monotonicity-based ranking rules focused on the search for a profile of rankings with a monotone representation of votes. We consider here the special case where each voter is assumed to provide a ranking with ties on the set of candidates, which is a common situation in real-life problems where voters might consider two or more candidates equally suitable [117]. This situation subsumes a three-way decision [168], where each voter needs to decide whether 'candidate $a$ is better than candidate $b$ ', 'candidate $b$ is better than candidate $a$ ' or 'candidates $a$ and $b$ are equally suitable'. This type of decision can be seen as a representation of bipolar information [34] on a three-label bipolar qualitative scale. As each voter directly provides a ranking with ties on the set of candidates, the relations 'the first candidate is better than the second candidate' (and its transpose) and 'both candidates are equally suitable' are considered to be transitive.

In this case, every voter is allowed to provide a weak order relation $\succsim_{j}$ on $\mathscr{C}$, i.e., a complete and transitive relation on $\mathscr{C}$ that might not be antisymmetric. Any weak order relation $\succsim$ 
can be written as the union of two relations $\succ$ and $\sim$, where:

$$
\begin{aligned}
& \succ=\left\{\left(a_{i_{1}}, a_{i_{2}}\right) \in \mathscr{C}^{2} \mid\left(a_{i_{1}} \succsim a_{i_{2}}\right) \wedge\left(a_{i_{2}} \succsim a_{i_{1}}\right)\right\}, \\
& \sim=\left\{\left(a_{i_{1}}, a_{i_{2}}\right) \in \mathscr{C}^{2} \mid\left(a_{i_{1}} \succsim a_{i_{2}}\right) \wedge\left(a_{i_{2}} \succsim a_{i_{1}}\right)\right\} .
\end{aligned}
$$

The relation $\succ$, called ranking with ties (or strict weak order relation), is irreflexive, transitive and antisymmetric and the relation $\sim$, called indifference relation, is reflexive, transitive and symmetric. Due to the completeness of a weak order relation, the weak order relation $\succsim_{j}$ on $\mathscr{C}$ expressed by a voter and the corresponding ranking with ties $\succ_{j}$ are used interchangeably 1

The set of all rankings with ties on $\mathscr{C}$ is denoted by $\mathcal{L}^{*}(\mathscr{C})$. As a ranking is a particular case of a ranking with ties, it obviously holds that $\mathcal{L}(\mathscr{C}) \subseteq \mathcal{L}^{*}(\mathscr{C})$. The list of $r$ rankings with ties given by the voters is called a profile of rankings with ties and is denoted by $\mathscr{R}^{*}=\left(\succ_{j}\right)_{j=1}^{r}$. Obviously, it holds that $\mathscr{R}^{*} \in \mathcal{L}^{*}(\mathscr{C})^{r}$.

In the following, we will see how the representations of votes analysed in the previous chapters are affected by ties.

\subsection{The weak-scorix}

In case a ranking with ties is provided, the position of a candidate is no longer determined by a number, but rather by an interval, where the left endpoint equals the best position of the candidate in any linear extension of the ranking with ties and the right endpoint equals the worst position of the candidate in any linear extension of the ranking with ties.

Definition 8.1 Let $\mathscr{C}$ be a set of $k$ candidates. For a weak order relation $\succsim$ on $\mathscr{C}$, the position $P_{\succ}\left(a_{i}\right)$ of candidate $a_{i}$ is defined as the interval $\left[\ell_{1}, \ell_{2}\right]$, where

$$
\begin{aligned}
& \ell_{1}=\#\left\{j \in\{1, \ldots, k\} \mid a_{j} \succ a_{i}\right\}+1, \\
& \ell_{2}=k-\#\left\{j \in\{1, \ldots, k\} \mid a_{i} \succ a_{j}\right\} .
\end{aligned}
$$

All intervals of this form are called interval positions.

\footnotetext{
${ }^{1}$ When a strict weak order relation is understood as a ranking with ties, the incomparable elements in the ranking with ties correspond to indifferent elements in the weak order relation.
} 
Interval positions are based on the generalization of the Borda count to rankings with ties. Several extensions of the Borda count have been proposed (see, for instance [50]). Here, we have considered a similar methodology to the two-valued approach discussed in [15].

Example 8.2 Let $\mathscr{C}=\{a, b, c, d\}$ be a set of candidates and $a \succ b \sim c \succ d$ be a weak order relation on $\mathscr{C}$. Note that there are two linear extensions of $a \succ b \sim c \succ d: a \succ b \succ c \succ d$ and $a \succ c \succ b \succ d$. As it is always ranked at the first position, the position of candidate $a$ in $\succsim$ is the (degenerated) interval $[1,1]$. Analogously, as it is always ranked at the last position, the position of candidate $d$ in $\succsim$ is the (degenerated) interval [4,4]. As they are ranked, in the best case scenario, at the second position and, in the worst case scenario, at the third position, the interval position of both candidates $b$ and $c$ in $\succsim$ is the interval $[2,3]$.

Remark 8.3 The set $\mathcal{P}_{k}$ of all interval positions for a set of $k$ candidates is denoted by $\mathcal{P}_{k}$, and it can be characterized in the following way ${ }^{2}$ :

$$
\mathcal{P}_{k}=\left\{\left[\ell_{1}, \ell_{2}\right] \in I(\mathbb{N}) \mid \ell_{1} \leq \ell_{2} \leq k\right\}=I(\{1, \ldots, k\})
$$

An intuitive order relation on $\mathcal{P}_{k}$ is defined according to how good a candidate is ranked in a given ranking with ties.

Proposition 8.4 Let $\mathcal{P}_{k}$ be the set of all interval positions for a set of $k$ candidates. The relation $\leq_{\mathcal{P}_{k}}$ defined as

$$
\leq_{\mathcal{P}_{k}}=\left\{\left(\left[\ell_{1}, \ell_{2}\right],\left[\ell_{1}^{\prime}, \ell_{2}^{\prime}\right]\right) \in \mathcal{P}_{k}^{2} \mid \ell_{1} \geq \ell_{1}^{\prime} \wedge \ell_{2} \geq \ell_{2}^{\prime}\right\}
$$

is an order relation on $\mathcal{P}_{k}$.

Proof: The proof of this proposition is immediate as $\leq_{\mathcal{P}_{k}}$ coincides with the converse of the partial order on intervals induced by the usual product order on $\mathbb{N}^{2}$.

In Figure 8.1, the Hasse diagram of the order relation $\leq_{\mathcal{P}_{k}}$ is shown for a set of four candidates.

\footnotetext{
${ }^{2}$ Throughout this chapter, $I(X)$ refers to the set of intervals in a totally ordered set $X$ and $\mathbb{N}$ refers to the set of natural numbers, not including zero.
} 


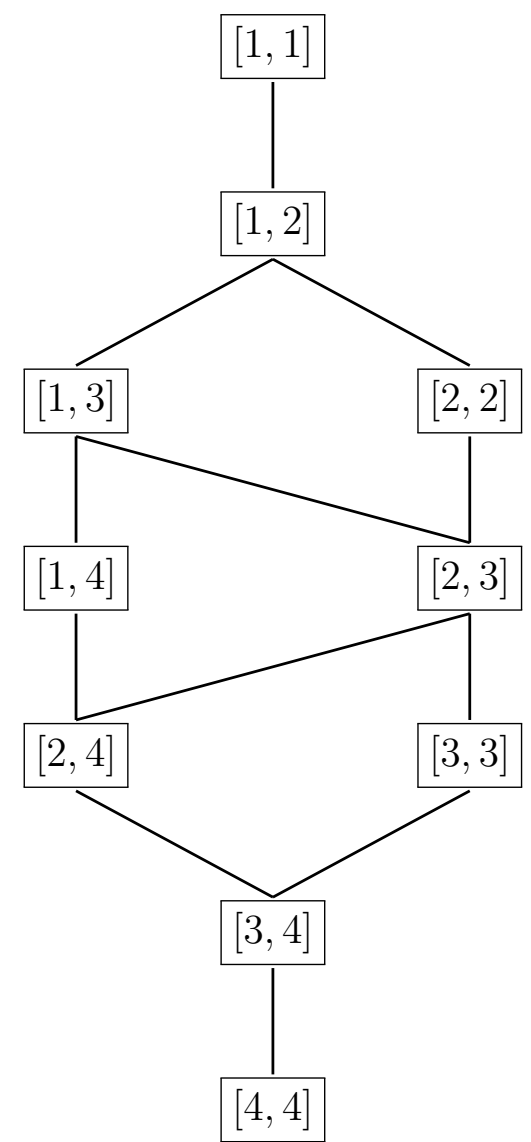

Figure 8.1: Hasse diagram of the order relation $\leq_{\mathcal{P}_{k}}$.

Remark 8.5 When restricting to the case where ties are not allowed, it holds that, for any candidate, its interval position $\left[\ell_{1}, \ell_{2}\right]$ reduces to a single value, i.e., $\ell_{1}=\ell_{2}$. Furthermore, as the position $\ell$ in a ranking is the interval position $[\ell, \ell]$ when a ranking is interpreted as a ranking with ties, it holds that $[\ell, \ell] \leq_{\mathcal{P}_{k}}\left[\ell^{\prime}, \ell^{\prime}\right]$ if and only if $\ell \geq \ell^{\prime}$. Hence, the order relation $\leq_{\mathcal{P}_{k}}$ is a total order relation when restricting to rankings without ties.

The scorix can no longer be defined in the form of a matrix for the case of rankings with ties. Instead, we define the weak-scorix.

Definition 8.6 Let $\mathscr{C}$ be a set of $k$ candidates and $r$ be the number of voters. A function $S^{*}: \mathscr{C} \times \mathcal{P}_{k} \rightarrow\{0,1, \ldots, r\}$ is called a weak-scorix (plural weak-scorices) on $\mathscr{C}$ if there exists a profile $\mathscr{R}^{*}$ of $r$ rankings with ties on $\mathscr{C}$ such that, for any $a_{i} \in \mathscr{C}$ and any $\left[\ell_{1}, \ell_{2}\right] \in \mathcal{P}_{k}$, 
it holds that

$$
S^{*}\left(a_{i},\left[\ell_{1}, \ell_{2}\right]\right)=\#\left\{j \in\{1, \ldots, r\} \mid P_{j}\left(a_{i}\right)=\left[\ell_{1}, \ell_{2}\right]\right\} .
$$

The set of all weak-scorices on $\mathscr{C}$ induced by any profile of $r$ rankings with ties on $\mathscr{C}$ is denoted by $\mathcal{S}_{r}^{*}(\mathscr{C})$.

Similarly to monotonicity of a scorix, we define monotonicity of a weak-scorix.

Definition 8.7 Let $\mathscr{C}$ be a set of $k$ candidates and $r$ be the number of voters. A weakscorix $S^{*} \in \mathcal{S}_{r}^{*}(\mathscr{C})$ is said to be monotone w.r.t. a ranking $\succ$ on $\mathscr{C}$ if, for any $a_{i_{1}}, a_{i_{2}} \in \mathscr{C}$ such that $a_{i_{1}} \succ a_{i_{2}}$ and any $\left[\ell_{1}, \ell_{2}\right] \in \mathcal{P}_{k}$, it holds that

$$
\sum_{\substack{\left[\ell_{1}^{\prime}, \ell_{2}^{\prime}\right] \in \mathcal{P}_{k} \\\left[\ell_{1}^{\prime}, \ell_{2}^{\prime}\right] \leq \mathcal{P}_{k}\left[\ell_{1}, \ell_{2}\right]}} S^{*}\left(a_{i_{1}},\left[\ell_{1}^{\prime}, \ell_{2}^{\prime}\right]\right) \leq \sum_{\substack{\left[\ell_{1}^{\prime}, \ell_{2}^{\prime}\right] \in \mathcal{P}_{k} \\\left[\ell_{1}^{\prime}\right] \in \mathcal{P}_{k} \\\left[\ell_{1}, \ell_{2}\right] \leq_{\mathcal{P}_{k}}\left[\ell_{1}^{\prime}, \ell_{2}^{\prime}\right]}} S^{*}\left(a_{i_{1}},\left[\ell_{1}^{\prime}, \ell_{2}^{\prime}\right]\right) \geq \sum_{\substack{\ell_{k}\left[\ell_{1}, \ell_{2}\right] \\\left[\ell_{1}^{\prime}, \ell_{2}^{\prime}\right] \in \mathcal{P}_{k} \\\left[\ell_{1}, \ell_{2}\right] \mathcal{P}_{k}\left[\ell_{1}^{\prime}, \ell_{2}^{\prime}\right]}} S^{*}\left(a_{i_{2}},\left[\ell_{1}^{\prime}, \ell_{2}^{\prime}\right]\right),\left[\ell_{i_{2}},\left[\ell_{1}^{\prime}, \ell_{2}^{\prime}\right]\right) .
$$

Again, the weak-scorix induced by most profiles of rankings with ties is not monotone w.r.t. any ranking. The set of all profiles of rankings with ties with a monotone weak-scorix can also be understood as a consensus state for the rationalisation of ranking rules.

\subsection{The weak-votrix}

Among the three main representations of votes discussed in this dissertation (scorix, votrix and votex), the votrix is the least affected by ties in the sense that the definition of the given representation of votes remains almost the same when rankings with ties are considered.

Definition 8.8 Let $\mathscr{C}$ be a set of $k$ candidates and $r$ be the number of voters. A matrix $V^{*} \in\{0,1, \ldots, r\}^{k \times k}$ is called a weak-votrix (plural votrices) on $\mathscr{C}$ if there exists a profile $\mathscr{R}^{*}$ of $r$ rankings with ties on $\mathscr{C}$ such that, for any $a_{i_{1}}, a_{i_{2}} \in \mathscr{C}$, it holds that

$$
V_{i_{1} i_{2}}^{*}=\#\left\{j \in\{1, \ldots, r\} \mid a_{i_{1}} \succ_{j} a_{i_{2}}\right\} .
$$

The set of all weak-votrices on $\mathscr{C}$ induced by any profile of $r$ rankings with ties on $\mathscr{C}$ is denoted by $\mathcal{V}_{r}^{*}(\mathscr{C})$. 
The notion of monotonicity of a votrix is easily extended to weak-votrices.

Definition 8.9 Let $\mathscr{C}$ be a set of $k$ candidates and $r$ be the number of voters. A weakvotrix $V^{*}$ is said to be monotone w.r.t. a ranking $\succ$ on $\mathscr{C}$ (with corresponding $\sqsupset$ ) if, for any $\left(a_{i_{1}}, a_{j_{1}}\right),\left(a_{i_{2}}, a_{j_{2}}\right) \in \mathscr{C}_{\neq}^{2}$ such that $\left(a_{i_{1}}, a_{j_{1}}\right) \sqsupset\left(a_{i_{2}}, a_{j_{2}}\right)$, it holds that

$$
\begin{aligned}
V_{i_{1} j_{1}}^{*} & \geq V_{i_{2} j_{2}}^{*}, \\
V_{j_{1} i_{1}}^{*} & \leq V_{j_{2} i_{2}}^{*} .
\end{aligned}
$$

Again, the weak-votrix induced by most profiles of rankings with ties is not monotone w.r.t. any ranking. The set of all profiles of rankings with ties with a monotone weak-votrix can also be understood as a consensus state for the rationalisation of ranking rules.

\subsection{The weak-votex}

In order to define the 'relative position' of a candidate $a_{i_{1}}$ w.r.t. another candidate $a_{i_{2}}$ in a ranking with ties $\succ$, the sizes of the equivalence classes in $\succsim$ of both candidates $a_{i_{1}}$ and $a_{i_{2}}$ need to be taken into account. For any candidate $a \in \mathscr{C}$, we denote by $[a]_{\succsim}$ the equivalence class of candidate $a$ in the weak order relation $\succsim$, i.e., $[a]_{\succsim}=\left\{a^{\prime} \in \mathscr{C} \mid a \sim a^{\prime}\right\}$.

The relative position of $a_{i_{1}}$ w.r.t. $a_{i_{2}}$ depends on three values: the size of the largest equivalence class among $\left[a_{i_{1}}\right]_{\succsim}$ and $\left[a_{i_{2}}\right]_{\succsim}$, the size of the smallest equivalence class among $\left[a_{i_{1}}\right]_{\succsim}$ and $\left[a_{i_{2}}\right]_{\succsim}$, and the relative position of both equivalence classes defined as

$$
P_{\succsim}\left(\left[a_{i_{1}}\right]_{\succsim},\left[a_{i_{2}}\right]_{\succsim}\right)= \begin{cases}\#\left\{i \in\{1, \ldots, k\} \mid a_{i_{1}} \succ a_{i} \succ a_{i_{2}}\right\}+1 & , \text { if } a_{i_{1}} \succ a_{i_{2}}, \\ 0 & , \text { if } a_{i_{1}} \sim a_{i_{2}}, \\ -\#\left\{i \in\{1, \ldots, k\} \mid a_{i_{2}} \succ a_{i} \succ a_{i_{1}}\right\}-1 & \text {, if } a_{i_{2}} \succ a_{i_{1}} .\end{cases}
$$

Definition 8.10 Let $\mathscr{C}$ be a set of $k$ candidates. For a weak order relation $\succsim$ on $\mathscr{C}$, the relative position of candidate $a_{i_{1}}$ w.r.t. candidate $a_{i_{2}}$ is defined as the triplet:

$$
\left(s_{1}, s_{2}, p\right)=\left(\max \left(\#\left[a_{i_{1}}\right]_{\succsim}, \#\left[a_{i_{2}}\right]_{\succsim}\right), \min \left(\#\left[a_{i_{1}}\right]_{\succsim}, \#\left[a_{i_{2}}\right]_{\succsim}\right), P_{\succsim}\left(\left[a_{i_{1}}\right]_{\succsim},\left[a_{i_{2}}\right]_{\succsim}\right)\right),
$$

in case $P_{\succsim}\left(\left[a_{i_{1}}\right]_{\succsim},\left[a_{i_{2}}\right]_{\succsim}\right) \neq 0$, or, otherwise, as the triplet $\left(s_{1}, s_{2}, p\right)=(0,0,0)$. All triplets of this form are called positional triplets. The set of all positional triplets for a set of $k$ candidates is denoted by $\mathcal{B}_{k}$. 
Remark 8.11 Note that $\mathcal{B}_{k}$ can be characterized in the following way:

$$
\mathcal{B}_{k}=\left\{\left(s_{1}, s_{2}, p\right) \in \mathbb{N}^{2} \times \mathbb{Z} \mid\left(s_{1} \geq s_{2}\right) \wedge\left(s_{1}+s_{2}+|p| \leq k+1\right) \wedge(p \neq 0)\right\} \cup\{(0,0,0)\} .
$$

In the following example we illustrate the notion of a positional triplet.

Example 8.12 Let $\mathscr{C}=\{a, b, c, d\}$ be a set of candidates and $a \succ b \sim c \succ d$ be a weak order relation on $\mathscr{C}$. For candidates a and $d$, the size of both equivalence classes equals one $\left(\#[a]_{\succsim}=\#[d]_{\succsim}=1\right)$ and the relative position of both equivalence classes equals three due to the fact that there are two candidates strictly in between $a$ and $d\left(P_{\succsim}\left([a]_{\succsim},[d]_{\succsim}\right)=3\right)$. Therefore, the relative position of a w.r.t. $d$ is $(1,1,3)$. Analogously, the relative position of $d$ w.r.t. $a$ is $(1,1,-3)$. For candidates $a$ and $b$, the size of the equivalence class of $a$ equals one $\left(\#[a]_{\succsim}=1\right)$, the size of the equivalence class of $b$ equals two $\left(\#[b]_{\succsim}=2\right)$ and the relative position of both equivalence classes equals one due to the fact that there are no candidates strictly in between $a$ and $b\left(P_{\succsim}\left([a]_{\succsim},[b]_{\succsim}\right)=1\right)$. Therefore, the relative position of a w.r.t. $b$ is $(2,1,1)$. Analogously, the relative position of $b$ w.r.t. a is $(2,1,-1)$. As candidates $b$ and $c$ belong to the same equivalence class, the relative position of $b$ w.r.t. $c$ is $(0,0,0)$. Analogously, the relative position of $c$ w.r.t. $b$ also is $(0,0,0)$.

The set of all positional triplets $\left(s_{1}, s_{2}, p\right) \in \mathcal{B}_{k}$ such that $p \geq 1$ is denoted by $\mathcal{B}_{k}^{+}$, the (singleton) set of all positional triplets $\left(s_{1}, s_{2}, p\right) \in \mathcal{B}_{k}$ such that $p=0$ is denoted by $\mathcal{B}_{k}^{0}$ and the set of all positional triplets $\left(s_{1}, s_{2}, p\right) \in \mathcal{B}_{k}$ such that $p \leq-1$ is denoted by $\mathcal{B}_{k}^{-}$. It obviously holds that $\mathcal{B}_{k}^{+}, \mathcal{B}_{k}^{0}$ and $\mathcal{B}_{k}^{-}$are disjoint sets and

$$
\mathcal{B}_{k}=\mathcal{B}_{k}^{+} \cup \mathcal{B}_{k}^{0} \cup \mathcal{B}_{k}^{-} \text {. }
$$

An intuitive order relation on $\mathcal{B}_{k}$ is defined according to how far apart two candidates are in a given ranking with ties. In order to do so, we will first define three order relations on $\mathcal{B}_{k}^{+}, \mathcal{B}_{k}^{0}$ and $\mathcal{B}_{k}^{-}$separately. The order relation on $\mathcal{B}_{k}^{+}$follows from the intuition that the addition (resp. removal) of a candidate strictly in between $a$ and $b$ or in the equivalence class of either $a$ or $b$ should make the relative position of $a$ w.r.t. $b$ to increase (resp. decrease). The order relation on $\mathcal{B}_{k}^{0}$ is trivially determined due to the fact that $\mathcal{B}_{k}^{0}$ is a singleton. The order relation on $\mathcal{B}_{k}^{-}$should be analogous to $\mathcal{B}_{k}^{+}$due to the fact that the relative position of $a$ w.r.t. $b$ and the relative position of $b$ w.r.t. $a$ play opposite roles. In that way, the order relation on $\mathcal{B}_{k}$ should be defined as the linear sum of the order relations on $\mathcal{B}_{k}^{-}, \mathcal{B}_{k}^{0}$ and $\mathcal{B}_{k}^{+}$. 
Proposition 8.13 Let $\mathcal{B}_{k}$ be the set of all positional triplets for a set of $k$ candidates.

(i) The relation $\leq_{\mathcal{B}_{k}}^{+}$, defined as

$$
\leq_{\mathcal{B}_{k}}^{+}=\left\{\begin{array}{l|l}
\left(\left(s_{1}, s_{2}, p\right),\left(s_{1}^{\prime}, s_{2}^{\prime}, p^{\prime}\right)\right) \in\left(\mathcal{B}_{k}^{+}\right)^{2} & \begin{array}{l}
\left(p \leq p^{\prime}\right) \\
\wedge\left(p+s_{1} \leq p^{\prime}+s_{1}^{\prime}\right) \\
\wedge\left(p+s_{2} \leq p^{\prime}+s_{2}^{\prime}\right) \\
\wedge\left(p+s_{1}+s_{2} \leq p^{\prime}+s_{1}^{\prime}+s_{2}^{\prime}\right)
\end{array}
\end{array}\right\},
$$

is an order relation on $\mathcal{B}_{k}^{+}$.

(ii) The relation $\leq_{\mathcal{B}_{k}}^{0}$, defined as $\leq_{\mathcal{B}_{k}}^{0}=\left(\mathcal{B}_{k}^{0}\right)^{2}$, is an order relation on $\mathcal{B}_{k}^{0}$.

(iii) The relation $\leq_{\overline{\mathcal{B}}_{k}}^{-}$, defined as

$$
\leq_{\overline{\mathcal{B}}_{k}}^{-}=\left\{\left(\left(s_{1}, s_{2}, p\right),\left(s_{1}^{\prime}, s_{2}^{\prime}, p^{\prime}\right)\right) \in\left(\mathcal{B}_{k}^{-}\right)^{2} \mid\left(\left(s_{1}^{\prime}, s_{2}^{\prime},-p^{\prime}\right),\left(s_{1}, s_{2},-p\right)\right) \in \leq_{\mathcal{B}_{k}}^{+}\right\}
$$

is an order relation on $\mathcal{B}_{k}^{-}$.

(iv) The relation $\leq_{\mathcal{B}_{k}}$, defined as

$$
\leq_{\mathcal{B}_{k}}=\leq_{\mathcal{B}_{k}}^{+} \cup \leq_{\mathcal{B}_{k}}^{0} \cup \leq_{\mathcal{B}_{k}}^{-} \cup\left(\mathcal{B}_{k}^{-} \times \mathcal{B}_{k}^{0}\right) \cup\left(\mathcal{B}_{k}^{-} \times \mathcal{B}_{k}^{+}\right) \cup\left(\mathcal{B}_{k}^{0} \times \mathcal{B}_{k}^{+}\right)
$$

is an order relation on $\mathcal{B}_{k}$.

Proof: If the three relations $\leq_{\mathcal{B}_{k}}^{+}, \leq_{\mathcal{B}_{k}}^{0}$ and $\leq_{\mathcal{B}_{k}}^{-}$are order relations on, respectively, $\mathcal{B}_{k}^{+}$, $\mathcal{B}_{k}^{0}$ and $\mathcal{B}_{k}^{-}$, then, as we are considering the linear sum (see [41]) of three disjoint partially ordered sets, $\leq_{\mathcal{B}_{k}}$ is an order relation on $\mathcal{B}_{k}$. Trivially, $\leq_{\mathcal{B}_{k}}^{0}$ is an order relation on $\mathcal{B}_{k}^{0}$. Let us prove that $\leq_{\mathcal{B}_{k}}^{+}$and $\leq_{\mathcal{B}_{k}}$ are reflexive, antisymmetric and transitive relations.

Reflexivity: evident.

Antisymmetry: for any $\left(s_{1}, s_{2}, p\right),\left(s_{1}^{\prime}, s_{2}^{\prime}, p^{\prime}\right) \in \mathcal{B}_{k}^{+}$such that $\left(s_{1}, s_{2}, p\right) \leq_{\mathcal{B}_{k}}^{+}\left(s_{1}^{\prime}, s_{2}^{\prime}, p^{\prime}\right)$ and $\left(s_{1}^{\prime}, s_{2}^{\prime}, p^{\prime}\right) \leq_{\mathcal{B}_{k}}^{+}\left(s_{1}, s_{2}, p\right)$, it holds that

$$
\begin{aligned}
& \left(p \leq p^{\prime}\right) \wedge\left(p^{\prime} \leq p\right) \text { which implies } p=p^{\prime}, \\
& \left(p+s_{1} \leq p^{\prime}+s_{1}^{\prime}\right) \wedge\left(p^{\prime}+s_{1}^{\prime} \leq p+s_{1}\right) \wedge\left(p=p^{\prime}\right) \text { which implies } s_{1}=s_{1}^{\prime}, \\
& \left(p+s_{2} \leq p^{\prime}+s_{2}^{\prime}\right) \wedge\left(p^{\prime}+s_{2}^{\prime} \leq p+s_{2}\right) \wedge\left(p=p^{\prime}\right) \text { which implies } s_{2}=s_{2}^{\prime} .
\end{aligned}
$$

Therefore, we conclude that $\left(s_{1}, s_{2}, p\right)=\left(s_{1}^{\prime}, s_{2}^{\prime}, p^{\prime}\right)$. 
For any $\left(s_{1}, s_{2}, p\right),\left(s_{1}^{\prime}, s_{2}^{\prime}, p^{\prime}\right) \in \mathcal{B}_{k}^{-}$such that $\left(s_{1}, s_{2}, p\right) \leq_{\mathcal{B}_{k}}^{-}\left(s_{1}^{\prime}, s_{2}^{\prime}, p^{\prime}\right)$ and $\left(s_{1}^{\prime}, s_{2}^{\prime}, p^{\prime}\right) \leq_{\mathcal{B}_{k}}^{-}$ $\left(s_{1}, s_{2}, p\right)$, it holds that $\left(s_{1}^{\prime}, s_{2}^{\prime},-p^{\prime}\right) \leq_{\mathcal{B}_{k}}^{+}\left(s_{1}, s_{2},-p\right)$ and $\left(s_{1}, s_{2},-p\right) \leq_{\mathcal{B}_{k}}^{+}\left(s_{1}^{\prime}, s_{2}^{\prime},-p^{\prime}\right)$. As $\leq_{\mathcal{B}_{k}}^{+}$is antisymmetric, it holds that $\left(s_{1}, s_{2},-p\right)=\left(s_{1}^{\prime}, s_{2}^{\prime},-p^{\prime}\right)$ and, therefore, we conclude that $\left(s_{1}, s_{2}, p\right)=\left(s_{1}^{\prime}, s_{2}^{\prime}, p^{\prime}\right)$.

Transitivity: for any $\left(s_{1}, s_{2}, p\right),\left(s_{1}^{\prime}, s_{2}^{\prime}, p^{\prime}\right),\left(s_{1}^{\prime \prime}, s_{2}^{\prime \prime}, p^{\prime \prime}\right) \in \mathcal{B}_{k}^{+}$such that $\left(s_{1}, s_{2}, p\right) \leq_{\mathcal{B}_{k}}^{+}\left(s_{1}^{\prime}, s_{2}^{\prime}, p^{\prime}\right)$ and $\left(s_{1}^{\prime}, s_{2}^{\prime}, p^{\prime}\right) \leq_{\mathcal{B}_{k}}^{+}\left(s_{1}^{\prime \prime}, s_{2}^{\prime \prime}, p^{\prime \prime}\right)$, it holds that

$$
\begin{aligned}
& \left(p \leq p^{\prime}\right) \wedge\left(p^{\prime} \leq p^{\prime \prime}\right) \text { which implies } p \leq p^{\prime \prime}, \\
& \left(p+s_{1} \leq p^{\prime}+s_{1}^{\prime}\right) \wedge\left(p^{\prime}+s_{1}^{\prime} \leq p^{\prime \prime}+s_{1}^{\prime \prime}\right) \text { which implies } p+s_{1} \leq p^{\prime \prime}+s_{1}^{\prime \prime}, \\
& \left(p+s_{2} \leq p^{\prime}+s_{2}^{\prime}\right) \wedge\left(p^{\prime}+s_{2}^{\prime} \leq p^{\prime \prime}+s_{2}^{\prime \prime}\right) \text { which implies } p+s_{2} \leq p^{\prime \prime}+s_{2}^{\prime \prime}, \\
& \left(p+s_{1}+s_{2} \leq p^{\prime}+s_{1}^{\prime}+s_{2}^{\prime}\right) \wedge\left(p^{\prime}+s_{1}^{\prime}+s_{2}^{\prime} \leq p^{\prime \prime}+s_{1}^{\prime \prime}+s_{2}^{\prime \prime}\right) \\
& \quad \text { which implies } p^{\prime}+s_{1}+s_{2} \leq p^{\prime \prime}+s_{1}^{\prime \prime}+s_{2}^{\prime \prime} .
\end{aligned}
$$

Therefore, we conclude that $\left(s_{1}, s_{2}, p\right) \leq_{\mathcal{B}_{k}}^{+}\left(s_{1}^{\prime \prime}, s_{2}^{\prime \prime}, p^{\prime \prime}\right)$.

For any $\left(s_{1}, s_{2}, p\right),\left(s_{1}^{\prime}, s_{2}^{\prime}, p^{\prime}\right),\left(s_{1}^{\prime \prime}, s_{2}^{\prime \prime}, p^{\prime \prime}\right) \in \mathcal{B}_{k}^{-}$such that $\left(s_{1}, s_{2}, p\right) \leq_{\mathcal{B}_{k}}^{-}\left(s_{1}^{\prime}, s_{2}^{\prime}, p^{\prime}\right)$ and $\left(s_{1}^{\prime}, s_{2}^{\prime}, p^{\prime}\right) \leq_{\mathcal{B}_{k}}^{-}\left(s_{1}^{\prime \prime}, s_{2}^{\prime \prime}, p^{\prime \prime}\right)$, it holds that $\left(s_{1}^{\prime}, s_{2}^{\prime},-p^{\prime}\right) \leq_{\mathcal{B}_{k}}^{+}\left(s_{1}, s_{2},-p\right)$ and $\left(s_{1}^{\prime \prime}, s_{2}^{\prime \prime},-p^{\prime \prime}\right) \leq_{\mathcal{B}_{k}}^{+}$ $\left(s_{1}^{\prime}, s_{2}^{\prime},-p^{\prime}\right)$. As $\leq_{\mathcal{B}_{k}}^{+}$is transitive, it holds that $\left(s_{1}^{\prime \prime}, s_{2}^{\prime \prime},-p^{\prime \prime}\right) \leq_{\mathcal{B}_{k}}^{+}\left(s_{1}, s_{2},-p\right)$. Therefore, we conclude that $\left(s_{1}, s_{2}, p\right) \leq_{\mathcal{B}_{k}}\left(s_{1}^{\prime \prime}, s_{2}^{\prime \prime}, p^{\prime \prime}\right)$.

In Figure 8.2, the Hasse diagrams of the order relations $\leq_{\mathcal{B}_{k}}^{+}, \leq_{\mathcal{B}_{k}}^{0}$ and $\leq_{\mathcal{B}_{k}}^{-}$are shown for a set of four candidates. The considered order relation on $\mathcal{B}_{k}$ is the one given by the linear sum of the three disjoint partially ordered sets $\left(\mathcal{B}_{k}^{-}, \leq_{\mathcal{B}_{k}}^{-}\right),\left(\mathcal{B}_{k}^{0}, \leq_{\mathcal{B}_{k}}^{0}\right)$ and $\left(\mathcal{B}_{k}^{+}, \leq_{\mathcal{B}_{k}}^{+}\right)$. The Hasse diagram of the order relation $\leq_{\mathcal{B}_{k}}$ is shown in Figure 8.3. Note that the relative position of two candidates is smaller than or equal to the relative position of two other candidates if the first two candidates are closer in the given weak order relation than the second couple of candidates.

Remark 8.14 When restricting to the case where ties are not allowed, it holds that $\max \left(\#\left[a_{i_{1}}\right]_{\succsim}, \#\left[a_{i_{2}}\right]_{\succsim}\right.$ $\min \left(\#\left[a_{i_{1}}\right]_{\succsim}, \#\left[a_{i_{2}}\right]_{\succsim}\right)=1$ and $P_{\succsim}\left(\left[a_{i_{1}}\right]_{\succsim},\left[a_{i_{2}}\right]_{\succsim}\right) \neq 0$ (for any two different candidates $\left.a_{i_{1}}, a_{i_{2}} \in \mathscr{C}\right)$.

In addition, it holds that $p \leq p^{\prime}$ if and only if $(1,1, p) \leq_{\mathcal{B}_{k}}\left(1,1, p^{\prime}\right)$. As the relative position $p$ in the sense of [124] is the relative position $(1,1, p)$ in the sense of this section, the usual order relation on relative positions defined in 124] is preserved. The order relation $\leq_{\mathcal{B}_{k}}$ is a total order relation when restricting to rankings without ties $\left(s_{1}=s_{2}=1\right)$. 


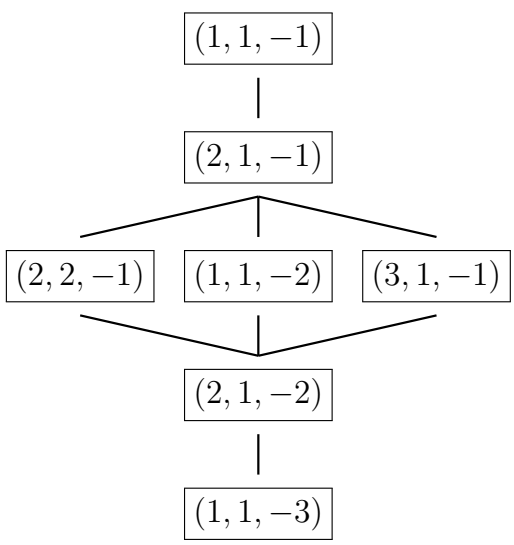

(a)

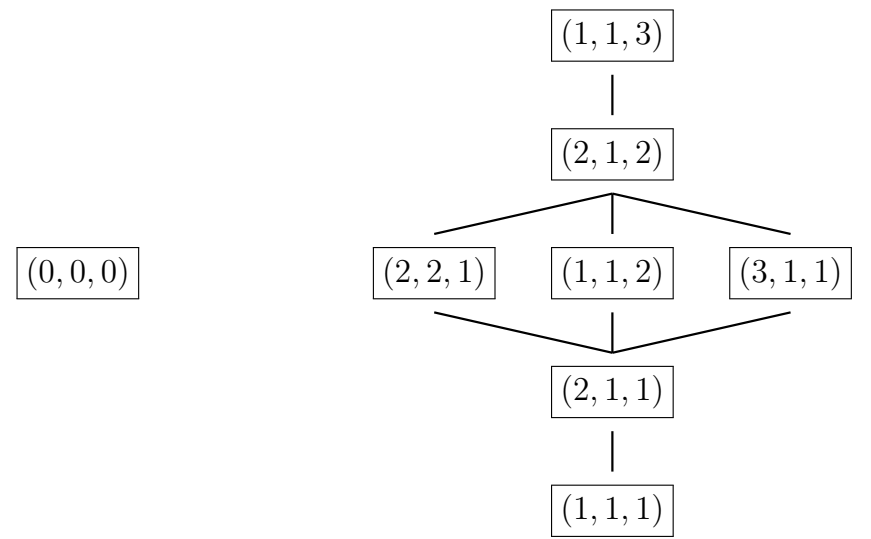

(b)

(c)

Figure 8.2: Hasse diagrams of the order relations $\leq_{\overline{\mathcal{B}}_{k}}^{-}(\mathbf{a}), \leq_{\mathcal{B}_{k}}^{0}(\mathbf{b})$ and $\leq_{\mathcal{B}_{k}}^{+}$ (c).

Gathering the information given by all the voters, we denote by $n_{\left(s_{1}, s_{2}, p\right)}\left(a_{i_{1}}, a_{i_{2}}\right)$ the (absolute) frequency of the relative position $\left(s_{1}, s_{2}, p\right) \in \mathcal{B}_{k}$ for the couple $\left(a_{i_{1}}, a_{i_{2}}\right) \in \mathscr{C}_{\neq}^{2}$, i.e., the number of voters considering that the relative position of candidate $a_{i_{1}}$ w.r.t. candidate $a_{i_{2}}$ equals $\left(s_{1}, s_{2}, p\right)$. The vector $\left(n_{\left(s_{1}, s_{2}, p\right)}\left(a_{i_{1}}, a_{i_{2}}\right)\right)_{\left(s_{1}, s_{2}, p\right) \in \mathcal{B}_{k}}$ is called the frequency distribution of the couple $\left(a_{i_{1}}, a_{i_{2}}\right)$.

As $\leq_{\mathcal{B}_{k}}$ does not define a total order relation on $\mathcal{B}_{k}$ but a partial order relation, two cumulative frequency distributions (instead of one) are associated with a frequency distribution: a top-down and a bottom-up one. For any $\left(s_{1}, s_{2}, p\right) \in \mathcal{B}_{k}$, the top-down cumulative frequency distribution of the couple $\left(a_{i_{1}}, a_{i_{2}}\right) \in \mathscr{C}_{\neq}^{2}$ is given by

$$
\bar{n}_{\left(s_{1}, s_{2}, p\right)}\left(a_{i_{1}}, a_{i_{2}}\right)=\sum_{\substack{\left(s_{1}^{\prime}, s_{2}^{\prime}, p^{\prime}\right) \in \mathcal{B}_{k} \\\left(s_{1}, s_{2}, p\right) \leq \mathcal{B}_{k}\left(s_{1}^{\prime}, s_{2}^{\prime}, p^{\prime}\right)}} n_{\left(s_{1}^{\prime}, s_{2}^{\prime}, p^{\prime}\right)}\left(a_{i_{1}}, a_{i_{2}}\right) .
$$

Analogously, for any $\left(s_{1}, s_{2}, p\right) \in \mathcal{B}_{k}$, the bottom-up cumulative frequency distribution of the couple $\left(a_{i_{1}}, a_{i_{2}}\right) \in \mathscr{C}_{\neq}^{2}$ is given by

$$
\underline{n}_{\left(s_{1}, s_{2}, p\right)}\left(a_{i_{1}}, a_{i_{2}}\right)=\sum_{\substack{\left(s_{1}^{\prime}, s_{2}^{\prime}, p^{\prime}\right) \in \mathcal{B}_{k} \\\left(s_{1}^{\prime}, s_{2}^{\prime}, p^{\prime}\right) \leq \mathcal{B}_{k}\left(s_{1}, s_{2}, p\right)}} n_{\left(s_{1}^{\prime}, s_{2}^{\prime}, p^{\prime}\right)}\left(a_{i_{1}}, a_{i_{2}}\right) .
$$

In order to illustrate how the frequency distribution given by a profile of rankings with ties and the corresponding top-down and bottom-up cumulative frequency distributions can be obtained, we consider the following example. 


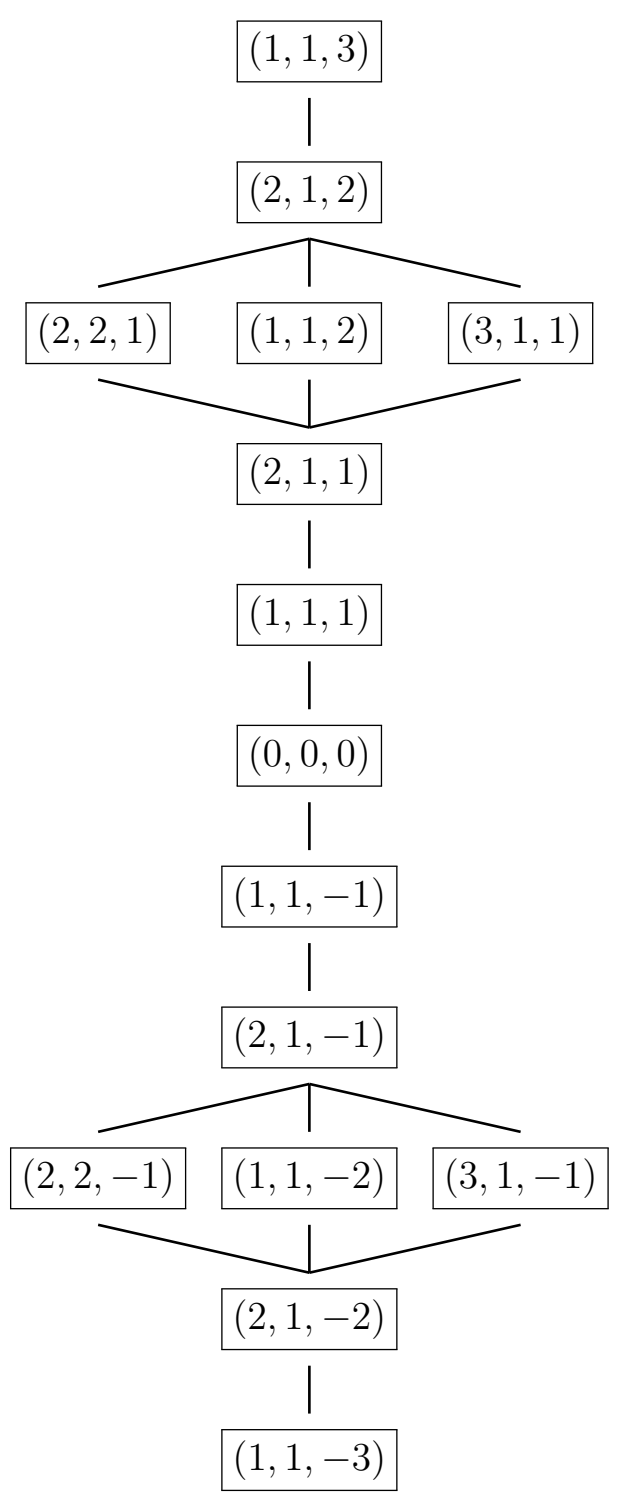

Figure 8.3: Hasse diagram of the order relation $\leq_{\mathcal{B}_{k}}$.

Example 8.15 Let $\mathscr{C}=\{a, b, c, d\}$ be a set of candidates and $\mathscr{R}^{*}=\left\{\succ_{1}, \succ_{2}, \succ_{3}\right\}$ be a profile of three rankings with ties with $\succsim_{1}: a \succ b \sim c \succ d, \succsim_{2}: d \succ a \sim c \succ b$ and $\succsim_{3}: a \sim b \sim d \succ c$.

As the equivalence classes of both $a$ and $d$ have size one in $\succsim_{1}$ and the relative position of the first equivalence class w.r.t. the second one is three, it holds that $n_{(1,1,3)}(a, d)=1$. As the equivalence class of a has size two and the one of $d$ has size one in $\succsim_{2}$ and the relative position of the first equivalence class w.r.t. the second one is (minus) one, it holds that 


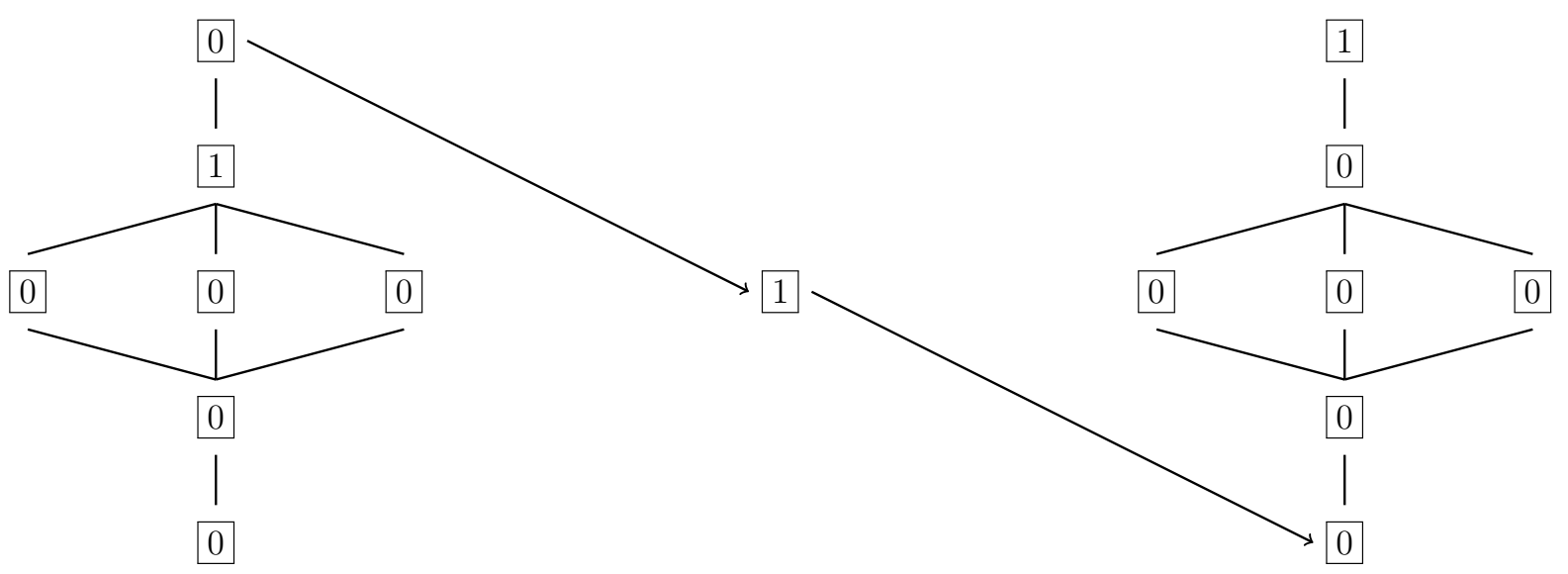

Figure 8.4: Frequency distribution of the couple $(a, d)$.

$n_{(2,1,-1)}(a, d)=1$. Finally, as the equivalence classes of $a$ and $d$ coincide in $\succsim_{3}$, it holds that $n_{(0,0,0)}(a, d)=1$. Of course, $n_{\left(s_{1}, s_{2}, p\right)}(a, d)=0$ for any other $\left(s_{1}, s_{2}, p\right) \in \mathcal{B}_{k}$. Thus, the frequency distribution ${ }^{3}$ of relative positions of the couple $(a, d)$ is shown in Figure 8.4 .

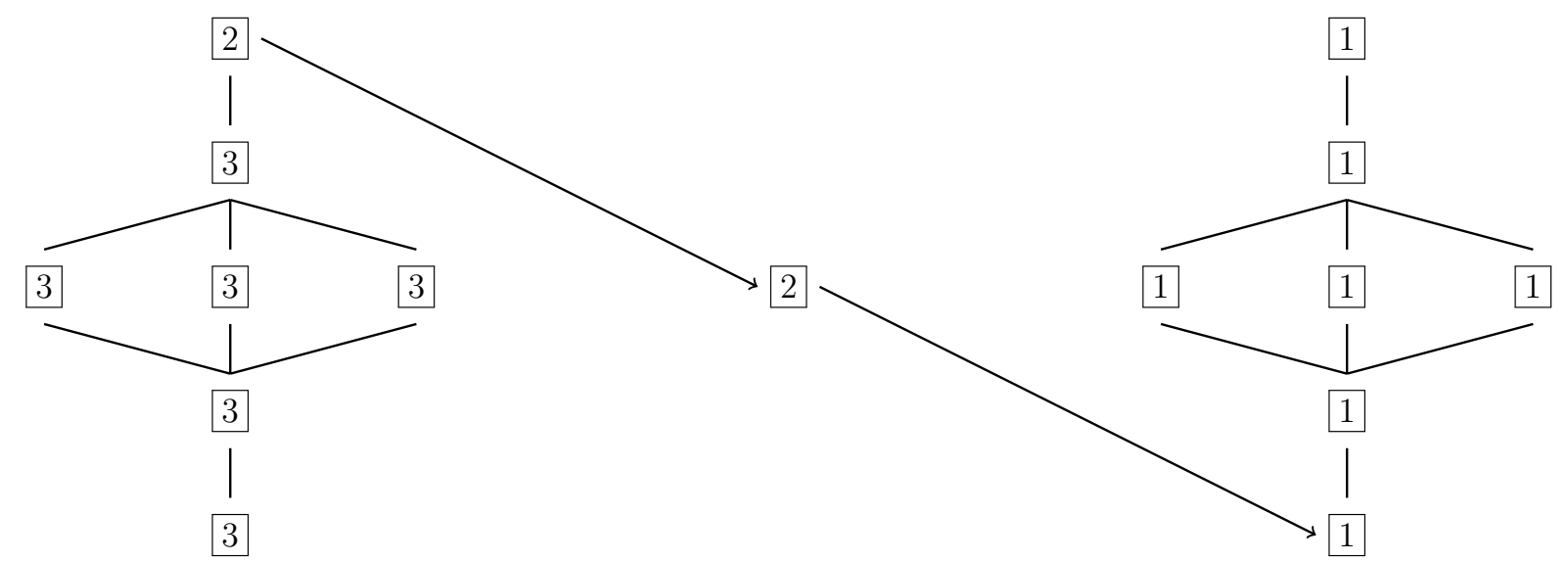

Figure 8.5: Top-down cumulative frequency distribution of the couple $(a, d)$.

The top-down and bottom-up cumulative frequency distributions of the couple $(a, d)$ are shown in Figures 8.5 and 8.6 , respectively.

\footnotetext{
${ }^{3}$ Due to the lack of available space, in Figures 8.4 8.6. the frequency distribution is shown over the Hasse diagrams of Figure 8.2 and not over the Hasse diagram of Figure 8.3 . Two arrows have been added for indicating that $(1,1,-1) \leq_{\mathcal{B}_{k}}(0,0,0)$ and that $(0,0,0) \leq_{\mathcal{B}_{k}}(1,1,1)$.
} 


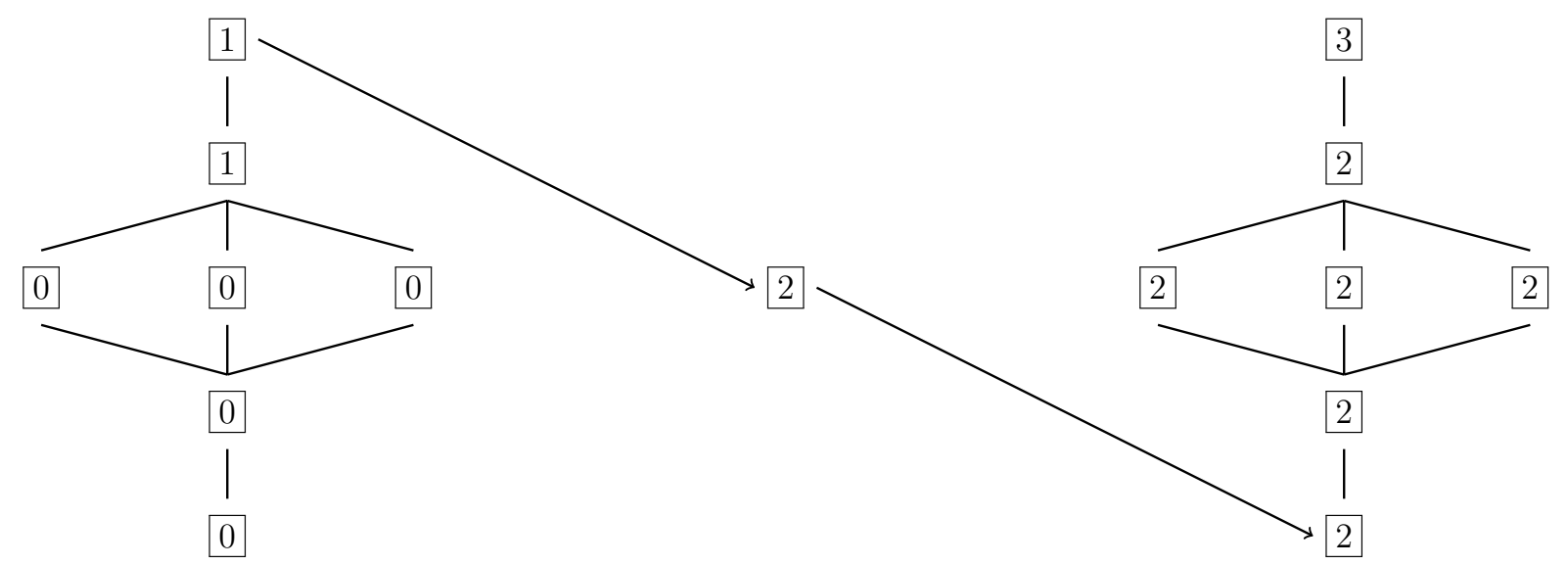

Figure 8.6: Bottom-up cumulative frequency distribution of the couple $(a, d)$.

Finally, the frequency distributions of all couples of candidates are given in Tables 8.1 and 8.2.

\begin{tabular}{|c|ccccccc|}
\hline$\left(s_{1}, s_{2}, p\right)$ & $(1,1,-3)$ & $(2,1,-2)$ & $(2,2,-1)$ & $(1,1,-2)$ & $(3,1,-1)$ & $(2,1,-1)$ & $(1,1,-1)$ \\
\hline$n_{\left(s_{1}, s_{2}, p\right)}(a, b)$ & 0 & 0 & 0 & 0 & 0 & 0 & 0 \\
\hline$n_{\left(s_{1}, s_{2}, p\right)}(a, c)$ & 0 & 0 & 0 & 0 & 0 & 0 & 0 \\
\hline$n_{\left(s_{1}, s_{2}, p\right)}(a, d)$ & 0 & 0 & 0 & 0 & 0 & 1 & 0 \\
\hline$n_{\left(s_{1}, s_{2}, p\right)}(b, a)$ & 0 & 0 & 0 & 0 & 0 & 2 & 0 \\
\hline$n_{\left(s_{1}, s_{2}, p\right)}(b, c)$ & 0 & 0 & 0 & 0 & 0 & 1 & 0 \\
\hline$n_{\left(s_{1}, s_{2}, p\right)}(b, d)$ & 1 & 0 & 0 & 0 & 0 & 0 & 0 \\
\hline$n_{\left(s_{1}, s_{2}, p\right)}(c, a)$ & 0 & 0 & 0 & 0 & 1 & 1 & 0 \\
\hline$n_{\left(s_{1}, s_{2}, p\right)}(c, b)$ & 0 & 0 & 0 & 0 & 1 & 0 & 0 \\
\hline$n_{\left(s_{1}, s_{2}, p\right)}(c, d)$ & 0 & 0 & 0 & 0 & 1 & 1 & 0 \\
\hline$n_{\left(s_{1}, s_{2}, p\right)}(d, a)$ & 1 & 0 & 0 & 0 & 0 & 0 & 0 \\
\hline$n_{\left(s_{1}, s_{2}, p\right)}(d, b)$ & 0 & 0 & 0 & 0 & 0 & 1 & 0 \\
\hline$n_{\left(s_{1}, s_{2}, p\right)}(d, c)$ & 0 & 0 & 0 & 0 & 0 & 1 & 0 \\
\hline
\end{tabular}

Table 8.1: Frequency distribution of all couples in $\mathscr{C}_{\neq}^{2}$ (first part).

As can be seen in Example 8.15, any profile of rankings with ties $\mathscr{R}^{*}$ defines a function of the form $W^{*}: \mathscr{C}_{\neq}^{2} \rightarrow\{0,1, \ldots, r\}^{\# \mathcal{B}_{k}}$. This function will be henceforth called the weak-votex (plural weak-votices) induced by the profile of rankings with ties $\mathscr{R}^{*}$. 


\begin{tabular}{|c|cccccccc|}
\hline$\left(s_{1}, s_{2}, p\right)$ & $(0,0,0)$ & $(1,1,1)$ & $(2,1,1)$ & $(2,2,1)$ & $(1,1,2)$ & $(3,1,1)$ & $(2,1,2)$ & $(1,1,3)$ \\
\hline$n_{\left(s_{1}, s_{2}, p\right)}(a, b)$ & 1 & 0 & 2 & 0 & 0 & 0 & 0 & 0 \\
\hline$n_{\left(s_{1}, s_{2}, p\right)}(a, c)$ & 1 & 0 & 1 & 0 & 0 & 1 & 0 & 0 \\
\hline$n_{\left(s_{1}, s_{2}, p\right)}(a, d)$ & 1 & 0 & 0 & 0 & 0 & 0 & 0 & 1 \\
\hline$n_{\left(s_{1}, s_{2}, p\right)}(b, a)$ & 1 & 0 & 0 & 0 & 0 & 0 & 0 & 0 \\
\hline$n_{\left(s_{1}, s_{2}, p\right)}(b, c)$ & 1 & 0 & 0 & 0 & 0 & 1 & 0 & 0 \\
\hline$n_{\left(s_{1}, s_{2}, p\right)}(b, d)$ & 1 & 0 & 1 & 0 & 0 & 0 & 0 & 0 \\
\hline$n_{\left(s_{1}, s_{2}, p\right)}(c, a)$ & 1 & 0 & 0 & 0 & 0 & 0 & 0 & 0 \\
\hline$n_{\left(s_{1}, s_{2}, p\right)}(c, b)$ & 1 & 0 & 1 & 0 & 0 & 0 & 0 & 0 \\
\hline$n_{\left(s_{1}, s_{2}, p\right)}(c, d)$ & 0 & 0 & 1 & 0 & 0 & 0 & 0 & 0 \\
\hline$n_{\left(s_{1}, s_{2}, p\right)}(d, a)$ & 1 & 0 & 1 & 0 & 0 & 0 & 0 & 0 \\
\hline$n_{\left(s_{1}, s_{2}, p\right)}(d, b)$ & 1 & 0 & 0 & 0 & 0 & 0 & 0 & 1 \\
\hline$n_{\left(s_{1}, s_{2}, p\right)}(d, c)$ & 0 & 0 & 1 & 0 & 0 & 1 & 0 & 0 \\
\hline
\end{tabular}

Table 8.2: Frequency distribution of all couples in $\mathscr{C}_{\neq}^{2}$ (second part).

Definition 8.16 Let $\mathscr{C}$ be a set of $k$ candidates and $r$ be the number of voters. A function $W^{*}: \mathscr{C}_{\neq}^{2} \rightarrow\{0,1, \ldots, r\}^{\# \mathcal{B}_{k}}$ is called a weak-votex (plural weak-votices) on $\mathscr{C}$ if there exists a profile $\mathscr{R}^{*}$ of $r$ rankings with ties on $\mathscr{C}$ such that, for any $\left(a_{i_{1}}, a_{i_{2}}\right) \in \mathscr{C}_{\neq}^{2}$ and any $\left(s_{1}, s_{2}, p\right) \in \mathcal{B}_{k}$, it holds that

$$
W^{*}\left(a_{i_{1}}, a_{i_{2}}\right)=\left(n_{\left(s_{1}, s_{2}, p\right)}\left(a_{i_{1}}, a_{i_{2}}\right)\right)_{\left(s_{1}, s_{2}, p\right) \in \mathcal{B}_{k}} .
$$

The set of all weak-votices on $\mathscr{C}$ induced by any profile of $r$ rankings with ties on $\mathscr{C}$ is denoted by $\mathcal{W}_{r}^{*}(\mathscr{C})$.

Similarly to the dominance relation defined on votices, we define a dominance relation on weak-votices.

The strict partial order relation $\sqsupset$ between couples of candidates associated to a given ranking $\succ$ on $\mathscr{C}$ is used to define monotonicity of the weak-votex w.r.t. this ranking.

Definition 8.17 Let $\mathscr{C}$ be a set of $k$ candidates and $r$ be the number of voters. A weakvotex $W^{*} \in \mathcal{W}_{r}^{*}(\mathscr{C})$ is said to be monotone w.r.t. a ranking $\succ$ on $\mathscr{C}$ (with corresponding $\sqsupset$ ) if, for any $\left(a_{i_{1}}, a_{j_{1}}\right),\left(a_{i_{2}}, a_{j_{2}}\right) \in \mathscr{C}_{\neq}^{2}$ such that $\left(a_{i_{1}}, a_{j_{1}}\right) \sqsupset\left(a_{i_{2}}, a_{j_{2}}\right)$ and any $\left(s_{1}, s_{2}, p\right) \in \mathcal{B}_{k}$, it holds that the following two conditions are satisfied: 
(i) Dominance w.r.t. the top-down cumulative frequency distribution:

$$
\bar{n}_{\left(s_{1}, s_{2}, p\right)}\left(a_{i_{1}}, a_{j_{1}}\right) \geq \bar{n}_{\left(s_{1}, s_{2}, p\right)}\left(a_{i_{2}}, a_{j_{2}}\right) .
$$

(ii) Dominance w.r.t. the bottom-up cumulative frequency distribution:

$$
\underline{n}_{\left(s_{1}, s_{2}, p\right)}\left(a_{i_{1}}, a_{j_{1}}\right) \leq \underline{n}_{\left(s_{1}, s_{2}, p\right)}\left(a_{i_{2}}, a_{j_{2}}\right),
$$

Remark 8.18 In case a total order relation on $\mathcal{B}_{k}$ would be considered, dominance w.r.t. the top-down cumulative frequency distribution and dominance w.r.t. the bottom-up cumulative frequency distribution would coincide. However, as $\leq_{\mathcal{B}_{k}}$ is a partial order relation, dominance w.r.t. the top-down cumulative frequency distribution and dominance w.r.t. the bottom-up cumulative frequency distribution might not coincide.

Again, the weak-votex induced by most profiles of rankings with ties is not monotone w.r.t. any ranking. The set of all profiles of rankings with ties with a monotone weak-votex can also be understood as a consensus state for the rationalisation of ranking rules.

\subsection{The profile of rankings with ties}

In case voters express rankings with ties instead of rankings, monotonicity of the profile of rankings with ties does not follow immediately from the definition of monotonicity of the profile of rankings. The relation $\geqq$ needs to be extended to $\mathcal{L}^{*}(\mathscr{C})$. In order to do so, we note that, for a ranking with ties $\succ_{i}$, the conditions $a_{i_{1}} \succ_{i} a_{i_{2}}$ and $a_{i_{2}} \nsucc_{i} a_{i_{1}}$ are no longer equivalent. Therefore, the former unique condition for rankings (without ties) now needs to be divided in two parts.

Proposition 8.19 Let $\mathscr{C}$ be a set of $k$ candidates and $\succ$ be a ranking on $\mathscr{C}$. The relation $\unrhd$ defined as

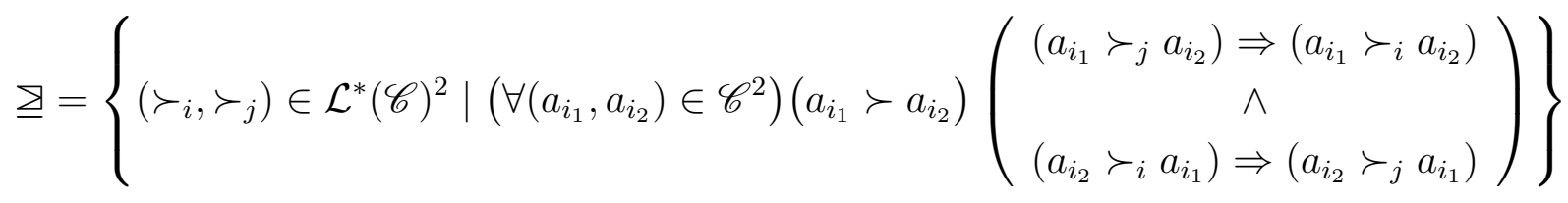

is an order relation on $\mathcal{L}^{*}(\mathscr{C})$. 
Proof: We prove that $\geqq$ satisfies reflexivity, antisymmetry and transitivity.

Reflexivity: holds trivially.

Antisymmetry: for any $\succ_{i}, \succ_{j} \in \mathcal{L}^{*}(\mathscr{C})$, if $\succ_{i} \geqq \succ_{j}$ and $\succ_{j} \succ_{i}$, then it holds that:

$$
\left(\forall\left(a_{i_{1}}, a_{i_{2}}\right) \in \mathscr{C}^{2}\right)\left(a_{i_{1}} \succ a_{i_{2}}\right)\left(\begin{array}{c}
\left(a_{i_{1}} \succ_{j} a_{i_{2}}\right) \Rightarrow\left(a_{i_{1}} \succ_{i} a_{i_{2}}\right) \\
\left(a_{i_{2}} \succ_{i} a_{i_{1}}\right) \Rightarrow\left(a_{i_{2}} \succ_{j} a_{i_{1}}\right)
\end{array}\right),
$$

and

$$
\left(\forall\left(a_{i_{1}}, a_{i_{2}}\right) \in \mathscr{C}^{2}\right)\left(a_{i_{1}} \succ a_{i_{2}}\right)\left(\begin{array}{c}
\left(a_{i_{1}} \succ_{i} a_{i_{2}}\right) \Rightarrow\left(a_{i_{1}} \succ_{j} a_{i_{2}}\right) \\
\left(a_{i_{2}} \succ_{j} a_{i_{1}}\right) \Rightarrow\left(a_{i_{2}} \succ_{i} a_{i_{1}}\right)
\end{array}\right) .
$$

Therefore, for any $a_{i_{1}}, a_{i_{2}} \in \mathscr{C}$ such that $a_{i_{1}} \succ a_{i_{2}}$, it holds that

$$
\left(\left(a_{i_{1}} \succ_{i} a_{i_{2}}\right) \Leftrightarrow\left(a_{i_{1}} \succ_{j} a_{i_{2}}\right)\right) \wedge\left(\left(a_{i_{2}} \succ_{i} a_{i_{1}}\right) \Leftrightarrow\left(a_{i_{2}} \succ_{j} a_{i_{1}}\right)\right) .
$$

As $\succeq$ is complete, if $a_{i_{1}} \nsucc a_{i_{2}}$, then it holds that $a_{i_{1}}=a_{i_{2}}$ or $a_{i_{2}} \succ a_{i_{1}}$. The case $a_{i_{2}} \succ a_{i_{1}}$ is equivalent to the case $a_{i_{2}} \succ a_{i_{1}}$ as both $a_{i_{1}}$ and $a_{i_{2}}$ play a symmetric role in Eq. (8.1). The case $a_{i_{1}}=a_{i_{2}}$ is immediate due to the fact that both $\succ_{i}$ and $\succ_{j}$ are irreflexive by definition of ranking. Hence, for any $a_{i_{1}}, a_{i_{2}} \in \mathscr{C}$, it holds that

$$
\left(a_{i_{1}} \succ_{i} a_{i_{2}}\right) \Leftrightarrow\left(a_{i_{1}} \succ_{j} a_{i_{2}}\right)
$$

i.e., it holds that $\succ_{i}=\succ_{j}$.

Transitivity: for any $\succ_{i}, \succ_{j}, \succ_{\ell} \in \mathcal{L}^{*}(\mathscr{C})$, if $\succ_{i} \geqq \succ_{j}$ and $\succ_{j} \geqq \succ_{\ell}$, then it holds that

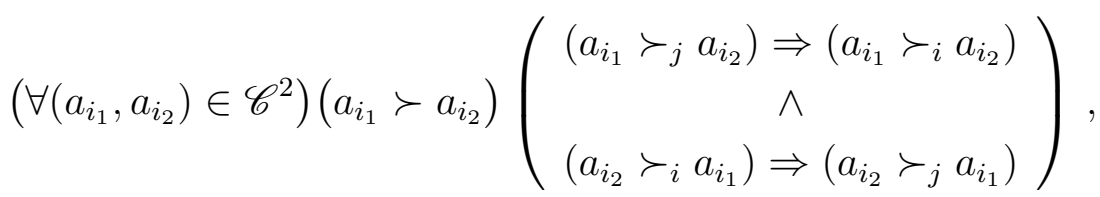

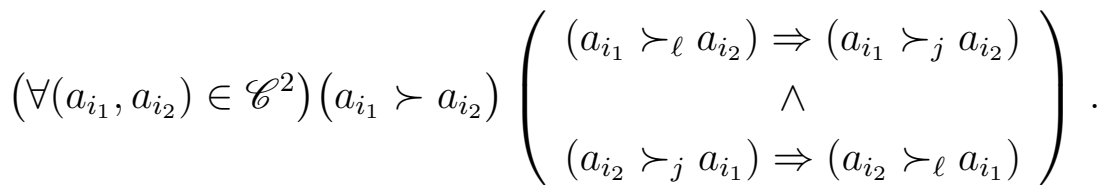

Hence, for any $a_{i_{1}}, a_{i_{2}} \in \mathscr{C}$ such that $a_{i_{1}} \succ a_{i_{2}}$, it holds that

$$
\begin{aligned}
& \left(a_{i_{1}} \succ_{\ell} a_{i_{2}}\right) \Rightarrow\left(a_{i_{1}} \succ_{j} a_{i_{2}}\right) \Rightarrow\left(a_{i_{1}} \succ_{i} a_{i_{2}}\right) \\
& \left(a_{i_{2}} \succ_{i} a_{i_{1}}\right) \Rightarrow\left(a_{i_{2}} \succ_{j} a_{i_{1}}\right) \Rightarrow\left(a_{i_{2}} \succ_{\ell} a_{i_{1}}\right)
\end{aligned},
$$

i.e., it holds that $\succ_{i} \geqq \succ_{\ell}$.

Thus, $\geqq$ is an order relation on $\mathcal{L}^{*}(\mathscr{C})$. 
Remark 8.20 For any ranking $\succ$ on $\mathscr{C}$, the restriction of the relation $\geqq$ on $\mathcal{L}^{*}(\mathscr{C})$ to $\mathcal{L}(\mathscr{C})$ coincides with the relation $\geqq$ on $\mathcal{L}(\mathscr{C})$. Therefore, the use of the same notation is justified.

Figure 8.7 displays the Hasse diagram of the order relation $\geqq$ on $\mathcal{L}^{*}(\mathscr{C})$ for the set of candidates $\mathscr{C}=\{a, b, c\}$ and the ranking $a \succ b \succ c$. Note that this Hasse diagram coincides with that used by Kemeny [80] for defining a distance function on $\mathcal{L}^{*}(\mathscr{C})$.

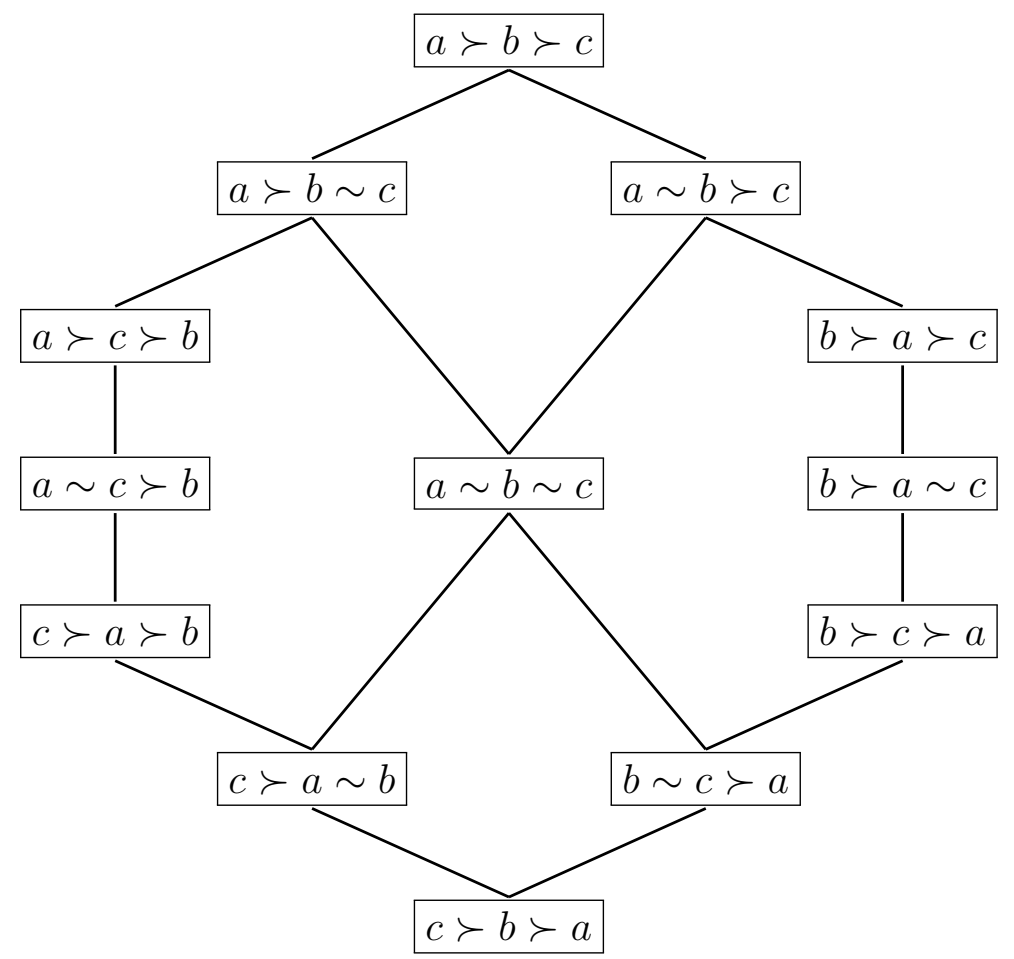

Figure 8.7: Hasse diagram of the order relation $\geqq$ on $\mathcal{L}^{*}(\mathscr{C})$ for the ranking $a \succ b \succ c$.

Unfortunately, this diagram may be inappropriate for modelling real-world problems. For any $\left(a_{i_{1}}, a_{i_{2}}\right) \in \mathscr{C}_{\neq}^{2}$, voters might show inclination towards either $a_{i_{1}} \succ a_{i_{2}}$ and $a_{i_{2}} \succ a_{i_{1}}$ or $a_{i_{1}} \sim a_{i_{2}}$. In order to model this inclination, we introduce here the notions of signature and ordered signature.

Definition 8.21 Let $\mathscr{C}$ be a set of $k$ candidates. The signature $\mathscr{S}$ of a ranking with ties $\succ$ on $\mathscr{C}$, denoted by $\mathscr{S}(\succ)$, is a vector where the $i$-th component equals the size of the $i$-th equivalence class in $\succ$. The set of all the signatures on $\mathscr{C}$ is denoted by $\mathbb{S}(\mathscr{C})$. 
Definition 8.22 Let $\mathscr{C}$ be a set of $k$ candidates. The ordered signature $\mathscr{O}$ of a ranking with ties $\succ$ on $\mathscr{C}$, denoted by $\mathscr{O}(\succ)$, is a vector where the $i$-th component equals the size of the $i$-th largest equivalence class in $\succ$. The set of all the ordered signatures on $\mathscr{C}$ is denoted by $\mathbb{O}(\mathscr{C})$.

Remark 8.23 Each signature $\mathscr{S} \in \mathbb{S}(\mathscr{C})$ leads to a unique ordered signature $\mathscr{O} \in \mathbb{O}(\mathscr{C})$. Note that the lengths of $\mathscr{S}$ and $\mathscr{O}$ coincide. The fact that the signature $\mathscr{S}$ leads to the ordered signature $\mathscr{O}$ is denoted by $\mathscr{S} \rightsquigarrow \mathscr{O}$.

The notions of signature and ordered signature are illustrated in the following example.

Example 8.24 Consider the set of candidates $\mathscr{C}=\{a, b, c\}$. The signature of the ranking with ties $a \succ b \succ c$ is the vector $(1,1,1)$ and its ordered signature is $(1,1,1)$. Therefore, it holds that

$$
\mathscr{S}(a \succ b \succ c)=\mathscr{O}(a \succ b \succ c)=(1,1,1) .
$$

Analogously, the signature of the ranking with ties $a \succ b \sim c$ is the vector $(1,2)$ and its ordered signature is $(2,1)$. Therefore, it holds that

$$
\mathscr{S}(a \succ b \sim c)=(1,2) \quad \text { and } \quad \mathscr{O}(a \succ b \sim c)=(2,1) .
$$

In general, the set of all signatures on $\mathscr{C}$ is given by:

$$
\mathbb{S}(\mathscr{C})=\{(1,1,1),(1,2),(2,1),(3)\}
$$

Analogously, the set of all ordered signatures on $\mathscr{C}$ is given by:

$$
\mathbb{O}(\mathscr{C})=\{(1,1,1),(2,1),(3)\}
$$

We prove the following lemma, which will be useful in the upcoming proofs.

Lemma 8.25 Let $E$ be an equivalence relation and $R$ be an order relation on a set $X$. The relation $E \cap R$ is an order relation on $X$.

Proof: Reflexivity follows immediately from the reflexivity of both $E$ and $R$. 
Antisymmetry. Suppose that $E \cap R$ is not antisymmetric. Therefore, there exist two different elements $x, y \in X$ such that $x(E \cap R) y$ and $y(E \cap R) x$. It follows that $x R y$ and $y R x$, a contradiction with the fact that $R$ is antisymmetric.

Transitivity. Suppose that $E \cap R$ is not transitive. Therefore, there exist three elements $x, y, z \in X$ such that $x(E \cap R) y$ and $y(E \cap R) z$ but such that $x(E \cap R)^{c} z$. On the one hand, it holds that $x E y$ and $y E z$ and, therefore, that $x E z$, due to the transitivity of an equivalence relation. On the other hand, it holds that $x R y$ and $y R z$ and, therefore, that $x R z$, due to the transitivity of an order relation. We conclude that $x(E \cap R) z$, a contradiction.

These (ordered) signatures can be used for defining two natural order relations $\mathcal{L}^{*}(\mathscr{C})$. In the first order relation, only couples of rankings with ties belonging to $\geqq$ and that have the same signature are considered comparable elements, while, in the second order relation, only couples of rankings with ties belonging to $\geqq$ and that have the same ordered signature are considered comparable elements.

Proposition 8.26 Let $\mathscr{C}$ be a set of $k$ candidates and $\succ$ be a ranking on $\mathscr{C}$. The relation $\unrhd^{\mathbb{S}}$ defined as

$$
\varliminf^{\mathbb{S}}=\geqq \cap\left\{\left(\succ_{i}, \succ_{j}\right) \in \mathcal{L}^{*}(\mathscr{C})^{2} \mid \mathscr{S}\left(\succ_{i}\right)=\mathscr{S}\left(\succ_{j}\right)\right\},
$$

is an order relation on $\mathcal{L}^{*}(\mathscr{C})$.

\section{Proof:}

The proof follows immediately from Lemma 8.25 .

Figure 8.8 displays the Hasse diagram of the order relation $\unlhd^{\mathbb{S}}$ on $\mathcal{L}^{*}(\mathscr{C})$ for the set of candidates $\mathscr{C}=\{a, b, c\}$ and the ranking $a \succ b \succ c$. Note that we use dashed lines for separating sets of incomparable rankings with ties.

Proposition 8.27 Let $\mathscr{C}$ be a set of $k$ candidates and $\succ$ be a ranking on $\mathscr{C}$. The relation $\geqq^{\mathbb{O}}$ defined as

$$
\varliminf^{\mathbb{O}}=\geqq \cap\left\{\left(\succ_{i}, \succ_{j}\right) \in \mathcal{L}^{*}(\mathscr{C})^{2} \mid \mathscr{O}\left(\succ_{i}\right)=\mathscr{O}\left(\succ_{j}\right)\right\},
$$

is an order relation on $\mathcal{L}^{*}(\mathscr{C})$. 

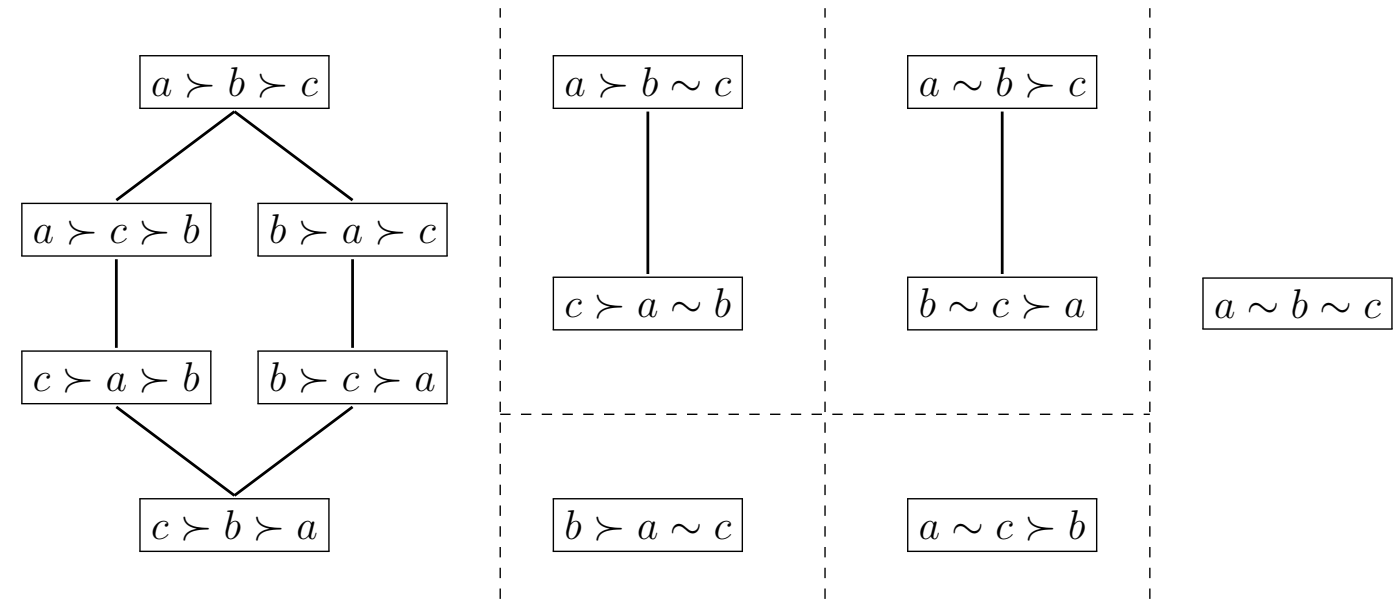

Figure 8.8: Hasse diagram of the order relation $\unrhd^{\mathbb{S}}$ on $\mathcal{L}^{*}(\mathscr{C})$ for the ranking $a \succ b \succ c$.

\section{Proof:}

The proof follows immediately from Lemma 8.25.

Figure 8.9 displays the Hasse diagram of the order relation $\unrhd^{\mathbb{O}}$ on $\mathcal{L}^{*}(\mathscr{C})$ for the set of candidates $\mathscr{C}=\{a, b, c\}$ and the ranking $a \succ b \succ c$. Note that, as in Figure 8.8, we use dashed lines for separating sets of incomparable rankings with ties.
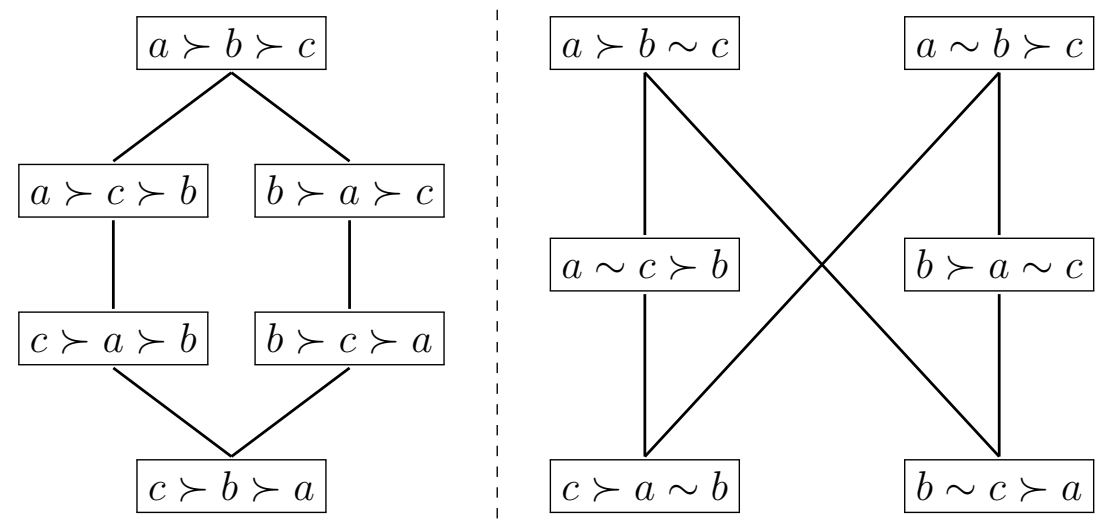

$a \sim b \sim c$

Figure 8.9: Hasse diagram of the order relation $\unrhd^{\mathbb{O}}$ on $\mathcal{L}^{*}(\mathscr{C})$ for the ranking $a \succ b \succ c$.

Of course, there is an immediate relation between the three relations $\unrhd, \underline{\mathbb{S}}^{\mathbb{S}}$ and $\unrhd^{\mathbb{Q}}$. 
Proposition 8.28 Let $\mathscr{C}$ be a set of $k$ candidates and $\succ$ be a ranking on $\mathscr{C}$. The following statement holds:

$$
\unrhd^{\mathbb{S}} \subseteq \unrhd^{\mathbb{O}} \subseteq \unrhd
$$

Proof: The fact that $\unrhd^{\mathbb{O}} \subseteq \geqq$ is straightforward by definition of $\unrhd^{\mathbb{O}}$. The fact that $\unrhd^{\mathbb{S}} \subseteq \unrhd^{\mathbb{O}}$ follows from the fact that, if two rankings with ties have the same signature, then they have the same ordered signature.

Consider the relation $\gg^{\mathbb{S}}$ on $\mathbb{S}(\mathscr{C})$, where ' $\mathscr{S}_{1} \gg^{\mathbb{S}} \mathscr{S}_{2}$ ' represents that the length of the signature $\mathscr{S}_{1}$ equals the length of the signature $\mathscr{S}_{2}$ plus one and, at the same time, the signature $\mathscr{S}_{2}$ is obtained by merging two consecutive components of $\mathscr{S}_{1}$. For instance, the signature $(1,2)$ is obtained by merging the last two components of the signature $(1,1,1)$, therefore $(1,1,1) \gg^{\mathbb{S}}(1,2)$. This relation is used for defining a natural order relation on $\mathbb{S}(\mathscr{C})$.

Proposition 8.29 Let $\mathscr{C}$ be a set of $k$ candidates. The relation $\geq^{\mathbb{S}}$, defined as the preorder closur $\rfloor^{4}$ of $\gg^{\mathbb{S}}$, is an order relation on $\mathbb{S}(\mathscr{C})$.

Proof: By definition of pre-order closure, $\geq^{\mathbb{S}}$ obviously is reflexive and transitive. We need to prove that $\geq{ }^{\mathbb{S}}$ satisfies antisymmetry.

By definition, all couples in $\gg^{\mathbb{S}}$ satisfy that the length of the first signature in the couple is greater than the length of the second signature in the couple. Note that this property is preserved by the smallest transitive relation containing $\gg^{\mathbb{S}}$. Therefore, when considering the pre-order closure of $\gg^{\mathbb{S}}$, it only holds that $\mathscr{S} \geq^{\mathbb{S}} \mathscr{S}$ and $\mathscr{S} \geq^{\mathbb{S}} \mathscr{S}$ in case $\mathscr{S}=\mathscr{S}$. We conclude that $\geq^{\mathbb{S}}$ satisfies the antisymmetry property.

Figure 8.10 displays the Hasse diagram of the order relation $\geq^{\mathbb{S}}$ on $\mathbb{S}(\mathscr{C})$ for a set $\mathscr{C}$ of three and of four candidates.

Analogously, a natural order relation can be defined for ordered signatures. Consider the relation $\gg^{\mathbb{O}}$ on $\mathbb{O}(\mathscr{C})$, where ' $\mathscr{O}_{1} \gg^{\mathbb{O}} \mathscr{O}_{2}$ ' represents that there exist two signatures $\mathscr{S}_{1}, \mathscr{S}_{2} \in \mathbb{S}(\mathscr{C})$ such that $\mathscr{S}_{1} \rightsquigarrow \mathscr{O}_{1}, \mathscr{S}_{2} \rightsquigarrow \mathscr{O}_{2}$ and $\mathscr{S}_{1} \gg^{\mathbb{S}} \mathscr{S}_{2}$. For instance, for the ordered signature $(2,1)$ and $(1,1,1)$, it holds that $(1,2) \rightsquigarrow(2,1),(1,1,1) \rightsquigarrow(1,1,1)$ and

\footnotetext{
${ }^{4}$ The pre-order closure of a relation $R$ is the smallest reflexive and transitive relation containing $R$.
} 

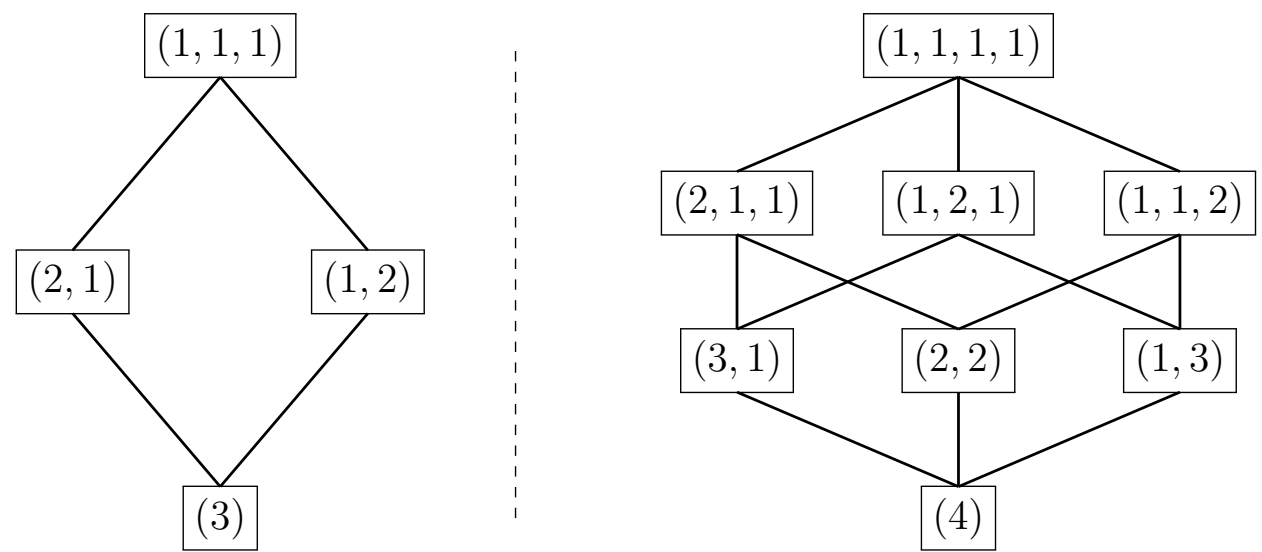

Figure 8.10: Hasse diagram of the order relation $\geq^{\mathbb{S}}$ on $\mathbb{S}(\mathscr{C})$ for a set $\mathscr{C}$ of three (left) and of four (right) candidates.

$(1,1,1) \gg^{\mathbb{S}}(1,2)$. Therefore, it holds that $(1,1,1) \gg^{\mathbb{O}}(2,1)$. This relation is used for defining a natural order relation on $\mathbb{O}(\mathscr{C})$.

Proposition 8.30 Let $\mathscr{C}$ be a set of $k$ candidates. The relation $\geq^{\mathbb{O}}$, defined as the preorder closure of $\gg^{\mathbb{O}}$, is an order relation on $\mathbb{O}(\mathscr{C})$.

Proof: By definition of pre-order closure, $\geq^{\mathbb{1}}$ obviously is reflexive and transitive. We need to prove that $\geq{ }^{\mathbb{0}}$ satisfies antisymmetry.

By definition, all couples in $\gg^{\mathbb{O}}$ satisfy that the length of the first ordered signature in the couple is greater than the length of the second ordered signature in the couple. Therefore, as $\geq^{\mathbb{S}}$ is only defined for couples where the length of the first signature in the couple is greater than the length of the second signature in the couple (or for couples where both signatures coincide), there exists no couple $\left(\mathscr{O}_{1}, \mathscr{O}_{2}\right) \in \mathbb{O}(\mathscr{C})^{2}$ such that $\mathscr{O}_{1} \geq \mathbb{O} \mathscr{O}_{2}$ and $\mathscr{O}_{2} \geq \mathbb{D} \mathscr{O}_{1}$.

Figure 8.11 displays the Hasse diagram of the order relation $\geq^{\mathbb{O}}$ on $\mathbb{O}(\mathscr{C})$ for a set $\mathscr{C}$ of three and of four candidates.

The following result follows immediately. 

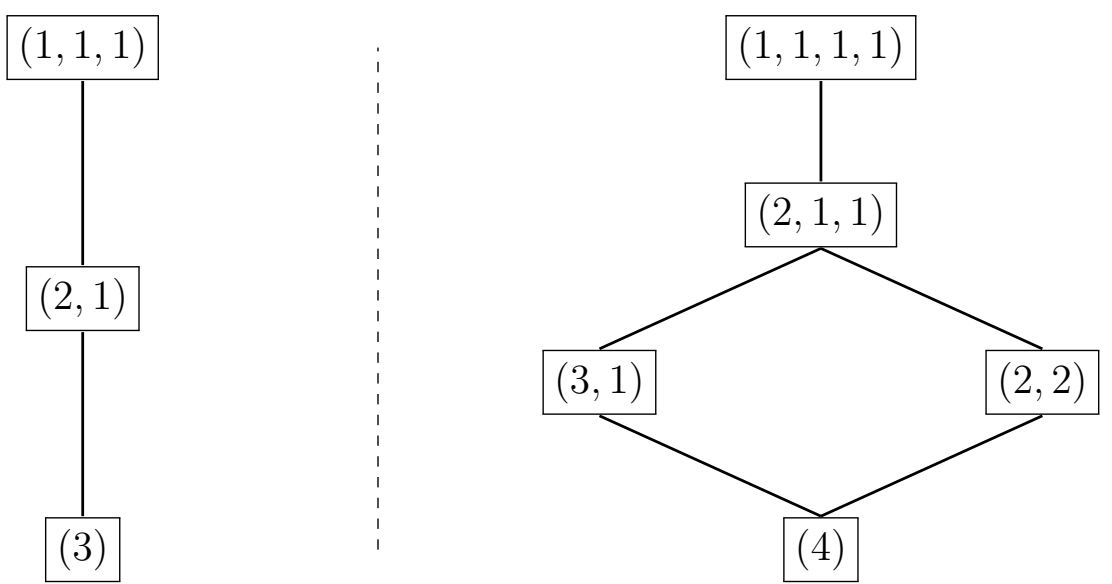

Figure 8.11: Hasse diagram of the order relation $\geq^{\mathbb{D}}$ on $\mathbb{O}(\mathscr{C})$ for a set $\mathscr{C}$ of three (left) and of four (right) candidates.

Corollary 8.31 Let $\mathscr{C}$ be a set of $k$ candidates. For any $\mathscr{S}_{1}, \mathscr{S}_{2} \in \mathbb{S}(\mathscr{C})$ and $\mathscr{O}_{1}, \mathscr{O}_{2} \in$ $\mathbb{O}(\mathscr{C})$ such that $\mathscr{S}_{1} \rightsquigarrow \mathscr{O}_{1}$ and $\mathscr{S}_{2} \rightsquigarrow \mathscr{O}_{2}$, it holds that

$$
\mathscr{S}_{1} \geq^{\mathbb{S}} \mathscr{S}_{2} \Rightarrow \mathscr{O}_{1} \geq^{\mathbb{D}} \mathscr{O}_{2}
$$

We prove the following lemma, which will be useful in the upcoming proofs.

Lemma 8.32 Let $R$ and $S$ be two order relations on a set $X$. The relation $R \cap S$ is an order relation on $X$.

Proof: Reflexivity follows immediately from the reflexivity of both $R$ and $S$.

Antisymmetry. Suppose that $R \cap S$ is not antisymmetric. Therefore, there exist two different elements $x, y \in X$ such that $x(R \cap S) y$ and $y(R \cap S) x$. It follows that $x R y$ and $y R x$, a contradiction with the fact that $R$ is antisymmetric.

Transitivity. Suppose that $R \cap S$ is not transitive. Therefore, there exist three elements $x, y, x \in X$ such that $x(R \cap S) y$ and $y(R \cap S) z$ but such that $x(R \cap S)^{c} z$. On the one hand, it holds that $x R y$ and $y R z$ and, therefore, that $x R z$, due to the transitivity of an order relation. On the other hand, it holds that $x S y$ and $y S z$ and, therefore, that $x S z$, due to the transitivity of an order relation. We conclude that $x(R \cap S) z$, a contradiction. 
These order relations on the set of (ordered) signatures can be used for defining four natural order relations $\mathcal{L}^{*}(\mathscr{C})$, where only couples of rankings with ties belonging to sqsupseteqbis and satisfying these additional requirements are considered comparable elements.

Proposition 8.33 Let $\mathscr{C}$ be a set of $k$ candidates and $\succ$ be a ranking on $\mathscr{C}$. The relations defined as

$$
\begin{aligned}
& \unrhd^{\mathbb{S}_{\downarrow}}=\geqq \cap\left\{\left(\succ_{i}, \succ_{j}\right) \in \mathcal{L}^{*}(\mathscr{C})^{2} \mid \mathscr{S}\left(\succ_{i}\right) \geq^{\mathbb{S}} \mathscr{S}\left(\succ_{j}\right)\right\}, \\
& \unrhd^{\mathbb{S}_{\uparrow}}=\geqq \cap\left\{\left(\succ_{i}, \succ_{j}\right) \in \mathcal{L}^{*}(\mathscr{C})^{2} \mid \mathscr{S}\left(\succ_{j}\right) \geq^{\mathbb{S}} \mathscr{S}\left(\succ_{i}\right)\right\}, \\
& \underline{\Xi}^{\mathbb{O}_{\downarrow}}=\geqq \cap\left\{\left(\succ_{i}, \succ_{j}\right) \in \mathcal{L}^{*}(\mathscr{C})^{2} \mid \mathscr{O}\left(\succ_{i}\right) \geq^{\mathbb{O}} \mathscr{O}\left(\succ_{j}\right)\right\}, \\
& \unrhd^{\mathbb{O}_{\uparrow}}=\geqq \cap\left\{\left(\succ_{i}, \succ_{j}\right) \in \mathcal{L}^{*}(\mathscr{C})^{2} \mid \mathscr{O}\left(\succ_{j}\right) \geq^{\mathbb{O}} \mathscr{O}\left(\succ_{i}\right)\right\},
\end{aligned}
$$

are four order relations on $\mathcal{L}^{*}(\mathscr{C})$.

\section{Proof:}

The proof follows immediately from Lemma 8.32 .

Figure 8.12 displays the Hasse diagram of the order relation $\unrhd^{\mathbb{O}_{\downarrow}}$ on $\mathcal{L}^{*}(\mathscr{C})$ for the set of candidates $\mathscr{C}=\{a, b, c\}$ and the ranking $a \succ b \succ c$.

Figure 8.13 displays the relation between the different order relations on $\mathcal{L}^{*}(\mathscr{C})$ introduced in this section.

From now on, we denote by $\mathbf{n}_{\mathscr{R}} *$ the vector of absolute frequencies of the profile of rankings with ties $\mathscr{R}^{*}$, where $\mathbf{n}_{\mathscr{R}^{*}}(i)$ is the absolute frequency of the $i$-th ranking with ties in $\mathcal{L}^{*}(\mathscr{C})$, i.e., the number of voters that expressed the $i$-th ranking with ties in $\mathcal{L}^{*}(\mathscr{C})$ in the profile of rankings with ties $\mathscr{R}^{*}$.

Under the assumption that there exists a true ranking $\succ$ on $\mathscr{C}$, it seems natural that the vector of absolute frequencies of the given profile of rankings with ties should be decreasing on the Hasse diagram of the order relation $\geqq$ (note that now seven different diagrams are considered). A profile of rankings with ties satisfying this property is said to be monotone w.r.t. the ranking $\succ$.

Definition 8.34 Let $\mathscr{C}$ be a set of $k$ candidates, $r$ be the number of voters and $\succ$ be a ranking on $\mathscr{C}$. 


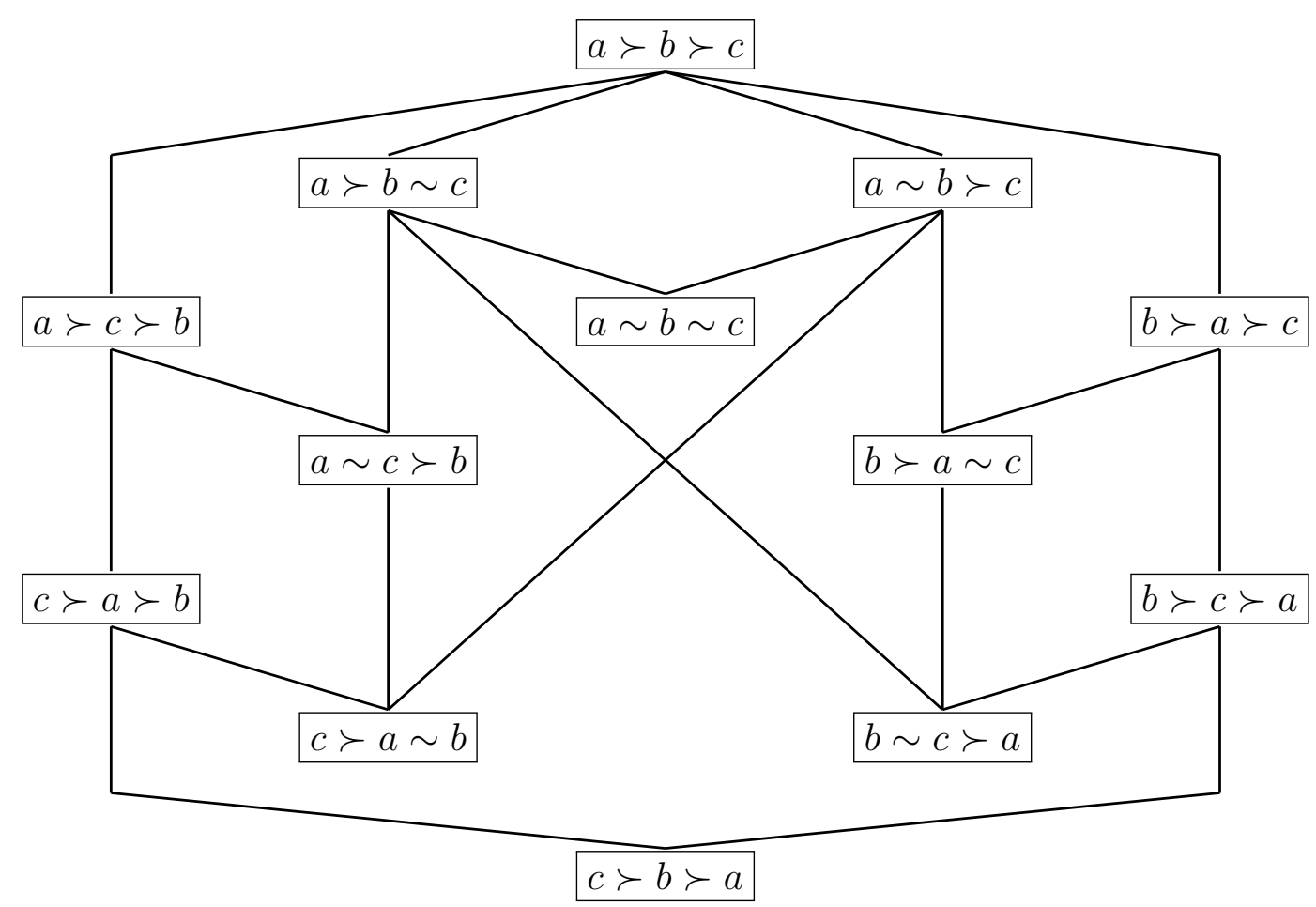

Figure 8.12: Hasse diagram of the order relation $\unrhd^{\mathbb{O}_{\downarrow}}$ on $\mathcal{L}^{*}(\mathscr{C})$ for the ranking $a \succ b \succ c$.

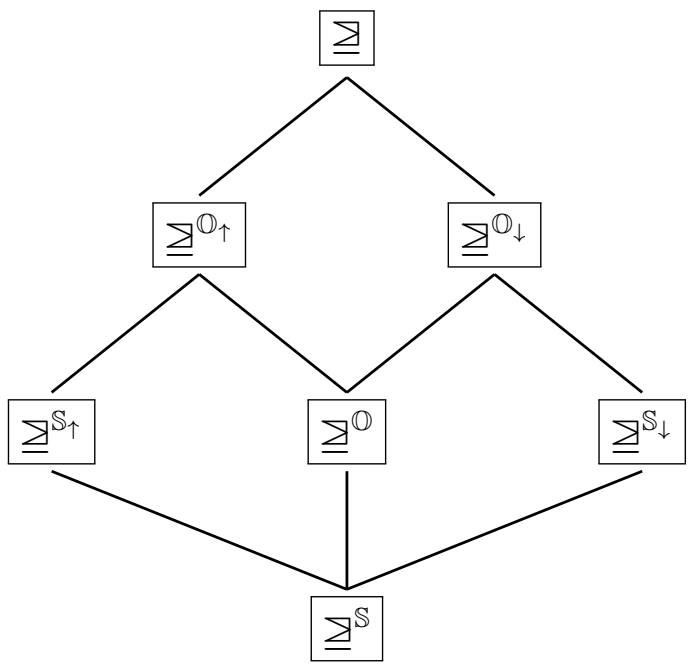

Figure 8.13: Relation between different order relations on $\mathcal{L}^{*}(\mathscr{C})$. 
(i) A profile $\mathscr{R}^{*}$ of rankings with ties on $\mathscr{C}$ is said to be monotone w.r.t. $\succ$ if, for any $\succ_{i}, \succ_{j} \in \mathcal{L}^{*}(\mathscr{C})$, it holds that

$$
\left(\succ_{i} \succ_{j}\right) \Rightarrow\left(\mathbf{n}_{\mathscr{R}^{*}}(i) \geq \mathbf{n}_{\mathscr{R}^{*}}(j)\right)
$$

(ii) A profile $\mathscr{R}^{*}$ of rankings with ties on $\mathscr{C}$ is said to be $\mathbb{O}_{\downarrow}$-monotone w.r.t. $\succ$ if, for any $\succ_{i}, \succ_{j} \in \mathcal{L}^{*}(\mathscr{C})$, it holds that

$$
\left(\succ_{i} \unrhd^{\mathbb{O}_{\downarrow}} \succ_{j}\right) \Rightarrow\left(\mathbf{n}_{\mathscr{R} *}(i) \geq \mathbf{n}_{\mathscr{R} *}(j)\right)
$$

(iii) A profile $\mathscr{R}^{*}$ of rankings with ties on $\mathscr{C}$ is said to be $\mathbb{O}_{\uparrow}$-monotone w.r.t. $\succ$ if, for any $\succ_{i}, \succ_{j} \in \mathcal{L}^{*}(\mathscr{C})$, it holds that

$$
\left(\succ_{i} \unrhd^{\mathbb{O}_{\uparrow}} \succ_{j}\right) \Rightarrow\left(\mathbf{n}_{\mathscr{R}^{*}}(i) \geq \mathbf{n}_{\mathscr{R}^{*}}(j)\right)
$$

(iv) A profile $\mathscr{R}^{*}$ of rankings with ties on $\mathscr{C}$ is said to be $\mathbb{O}$-monotone w.r.t. $\succ$ if, for any $\succ_{i}, \succ_{j} \in \mathcal{L}^{*}(\mathscr{C})$, it holds that

$$
\left(\succ_{i} \unrhd^{\mathbb{O}} \succ_{j}\right) \Rightarrow\left(\mathbf{n}_{\mathscr{R}^{*}}(i) \geq \mathbf{n}_{\mathscr{R}^{*}}(j)\right)
$$

(v) A profile $\mathscr{R}^{*}$ of rankings with ties on $\mathscr{C}$ is said to be $\mathbb{S}_{\downarrow}$-monotone w.r.t. $\succ$ if, for any $\succ_{i}, \succ_{j} \in \mathcal{L}^{*}(\mathscr{C})$, it holds that

$$
\left(\succ_{i} \unrhd^{\mathbb{S}_{\downarrow}} \succ_{j}\right) \Rightarrow\left(\mathbf{n}_{\mathscr{R}^{*}}(i) \geq \mathbf{n}_{\mathscr{R}^{*}}(j)\right)
$$

(vi) A profile $\mathscr{R}^{*}$ of rankings with ties on $\mathscr{C}$ is said to be $\mathbb{S}_{\uparrow}$-monotone w.r.t. $\succ$ if, for any $\succ_{i}, \succ_{j} \in \mathcal{L}^{*}(\mathscr{C})$, it holds that

$$
\left(\succ_{i} \underline{\Xi}^{\mathbb{S} \uparrow} \succ_{j}\right) \Rightarrow\left(\mathbf{n}_{\mathscr{R}^{*}}(i) \geq \mathbf{n}_{\mathscr{R}^{*}}(j)\right)
$$

(vii) A profile $\mathscr{R}^{*}$ of rankings with ties on $\mathscr{C}$ is said to be $\mathbb{S}$-monotone w.r.t. $\succ$ if, for any $\succ_{i}, \succ_{j} \in \mathcal{L}^{*}(\mathscr{C})$, it holds that

$$
\left(\succ_{i} \unrhd^{\mathbb{S}} \succ_{j}\right) \Rightarrow\left(\mathbf{n}_{\mathscr{R}^{*}}(i) \geq \mathbf{n}_{\mathscr{R}^{*}}(j)\right)
$$

In general, in case the considered order relation on $\mathcal{L}^{*}(\mathscr{C})$ is not specified, we talk about *-monotonicity w.r.t. a ranking on the set of candidates. 


\subsection{Search for monotonicity of representations of votes affected by ties}

When the chosen representation of votes is not monotone w.r.t. any ranking, the goal is to look for a new profile of rankings with ties with a monotone representation of votes w.r.t. at least one ranking. Obviously, among all the profiles of rankings with ties with a monotone representation of votes, we want to find the closest to the given one. This closeness can be measured by means of a monometric.

The notion of betweenness to be considered between profiles of rankings with ties is based on the Kemeny distance function [80] on rankings with ties? which is an extension of the Kendall distance function [82] on rankings.

Definition 8.35 Let $\mathscr{C}$ be a set of $k$ candidates. For any two weak order relations $\succsim_{1}$ and $\succsim_{2}$ on $\mathscr{C}$, the Kemeny distance between $\succsim_{1}$ and $\succsim_{2}$ is defined as

$$
\begin{aligned}
K\left(\succsim_{1}, \succsim_{2}\right)= & \#\left\{\left(a_{i_{1}}, a_{i_{2}}\right) \in \mathscr{C}_{\neq}^{2} \mid a_{i_{1}} \succ_{1} a_{i_{2}}, a_{i_{2}} \succ_{2} a_{i_{1}}\right\} \\
& +\frac{1}{2} \#\left\{\left(a_{i_{1}}, a_{i_{2}}\right) \in \mathscr{C}_{\neq}^{2} \mid a_{i_{1}} \succ_{1} a_{i_{2}}, a_{i_{1}} \sim_{2} a_{i_{2}}\right\} \\
& +\frac{1}{2} \#\left\{\left(a_{i_{1}}, a_{i_{2}}\right) \in \mathscr{C}_{\neq}^{2} \mid a_{i_{1}} \sim_{1} a_{i_{2}}, a_{i_{1}} \succ_{2} a_{i_{2}}\right\} .
\end{aligned}
$$

Remark 8.36 For any two rankings with ties $\succ_{1}$ and $\succ_{2}$ on $\mathscr{C}$, we interchangeably write $K\left(\succsim_{1}, \succsim_{2}\right)$ or $K\left(\succ_{1}, \succ_{2}\right)$.

Considering the Kemeny distance function on $\mathcal{L}^{*}(\mathscr{C})$, the following betweenness relation on $\mathcal{L}^{*}(\mathscr{C})$ can be defined.

Definition 8.37 Let $\mathscr{C}$ be a set of $k$ candidates and $r$ be the number of voters.

(i) The betweenness relation $S$ on $\mathcal{L}^{*}(\mathscr{C})$ is defined as

$$
S=\left\{\left(\succ_{1}, \succ_{2}, \succ_{3}\right) \in \mathcal{L}^{*}(\mathscr{C})^{3} \mid K\left(\succ_{1}, \succ_{3}\right)=K\left(\succ_{1}, \succ_{2}\right)+K\left(\succ_{2}, \succ_{3}\right)\right\} .
$$

\footnotetext{
${ }^{5}$ The original Kemeny distance function proposed in [80] is the double of the distance function used in this dissertation. However, in order to maintain a consistency with the Kendall distance function 82 ] when restricted to rankings, this rescaled distance function will be considered.
} 
(ii) The betweenness relation $R$ on $\mathcal{L}^{*}(\mathscr{C})^{r}$ is defined as

$$
R=\left\{\left(\mathscr{R}_{1}^{*}, \mathscr{R}_{2}^{*}, \mathscr{R}_{3}^{*}\right) \in\left(\mathcal{L}^{*}(\mathscr{C})^{r}\right)^{3} \mid(\forall i \in\{1, \ldots, r\})\left(\left(\succ_{i}^{1}, \succ_{i}^{2}, \succ_{i}^{3}\right) \in S\right)\right\},
$$

where, for any $i \in\{1, \ldots, r\}$ and any $j \in\{1,2,3\}, \succ_{i}^{j}$ represents the $i$-th ranking with ties in $\mathscr{R}_{j}^{*}$.

In order to ease notations, for any $\mathscr{R}_{1}^{*}, \mathscr{R}_{2}^{*}, \mathscr{R}_{3}^{*} \in \mathcal{L}^{*}(\mathscr{C})^{r},\left(\mathscr{R}_{1}^{*}, \mathscr{R}_{2}^{*}, \mathscr{R}_{3}^{*}\right) \in R$ is denoted by $\left[\mathscr{R}_{1}^{*}, \mathscr{R}_{2}^{*}, \mathscr{R}_{3}^{*}\right]$. As a ranking with ties is a particular case of a profile of rankings with ties, for any $\succ_{1}, \succ_{2}, \succ_{3} \in \mathcal{L}^{*}(\mathscr{C}),\left(\succ_{1}, \succ_{2}, \succ_{3}\right) \in S$ is also denoted by $\left[\succ_{1}, \succ_{2}, \succ_{3}\right]$.

Definition 8.38 Let $\mathscr{C}$ be a set of $k$ candidates and $r$ be the number of voters. A function $M: \mathcal{L}^{*}(\mathscr{C})^{r} \times \mathcal{L}^{*}(\mathscr{C})^{r} \rightarrow \mathbb{R}$ is called a monometric if it satisfies the following three properties:

(i) Non-negativity: for any $\mathscr{R}^{*}, \mathscr{R}^{* \prime} \in \mathcal{L}^{*}(\mathscr{C})^{r}$, it holds that $M\left(\mathscr{R}^{*}, \mathscr{R}^{* \prime}\right) \geq 0$.

(ii) Coincidence: for any $\mathscr{R}^{*}, \mathscr{R}^{* \prime} \in \mathcal{L}^{*}(\mathscr{C})^{r}$, it holds that $M\left(\mathscr{R}^{*}, \mathscr{R}^{* \prime}\right)=0 \Leftrightarrow \mathscr{R}^{*}=$ $\mathscr{R}^{* \prime}$.

(iii) Compatibility: for any $\mathscr{R}^{*}, \mathscr{R}^{* \prime}, \mathscr{R}^{* \prime \prime} \in \mathcal{L}^{*}(\mathscr{C})^{r}$ such that $\left[\mathscr{R}^{*}, \mathscr{R}^{* \prime}, \mathscr{R}^{* \prime \prime}\right]$, it holds that $M\left(\mathscr{R}^{*}, \mathscr{R}^{* \prime}\right) \leq M\left(\mathscr{R}^{*}, \mathscr{R}^{* \prime \prime}\right)$.

Several monometrics can be considered w.r.t. the betweenness relation based on the Kemeny distance function between rankings with ties. In the following, three intuitive examples are provided:

(i) $M_{1}\left(\mathscr{R}_{1}^{*}, \mathscr{R}_{2}^{*}\right)=\sum_{i=1}^{r} K\left(\succ_{i}^{1}, \succ_{i}^{2}\right)$.

(ii) $M_{2}\left(\mathscr{R}_{1}^{*}, \mathscr{R}_{2}^{*}\right)=\sum_{i=1}^{r} 1-\delta\left(\succ_{i}^{1}, \succ_{i}^{2}\right)$, where $\delta\left(\succ, \succ^{\prime}\right)$ equals one if $\succ=\succ^{\prime}$ and zero otherwise. $M_{2}$ counts the number of rankings with ties that differ from a profile of rankings with ties to another one.

(iii) $M_{3}\left(\mathscr{R}_{1}^{*}, \mathscr{R}_{2}^{*}\right)= \begin{cases}M_{1}\left(\mathscr{R}_{1}^{*}, \mathscr{R}_{2}^{*}\right) & , \text { if } \succ_{i}^{1} \subseteq \succ_{i}^{2} \text { for any } i \\ \frac{r k(k-1)}{2} & , \text { otherwise }\end{cases}$

$M_{3}$ counts the number of ties that are broken in the profile in case that $\mathscr{R}_{2}^{*}$ can be reached from $\mathscr{R}_{1}^{*}$ by only breaking ties. Otherwise, it is equal to an upper bound of $M_{1}$. 
A procedure for hierarchically combining monometrics was analysed in [126].

After fixing a monometric, the search for a profile of rankings with ties with a monotone representation of votes w.r.t. $\succ$ that is closest to the profile of rankings with ties given by the voters can be addressed. However, under some circumstances, all ties may need to be avoided. In that case, the search for a closest profile of rankings with a monotone representation of votes should be addressed.

Definition 8.39 Let $\mathscr{C}$ be a set of $k$ candidates and $\mathscr{R}$ be the profile of $r$ rankings on $\mathscr{C}$ given by the voters. Let $M: \mathcal{L}(\mathscr{C})^{r} \times \mathcal{L}(\mathscr{C})^{r} \rightarrow \mathbb{R}$ be a monometric and consider a fixed representation of votes.

(i) A closest profile of rankings with ties with a monotone representation of votes is a profile of rankings with ties $\mathscr{R}^{\prime *}$ such that there exists a ranking $\succ$ w.r.t. which the representation of votes induced by $\mathscr{R}^{* *}$ is monotone and for which it holds that there exists no profile of rankings with ties $\mathscr{R}^{\prime \prime *}$ such that there exists a ranking $\succ$ w.r.t. which the representation of votes induced by $\mathscr{R}^{\prime \prime *}$ is monotone, while $M\left(\mathscr{R}^{*}, \mathscr{R}^{\prime \prime *}\right)<$ $M\left(\mathscr{R}^{*}, \mathscr{R}^{\prime *}\right)$.

(ii) A closest profile of rankings with a monotone representation of votes is a profile of rankings $\mathscr{R}^{\prime}$ such that there exists a ranking $\succ$ w.r.t. which the representation of votes induced by $\mathscr{R}^{\prime}$ is monotone and for which it holds that there exists no profile of rankings $\mathscr{R}^{\prime \prime}$ such that there exists a ranking $\succ$ w.r.t. which the representation of votes induced by $\mathscr{R}^{\prime \prime}$ is monotone, while $M\left(\mathscr{R}, \mathscr{R}^{\prime \prime}\right)<M\left(\mathscr{R}, \mathscr{R}^{\prime}\right)$.

As $\mathcal{L}^{*}(\mathscr{C})^{r}$ and $\mathcal{L}(\mathscr{C})^{r}$ are finite, the existence of both a closest profile of rankings with ties with a monotone representation of votes and a closest profile of rankings with a monotone representation of votes is assured. Unfortunately, the uniqueness cannot be assured.

As a profile of rankings (without ties) is a particular case of a profile of rankings with ties, the following result is straightforward.

Proposition 8.40 Let $\mathscr{C}$ be a set of $k$ candidates and $\mathscr{R}^{*} \in \mathcal{L}^{*}(\mathscr{C})^{r}$ be the profile of $r$ rankings with ties given by the voters. Let $M: \mathcal{L}^{*}(\mathscr{C})^{r} \times \mathcal{L}^{*}(\mathscr{C})^{r} \rightarrow \mathbb{R}$ be a monometric and consider a fixed representation of votes. For any closest profile of rankings with ties $\mathscr{R}^{* \prime}$ with a monotone representation of votes and any closest profile of rankings $\mathscr{R}^{\prime}$ with a monotone representation of votes, it holds that $M\left(\mathscr{R}^{*}, \mathscr{R}^{* \prime}\right) \leq M\left(\mathscr{R}^{*}, \mathscr{R}^{\prime}\right)$. 
Now, two kinds of optimal rankings can be defined: a weak-optimal ranking and a totaloptimal ranking. On the one hand, a weak-optimal ranking is one whose corresponding closest profile of rankings with ties with a monotone representation of votes is closest to the profile of rankings with ties given by the voters. On the other hand, a total-optimal ranking is one whose corresponding closest profile of rankings with a monotone representation of votes is closest to the profile of rankings with ties given by the voters.

Definition 8.41 Let $\mathscr{C}$ be a set of $k$ candidates and $\mathscr{R}^{*}$ be the profile of $r$ rankings with ties on $\mathscr{C}$ given by the voters. Let $M: \mathcal{L}^{*}(\mathscr{C})^{r} \times \mathcal{L}^{*}(\mathscr{C})^{r} \rightarrow \mathbb{R}$ be a monometric.

(i) A weak-optimal ranking $\succ$ is a ranking imposing monotonicity on at least one closest profile of rankings with ties with a monotone representation of votes.

(ii) A total-optimal ranking $\succ$ is a ranking imposing monotonicity on at least one closest profile of rankings with a monotone representation of votes.

Obviously, the existence of both a weak-optimal ranking and a total-optimal ranking is assured. Unfortunately, the uniqueness cannot be assured.

The extension of the method of Kemeny to rankings with ties (and to tournaments and to binary relations) is a common problem in social choice [8, 21, 30, 31, usually referred to as the search for 'median orders' or 'median relations'. These extensions are obtained by allowing the relations in the profile to be rankings with ties (or tournaments, or binary relations) or by allowing the winning relations to be rankings with ties (or tournaments, or binary relations). This approach differs from the one followed in this dissertation (it suffices to see that the method of Kemeny does not need to coincide with the search for monotonicity of a representation of votes).

\subsection{Relation with the antisymmetric case}

A profile of rankings is a special case of a profile of rankings with ties. The methods recalled in Chapter 6 based on the search for monotonicity of a representation of votes should then coincide with the methods introduced in this chapter when restricted to the case where voters are not allowed to express ties in their preferences. 
Any scorix has a weak-scorix representation when the corresponding profile of rankings is considered as a profile of rankings with ties.

Proposition 8.42 Let $\mathscr{C}$ be a set of $k$ candidates and $\mathscr{R} \in \mathcal{L}(\mathscr{C})^{r}$ be the profile of $r$ rankings on $\mathscr{C}$ given by the voters. Let us denote by $S_{\mathscr{R}} \in \mathcal{S}_{r}(\mathscr{C})$ the scorix of $\mathscr{R}$ and by $S_{\mathscr{R}}^{*} \in \mathcal{S}_{r}^{*}(\mathscr{C})$ the weak-scorix of $\mathscr{R}$. For any $a_{i} \in \mathscr{C}$, it holds that

(i) for any $\left[\ell_{1}, \ell_{2}\right] \in \mathcal{P}_{k}$ such that $\ell_{1}=\ell_{2}$,

$$
S_{\mathscr{R}}^{*}\left(a_{i},\left[\ell_{1}, \ell_{2}\right]\right)=\left(S_{\mathscr{R}}\right)_{i \ell_{1}},
$$

(ii) for any $\left[\ell_{1}, \ell_{2}\right] \in \mathcal{P}_{k}$ such that $\ell_{1} \neq \ell_{2}$,

$$
S_{\mathscr{R}}^{*}\left(a_{i},\left[\ell_{1}, \ell_{2}\right]\right)=0 .
$$

Any votrix has a weak-votrix representation when the corresponding profile of rankings is considered as a profile of rankings with ties.

Proposition 8.43 Let $\mathscr{C}$ be a set of $k$ candidates and $\mathscr{R} \in \mathcal{L}(\mathscr{C})^{r}$ be the profile of $r$ rankings on $\mathscr{C}$ given by the voters. Let us denote by $V_{\mathscr{R}} \in \mathcal{V}_{r}(\mathscr{C})$ the votrix of $\mathscr{R}$ and by $V_{\mathscr{R}}^{*} \in \mathcal{V}_{r}^{*}(\mathscr{C})$ the weak-votrix of $\mathscr{R}$. For any $\left(a_{i_{1}}, a_{i_{2}}\right) \in \mathscr{C}_{\neq}^{2}$, it holds that

$$
\left(V_{\mathscr{R}}^{*}\right)_{i_{1} i_{2}}=\left(V_{\mathscr{R}}\right)_{i_{1} i_{2}}
$$

Any votex has a weak-votex representation when the corresponding profile of rankings is considered as a profile of rankings with ties.

Proposition 8.44 Let $\mathscr{C}$ be a set of $k$ candidates and $\mathscr{R} \in \mathcal{L}(\mathscr{C})^{r}$ be the profile of $r$ rankings on $\mathscr{C}$ given by the voters. Let us denote by $W_{\mathscr{R}} \in \mathcal{W}_{r}(\mathscr{C})$ the votex of $\mathscr{R}$ and by $W_{\mathscr{R}}^{*} \in \mathcal{W}_{r}^{*}(\mathscr{C})$ the weak-votex of $\mathscr{R}$. For any $\left(a_{i_{1}}, a_{i_{2}}\right) \in \mathscr{C}_{\neq}^{2}$, it holds that

(i) for any $\left(s_{1}, s_{2}, p\right) \in \mathcal{B}_{k}$ such that $s_{1}=s_{2}=1$ and $p \neq 0$,

$$
W_{\mathscr{R}}^{*}\left(a_{i_{1}}, a_{i_{2}}\right)(1,1, p)=W_{\mathscr{R}}\left(a_{i_{1}}, a_{i_{2}}\right)(p),
$$

(ii) for any $\left(s_{1}, s_{2}, p\right) \in \mathcal{B}_{k}$ such that $s_{1} \neq 1$ or $p=0$,

$$
W_{\mathscr{R}}^{*}\left(a_{i_{1}}, a_{i_{2}}\right)\left(s_{1}, s_{2}, p\right)=0 .
$$


It follows from the previous propositions that the scorix/votrix/votex of a profile $\mathscr{R}$ of rankings on $\mathscr{C}$ is monotone w.r.t. a ranking $\succ$ on $\mathscr{C}$ if and only if the weak-scorix/votrix/votex of $\mathscr{R}$ is monotone w.r.t. $\succ$.

Proposition 8.45 Let $\mathscr{C}$ be a set of $k$ candidates and $\mathscr{R} \in \mathcal{L}(\mathscr{C})^{r}$ be the profile of $r$ rankings on $\mathscr{C}$ given by the voters. Let us denote by $S_{\mathscr{R}} \in \mathcal{S}_{r}(\mathscr{C})$ the scorix of $\mathscr{R}$, by $S_{\mathscr{R}}^{*} \in \mathcal{S}_{r}^{*}(\mathscr{C})$ the weak-scorix of $\mathscr{R}$, by $V_{\mathscr{R}} \in \mathcal{V}_{r}(\mathscr{C})$ the votrix of $\mathscr{R}$, by $V_{\mathscr{R}}^{*} \in \mathcal{V}_{r}^{*}(\mathscr{C})$ the weak-votrix of $\mathscr{R}$, by $W_{\mathscr{R}} \in \mathcal{W}_{r}(\mathscr{C})$ the votex of $\mathscr{R}$ and by $W_{\mathscr{R}}^{*} \in \mathcal{W}_{r}^{*}(\mathscr{C})$ the weak-votex of $\mathscr{R}$. For any ranking $\succ$ on $\mathscr{C}$, the following three equivalences hold:

(i) $S_{\mathscr{R}}$ is monotone w.r.t. $\succ$ if and only if $S_{\mathscr{R}}^{*}$ is monotone w.r.t. $\succ$.

(ii) $V_{\mathscr{R}}$ is monotone w.r.t. $\succ$ if and only if $V_{\mathscr{R}}^{*}$ is monotone w.r.t. $\succ$.

(iii) $W_{\mathscr{R}}$ is monotone w.r.t. $\succ$ if and only if $W_{\mathscr{R}}^{*}$ is monotone w.r.t. $\succ$.

(iv) $\mathscr{R}$ is monotone w.r.t. $\succ$ if and only if $\mathscr{R}^{*}$ is $\mathbb{S}$-monotone (or $\mathbb{O}$-monotone) w.r.t. $\succ$.

Proof: (i) As $\leq$ is a total order relation on $\{1, \ldots, k\}$ and it holds that $[\ell, \ell] \leq_{\mathcal{P}_{k}}\left[\ell^{\prime}, \ell^{\prime}\right]$ if and only if $\ell \geq \ell^{\prime}$, the equivalence follows from Proposition 8.42 .

(ii) Follows immediately from Proposition 8.43.

(iii) As $\leq$ is a total order relation on $\mathcal{K}$ and it holds that $(1,1, p) \leq_{\mathcal{B}_{k}}\left(1,1, p^{\prime}\right)$ if and only if $p \leq p^{\prime}$, the equivalence follows from Proposition 8.44 .

(iv) Follows immediately from the definition of $\unrhd^{\mathbb{S}}$ and $\unrhd^{\mathbb{O}}$

The chosen monometric on $\mathcal{L}^{*}(\mathscr{C})^{r}$ needs to coincide with the monometric on $\mathcal{L}(\mathscr{C})^{r}$ when restricted to profiles of rankings.

Definition 8.46 Let $\mathscr{C}$ be a set of $k$ candidates and $r$ be the number of voters. A monometric $M: \mathcal{L}(\mathscr{C})^{r} \times \mathcal{L}(\mathscr{C})^{r} \rightarrow \mathbb{R}$ is called the restriction of a monometric $M^{*}$ : $\mathcal{L}^{*}(\mathscr{C})^{r} \times \mathcal{L}^{*}(\mathscr{C})^{r} \rightarrow \mathbb{R}$ to $\mathcal{L}(\mathscr{C})^{r}$ if, for any two profiles of rankings $\mathscr{R}$ and $\mathscr{R}^{\prime}$, it holds that $M\left(\mathscr{R}, \mathscr{R}^{\prime}\right)=M^{*}\left(\mathscr{R}, \mathscr{R}^{\prime}\right)$.

Remark 8.47 The restriction of a monometric $M^{*}: \mathcal{L}^{*}(\mathscr{C})^{r} \times \mathcal{L}^{*}(\mathscr{C})^{r} \rightarrow \mathbb{R}$ to $\mathcal{L}(\mathscr{C})^{r}$ is unique. 
In case a monometric on $\mathcal{L}^{*}(\mathscr{C})^{r}$ and its restriction to $\mathcal{L}(\mathscr{C})^{r}$ are considered, it is straightforward to see that the search for a closest profile of rankings with a monotone representation of votes in the set of profiles of rankings is equivalent to the search for a closest profile of rankings with a monotone representation of votes in the set of profiles of rankings with ties.

Proposition 8.48 Let $\mathscr{C}$ be a set of $k$ candidates, $r$ be the number of voters, $\succ$ be a ranking on $\mathscr{C}, \mathscr{R} \in \mathcal{L}(\mathscr{C})^{r}$ be a profile of rankings and $M: \mathcal{L}(\mathscr{C})^{r} \times \mathcal{L}(\mathscr{C})^{r} \rightarrow \mathbb{R}$ be the restriction of a monometric $M^{*}: \mathcal{L}^{*}(\mathscr{C})^{r} \times \mathcal{L}^{*}(\mathscr{C})^{r} \rightarrow \mathbb{R}$ to $\mathcal{L}(\mathscr{C})^{r}$. A profile of rankings $\mathscr{R}^{\prime} \in \mathcal{L}(\mathscr{C})^{r}$ is a closest profile of rankings with a monotone representation of votes w.r.t. $\succ$ considering $M^{*}$ if and only if it is a closest profile of rankings with a monotone representation of votes w.r.t. $\succ$ considering $M$.

We conclude that the methods introduced in the previous chapters coincide with the one introduced in this chapter when restricted to the search for total-optimal rankings.

Corollary 8.49 Let $\mathscr{C}$ be a set of $k$ candidates, $\mathscr{R}$ be the profile of $r$ rankings on $\mathscr{C}$ given by the voters and $M: \mathcal{L}(\mathscr{C})^{r} \times \mathcal{L}(\mathscr{C})^{r} \rightarrow \mathbb{R}$ be the restriction of a monometric $M^{*}: \mathcal{L}^{*}(\mathscr{C})^{r} \times \mathcal{L}^{*}(\mathscr{C})^{r} \rightarrow \mathbb{R}$ to $\mathcal{L}(\mathscr{C})^{r}$. A ranking $\succ$ on $\mathscr{C}$ is an optimal ranking if and only if it is a total-optimal ranking. 


\section{CHAPTER 9}

\section{Winning candidates}

In this chapter, we do not discuss the problem of the aggregation of rankings, but, instead, we address the problem of identifying the best candidate on the set of candidates. There are some situations in which the selection of a winning candidate is easy, for instance, when every voter agrees on the candidate that needs to be selected as the winner. Such candidate is called the unanimous winner [6]. Unfortunately, the unanimous winner usually does not exist. In case more than half of the voters agree on the candidate that should be selected as the winner, one talks about the majority! winner [107]. Obviously, the majority winner might not exist either. Based only on the head-to-head comparisons between candidates, Condorcet [35] advocated a weaker condition than that of the unanimous or the majority winner: if a candidate is preferred by more than half of the voters to each of the other candidates, then it should be selected as the winner; said candidate is referred to as the Condorcet winner. The existence of the Condorcet winner is neither assured due to the more than famous 'voting paradox' where, although all the voters provide transitive preferences on the set of candidates, the collective preference might be cyclic.

Although the existence of the unanimous winner entails the existence of an undoubtedly clear winner, we find several examples where selecting the majority winner or the Condorcet winner is more than questionable. This is due to the fact that both the majority winner and the Condorcet winner disregard an important part of the preferences of the voters; quoting a well-known phrase by Sen [150], “... the method of majority decision takes no

\footnotetext{
${ }^{1}$ The concept of a majority winner is not related with that of the (simple) majority rule [54, 64, 76, 95, [149] whenever more than two candidates are considered.
} 
account of intensities of preference, and it is certainly arguable that what matters is not merely the number who prefer $x$ to $y$ and the number who prefer $y$ to $x$, but also by how much each prefers one alternative to the other". Although Sen's statement tries to call attention to the need of requiring voters to express intensities in their preferences, there is also some unconsidered intensity of preference when voters express rankings on the set of candidates. After all, when a voter is providing a ranking $x \succ y \succ z$, he is actually declaring that he supports $x$ over $z$ stronger than both $x$ over $y$ and $y$ over $z$. PérezFernández et al. 124] proposed a new representation of votes capturing this information: the votex. Another way of exploiting all the information provided by the voters is to focus not only on the "the number who prefer $x$ to $y$ ", but also on the number who prefer $x$ and $y$ to any other candidate $z$. We will explore this direction throughout this section resulting in a new type of winner: the pairwise winner [120]. The quasipositional winner, which is the winner given by the positional rule [52, 98, and a stronger version - the positional winner [118] - will also be analysed.

\subsection{On being better than another candidate}

In this section, we discuss three motivations for assuring that a candidate is better than another candidate considering either positional or pairwise information.

\subsubsection{On being quasipositionally better than another candidate}

In case the vector of positions of a candidate (strictly) dominates the vector of positions of another candidate, the first candidate will always be ranked at a better position than the other candidate by any ranking rule. The first candidate is then considered to be quasipositionally as good as the second candidate.

Definition 9.1 Let $\mathscr{C}$ be a set of $k$ candidates, $\mathscr{R}$ be the profile of $r$ rankings on $\mathscr{C}$ given by the voters and $S$ be the scorix induced by $\mathscr{R}$. For any $a_{i_{1}}, a_{i_{2}} \in \mathscr{C}, a_{i_{1}}$ is said to be quasipositionally as good as $a_{i_{2}}$, denoted by $a_{i_{1}} \gtrsim_{B} a_{i_{2}}$, if $S_{i_{1}} \unrhd_{1} S_{i_{2}}$.

\footnotetext{
${ }^{2}$ The subindex ' $B$ ' stands from Borda, the forefather of the exploitation of quasipositional information in the field of social choice theory.
} 
If $a_{i_{1}} \gtrsim_{B} a_{i_{2}}$ and $S_{i_{1}} \triangleright_{1} S_{i_{2}}$, then $a_{i_{1}}$ is said to be quasipositionally better than $a_{i_{2}}$, denoted by $a_{i_{1}}>_{B} a_{i_{2}}$. The reflexive closurt $\mathrm{f}^{3}$ of the relation $\gg_{B}$ is denoted by $\geq_{B}$.

Of course, being quasipositionally better than or equal to another candidate implies being quasipositionally as good as this candidate.

Proposition 9.2 Let $\mathscr{C}$ be a set of $k$ candidates and $\mathscr{R}$ be the profile of $r$ rankings on $\mathscr{C}$ given by the voters. For any $a_{i_{1}}, a_{i_{2}} \in \mathscr{C}$, it holds that $a_{i_{1}} \unrhd_{B} a_{i_{2}}$ implies that $a_{i_{1}} \gtrsim_{B} a_{i_{2}}$.

Note that the fact that a candidate is quasipositionally better than another candidate trivially implies that the Borda score $\mathrm{f}^{4}$ of the first candidate is greater than the Borda score of the second candidate.

Proposition 9.3 Let $\mathscr{C}$ be a set of $k$ candidates and $\mathscr{R}$ be the profile of $r$ rankings on $\mathscr{C}$ given by the voters. For any $a_{i_{1}}, a_{i_{2}} \in \mathscr{C}$ such that $a_{i_{1}}>_{B} a_{i_{2}}$, it holds that $B\left(a_{i_{1}}\right)>B\left(a_{i_{2}}\right)$.

From now on, we focus on the relation $\geq_{B}$ as it leads to an order relation on the set of candidates instead of a preorder relation.

Proposition 9.4 Let $\mathscr{C}$ be a set of $k$ candidates and $\mathscr{R}$ be the profile of $r$ rankings on $\mathscr{C}$ given by the voters. The relation $\geq_{B}$ is an order relation on $\mathscr{C}$.

Proof: By definition of $\unrhd_{1}$, the relation $>_{B}$ obviously is transitive. As we consider the reflexive closure of an irreflexive relation, the reflexivity and the antisymmetry are also satisfied.

The previous definitions are illustrated in the following example.

Example 9.5 Consider a set of four candidates $\mathscr{C}=\{a, b, c, d\}$ and the profile of rankings $\mathscr{R}=\left(\succ_{i}\right)_{i=1}^{14}$ provided by fourteen voters shown in Table 9.1.

\footnotetext{
${ }^{3}$ The reflexive closure of a relation $R$ is the smallest reflexive relation containing $R$.

${ }^{4}$ The Borda score $B\left(a_{i}\right)$ of candidate $a_{i}$ is defined as the number of times that a candidate is ranked at a worse position than candidate $a_{i}$ in the profile of rankings given by the voters, i.e., $B\left(a_{i}\right)=\sum_{a_{\ell} \in \mathscr{C} \backslash\left\{a_{i}\right\}} V_{i \ell}$.
} 


\begin{tabular}{|c|c|}
\hline$\# \succ_{i}$ & Rankings on $\mathscr{C}$ \\
\hline 6 & $c \succ b \succ a \succ d$ \\
5 & $a \succ d \succ b \succ c$ \\
3 & $b \succ a \succ d \succ c$ \\
\hline
\end{tabular}

Table 9.1: Frequency of the rankings on $\mathscr{C}$ expressed by 14 voters.

The scorix induced by $\mathscr{R}$ is given by:

$$
S_{\mathscr{R}}=\left(\begin{array}{llll}
5 & 3 & 6 & 0 \\
3 & 6 & 5 & 0 \\
6 & 0 & 0 & 8 \\
0 & 5 & 3 & 6
\end{array}\right) .
$$

Note that, for instance, considering candidates a and d, it holds that:

$$
\begin{gathered}
5=S_{11}>S_{41}=0 \\
8=S_{11}+S_{12}>S_{41}+S_{42}=5, \\
14=S_{11}+S_{12}+S_{13}>S_{41}+S_{42}+S_{43}=8 .
\end{gathered}
$$

Thus, it holds that $a>_{B}$ d. In general, $>_{B}$ is given by:

$$
>_{B}=\{(a, d),(b, d)\} .
$$

Obviously, $\geq_{B}$ is given by:

$$
\geq_{B}=\{(a, a),(a, d),(b, b),(b, d),(c, c),(d, d)\} .
$$

This leads to the partially ordered set with the Hasse diagram in Figure 9.1.

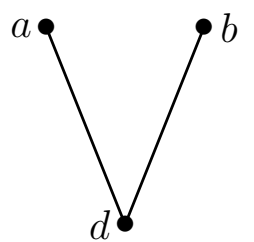

Figure 9.1: Hasse diagram of the order relation $\geq_{B}$. 


\subsubsection{On being positionally better than another candidate}

Based on the notion of sub-scorix, we define the notion of being quasipositionally better than another candidate.

Definition 9.6 Let $\mathscr{C}$ be a set of $k$ candidates, $\mathscr{R}$ be the profile of $r$ rankings on $\mathscr{C}$ given by the voters and $S$ be the scorix induced by $\mathscr{R}$. For any $a_{i_{1}}, a_{i_{2}} \in \mathscr{C}, a_{i_{1}}$ is said to be positionally as good as $a_{i_{2}}$, denoted by $y^{5} a_{i_{1}} \gtrsim_{R} a_{i_{2}}$, if, for any $\mathscr{C}^{\prime} \subseteq \mathscr{C}$ such that $a_{i_{1}}, a_{i_{2}} \in \mathscr{C}^{\prime}$ and the corresponding sub-scorix of $S$ on $\mathscr{C}^{\prime}$, it holds that $S_{i_{1}}^{\prime} \unrhd_{1} S_{i_{2}}^{\prime}$.

If, for any $\mathscr{C}^{\prime} \subseteq \mathscr{C}$ such that $a_{i_{1}}, a_{i_{2}} \in \mathscr{C}^{\prime}$ and the corresponding sub-scorix of $S$ on $\mathscr{C}^{\prime}$, it holds that $S_{i_{1}}^{\prime} \triangleright_{1} S_{i_{2}}^{\prime}$, then $a_{i_{1}}$ is said to be positionally better than $a_{i_{2}}$, denoted by $a_{i_{1}}>_{R} a_{i_{2}}$. The reflexive closure of the relation $>_{R}$ is denoted by $\geq_{R}$.

Of course, being positionally better than or equal to another candidate implies being positionally as good as this candidate.

Proposition 9.7 Let $\mathscr{C}$ be a set of $k$ candidates and $\mathscr{R}$ be the profile of $r$ rankings on $\mathscr{C}$ given by the voters. For any $a_{i_{1}}, a_{i_{2}} \in \mathscr{C}$, it holds that $a_{i_{1}} \geq_{R} a_{i_{2}}$ implies that $a_{i_{1}} \gtrsim_{R} a_{i_{2}}$.

Note that the fact that a candidate is positionally better than another candidate trivially implies that the candidate is quasipositionally better than another candidate. The same result holds for the relations 'being positionally as good' as and 'being quasipositionally as good as'.

Proposition 9.8 Let $\mathscr{C}$ be a set of $k$ candidates and $\mathscr{R}$ be the profile of $r$ rankings on $\mathscr{C}$ given by the voters. For any $a_{i_{1}}, a_{i_{2}} \in \mathscr{C}$ such that $a_{i_{1}} \gtrsim_{R} a_{i_{2}}$, it holds that $a_{i_{1}} \gtrsim_{B} a_{i_{2}}$. Moreover, for any $a_{i_{1}}, a_{i_{2}} \in \mathscr{C}$ such that $a_{i_{1}}>_{R} a_{i_{2}}$, it holds that $a_{i_{1}}>_{B} a_{i_{2}}$.

From now on, we focus on the relation $\geq_{R}$ as it leads to an order relation on the set of candidates instead of a preorder relation.

\footnotetext{
${ }^{5}$ The subindex ' $R$ ' stands from 'recursive' monotonicity of the scorix.
} 
Proposition 9.9 Let $\mathscr{C}$ be a set of $k$ candidates and $\mathscr{R}$ be the profile of $r$ rankings on $\mathscr{C}$ given by the voters. The relation $\geq_{R}$ is an order relation on $\mathscr{C}$.

Proof: By definition of $\unrhd_{1}$, the relation $>_{R}$ obviously is transitive. As we consider the reflexive closure of an irreflexive relation, the reflexivity and the antisymmetry are also satisfied.

The previous definitions are illustrated in the following example.

Example 9.10 Consider the set of four candidates $\mathscr{C}=\{a, b, c, d\}$ and the profile of rankings $\mathscr{R}=\left(\succ_{i}\right)_{i=1}^{14}$ provided in Example 9.5. As a result of Proposition 9.8, it holds that $>_{R} \subseteq>_{B}=\{(a, d),(b, d)\}$.

We check whether $(a, d)$ belongs to $\gg_{R}$. First, the scorix induced by the restriction of $\mathscr{R}$ to $\mathscr{C}_{a b d}=\{a, b, d\}$ is given by:

$$
S_{a b d}=\left(\begin{array}{ccc}
5 & 9 & 0 \\
9 & 0 & 5 \\
0 & 5 & 9
\end{array}\right) .
$$

Note that, for candidates a and $d$, it holds that:

$$
\begin{gathered}
5=S_{11}>S_{31}=0 \\
14=S_{11}+S_{12}>S_{31}+S_{32}=5 .
\end{gathered}
$$

Second, the scorix induced by the restriction of $\mathscr{R}$ to $\mathscr{C}_{\text {acd }}=\{a, c, d\}$ is given by:

$$
S_{a c d}=\left(\begin{array}{ccc}
8 & 6 & 0 \\
6 & 0 & 8 \\
0 & 8 & 6
\end{array}\right) .
$$

Note that, for candidates $a$ and $d$, it holds that:

$$
\begin{gathered}
8=S_{11}>S_{31}=0 \\
14=S_{11}+S_{12}>S_{31}+S_{32}=8 .
\end{gathered}
$$

Third, the scorix induced by the restriction of $\mathscr{R}$ to $\mathscr{C}_{a d}=\{a, d\}$ is given by:

$$
S_{a d}=\left(\begin{array}{cc}
14 & 0 \\
0 & 14
\end{array}\right) .
$$


Note that, for candidates a and $d$, it holds that:

$$
14=S_{11}>S_{21}=0 .
$$

Thus, $(a, d) \in>_{R}$.

However, it holds that $(b, d) \notin>_{R}$ in case $\mathscr{C}_{b c d}=\{b, c, d\}$ is considered. The scorix induced by the restriction of $\mathscr{R}$ to $\mathscr{C}_{b c d}$ is given by:

$$
S_{b c d}=\left(\begin{array}{ccc}
3 & 11 & 0 \\
6 & 0 & 8 \\
5 & 3 & 6
\end{array}\right) .
$$

Note that, for candidates $b$ and $d$, it holds that:

$$
3=S_{11} \ngtr S_{31}=5 .
$$

Thus, it holds that $>_{R}$ is given by:

$$
>_{R}=\{(a, d)\}
$$

Obviously, $\geq_{R}$ is given by:

$$
\geq_{R}=\{(a, a),(a, d),(b, b),(c, c),(d, d)\} .
$$

This leads to the partially ordered set with the Hasse diagram in Figure 9.2.

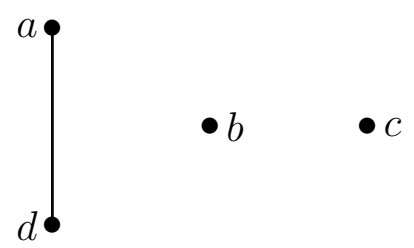

Figure 9.2: Hasse diagram of the order relation $\geq_{R}$.

\subsubsection{On being pairwisely better than another candidate}

In social choice theory, researchers have focused too strongly on the notion of a candidate that beats another one (the strength of support of the first candidate over the second 
one is greater than the strength of support of the second candidate over the first one) following the ideas of Condorcet [35]. Nevertheless, this notion disregards the comparisons with all the other candidates leading sometimes to the well-known voting paradox (also known as Condorcet's paradox) where a first candidate beats a second candidate, this second candidate beats a third candidate but this third candidate beats the first candidate. Several ways of avoiding the voting paradox have been analysed; obtaining choice sets [22] such as the Smith Set [70, 152] or the Schwarz set [148] and the use of beatpaths [147] being the most prominent ones. With the intention of avoiding such paradox, we propose here to also consider the relations with all the other candidates when comparing a candidate with another one.

Definition 9.11 Let $\mathscr{C}$ be a set of $k$ candidates and $\mathscr{R}$ be the profile of $r$ rankings on $\mathscr{C}$ given by the voters. For any $a_{i_{1}}, a_{i_{2}} \in \mathscr{C}, a_{i_{1}}$ is said to be pairwisely as good as $a_{i_{2}}$, denoted $b y^{6} a_{i_{1}} \gtrsim_{C} a_{i_{2}}$, if the following conditions hold:

(i) $V_{i_{1} i_{2}} \geq V_{i_{2} i_{1}}$,

(ii) $V_{i_{1} \ell} \geq V_{i_{2} \ell}$, for any $a_{\ell} \in \mathscr{C} \backslash\left\{a_{i_{1}}, a_{i_{2}}\right\}$,

(iii) $V_{\ell i_{1}} \leq V_{\ell i_{2}}$, for any $a_{\ell} \in \mathscr{C} \backslash\left\{a_{i_{1}}, a_{i_{2}}\right\}$.

If $a_{i_{1}} \gtrsim_{C} a_{i_{2}}$ and $V_{i_{1} i_{2}}>V_{i_{2} i_{1}}$, then $a_{i_{1}}$ is said to be pairwisely better than $a_{i_{2}}$, denoted by $a_{i_{1}} \gg_{C} a_{i_{2}}$. The reflexive closure of the relation $\gg_{C}$ is denoted by $\gtrsim_{C}$.

Remark 9.12 In case we restrict our attention to the case where voters express rankings (without ties) on the set of candidates, conditions (ii) and (iii) are equivalent.

Remark 9.13 Dutta and Laslier [47] generalized the covering relation, which is an old acquaintance for scholars of social choice [59, 103, 129] in the context of (weak) tournaments, by introducing comparison functions. In this generalized case, the relation 'being pairwisely better than' and the covering relation turn out to be equivalent in case the following two conditions hold: (a) each voter expresses a ranking (without ties) on the set of candidates (forcing conditions (ii) and (iii) to be equivalent) and (b) the considered comparison function $g$ is defined as $g\left(a_{i_{1}}, a_{i_{2}}\right)=V_{i_{1} i_{2}}-V_{i_{2} i_{1}}$.

\footnotetext{
${ }^{6}$ The subindex ' $C$ ' stands from Condorcet, the forefather of the exploitation of pairwise information in the field of social choice theory.
} 
Of course, being pairwisely better than or equal to another candidate implies being pairwisely as good as this candidate. The converse is also true for an odd number of voters.

Proposition 9.14 Let $\mathscr{C}$ be a set of $k$ candidates and $\mathscr{R}$ be the profile of $r$ rankings on $\mathscr{C}$ given by the voters. For any $a_{i_{1}}, a_{i_{2}} \in \mathscr{C}$, the following two statements hold:

(i) $a_{i_{1}} \unrhd_{C} a_{i_{2}}$ implies that $a_{i_{1}} \gtrsim_{C} a_{i_{2}}$.

(ii) If $r$ is an odd number, then it holds that $a_{i_{1}} \geq_{C} a_{i_{2}}$ if and only if $a_{i_{1}} \gtrsim_{C} a_{i_{2}}$.

Proof: Statement (i) is evident by definition. Statement (ii) follows from the fact that both $V_{i_{1} i_{2}}$ and $V_{i_{2} i_{1}}$ are natural numbers and, at the same time, $V_{i_{1} i_{2}}+V_{i_{2} i_{1}}=r$. Therefore, it holds that $V_{i_{1} i_{2}} \geq V_{i_{2} i_{1}}$ if and only if $V_{i_{1} i_{2}}>V_{i_{2} i_{1}}$.

Note that the fact that a candidate is pairwisely better than another candidate implies that the Borda score of the first candidate is greater than the Borda score of the second candidate.

Proposition 9.15 Let $\mathscr{C}$ be a set of $k$ candidates and $\mathscr{R}$ be the profile of $r$ rankings on $\mathscr{C}$ given by the voters. For any $a_{i_{1}}, a_{i_{2}} \in \mathscr{C}$ such that $a_{i_{1}}>_{C} a_{i_{2}}$, it holds that $B\left(a_{i_{1}}\right)>B\left(a_{i_{2}}\right)$.

Proof: As $a_{i_{1}}>_{C} a_{i_{2}}$, it follows that,

$$
\begin{aligned}
B\left(a_{i_{1}}\right) & =\sum_{a_{\ell} \in \mathscr{C} \backslash\left\{a_{i_{1}}\right\}} V_{i_{1} \ell} \\
& =V_{i_{1} i_{2}}+\sum_{a_{\ell} \in \mathscr{C} \backslash\left\{a_{i_{1}}, a_{i_{2}}\right\}} V_{i_{1} \ell} \\
> & V_{i_{2} i_{1}}+\sum_{a_{\ell} \in \mathscr{C} \backslash\left\{a_{i_{1}}, a_{i_{2}}\right\}} V_{i_{2} \ell} \\
& =\sum_{a_{\ell} \in \mathscr{C} \backslash\left\{a_{i_{2}}\right\}} V_{i_{2} \ell} \\
& =B\left(a_{i_{2}}\right) .
\end{aligned}
$$

We conclude that $B\left(a_{i_{1}}\right)>B\left(a_{i_{2}}\right)$. 
From now on, we focus on the relation $\geq_{C}$ as it leads to an order relation on the set of candidates, while the relation $\gtrsim_{C}$ leads to a pre-order relation only. Note that, although Condorcet's proposal might not be transitive, the relation $\geq_{C}$ is transitive and it avoids the voting paradox.

Proposition 9.16 Let $\mathscr{C}$ be a set of $k$ candidates and $\mathscr{R}$ be the profile of $r$ rankings on $\mathscr{C}$ given by the voters. The relation $\geq_{C}$ is an order relation on $\mathscr{C}$.

Proof: We prove that $\geq_{C}$ is reflexive, antisymmetric and transitive.

(a) Reflexivity: as $\geq_{C}$ is defined as the reflexive closure of $\gg_{C}$, it trivially holds that $\geq_{C}$ is reflexive.

(b) Antisymmetry: as $>_{C}$ obviously is asymmetric, $\geq_{C}$ is antisymmetric.

(c) Transitivity: for any $a_{i_{1}}, a_{i_{2}}, a_{i_{3}} \in \mathscr{C}$ such that $a_{i_{1}} \unrhd_{C} a_{i_{2}}$ and that $a_{i_{2}} \unrhd_{C} a_{i_{3}}$, we need to prove that $a_{i_{1}} \geq_{C} a_{i_{3}}$. If $a_{i_{1}}=a_{i_{2}}$ or $a_{i_{1}}=a_{i_{3}}$ or $a_{i_{2}}=a_{i_{3}}$, then it is trivial. Consider the case where $a_{i_{1}} \neq a_{i_{2}} \neq a_{i_{3}} \neq a_{i_{1}}$ (only necessary if $k \geq 3$ ). First, as $a_{i_{1}}>_{C} a_{i_{2}}$ and as $a_{i_{3}} \in \mathscr{C} \backslash\left\{a_{i_{1}}, a_{i_{2}}\right\}$, it holds that $V_{i_{1} i_{3}} \geq V_{i_{2} i_{3}}$ and, therefore, that $V_{i_{3} i_{1}} \leq V_{i_{3} i_{2}}$. Furthermore, as $a_{i_{2}}>_{C} a_{i_{3}}$, it holds that $V_{i_{2} i_{3}}>V_{i_{3} i_{2}}$. Thus, it holds that:

$$
V_{i_{1} i_{3}} \geq V_{i_{2} i_{3}}>V_{i_{3} i_{2}} \geq V_{i_{3} i_{1}}
$$

Second, for any $a_{\ell} \in \mathscr{C} \backslash\left\{a_{i_{1}}, a_{i_{3}}\right\}$, we need to prove that

$$
V_{i_{1} \ell} \geq V_{i_{3} \ell}
$$

We distinguish two cases: $\ell=i_{2}$ and $\ell \neq i_{2}$.

(i) If $\ell=i_{2}$, then it follows from $a_{i_{1}}>_{C} a_{i_{2}}$ and $a_{i_{2}}>_{C} a_{i_{3}}$ that

$$
V_{i_{1} i_{2}} \geq \frac{r}{2} \geq V_{i_{3} i_{2}}
$$

(ii) If $\ell \neq i_{2}$ (only necessary if $k \geq 4$ ), then it follows from $a_{i_{1}}>_{C} a_{i_{2}}$ and $a_{i_{2}}>_{C} a_{i_{3}}$ that

$$
V_{i_{1} \ell} \geq V_{i_{2} \ell} \geq V_{i_{3} \ell}
$$

Thus, it holds that $a_{i_{1}}>_{C} a_{i_{3}}$ and, therefore, $\geq_{C}$ is transitive. 
We conclude that $\geq_{C}$ is an order relation on $\mathscr{C}$.

As a consequence of this proposition, we conclude that the voting paradox results from the fact that the relation of the candidates with respect to all the other ones is disregarded in Condorcet's proposal.

Example 9.17 Consider a set of four candidates $\mathscr{C}=\{a, b, c, d\}$ and the profile of rankings $\mathscr{R}=\left(\succ_{i}\right)_{i=1}^{14}$ provided by fourteen voters shown in Table 9.2.

\begin{tabular}{|c|c|}
\hline$\# \succ_{i}$ & Rankings on $\mathscr{C}$ \\
\hline 6 & $c \succ b \succ a \succ d$ \\
5 & $a \succ d \succ b \succ c$ \\
3 & $b \succ a \succ d \succ c$ \\
\hline
\end{tabular}

Table 9.2: Frequency of the rankings on $\mathscr{C}$ expressed by 14 voters.

The votrix induced by $\mathscr{R}$ is given by:

$$
V_{\mathscr{R}}=\left(\begin{array}{cccc}
0 & 5 & 8 & 14 \\
9 & 0 & 8 & 9 \\
6 & 6 & 0 & 6 \\
0 & 5 & 8 & 0
\end{array}\right) .
$$

Note that, for instance, considering candidates a and $d$, it holds that:

$$
\begin{aligned}
14 & =V(a, d)>V(d, a)=0, \\
5 & =V(a, b) \geq V(d, b)=5, \\
8 & =V(a, c) \geq V(d, c)=8 .
\end{aligned}
$$

Thus, it holds that $a>_{C}$ d. In general, $\gg_{C}$ is given by:

$$
>_{C}=\{(a, d),(b, c),(b, d)\} .
$$

Obviously, $\geq_{C}$ is given by:

$$
\geq_{C}=\{(a, a),(a, d),(b, b),(b, c),(b, d),(c, c),(d, d)\} .
$$

This leads to the partially ordered set with the Hasse diagram in Figure 9.3. 


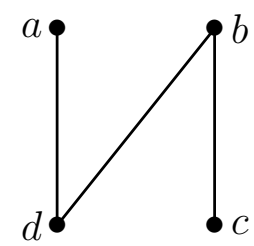

Figure 9.3: Hasse diagram of the order relation $\geq_{C}$.

\subsection{On being the best candidate}

In this section, we discuss several existing notions of winning candidate. We also propose two new types of winner, positioning them with respect to the rest of existing notions.

\subsubsection{Some existing notions of best candidate}

The choice of the winner of an election is an easy problem when all the voters agree on which candidate is the best. In that case, one talks about a unanimous winner [6].

Definition 9.18 Let $\mathscr{C}$ be a set of $k$ candidates and $\mathscr{R}$ be the profile of $r$ rankings on $\mathscr{C}$ given by the voters. A candidate $a_{i_{1}}$ is called the unanimous winner if, for any other candidate $a_{i_{2}} \in \mathscr{C} \backslash\left\{a_{i_{1}}\right\}$, it holds that $V_{i_{1} i_{2}}=r$.

However, the existence of a unanimous winner does not hold in almost any election. Instead, a weaker version is often considered requiring that more than half of the voters agree on the candidate that should be selected as the winner [107].

Definition 9.19 Let $\mathscr{C}$ be a set of $k$ candidates and $\mathscr{R}$ be the profile of $r$ rankings on $\mathscr{C}$ given by the voters. A candidate $a_{i_{1}}$ is called the majority winner if it holds that

$$
\#\left\{j \in\{1, \ldots, r\} \mid\left(\forall a_{i_{2}} \in \mathscr{C} \backslash\left\{a_{i_{1}}\right\}\right)\left(a_{i_{1}} \succ_{j} a_{i_{2}}\right)\right\}>\frac{r}{2} .
$$

Nevertheless, the existence of such a majority winner usually does not hold when more than two candidates are considered. 
In 1781, Borda [18] proposed to exploit the positions at which every candidate is ranked in order to determine a new type of winner: the Borda winner.

Definition 9.20 Let $\mathscr{C}$ be a set of $k$ candidates and $\mathscr{R}$ be the profile of $r$ rankings on $\mathscr{C}$ given by the voters. A candidate $a_{i_{1}}$ is called the Borda winner if, for any other candidate $a_{i_{2}} \in \mathscr{C} \backslash\left\{a_{i_{1}}\right\}$, it holds that $B\left(a_{i_{1}}\right)>B\left(a_{i_{2}}\right)$.

Remark 9.21 Some authors consider that a Borda winner is one that maximizes the Borda score, without requiring this maximum to be unique. Here, we require the Borda winner to be unique and, therefore, it is not assured to exist.

In 1785, Condorcet [35] gave another condition that at first sight seems to be sufficient for selecting a candidate as the winner. He advocated that, if a candidate is preferred to each of the other candidates by more than half of the voters, then it should be selected as the winner.

Definition 9.22 Let $\mathscr{C}$ be a set of $k$ candidates and $\mathscr{R}$ be the profile of $r$ rankings on $\mathscr{C}$ given by the voters. A candidate $a_{i_{1}}$ is called the Condorcet winner if, for any other candidate $a_{i_{2}} \in \mathscr{C} \backslash\left\{a_{i_{1}}\right\}$, it holds that $V_{i_{1} i_{2}}>\frac{r}{2}$.

Remark 9.23 Some authors consider that a Condorcet winner $a_{i_{1}}$ is one that satisfies that, for any other candidate $a_{i_{2}} \in \mathscr{C} \backslash\left\{a_{i_{1}}\right\}$, it holds that $V_{i_{1} i_{2}}>V_{i_{2} i_{1}}$. Note that both definitions are equivalent in the main setting considered in this dissertation where each voter expresses his/her preferences in the form of a ranking on the set of candidates.

However, as it disregards a big part of the information provided by the voters, this concept has withstood a big criticism from part of the scientific community [18, 142]. In the following example, we provide a case where the suitability of the Condorcet winner may be questioned.

Example 9.24 Consider a set of four candidates $\mathscr{C}=\{a, b, c, d\}$ and the profile of rankings $\mathscr{R}=\left(\succ_{i}\right)_{i=1}^{101}$ provided by 101 voters shown in Table 9.3. 


\begin{tabular}{|c|c|}
\hline$\# \succ_{i}$ & Rankings on $\mathscr{C}$ \\
\hline 51 & $a \succ b \succ c \succ d$ \\
50 & $b \succ c \succ d \succ a$ \\
\hline
\end{tabular}

Table 9.3: Frequency of the rankings on $\mathscr{C}$ expressed by 101 voters.

The votrix induced by $\mathscr{R}$ is given by:

$$
V_{\mathscr{R}}=\left(\begin{array}{cccc}
0 & 51 & 51 & 51 \\
50 & 0 & 101 & 101 \\
50 & 0 & 0 & 101 \\
50 & 0 & 0 & 0
\end{array}\right) .
$$

Note that although candidate $b$ - which is the Borda winner - seems to be the most reasonable choice, candidate a is the Condorcet winner (and the majority winner). However, it clearly is a questionable choice for this particular example. This counter-intuitive result derives from the fact that candidate $b$ is pairwisely better than candidates $c$ and $d$ and just a small change of one of the rankings $a \succ b \succ c \succ d$ into $b \succ a \succ c \succ d$ will also make $b$ pairwisely better than a. Many more changes are needed for turning candidate a pairwisely better than candidates $b$ and $c$.

\subsubsection{On being the quasipositionally best candidate}

The quasipositional winner is a candidate that is quasipositionally better than each candidate in the set of candidates.

Definition 9.25 Let $\mathscr{C}$ be a set of $k$ candidates and $\mathscr{R}$ be the profile of $r$ rankings on $\mathscr{C}$ given by the voters. A candidate $a_{i_{1}}$ is called the quasipositional winner if, for any other candidate $a_{i_{2}} \in \mathscr{C} \backslash\left\{a_{i_{1}}\right\}$, it holds that $a_{i_{1}} \gg_{B} a_{i_{2}}$.

It is known that the unanimous winner, the majority winner, the Borda winner and the Condorcet winner are not assured to exist but, if they do exist, they are unique. Note that this is also the case for the quasipositional winner.

Proposition 9.26 Let $\mathscr{C}$ be a set of $k$ candidates and $\mathscr{R}$ be the profile of $r$ rankings on $\mathscr{C}$ given by the voters. 
(i) A quasipositional winner might not exist.

(ii) If a quasipositional winner exists, then it is unique.

\section{Proof:}

Statement (i). It suffices to consider the profile of rankings given in Example 9.5 and the Hasse diagram represented in Figure 9.1.

Statement (ii). Suppose that $a_{i_{1}}$ and $a_{i_{2}}$ are two different quasipositional winners. It then holds that $a_{i_{1}}>_{B} a_{i_{2}}$ and $a_{i_{2}}>_{B} a_{i_{1}}$. By definition of $>_{B}$, it holds that $S_{i_{1} \ell}=S_{i_{2} \ell}$, for any $\ell \in\{1, \ldots, k\}$ and, at the same time, there exist $\ell_{1}, \ell_{2} \in\{1, \ldots, k\}$ such that $S_{i_{1} \ell_{1}}>S_{i_{2} \ell_{1}}$ and $S_{i_{1} \ell_{2}}<S_{i_{2} \ell_{2}}$, a contradiction.

Therefore, if a quasipositional winner exists, it is unique.

Evidently, the notion of quasipositional winner is a stronger notion than that of Borda winner and a weaker notion than that of unanimous winner.

Theorem 9.27 Let $\mathscr{C}$ be a set of $k$ candidates and $\mathscr{R}$ be the profile of $r$ rankings on $\mathscr{C}$ given by the voters.

(i) If the unanimous winner exists, then the quasipositional exists and it coincides with the unanimous winner.

(ii) If the quasipositional winner exists, then the unanimous winner might not exist.

\section{Proof:}

Statement (i). If a candidate $a_{i_{1}}$ is the unanimous winner, then it is ranked at the first position by every voter. For any other candidate $a_{i_{2}} \in \mathscr{C} \backslash\left\{a_{i_{1}}\right\}$, it trivially holds that $r=S_{i_{1} 1}>S_{i_{2} 1}=0$. Furthermore, for any $\ell \in\{2, \ldots, k\}$, it holds that $r=\sum_{j=1}^{\ell} S_{i_{1} \ell} \geq$ $\sum_{j=1}^{\ell} S_{i_{2} \ell}$. Thus, for any other candidate $a_{i_{2}} \in \mathscr{C} \backslash\left\{a_{i_{1}}\right\}$, it holds that $a_{i_{1}}>_{B} a_{i_{2}}$. Therefore, if the unanimous winner $a_{i_{1}}$ exists, then the quasipositional winner exists and it coincides with $a_{i_{1}}$.

Statement (ii). Consider the set of candidates $\mathscr{C}=\{a, b, c\}$ and the profile of rankings shown in Table 9.4 . 


\begin{tabular}{|c|c|}
\hline$\# \succ_{i}$ & Rankings on $\mathscr{C}$ \\
\hline 9 & $a \succ b \succ c$ \\
1 & $c \succ a \succ b$ \\
\hline
\end{tabular}

Table 9.4: Frequency of the rankings on $\mathscr{C}=\{a, b, c\}$ expressed by 10 voters.

Clearly, candidate $a$ is the quasipositional winner, but there does not exist a unanimous winner.

Theorem 9.28 Let $\mathscr{C}$ be a set of $k$ candidates and $\mathscr{R}$ be the profile of $r$ rankings on $\mathscr{C}$ given by the voters.

(i) If the quasipositional winner exists, then the Borda winner exists and it coincides with the pairwise winner.

(ii) If the Borda winner exists, then the quasipositional winner might not exist.

\section{Proof:}

Statement (i). Note that the Borda score of any candidate $a_{i} \in \mathscr{C}$ can be expressed in the following way as a linear combination of the components of the vector of positions of $a_{i}$ :

$$
B\left(a_{i}\right)=\sum_{\ell=1}^{k}(k-\ell) S_{i \ell} .
$$

We conclude that, as the vector of positions of candidate $a_{i}$ dominates the vector of positions of any other candidate (due to the fact that it is the quasipositional winner), then the Borda score of candidate $a_{i}$ is greater than the Borda score of any other candidate.

Statement (ii) immediately follows from Example 9.24 .

In Theorems 9.27 and 9.28 , we described the relation between the notions of unanimous winner, quasipositional winner and Borda winner. We will now prove that there is no immediate relation between the existence of the quasipositional winner and the existence of the Condorcet winner nor the majority winner. Note that, in case both the majority winner and the quasipositional winner exist, then they need to coincide. However, this is not the case for the quasipositional winner and the Condorcet winner. 
Theorem 9.29 Let $\mathscr{C}$ be a set of $k$ candidates and $\mathscr{R}$ be the profile of $r$ rankings on $\mathscr{C}$ given by the voters.

(i) If the quasipositional winner exists, then the majority winner might not exist.

(ii) If the majority winner exists, then the quasipositional winner might not exist.

(iii) If both the majority winner and the quasipositional winner exist, then they coincide.

\section{Proof:}

Statement (i). Consider the set of candidates $\mathscr{C}=\{a, b, c\}$ and the profile of rankings shown in Table 9.5.

\begin{tabular}{|c|c|}
\hline$\# \succ_{i}$ & Rankings on $\mathscr{C}$ \\
\hline 4 & $a \succ b \succ c$ \\
3 & $b \succ a \succ c$ \\
3 & $c \succ a \succ b$ \\
\hline
\end{tabular}

Table 9.5: Frequency of the rankings on $\mathscr{C}=\{a, b, c\}$ expressed by 10 voters.

Clearly, candidate $a$ is the quasipositional winner, but there does not exist a majority winner.

Statement (ii) immediately follows from Example 9.24 .

Statement (iii). Note that if the majority winner exists, then the first component of the vector of positions of this candidate needs to be greater than half of the number of voters. Therefore, this vector of positions cannot be dominated by any other vector of positions. We conclude that, if the quasipositional winner also exists, then the majority candidate and the quasipositional winner coincide.

Theorem 9.30 Let $\mathscr{C}$ be a set of $k$ candidates and $\mathscr{R}$ be the profile of $r$ rankings on $\mathscr{C}$ given by the voters.

(i) If the quasipositional winner exists, then the Condorcet winner might not exist.

(ii) If the Condorcet winner exists, then the quasipositional winner might not exist. 
(iii) If both the Condorcet winner and the quasipositional winner exist, then they might not coincide.

\section{Proof:}

Statement (i). Consider the set of candidates $\mathscr{C}=\{a, b, c\}$ and the profile of rankings shown in Table 9.6 .

\begin{tabular}{|c|c|}
\hline$\# \succ_{i}$ & Rankings on $\mathscr{C}$ \\
\hline 4 & $a \succ c \succ b$ \\
3 & $b \succ a \succ c$ \\
3 & $c \succ b \succ a$ \\
\hline
\end{tabular}

Table 9.6: Frequency of the rankings on $\mathscr{C}=\{a, b, c\}$ expressed by 10 voters.

Clearly, candidate $a$ is the quasipositional winner, but there does not exist a Condorcet winner.

Statement (ii) immediately follows from Example 9.24 .

Statement (iii). Consider the set of candidates $\mathscr{C}=\{a, b, c, d\}$ and the profile of rankings shown in Table 9.7 .

\begin{tabular}{|c|c|}
\hline$\# \succ_{i}$ & Rankings on $\mathscr{C}$ \\
\hline 4 & $a \succ c \succ b \succ d$ \\
3 & $b \succ a \succ c \succ d$ \\
3 & $d \succ b \succ a \succ c$ \\
\hline
\end{tabular}

Table 9.7: Frequency of the rankings on $\mathscr{C}=\{a, b, c\}$ expressed by 10 voters.

Note that candidate $a$ is the quasipositional winner and candidate $b$ is the Condorcet winner.

\subsubsection{On being the positionally best candidate}

The positional winner is a candidate that is positionally better than each candidate in the set of candidates. 
Definition 9.31 Let $\mathscr{C}$ be a set of $k$ candidates and $\mathscr{R}$ be the profile of $r$ rankings on $\mathscr{C}$ given by the voters. A candidate $a_{i_{1}}$ is called the positional winner if, for any other candidate $a_{i_{2}} \in \mathscr{C} \backslash\left\{a_{i_{1}}\right\}$, it holds that $a_{i_{1}}>_{R} a_{i_{2}}$.

The positional winner is not assured to exist but, if it does exist, it is unique.

Proposition 9.32 Let $\mathscr{C}$ be a set of $k$ candidates and $\mathscr{R}$ be the profile of $r$ rankings on $\mathscr{C}$ given by the voters.

(i) A positional winner might not exist.

(ii) If a positional winner exists, then it is unique.

\section{Proof:}

Statement (i). It suffices to consider the profile of rankings given in Example 9.10 and the Hasse diagram represented in Figure 9.2.

Statement (ii). Suppose that $a_{i_{1}}$ and $a_{i_{2}}$ are two different positional winners. It then holds that $a_{i_{1}}>_{R} a_{i_{2}}$ and $a_{i_{2}}>_{R} a_{i_{1}}$. Therefore, as a result of Proposition 9.8, it holds that $a_{i_{1}}>_{B} a_{i_{2}}$ and $a_{i_{2}}>_{B} a_{i_{1}}$. By definition of $>_{B}$, it holds that $S_{i_{1} \ell}=S_{i_{2} \ell}$, for any $\ell \in\{1, \ldots, k\}$ and, at the same time, there exist $\ell_{1}, \ell_{2} \in\{1, \ldots, k\}$ such that $S_{i_{1} \ell_{1}}>S_{i_{2} \ell_{1}}$ and $S_{i_{1} \ell_{2}}<S_{i_{2} \ell_{2}}$, a contradiction.

Therefore, if a positional winner exists, it is unique.

Evidently, the notion of positional winner is a stronger notion than that of quasipositional winner (and therefore than that of Borda winner) and a weaker notion than that of unanimous winner.

Theorem 9.33 Let $\mathscr{C}$ be a set of $k$ candidates and $\mathscr{R}$ be the profile of $r$ rankings on $\mathscr{C}$ given by the voters.

(i) If the unanimous winner exists, then the positional exists and it coincides with the unanimous winner.

(ii) If the positional winner exists, then the unanimous winner might not exist. 


\section{Proof:}

Statement (i). If a candidate $a_{i_{1}}$ is the unanimous winner, then it is ranked at the first position by every voter. For any other candidate $a_{i_{2}} \in \mathscr{C} \backslash\left\{a_{i_{1}}\right\}$, it trivially holds that $r=S_{i_{1} 1}>S_{i_{2} 1}=0$. Furthermore, for any $\ell \in\{2, \ldots, k\}$, it holds that $r=\sum_{j=1}^{\ell} S_{i_{1} \ell} \geq$ $\sum_{j=1}^{\ell} S_{i_{2} \ell}$. The previous result trivially holds for any sub-scorix of $S$ on any subset of the set of candidates containing $a_{i_{1}}$ and $a_{i_{2}}$. Thus, for any other candidate $a_{i_{2}} \in \mathscr{C} \backslash\left\{a_{i_{1}}\right\}$, it holds that $a_{i_{1}}>_{R} a_{i_{2}}$. Therefore, if the unanimous winner $a_{i_{1}}$ exists, then the positional winner exists and it coincides with $a_{i_{1}}$.

Statement (ii). Consider the set of candidates $\mathscr{C}=\{a, b, c\}$ and the profile of rankings shown in Table 9.8

\begin{tabular}{|c|c|}
\hline$\# \succ_{i}$ & Rankings on $\mathscr{C}$ \\
\hline 9 & $a \succ b \succ c$ \\
1 & $c \succ a \succ b$ \\
\hline
\end{tabular}

Table 9.8: Frequency of the rankings on $\mathscr{C}=\{a, b, c\}$ expressed by 10 voters.

Clearly, candidate $a$ is the positional winner, but there does not exist a unanimous winner.

Theorem 9.34 Let $\mathscr{C}$ be a set of $k$ candidates and $\mathscr{R}$ be the profile of $r$ rankings on $\mathscr{C}$ given by the voters.

(i) If the positional winner exists, then the quasipositional winner exists and it coincides with the positional winner.

(ii) If the quasipositional winner exists, then the quasipositional winner might not exist.

\section{Proof:}

Statement (i) trivially follows from Proposition 9.8 .

Statement (ii). Consider the set of candidates $\mathscr{C}=\{a, b, c\}$ and the profile of rankings shown in Table 9.9. 


\begin{tabular}{|c|c|}
\hline$\# \succ_{i}$ & Rankings on $\mathscr{C}$ \\
\hline 4 & $a \succ b \succ c$ \\
3 & $b \succ c \succ a$ \\
3 & $c \succ a \succ b$ \\
\hline
\end{tabular}

Table 9.9: Frequency of the rankings on $\mathscr{C}=\{a, b, c\}$ expressed by 10 voters.

Clearly, candidate $a$ is the quasipositional winner, but there does not exist a positional winner (it suffices to consider the sub-scorix on $\mathscr{C}^{\prime}=\{a, c\}$ to see that $a$ is not positionally better than $c)$.

In Theorems 9.33 and 9.34 , we described the relation between the notions of unanimous winner, positional winner and quasipositional winner. The positional winner is understood as a natural link between the Borda winner and the Condorcet winner, leading to the same social outcome.

Theorem 9.35 Let $\mathscr{C}$ be a set of $k$ candidates and $\mathscr{R}$ be the profile of $r$ rankings on $\mathscr{C}$ given by the voters.

(i) If the positional winner exists, then the Condorcet winner exists and it coincides with the positional winner.

(ii) If the Condorcet winner exists, then the positional winner might not exist.

\section{Proof:}

Statement (i). Note that a consequence of the relation $>_{R}$ is that, for any $a_{i_{1}}, a_{i_{2}} \in \mathscr{C}$ with $a_{i_{1}} \gg_{R} a_{i_{2}}$ and any $\mathscr{C}^{\prime} \subseteq \mathscr{C}$ such that $a_{i_{1}}, a_{i_{2}} \in \mathscr{C}^{\prime}$, it holds that $S_{i_{1}}^{\prime} \triangleright_{1} S_{i_{2}}^{\prime}$, where $S^{\prime}$ is the corresponding sub-scorix of $S$ on $\mathscr{C}^{\prime}$. The result immediately follows by considering $\mathscr{C}^{\prime}=\left\{a_{i_{1}}, a_{i_{2}}\right\}$. Therefore, if the positional winner exists, then the Condorcet winner exists and it coincides with $a_{i_{1}}$.

Statement (ii). Immediately follows from Example 9.24 .

As a result of Theorem 9.35, we conclude that every Condorcet method will select the positional winner (in case it exists) as the winner of the election. 
Corollary 9.36 Let $\mathscr{C}$ be a set of $k$ candidates and $\mathscr{R}$ be the profile of $r$ rankings on $\mathscr{C}$ given by the voters. If a candidate $a_{i} \in \mathscr{C}$ is the positional winner, then $a_{i}$ will be the winner for any Condorcet method.

Therefore, the most well-known Condorcet methods, such as the ones due to Kemeny [80], Copeland [40], Tideman [157], Dodgson [46] or Schulze [147], will select the positional winner (in case it exists) as the winner.

We will now prove that there is no immediate relation between the existence of the positional winner and the existence of the majority winner. Note that, in case both the majority winner and the positional winner exist, then they need to coincide.

Theorem 9.37 Let $\mathscr{C}$ be a set of $k$ candidates and $\mathscr{R}$ be the profile of $r$ rankings on $\mathscr{C}$ given by the voters.

(i) If the positional winner exists, then the majority winner might not exist.

(ii) If the majority winner exists, then the positional winner might not exist.

(iii) If both the majority winner and the quasipositional winner exist, then they coincide.

\section{Proof:}

Statement (i). Consider the set of candidates $\mathscr{C}=\{a, b, c\}$ and the profile of rankings shown in Table 9.10,

\begin{tabular}{|c|c|}
\hline$\# \succ_{i}$ & Rankings on $\mathscr{C}$ \\
\hline 4 & $a \succ b \succ c$ \\
4 & $a \succ c \succ b$ \\
3 & $b \succ a \succ c$ \\
3 & $b \succ c \succ a$ \\
3 & $c \succ a \succ b$ \\
3 & $c \succ b \succ a$ \\
\hline
\end{tabular}

Table 9.10: Frequency of the rankings on $\mathscr{C}=\{a, b, c\}$ expressed by 20 voters.

Candidate $a$ is the positional winner, but there does not exist a majority winner. 
Statement (ii) immediately follows from Example 9.24 .

Statement (iii). Note that if the majority winner exists, then it coincides with the Condorcet winner. As a result of Theorem 9.35, if the positional winner exists, then it coincides with the Condorcer winner. We conclude that, if both the majority winner and the quasipositional winner exist, then they coincide.

\subsubsection{On being the pairwisely best candidate}

From Example 9.24 we conclude that, although the Condorcet winner may seem a natural choice when it exists, it may also be subject of controversy. We propose here a stronger version of the Condorcet winner, the pairwise winner, which is a candidate that is pairwisely better than each candidate in the set of candidates.

Definition 9.38 Let $\mathscr{C}$ be a set of $k$ candidates and $\mathscr{R}$ be the profile of $r$ rankings on $\mathscr{C}$ given by the voters. A candidate $a_{i_{1}}$ is called the pairwise winner if, for any other candidate $a_{i_{2}} \in \mathscr{C} \backslash\left\{a_{i_{1}}\right\}$, it holds that $a_{i_{1}}>_{C} a_{i_{2}}$.

The pairwise winner is not assured to exist but, if it does exist, it is unique.

Proposition 9.39 Let $\mathscr{C}$ be a set of $k$ candidates and $\mathscr{R}$ be the profile of $r$ rankings on $\mathscr{C}$ given by the voters.

(i) A pairwise winner might not exist.

(ii) If a pairwise winner exists, then it is unique.

\section{Proof:}

Statement (i). It suffices to consider the profile of rankings given in Example 9.17 and the Hasse diagram represented in Figure 9.3.

Statement (ii). Suppose that $a_{i_{1}}$ and $a_{i_{2}}$ are two different pairwise winners. It then holds that $a_{i_{1}}>_{C} a_{i_{2}}$ and $a_{i_{2}}>_{C} a_{i_{1}}$. By definition of $>_{C}$, it holds that $V_{i_{1} i_{2}}>V_{i_{2} i_{1}}$ and that $V_{i_{2} i_{1}}>V_{i_{1} i_{2}}$, a contradiction. 
Therefore, if a pairwise winner exists, it is unique.

Evidently, the notion of pairwise winner is a stronger notion than that of Condorcet winner and a weaker notion than that of unanimous winner.

Theorem 9.40 Let $\mathscr{C}$ be a set of $k$ candidates and $\mathscr{R}$ be the profile of $r$ rankings on $\mathscr{C}$ given by the voters.

(i) If the unanimous winner exists, then the pairwise winner exists and it coincides with the unanimous winner.

(ii) If the pairwise winner exists, then the unanimous winner might not exist.

\section{Proof:}

Statement (i). If a candidate $a_{i_{1}}$ is the unanimous winner, then it is ranked at the first position by every voter. For any other candidate $a_{i_{2}} \in \mathscr{C} \backslash\left\{a_{i_{1}}\right\}$, it trivially holds that $r=V_{i_{1} i_{2}}>V_{i_{2} i_{1}}=0$. Furthermore, for any $a_{\ell} \in \mathscr{C} \backslash\left\{a_{i_{1}}, a_{i_{2}}\right\}$, it holds that $r=V_{i_{1} \ell} \geq V_{i_{2} \ell}$. Thus, for any other candidate $a_{i_{2}} \in \mathscr{C} \backslash\left\{a_{i_{1}}\right\}$, it holds that $a_{i_{1}}>_{C} a_{i_{2}}$. Therefore, if the unanimous winner $a_{i_{1}}$ exists, then the pairwise winner exists and it coincides with $a_{i_{1}}$.

Statement (ii). Consider the set of candidates $\mathscr{C}=\{a, b, c\}$ and the profile of rankings shown in Table 9.11.

\begin{tabular}{|c|c|}
\hline$\# \succ_{i}$ & Rankings on $\mathscr{C}$ \\
\hline 4 & $a \succ b \succ c$ \\
3 & $b \succ a \succ c$ \\
3 & $c \succ a \succ b$ \\
\hline
\end{tabular}

Table 9.11: Frequency of the rankings on $\mathscr{C}=\{a, b, c\}$ expressed by 10 voters.

Clearly, candidate $a$ is the pairwise winner, but there does not exist a unanimous winner.

Theorem 9.41 Let $\mathscr{C}$ be a set of $k$ candidates and $\mathscr{R}$ be the profile of $r$ rankings on $\mathscr{C}$ given by the voters. 
(i) If the pairwise winner exists, then the Condorcet winner exists and it coincides with the pairwise winner.

(ii) If the Condorcet winner exists, then the pairwise winner might not exist.

\section{Proof:}

Statement (i). Note that the first condition of the relation $>_{C}$ is that $a_{i_{1}}>_{C} a_{i_{2}}$ implies that $V_{i_{1} i_{2}}>\frac{r}{2}>V_{i_{2} i_{1}}$. Therefore, if the pairwise winner exists, then the Condorcet winner exists and it coincides with $a_{i_{1}}$.

Statement (ii). Immediately follows from Example 9.24 .

As a result of Theorem 9.41, we conclude that every Condorcet method will select the pairwise winner (in case it exists) as the winner of the election.

Corollary 9.42 Let $\mathscr{C}$ be a set of $k$ candidates and $\mathscr{R}$ be the profile of $r$ rankings on $\mathscr{C}$ given by the voters. If a candidate $a_{i} \in \mathscr{C}$ is the pairwise winner, then $a_{i}$ will be the winner for any Condorcet method.

Therefore, the most well-known Condorcet methods, such as the ones due to Kemeny [80], Copeland [40], Tideman [157], Dodgson [46] or Schulze [147], will select the pairwise winner (in case it exists) as the winner.

In Theorems 9.40 and 9.41, we described the relation between the notions of unanimous winner, pairwise winner and Condorcet winner. It is clear that the majority winner also lies between the unanimous winner and the Condorcet winner. We will now prove that, although they need to coincide if both exist, there is no immediate relation between the existence of the pairwise winner and the existence of the majority winner.

Theorem 9.43 Let $\mathscr{C}$ be a set of $k$ candidates and $\mathscr{R}$ be the profile of $r$ rankings on $\mathscr{C}$ given by the voters.

(i) If the pairwise winner exists, then the majority winner might not exist.

(ii) If the majority winner exists, then the pairwise winner might not exist. 
(iii) If both the majority winner and the pairwise winner exist, then they coincide.

\section{Proof:}

Statement (i). Consider the set of candidates $\mathscr{C}=\{a, b, c\}$ and the profile of rankings shown in Table 9.11. Clearly, candidate $a$ is the pairwise winner, but there does not exist a majority winner.

Statement (ii) immediately follows from Example 9.24 .

Statement (iii). Note that if the majority winner $a_{M}$ exists, then the Condorcet winner also exists and it coincides with $a_{M}$. As a result of Theorem 9.41, we know that, if the pairwise winner $a_{P}$ exists, then the Condorcet winner also exists and it coincides with $a_{P}$. Due to the uniqueness of the Condorcet winner, both the majority winner and the pairwise winner coincide when they exist.

An even more surprising observation is that the pairwise winner also turns out to be a stronger winner than the Borda winner. This implies that the pairwise winner is a type of winner that finally unites the works of Borda and Condorcet, assuring that both the Borda winner and the Condorcet winner lead to the same social outcome.

Theorem 9.44 Let $\mathscr{C}$ be a set of $k$ candidates and $\mathscr{R}$ be the profile of $r$ rankings on $\mathscr{C}$ given by the voters.

(i) If the pairwise winner exists, then the Borda winner exists and it coincides with the pairwise winner.

(ii) If the Borda winner exists, then the pairwise winner might not exist.

\section{Proof:}

Statement (i). Let $a_{P}$ be the pairwise winner. By definition, for any other candidate $a_{i} \in \mathscr{C} \backslash\left\{a_{P}\right\}$, it holds that $a_{P}>_{C} a_{i}$. As a result of Proposition 9.15, it follows that $B\left(a_{P}\right)>B\left(a_{i}\right)$. We conclude that $a_{P}$ is the Borda winner.

Statement (ii) immediately follows from Example 9.24 . 
Although it obeys a stronger condition than that of the Borda and Condorcet winners, the pairwise winner is the actual cornerstone of social choice theory; it is under the absence of the pairwise winner when the need of making a decision arises.

\subsubsection{Relation between the (quasi)positional and the pairwise winners}

We conclude this section by comparing the (quasi)positional winner and the pairwise winner.

Theorem 9.45 Let $\mathscr{C}$ be a set of $k$ candidates and $\mathscr{R}$ be the profile of $r$ rankings on $\mathscr{C}$ given by the voters.

(i) If the pairwise winner exists, then the quasipositional winner might not exist.

(ii) If the quasipositional winner exists, then the pairwise winner might not exist.

(iii) If both the quasipositional winner and the pairwise winner exist, then they coincide.

\section{Proof:}

Statement (i). Consider the set of candidates $\mathscr{C}=\{a, b, c\}$ and the profile of rankings shown in Table 9.12 .

\begin{tabular}{|c|c|}
\hline$\# \succ_{i}$ & Rankings on $\mathscr{C}$ \\
\hline 4 & $b \succ a \succ c$ \\
3 & $a \succ c \succ b$ \\
3 & $c \succ a \succ b$ \\
\hline
\end{tabular}

Table 9.12: Frequency of the rankings on $\mathscr{C}=\{a, b, c\}$ expressed by 10 voters.

Clearly, candidate $a$ is the pairwise winner, but there does not exist a quasipositional winner.

Statement (ii). Consider the set of candidates $\mathscr{C}=\{a, b, c\}$ and the profile of rankings shown in Table 9.13 . 


\begin{tabular}{|c|c|}
\hline$\# \succ_{i}$ & Rankings on $\mathscr{C}$ \\
\hline 5 & $a \succ b \succ c$ \\
2 & $b \succ c \succ a$ \\
2 & $c \succ a \succ b$ \\
1 & $b \succ a \succ c$ \\
\hline
\end{tabular}

Table 9.13: Frequency of the rankings on $\mathscr{C}=\{a, b, c\}$ expressed by 10 voters.

Clearly, candidate $a$ is the quasipositional winner, but there does not exist a pairwise winner.

Statement (iii). As a result of Theorem 9.28, we know that, if the quasipositional winner exists, then the Borda winner exists and it coincides with the quasipositional winner. As a result of Theorem 9.44, we know that, if the pairwise winner exists, then the Borda winner exists and it coincides with the pairwise winner. We conclude that, if both the quasipositional winner and the pairwise winner exist, then the Borda winner exists and all three need to coincide.

Theorem 9.46 Let $\mathscr{C}$ be a set of $k$ candidates and $\mathscr{R}$ be the profile of $r$ rankings on $\mathscr{C}$ given by the voters.

(i) If the pairwise winner exists, then the positional winner might not exist.

(ii) If the positional winner exists, then the pairwise winner might not exist.

(iii) If both the positional winner and the pairwise winner exist, then they coincide.

\section{Proof:}

Statement (i). Consider the set of candidates $\mathscr{C}=\{a, b, c\}$ and the profile of rankings shown in Table 9.12. Clearly, candidate $a$ is the pairwise winner, but there does not exist a positional winner.

Statement (ii). Consider the set of candidates $\mathscr{C}=\{a, b, c\}$ and the profile of rankings shown in Table 9.13. Clearly, candidate $a$ is the positional winner, but there does not exist a pairwise winner. 
Statement (iii). As a result of Theorems 9.28 and 9.34, we know that, if the positional winner exists, then the Borda winner exists and it coincides with the positional winner. As a result of Theorem 9.44, we know that, if the pairwise winner exists, then the Borda winner exists and it coincides with the pairwise winner. We conclude that, if both the positional winner and the pairwise winner exist, then the Borda winner exists and all three need to coincide.

Figure 9.4 displays the relation between the different types of winners analysed in this chapter. In this figure, an arrow indicates that, if the winner from which the arrow starts exists, then the winner to which the arrow points also exists and it coincides with the former.

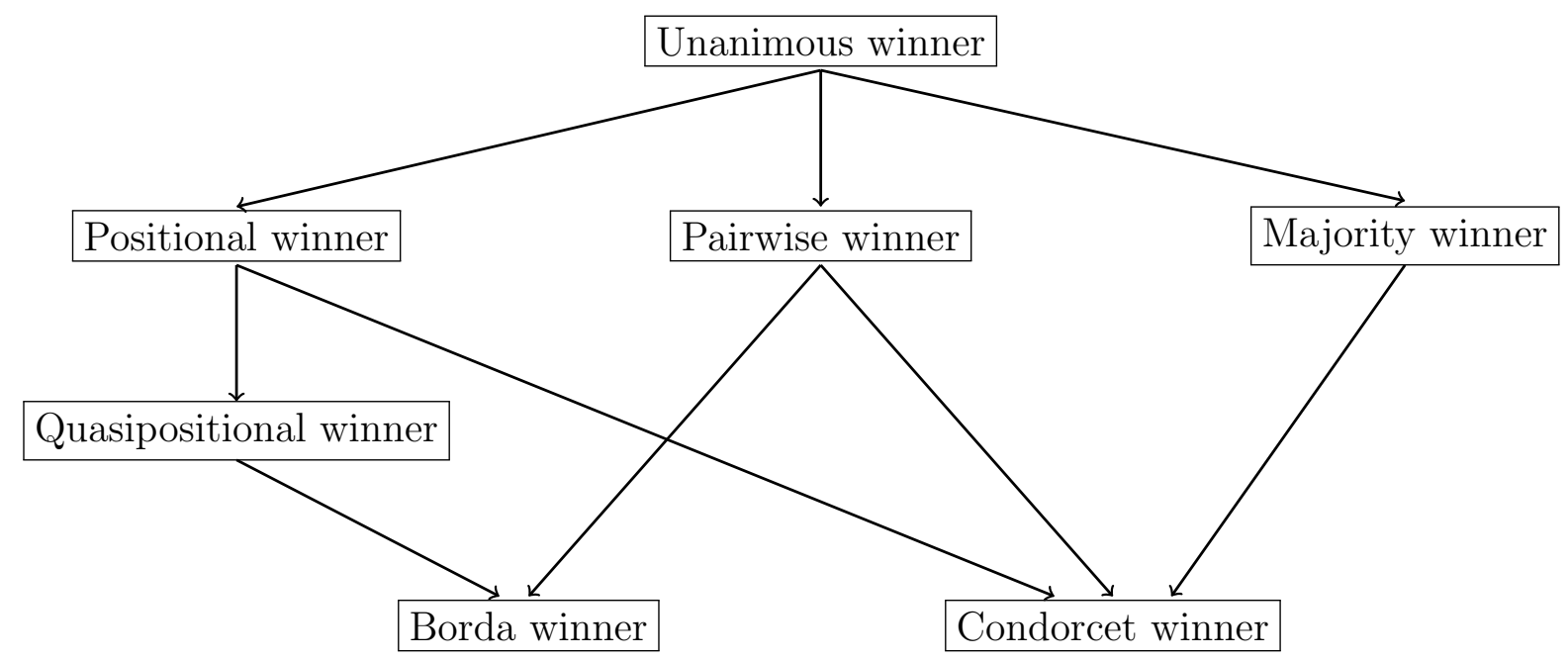

Figure 9.4: Relation between the different types of winners.

\subsection{On being a potential best candidate}

In this section, we discuss what candidates could be considered potential winning candidates considering either quasipositional or pairwise information. In order to do so, we define the undominated sets for the relations $>_{B},>_{R}$ and $>_{C}$. 


\subsubsection{The quasipositionally undominated set}

In case the quasipositional winner does not exist, a natural solution we propose to consider the quasipositionally undominated set, i.e., the set of all elements for which there exists no quasipositionally better element.

Definition 9.47 Let $\mathscr{C}$ be a set of $k$ candidates and $\mathscr{R}$ be the profile of $r$ rankings on $\mathscr{C}$ given by the voters. The quasipositionally undominated set is the subset $\mathscr{U}_{B}$ of $\mathscr{C}$ defined as

$$
\mathscr{U}_{B}=\left\{a_{i_{1}} \in \mathscr{C} \mid\left(\nexists a_{i_{2}} \in \mathscr{C}\right)\left(a_{i_{2}}>_{B} a_{i_{1}}\right)\right\}
$$

Note that there always exists at least one candidate that belongs to the quasipositionally undominated set.

Proposition 9.48 Let $\mathscr{C}$ be a set of $k$ candidates and $\mathscr{R}$ be the profile of $r$ rankings on $\mathscr{C}$ given by the voters. It holds that $\mathscr{U}_{B} \neq \emptyset$.

Proof: The result is straightforward due to the fact that $\geq_{B}$ defines an order relation on $\mathscr{C}$, that $\mathscr{C}$ is a finite set and that the quasipositionally undominated set coincides with the set of maximal elements of $\geq_{B}$.

The existence of the quasipositional winner is equivalent to the fact that the quasipositionally undominated set is a singleton.

Proposition 9.49 Let $\mathscr{C}$ be a set of $k$ candidates and $\mathscr{R}$ be the profile of $r$ rankings on $\mathscr{C}$ given by the voters. A candidate $a_{P}$ is the quasipositional winner if and only if $\mathscr{U}_{B}=\left\{a_{P}\right\}$.

Proof: Let $a_{P}$ be the quasipositional winner. By definition of the quasipositional winner, for any other candidate $a_{i} \in \mathscr{C} \backslash\left\{a_{P}\right\}$, it holds that $a_{P}>_{B} a_{i}$. It obviously follows that the unique element that can belong to the quasipositionally undominated set is $a_{P}$. As a result of Proposition 9.48, we know that $\mathscr{U}_{B} \neq \emptyset$. Therefore, we conclude that $\mathscr{U}_{B}=\left\{a_{P}\right\}$.

Let $\mathscr{U}_{B}=\left\{a_{P}\right\}$. Suppose that $a_{P}$ is not the quasipositional winner. Therefore, there exists $a_{i_{1}} \in \mathscr{C} \backslash\left\{a_{P}\right\}$ such that it does not hold that $a_{P}>_{B} a_{i_{1}}$. As $a_{i_{1}} \notin \mathscr{U}_{B}$ and $>_{B}$ is an order 
relation on a finite set, there exists a maximal element $a_{i_{2}} \in \mathscr{C} \backslash\left\{a_{P}, a_{i_{1}}\right\}$ of $>_{B}$ such that $a_{i_{2}} \gg_{B} a_{i_{1}}$. Note that $a_{i_{2}}$ is a maximal element of $>_{B}$ not belonging to $\mathscr{U}_{B}$, a contradiction.

\subsubsection{The positionally undominated set}

As discussed in the previous sections, the positional winner seems to be an intuitive winner in case it exists. In case it does not exist, a natural solution is to consider the positionally undominated set, i.e., the set of all elements for which there exists no positionally better element. This set is always not empty and, as we will show next, in case the positional winner does not exist, contains at least two elements. As it likely is a proper subset of the set of candidates itself, the choice of the best candidate can be restricted to that subset.

Definition 9.50 Let $\mathscr{C}$ be a set of $k$ candidates and $\mathscr{R}$ be the profile of $r$ rankings on $\mathscr{C}$ given by the voters. The positionally undominated set is the subset $\mathscr{U}_{R}$ of $\mathscr{C}$ defined as

$$
\mathscr{U}_{R}=\left\{a_{i_{1}} \in \mathscr{C} \mid\left(\nexists a_{i_{2}} \in \mathscr{C}\right)\left(a_{i_{2}}>_{R} a_{i_{1}}\right)\right\} .
$$

Remark 9.51 As a result of Proposition 9.8, we know that $>_{R} \subseteq>_{B}$. Therefore, it trivially holds that

$$
\mathscr{U}_{B} \subseteq \mathscr{U}_{R}
$$

Note that there always exists at least one candidate that belongs to the positionally undominated set.

Proposition 9.52 Let $\mathscr{C}$ be a set of $k$ candidates and $\mathscr{R}$ be the profile of $r$ rankings on $\mathscr{C}$ given by the voters. It holds that $\mathscr{U}_{R} \neq \emptyset$.

Proof: The result is straightforward due to the fact that $\geq_{R}$ defines an order relation on $\mathscr{C}$, that $\mathscr{C}$ is a finite set and that the positionally undominated set coincides with the set of maximal elements of $\geq_{R}$.

The existence of the positional winner is equivalent to the fact that the positionally undominated set is a singleton. 
Proposition 9.53 Let $\mathscr{C}$ be a set of $k$ candidates and $\mathscr{R}$ be the profile of $r$ rankings on $\mathscr{C}$ given by the voters. A candidate $a_{P}$ is the positional winner if and only if $\mathscr{U}_{R}=\left\{a_{P}\right\}$.

Proof: Let $a_{P}$ be the positional winner. By definition of the positional winner, for any other candidate $a_{i} \in \mathscr{C} \backslash\left\{a_{P}\right\}$, it holds that $a_{P}>_{R} a_{i}$. It obviously follows that the unique element that can belong to the positionally undominated set is $a_{P}$. As a result of Proposition 9.52, we know that $\mathscr{U}_{R} \neq \emptyset$. Therefore, we conclude that $\mathscr{U}_{R}=\left\{a_{P}\right\}$.

Let $\mathscr{U}_{R}=\left\{a_{P}\right\}$. Suppose that $a_{P}$ is not the positional winner. Therefore, there exists $a_{i_{1}} \in \mathscr{C} \backslash\left\{a_{P}\right\}$ such that it does not hold that $a_{P}>_{R} a_{i_{1}}$. As $a_{i_{1}} \notin \mathscr{U}_{R}$ and $>_{R}$ is an order relation on a finite set, there exists a maximal element $a_{i_{2}} \in \mathscr{C} \backslash\left\{a_{P}, a_{i_{1}}\right\}$ of $>_{R}$ such that $a_{i_{2}}>_{R} a_{i_{1}}$. Note that $a_{i_{2}}$ is a maximal element of $>_{R}$ not belonging to $\mathscr{U}_{R}$, a contradiction.

\subsubsection{The pairwisely undominated set}

Similarly, the pairwise winner also seems to be an intuitive winner in case it exists. In case it does not exist, a natural solution is to consider the pairwisely undominated set, i.e., the set of all elements for which there exists no pairwisely better element. Like the (quasi)positionally undominated set, the pairwisely undominated set is always not empty and, in case the pairwise winner does not exist, contains at least two elements.

Definition 9.54 Let $\mathscr{C}$ be a set of $k$ candidates and $\mathscr{R}$ be the profile of $r$ rankings on $\mathscr{C}$ given by the voters. The pairwisely undominated set is the subset $\mathscr{U}_{C}$ of $\mathscr{C}$ defined as

$$
\mathscr{U}_{C}=\left\{a_{i_{1}} \in \mathscr{C} \mid\left(\nexists a_{i_{2}} \in \mathscr{C}\right)\left(a_{i_{2}}>_{C} a_{i_{1}}\right)\right\} .
$$

Note that there always exists at least one candidate that belongs to the pairwisely undominated set.

Proposition 9.55 Let $\mathscr{C}$ be a set of $k$ candidates and $\mathscr{R}$ be the profile of $r$ rankings on $\mathscr{C}$ given by the voters. It holds that $\mathscr{U}_{C} \neq \emptyset$.

Proof: The result is straightforward due to the fact that $\geq_{C}$ defines an order relation on $\mathscr{C}$, that $\mathscr{C}$ is a finite set and that the pairwisely undominated set coincides with the set of 
maximal elements of $\gtrsim_{C}$.

The existence of the pairwise winner is equivalent to the fact that the pairwisely undominated set is a singleton.

Proposition 9.56 Let $\mathscr{C}$ be a set of $k$ candidates and $\mathscr{R}$ be the profile of $r$ rankings on $\mathscr{C}$ given by the voters. A candidate $a_{P}$ is the pairwise winner if and only if $\mathscr{U}_{C}=\left\{a_{P}\right\}$.

Proof: Let $a_{P}$ be the pairwise winner. By definition of the pairwise winner, for any other candidate $a_{i} \in \mathscr{C} \backslash\left\{a_{P}\right\}$, it holds that $a_{P} \gg_{C} a_{i}$. It obviously follows that the unique element that can belong to the pairwisely undominated set is $a_{P}$. As a result of Proposition 9.55, we know that $\mathscr{U}_{C} \neq \emptyset$. Therefore, we conclude that $\mathscr{U}_{C}=\left\{a_{P}\right\}$.

Let $\mathscr{U}_{C}=\left\{a_{P}\right\}$. Suppose that $a_{P}$ is not the pairwise winner. Therefore, there exists $a_{i_{1}} \in \mathscr{C} \backslash\left\{a_{P}\right\}$ such that it does not hold that $a_{P}>_{C} a_{i_{1}}$. As $a_{i_{1}} \notin \mathscr{U}_{C}$ and $>_{C}$ is an order relation on a finite set, there exists a maximal element $a_{i_{2}} \in \mathscr{C} \backslash\left\{a_{P}, a_{i_{1}}\right\}$ of $>_{C}$ such that $a_{i_{2}} \gg_{C} a_{i_{1}}$. Note that $a_{i_{2}}$ is a maximal element of $>_{C}$ not belonging to $\mathscr{U}_{C}$, a contradiction.

\subsection{On being the best ranking}

In this section, we discuss the case where the partial order relations "being quasipositionally as good as" and "being pairwisely as good as" turn out to be total order relations on the set of candidates.

\subsubsection{On being the quasipositionally best ranking}

The fact that the scorix is monotone w.r.t. a ranking $\succ$ on the set of candidates turns out to be equivalent to the fact that every two candidates $a_{i_{1}}, a_{i_{2}} \in \mathscr{C}$ such that $a_{i_{1}} \succ a_{i_{2}}$ satisfy that $a_{i_{1}}$ is quasipositionally as good as $a_{i_{2}}$. 
Proposition 9.57 Let $\mathscr{C}$ be a set of $k$ candidates, $\mathscr{R}$ be the profile of $r$ rankings on $\mathscr{C}$ given by the voters, $S$ be the scorix induced by $\mathscr{R}$ and $\succ$ be a ranking on $\mathscr{C}$. The following two statements are equivalent:

(i) $S$ is monotone w.r.t. $\succ$.

(ii) For any $a_{i_{1}}, a_{i_{2}} \in \mathscr{C}$ such that $a_{i_{1}} \succ a_{i_{2}}$, it holds that $a_{i_{1}} \gtrsim_{B} a_{i_{2}}$.

Proof: The proof is straightforward by definition of monotonicity of the scorix and of the relation 'being quasipositionally as good as'.

Unfortunately, monotonicity of the scorix might hold w.r.t. different rankings on the set of candidates. Instead of the relation 'being quasipositionally as good as', we propose to consider the relation 'being quasipositionally better than' in the characterization of monotonicity of the scorix given in Proposition 9.57, leading to a strict version of the property of monotonicity of the scorix. This strictness leads to the uniqueness of the ranking w.r.t. which monotonicity of the scorix holds, something that was not assured before.

Definition 9.58 Let $\mathscr{C}$ be a set of $k$ candidates and $\mathscr{R}$ be the profile of $r$ rankings on $\mathscr{C}$ given by the voters. The scorix $S$ induced by $\mathscr{R}$ is said to be strictly monotone w.r.t. a ranking $\succ$ on $\mathscr{C}$ if, for any $a_{i_{1}}, a_{i_{2}} \in \mathscr{C}$ such that $a_{i_{1}} \succ a_{i_{2}}$, it holds that $a_{i_{1}} \gg_{B} a_{i_{2}}$.

Strict monotonicity of the scorix can be understood as the requirement for the relation $>_{B}$ to be a strict total order relation on the set of candidates. This property results in an intuitive condition for the Borda ranking to exist.

Theorem 9.59 Let $\mathscr{C}$ be a set of $k$ candidates, $\mathscr{R}$ be the profile of $r$ rankings on $\mathscr{C}$ given by the voters and $S$ be the scorix induced by $\mathscr{R}$. If $S$ is strictly monotone w.r.t. a ranking $\succ$ on $\mathscr{C}$, then $\succ$ is the Borda ranking.

Proof: It follows from the fact that, for any $a_{i_{1}}, a_{i_{2}} \in \mathscr{C}, a_{i_{1}}>_{B} a_{i_{2}}$ implies that $B\left(a_{i_{1}}\right)>B\left(a_{i_{2}}\right)$. 
The search for the ranking that is the closest to imposing strict monotonicity on the scorix will lead to the definition of different ranking rules depending on the chosen notion of closeness.

\subsubsection{On being the positionally best ranking}

The fact that the scorix is recursively monotone w.r.t. a ranking $\succ$ on the set of candidates turns out to be equivalent to the fact that every two candidates $a_{i_{1}}, a_{i_{2}} \in \mathscr{C}$ such that $a_{i_{1}} \succ a_{i_{2}}$ satisfy that $a_{i_{1}}$ is positionally as good as $a_{i_{2}}$.

Proposition 9.60 Let $\mathscr{C}$ be a set of $k$ candidates, $\mathscr{R}$ be the profile of $r$ rankings on $\mathscr{C}$ given by the voters, $S$ be the scorix induced by $\mathscr{R}$ and $\succ$ be a ranking on $\mathscr{C}$. The following two statements are equivalent:

(i) $S$ is recursively monotone w.r.t. $\succ$.

(ii) For any $a_{i_{1}}, a_{i_{2}} \in \mathscr{C}$ such that $a_{i_{1}} \succ a_{i_{2}}$, it holds that $a_{i_{1}} \gtrsim_{R} a_{i_{2}}$.

Proof: The proof is straightforward by definition of recursive monotonicity of the scorix and of the relation 'being positionally as good as'.

Analogously, the fact that the scorix is strictly recursively monotone w.r.t. a ranking $\succ$ on the set of candidates turns out to be equivalent to the fact that every two candidates $a_{i_{1}}, a_{i_{2}} \in \mathscr{C}$ such that $a_{i_{1}} \succ a_{i_{2}}$ satisfy that $a_{i_{1}}$ is positionally better than $a_{i_{2}}$.

Proposition 9.61 Let $\mathscr{C}$ be a set of $k$ candidates, $\mathscr{R}$ be the profile of $r$ rankings on $\mathscr{C}$ given by the voters, $S$ be the scorix induced by $\mathscr{R}$ and $\succ$ be a ranking on $\mathscr{C}$. The following two statements are equivalent:

(i) $S$ is strictly recursively monotone w.r.t. $\succ$.

(ii) For any $a_{i_{1}}, a_{i_{2}} \in \mathscr{C}$ such that $a_{i_{1}} \succ a_{i_{2}}$, it holds that $a_{i_{1}} \gg_{R} a_{i_{2}}$.

Proof: The proof is straightforward by definition of strict recursive monotonicity of the scorix and of the relation 'being positionally better than'. 
Strict monotonicity of the scorix can be understood as the requirement for the relation $>_{R}$ to be a strict total order relation on the set of candidates. This property results in an intuitive condition for the Borda ranking and the Condorcet ranking to finally agree.

Theorem 9.62 Let $\mathscr{C}$ be a set of $k$ candidates, $\mathscr{R}$ be the profile of $r$ rankings on $\mathscr{C}$ given by the voters and $S$ be the scorix induced by $\mathscr{R}$. If $S$ is strictly recursively monotone w.r.t. a ranking $\succ$ on $\mathscr{C}$, then $\succ$ is both the Borda ranking and the Condorcet ranking.

Proof: It follows from the fact that, for any $a_{i_{1}}, a_{i_{2}} \in \mathscr{C}, a_{i_{1}}>_{R} a_{i_{2}}$ implies that $B\left(a_{i_{1}}\right)>B\left(a_{i_{2}}\right)$ and $V_{i_{1} i_{2}}>V_{i_{2} i_{1}}$.

The search for the ranking that is the closest to imposing strict recursive monotonicity on the scorix will lead to the definition of different ranking rules depending on the chosen notion of closeness.

\subsubsection{On being the pairwisely best ranking}

The fact that the votrix is monotone w.r.t. a ranking $\succ$ on the set of candidates turns out to be equivalent to the fact that every two candidates $a_{i_{1}}, a_{i_{2}} \in \mathscr{C}$ such that $a_{i_{1}} \succ a_{i_{2}}$ satisfy that $a_{i_{1}}$ is pairwisely as good as $a_{i_{2}}$.

Proposition 9.63 Let $\mathscr{C}$ be a set of $k$ candidates, $\mathscr{R}$ be the profile of $r$ rankings on $\mathscr{C}$ given by the voters, $V$ be the votrix induced by $\mathscr{R}$ and $\succ$ be a ranking on $\mathscr{C}$. The following two statements are equivalent:

(i) $V$ is monotone w.r.t. $\succ$.

(ii) For any $a_{i_{1}}, a_{i_{2}} \in \mathscr{C}$ such that $a_{i_{1}} \succ a_{i_{2}}$, it holds that $a_{i_{1}} \gtrsim_{C} a_{i_{2}}$.

Proof: The implication (i) $\Rightarrow$ (ii). Firstly, for any $a_{i_{1}}, a_{i_{2}} \in \mathscr{C}$ such that $a_{i_{1}} \succ a_{i_{2}}$, it holds that $\left(a_{i_{1}}, a_{i_{2}}\right) \sqsupset\left(a_{i_{2}}, a_{i_{1}}\right)$. Therefore, as $V$ is monotone w.r.t. $\succ$, it holds that $V_{i_{1} i_{2}} \geq V_{i_{2} i_{1}}$. Secondly, for any $a_{i_{1}}, a_{i_{2}} \in \mathscr{C}$ such that $a_{i_{1}} \succ a_{i_{2}}$ and any $a_{i_{3}} \in \mathscr{C} \backslash\left\{a_{i_{1}}, a_{i_{2}}\right\}$, it holds that 
$\left(a_{i_{1}}, a_{i_{3}}\right) \sqsupset\left(a_{i_{2}}, a_{i_{3}}\right)$. Therefore, as $V$ is monotone w.r.t. $\succ$, it holds that $V_{i_{1} i_{3}} \geq V_{i_{2} i_{3}}$. Thus, we conclude that, for any $a_{i_{1}}, a_{i_{2}} \in \mathscr{C}$ such that $a_{i_{1}} \succ a_{i_{2}}$, it holds that $a_{i_{1}} \gtrsim_{C} a_{i_{2}}$.

The implication (ii) $\Rightarrow\left(\right.$ i). Let us assume that, for any $a_{i_{1}}, a_{i_{2}} \in \mathscr{C}$ such that $a_{i_{1}} \succ a_{i_{2}}$, it holds that $a_{i_{1}} \gtrsim_{C} a_{i_{2}}$. By definition of $\sqsupset$, for any $\left(a_{i_{1}}, a_{j_{1}}\right),\left(a_{i_{2}}, a_{j_{2}}\right) \in \mathscr{C}_{\neq}^{2}$ such that $\left(a_{i_{1}}, a_{j_{1}}\right) \sqsupset\left(a_{i_{2}}, a_{j_{2}}\right)$, it holds that

$$
\left(a_{i_{1}} \succeq a_{i_{2}}\right) \wedge\left(a_{j_{2}} \succeq a_{j_{1}}\right) \wedge\left(a_{i_{1}} \succ a_{i_{2}} \vee a_{j_{2}} \succ a_{j_{1}}\right)
$$

We distinguish three cases: if $a_{i_{1}} \succ a_{i_{2}}$ and $a_{j_{2}} \succ a_{j_{1}}$, then it holds that $a_{i_{1}} \gtrsim_{C} a_{i_{2}}$ and $a_{j_{2}} \gtrsim_{C} a_{j_{1}}$, therefore it holds that $V_{i_{1} j_{1}} \geq V_{i_{2} j_{1}} \geq V_{i_{2} j_{2}}$; if $a_{i_{1}} \succ a_{i_{2}}$ and $a_{j_{2}}=a_{j_{1}}$, then it holds that $a_{i_{1}} \gtrsim_{C} a_{i_{2}}$, therefore it holds that $V_{i_{1} j_{1}} \geq V_{i_{2} j_{1}}=V_{i_{2} j_{2}}$; if $a_{i_{1}}=a_{i_{2}}$ and $a_{j_{2}} \succ a_{j_{1}}$, then it holds that $a_{j_{2}} \gtrsim_{C} a_{j_{1}}$, therefore it holds that $V_{i_{1} j_{1}}=V_{i_{2} j_{1}} \geq V_{i_{2} j_{2}}$. We conclude that $V_{i_{1} j_{1}} \geq V_{i_{2} j_{2}}$ and, therefore, $V$ is monotone w.r.t. $\succ$.

Unfortunately, monotonicity of the votrix might hold w.r.t. different rankings on the set of candidates. Instead of the relation 'being pairwisely as good as', we propose to consider the relation 'being pairwisely better than' in the characterization of monotonicity of the votrix given in Proposition 9.63, leading to a strict version of the property of monotonicity of the votrix. This strictness leads to the uniqueness of the ranking w.r.t. which monotonicity of the votrix holds, something that was not assured before.

Definition 9.64 Let $\mathscr{C}$ be a set of $k$ candidates and $\mathscr{R}$ be the profile of $r$ rankings on $\mathscr{C}$ given by the voters. The votrix $V$ induced by $\mathscr{R}$ is said to be strictly monotone w.r.t. a ranking $\succ$ on $\mathscr{C}$ if, for any $a_{i_{1}}, a_{i_{2}} \in \mathscr{C}$ such that $a_{i_{1}} \succ a_{i_{2}}$, it holds that $a_{i_{1}}>_{C} a_{i_{2}}$.

Strict monotonicity of the votrix can be understood as the requirement for the relation $>$ to be a strict total order relation on the set of candidates. This property results in an intuitive condition for the Borda ranking and the Condorcet ranking to finally agree.

Theorem 9.65 Let $\mathscr{C}$ be a set of $k$ candidates, $\mathscr{R}$ be the profile of $r$ rankings on $\mathscr{C}$ given by the voters and $V$ be the votrix induced by $\mathscr{R}$. If $V$ is strictly monotone w.r.t. a ranking $\succ$ on $\mathscr{C}$, then $\succ$ is both the Borda ranking and the Condorcet ranking.

Proof: From Proposition 9.15, it follows that, for any $a_{i_{1}}, a_{i_{2}} \in \mathscr{C}, a_{i_{1}}>a_{i_{2}}$ implies that $B\left(a_{i_{1}}\right)>B\left(a_{i_{2}}\right)$. In addition, by definition of $>$, it follows that, for any $a_{i_{1}}, a_{i_{2}} \in \mathscr{C}$, 
$a_{i_{1}}>a_{i_{2}}$ implies that $V_{i_{1} i_{2}}>V_{i_{2} i_{1}}$. We conclude that $\succ$ is both the Borda ranking and the Condorcet ranking.

The search for the ranking that is the closest to imposing strict monotonicity on the votrix will lead to the definition of different ranking rules depending on the chosen notion of closeness.

\subsection{A new social choice function}

As discussed in this chapter, the pairwise winner is a natural type of winner finally uniting the Borda winner and the Condorcet winner. In this section, we recall part of the work in [120], where we introduced a social choice function that selects the candidate(s) that is the closest to becoming the pairwise winner. Of course, this closeness can be measured in many different ways. For instance, the search for the Condorcet winner is addressed by means of a distance function at the votrix level and of a distance function at the profile level by two prominent methods: Condorcet's least-reversal method [99] and the method of Dodgson [46].

Here, we consider to assign a score to each candidate $a_{i}$ according to how close it is to becoming the pairwise winner. In order to fulfill the conditions of Definition 9.11, we need to increase $V_{i \ell}$ until the following three conditions hold: (a) $V_{i \ell}$ is greater than $V_{\ell i}$ (i.e., it is greater than or equal to $\frac{r}{2}+1$, in case $r$ is even, or $\frac{r+1}{2}$, in case $r$ is odd), for any other candidate $a_{\ell}$; (b) $V_{i \ell}$ is greater than or equal to $V_{t \ell}$, for any other candidates $a_{\ell}$ and $a_{t}$; (c) $V_{\ell i}$ is smaller than or equal to $V_{\ell t}$, for any other candidates $a_{\ell}$ and $a_{t}$. Analogously, we need to decrease the counterpart $V_{\ell i}$ until the following three conditions hold: (a) $V_{\ell i}$ is smaller than $V_{i \ell}$; (b) $V_{\ell i}$ is smaller than or equal to $V_{\ell t}$, for any other candidates $a_{\ell}$ and $a_{t}$; (c) $V_{i \ell}$ is greater than or equal to $V_{t \ell}$, for any other candidates $a_{\ell}$ and $a_{t}$. The score for each candidate $a_{i}$ is then given by $]^{7}$

$$
\begin{aligned}
s\left(a_{i}\right)= & \frac{1}{2} \sum_{a_{\ell} \in \mathscr{C} \backslash\left\{a_{i}\right\}}\left|V_{i \ell}-\max \left(\frac{r}{2}+1, \max _{a_{t} \in \mathscr{C} \backslash\left\{a_{i}, a_{\ell}\right\}} V_{t \ell}\right)\right|_{-} \\
& +\frac{1}{2} \sum_{a_{\ell} \in \mathscr{C} \backslash\left\{a_{i}\right\}}\left|\min \left(\frac{r}{2}-1, \min _{a_{t} \in \mathscr{C} \backslash\left\{a_{i}, a_{\ell}\right\}} V_{t \ell}\right)-V_{\ell i}\right|_{-},
\end{aligned}
$$

\footnotetext{
${ }^{7}$ We denote the negative part of $x$ by $|x|_{-}=-\min (x, 0)$.
} 
in case $r$ is an even number, and

$$
\begin{aligned}
s\left(a_{i}\right)= & \frac{1}{2} \sum_{a_{\ell} \in \mathscr{C} \backslash\left\{a_{i}\right\}}\left|V_{i \ell}-\max \left(\frac{r+1}{2}, \max _{a_{t} \in \mathscr{C} \backslash\left\{a_{i}, a_{\ell}\right\}} V_{t \ell}\right)\right|_{-} \\
& +\frac{1}{2} \sum_{a_{\ell} \in \mathscr{C} \backslash\left\{a_{i}\right\}}\left|\min \left(\frac{r-1}{2}, \min _{a_{t} \in \mathscr{C} \backslash\left\{a_{i}, a_{\ell}\right\}} V_{t \ell}\right)-V_{\ell i}\right|_{-},
\end{aligned}
$$

in case $r$ is an odd number.

In the setting where each voter provides a ranking (without ties) on the set of candidates, the terms corresponding to the same index are identical in both sums in each of the above expressions due to the fact that $V_{i \ell}=r-V_{\ell i}$, for any two candidates $a_{i}, a_{\ell} \in \mathscr{C}$. Moreover, the reader can easily check that both $\max _{a_{t} \in \mathscr{C} \backslash\left\{a_{i}, a_{\ell}\right\}} V_{t \ell}$ and $\max _{a_{t} \in \mathscr{C}} V_{t \ell}$ lead to the same result. We sketch the proof for the case where $r$ is an even number (the case where $r$ is an odd number is analogous). It suffices to distinguish three cases:

(a) $\frac{r}{2}+1 \geq V_{i \ell}>\max _{a_{t} \in \mathscr{C} \backslash\left\{a_{i}, a_{\ell}\right\}} V_{t \ell}$, where it holds that $\left|V_{i \ell}-\max \left(\frac{r}{2}+1, \max _{a_{t} \in \mathscr{C} \backslash\left\{a_{i}, a_{\ell}\right\}} V_{t \ell}\right)\right|_{-}=\left|V_{i \ell}-\frac{r}{2}-1\right|_{-}=\left|V_{i \ell}-\max \left(\frac{r}{2}+1, \max _{a_{t} \in \mathscr{C}} V_{t \ell}\right)\right|_{-} ;$

(b) $V_{i \ell} \geq \frac{r}{2}+1 \geq \max _{a_{t} \in \mathscr{C} \backslash\left\{a_{i}, a_{\ell}\right\}} V_{t \ell}\left(\right.$ with $V_{i \ell}>\max _{a_{t} \in \mathscr{C} \backslash\left\{a_{i}, a_{\ell}\right\}} V_{t \ell}$ ), where it holds that

$$
\left|V_{i \ell}-\max \left(\frac{r}{2}+1, \max _{a_{t} \in \mathscr{C} \backslash\left\{a_{i}, a_{\ell}\right\}} V_{t \ell}\right)\right|_{-}=0=\left|V_{i \ell}-\max \left(\frac{r}{2}+1, \max _{a_{t} \in \mathscr{C}} V_{t \ell}\right)\right|_{-} ;
$$

(c) $V_{i \ell}>\max _{a_{t} \in \mathscr{C} \backslash\left\{a_{i}, a_{\ell}\right\}} V_{t \ell} \geq \frac{r}{2}+1$, where it holds that

$$
\left|V_{i \ell}-\max \left(\frac{r}{2}+1, \max _{a_{t} \in \mathscr{C} \backslash\left\{a_{i}, a_{\ell}\right\}} V_{t \ell}\right)\right|_{-}=0=\left|V_{i \ell}-\max \left(\frac{r}{2}+1, \max _{a_{t} \in \mathscr{C}} V_{t \ell}\right)\right|_{-} .
$$

Therefore, the above expressions can be simplified to

$$
s\left(a_{i}\right)=\sum_{a_{\ell} \in \mathscr{C} \backslash\left\{a_{i}\right\}} K_{\ell}-V_{i \ell},
$$

where $K_{\ell}=\max \left(\frac{r}{2}+1, \max _{a_{t} \in \mathscr{C}} V_{t \ell}\right)$ in case $r$ is an even number, and $K_{\ell}=\max \left(\frac{r+1}{2}, \max _{a_{t} \in \mathscr{C}} V_{t \ell}\right)$ in case $r$ is an odd number.

Obviously, in case the pairwise winner $a_{P}$ exists, its score $s\left(a_{P}\right)$ equals zero. In the following example, we illustrate how the score of a candidate can be easily obtained by just analysing the corresponding votrix. 
Example 9.66 The votrix induced by the profile of rankings listed in Example 9.17 is given by:

$$
V_{\mathscr{R}}=\left(\begin{array}{cccc}
0 & 5 & 8 & 14 \\
9 & 0 & 8 & 9 \\
6 & 6 & 0 & 6 \\
0 & 5 & 8 & 0
\end{array}\right) .
$$

The score of the $i$-th candidate equals the sum (for all columns $j \neq i$ ) of the costs of making the element at the $i$-th row and $j$-th column the maximum of the column and, at the same time, greater than half of the number of voters. We illustrate how to obtain the score of candidate a. For the second column we need to increase five until it becomes the maximum of the column (six) and, at the same time, it becomes greater than half of the number of voters, i.e., it becomes at least eight. Therefore, for the second column, we need to increase five until it becomes eight, leading to a cost of three. Note that, for the third column, eight is already the maximum of the column and at the same time greater than half of the number of voters and, for the fourth column, fourteen is already the maximum of the column and at the same time greater than half of the number of voters. Therefore, the score of candidate a equals

$$
s(a)=3+0+0=3 .
$$

Analogously, the scores of the other candidates are $s(b)=5, s(c)=13$ and $s(d)=12$.

We suggest that a candidate should be elected a winner if it minimizes the score given by Eq. (9.1), i.e., a candidate $a_{i}$ is a winning candidate if it belongs to

$$
\mathscr{A}=\arg \min _{a_{i} \in \mathscr{C}} s\left(a_{i}\right)
$$

Remark 9.67 As $s\left(a_{i}\right)=-B\left(a_{i}\right)-K_{i}+\sum_{a_{\ell} \in \mathscr{C}} K_{\ell}$, the proposed method can be understood as a modified version of the Borda method, where, for any candidate $a_{i}$, the term $K_{i}$ is added to the Borda score:

$$
\mathscr{A}=\arg \max _{a_{i} \in \mathscr{C}} B\left(a_{i}\right)+K_{i}
$$

Remark 9.68 The search for a pairwise winner in the same fashion as the method of Dodgson searches for a Condorcet winner (i.e., by minimizing the number of consecutive candidates that need to be switched in the profile of rankings) has also been implemented. 
This has resulted in the same scores in most of the analysed profiles. Nevertheless, this was not always the case, for instance, in case we consider the set of candidates $\mathscr{C}=\{a, b, c, d\}$ and the profile of rankings listed in Table 9.14.

\begin{tabular}{|c|c|}
\hline$\# \succ_{i}$ & Rankings on $\mathscr{C}$ \\
\hline 26 & $c \succ d \succ a \succ b$ \\
25 & $a \succ b \succ d \succ c$ \\
25 & $b \succ a \succ d \succ c$ \\
25 & $c \succ b \succ a \succ d$ \\
\hline
\end{tabular}

Table 9.14: Frequency of the rankings on $\mathscr{C}=\{a, b, c, d\}$ expressed by 101 voters.

The unique minimizer of Eq. (9.2) is candidate a, while the set of minimizers for the search for a pairwise winner by minimizing the number of consecutive candidates that are switched in the profile of rankings is $\{a, b\}$.

In case a candidate $a_{i_{1}}$ is pairwisely better than another candidate $a_{i_{2}}$, the score of $a_{i_{1}}$ is smaller than the score of $a_{i_{2}}$.

Proposition 9.69 Let $\mathscr{C}$ be a set of $k$ candidates and $\mathscr{R}$ be the profile of $r$ rankings on $\mathscr{C}$ given by the voters. For any $a_{i_{1}}, a_{i_{2}} \in \mathscr{C}, a_{i_{1}}>_{C} a_{i_{2}}$ implies that $s\left(a_{i_{1}}\right)<s\left(a_{i_{2}}\right)$.

Proof: Let $a_{i_{1}}, a_{i_{2}} \in \mathscr{C}$ be such that $a_{i_{1}}>_{C} a_{i_{2}}$. As $a_{i_{1}}>_{C} a_{i_{2}}$, it follows that, for any $a_{\ell} \in \mathscr{C} \backslash\left\{a_{i_{1}}, a_{i_{2}}\right\}$

$$
K_{\ell}-V_{i_{1} \ell} \leq K_{\ell}-V_{i_{2} \ell}
$$

It immediately follows that

$$
\sum_{a_{\ell} \in \mathscr{C} \backslash\left\{a_{i_{1}}, a_{i_{2}}\right\}} K_{\ell}-V_{i_{1} \ell} \leq \sum_{a_{\ell} \in \mathscr{C} \backslash\left\{a_{i_{1}}, a_{i_{2}}\right\}} K_{\ell}-V_{i_{2} \ell} .
$$

As $s\left(a_{i}\right)=\sum_{a_{\ell} \in \mathscr{C} \backslash\left\{a_{i}\right\}} K_{\ell}-V_{i \ell}$, in order for $s\left(a_{i_{1}}\right)<s\left(a_{i_{2}}\right)$ to hold, it would suffice that

$$
K_{i_{1}}-V_{i_{2} i_{1}}<K_{i_{2}}-V_{i_{1} i_{2}}
$$

Unfortunately, this may not be the case. However, it is also sufficient to prove that

$$
K_{i_{1}}-V_{i_{2} i_{1}}+K_{\ell}-V_{i_{1} \ell}<K_{i_{2}}-V_{i_{1} i_{2}}+K_{\ell}-V_{i_{2} \ell},
$$


for some appropriate index $\ell$, or

$$
K_{i_{1}}-V_{i_{2} i_{1}}+K_{\ell_{1}}-V_{i_{1} \ell_{1}}+K_{\ell_{2}}-V_{i_{1} \ell_{2}}<K_{i_{2}}-V_{i_{1} i_{2}}+K_{\ell_{1}}-V_{i_{2} \ell_{1}}+K_{\ell_{2}}-V_{i_{2} \ell_{2}},
$$

for some appropriate indices $\ell_{1}$ and $\ell_{2}$.

Let $T_{1}$ be an index $t$ maximizing $V_{t i_{1}}$ and $T_{2}$ be an index $t$ maximizing $V_{t i_{2}}$ (we assume here $k>2$, otherwise this proof is unnecessary). We distinguish five cases: ${ }^{8}$ (a) $T_{1}=i_{2}$ and $T_{2}=i_{1}$; (b) $T_{1}=i_{2}$ and $T_{2} \notin\left\{i_{1}, i_{2}\right\} ;$ (c) $T_{1} \notin\left\{i_{1}, i_{2}\right\}$ and $T_{2}=i_{1}$; (d) $T_{1}, T_{2} \notin\left\{i_{1}, i_{2}\right\}$ and $T_{1}=T_{2}$; (e) $T_{1}, T_{2} \notin\left\{i_{1}, i_{2}\right\}$ and $T_{1} \neq T_{2}$ (only needed in case $k>3$ ).

The cases (a) and (c) trivially lead to $s\left(a_{i_{1}}\right)<s\left(a_{i_{2}}\right)$.

The case (b). It holds that $K_{i_{1}}=\frac{r}{2}+1$ (in case $r$ is even) or $K_{i_{1}}=\frac{r+1}{2}$ (in case $r$ is odd) and, due to the fact that $V_{i_{1} i_{2}}>\frac{r}{2}$, it follows that $K_{i_{2}}=V_{T_{2} i_{2}}=r-V_{i_{2} T_{2}}$. We will prove that the strict inequality

$$
K_{i_{2}}-V_{i_{1} i_{2}}+K_{T_{2}}-V_{i_{1} T_{2}}<K_{i_{1}}-V_{i_{2} i_{1}}+K_{T_{2}}-V_{i_{2} T_{2}}
$$

holds, or, equivalently, in case $r$ is even (the case $r$ is odd is analogous),

$$
V_{i_{2} i_{1}}-V_{i_{1} i_{2}}+\frac{r}{2}-1-V_{i_{1} T_{2}}<0 .
$$

This indeed holds due to the fact that $V_{i_{2} i_{1}}-V_{i_{1} i_{2}}<0$ and $V_{T_{2} i_{1}} \leq V_{T_{1} i_{1}} \leq \frac{r}{2}+1$. We conclude that the strict inequality in Eq. (9.4) holds and, combining Eqs. (9.3) and (9.4), it follows that $s\left(a_{i_{1}}\right)<s\left(a_{i_{2}}\right)$.

The case $(\mathrm{d})$. Let $T=T_{1}=T_{2}$. We will prove that the strict inequality

$$
K_{i_{2}}-V_{i_{1} i_{2}}+K_{T}-V_{i_{1} T}<K_{i_{1}}-V_{i_{2} i_{1}}+K_{T}-V_{i_{2} T}
$$

holds, or, equivalently,

$$
K_{i_{2}}-K_{i_{1}}+V_{i_{2} i_{1}}-V_{i_{1} i_{2}}+V_{i_{2} T}-V_{i_{1} T}<0 .
$$

Note that $K_{i_{1}}=V_{T i_{1}}$ or $K_{i_{1}}=\frac{r}{2}+1$ (in case $r$ is even) or $K_{i_{1}}=\frac{r+1}{2}$ (in case $r$ is odd) and, due to the fact that $V_{i_{1} i_{2}}^{2}>\frac{r}{2}$, it follows that $K_{i_{2}}=V_{T i_{2}}=r-V_{i_{2} T}$. In case $K_{i_{1}}=V_{T i_{1}}=r-V_{i_{1} T}$, Eq. (9.6) leads to:

$$
V_{i_{2} i_{1}}-V_{i_{1} i_{2}}+V_{i_{2} T}-V_{i_{1} T}<0 .
$$

\footnotetext{
${ }^{8}$ Note that the case where $T_{1}=i_{1}$ and $T_{2}=i_{2}$ does not need to be considered because $V_{i i}=0$.
} 
This indeed holds due to the fact that $V_{i_{2} i_{1}}-V_{i_{1} i_{2}}<0$ and $V_{T i_{1}} \leq V_{T i_{2}}$. In case $K_{i_{1}}=\frac{r}{2}+1$ (the case $K_{i_{1}}=\frac{r+1}{2}$ is analogous), Eq. (9.6) leads to:

$$
V_{i_{2} i_{1}}-V_{i_{1} i_{2}}+\frac{r}{2}-1-V_{i_{1} T}<0 .
$$

This indeed holds due to the fact that $V_{i_{2} i_{1}}-V_{i_{1} i_{2}}<0$ and $V_{T_{1} i_{1}} \leq \frac{r}{2}+1$. We conclude that the strict inequality in Eq. (9.5) holds and, combining Eqs. (9.3) and (9.5), it follows that $s\left(a_{i_{1}}\right)<s\left(a_{i_{2}}\right)$.

The case (e). We will prove that the strict inequality

$$
K_{i_{2}}-V_{i_{1} i_{2}}+K_{T_{1}}-V_{i_{1} T_{1}}+K_{T_{2}}-V_{i_{1} T_{2}}<K_{i_{1}}-V_{i_{2} i_{1}}+K_{T_{1}}-V_{i_{2} T_{1}}+K_{T_{2}}-V_{i_{2} T_{2}}
$$

holds, or, equivalently,

$$
K_{i_{2}}-K_{i_{1}}+V_{i_{2} i_{1}}-V_{i_{1} i_{2}}+V_{i_{2} T_{1}}-V_{i_{1} T_{1}}+V_{i_{2} T_{2}}-V_{i_{1} T_{2}}<0 .
$$

Note that $K_{i_{1}}=V_{T_{1} i_{1}}$ or $K_{i_{1}}=\frac{r}{2}+1$ (in case $r$ is even) or $K_{i_{1}}=\frac{r+1}{2}$ (in case $r$ is odd) and, due to the fact that $V_{i_{1} i_{2}}>\frac{r}{2}$, it follows that $K_{i_{2}}=V_{T_{2} i_{2}}=r-V_{i_{2} T_{2}}$. In case $K_{i_{1}}=V_{T_{1} i_{1}}=r-V_{i_{1} T_{1}}$, Eq. 9.8 leads to:

$$
V_{i_{2} i_{1}}-V_{i_{1} i_{2}}+V_{i_{2} T_{1}}-V_{i_{1} T_{2}}<0 .
$$

This indeed holds due to the fact that $V_{i_{2} i_{1}}-V_{i_{1} i_{2}}<0$ and $V_{T_{2} i_{1}} \leq V_{T_{1} i_{1}} \leq V_{T_{1} i_{2}}$. In case $K_{i_{1}}=\frac{r}{2}+1$ (the case $K_{i_{1}}=\frac{r+1}{2}$ is analogous), Eq. 9.8 leads to:

$$
V_{i_{2} i_{1}}-V_{i_{1} i_{2}}+V_{i_{2} T_{1}}-V_{i_{1} T_{2}}+\frac{r}{2}-1-V_{i_{1} T_{1}}<0 .
$$

This indeed holds due to the fact that $V_{i_{2} i_{1}}-V_{i_{1} i_{2}}<0, V_{T_{2} i_{1}} \leq V_{T_{1} i_{1}} \leq V_{T_{1} i_{2}}$ and $V_{T_{1} i_{1}} \leq$ $\frac{r}{2}+1$.

We conclude that the strict inequality in Eq. (9.7) holds and, combining Eqs. (9.3) and (9.7), it follows that $s\left(a_{i_{1}}\right)<s\left(a_{i_{2}}\right)$.

The preceding proposition implies that the winner(s) needs to belong to the pairwisely undominated set.

Corollary 9.70 Let $\mathscr{C}$ be a set of $k$ candidates and $\mathscr{R}$ be the profile of $r$ rankings on $\mathscr{C}$ given by the voters. It holds that $\mathscr{A} \subseteq \mathscr{U}_{C}$. 
Corollary 9.71 Let $\mathscr{C}$ be a set of $k$ candidates and $\mathscr{R}$ be the profile of $r$ rankings on $\mathscr{C}$ given by the voters. If the pairwise winner $a_{P}$ exists, then it holds that $s\left(a_{P}\right)=0<s\left(a_{i}\right)$, for any $a_{i} \in \mathscr{C} \backslash\left\{a_{P}\right\}$, and $\mathscr{A}=\mathscr{U}_{C}=\left\{a_{P}\right\}$.

Example 9.72 For the profile of rankings listed in Example 9.17, the scores associated with each candidate are the following:

$$
\begin{aligned}
& s(a)=3, \\
& s(b)=5, \\
& s(c)=13, \\
& s(d)=12 .
\end{aligned}
$$

Therefore, candidate $a$, which belongs to the pairwisely undominated set $\mathscr{U}_{C}=\{a, b\}$, should be considered the winner in Example 9.17.

For the profile of rankings listed in Example 9.24, the scores associated with each candidate are the following:

$$
\begin{aligned}
& s(a)=100, \\
& s(b)=1, \\
& s(c)=52, \\
& s(d)=153 .
\end{aligned}
$$

Therefore, candidate $b$, which belongs to the pairwisely undominated set $\mathscr{U}_{C}=\{a, b\}$, should be considered the winner in Example 9.24.

In the following, we analyse some intuitive properties of the proposed method.

Proposition 9.73 Let $\mathscr{C}$ be a set of $k$ candidates and $\mathscr{R}$ be the profile of $r$ rankings on $\mathscr{C}$ given by the voters. The search for the candidate(s) that minimizes Eq. (9.2)

(i) satisfies the non-dictatorship criterion, i.e., there is no voter whose winning candidate is always elected winner;

(ii) satisfies the non-imposition criterion, i.e., every candidate can be elected winner if unanimously desired by the group; 
(iii) satisfies the anonymity criterion, i.e., reassigning the rankings over the voters does not change the outcome;

(iv) satisfies the neutrality criterion, i.e., if some permutation of the candidates is applied to each voter's ranking, the winner should be the result of this same permutation;

(v) is not a Condorcet method (is not Condorcet consistent), i.e., the Condorcet winner is not always elected the winner in case of existence;

(vi) is not Borda consistent, i.e., the Borda winner is not always elected the winner in case of existence;

(vii) does not satisfy the majority criterion, i.e., the majority winner is not always elected the winner in case of existence;

(viii) is not independent of clones, i.e., the winner could change due to the addition of a non-winning candidate who is a clone of a candidate already present;

(ix) satisfies the monotonicity criterion, i.e., a winner remains a winner in case it is raised in the rankings of some of the voters;

(x) does not satisfy the homogeneity criterion, i.e., the set of winning candidates may not remain the same in case the rankings of the voters are repeated a finite number of times.

Proof: Statements (i), (ii), (iii) and (iv) are straightforward.

Statements (v) and (vii) follow from Example 9.24. As discussed in Example 9.72, the winner for the profile of rankings given in Example 9.24 is candidate $b$, while candidate $a$ is the majority winner, and thus the Condorcet winner.

Statement (vi) follows from the following example. Consider the set of candidates $\mathscr{C}=$ $\{a, b, c, d\}$ and the profile of rankings shown in Table 9.15. The Borda winner is candidate $d$, while the candidate minimizing Eq. $(9.2)$ is candidate $a$.

Statement (viii) follows from the following example. Consider the set of candidates $\mathscr{C}=$ $\{a, b, c\}$ and the profile of rankings shown in Table 9.16 (left). Candidate $a$ is the pairwise winner, therefore it is the unique candidate minimizing Eq. (9.2). Nevertheless, if we add 


\begin{tabular}{|c|c|}
\hline$\# \succ_{i}$ & Rankings on $\mathscr{C}$ \\
\hline 8 & $a \succ b \succ c \succ d$ \\
5 & $a \succ c \succ b \succ d$ \\
5 & $b \succ d \succ c \succ a$ \\
5 & $c \succ d \succ b \succ a$ \\
5 & $d \succ b \succ a \succ c$ \\
5 & $d \succ c \succ a \succ b$ \\
1 & $d \succ a \succ b \succ c$ \\
\hline
\end{tabular}

Table 9.15: Frequency of the rankings on $\mathscr{C}=\{a, b, c, d\}$ expressed by 34 voters (statement (vi)).

a candidate $d$ that is a clone of candidate $b$, the resulting profile will be the one shown in Table 9.16 (right). The candidate minimizing Eq. 9.2 is now candidate $b$.

\begin{tabular}{|c|c||c|c|}
\hline$\# \succ_{i}$ & Rankings on $\mathscr{C}$ & $\# \succ_{i}$ & Rankings on $\mathscr{C}$ \\
\hline 2 & $a \succ b \succ c$ & 2 & $a \succ b \succ d \succ c$ \\
1 & $a \succ c \succ b$ & 1 & $a \succ c \succ b \succ d$ \\
1 & $b \succ a \succ c$ & 1 & $b \succ d \succ a \succ c$ \\
1 & $b \succ c \succ a$ & 1 & $b \succ d \succ c \succ a$ \\
1 & $c \succ a \succ b$ & 1 & $c \succ a \succ b \succ d$ \\
1 & $c \succ b \succ a$ & 1 & $c \succ b \succ d \succ a$ \\
\hline
\end{tabular}

Table 9.16: Frequency of the rankings on $\mathscr{C}=\{a, b, c\}$ (left) and on $\mathscr{C}=$ $\{a, b, c, d\}$ (right) expressed by 7 voters (statement (viii)).

Statement (ix). We prove that, after improving a winning candidate $a_{i_{1}}$ exactly one position in one of the rankings, this candidate remains being a winner. The monotonicity criterion would then be satisfied. Let us denote by $S, V$ and $K$ the terms related to the original profile and by $S^{\prime}, V^{\prime}$ and $K^{\prime}$ the terms related to the profile obtained by raising candidate $a_{i_{1}}$ exactly one position in one of the rankings. Suppose that there exists another candidate $a_{i_{2}}$ satisfying that $s\left(a_{i_{2}}\right) \geq s\left(a_{i_{1}}\right)$, while $S^{\prime}\left(a_{i_{2}}\right)<S^{\prime}\left(a_{i_{1}}\right)$. Note that it holds that

$$
\sum_{a_{\ell} \in \mathscr{C} \backslash\left\{a_{i_{1}}\right\}}-V_{i_{1} \ell}=-1+\sum_{a_{\ell} \in \mathscr{C} \backslash\left\{a_{i_{1}}\right\}}-V_{i_{1} \ell}^{\prime},
$$


while

$$
\sum_{a_{\ell} \in \mathscr{C} \backslash\left\{a_{i_{2}}\right\}}-V_{i_{2} \ell} \geq \sum_{a_{\ell} \in \mathscr{C} \backslash\left\{a_{i_{2}}\right\}}-V_{i_{2} \ell}^{\prime} .
$$

This implies that, for $S^{\prime}\left(a_{i_{2}}\right)<S^{\prime}\left(a_{i_{1}}\right)$ to hold, it should hold that

$$
K_{i_{2}}^{\prime}<K_{i_{2}}-1
$$

which is a contradiction as the maximum of the $i_{2}$-th column has increased at most one unit.

Statement (x) follows from the following example. Consider the set of candidates $\mathscr{C}=$ $\{a, b, c, d\}$ and the profile of rankings shown in Table 9.17. The set of candidates minimizing Eq. 9.2 is $\{a, b\}$. Nevertheless, in case we repeat the profile twice, the unique candidate minimizing Eq. 9.2 is $a$.

\begin{tabular}{|c|c|}
\hline$\# \succ_{i}$ & Rankings on $\mathscr{C}$ \\
\hline 4 & $b \succ a \succ d \succ c$ \\
2 & $c \succ a \succ b \succ d$ \\
1 & $a \succ c \succ d \succ b$ \\
1 & $b \succ c \succ a \succ d$ \\
1 & $c \succ a \succ d \succ b$ \\
1 & $d \succ c \succ b \succ a$ \\
\hline
\end{tabular}

Table 9.17: Frequency of the rankings on $\mathscr{C}=\{a, b, c, d\}$ expressed by 10 voters (statement $(\mathrm{x})$ ).

In this chapter, we have identified seven different types of winning candidates. A study of the relations between all these types of winner has also been addressed. We concluded this section by proposing a social choice rule based on the search for the pairwise winner, resulting in a Borda-like method where a correction term is added to the Borda count. 


\section{PART III}

THE APPLICATION 



\section{CHAPTER 10}

\section{Case studies}

The aggregation of rankings has been addressed in many scientific disciplines, including medicine [91], consumer preference analysis [162], computer science [48], management science [173] and social choice theory [6, 14]. Nevertheless, due to the natural interpretation of the aggregation of rankings as a voting procedure, social choice theory is considered (one of) the most prominent field of application. All these scientific disciplines represent some of the possible large-scale applications of the contents of this dissertation. Indeed, the theoretical development in this dissertation should be understood as a necessary tool for proposing a solution for ranking all available alternatives/rankings, which is the ultimate goal of this research.

Throughout this chapter, we analyse three different real-life problems: an ecosystem management problem where the goal is to rank four plans according to their perceived suitability for the Lar rangeland in Iran; an environmental decision making problem where the goal is to identify the best estimation method for the production of orange trees in the Argentinian province of Corrientes; and a consumer preference analysis problem where the goal is to check whether or not consumers are able to distinguish between different degrees of spoilage of some cod fish samples at the Laboratory of Food Microbiology and Food Preservation at Ghent University, Belgium. In each of the three cases, we search for all different consensus states and analyse the obtained results. Due to its natural interpretation, the Kendall distance function is always considered in this chapter. 


\subsection{Lar rangeland in Iran}

The methodologies discussed in this dissertation are applied here to a dataset coming from a decision making problem concerning the management of the Lar rangeland in Iran, an area of great local economic, ecological and social importance. When drawing up new management plans, the Iranian government asked $r=31$ representatives of different stakeholder groups to rank four plans according to their perceived suitability on different criteria. For more details, we refer to [173].

\subsubsection{The wildlife diversity criterion}

In Table 10.1, we list the rankings given by the voters in the Lar rangeland decision problem corresponding to the wildlife diversity criterion. We see that a majority of voters has expressed the ranking $a \succ b \succ c \succ d$. However, an optimal ranking could differ from the most frequent ranking.

\begin{tabular}{|c|c|}
\hline$\# \succ_{i}$ & Rankings on $\mathscr{C}$ \\
\hline 18 & $a \succ b \succ c \succ d$ \\
3 & $c \succ d \succ b \succ a$ \\
3 & $d \succ c \succ b \succ a$ \\
2 & $b \succ a \succ c \succ d$ \\
1 & $b \succ c \succ a \succ d$ \\
1 & $b \succ c \succ d \succ a$ \\
1 & $b \succ d \succ c \succ a$ \\
1 & $c \succ b \succ d \succ a$ \\
1 & $d \succ b \succ c \succ a$ \\
\hline
\end{tabular}

Table 10.1: Expressed rankings and their frequency for the Wildlife Diversity Criterion in the Lar rangeland decision problem.

The scorix induced by the profile of rankings given by the voters is:

$$
S=\left(\begin{array}{cccc}
18 & 2 & 1 & 10 \\
5 & 20 & 6 & 0 \\
4 & 5 & 22 & 0 \\
4 & 4 & 2 & 21
\end{array}\right)
$$


We see that $S$ is not (recursively) monotone w.r.t. any ranking on the set of candidates due to the fact that the vector of positions of candidate $a$ does not dominate the vector of positions of candidate $b(18+2<5+20)$ and, at the same time, the vector of positions of candidate $b$ does not dominate the vector of positions of candidate $a(18>5)$.

The relation $>_{B}$ is given by:

$$
>_{B}=\{(a, d),(b, c),(b, d),(c, d)\}
$$

Evidently, $\geq_{B}$ is given by:

$$
\geq_{B}=\{(a, a),(a, d),(b, b),(b, c),(b, d),(c, c),(c, d),(d, d)\} .
$$

Figure 10.1 displays the Hasse diagram of the order relation $\geq_{B}$. Note that the quasipositionally undominated set is here given by $\mathscr{U}_{B}=\{a, b\}$.

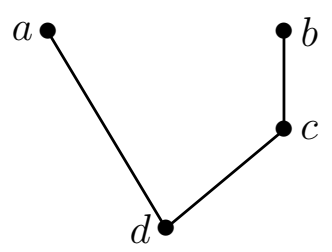

Figure 10.1: Hasse diagram of the order relation $\geq_{B}$.

Similarly, the relation $>_{R}$ is given by:

$$
>_{R}=\{(a, d),(c, d)\}
$$

Evidently, $\geq_{R}$ is given by:

$$
\geq_{R}=\{(a, a),(a, d),(b, b),(c, c),(c, d),(d, d)\}
$$

Figure 10.2 displays the Hasse diagram of the order relation $\geq_{R}$. Note that the positionally undominated set is here given by $\mathscr{U}_{B}=\{a, b, c\}$.

In Table 10.2, the costs of imposing monotonicity and recursive monotonicity on the scorix w.r.t. each possible ranking are listed. We conclude that $a \succ b \succ c \succ d$ is the ranking w.r.t. which the closest profile of rankings with a monotone scorix and the closest profile of rankings with a recursively monotone scorix lead to the lowest cost (11 and 14). Of course, the ranking $a \succ b \succ c \succ d$ linearly extends the strict partial order relation given by $>_{B}$. 


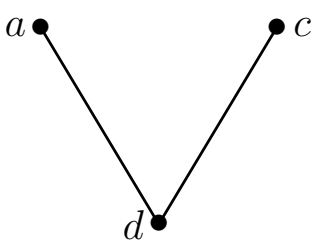

Figure 10.2: Hasse diagram of the order relation $\geq_{R}$.

\begin{tabular}{|c|c|c||c|c|c|}
\hline Ranking & Cost S & Cost RS & Ranking & Cost S & Cost RS \\
\hline$a \succ b \succ c \succ d$ & $\mathbf{1 1}$ & $\mathbf{1 4}$ & $c \succ a \succ b \succ d$ & 21 & 25 \\
$a \succ b \succ d \succ c$ & 20 & 21 & $c \succ a \succ d \succ b$ & 33 & 35 \\
$a \succ c \succ b \succ d$ & 17 & 21 & $c \succ b \succ a \succ d$ & 18 & 23 \\
$a \succ c \succ d \succ b$ & 28 & 32 & $c \succ b \succ d \succ a$ & 34 & 39 \\
$a \succ d \succ b \succ c$ & 28 & 31 & $c \succ d \succ a \succ b$ & 39 & 42 \\
$a \succ d \succ c \succ b$ & 28 & 34 & $c \succ d \succ b \succ a$ & 40 & 45 \\
$b \succ a \succ c \succ d$ & 15 & 15 & $d \succ a \succ b \succ c$ & 38 & 40 \\
$b \succ a \succ d \succ c$ & 19 & 19 & $d \succ a \succ c \succ b$ & 39 & 42 \\
$b \succ c \succ a \succ d$ & 17 & 21 & $d \succ b \succ a \succ c$ & 39 & 42 \\
$b \succ c \succ d \succ a$ & 30 & 31 & $d \succ b \succ c \succ a$ & 41 & 45 \\
$b \succ d \succ a \succ c$ & 29 & 29 & $d \succ c \succ a \succ b$ & 41 & 46 \\
$b \succ d \succ c \succ a$ & 33 & 35 & $d \succ c \succ b \succ a$ & 41 & 48 \\
\hline
\end{tabular}

Table 10.2: Cost of a closest profile of rankings with a monotone scorix (Cost S) and with a recursively monotone scorix (Cost RS) w.r.t. each possible ranking on $\mathscr{C}$.

As expected due to Theorem 5.13, the cost of imposing monotonicity on the scorix is always lower than or equal to the cost of imposing recursive monotonicity on the scorix. Nevertheless, the fact that the cost of imposing monotonicity on the scorix w.r.t. a ranking $\succ$ is lower than the cost of imposing recursive monotonicity on the scorix w.r.t. another ranking $\succ^{\prime}$ does not imply that the cost of imposing recursive monotonicity on the scorix w.r.t. $\succ$ is lower than the cost of imposing monotonicity w.r.t. $\succ^{\prime}$. For instance, the cost of imposing monotonicity on the scorix w.r.t. the ranking $c \succ b \succ a \succ d$ (18) is lower than the cost of imposing monotonicity on the scorix w.r.t. the ranking $a \succ b \succ d \succ c(20)$; nevertheless, the cost of imposing recursive monotonicity on the scorix w.r.t. the ranking $c \succ b \succ a \succ d$ (23) is greater than the cost of imposing recursive monotonicity on the scorix w.r.t. the ranking $a \succ b \succ d \succ c(21)$. 
In Table 10.3, a closest profile of rankings with a monotone scorix (left) w.r.t. the ranking $a \succ b \succ c \succ d$ and a closest profile of rankings with a recursively monotone scorix (right) w.r.t. the ranking $a \succ b \succ c \succ d$ are shown.

\begin{tabular}{|c|c|c|c|}
\hline$\# \succ_{i}$ & Rankings on $\mathscr{C}$ & $\# \succ_{i}$ & Rankings on $\mathscr{C}$ \\
\hline 18 & $a \succ b \succ c \succ d$ & 16 & $a \succ b \succ c \succ d$ \\
\hline 2 & $b \succ a \succ c \succ d$ & 3 & $b \succ a \succ c \succ d$ \\
\hline 2 & $b \succ d \succ a \succ c$ & 2 & $a \succ b \succ d \succ c$ \\
\hline 2 & $c \succ d \succ b \succ a$ & 2 & $c \succ a \succ d \succ b$ \\
\hline 2 & $d \succ c \succ a \succ b$ & 1 & $b \succ c \succ d \succ a$ \\
\hline 1 & $b \succ c \succ a \succ d$ & 1 & $b \succ d \succ c \succ a$ \\
\hline 1 & $c \succ a \succ b \succ d$ & 1 & $c \succ b \succ d \succ a$ \\
\hline 1 & $c \succ a \succ d \succ b$ & 1 & $c \succ d \succ a \succ b$ \\
\hline 1 & $d \succ a \succ b \succ c$ & 1 & $d \succ a \succ b \succ c$ \\
\hline \multirow[t]{3}{*}{1} & $d \succ c \succ b \succ a$ & 1 & $d \succ a \succ c \succ b$ \\
\hline & & 1 & $d \succ b \succ a \succ c$ \\
\hline & & 1 & $d \succ c \succ b \succ a$ \\
\hline
\end{tabular}

Table 10.3: Closest profile of rankings with a monotone scorix (left) and with a recursively monotone scorix (right) w.r.t. the ranking $a \succ b \succ c \succ d$.

The scorix induced by the closest profile of rankings with a recursively monotone scorix listed in Table 10.3 is the following:

$$
S^{\prime}=\left(\begin{array}{cccc}
18 & 7 & 2 & 4 \\
5 & 20 & 2 & 4 \\
4 & 2 & 21 & 4 \\
4 & 2 & 6 & 19
\end{array}\right)
$$

We easily see that $S^{\prime}$ is monotone w.r.t. the ranking $a \succ b \succ c \succ d$. The four sub-scorices associated with the restriction of the profile of rankings to a subset of the set of candidates of cardinality three are the following:

$$
S_{a b c}=\left(\begin{array}{ccc}
20 & 7 & 4 \\
6 & 21 & 4 \\
5 & 3 & 23
\end{array}\right)
$$


which is monotone w.r.t. $a \succ b \succ c$;

$$
S_{a b d}=\left(\begin{array}{ccc}
20 & 6 & 5 \\
6 & 20 & 5 \\
5 & 5 & 21
\end{array}\right),
$$

which is monotone w.r.t. $a \succ b \succ d$;

$$
S_{a c d}=\left(\begin{array}{ccc}
21 & 5 & 5 \\
5 & 21 & 5 \\
5 & 5 & 21
\end{array}\right),
$$

which is monotone w.r.t. $a \succ c \succ d$; and

$$
S_{b c d}=\left(\begin{array}{ccc}
23 & 3 & 5 \\
4 & 22 & 5 \\
4 & 6 & 21
\end{array}\right),
$$

which is monotone w.r.t. $b \succ c \succ d$.

In order to prove the monotonicity of the six sub-scorices associated with the restriction of the profile of rankings to a subset of the set of candidates of cardinality two, it suffices to see that $a \succ b \succ c \succ d$ is a weak Condorcet ranking (Lemma 5.11). The votrix induced by the closest profile of rankings with a recursively monotone scorix listed in Table 10.3 is the following:

$$
V^{\prime}=\left(\begin{array}{cccc}
0 & 23 & 24 & 23 \\
8 & 0 & 25 & 24 \\
7 & 6 & 0 & 24 \\
8 & 7 & 7 & 0
\end{array}\right) .
$$

As $a \succ b \succ c \succ d$ is a ranking such that every candidate is preferred by not less than half of the voters to all the candidates ranked at a worse position, we conclude that $a \succ b \succ c \succ d$ is a weak Condorcet ranking. Therefore, the six sub-scorices associated with the restriction of the profile of rankings to a subset of the set of candidates of cardinality two are monotone. We conclude that $S^{\prime}$ is recursively monoton $\oint^{1}$ w.r.t. $a \succ b \succ c \succ d$.

The votrix induced by the profile of rankings given by the voters is:

$$
V=\left(\begin{array}{cccc}
0 & 18 & 20 & 21 \\
13 & 0 & 24 & 24 \\
11 & 7 & 0 & 26 \\
10 & 7 & 5 & 0
\end{array}\right)
$$

\footnotetext{
${ }^{1} S^{\prime}$ actually is strictly recursively monotone w.r.t. $a \succ b \succ c \succ d$.
} 
We see that $V$ is not monotone w.r.t. any ranking on the set of candidates due to the fact that $a \succ b \succ c \succ d$ is the Condorcet ranking but, at the same time, $V_{a d}=21<24=V_{b d}$.

The relation $>_{C}$ is given by:

$$
>_{C}=\{(a, d),(b, d),(c, d)\}
$$

Evidently, $\geq_{C}$ is given by:

$$
\geq_{C}=\{(a, a),(a, d),(b, b),(b, d),(c, c),(c, d),(d, d)\} .
$$

Figure 10.3 displays the Hasse diagram of the order relation $\geq_{C}$.

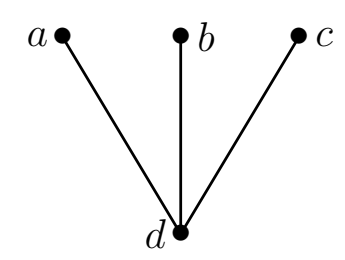

Figure 10.3: Hasse diagram of the order relation $\geq_{C}$.

Note that the pairwisely undominated set is here given by $\mathscr{U}_{C}=\{a, b, c\}$. Applying the Borda-like social choice function introduced at the end of Chapter 9, the scores associated with each candidate are the following:

$$
\begin{aligned}
& s(a)=9, \\
& s(b)=5, \\
& s(c)=16, \\
& s(d)=36 .
\end{aligned}
$$

Therefore, candidate $b$ is the winning candidate according to the search for a pairwise winner.

The votex induced by the profile of rankings given by the voters is:

$$
\begin{array}{rl|rl}
W: \mathscr{C}_{\neq}^{2} & \longrightarrow\{0, \ldots, 31\}^{6} & W: \mathscr{C}_{\neq}^{2} & \rightarrow\{, \ldots, 31\}^{6} \\
W(a, b) & =(2,3,8,18,0,0) & W(b, c) & =(0,3,4,21,3,0) \\
W(a, c) & =(4,4,3,2,18,0) & W(b, d) & =(0,3,4,2,19,3) \\
W(a, d) & =(4,4,2,1,2,18) & W(c, d) & =(0,1,4,24,2,0)
\end{array}
$$


As the votrix is not monotone w.r.t. any ranking on the set of candidates, we conclude that the votex induced by the profile of rankings given by the voters is not monotone w.r.t. any ranking either (see Theorem 5.23).

In Table 10.4, the costs of imposing monotonicity on the votrix and on the votex w.r.t. each possible ranking are listed. We conclude that $b \succ a \succ c \succ d$ is the ranking w.r.t. which the closest profile of rankings with a monotone votrix leads to the lowest cost (8), while $a \succ b \succ c \succ d$ is the ranking w.r.t. which the closest profile of rankings with a monotone votex leads to the lowest cost (16). As expected due to Theorem 5.23, the cost of imposing monotonicity on the votrix is always lower than or equal to the cost of imposing monotonicity on the votex. Nevertheless, the ranking leading to the lowest cost does not need to coincide for both the search for monotonicity of the votrix and of the votex, as can be seen in this example. Note that both $a \succ b \succ c \succ d$ and $b \succ a \succ c \succ d$ linearly extend the strict partial order relation given by $>_{C}$.

\begin{tabular}{|c|c|c||c|c|c|}
\hline Ranking & Cost V & Cost W & Ranking & Cost V & Cost W \\
\hline$a \succ b \succ c \succ d$ & 9 & $\mathbf{1 6}$ & $c \succ a \succ b \succ d$ & 19 & 33 \\
$a \succ b \succ d \succ c$ & 18 & 26 & $c \succ a \succ d \succ b$ & 36 & 41 \\
$a \succ c \succ b \succ d$ & 16 & 25 & $c \succ b \succ a \succ d$ & 17 & 29 \\
$a \succ c \succ d \succ b$ & 31 & 36 & $c \succ b \succ d \succ a$ & 41 & 41 \\
$a \succ d \succ b \succ c$ & 31 & 39 & $c \succ d \succ a \succ b$ & 41 & 46 \\
$a \succ d \succ c \succ b$ & 32 & 40 & $c \succ d \succ b \succ a$ & 42 & 47 \\
$b \succ a \succ c \succ d$ & $\mathbf{8}$ & 21 & $d \succ a \succ b \succ c$ & 36 & 45 \\
$b \succ a \succ d \succ c$ & 15 & 30 & $d \succ a \succ c \succ b$ & 41 & 46 \\
$b \succ c \succ a \succ d$ & 18 & 29 & $d \succ b \succ a \succ c$ & 37 & 46 \\
$b \succ c \succ d \succ a$ & 32 & 37 & $d \succ b \succ c \succ a$ & 42 & 48 \\
$b \succ d \succ a \succ c$ & 32 & 39 & $d \succ c \succ a \succ b$ & 42 & 48 \\
$b \succ d \succ c \succ a$ & 33 & 41 & $d \succ c \succ b \succ a$ & 43 & 48 \\
\hline
\end{tabular}

Table 10.4: Cost of a closest profile of rankings with a monotone votrix (Cost $\mathrm{V})$ and with a monotone votex (Cost W) w.r.t. each possible ranking on $\mathscr{C}$.

In Table 10.5, a closest profile of rankings with a monotone votrix (left) w.r.t. the ranking $b \succ a \succ c \succ d$ and a closest profile of rankings with a monotone votex (right) w.r.t. the ranking $a \succ b \succ c \succ d$ are shown. 


\begin{tabular}{|c|c||c|c|}
\hline$\# \succ_{i}$ & Rankings on $\mathscr{C}$ & $\# \succ_{i}$ & Rankings on $\mathscr{C}$ \\
\hline 11 & $a \succ b \succ c \succ d$ & 17 & $a \succ b \succ c \succ d$ \\
5 & $b \succ a \succ c \succ d$ & 2 & $b \succ a \succ c \succ d$ \\
4 & $a \succ b \succ d \succ c$ & 1 & $a \succ b \succ d \succ c$ \\
3 & $c \succ d \succ b \succ a$ & 1 & $a \succ c \succ b \succ d$ \\
3 & $d \succ c \succ b \succ a$ & 1 & $a \succ d \succ c \succ b$ \\
2 & $b \succ d \succ c \succ a$ & 1 & $b \succ c \succ a \succ d$ \\
1 & $b \succ c \succ a \succ d$ & 1 & $b \succ c \succ d \succ a$ \\
1 & $c \succ b \succ d \succ a$ & 1 & $b \succ d \succ a \succ c$ \\
1 & $d \succ b \succ c \succ a$ & 1 & $c \succ a \succ b \succ d$ \\
& & 1 & $c \succ a \succ d \succ b$ \\
& & 1 & $c \succ d \succ b \succ a$ \\
& & 1 & $d \succ a \succ c \succ b$ \\
& & 1 & $d \succ b \succ a \succ c$ \\
& & $d \succ c \succ b \succ a$ \\
\hline
\end{tabular}

Table 10.5: Closest profile of rankings with a monotone votrix (left) w.r.t. the ranking $b \succ a \succ c \succ d$ and with a monotone votex (right) w.r.t. the ranking $a \succ b \succ c \succ d$.

The votrix associated with the closest profile of rankings with a monotone votrix listed in Table 10.5 is the following:

$$
V^{\prime}=\left(\begin{array}{cccc}
0 & 15 & 20 & 21 \\
16 & 0 & 24 & 24 \\
11 & 7 & 0 & 21 \\
10 & 7 & 10 & 0
\end{array}\right)
$$

We easily see that $V^{\prime}$ is monotone w.r.t. the ranking $b \succ a \succ c \succ d$.

Analogously, the votex associated with the closest profile of rankings with a monotone votex listed in Table 10.5 is the following:

$$
\begin{aligned}
& W^{\prime}: \quad \mathscr{C}_{\neq}^{2} \quad \longrightarrow \quad\{0, \ldots, 31\}^{6} \\
& W(a, b)=(1,2,5,19,3,1) \\
& W(a, c)=(1,2,3,6,18,1) \\
& W(a, d)=(1,2,3,3,4,18) \\
& W^{\prime}: \quad \mathscr{C}_{\neq}^{2} \quad \longrightarrow \quad\{0, \ldots, 31\}^{6} \\
& W(b, c)=(1,2,4,19,4,1) \\
& W(b, d)=(1,2,3,4,18,3) \\
& W(c, d)=(1,2,3,21,3,1)
\end{aligned}
$$

We easily see that $W^{\prime}$ is monotone w.r.t. the ranking $a \succ b \succ c \succ d$. 
Figure 10.4 displays the given profile of rankings represented on the diagram of $\geqq$ (where $\succ$ represents the ranking $a \succ b \succ c \succ d)$. Note that the profile of rankings is not monotone w.r.t. the ranking $a \succ b \succ c \succ d$. As $a \succ b \succ c \succ d$ is the most frequent ranking, the profile of rankings cannot be monotone w.r.t. any other ranking on the set of candidates. Nevertheless, although there are some violations of monotonicity, the cost of imposing monotonicity w.r.t. the ranking $a \succ b \succ c \succ d$ should be 'low'.

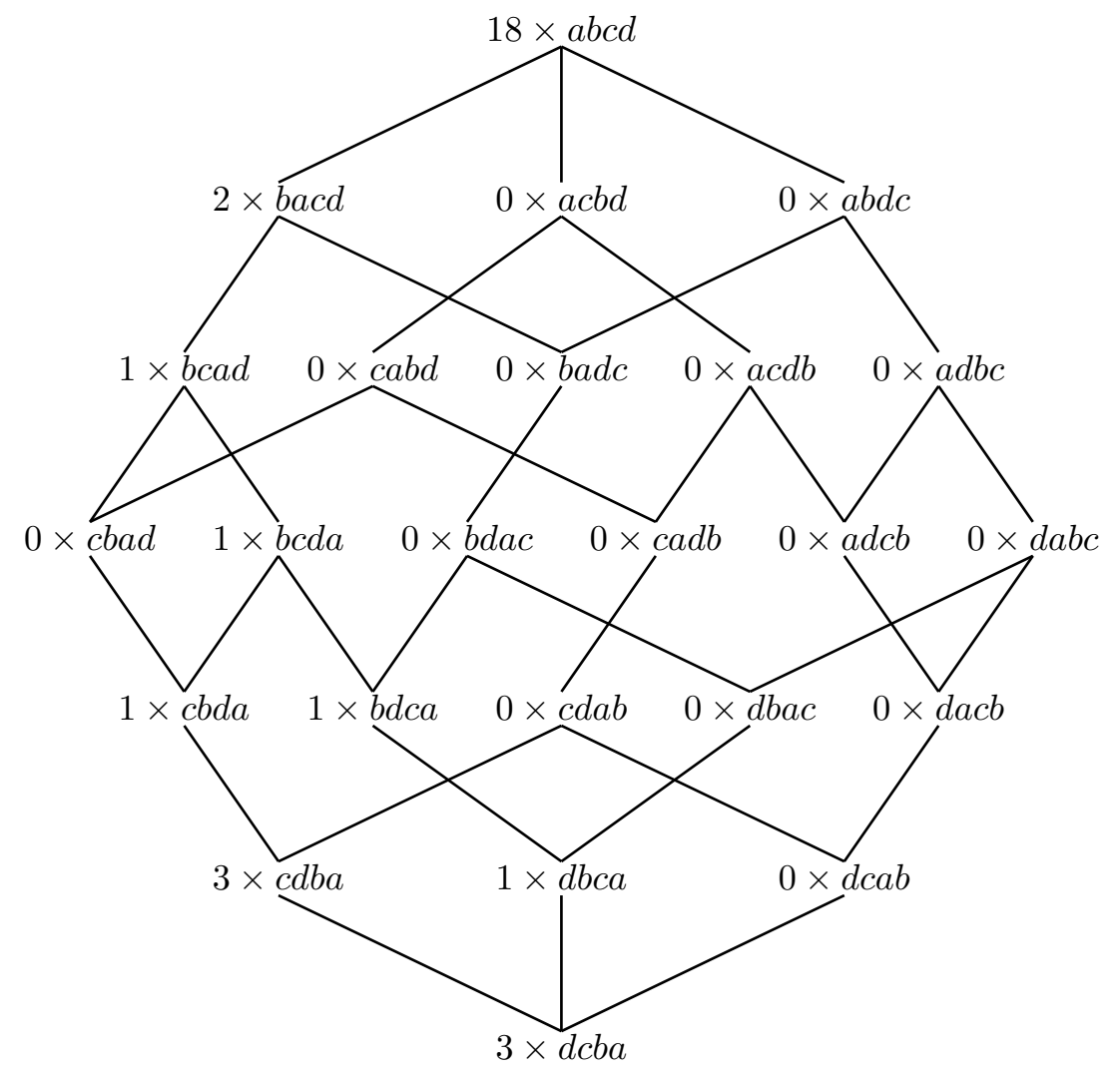

Figure 10.4: Frequencies of Table 10.1 represented on the Hasse diagram of the order relation $\geqq$ for the ranking $a \succ b \succ c \succ d$.

In Table 10.6, the costs of imposing monotonicity on the profile w.r.t. each possible ranking are listed. We conclude that $a \succ b \succ c \succ d$ is the ranking w.r.t. which the closest monotone profile of rankings leads to the lowest cost (19).

Figure 10.5 displays the closest monotone profile of rankings w.r.t. the ranking $a \succ b \succ$ $c \succ d$ represented on the diagram of $\geqq$ (where $\succ$ represents the ranking $a \succ b \succ c \succ d$ ). As it is decreasing on the diagram, we clearly see that this profile of rankings is monotone w.r.t. the ranking $a \succ b \succ c \succ d$. 


\begin{tabular}{|c|c||c|c||c|c||c|c|}
\hline Ranking & Cost & Ranking & Cost & Ranking & Cost & Ranking & Cost \\
\hline$a \succ b \succ c \succ d$ & $\mathbf{1 9}$ & $b \succ a \succ c \succ d$ & 20 & $c \succ a \succ b \succ d$ & 26 & $d \succ a \succ b \succ c$ & 38 \\
$a \succ b \succ d \succ c$ & 29 & $b \succ a \succ d \succ c$ & 28 & $c \succ a \succ d \succ b$ & 35 & $d \succ a \succ c \succ b$ & 45 \\
$a \succ c \succ b \succ d$ & 25 & $b \succ c \succ a \succ d$ & 24 & $c \succ b \succ a \succ d$ & 30 & $d \succ b \succ a \succ c$ & 42 \\
$a \succ c \succ d \succ b$ & 32 & $b \succ c \succ d \succ a$ & 30 & $c \succ b \succ d \succ a$ & 35 & $d \succ b \succ c \succ a$ & 44 \\
$a \succ d \succ b \succ c$ & 36 & $b \succ d \succ a \succ c$ & 33 & $c \succ d \succ a \succ b$ & 40 & $d \succ c \succ a \succ b$ & 49 \\
$a \succ d \succ c \succ b$ & 41 & $b \succ d \succ c \succ a$ & 37 & $c \succ d \succ b \succ a$ & 43 & $d \succ c \succ b \succ a$ & 52 \\
\hline
\end{tabular}

Table 10.6: Cost of a closest monotone profile of rankings w.r.t. each possible ranking on $\mathscr{C}$.

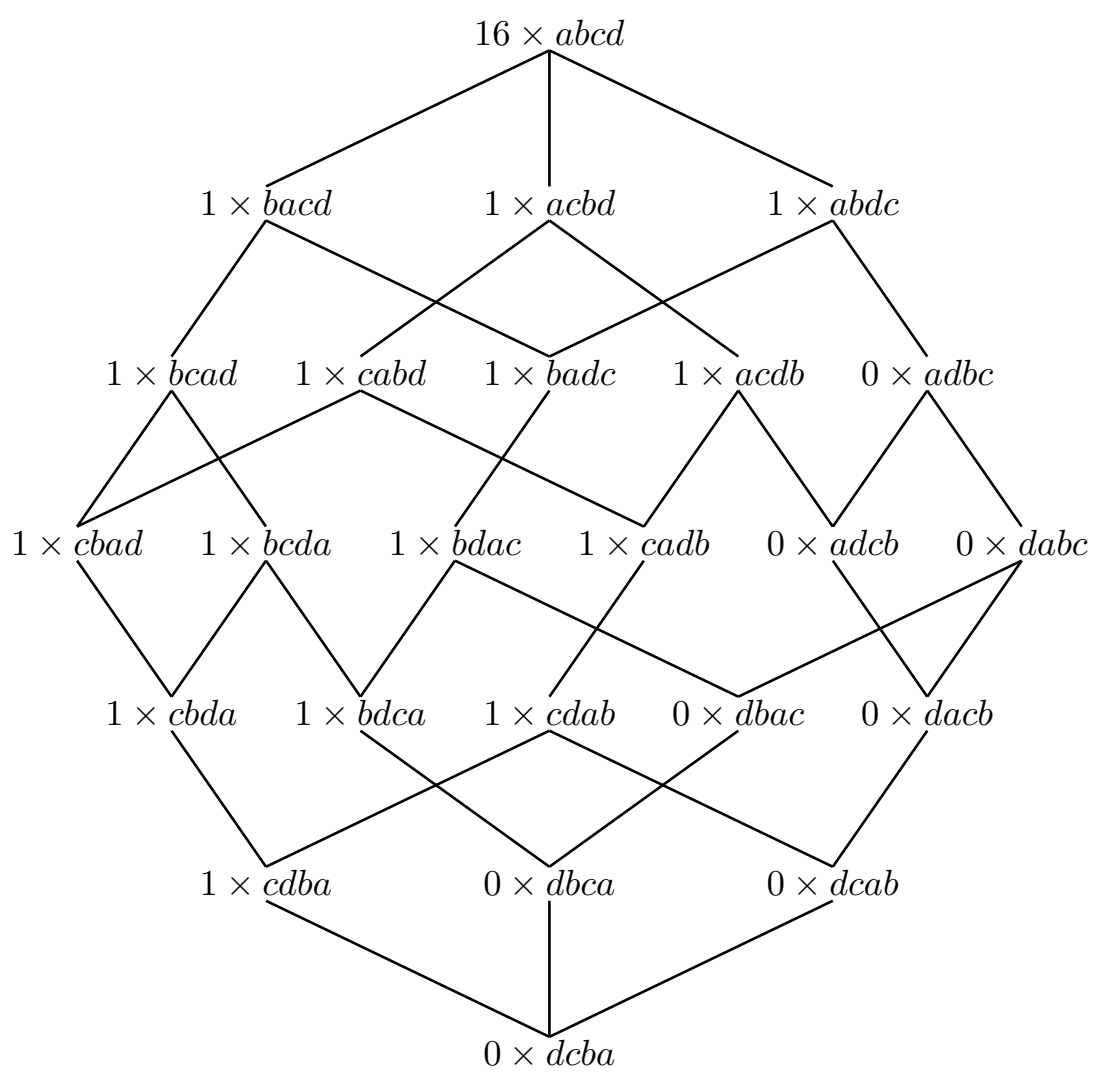

Figure 10.5: Frequencies of the closest monotone profile of rankings w.r.t. $a \succ$ $b \succ c \succ d$ represented on the Hasse diagram of the order relation $\geqq$.

The last analysis for the Lar rangeland problem is linked to the search for a Condorcet ranking, acclamation and unanimity. As previously discussed, the ranking $a \succ b \succ c \succ d$ is the Condorcet ranking for the profile of rankings given by the voters. Obviously, the 
ranking that leads to the lowest cost (zero) when searching for a Condorcet ranking is the ranking $a \succ b \succ c \succ d$. Under the existence of a Condorcet ranking, the ranking that leads to the lowest cost (53) when searching for unanimity (which is the winner according to the method of Kemeny) always coincides with this Condorcet ranking. It remains to identify the ranking that leads to the lowest cost when searching for acclamation.

In Table 10.7, the costs associated with the search for a Condorcet ranking, acclamation and unanimity w.r.t. each possible ranking are listed. We conclude that $a \succ b \succ c \succ d$ is the ranking that leads to the lowest cost in all three cases.

\begin{tabular}{|c|c|c|c||c|c|c|c|}
\hline Ranking & Cost C & Cost A & Cost U & Ranking & Cost C & Cost A & Cost U \\
\hline$a \succ b \succ c \succ d$ & $\mathbf{0}$ & $\mathbf{2 2}$ & $\mathbf{5 3}$ & $c \succ a \succ b \succ d$ & 14 & 34 & 79 \\
$a \succ b \succ d \succ c$ & 11 & 31 & 74 & $c \succ a \succ d \succ b$ & 23 & 41 & 96 \\
$a \succ c \succ b \succ d$ & 9 & 28 & 70 & $c \succ b \succ a \succ d$ & 17 & 32 & 84 \\
$a \succ c \succ d \succ b$ & 18 & 40 & 87 & $c \succ b \succ d \succ a$ & 23 & 44 & 95 \\
$a \succ d \succ b \succ c$ & 20 & 41 & 91 & $c \succ d \succ a \succ b$ & 29 & 49 & 107 \\
$a \succ d \succ c \succ b$ & 29 & 43 & 108 & $c \succ d \succ b \succ a$ & 32 & 50 & 112 \\
$b \succ a \succ c \succ d$ & 3 & 25 & 58 & $d \succ a \succ b \succ c$ & 26 & 48 & 102 \\
$b \succ a \succ d \succ c$ & 14 & 32 & 79 & $d \succ a \succ c \succ b$ & 35 & 49 & 119 \\
$b \succ c \succ a \succ d$ & 8 & 31 & 67 & $d \succ b \succ a \succ c$ & 29 & 49 & 107 \\
$b \succ c \succ d \succ a$ & 14 & 40 & 78 & $d \succ b \succ c \succ a$ & 34 & 53 & 116 \\
$b \succ d \succ a \succ c$ & 20 & 42 & 90 & $d \succ c \succ a \succ b$ & 40 & 52 & 128 \\
$b \succ d \succ c \succ a$ & 25 & 44 & 99 & $d \succ c \succ b \succ a$ & 43 & 54 & 133 \\
\hline
\end{tabular}

Table 10.7: Cost of a closest profile with a Condorcet ranking (Cost C), a closest acclaimed profile (Cost A) and a closest unanimous profile (Cost U) w.r.t. each possible ranking on $\mathscr{C}$.

As expected due to Theorem 5.32 , the cost associated with the search for a Condorcet ranking is always lower than or equal to the cost associated with the search for acclamation. As also expected due to Theorem 5.32, the cost associated with the search for acclamation is always lower than or equal to the cost associated with the search for unanimity. Nevertheless, as discussed in Chapter 7, the ranking leading to the lowest cost does not need to coincide for the three searches, although this is the case in this example.

We conclude this section by analysing the optimal ranking w.r.t. the search for each of the considered consensus states. Note that, as listed in Table 10.8, the optimal ranking 
according to the search for all consensus states - but monotonicity of the votrix - is the ranking $a \succ b \succ c \succ d$. Note that the optimal ranking according to the search for monotonicity of the votrix is the ranking $b \succ a \succ c \succ d$.

\begin{tabular}{|c|c||c|c|}
\hline Search for & Optimal ranking & Search for & Optimal ranking \\
\hline Monotone scorix & $a \succ b \succ c \succ d$ & Rec. monotone scorix & $a \succ b \succ c \succ d$ \\
\hline Monotone votrix & $b \succ a \succ c \succ d$ & Monotone votex & $a \succ b \succ c \succ d$ \\
\hline Monotone profile & $a \succ b \succ c \succ d$ & Condorcet ranking & $a \succ b \succ c \succ d$ \\
\hline Acclamation & $a \succ b \succ c \succ d$ & Unanimity & $a \succ b \succ c \succ d$ \\
\hline
\end{tabular}

Table 10.8: Optimal ranking w.r.t. the search for all the considered consensus states.

From the results of this case study it follows that the ranking $a \succ b \succ c \succ d$ seems to be the most natural ranking of the four plans proposed for the Lar rangeland. After all, it is the optimal ranking according to the search for seven out of eight consensus states and it is only one unit of cost away from being optimal according to the other one.

For this case study, there does not exist a unanimous winner. A quasipositional winner, a positional winner or a pairwise winner does not exist either. However, the majority winner, the Condorcet winner and the Borda winner exist. Candidate $a$ is both the majority winner and the Condorcet winner, while candidate $b$ is the Borda winner.

\subsubsection{Allowing for ties}

For the four available plans in the Lar rangeland problem, some representatives of the stakeholder groups considered that, sometimes, two or more plans were almost equally suitable for the management of the rangeland. In order to prevent them from taking an arbitrary decision when ranking the plans, the representatives of the stakeholder groups were also asked to provide rankings with ties whenever they considered one candidate was as equally suitable as another candidate. This profile of rankings with ties is listed in Table 10.9.

At this point, the goal is to look for the set of (weak/total)-optimal rankings. In Table 10.10, the cost associated to a closest profile of rankings and to a closest profile of 


\begin{tabular}{|c|c||c|c|}
\hline$\#_{i} \succsim_{i}$ & Ranking with ties & $\# \succsim_{i}$ & Ranking with ties \\
\hline 7 & $a \succ b \succ c \succ d$ & 6 & $a \sim b \sim c \sim d$ \\
3 & $a \succ b \succ c \sim d$ & 3 & $a \succ b \sim c \sim d$ \\
3 & $a \sim b \succ c \succ d$ & 2 & $c \sim d \succ a \sim b$ \\
1 & $a \sim b \succ c \sim d$ & 1 & $c \succ a \sim b \sim d$ \\
1 & $c \succ d \succ a \sim b$ & 1 & $b \succ a \succ c \succ d$ \\
1 & $d \succ c \succ b \succ a$ & 1 & $d \succ a \sim b \sim c$ \\
1 & $b \succ c \succ a \sim d$ & & \\
\hline
\end{tabular}

Table 10.9: Expressed rankings with ties and their frequency for the Wildlife Diversity Criterion in the Lar rangeland decision problem.

rankings with ties with a monotone representation of votes w.r.t. each possible ranking and its associated cost are listed.

As expected due to Proposition 8.40, the cost associated to a closest profile of rankings with ties with a monotone weak-scorix/votrix/votex is always lower than or equal to the cost of a closest profile of rankings with a monotone scorix/votrix/votex. Furthermore, as monotonicity of the votex implies monotonicity of the votrix [124], the cost associated to the (weak-)votrix is always lower than or equal to the cost associated to the (weak-)votex.

We conclude that the ranking $a \succ b \succ c \succ d$ clearly is the weak-optimal ranking considering either the monotonicity of the scorix, votrix or votex. Nevertheless, we see that, although it still is the total-optimal ranking considering either the monotonicity of the scorix, votrix or votex, there are also other total-optimal rankings. This is due to the fact that the minimum cost of changing the given profile of rankings with ties into an arbitrary profile of rankings (without ties) equals 32.5. In that way, we impose, with a minimum cost, monotonicity on the scorix w.r.t. the rankings $a \succ b \succ c \succ d, a \succ b \succ d \succ c$ and $a \succ c \succ b \succ d$ and on the votrix w.r.t. the rankings $a \succ b \succ c \succ d, a \succ b \succ d \succ c, b \succ a \succ c \succ d$ and $b \succ a \succ d \succ c$. This is caused due to the six appearances of the ranking with ties $a \sim b \sim c \sim d$ in the profile of rankings with ties given by the experts.

In Table 10.11, the cost associated to a closest $*$-monoton $2^{2}$ profile of rankings with ties w.r.t. each possible ranking and its associated cost are listed. We note that the compar-

\footnotetext{
${ }^{2}$ The notation $*$-monotone is a shorthand for referring to any of $\geqq$-monotone, $\unrhd^{\mathbb{Q} \uparrow}$-monotone, $\unrhd^{\mathbb{O} \downarrow_{-}}$ monotone, $\geqq^{\mathbb{Q}}$-monotone, $\geqq^{\mathbb{S} \uparrow}$-monotone, $\geqq^{\mathbb{S} \downarrow}$-monotone or $\geqq^{\mathbb{S}}$-monotone.
} 


\begin{tabular}{|c|c|c|c|c|c|c|}
\hline Ranking & Cost WS & Cost TS & Cost WV & Cost TV & Cost WW & Cost TW \\
\hline$a \succ b \succ c \succ d$ & 1.5 & 32.5 & 3.5 & 32.5 & 9 & 32.5 \\
\hline$a \succ b \succ d \succ c$ & 7 & 32.5 & 7.5 & 32.5 & 14 & 34.5 \\
\hline$a \succ c \succ b \succ d$ & 7.5 & 32.5 & 10.5 & 33.5 & 17.5 & 36.5 \\
\hline$a \succ c \succ d \succ b$ & 16.5 & 34.5 & 19.5 & 34.5 & 23.5 & 41.5 \\
\hline$a \succ d \succ b \succ c$ & 17 & 35.5 & 21 & 33.5 & 25 & 41.5 \\
\hline$a \succ d \succ c \succ b$ & 17 & 36.5 & 20 & 34.5 & 25 & 43.5 \\
\hline$b \succ a \succ c \succ d$ & 8 & 33.5 & 8.5 & 32.5 & 14 & 34.5 \\
\hline$b \succ a \succ d \succ c$ & 13.5 & 33.5 & 13.5 & 32.5 & 19.5 & 35.5 \\
\hline$b \succ c \succ a \succ d$ & 16.5 & 37.5 & 20 & 35.5 & 24.5 & 41.5 \\
\hline$b \succ c \succ d \succ a$ & 27 & 41.5 & 29 & 38.5 & 32.5 & 48.5 \\
\hline$b \succ d \succ a \succ c$ & 25.5 & 39.5 & 29 & 36.5 & 32 & 45.5 \\
\hline$b \succ d \succ c \succ a$ & 27.5 & 43.5 & 29 & 38.5 & 33 & 48.5 \\
\hline$c \succ a \succ b \succ d$ & 16 & 35.5 & 18.5 & 36.5 & 23 & 41.5 \\
\hline$c \succ a \succ d \succ b$ & 24.5 & 39.5 & 25.5 & 39.5 & 29 & 46.5 \\
\hline$c \succ b \succ a \succ d$ & 16.5 & 37.5 & 18.5 & 36.5 & 24 & 43.5 \\
\hline$c \succ b \succ d \succ a$ & 29 & 42.5 & 34.5 & 39.5 & 34.5 & 50.5 \\
\hline$c \succ d \succ a \succ b$ & 29.5 & 43.5 & 34.5 & 40.5 & 35.5 & 51.5 \\
\hline$c \succ d \succ b \succ a$ & 29.5 & 44.5 & 34.5 & 40.5 & 35.5 & 52.5 \\
\hline$d \succ a \succ b \succ c$ & 27 & 40.5 & 29.5 & 37.5 & 33 & 47.5 \\
\hline$d \succ a \succ c \succ b$ & 29 & 43.5 & 34.5 & 39.5 & 34.5 & 50.5 \\
\hline$d \succ b \succ a \succ c$ & 27.5 & 42.5 & 29.5 & 37.5 & 34 & 48.5 \\
\hline$d \succ b \succ c \succ a$ & 29.5 & 46.5 & 34.5 & 39.5 & 37 & 53.5 \\
\hline$d \succ c \succ a \succ b$ & 29.5 & 45.5 & 34.5 & 40.5 & 35.5 & 52.5 \\
\hline$d \succ c \succ b \succ a$ & 29.5 & 46.5 & 34.5 & 40.5 & 36.5 & 53.5 \\
\hline
\end{tabular}

Table 10.10: Cost of a closest profile of rankings (with ties) with a monotone weak-scorix (Cost WS), with a monotone scorix (Cost TS), with a monotone weak-votrix (Cost WV), with a monotone votrix (Cost V), with a monotone weak-votex (Cost WW) and with a monotone votex (Cost TW) w.r.t. each possible ranking on $\mathscr{C}$.

ison of costs in different columns respects the relations between different order relations illustrated in Figure 8.13 . 


\begin{tabular}{|c|c|c|c|c|c|c|c|}
\hline Ranking & Cost $\unrhd$ & Cost $\unrhd^{\mathbb{O} \uparrow}$ & Cost $\unrhd^{\mathbb{O} \downarrow}$ & Cost $\unrhd^{\mathbb{O}}$ & Cost $\unrhd^{\mathbb{S} \uparrow}$ & Cost $\unrhd^{\mathbb{S} \downarrow}$ & Cost $\unrhd^{\mathbb{S}}$ \\
\hline$a \succ b \succ c \succ d$ & 25.5 & 8.5 & 22.5 & 8.5 & 5.5 & 21.5 & 5.5 \\
\hline$a \succ b \succ d \succ c$ & 32.5 & 14 & 29.5 & 14 & 11.5 & 29 & 11 \\
\hline$a \succ c \succ b \succ d$ & 29 & 10.5 & 27.5 & 10.5 & 9.5 & 25.5 & 9.5 \\
\hline$a \succ c \succ d \succ b$ & 29.5 & 13.5 & 29.5 & 13.5 & 7 & 26 & 7 \\
\hline$a \succ d \succ b \succ c$ & 36 & 19 & 32.5 & 19 & 11.5 & 31.5 & 11 \\
\hline$a \succ d \succ c \succ b$ & 34.5 & 16 & 33 & 16 & 12.5 & 32 & 12.5 \\
\hline$b \succ a \succ c \succ d$ & 30.5 & 11.5 & 28 & 11.5 & 10.5 & 26.5 & 10.5 \\
\hline$b \succ a \succ d \succ c$ & 36.5 & 17 & 32.5 & 17 & 13 & 32 & 13 \\
\hline$b \succ c \succ a \succ d$ & 34 & 17.5 & 31.5 & 17.5 & 11 & 29.5 & 11 \\
\hline$b \succ c \succ d \succ a$ & 37.5 & 16.5 & 36.5 & 16.5 & 9.5 & 32 & 9.5 \\
\hline$b \succ d \succ a \succ c$ & 37.5 & 21 & 35 & 21 & 16.5 & 34 & 16.5 \\
\hline$b \succ d \succ c \succ a$ & 42.5 & 17.5 & 41.5 & 17.5 & 14.5 & 37.5 & 14.5 \\
\hline$c \succ a \succ b \succ d$ & 32 & 13 & 30.5 & 13 & 7.5 & 29 & 7.5 \\
\hline$c \succ a \succ d \succ b$ & 36.5 & 22 & 34 & 22 & 13.5 & 33 & 13.5 \\
\hline$c \succ b \succ a \succ d$ & 35 & 15 & 33.5 & 15 & 11.5 & 32 & 11.5 \\
\hline$c \succ b \succ d \succ a$ & 41.5 & 25.5 & 40 & 25.5 & 23.5 & 36.5 & 23.5 \\
\hline$c \succ d \succ a \succ b$ & 39.5 & 27 & 38 & 27 & 19.5 & 37.5 & 19 \\
\hline$c \succ d \succ b \succ a$ & 49.5 & 30 & 46 & 30 & 23.5 & 41 & 23.5 \\
\hline$d \succ a \succ b \succ c$ & 40 & 16 & 37 & 16 & 8 & 34.5 & 8 \\
\hline$d \succ a \succ c \succ b$ & 42.5 & 25 & 40 & 25 & 23.5 & 38.5 & 23.5 \\
\hline$d \succ b \succ a \succ c$ & 41.5 & 17.5 & 40 & 17.5 & 12 & 38 & 12 \\
\hline$d \succ b \succ c \succ a$ & 50 & 35.5 & 48 & 35 & 34 & 44 & 33 \\
\hline$d \succ c \succ a \succ b$ & 50 & 30 & 47 & 30 & 23.5 & 43 & 23.5 \\
\hline$d \succ c \succ b \succ a$ & 61 & 36.5 & 51 & 36.5 & 34.5 & 49 & 34.5 \\
\hline
\end{tabular}

Table 10.11: Cost of a closest $*$-monotone profile of rankings with ties w.r.t. each possible ranking on $\mathscr{C}$.

We conclude that the ranking $a \succ b \succ c \succ d$ is the ranking that is the closest to imposing all types of $*$-monotonicity. 


\subsection{Orange harvest in Argentina}

In this section, the proposed methodology is illustrated on a decision making problem concerning the orange harvest in the Argentinian province of Corrientes. The farmers of this province have four different methods (from now on referred to as candidates and denoted by $a, b, c$ and $d$ ) to estimate the production of an orange tree based on, among other factors, the amount of fallen pieces of fruit and the volume of its crown. Although the original number of orange trees in the considered orchards equals 2462, as part of a preliminary process of data cleaning, trees with a missing value for at least one of the methods were disregarded, yielding estimates for 1565 trees for further analysis.

In order to reduce the cost associated with the estimation, all these factors are measured inaccurately, leading to estimates with a varying degree of error. A quick glance at the data shows that the average production of each tree equals 586.51, while the average estimation error embarrassingly equals 378.79. The number of outliers is overwhelming. Rather than being the exception, they may be considered the standard. This issue hinders the ranking of the methods by means of techniques based on the analysis of the estimation errors. Therefore, the farmers tend to disregard the value of the estimates and exclusively consider the ordinal part of the data.

After the harvest, farmers ranked the four considered methods according to how accurate they were estimating the production of each tree. The final goal is to rank the four methods according to their accuracy for estimating the production of the local orange trees in order to decide what method should be used during the next harvest season. In that way, we do not consider here a voting problem where each voter expresses his/her personal preferences on the set of candidates, but an identification problem where the goal is to recognize the unknown true ranking on the set of candidates. For more details, we refer to [45].

In Table 10.12, the expressed $r=1565$ rankings and their frequency are provided. Note that the profile of rankings is not monotone w.r.t. any ranking on the set of candidates.

It should be remarked that the most frequent ranking in the profile is the ranking $c \succ d \succ$ $b \succ a$. However, as the profile of rankings is not monotone w.r.t. any ranking on the set of candidates, the optimal ranking could differ from the most frequent ranking. It should also be remarked that, as we observe from the low values of the first column and the two first rows of Table 10.12, method $a$ clearly is less accurate than the other three methods. 


\begin{tabular}{|c|c||c|c||c|c||c|c|}
\hline Ranking & Freq. & Ranking & Freq. & Ranking & Freq. & Ranking & Freq. \\
\hline$a \succ b \succ c \succ d$ & 3 & $b \succ a \succ c \succ d$ & 8 & $c \succ a \succ b \succ d$ & 10 & $d \succ a \succ b \succ c$ & 6 \\
$a \succ b \succ d \succ c$ & 2 & $b \succ a \succ d \succ c$ & 11 & $c \succ a \succ d \succ b$ & 17 & $d \succ a \succ c \succ b$ & 12 \\
$a \succ c \succ b \succ d$ & 1 & $b \succ c \succ a \succ d$ & 76 & $c \succ b \succ a \succ d$ & 98 & $d \succ b \succ a \succ c$ & 71 \\
$a \succ c \succ d \succ b$ & 0 & $b \succ c \succ d \succ a$ & 135 & $c \succ b \succ d \succ a$ & 165 & $d \succ b \succ c \succ a$ & 148 \\
$a \succ d \succ b \succ c$ & 1 & $b \succ d \succ a \succ c$ & 60 & $c \succ d \succ a \succ b$ & 113 & $d \succ c \succ a \succ b$ & 111 \\
$a \succ d \succ c \succ b$ & 0 & $b \succ d \succ c \succ a$ & 134 & $c \succ d \succ b \succ a$ & 204 & $d \succ c \succ b \succ a$ & 179 \\
\hline
\end{tabular}

Table 10.12: Expressed rankings and their frequency for the orange harvest problem in the Argentinian province of Corrientes.

The scorix induced by the profile of rankings given by the voters is:

$$
S=\left(\begin{array}{cccc}
7 & 64 & 529 & 965 \\
424 & 487 & 401 & 253 \\
607 & 502 & 305 & 151 \\
527 & 512 & 330 & 196
\end{array}\right)
$$

We easily see that $S$ is monotone w.r.t. the ranking $c \succ d \succ b \succ a$. Actually, $S$ is recursively monotone w.r.t. $c \succ d \succ b \succ a$.

The relation $>_{B}$ is given by:

$$
>_{B}=\{(b, a),(c, a),(c, b),(c, d),(d, a),(d, b)\} .
$$

Actually, $>_{B}$ is the ranking $c \succ d \succ b \succ a$. This result follows from the fact that the scorix is monotone w.r.t. the ranking $c \succ d \succ b \succ a$. We conclude that candidate $c$ is the quasipositional winner.

Evidently, $\geq_{B}$ is given by:

$$
\geq_{B}=\{(a, a),(b, a),(b, b),(c, a),(c, b),(c, c),(c, d),(d, a),(d, b),(d, d)\} .
$$

Figure 10.6 displays the Hasse diagram of the order relation $\geq_{B}$. Note that the quasipositionally undominated set is here given by $\mathscr{U}_{B}=\{c\}$.

Similarly, the relation $>_{R}$ is given by:

$$
>_{R}=\{(b, a),(c, a),(c, b),(c, d),(d, a),(d, b)\} .
$$




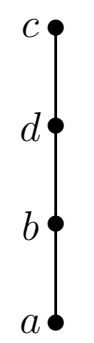

Figure 10.6: Hasse diagram of the order relation $\geq_{B}$.

Actually, $>_{R}$ is the ranking $c \succ d \succ b \succ a$. This result follows from the fact that the scorix is recursively monotone w.r.t. the ranking $c \succ d \succ b \succ a$. We conclude that candidate $c$ is the positional winner.

Evidently, $\geq_{R}$ is given by:

$$
\geq_{R}=\{(a, a),(b, a),(b, b),(c, a),(c, b),(c, c),(c, d),(d, a),(d, b),(d, d)\} .
$$

Figure 10.7 displays the Hasse diagram of the order relation $\geq_{R}$. Note that the positionally undominated set is here given by $\mathscr{U}_{R}=\{c\}$.

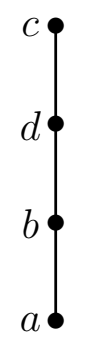

Figure 10.7: Hasse diagram of the order relation $\geq_{R}$.

In Table 10.13 , the costs of imposing monotonicity and recursive monotonicity on the scorix w.r.t. each possible ranking are listed. Obviously, as the scorix is already monotone w.r.t. this ranking, we conclude that $c \succ d \succ b \succ a$ is the ranking w.r.t. which the closest profile of rankings with a (recursively) monotone scorix leads to the lowest cost (zero).

Note that, as expected due to Theorem 5.13, the cost of imposing monotonicity on the scorix is always lower than or equal to the cost of imposing recursive monotonicity on the scorix. We also note that all rankings where candidate $a$ is not ranked at the last position lead to a really big cost. 


\begin{tabular}{|c|c|c||c|c|c|}
\hline Ranking & Cost S & Cost RS & Ranking & Cost S & Cost RS \\
\hline$a \succ b \succ c \succ d$ & 1671 & 1925 & $c \succ a \succ b \succ d$ & 1457 & 1702 \\
$a \succ b \succ d \succ c$ & 1671 & 1928 & $c \succ a \succ d \succ b$ & 1457 & 1582 \\
$a \succ c \succ b \succ d$ & 1671 & 1878 & $c \succ b \succ a \succ d$ & 1375 & 1399 \\
$a \succ c \succ d \succ b$ & 1671 & 1777 & $c \succ b \succ d \succ a$ & 151 & 151 \\
$a \succ d \succ b \succ c$ & 1671 & 1926 & $c \succ d \succ a \succ b$ & 1185 & 1185 \\
$a \succ d \succ c \succ b$ & 1671 & 1845 & $c \succ d \succ b \succ a$ & $\mathbf{0}$ & $\mathbf{0}$ \\
$b \succ a \succ c \succ d$ & 1605 & 1889 & $d \succ a \succ b \succ c$ & 1525 & 1845 \\
$b \succ a \succ d \succ c$ & 1605 & 1925 & $d \succ a \succ c \succ b$ & 1525 & 1690 \\
$b \succ c \succ a \succ d$ & 1410 & 1523 & $d \succ b \succ a \succ c$ & 1492 & 1531 \\
$b \succ c \succ d \succ a$ & 283 & 313 & $d \succ b \succ c \succ a$ & 262 & 288 \\
$b \succ d \succ a \succ c$ & 1508 & 1605 & $d \succ c \succ a \succ b$ & 1195 & 1239 \\
$b \succ d \succ c \succ a$ & 283 & 356 & $d \succ c \succ b \succ a$ & 104 & 105 \\
\hline
\end{tabular}

Table 10.13: Cost of a closest profile of rankings with a monotone scorix (Cost S) and with a recursively monotone scorix (Cost RS) w.r.t. each possible ranking on $\mathscr{C}$.

The votrix induced by the profile of rankings given by the voters is:

$$
V=\left(\begin{array}{cccc}
0 & 276 & 175 & 227 \\
1289 & 0 & 655 & 703 \\
1390 & 910 & 0 & 830 \\
1338 & 862 & 735 & 0
\end{array}\right)
$$

We see that $V$ is monotone w.r.t. the ranking $c \succ d \succ b \succ a$.

The relation $>_{C}$ is given by:

$$
>_{C}=\{(b, a),(c, a),(c, b),(c, d),(d, a),(d, b)\} .
$$

Actually, $>_{C}$ is the ranking $c \succ d \succ b \succ a$. This result follows from the fact that the votrix is monotone w.r.t. the ranking $c \succ d \succ b \succ a$. We conclude that candidate $c$ is the pairwise winner.

Evidently, $\geq_{C}$ is given by:

$$
\geq_{C}=\{(a, a),(b, a),(b, b),(c, a),(c, b),(c, c),(c, d),(d, a),(d, b),(d, d)\} .
$$




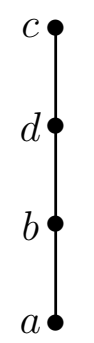

Figure 10.8: Hasse diagram of the order relation $\geq_{C}$.

Figure 10.8 displays the Hasse diagram of the order relation $\unrhd_{C}$.

Note that the pairwisely undominated set is here given by $\mathscr{U}_{C}=\{c\}$.

The votex induced by the profile of rankings given by the voters is:

$$
\begin{array}{rlc}
W: \mathscr{C}_{\neq}^{2} & \longrightarrow & \{0, \ldots, 1565\}^{6} \\
W(a, b) & = & (269,449,571,245,31,0) \\
W(a, c) & = & (369,525,496,152,20,3) \\
W(a, d) & = & (327,520,491,203,20,4) \\
W(b, c) & = & (130,325,455,369,215,71) \\
W(b, d) & = & (123,298,441,372,247,84) \\
W(c, d) & =(77,221,437,463,259,108)
\end{array}
$$

Although the votrix is monotone w.r.t. the ranking $c \succ d \succ b \succ a$, the votex is not monotone w.r.t. any ranking. Note that the votex is not monotone w.r.t. the ranking $c \succ d \succ b \succ a$ since candidate $b$ is never ranked at the last position at the same time that $a$ is ranked at the first position, but candidate $c$ is ranked three times at the last position at the same time that $a$ is ranked at the first position. As the votrix is only monotone w.r.t. the ranking $c \succ d \succ b \succ a$, the votex cannot be monotone w.r.t. any other ranking on the set of candidates (see Theorem 5.23).

In Table 10.14, the costs of imposing monotonicity on the votrix and on the votex w.r.t. each possible ranking are listed. Note that, as expected due to Theorem 5.23 , the cost of imposing monotonicity on the votrix is always lower than or equal to the cost of imposing monotonicity on the votex. We conclude that $c \succ d \succ b \succ a$ is the ranking w.r.t. which the closest profile of rankings with a monotone votrix/votex leads to the lowest cost (zero/three). 


\begin{tabular}{|c|c|c||c|c|c|}
\hline Ranking & Cost V & Cost W & Ranking & Cost V & Cost W \\
\hline$a \succ b \succ c \succ d$ & 1926 & 1927 & $c \succ a \succ b \succ d$ & 1703 & 1703 \\
$a \succ b \succ d \succ c$ & 1927 & 1928 & $c \succ a \succ d \succ b$ & 1702 & 1702 \\
$a \succ c \succ b \succ d$ & 1925 & 1925 & $c \succ b \succ a \succ d$ & 1702 & 1702 \\
$a \succ c \succ d \succ b$ & 1845 & 1845 & $c \succ b \succ d \succ a$ & 209 & 211 \\
$a \succ d \succ b \succ c$ & 1926 & 1927 & $c \succ d \succ a \succ b$ & 1463 & 1463 \\
$a \succ d \succ c \succ b$ & 1846 & 1846 & $c \succ d \succ b \succ a$ & $\mathbf{0}$ & $\mathbf{3}$ \\
$b \succ a \succ c \succ d$ & 1925 & 1926 & $d \succ a \succ b \succ c$ & 1846 & 1846 \\
$b \succ a \succ d \succ c$ & 1926 & 1927 & $d \succ a \succ c \succ b$ & 1845 & 1845 \\
$b \succ c \succ a \succ d$ & 1830 & 1830 & $d \succ b \succ a \succ c$ & 1845 & 1845 \\
$b \succ c \succ d \succ a$ & 356 & 356 & $d \succ b \succ c \succ a$ & 356 & 356 \\
$b \succ d \succ a \succ c$ & 1925 & 1925 & $d \succ c \succ a \succ b$ & 1511 & 1511 \\
$b \succ d \succ c \succ a$ & 357 & 357 & $d \succ c \succ b \succ a$ & 148 & 148 \\
\hline
\end{tabular}

Table 10.14: Cost of a closest profile of rankings with a monotone votrix $($ Cost V) and with a monotone votex (Cost W) w.r.t. each possible ranking on $\mathscr{C}$.

Figure 10.9 displays the given profile of rankings represented on the diagram of $\geqq$ (where $\succ$ represents the ranking $c \succ d \succ b \succ a$ ). Note that the profile of rankings is not monotone w.r.t. the ranking $c \succ d \succ b \succ a$. As $c \succ d \succ b \succ a$ is the most frequent ranking, the profile of rankings cannot be monotone w.r.t. any other ranking on the set of candidates. Nevertheless, although there are some violations of monotonicity, the cost of imposing monotonicity w.r.t. the ranking $c \succ d \succ b \succ a$ should be 'low'.

In Table 10.15, the costs of imposing monotonicity on the profile w.r.t. each possible ranking are listed. We conclude that $c \succ d \succ b \succ a$ is the ranking w.r.t. which the closest monotone profile of rankings leads to the lowest cost (five).

The last analysis for the orange harvest problem is linked to the search for a Condorcet ranking, acclamation and unanimity. As the votrix induced by the profile of rankings given by the voters is monotone w.r.t. the ranking $c \succ d \succ b \succ a$, the ranking $c \succ d \succ b \succ a$ is the Condorcet ranking for the profile of rankings given by the voters. Obviously, the ranking that leads to the lowest cost (zero) when searching for a Condorcet ranking is the ranking $c \succ d \succ b \succ a$. Under the existence of a Condorcet ranking, the ranking that leads to the lowest cost (2771) when searching for unanimity (which is the winner according 


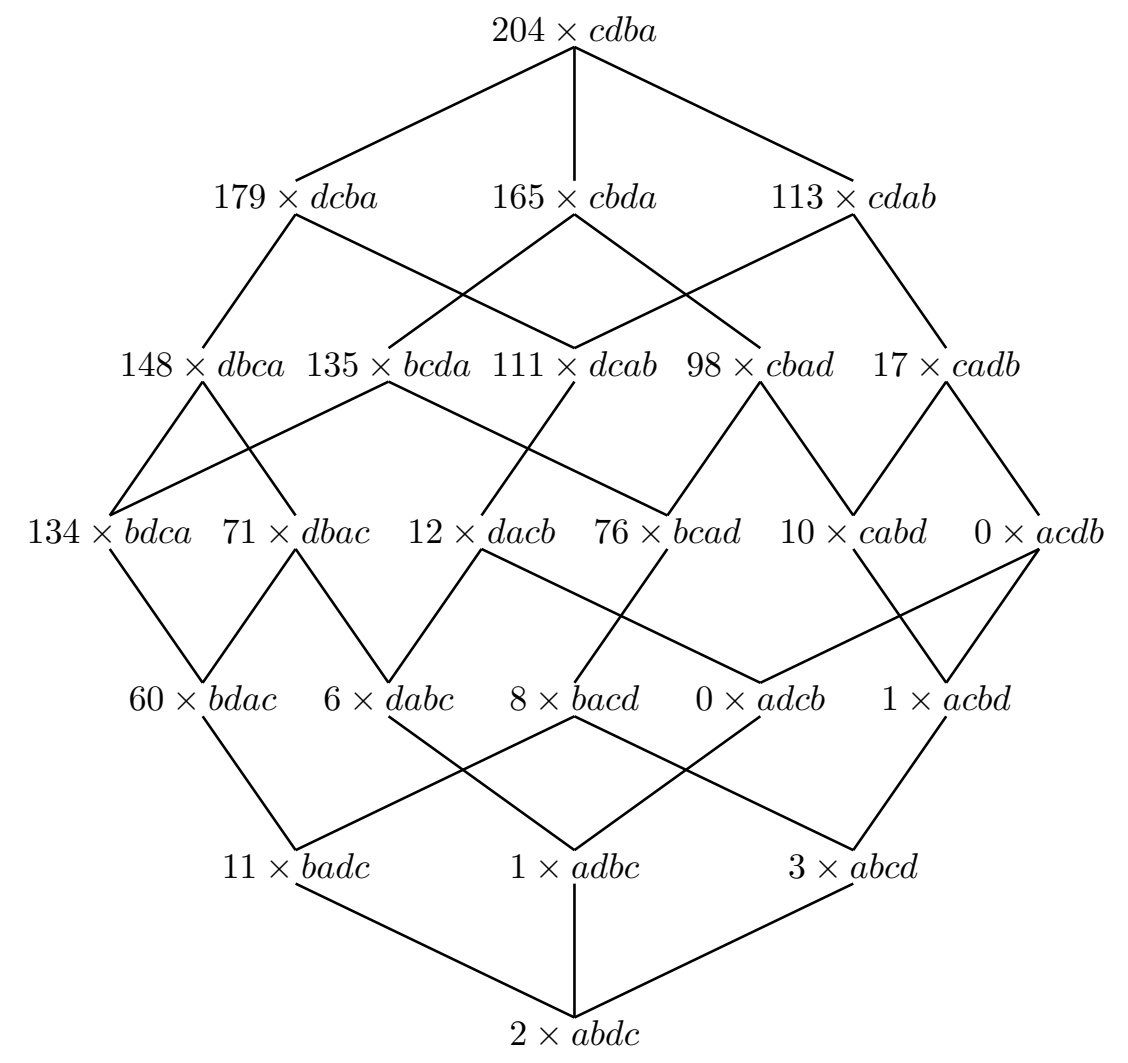

Figure 10.9: Frequencies of Table 10.12 represented on the Hasse diagram of the order relation $\geqq$ for the ranking $c \succ d \succ b \succ a$.

\begin{tabular}{|c|c||c|c||c|c||c|c|}
\hline Ranking & Cost & Ranking & Cost & Ranking & Cost & Ranking & Cost \\
\hline$a \succ b \succ c \succ d$ & 1883 & $b \succ a \succ c \succ d$ & 1374 & $c \succ a \succ b \succ d$ & 973 & $d \succ a \succ b \succ c$ & 1216 \\
$a \succ b \succ d \succ c$ & 1931 & $b \succ a \succ d \succ c$ & 1424 & $c \succ a \succ d \succ b$ & 891 & $d \succ a \succ c \succ b$ & 1086 \\
$a \succ c \succ b \succ d$ & 1712 & $b \succ c \succ a \succ d$ & 669 & $c \succ b \succ a \succ d$ & 593 & $d \succ b \succ a \succ c$ & 788 \\
$a \succ c \succ d \succ b$ & 1676 & $b \succ c \succ d \succ a$ & 139 & $c \succ b \succ d \succ a$ & 46 & $d \succ b \succ c \succ a$ & 105 \\
$a \succ d \succ b \succ c$ & 1852 & $b \succ d \succ a \succ c$ & 840 & $c \succ d \succ a \succ b$ & 341 & $d \succ c \succ a \succ b$ & 367 \\
$a \succ d \succ c \succ b$ & 1699 & $b \succ d \succ c \succ a$ & 161 & $c \succ d \succ b \succ a$ & $\mathbf{6}$ & $d \succ c \succ b \succ a$ & 20 \\
\hline
\end{tabular}

Table 10.15: Cost of a closest monotone profile of rankings w.r.t. each possible ranking on $\mathscr{C}$.

to the method of Kemeny) always coincides with this Condorcet ranking. It remains to identify the ranking that leads to the lowest cost when searching for acclamation. 
In Table 10.16, the costs associated with the search for a Condorcet ranking, acclamation and unanimity w.r.t. each possible ranking are listed. We conclude that $c \succ d \succ b \succ a$ is the ranking that leads to the lowest cost in all three cases.

\begin{tabular}{|c|c|c|c||c|c|c|c|}
\hline Ranking & Cost C & Cost A & Cost U & Ranking & Cost C & Cost A & Cost U \\
\hline$a \succ b \succ c \succ d$ & 1879 & 1928 & 6524 & $c \succ a \succ b \succ d$ & 1143 & 1703 & 5054 \\
$a \succ b \succ d \succ c$ & 1927 & 1933 & 6619 & $c \succ a \succ d \succ b$ & 1063 & 1702 & 4895 \\
$a \succ c \succ b \succ d$ & 1751 & 1925 & 6269 & $c \succ b \succ a \succ d$ & 636 & 1702 & 4041 \\
$a \succ c \succ d \succ b$ & 1671 & 1845 & 6110 & $c \succ b \succ d \succ a$ & 80 & 212 & 2930 \\
$a \succ d \succ b \succ c$ & 1847 & 1932 & 6460 & $c \succ d \succ a \succ b$ & 507 & 1463 & 3784 \\
$a \succ d \succ c \succ b$ & 1719 & 1846 & 6205 & $c \succ d \succ b \succ a$ & $\mathbf{0}$ & $\mathbf{6}$ & $\mathbf{2 7 7 1}$ \\
$b \succ a \succ c \succ d$ & 1372 & 1927 & 5511 & $d \succ a \succ b \succ c$ & 1291 & 1846 & 5349 \\
$b \succ a \succ d \succ c$ & 1420 & 1928 & 5606 & $d \succ a \succ c \succ b$ & 1163 & 1845 & 5094 \\
$b \succ c \succ a \succ d$ & 764 & 1830 & 4296 & $d \succ b \succ a \succ c$ & 784 & 1845 & 4336 \\
$b \succ c \succ d \succ a$ & 208 & 356 & 3185 & $d \succ b \succ c \succ a$ & 176 & 356 & 3121 \\
$b \succ d \succ a \succ c$ & 864 & 1925 & 4495 & $d \succ c \succ a \succ b$ & 555 & 1511 & 3879 \\
$b \succ d \succ c \succ a$ & 256 & 357 & 3280 & $d \succ c \succ b \succ a$ & 48 & 148 & 2866 \\
\hline
\end{tabular}

Table 10.16: Cost of a closest profile with a Condorcet ranking (Cost C), a closest acclaimed profile (Cost A) and a closest unanimous profile (Cost U) w.r.t. each possible ranking on $\mathscr{C}$.

As expected due to Theorem 5.32 , the cost associated with the search for a Condorcet ranking is always lower than or equal to the cost associated with the search for acclamation. As also expected due to Theorem 5.32, the cost associated with the search for acclamation is always lower than or equal to the cost associated with the search for unanimity. Nevertheless, as discussed in Chapter 7, the ranking leading to the lowest cost does not need to coincide for the three searches, although this is the case in this example.

We conclude this section by analysing the optimal ranking w.r.t. the search for each of the considered consensus states. Note that, as listed in Table 10.17, the optimal ranking according to the search for all consensus states is the ranking $c \succ d \succ b \succ a$.

From the results of this case study it follows that the ranking $c \succ d \succ b \succ a$ clearly is the most natural ranking of the four methods used by the farmers in Corrientes. 


\begin{tabular}{|c|c||c|c|}
\hline Search for & Optimal ranking & Search for & Optimal ranking \\
\hline Monotone scorix & $c \succ d \succ b \succ a$ & Rec. monotone scorix & $c \succ d \succ b \succ a$ \\
\hline Monotone votrix & $c \succ d \succ b \succ a$ & Monotone votex & $c \succ d \succ b \succ a$ \\
\hline Monotone profile & $c \succ d \succ b \succ a$ & Condorcet ranking & $c \succ d \succ b \succ a$ \\
\hline Acclamation & $c \succ d \succ b \succ a$ & Unanimity & $c \succ d \succ b \succ a$ \\
\hline
\end{tabular}

Table 10.17: Optimal ranking w.r.t. the search for all the considered consensus states.

For this case study, there does not exist a unanimous winner, nor a majority winner. However, candidate $c$ is the quasipositional winner, the positional winner, the pairwise winner, the Condorcet winner and the Borda winner.

\subsection{Food spoilage in Belgium}

In this section, we apply the methodology proposed in this dissertation to a dataset of an acceptance test that measures the degree of freshness of samples of cod fish [87, 127]. At the Laboratory of Food Microbiology and Food Preservation at Ghent University, $r=9$ experts were asked to give their appreciation of the cod fish samples by ranking them, in terms of perceived freshness based on their smell.

Four different samples of cod fish were considered. We denote by a the zero-days-old sample, $b$ the three-days-old sample, $c$ the five-days-old sample and $d$ the seven-days-old sample. In Table 10.18, the expressed rankings and their frequency are provided.

\begin{tabular}{|c|c|}
\hline$\# \succ_{i}$ & Rankings on $\mathscr{C}$ \\
\hline 4 & $a \succ b \succ c \succ d$ \\
2 & $a \succ b \succ d \succ c$ \\
1 & $a \succ c \succ b \succ d$ \\
1 & $d \succ b \succ a \succ c$ \\
1 & $d \succ c \succ b \succ a$ \\
\hline
\end{tabular}

Table 10.18: Expressed rankings and their frequency for the food spoilage problem. 
Note that the problem considered in this section has a different nature than the problems considered in the previous two sections. While the true ranking on the set of candidates is totally unknown in the Lar rangeland and the orange harvest problem, here, the freshness of each sample is known and, therefore, samples can actually be ranked according to the known freshness.

The scorix induced by the profile of rankings given by the voters is:

$$
S=\left(\begin{array}{llll}
7 & 0 & 1 & 1 \\
0 & 7 & 2 & 0 \\
0 & 2 & 4 & 3 \\
2 & 0 & 2 & 5
\end{array}\right)
$$

We see that $S$ is not (recursively) monotone w.r.t. any ranking on the set of candidates due to the fact that the vector of positions of candidate $b$ does not dominate the vector of positions of candidate $d(0<2)$ and, at the same time, the vector of positions of candidate $d$ does not dominate the vector of positions of candidate $b(0+7>2+0)$.

The relation $>_{B}$ is given by:

$$
>_{B}=\{(a, c),(a, d),(b, c)\}
$$

Evidently, $\geq_{B}$ is given by:

$$
\geq_{B}=\{(a, a),(a, c),(a, d),(b, b),(b, c),(c, c),(d, d)\} .
$$

Figure 10.10 displays the Hasse diagram of the order relation $\geq_{B}$. Note that the quasipositionally undominated set is here given by $\mathscr{U}_{B}=\{a, b\}$.

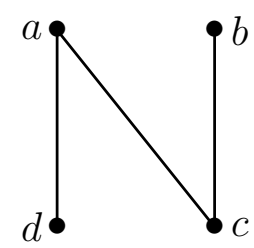

Figure 10.10: Hasse diagram of the order relation $\geq_{B}$.

Similarly, the relation $>_{R}$ is given by:

$$
>_{R}=\{(a, c),(a, d),(b, c)\}
$$


Evidently, $\geq_{R}$ is given by:

$$
\geq_{R}=\{(a, a),(a, c),(a, d),(b, b),(b, c),(c, c),(d, d)\} .
$$

Figure 10.11 displays the Hasse diagram of the order relation $\geq_{R}$. Note that the positionally undominated set is here given by $\mathscr{U}_{R}=\{a, b\}$.

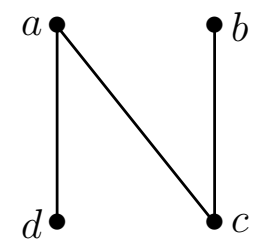

Figure 10.11: Hasse diagram of the order relation $\geq_{R}$.

In Table 10.19, the costs of imposing monotonicity and recursive monotonicity on the scorix w.r.t. each possible ranking are listed. We conclude that both rankings $a \succ b \succ c \succ d$ and $a \succ b \succ d \succ c$ are a ranking w.r.t. which the closest profile of rankings with a (recursively) monotone scorix leads to the lowest cost (three). Of course, both rankings $a \succ b \succ c \succ d$ and $a \succ b \succ c \succ d$ linearly extend the strict partial order relation given by $>_{B}$.

Note that, as expected due to Theorem 5.13, the cost of imposing monotonicity on the scorix is always lower than or equal to the cost of imposing recursive monotonicity on the scorix.

The votrix induced by the profile of rankings given by the voters is:

$$
V=\left(\begin{array}{llll}
0 & 7 & 8 & 7 \\
2 & 0 & 7 & 7 \\
1 & 2 & 0 & 5 \\
2 & 2 & 4 & 0
\end{array}\right)
$$

We see that $V$ is not monotone w.r.t. any ranking on the set of candidates. First, we note that the ranking $a \succ b \succ c \succ d$ is the Condorcet ranking. Therefore, the only ranking on the set of candidates w.r.t. the votrix can be monotone is the ranking $a \succ b \succ c \succ d$. Nevertheless, the number of voters preferring candidate $a$ to candidate $c$ (eight) is greater than the number of voters preferring candidate $a$ to candidate $d$ (seven).

The relation $>_{C}$ is given by:

$$
>_{C}=\{(a, b),(a, c),(a, d),(b, c),(b, d)\} .
$$




\begin{tabular}{|c|c|c||c|c|c|}
\hline Ranking & Cost S & Cost RS & Ranking & Cost S & Cost RS \\
\hline$a \succ b \succ c \succ d$ & $\mathbf{3}$ & $\mathbf{3}$ & $c \succ a \succ b \succ d$ & 11 & 12 \\
$a \succ b \succ d \succ c$ & $\mathbf{3}$ & $\mathbf{3}$ & $c \succ a \succ d \succ b$ & 13 & 13 \\
$a \succ c \succ b \succ d$ & 7 & 7 & $c \succ b \succ a \succ d$ & 12 & 13 \\
$a \succ c \succ d \succ b$ & 8 & 8 & $c \succ b \succ d \succ a$ & 14 & 15 \\
$a \succ d \succ b \succ c$ & 6 & 6 & $c \succ d \succ a \succ b$ & 14 & 16 \\
$a \succ d \succ c \succ b$ & 7 & 8 & $c \succ d \succ b \succ a$ & 15 & 16 \\
$b \succ a \succ c \succ d$ & 5 & 5 & $d \succ a \succ b \succ c$ & 11 & 11 \\
$b \succ a \succ d \succ c$ & 5 & 5 & $d \succ a \succ c \succ b$ & 13 & 14 \\
$b \succ c \succ a \succ d$ & 9 & 10 & $d \succ b \succ a \succ c$ & 12 & 12 \\
$b \succ c \succ d \succ a$ & 12 & 13 & $d \succ b \succ c \succ a$ & 14 & 16 \\
$b \succ d \succ a \succ c$ & 10 & 11 & $d \succ c \succ a \succ b$ & 14 & 16 \\
$b \succ d \succ c \succ a$ & 12 & 13 & $d \succ c \succ b \succ a$ & 15 & 17 \\
\hline
\end{tabular}

Table 10.19: Cost of a closest profile of rankings with a monotone scorix (Cost S) and with a recursively monotone scorix (Cost RS) w.r.t. each possible ranking on $\mathscr{C}$.

We conclude that candidate $a$ is the pairwise winner. Evidently, $\geq_{C}$ is given by:

$$
\geq_{C}=\{(a, a),(a, b),(a, c),(a, d),(b, b),(b, c),(b, d),(c, c),(d, d)\} .
$$

Figure 10.12 displays the Hasse diagram of the order relation $\geq_{C}$.

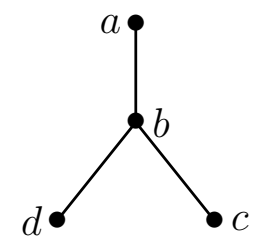

Figure 10.12: Hasse diagram of the order relation $\geq_{C}$.

Note that the pairwisely undominated set is here given by $\mathscr{U}_{C}=\{a\}$.

The votex induced by the profile of rankings given by the voters is:

$$
\begin{aligned}
& W: \quad \mathscr{C}_{\neq}^{2} \quad \longrightarrow \quad\{0, \ldots, 9\}^{6} \\
& W(a, b)=(0,0,2,6,1,0) \\
& W(a, c)=(0,1,0,2,4,2) \\
& W(a, d)=(1,1,0,0,2,5) \\
& \begin{aligned}
W: \mathscr{C}_{\neq}^{2} & \longrightarrow\{0, \ldots, 9\}^{6} \\
W(b, c) & =(0,0,2,4,3,0) \\
W(b, d) & =(0,1,1,3,4,0) \\
W(c, d) & =(1,0,3,4,1,0)
\end{aligned}
\end{aligned}
$$


As the votrix is not monotone w.r.t. any ranking on the set of candidates, we conclude that the votex induced by the profile of rankings given by the voters is not monotone w.r.t. any ranking either (see Theorem 5.23).

In Table 10.20, the costs of imposing monotonicity on the votrix and on the votex w.r.t. each possible ranking are listed. Note that, as expected due to Theorem 5.23 , the cost of imposing monotonicity on the votrix is always lower than or equal to the cost of imposing monotonicity on the votex. We conclude that both rankings $a \succ b \succ c \succ d$ and $a \succ b \succ d \succ$ $c$ are a ranking w.r.t. which the closest profile of rankings with a monotone votrix/votex leads to the lowest cost (one/six). Note that both $a \succ b \succ c \succ d$ and $a \succ b \succ d \succ c$ linearly extend the strict partial order relation given by $>_{C}$.

\begin{tabular}{|c|c|c||c|c|c|}
\hline Ranking & Cost V & Cost W & Ranking & Cost V & Cost W \\
\hline$a \succ b \succ c \succ d$ & $\mathbf{1}$ & $\mathbf{6}$ & $c \succ a \succ b \succ d$ & 11 & 15 \\
$a \succ b \succ d \succ c$ & $\mathbf{1}$ & $\mathbf{6}$ & $c \succ a \succ d \succ b$ & 14 & 15 \\
$a \succ c \succ b \succ d$ & 6 & 10 & $c \succ b \succ a \succ d$ & 12 & 16 \\
$a \succ c \succ d \succ b$ & 7 & 11 & $c \succ b \succ d \succ a$ & 15 & 19 \\
$a \succ d \succ b \succ c$ & 6 & 8 & $c \succ d \succ a \succ b$ & 15 & 18 \\
$a \succ d \succ c \succ b$ & 8 & 10 & $c \succ d \succ b \succ a$ & 15 & 21 \\
$b \succ a \succ c \succ d$ & 4 & 8 & $d \succ a \succ b \succ c$ & 12 & 14 \\
$b \succ a \succ d \succ c$ & 5 & 9 & $d \succ a \succ c \succ b$ & 15 & 16 \\
$b \succ c \succ a \succ d$ & 11 & 12 & $d \succ b \succ a \succ c$ & 13 & 16 \\
$b \succ c \succ d \succ a$ & 12 & 15 & $d \succ b \succ c \succ a$ & 16 & 20 \\
$b \succ d \succ a \succ c$ & 12 & 12 & $d \succ c \succ a \succ b$ & 16 & 20 \\
$b \succ d \succ c \succ a$ & 13 & 16 & $d \succ c \succ b \succ a$ & 17 & 22 \\
\hline
\end{tabular}

Table 10.20: Cost of a closest profile of rankings with a monotone votrix $($ Cost $\mathrm{V})$ and with a monotone votex (Cost W) w.r.t. each possible ranking on $\mathscr{C}$.

Figure 10.13 displays the given profile of rankings represented on the diagram of $\geqq$ (where $\succ$ represents the ranking $a \succ b \succ c \succ d$ ). Note that the profile of rankings is not monotone w.r.t. the ranking $a \succ b \succ c \succ d$. As $a \succ b \succ c \succ d$ is the most frequent ranking, the profile of rankings cannot be monotone w.r.t. any other ranking on the set of candidates. 


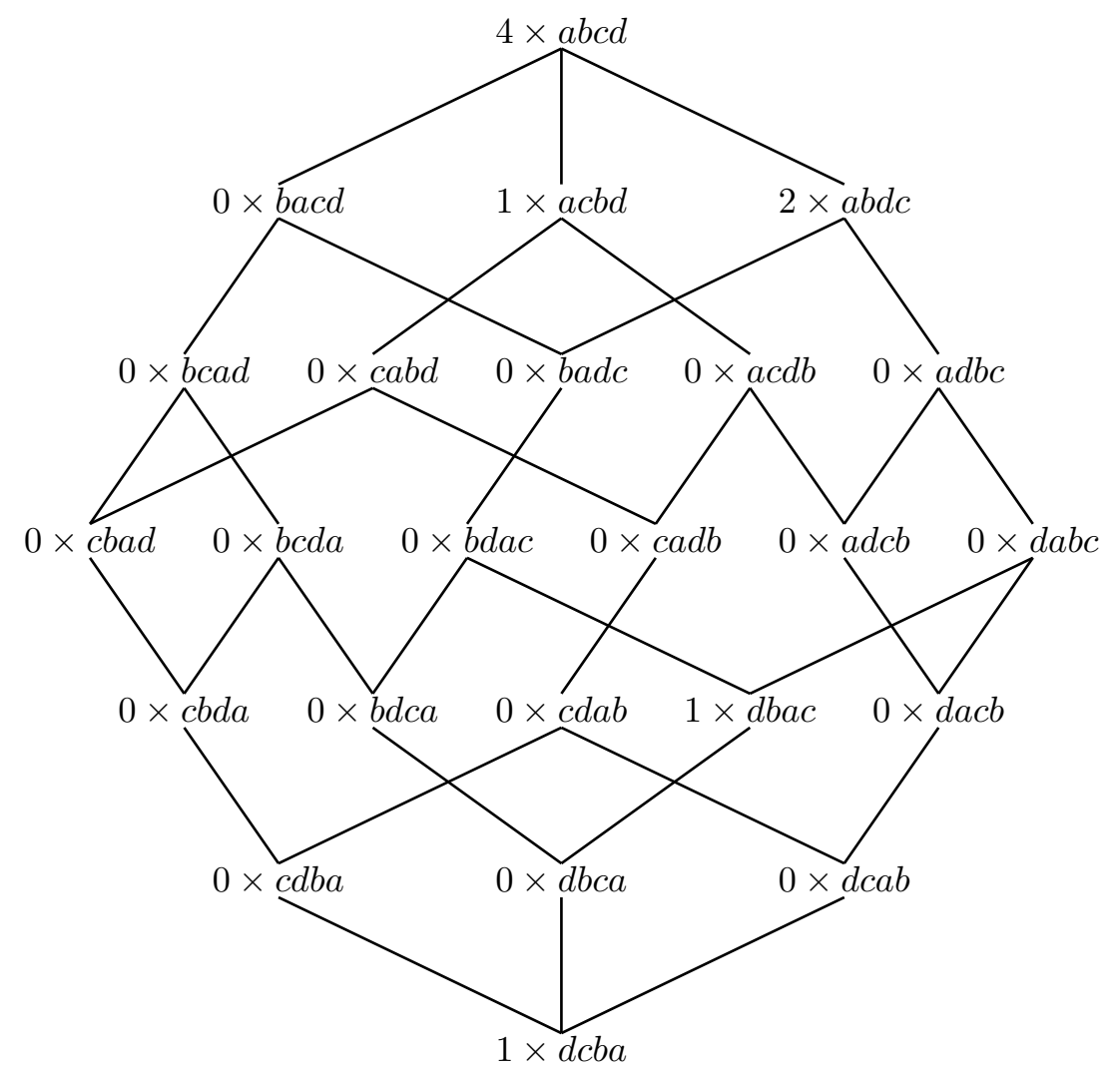

Figure 10.13: Frequencies of Table 10.18 represented on the Hasse diagram of the order relation $\geqq$ for the ranking $a \succ b \succ c \succ d$.

In Table 10.21, the costs of imposing monotonicity on the profile w.r.t. each possible ranking are listed. We conclude that $a \succ b \succ c \succ d$ is the ranking w.r.t. which the closest monotone profile of rankings leads to the lowest cost (five).

The last analysis for the orange harvest problem is linked to the search for a Condorcet ranking, acclamation and unanimity. As previously discussed, the ranking $a \succ b \succ c \succ d$ is the Condorcet ranking for the profile of rankings given by the voters. Obviously, the ranking that leads to the lowest cost (zero) when searching for a Condorcet ranking is the ranking $a \succ b \succ c \succ d$. Under the existence of a Condorcet ranking, the ranking that leads to the lowest cost (13) when searching for unanimity (which is the winner according to the method of Kemeny) always coincides with this Condorcet ranking. It remains to identify the ranking that leads to the lowest cost when searching for acclamation. 


\begin{tabular}{|c|c||c|c||c|c||c|c|}
\hline Ranking & Cost & Ranking & Cost & Ranking & Cost & Ranking & Cost \\
\hline$a \succ b \succ c \succ d$ & $\mathbf{5}$ & $b \succ a \succ c \succ d$ & 6 & $c \succ a \succ b \succ d$ & 12 & $d \succ a \succ b \succ c$ & 11 \\
$a \succ b \succ d \succ c$ & 6 & $b \succ a \succ d \succ c$ & 8 & $c \succ a \succ d \succ b$ & 13 & $d \succ a \succ c \succ b$ & 11 \\
$a \succ c \succ b \succ d$ & 7 & $b \succ c \succ a \succ d$ & 11 & $c \succ b \succ a \succ d$ & 13 & $d \succ b \succ a \succ c$ & 12 \\
$a \succ c \succ d \succ b$ & 10 & $b \succ c \succ d \succ a$ & 13 & $c \succ b \succ d \succ a$ & 17 & $d \succ b \succ c \succ a$ & 18 \\
$a \succ d \succ b \succ c$ & 9 & $b \succ d \succ a \succ c$ & 9 & $c \succ d \succ a \succ b$ & 17 & $d \succ c \succ a \succ b$ & 18 \\
$a \succ d \succ c \succ b$ & 11 & $b \succ d \succ c \succ a$ & 13 & $c \succ d \succ b \succ a$ & 22 & $d \succ c \succ b \succ a$ & 23 \\
\hline
\end{tabular}

Table 10.21: Cost of a closest monotone profile of rankings w.r.t. each possible ranking on $\mathscr{C}$.

In Table 10.22, the costs associated with the search for a Condorcet ranking, acclamation and unanimity w.r.t. each possible ranking are listed. We conclude that $a \succ b \succ c \succ d$ is the ranking that leads to the lowest cost in all three cases, but the ranking $a \succ b \succ d \succ c$ also leads to a lowest cost for the search for acclamation.

\begin{tabular}{|c|c|c|c||c|c|c|c|}
\hline Ranking & Cost C & Cost A & Cost U & Ranking & Cost C & Cost A & Cost U \\
\hline$a \succ b \succ c \succ d$ & $\mathbf{0}$ & $\mathbf{6}$ & $\mathbf{1 3}$ & $c \succ a \succ b \succ d$ & 7 & 17 & 25 \\
$a \succ b \succ d \succ c$ & 1 & $\mathbf{6}$ & 14 & $c \succ a \succ d \succ b$ & 10 & 18 & 30 \\
$a \succ c \succ b \succ d$ & 3 & 10 & 18 & $c \succ b \succ a \succ d$ & 10 & 18 & 30 \\
$a \succ c \succ d \succ b$ & 6 & 12 & 23 & $c \succ b \succ d \succ a$ & 13 & 21 & 35 \\
$a \succ d \succ b \succ c$ & 4 & 10 & 19 & $c \succ d \succ a \succ b$ & 13 & 21 & 35 \\
$a \succ d \succ c \succ b$ & 7 & 12 & 24 & $c \succ d \succ b \succ a$ & 16 & 26 & 40 \\
$b \succ a \succ c \succ d$ & 3 & 8 & 18 & $d \succ a \succ b \succ c$ & 7 & 14 & 24 \\
$b \succ a \succ d \succ c$ & 4 & 9 & 19 & $d \succ a \succ c \succ b$ & 10 & 17 & 29 \\
$b \succ c \succ a \succ d$ & 7 & 13 & 25 & $d \succ b \succ a \succ c$ & 10 & 17 & 29 \\
$b \succ c \succ d \succ a$ & 10 & 16 & 30 & $d \succ b \succ c \succ a$ & 14 & 22 & 36 \\
$b \succ d \succ a \succ c$ & 7 & 14 & 24 & $d \succ c \succ a \succ b$ & 14 & 22 & 36 \\
$b \succ d \succ c \succ a$ & 11 & 17 & 31 & $d \succ c \succ b \succ a$ & 17 & 27 & 41 \\
\hline
\end{tabular}

Table 10.22: Cost of a closest profile with a Condorcet ranking (Cost C), a closest acclaimed profile (Cost A) and a closest unanimous profile (Cost U) w.r.t. each possible ranking on $\mathscr{C}$.

As expected due to Theorem 5.32 , the cost associated with the search for a Condorcet ranking is always lower than or equal to the cost associated with the search for acclamation. 
As also expected due to Theorem 5.32 , the cost associated with the search for acclamation is always lower than or equal to the cost associated with the search for unanimity. Nevertheless, as discussed in Chapter 7 , the ranking leading to the lowest cost does not need to coincide for the three searches. For intance, we see that the search for acclamation leads to two optimal rankings, while the search for a Condorcet ranking and the search for unanimity lead to a unique optimal ranking.

We conclude this section by analysing the optimal ranking w.r.t. the search for each of the considered consensus states. As listed in Table 10.23 , the ranking $a \succ b \succ c \succ d$ is an optimal ranking according to the search for all consensus states. Nevertheless, the ranking $a \succ b \succ d \succ c$ is also an optimal ranking for the search for most consensus states.

\begin{tabular}{|c|c|c|c|}
\hline Search for & Optimal ranking & Search for & Optimal ranking \\
\hline Monotone scorix & $\begin{array}{l}a \succ b \succ c \succ d \\
a \succ b \succ d \succ c\end{array}$ & Rec. monotone scorix & $\begin{array}{l}a \succ b \succ c \succ d \\
a \succ b \succ d \succ c\end{array}$ \\
\hline Monotone votrix & $\begin{array}{l}a \succ b \succ c \succ d \\
a \succ b \succ d \succ c\end{array}$ & Monotone votex & $\begin{array}{l}a \succ b \succ c \succ d \\
a \succ b \succ d \succ c\end{array}$ \\
\hline Monotone profile & $a \succ b \succ c \succ d$ & Condorcet ranking & $a \succ b \succ c \succ d$ \\
\hline Acclamation & $\begin{array}{l}a \succ b \succ c \succ d \\
a \succ b \succ d \succ c\end{array}$ & Unanimity & $a \succ b \succ c \succ d$ \\
\hline
\end{tabular}

Table 10.23: Optimal ranking w.r.t. the search for all the considered consensus states.

From the results of this case study it follows that the ranking $a \succ b \succ c \succ d$ is the most natural ranking of the cod fish samples according to the perceived freshness. This result was already expected due to the fact that $a$ represents the zero-days-old sample, $b$ the three-days-old sample, $c$ the five-days-old sample and $d$ the seven-days-old sample. Nevertheless, the fact that the ranking $a \succ b \succ d \succ c$ is the optimal ranking according to the search for five out of eight consensus states - and it is only one unit of cost away from being optimal according the three other searches - may lead us to think that experts do not easily identify the difference in freshness between samples $c$ and $d$.

For this case study, there does not exist a unanimous winner. A quasipositional winner or a positional winner does not exist either. However, candidate $a$ is the pairwise winner, the majority winner, the Condorcet winner and the Borda winner. 


\section{PART IV}

EPILOGUE 



\section{CHAPTER 11}

\section{Conclusions and perspectives}

The field of social choice dates back to the eighteenth century, when Borda and Condorcet started an endless discussion about the use of either positional or pairwise information. Three centuries later, after countless axiomatic characterizations of ranking rules, impossibility theorems and many other study subjects that have called the attention of the scientific community, researchers still debate whether positional information is really sensitive to manipulation or pairwise information disregards the transitivity of the voters' preferences. Three centuries have passed and the scholars of social choice theory are still returning to the same old topics that led Borda and Condorcet to embark on more than one dialectic battle.

This eternal comeback makes us wonder how such an easy, intuitive and natural property as monotonicity - in the sense of this dissertation - has been disregarded through the years. It is not until a couple of years ago that Rademaker and De Baets pointed out that, for a ranking $a \succ b \succ c$ to represent a group's opinion, the number of voters preferring candidate $a$ to candidate $c$ should not be less than both the number of candidates preferring candidate $a$ to candidate $b$ and the number of voters preferring candidate $b$ to candidate c. In particular, they proposed a ranking rule that amounts to finding the ranking that is the closest to satisfying this natural property. The idea was undoubtedly revolutionary. Probably, Rademaker and De Baets did not know at the time that they just opened Pandora's box. Indeed, it resulted in the starting point of this PhD thesis and has led to an overwhelming number of open problems, which certainly matches the number of closed problems. 
This monotonicity property can be understood as a property of a particular representation of votes, the voting matrix or votrix. In general, a representation of votes is a construct that gathers the most important information given by the voters. Two representations of votes are historically considered in the field of social choice theory: the votrix and the scorix. One then wonders whether the notion of monotonicity can be translated to the scorix or not. As discussed in the very first chapters of this dissertation, the answer is affirmative. Monotonicity of the scorix can be understood as that, for a ranking $a \succ b \succ c$ to represent a group's opinion, the positions at which candidate $a$ is ranked should be better than both the positions at which candidate $b$ is ranked and the positions at which candidate $c$ is ranked; and, at the same time, the positions at which candidate $b$ is ranked should be better than the positions at which candidate $c$ is ranked. Monotonicity of the scorix did not result in such a revolutionary concept as monotonicity of the votrix, as several authors already analysed this property in the context of scoring ranking rules. Nevertheless, the search for monotonicity of the scorix did result in a novelty for social choice.

In general, the search for a property is a natural way of defining a ranking rule. Indeed, the method of Kemeny searches for the ranking that is the closest to becoming unanimous in the profile of rankings in terms of the Kendall distance function, the Borda count searches for the candidate that is the closest to becoming the unanimous winner in terms of the Kendall distance function, the plurality rule searches for the candidate that is the closest to becoming the unanimous winner in terms of the zero-one distance function, the method of Dodgson searches for the candidate that is the closest to becoming the Condorcet winner in terms of the Kendall tau distance function, etc. The set of all profiles of rankings satisfying certain property that obviously leads to a winning ranking (sometimes winning candidate) is referred to as a consensus state. The characterization of ranking rules by the minimization of the distance to a consensus state for some appropriate distance function is known by the name of distance rationalisation of ranking rules or metric rationalisation of ranking rules. However, as discussed in Chapter 3, we are not dealing with a notion of closeness in the most geometrical sense. The axioms of symmetry and, specially, the triangle inequality lack sense here. The role that the triangle inequality plays in giving a real meaning to a distance function is replaced by the preservation of a substantial betweenness relation. In that way, monometrics are introduced, leading to the monometric rationalisation of ranking rules.

By means of these newly introduced monometrics, we can now address the search for the best consensus state. But, what does 'best' mean here? Unanimity, as proposed by 
Kemeny, seems to be too restrictive. As unanimity tends not to hold in real-life elections, the search for unanimity turns out to highly depend on the chosen monometric. In most of the cases, just a change in the monometric may lead to a noteworthy change in the winning ranking. On the other hand, the presence of a Condorcet ranking seems to be too loose. As Saari explains in one of my favourite quotes in social choice: "the combination of the pairwise vote with the Condorcet terms loses the crucial fact that voters have transitive preferences. [...] An equally surprising assertion is that rather than being the standard, the Condorcet winner must be held suspect". Therefore, we need to find a consensus state that is considerably broader than unanimity, but that, at the same time, still leads to a consistent and undisputed winning ranking. Monotonicity comes again into play.

First, monotonicity of the scorix is proved to be a meeting point for all scoring ranking rules. Second, monotonicity of the votrix is proved to be a meeting point for most ranking rules based on pairwise information. Therefore, the intersection of both consensus states (w.r.t. the same ranking) turns out to be a meeting point for most ranking rules. Many other monotonicity-based consensus states are discussed in this dissertation. Recursive monotonicity of the scorix turns out to be a meeting point between the Borda and the Condorcet rankings. Monotonicity of the profile is linked to the real existence of a true ranking on the set of candidates, linked to the philosophy advocated by Rousseau and Condorcet where personal preferences are not considered and where we try to identify the 'general will'. This phylosophy is clearly described by Arrow: "each individual has two orderings, one which governs him in his everyday actions, and one which would be relevant under some ideal conditions and which is in some sense truer than the first ordering. It is the latter that is considered relevant to social choice, and it is assumed that there is complete unanimity with regard to the truer individual ordering". Monotonicity of other representations of votes, such as the votex and the beatpath matrix, has also been analysed. Throughout this dissertation, we advocate that acclamation, which is defined as the intersection of recursive monotonicity of the scorix, monotonicity of the votrix and monotonicity of the profile (w.r.t. the same ranking), leads to an agreement of most ranking rules and should be considered in the monometric rationalisation of ranking rules. Obviously, acclamation is a much broader consensus state than unanimity and its consideration will decrease the dependence of the ranking rule w.r.t. the chosen monometric.

The search for a consensus state is solved in this dissertation as an optimization problem, in particular as a well-known type of Integer Linear Programming problem: a transportation problem. Undoubtedly, there is still a lot of work to be done here. Our proposed 
solution leads to an inconvenient factorial time complexity, which complicates the computation of the optimization problem even for a moderately small number of candidates. Although similar problems are proved to be NP-hard problems, there is still many room for improvement here. Several approximations and pruning techniques have been proposed for the search for unanimity and for a Condorcet ranking, considerably decreasing the computational time. This suggests a clear future direction that will be addressed in the near future.

Monotonicity of both the scorix and the votrix is a global property that leads to an obvious winning ranking(s) on the set of candidates. In case either monotonicity of the scorix or monotonicity of the votrix does not hold, instead of directly searching for this monotonicity, we may be interested in analysing the underlying reasons why this monotonicity does not hold. In that way, based on the same principles of (recursive) monotonicity of the scorix, we can define a strict partial order relation on the set of candidates according to how good all candidates are in terms of positional information. When there exists a greatest element for this partial order relation, one talks about the positional winner, and the fact that the partial order relation is a total relation turns out to be equivalent to the fact that the scorix is (strictly) recursively monotone. Analogously, based on the same principles of monotonicity of the votrix, we can define a strict partial order relation on the set of candidates according to how good all candidates are in terms of pairwise information. When there exists a greatest element for this partial order relation, one talks about the pairwise winner, and the fact that the partial order relation is a total relation turns out to be equivalent to the fact that the votrix is (strictly) monotone.

Last, but definitely not least, we have discussed the potential applications of this dissertation. The aggregation of rankings has been addressed in many scientific disciplines, including medicine, consumer preference analysis, computer science, management science and social choice theory. Nevertheless, due to the natural interpretation of the aggregation of rankings as a voting procedure, social choice theory is considered the most prominent field of application. Here, we have considered three different real-life problems: an ecosystem management problem where the goal is to rank four plans according to their perceived suitability for the Lar rangeland in Iran; an environmental decision making problem where the goal is to identify the best estimation method for the production of orange trees in the Argentinian province of Corrientes; and a consumer preference analysis problem where the goal is to check whether or not consumers are able to distinguish between different degrees 
of spoilage of some cod fish samples at the Laboratory of Food Microbiology and Food Preservation at Ghent University, Belgium.

The methodology introduced in this dissertation has been exclusively analysed here for the particular case of the aggregation of rankings. Nevertheless, as part of a side joint project with the Laboratory of Food Microbiology and Food Preservation at Ghent University, a first approach to the aggregation of labels given on a qualitative scale has been addressed based on the notion of monotonicity. The aggregation of compositional data, directional data, strings and many other types of structured and/or unstructured data undoubtedly is a future research line. The introduction of betweenness relations, consensus states and monometrics in the field of aggregation results in promising tools that may help the understanding and progress in the study of the aggregation of such complex types of data. 


\section{Bibliography}

[1] A. Alnur, M. Meila, Experiments with Kemeny ranking: What works when?, Mathematical Social Sciences 64 (2012) 28-40.

[2] N. G. Andjiga, A. Y. Mekuko, I. Moyouwou, Metric rationalization of social welfare functions, Mathematical Social Sciences 72 (2014) 14-23.

[3] K. J. Arrow, A difficulty in the concept of social welfare, The Journal of Political Economy 58 (4) (1950) 328-346.

[4] K. J. Arrow, Social Choice and Individual Values, Wiley, New York, 1963.

[5] K. J. Arrow, H. Raynaud, Social Choice and Multicriterion Decision-Making, MIT Press Books, Cambridge, 1986.

[6] K. J. Arrow, A. K. Sen, K. Suzumura, Handbook of Social Choice and Welfare, vol. 1, North-Holland, Amsterdam, 2002.

[7] E. Baharad, S. Nitzan, The inverse plurality rule - an axiomatization, Social Choice and Welfare 25 (2005) 173-178.

[8] J. P. Barthelemy, B. Monjardet, The median procedure in cluster analysis and social choice theory, Mathematical Social Sciences 1 (1981) 235-267.

[9] J. Bartholdi, C. A. Tovey, A. Trick, Voting schemes for which it can be difficult to tell who won the election, Social Choice and Welfare 6 (1989) 157-165.

[10] V. S. Bawa, Optimal rules for ordering uncertain prospects, Journal of Financial Economics 2 (1) (1975) 95-121. 
[11] G. Beliakov, A. Pradera, T. Calvo, Aggregation Functions: A Guide for Practitioners, vol. 221 of Studies in Fuzziness and Soft Computing, Springer, Berlin, Heidelberg, 2007.

[12] A. Ben-David, Monotonicity maintenance in information-theoretic machine learning algorithms, Machine Learning 19 (1) (1995) 29-43.

[13] N. Betzler, M. R. Fellows, J. Guo, R. Niedermeier, F. A. Rosamond, Fixed-parameter algorithms for Kemeny rankings, Theoretical Computer Science 310 (2009) 45544570 .

[14] D. Black, The Theory of Committees and Elections, Cambridge University Press, Cambridge, 1958.

[15] D. Black, Partial justification of the Borda count, Public Choice 28 (1976) 1-15.

[16] K. P. Bogart, Preference structures I: Distances between transitive preference relations, Journal of Mathematical Sociology 3 (1) (1973) 49-67.

[17] K. P. Bogart, Preference structures II: Distances between asymmetric relations, SIAM Journal on Applied Mathematics 29 (2) (1975) 254-262.

[18] J. C. Borda, Mémoire sur les Élections au Scrutin, Histoire de l'Académie Royale des Sciences, Paris, 1781.

[19] H. Bouremel, R. Pérez-Fernández, L. Zedam, B. De Baets, The clone relation of a binary relation, Information Sciences 382-383 (2017) 308-325.

[20] S. Brams, P. C. Fishburn, Approval Voting, Birkhauser, Boston, 1983.

[21] F. Brandt, V. Conitzer, U. Endriss, J. Lang, A. D. Procaccia (eds.), Handbook of Computational Social Choice, Cambridge University Press, New York, 2016.

[22] F. Brandt, F. Fischer, P. Harrenstein, The computational complexity of choice sets, Mathematical Logic Quarterly 55 (4) (2009) 444-459.

[23] J. W. Bucklin, The grand junction plan of city government and its results, Annals of the American Academy of Political and Social Science 38 (3) (1911) 87-102.

[24] H. Bustince, G. Beliakov, G. P. Dimuro, B. Bedregal, R. Mesiar, On the definition of penalty functions in data aggregation, Fuzzy Sets and Systems, in press, http://dx.doi.org/10.1016/j.fss.2016.09.011. 
[25] T. Calvo, G. Beliakov, Aggregation functions based on penalties, Fuzzy Sets and Systems 161 (2010) 1420-1436.

[26] T. Calvo, R. Mesiar, R. R. Yager, Quantitative weights and aggregation, IEEE Transactions on Fuzzy Systems 12 (2004) 62-69.

[27] D. E. Campbell, S. I. Nitzan, Social compromise and social metrics, Social Choice and Welfare 3 (1986) 1-16.

[28] K. Cao-Van, B. De Baets, Growing decision trees in an ordinal setting, International Journal of Intelligent Systems 18 (7) (2003) 733-750.

[29] A. Cayley, Note on the theory of permutations, The London, Edinburgh, and Dublin Philosophical Magazine and Journal of Science 34 (232) (1849) 527-529.

[30] I. Charon, O. Hudry, A survey on the linear ordering problem for weighted or unweighted tournaments, 4OR 5 (2007) 5-60.

[31] I. Charon, O. Hudry, An updated survey on the linear ordering problem for weighted aor unweighted tournaments, Annals of Operations Research 175 (2010) 107-158.

[32] P. Y. Chebotarev, E. Shamis, Characterizations of scoring methods for preference aggregation, Annals of Operations Research 80 (1998) 299-332.

[33] F. Chiclana, F. Herrera, E. Herrera-Viedma, Integrating three representation models in fuzzy multipurpose decision making based on fuzzy preference relations, Fuzzy Sets and Systems 97 (1) (1998) 33-48.

[34] D. Ciucci, Orthopairs: A simple and widely used way to model uncertainty, Fundamenta Informaticae 108 (2011) 287-304.

[35] M. Condorcet, Essai sur l'Application de l'Analyse à la Probabilité des Décisions Rendues à la Pluralité des Voix, De l'Imprimerie Royale, Paris, 1785.

[36] V. Conitzer, A. Davenport, J. Kalagnanam, Improved bounds for computing Kemeny rankings, in: Proceedings of the 21st National Vonference on Artificial Intelligence, Boston, 2006.

[37] W. D. Cook, L. M. Seiford, Priority ranking and consensus formation, Management Science 24 (16) (1978) 1721-1732. 
[38] W. D. Cook, L. M. Seiford, On the Borda-Kendall consensus method for priority ranking problems, Management Science 28 (6) (1982) 621-637.

[39] C. H. Coombs, A Theory of Data, John Wiley, New York, 1957.

[40] A. H. Copeland, A reasonable social welfare function, Seminar on Applications of Mathematics to the Social Sciences, University of Michigan (1951).

[41] B. A. Davey, H. A. Priestley, Introduction to Lattices and Order, Cambridge University Press, Cambridge, 2002.

[42] B. De Baets, L. Zedam, A. Kheniche, A clone-based representation of the fuzzy tolerance or equivalence relations a strict order relation is compatible with, Fuzzy Sets and Systems 296 (2016) 35-50.

[43] K. De Loof, B. De Baets, H. De Meyer, On the random generation of monotone data sets, Information Processing Letters 107 (6) (2008) 216-220.

[44] M. M. Deza, E. Deza, Encyclopedia of Distances, Springer-Verlag Berlin Heidelberg, Berlin, 2009.

[45] I. Díaz, S. M. Mazza, E. F. Combarro, L. I. Giménez, J. E. Gaiad, Machine learning applied to the prediction of citrus production, Spanish Journal of Agricultural Research, to appear.

[46] C. L. Dodgson, A Method of Taking Votes on More than Two Issues, Clarendon Press, Oxford, 1876.

[47] B. Dutta, J.-F. Laslier, Comparison functions and choice correspondences, Social Choice and Welfare 16 (1999) 513-532.

[48] C. Dwork, R. Kumar, M. Naor, D. Sivakumar, Rank aggregation methods for the web, in: Proceedings of the 10th International Conference on World Wide Web, Hong Kong, 2001, pp. 613-622.

[49] E. Elkind, P. Faliszewski, A. Slinko, Distance rationalization of voting rules, Social Choice and Welfare 45 (2) (2015) 345-377.

[50] P. Emerson, The original Borda count and partial voting, Social Choice and Welfare 40 (2013) 353-358. 
[51] J. A. Ferejohn, D. M. Grether, On a class of rational social decision procedures, Journal of Economic Theory 8 (1974) 471-482.

[52] B. Fine, K. Fine, Social choice and individual ranking I, The Review of Economic Studies 41 (3) (1974) 303-322.

[53] B. Fine, K. Fine, Social choice and individual rankings II, The Review of Economic Studies 41 (4) (1974) 459-475.

[54] P. C. Fishburn, Conditions for simple majority decision functions with intransitive individual indifference, Journal of Economic Theory 2 (1970) 354-367.

[55] P. C. Fishburn, Betweenness, orders and interval graphs, Journal of Pure and Applied Algebra 1 (2) (1971) 159-178.

[56] P. C. Fishburn, A comparative analysis of group decision methods, Behavioral Science 16 (1971) 538-544.

[57] P. C. Fishburn, The Theory of Social Choice, Princeton University Press, New Jersey, 1973.

[58] P. C. Fishburn, Paradoxes of voting, The American Political Science Review 68 (2) (1974) 537-546.

[59] P. C. Fishburn, Condorcet social choice functions, SIAM Journal on Applied Mathematics 33 (3) (1977) 469-489.

[60] P. C. Fishburn, Monotonicity paradoxes in the theory of elections, Discrete Applied Mathematics 4 (2) (1982) 119-134.

[61] P. C. Fishburn, J. C. Falmagne, Binary choice probabilities and rankings, Economics Letters 31 (2) (1989) 113-117.

[62] M. Gagolewski, Data Fusion. Theory, Methods and Applications, Institute of Computer Science, Polish Academy of Sciences, Warsaw, 2015.

[63] M. Gagolewski, Penalty-based aggregation of multidimensional data, Fuzzy Sets and Systems, in press, http://dx.doi.org/10.1016/j.fss.2016.12.009.

[64] J. L. García-Lapresta, A general class of simple majority decision rules based on linguistic opinions, Information Sciences 176 (2006) 352-365. 
[65] J. L. García-Lapresta, B. Llamazares, Majority decisions based on difference of votes, Journal of Mathematical Economics 35 (3) (2001) 463-481.

[66] J. L. García-Lapresta, A. A. J. Marley, M. Martínez-Panero, Characterizing bestworst voting systems in the scoring context, Social Choice and Welfare 34 (2010) 487-496.

[67] P. Gärdenfors, Positionalist voting functions, Theory and Decision 4 (1) (1973) 1-24.

[68] A. Gibbard, Manipulation of voting schemes: A general result, Econometrica 41 (1973) 587-601.

[69] C. Girard, Acclamation voting in Sparta: An early use of approval voting, Studies in Choice and Welfare, Springer-Verlag, Berlin Heidelberg, 2010, pp. 15-17.

[70] I. J. Good, A note on Condorcet sets, Public Choice 10 (1971) 97-101.

[71] M. Grabisch, J.-L. Marichal, R. Mesiar, E. Pap, Aggregation Functions, Cambridge University Press, Cambridge, 2009.

[72] J. Hadar, W. R. Russell, Rules for ordering uncertain prospects, The American Economic Review (1969) 25-34.

[73] R. W. Hamming, Error detecting and error correcting codes, The Bell System Technical Journal 29 (2) (1950) 147-160.

[74] E. Hüllermeier, K. Brinker, Learning valued preference structures for solving classification problems, Fuzzy Sets and Systems 159 (18) (2008) 2337-2352.

[75] E. V. Huntington, J. R. Kline, Sets of independent postulates for betweenness, Transactions of the American Mathematical Society 18 (3) (1917) 301-325.

[76] K. Inada, The simple majority decision rule, Econometrica 37 (3) (1969) 490-506.

[77] M. Karpinski, W. Schudy, Faster algorithms for feedback arc set tournament, kemeny rank aggregation and betweenness tournament, in: O. Cheong, K.-Y. Chwa, K. Park (eds.), Algorithms and Computation, Springer, Heidelberg, 2010, pp. 3-14.

[78] J. S. Kelly, Minimal manipulability and local strategy-proofness, Social Choice and Welfare 5 (1) (1988) 81-85.

[79] J. S. Kelly, Interjacency, Social Choice and Welfare 6 (4) (1989) 331-335. 
[80] J. G. Kemeny, Mathematics without numbers, Daedalus 88 (4) (1959) 577-591.

[81] J. G. Kemeny, J. L. Snell, Mathematical Models in the Social Sciences, vol. 9, Ginn, New York, 1962.

[82] M. G. Kendall, A new measure of rank correlation, Biometrika 30 (1938) 81-93.

[83] C. Klamler, The Dodgson ranking and its relation to Kemeny's method and Slater's rule, Social Choice and Welfare 23 (2004) 91-102.

[84] D. Klingman, R. Russell, Solving constrained transportation problems, Operations Research 23 (1) (1975) 91-106.

[85] G. H. Kramer, A dynamical model of political equilibrium, Journal of Economic Theory 16 (2) (1977) 310-334.

[86] H. W. Kuhn, The Hungarian method for the assignment problem, Naval Research Logistics Quarterly 2 (1955) 83-97.

[87] L. Kuuliala, Y. Al hage, A.-G. Ioannidis, M. Sader, M. Vanderroost, B. De Meulenaer, F. Devlieghere, Microbiological, chemical and sensory spoilage analysis of raw atlantic cod (gadus morhua): towards an integrated optical sensor for monitoring food quality, in: Innovations in Food Packaging, Shelf Life and Food Safety, Abstracts, Erding, Germany, 2015.

[88] E. Lerer, S. Nitzan, Some general results on the metric rationalization for social decision rules, Journal of Economic Theory 37 (1985) 191-201.

[89] H. Levy, Stochastic Dominance: Investment Decision Making under Uncertainty, 3rd ed., Springer, Berlin, 2016.

[90] S. Lievens, B. De Baets, K. Cao-Van, A probabilistic framework for the design of instance-based supervised ranking algorithms in an ordinal setting, Annals of Operations Research 163 (1) (2008) 115-142.

[91] S. Lin, Rank aggregation methods, Wiley Interdisciplinary Reviews: Computational Statistics 2 (5) (2010) 555-570.

[92] B. Llamazares, T. Peña, Positional voting systems generated by cumulative standings functions, Group Decision and Negotiation 24 (5) (2015) 777-801. 
[93] B. Llamazares, T. Peña, Scoring rules and social choice properties: some characterizations, Theory and Decision 78 (3) (2015) 429-450.

[94] J. Marschak, Binary-choice constraints and random utility indicators, in: K. J. Arrow, S. Karlin, P. Suppes (eds.), Mathematical Methods in the Social Sciences, Standford University Press, Stanford, 1959, pp. 312-329.

[95] K. O. May, A set of independent necessary and sufficient conditions for simple majority decision, Econometrica 20 (1952) 680-684.

[96] I. McLean, A. B. Urken, Classics of Social Choice, University of Michigan Press, Ann Arbor, 1995.

[97] V. Merlin, The axiomatic characterizations of majority voting and scoring rules, Mathematical Social Sciences 161 (2003) 87-109.

[98] V. Merlin, M. Tataru, F. Valognes, On the likelihood of condorcet's profiles, Social Choice and Welfare 19 (2002) 193-206.

[99] T. Meskanen, H. Nurmi, Distance from consensus: A theme and variations, in: B. Simeone, F. Pukelsheim (eds.), Mathematics and Democracy. Recent Advances in Voting Systems and Collective Choice, Springer, Berlin, 2006, pp. 117-132.

[100] T. Meskanen, H. Nurmi, Closeness counts in social choice, in: M. Braham, F. Steffen (eds.), Power, Freedom, and Voting, Springer, Heidelberg, 2008, pp. 289-306.

[101] G. Monge, Mémoire sur la Théorie des Déblais et des Remblais, De l'Imprimerie Royale, Paris, 1781.

[102] H. Moulin, The Strategy of Social Choice, North-Holland, Amsterdam, 1983.

[103] H. Moulin, Choosing from a tournament, Social Choice and Welfare 3 (1986) 271-291.

[104] H. Moulin, Axioms of Cooperative Decision Making, Cambridge University Press, Cambridge, 1988.

[105] E. J. Nanson, Methods of election, Transactions and Proceedings of the Royal Society of Victoria XIX (1882) 197-240.

[106] S. Nitzan, Some measures of closeness to unanimity and their implications, Theory and Decision 13 (1981) 129-138. 
[107] H. Nurmi, Comparing Voting Systems, Reidel, Dordrecht, 1987.

[108] H. Nurmi, A comparison of some distance-based choice rules in ranking environments, Theory and Decision 57 (1) (2004) 5-24.

[109] H. Nurmi, Settings of consensual processes: Candidates, verdicts, policies, in: E. Herrera-Viedma, J. L. García-Lapresta, J. Kacprzyk, M. Fedrizzi, H. Nurmi, S. Zadrozny (eds.), Consensual Processes, Springer, Berlin, 2011, pp. 159-177.

[110] C. H. Papadimitriou, K. Steiglitz, Combinatorial Optimization: Algorithms and Complexity, Prentice-Hall, New Jersey, 1982.

[111] M. Pasch, Vorlesungen über neuere Geometrie, vol. 23, Teubner, Leipzig, Berlin, 1882.

[112] P. Pérez-Asurmendi, F. Chiclana, Linguistic majorities with difference in support, Applied Soft Computing 18 (2014) 196-208.

[113] R. Pérez-Fernández, P. Alonso, H. Bustince, I. Díaz, A. Jurio, S. Montes, Ordering finitely generated sets and finite interval-valued hesitant fuzzy sets, Information Sciences 325 (2015) 375-392.

[114] R. Pérez-Fernández, P. Alonso, H. Bustince, I. Díaz, S. Montes, Applications of finite interval-valued hesitant fuzzy preference relations in group decision making, Information Sciences 326 (2016) 89-101.

[115] R. Pérez-Fernández, P. Alonso, I. Díaz, S. Montes, Multi-factorial risk assessment: An approach based on fuzzy preference relations, Fuzzy Sets and Systems 278 (2015) $67-80$.

[116] R. Pérez-Fernández, P. Alonso, I. Díaz, S. Montes, B. De Baets, Monotonicity as a tool for differentiating between truth and optimality in the aggregation of rankings, Journal of Mathematical Psychology 77 (2017) 1-9.

[117] R. Pérez-Fernández, P. Alonso, I. Díaz, S. Montes, B. De Baets, Monotonicity-based consensus states for the monometric rationalisation of ranking rules and how they are affected by ties, submitted.

[118] R. Pérez-Fernández, B. De Baets, The positional winner: The alter ego of the pairwise winner, document in preparation. 
[119] R. Pérez-Fernández, B. De Baets, The acclamation consensus state and an associated ranking rule, submitted.

[120] R. Pérez-Fernández, B. De Baets, The pairwise winner: The missing link between the Borda winner and the Condorcet winner, submitted.

[121] R. Pérez-Fernández, B. De Baets, Recursive monotonicity of the scorix: Borda meets Condorcet, Group Decision and Negotiation, in press, http://dx.doi.org/10.1007/s10726-017-9525-y.

[122] R. Pérez-Fernández, B. De Baets, Representations of votes based on pairwise information: Monotonicity versus consistency, Information Sciences, pending minor revision.

[123] R. Pérez-Fernández, M. Rademaker, P. Alonso, I. Díaz, S. Montes, B. De Baets, Monotonicity-based ranking on the basis of multiple partially specified reciprocal relations, Fuzzy Sets and Systems, in press, http://dx.doi.org/10.1016/j.fss.2016.12.008.

[124] R. Pérez-Fernández, M. Rademaker, P. Alonso, I. Díaz, S. Montes, B. De Baets, Representations of votes facilitating monotonicity-based ranking rules: From votrix to votex, International Journal of Approximate Reasoning 73 (2016) 87-107.

[125] R. Pérez-Fernández, M. Rademaker, B. De Baets, The scorix: A popular representation of votes revisited, International Journal of Approximate Reasoning 78 (2016) $241-251$.

[126] R. Pérez-Fernández, M. Rademaker, B. De Baets, Monometrics and their role in the rationalisation of ranking rules, Information Fusion 34 (2017) 16-27.

[127] R. Pérez-Fernández, M. Sader, B. De Baets, Joint consensus evaluation of multiple objects on an ordinal scale: An approach driven by monotonicity, submitted.

[128] I. Perfilieva, B. De Baets, Fuzzy transforms of monotone functions with application to image compression, Information Sciences 180 (17) (2010) 3304-3315.

[129] J. E. Peris, B. Subiza, Condorcet choice correspondences for weak tournaments, Social Choice and Welfare 16 (1999) 217-231.

[130] E. Pitcher, M. F. Smiley, Transitivities of betweenness, Transactions of the American Mathematical Society 52 (1) (1942) 95-114. 
[131] M. Rademaker, B. De Baets, Aggregation of monotone reciprocal relations with application to group decision making, Fuzzy Sets and Systems 184 (1) (2011) 29-51.

[132] M. Rademaker, B. De Baets, Optimal restoration of stochastic monotonicity with respect to cumulative label frequency loss functions, Information Sciences 181 (4) (2011) $747-757$.

[133] M. Rademaker, B. De Baets, A ranking procedure based on a natural monotonicity constraint, Information Fusion 17 (2014) 74-82.

[134] M. Rademaker, B. De Baets, H. De Meyer, Loss optimal monotone relabeling of noisy multi-criteria data sets, Information Sciences 179 (24) (2009) 4089-4096.

[135] M. Rademaker, B. De Baets, H. De Meyer, Optimal monotone relabelling of partially non-monotone ordinal data, Optimization Methods and Software 27 (1) (2012) 17-31.

[136] M. Regenwetter, J. Dana, C. P. Davis-Stober, Transitivity of preferences, Psychological Review 118 (1) (2011) 42-56.

[137] J. T. Richelson, Running off empty: run-off point systems, Public Choice 35 (4) (1980) 457-468.

[138] J. Rieskamp, J. R. Busemeyer, B. A. Mellers, Extending the bounds of rationality: Evidence and theories of preferential choice, Journal of Economic Literature 44 (3) (2006) 631-661.

[139] J. J. Rousseau, Du Contrat Social, Marc Michel Rey, Amsterdam, 1762.

[140] C. Rowley, Borda, Jean-Charles de (1733-1799), in: J. Eatwell, M. Milgate, P. Newman (eds.), The New Palgrave: A Dictionary of Economics, Macmillan, London, 1987, pp. 262-263.

[141] D. G. Saari, Basic Geometry of Voting, Springer-Verlag, Heidelberg, 1995.

[142] D. G. Saari, Explaining all three-alternative voting outcomes, Journal of Economic Theory 87 (1999) 313-355.

[143] D. G. Saari, Mathematical structure of voting paradoxes I: Pairwise votes, Economic Theory 15 (2000) 1-53.

[144] D. G. Saari, Mathematical structure of voting paradoxes II: Positional voting, Economic Theory 15 (2000) 55-102. 
[145] D. G. Saari, T. J. McIntee, Connecting pairwise and positional election outcomes, Mathematical Social Sciences 66 (2013) 140-151.

[146] M. A. Satterthwaite, Strategy-proofness and Arrow's conditions: Existence and correspondence theorems for voting procedures and social welfare functions, Journal of Economic Theory 10 (2) (1975) 187-217.

[147] M. Schulze, A new monotonic, clone-independent, reversal symmetric, and Condorcet-consistent single-winner election method, Social Choice and Welfare 36 (2011) 267-303.

[148] T. Schwartz, Rationality and the myth of the maximum, Noûs 6 (2) (1972) 97-117.

[149] A. K. Sen, A possibility theorem on majority decisions, Econometrica 34 (2) (1966) 491-499.

[150] A. K. Sen, Collective Choice and Social Welfare, Holden-Day, San Francisco, 1970.

[151] P. B. Simpson, On defining areas of voter choice: Professor Tullock on stable voting, The Quarterly Journal of Economics 83 (1969) 478-490.

[152] J. H. Smith, Aggregation of preferences with variable electorate, Econometrica 41 (1973) 1027-1041.

[153] E. S. Staveley, Greek and Roman Voting and Elections, Cornell University Press, New York, 1972.

[154] W. E. Stein, P. J. Mizzi, R. C. Pfaffenberger, A stochastic dominance analysis of ranked voting systems with scoring, European Journal of Operational Research 74 (1) (1994) 78-85.

[155] M. Stepnicka, B. De Baets, Implication-based models of monotone fuzzy rule bases, Fuzzy Sets and Systems 232 (2013) 134-155.

[156] M. Stepnicka, B. De Baets, Interpolativity of at-least and at-most models of monotone single-input single-output fuzzy rule bases, Information Sciences 234 (2013) $16-28$.

[157] T. N. Tideman, Independence of clones as a criterion for voting rules, Social Choice and Welfare 4 (3) (1987) 185-206.

[158] A. Tversky, Features of similarity, Psychological Review 84 (4) (1977) 327-352. 
[159] A. Tversky, I. Gati, Similarity, separability, and the triangle inequality, Psychological Review 89 (2) (1982) 123-154.

[160] E. Van Broekhoven, B. De Baets, Monotone Mamdani-Assilian models under mean of maxima defuzzification, Fuzzy Sets and Systems 159 (21) (2008) 2819-2844.

[161] E. Van Broekhoven, B. De Baets, Only smooth rule bases can generate monotone Mamdani-Assilian models under center-of-gravity defuzzification, IEEE Transactions on Fuzzy Systems 17 (5) (2009) 1157-1174.

[162] P. Varela, A. Gastón, Sensory profiling, the blurred line between sensory and consumer science. a review of novel methods for product characterization, Food Research International 48 (2012) 893-908.

[163] J. Verwaeren, P. Van der Weeën, B. De Baets, A search grid for parameter optimization as a byproduct of model sensitivity analysis, Applied Mathematics and Computation 261 (2015) 8-27.

[164] J. Verwaeren, W. Waegeman, B. De Baets, Learning partial ordinal class memberships with kernel-based proportional odds models, Computational Statistics and Data Analysis 56 (4) (2012) 928-942.

[165] T. Wilkin, G. Beliakov, Weakly monotonic averaging functions, International Journal of Intelligent Systems 30 (2) (2015) 144-169.

[166] R. R. Yager, Toward a general theory of information aggregation, Information Sciences 68 (1993) 191-206.

[167] R. R. Yager, A. Rybalov, Understanding the median as a fusion operator, International Journal of General Systems 26 (1997) 239-263.

[168] Y. Y. Yao, An outline of a theory of three-way decisions, in: J. Yao, Y. Yang, R. Slowinski, S. Greco, H. Li, S. Mitra, L. Polkowski (eds.), Rough Sets and Current Trends in Computing, vol. 7413, Springer, Heidelberg, 2012, pp. 1-17.

[169] H. P. Young, Social choice scoring functions, SIAM Journal on Applied Mathematics 28 (4) (1975) 824-838.

[170] H. P. Young, Condorcet's theory of voting, American Political Science Review 82 (4) (1988) 1231-1244. 
[171] P. Young, Optimal voting rules, Journal of Economic Perspectives 9 (1995) 51-64.

[172] T. M. Zavist, T. N. Tideman, Complete independence of clones in the ranked pairs rule, Social Choice and Welfare 6 (2) (1989) 167-173.

[173] K. Zendehdel, M. Rademaker, B. De Baets, G. Van Huylenbroeck, Environmental decision making with conflicting social groups: A case study of the Lar rangeland in Iran, Journal of Arid Environments 74 (3) (2010) 394-402. 


\section{Summaries}

In the following, we provide a summary of this dissertation in English, Spanish and Dutch.

\subsection{English summary}

Social choice theory concerns the study of the conclusions that can be drawn from the preferences expressed by several voters over a set of candidates. In particular, throughout this dissertation, we consider the setting where each voter expresses a ranking on the set of candidates. Although at first sight it might appear to be an artificial theoretical problem with little application outside the election framework, nothing could be further from the truth. The application field results to be very rich, attracting the attention of many different fields such as social sciences, computer sciences, economical sciences, biological sciences and mathematical sciences.

In Chapter 1, we motivate the problem in the paragraph above and highlight the most important objectives of this research. We also provide an outline of this dissertation, together with some suggestions for its adequate reading.

In Chapter 2, we review the history of social choice theory, recalling the most prominent ranking rules. Plurality, the Borda count, scoring (ranking) rules, majority, the notions of Condorcet winner and Condorcet ranking, the method of Kemeny - among others are recalled and illustrated with some toy examples. It is supposed to serve as a tool for making this dissertation fully self-contained. We conclude the chapter by explaining a ranking procedure based on a natural monotonicity constraint proposed by Rademaker and De Baets, which was the starting point of this thesis. 
In Chapter 3, we review the metric rationalisation of ranking rules, which represents the characterization of ranking rules by minimizing the distance to a consensus state for some appropriate distance function. A consensus state is the set of all profiles of rankings satisfying a natural property that leads to an obvious winning ranking. Monometrics, which are closely related to distance functions or metrics, are introduced here. Like a distance function, a monometric satisfies the axioms of non-negativity and coincidence, but a monometric requires compatibility with a given betweenness relation and does not impose symmetry nor the triangle inequality. As discussed throughout this chapter, monometrics are a more suitable tool for the rationalisation of ranking rules than metrics, and will lead to the introduction of the monometric rationalisation of ranking rules.

In Chapter 4, we analyse different representations of votes, which are tools that play a key role in the field of social choice theory. Usually, the profile of rankings provided by the voters is compressed into these representations of votes gathering the most significant information. For instance, the Borda count, probably the most well-known ranking rule, reduces the rankings given by the voters to the scoring matrix (from now on referred to as the scorix), which is a matrix where the element at the $i$-th row and $j$-th column equals the number of times that the $i$-th candidate is ranked at the $j$-th position in the rankings given by the voters. Besides the Borda count, all other scoring ranking rules also reduce the rankings given by the voters to the scorix. Based on the ideas of Condorcet, another representation of votes that gathers a completely different type of information is also commonly used in social choice theory: the voting matrix (from now on referred to as the votrix). The votrix is a matrix where the element at the $i$-th row and $j$-th column equals the number of times that the $i$-th candidate is preferred to the $j$-th candidate in the rankings given by the voters. Relative positions between candidates are not explicitly gathered by the votrix, and we propose a new representation of votes gathering this hitherto unconsidered information: the votex. The beatpath matrix, which is a recently introduced representation of votes based on the notion of beatpath, is also briefly recalled in this chapter.

In Chapter 5, we discuss the notion of monotonicity of a representation of votes, which will be proven to be a cornerstone for social choice theory. Monotonicity is a common desired property in mathematical modelling exercises, and its importance has been acknowledged in several disciplines. However, real-life data is often imperfect and does not fully comply with the monotonicity hypothesis. In the field of social choice theory, the concept of monotonicity is an old acquaintance for scholars that can easily be traced back to the early 
1970s. This classical concept of monotonicity differs from the one advocated by Rademaker and De Baets (discussed in Chapter 2), that was the starting point of this thesis. Here, monotonicity is not understood as a property of the ranking rule. Rather, monotonicity is a property of the representation of votes that will serve as a tool for defining the ranking rule. Different monotonicity-based consensus states are discussed in this chapter. Most of them will be used - together with the presence of a Condorcet ranking and unanimity - for defining natural ranking rules. Special attention will be paid to acclamation: the consensus state formed by the intersection of recursive monotonicity of the scorix, monotonicity of the votrix and monotonicity of the profile (w.r.t. the same ranking).

In Chapter 6, we address the search for all different monotonicity-based consensus states as an optimization problem. This search is formalized as an Integer Linear Programming problem. We divide the chapter into two different sections. First, the search for monotonicity is addressed by making changes in the representation of votes. Second, the search for monotonicity is addressed by making changes in the profile of rankings. The search for the same property by means of a distance function at the representation level and of a distance function at the profile level is a common topic in the field of social choice theory. For instance, the search for a Condorcet winner is addressed by means of a distance function at the votrix level and of a distance function at the profile level by two well-known methods: Condorcet's least-reversal method and Dodgson's method.

In Chapter 7, we address two main study subjects. First, as Arrow stated in his well-known Impossibility Theorem, there is no ranking rule simultaneously satisfying all the properties that can be considered desirable. This leads to an increasing interest of the scientific community in the search for axiomatic characterizations of different ranking rules. Here, although we do not provide any axiomatic characterization, we discuss some properties satisfied by the ranking rules based on the search for monotonicity. Second, we discuss the independence of the search for all monotonicity-based consensus states described in this dissertation w.r.t. each other.

In Chapter 8, we consider the more general setting where each voter is assumed to provide a ranking with ties on the set of candidates instead of a ranking on the set of candidates. This situation subsumes a three-way decision, where each voter needs to decide whether 'candidate $a$ is better than candidate $b$ ', 'candidate $b$ is better than candidate $a$ ' or 'candidates $a$ and $b$ are equally suitable'. This type of decision can be seen as a representation of bipolar information on a three-label bipolar qualitative scale. As each voter directly 
provides a ranking with ties on the set of candidates, the three aforementioned relations are considered to be transitive.

In Chapter 9, we discuss different types of winning candidates. There are obviously some situations in which the selection of a winning candidate is easy; for instance, when every voter agrees on the candidate that should be the winner. Such candidate is called the unanimous winner, and, unfortunately, it usually does not exist in real-life elections. In case more than half of the voters agree on the candidate that should be the winner, one talks about the (absolute) majority winner. Obviously, the majority winner might not exist either. Based only on the head-to-head comparisons between candidates, Condorcet advocated a weaker condition than that of the unanimous or the majority winner: in case a candidate is preferred by more than half of the voters to each of the other candidates, then it should be the winner; said candidate is referred to as the Condorcet winner. The existence of the Condorcet winner is neither assured due to the more than famous 'voting paradox' where, although all the voters provide transitive preferences on the set of candidates, the collective preference might be cyclic. Also, the Borda winner, which is the candidate that maximizes the Borda score is often considered. In this chapter, two new types of winner are discussed: the (quasi)positional winner and the pairwise winner. These two winners will be understood as necessary conditions for the Borda winner and the Condorcet winner to finally agree.

In Chapter 10, we apply the methodology discussed in this dissertation to different real-life problems. Presidential elections (social choice), ranking of governmental plans (multiplecriteria decision analysis) and search for consensus among a group of experts (group decision making) are some of the possible large-scale applications. However, every daily decision where more than one judgement/criterion is considered turns into an immediate potential application. The content of this dissertation is mainly of interest to fields such as ecosystem management, sustainability assessment or consumer preference analysis. In particular, we consider three problems: an ecosystem management problem in Iran, an environmental decision making problem in Argentina and a consumer preference analysis problem in Belgium.

In Chapter 11, we end with some conclusions and a discussion on future research directions. 


\subsection{Spanish summary}

La teoría de la elección social estudia las conclusiones que se pueden sacar de las preferencias de distintos votantes sobre un conjunto de candidatos. En particular, a lo largo de esta memoria, consideramos el marco teórico en el que cada votante expresa un orden total o ranking de los candidatos. Aunque a primera vista pueda parecer un problema con una fuerte inclinación teórica y poca aplicación más allá del marco electoral, nada más lejos de la realidad. Pueden encontrarse numerosos ejemplos de aplicaciones en campos tan diferentes como las ciencias sociales, las ciencias de la computación, las ciencias económicas, las ciencias biológicas o las ciencias matemáticas.

En el Capítulo 1, planteamos el problema aquí considerado y explicamos los objetivos más importantes de esta investigación. En este capítulo, también esbozamos brevemente como debe ser leída esta memoria.

En el Capítulo 2, repasamos las reglas de ordenación más importantes en el campo de la teoría de la elección social, así como los conceptos de pluralidad, el recuento Borda, las reglas de puntuación, las reglas mayoritarias, las nociones de ganador y ranking de Condorcet y el método de Kemeny, entre otros. Todos estos conceptos son ilustrados por medio de ejemplos. El principal objetivo de este capítulo es hacer que esta memoria resulte comprensible en sí misma, sin recurrir a elementos explicativos adicionales. Concluimos con una explicación detallada de una regla de ordenación basada en la propiedad de monotonía propuesta por Rademaker y De Baets, que supuso el punto de partida de esta tesis.

En el Capítulo 3, repasamos la racionalización matemática de las reglas de ordenación, que es la rama de la teoría de la elección social que caracteriza las reglas de ordenación por medio de la minimización de la distancia a un estado de consenso para una determinada métrica. Un estado de consenso está formado por todas las listas de rankings que cumplen una propiedad que, de una manera natural, determina inequivocamente el ranking que debe ser considerado el ganador. En este capítulo introducimos el concepto de monométrica, un tipo de función que está íntimamente relacionado con las métricas. Al igual que las métricas, una monométrica cumple los axiomas de no-negatividad y coincidencia, pero require además la compatibilidad con una relación de 'intermediación' y no impone el axioma de simetría, ni la desigualdad triangular. Tal y como explicamos a lo largo de este capítulo, las monométricas son una herramienta más adecuada para la 
racionalización matemática de las reglas de ordenación que las propias métricas. Por este motivo introducimos la racionalización monométrica de las reglas de ordenación.

En el Capítulo 4, analizamos diferentes representaciones de votos, que son herramientas que juegan un papel clave en la teoría de la elección social. Normalmente, una representación de votos recoge la información más importante de la lista de rankings dada por los votantes. Por ejemplo, el recuento Borda, probablemente la regla de ordenación más conocida, reduce la lista de rankings dada por los votantes a la matriz de puntuación (de ahora en adelante referida como la scorix), que es una matriz en la que el elemento de la $i$-ésima fila y $j$-ésima columna representa el número de veces que el $i$-ésimo candidato es clasificado en la $j$-ésima posición. Además del recuento Borda, el resto de reglas de puntuación también reducen la lista de rankings dada por los votantes a la scorix. Basada en las ideas de Condorcet, otra representación de votos que recoge un tipo de información totalmente diferente es la matriz de votación (de ahora en adelante referida como la votrix), que es una matriz en la que el elemento de la $i$-ésima fila y $j$-ésima columna representa el número de veces en las que el $i$-ésimo candidato es preferido al $j$-ésimo candidato. Las posiciones relativas entre los candidatos no son explicitamente reflejadas en la votrix. Por esto proponemos en este capítulo una nueva representación de votos que tiene en cuenta este tipo de información: el votex. Además, también mencionamos otra conocida representación de votos: la matriz de rutas más fuertes.

En el Capítulo 5, definimos una propiedad que puede ser considerada como una piedra angular para la teoría de elección social: la monotonía de una representación de votos. La propiedad de monotonía es una propiedad deseable en problemas de modelización matemática y su importancia ha sido reconocida en gran cantidad de disciplinas. Desafortunadamente, las bases de datos procedentes de problemas reales suelen ser imperfectas, impidiendo que la propiedad de monotonía se cumpla. La noción de monotonía es una vieja amiga para los eruditos de la teoría de la elección social que ha sido analizada desde el principio de la década de 1970. Este concepto clásico de monotonía difiere de aquel propuesto por Rademaker y De Baets (del cual hablamos en el Capítulo 2) y que supuso el punto de partida de esta investigación. En este caso, el concepto de monotonía no es considerado una propiedad de una regla de decisión, sino una propiedad de las representaciones de votos. En este capítulo, proponemos diferentes estados de consenso basados en la propiedad de monotonía. La mayoría de ellos serán usados (junto con la presencia de un ranking de Condorcet y la propiedad de unanimidad) para definir distintas reglas de ordenación. Prestaremos especial atención al estado de consenso 'aclamación', que es definido como la 
intersección de los estados de consenso de monotonía recursiva de la scorix, monotonía de la votrix y monotonía de la lista de ranking (con respecto al mismo ranking)

En el Capítulo 6, proponemos un problema de optimización para la búsqueda de los distintos estados de consenso propuestos en el capítulo anterior. En particular, definimos un problema de programación lineal entera. Este capítulo está dividido en dos secciones diferentes. En primer lugar, abordamos la búsqueda de monotonía por medio de cambios en las distintas representaciones de votos. En segundo lugar, abordamos la búsqueda de monotonía por medio de cambios en la lista de rankings dada por los votantes. La búsqueda de la misma propiedad por medio de cambios en la representación de votos y de cambios en la lista de rankings es un problema común en la teoría de la elección social. Por ejemplo, dos métodos conocidos en el campo buscan un ganador de Condorcet por medio de cambios en la votrix (método de mínimas inversiones de Condorcet) y de cambios en la lista de rankings (método de Dodgson).

En el Capítulo 7, centramos nuestra atención en dos temas de caracter teórico. En primer lugar, tal y como Arrow manifestó en su conocido Teorema de Imposibilidad, no existe ninguna regla de ordenación que cumpla al mismo tiempo tres propiedades que pueden ser consideradas elementales. Esto conlleva un creciente interés por parte de la comunidad científica en la búsqueda de caracterizaciones axiomáticas de las diferentes reglas de ordenación. Aunque no proponemos una axiomatización de las reglas de ordenación propuestas en esta memoria, discutimos las propiedades que todas estas reglas de ordenación cumplen. En segundo lugar, probamos que todas las reglas de ordenación basadas en la búsqueda de un estado de consenso propuestas en esta investigación son independientes entre ellas.

En el Capítulo 8, consideramos el caso más general en el que cada votante expresa una ranking con empates en lugar de un ranking (sin empates). Esta situación subsume una decisión a tres bandas, donde cada votante debe decidir si 'el candidato $a$ es mejor que el candidato $b$ ', 'el candidato $b$ es mejor que el candidato $a$ ' o 'los candidatos $a$ y $b$ son igualmente buenos'. Como cada votante expresa directamente un ranking con empates de los candidatos, las tres relaciones ya mencionadas son consideradas transitivas.

En el Capítulo 9, mostramos distintas nociones de candidato ganador. En algunas situaciones, el candidato que debe ser erigido ganador es obvio. Por ejemplo, en caso de que todos los votantes estén de acuerdo en apoyar a un mismo candidato. En este caso nos referimos a un ganador unánime. Desafortunadamente, este tipo de ganador no suele existir en condiciones reales. En caso de que más de la mitad de los votantes estén de acuerdo en 
el candidato que debe ser erigido ganador, hablamos de un ganador (absolutamente) mayoritario. Obviamente, el ganador mayoritario tampoco tiene por qué existir. Basándose solo en las comparaciones dos-a-dos entre los distintos candidatos, Condorcet propuso una condición más débil que la existencia de un ganador unánime o mayoritario: en caso de que un candidato sea preferido por más de la mitad de los votantes a cada uno de los otros candidatos, debe ser erigido el ganador. Este tipo de ganador es conocido como el ganador de Condorcet. La existencia de un ganador de Condorcet no está asegurada debido a la más que conocida 'paradoja de la votación' donde, aunque todos los votantes expresan preferencias transitivas, la preferencia colectiva puede resultar cíclica. El ganador de Borda, que es aquel candidato que maximiza el recuento de Borda, es también habitualmente considerado en el campo de la teoría de la elección social. En este capítulo, dos nuevos tipos de ganador son propuestos: el ganador (quasi)posicional y el ganador por pares. Estos dos tipos de ganadores serán entendidos como condiciones suficientes para que los ganadores de Condorcet y Borda coincidan finalmente.

En el Capítulo 10, aplicamos los método propuestos en esta memoria a distintos problemas de la vida real. Elecciones presidenciales (teoría de la elección social), la ordenación de planes gubernamentales (análisis de decisión multi-criterio) y la búsqueda de consenso entre un grupo de expertos (toma de decisión grupal) son algunas de las posibles aplicaciones. Sin embargo, cualquier acción diaria donde se considere más de un criterio se convierte en una potencial aplicación de esta investigación. El contenido de esta memoria es de interés, principalmente, en campos como la gerencia de ecosistemas, la evaluación de sostenibilidad o el análisis de preferencias de los consumidores. En concreto, consideramos tres casos: un primero de gerencia de ecosistemas en Irán, un segundo de toma de decisiones medioambiental en Argentina y un tercero de análisis de preferencias de los consumidores en Bélgica.

En el Capítulo 11, terminamos con unas conclusiones y una pequeña discusion de futuras líneas de investigación.

\subsection{Dutch summary}

Sociale keuzetheorie behandelt de vraag welke conclusies getrokken kunnen worden uit de preferenties uitgedrukt door een aantal stemmers over een aantal kandidaten. Meer bepaald bekijken we in deze thesis de setting waarin elke stemmer een rangschikking van 
het set van kandidaten heeft uitgedrukt. Misschien lijkt dit op het eerste zicht een nogal artificieel-theoretisch probleem, maar het tegenovergestelde blijkt al gauw waar te zijn. De toepassingsgebieden zijn zeer diverse en relevant, zoals sociale wetenschappen, computerwetenschappen, economische wetenschappen, biologische wetenschappen en wiskunde.

In Hoofdstuk 1 motiveren we belangrijkheid van de probleemstelling en lichten we belangrijkste doelstellingen van deze thesis uit. Dit hoofdstuk bevat ook een overzicht van de opbouw van deze thesis, alsook enkele tips hoe deze best gelezen kan worden.

Hoofdstuk 2 bespreekt de geschiedenis van sociale keuzetheorie, waar we de meest belangrijke rangschikking regels even herhalen. Pluraliteit, Borda, scoring (rangschikking) regels, de meerderheidsregel, het concept 'Condorcet winnaar' en 'Condorcet winning ranking', de Kemeny methode en anderen worden hier uitgelegd en geillustreerd met kleine voorbeelden. Het doel van dit hoofdstuk is om van deze thesis een op zich staand geheel te maken, waar geen verdere naslagwerk bij te pas hoeft te komen. Op het einde van dit hoofdstuk bespreken we een rangschikking regel gebaseerd op een natuurlijke monotoniteit voorwaarde zoals geformuleerd door Rademaker en De Baets. Deze regel vormt het startpunt van deze thesis.

In Hoofdstuk 3 behandelen we het metriek-aspect van rangschikking regels, waar we rangschikking regels dus beschouwen als 'manieren om de afstand tot een staat van consensus te becijferen' voor enkele toepasselijke afstandsfuncties. Een staat van consensus is een set rangschikkingen waarvoor de winnende rangschikking evident is. Monometrics, sterk gerelateerd aan afstandsfuncties of metrieken, worden hier ge $\tilde{A}^{-}$ntroduceerd. Net als een afstandsfunctie vervult een monometriek the axima van niet-negativiteit en samenvalling, maar niet symmetrie of de driehoeksongelijkheid. In de plaats van die twee is een monometriek compatibel met een 'tussenin' relatie. Dit hoofdstuk beargumenteert dat monometrieken toepasselijker zijn dan afstandsfuncties om rangschikking regels te rationaliseren, en resulteert aldus in de invoering van de monometriek rationalisering van rangsschikkingsregels.

In Hoofdstuk 4 analyseren we verschillende manieren om stemmen te representeren, een essentieel en belangrijk deel van het onderzoeksdomein van sociale keuzetheorie. Meestal zal het profiel van rangschikkingen zoals uitgedrukt door de stemmers samengevat worden in een van deze representaties, om de meest belangrijke informatie makkelijk verwerkbaar te maken. De Borda telling bijvoorbeeld, wellicht de best gekende rangschikking regel, reduceert de rangschikkingen uitgedrukt door de stemmers tot de score matrix (vanaf nu 
de scorix genoemd), een matrix waarin een element in rij $i$ en kolom $j$ het aantal keer dat de $i$-de kandidaat op de $j$-de plaats weer gevonden werd in de rangschikkingen uitgedrukt door de stemmers. Niet enkel de Borda telling, maar ook alle andere scorende rangschikkings regels reduceren de stemmen tot de scorematrix. Een andere voorstelling van de uitgebrachte stemmen is de stemmen matrix (vanaf nu votrix genoemd), gebaseerd op de opvattingen van Condorcet en een gericht op een wezenlijk verschillend type informatie. De votrix is een matrix waarin het element in de $i$-de rij en de $j$-de kolom het aantal keer dat de $i$-de kandidaat verkozen wordt boven de $j$-de kandidaat in de rangschikkingen uitgedrukt door de stemmers. Relatieve posities tussen kandidaten blijven niet bewaard in de votrix, en we formuleren een nieuwe representatie die toelaat om deze vooralsnog ongebruikte informatie alsnog in rekening te brengen, en noemen deze representatie de votex. De beatpath matrix, een recentelijk geformuleerde manier representatie van stemmen gebaseerd op het beatpath concept, wordt ook even kort aangehaald in dit hoofdstuk.

Hoofdstuk 5 bespreekt de notie van monotoniteit in een representatie van stemmen, en we zullen bewijzen dat dit een hoeksteen van sociale keuzetheorie vormt. Monotoniteit is een vaak vereiste eigenschap in wiskundige modellering toepassingen, en de belangrijkheid ervan wordt in verschillende disciplines expliciet erkend. Maar data komende uit de realiteit is vaak niet foutloos en vervult dan ook niet de monotoniteitvereiste. In het onderzoeksdomein van social choice theorie vormt het een oude bekende die voor het eerst opdook in de jaren 70 van de vorige eeuw. Dit monotoniteit-concept verschilt van het concept onderschreven door RAdemaker en De Baets (besproken in Hoofdstuk 2), het startpunt van deze thesis. De monotoniteit bedoeld door Rademaker en De Baets is geen eigenschap van de rangschikking regel, maar eerder als een mogelijke eigenschap die vervuld kan worden door een representatie van de stemmen, en zal dienen als een onderdeel van de definitie van de rangschikkings regel. Verschillende monotoniteit-gebaseerde consensus regels worden besproken in dit hoofdstuk. De meeste van deze alsook 'de aanwezigheid van een Condorcet ranking' en 'unanimiteit' zullen gebruikt worden om intuïtieve rangschikkigns regels te formuleren. We zullen extra aandacht besteden aan acclamation: de consensus toestand waar recursieve monotoniteit van de scorix, monotoniteit van de votrix en monotoniteit van het profiel met betrekking tot een en dezelfde rangschikking, alledrie samen aanwezig zijn.

In Hoofdstuk 6 zullen we uiteenzetten hoe het kwantificeren van monotoniteit als een optimalisatievraagstuk bekeken kan worden. Meer bepaald kan deze vraag geformaliseerd worden als een Integer Lineair Programmeringsprobleem. Dit hoofdstuk bestaat uit twee verschillende secties. In de eerste aanpak zal de zoektocht naar een consensus toestand 
gebeuren worden door aanpassingen in de representatie van de stemmen uit te voeren. In de tweede aanpak zullen we aanpassingen in de stemmen zelf doorvoeren, dus vòò we de informatie reduceren tot representatie niveau. We zullen als voorbeeld bespreken hoe een Condorcet winnaar te bepalen wanneer we de afstandsfunctie op votrix-niveau berekenen en wanneer we die een afstandsfunctie op niveau van de stemmen berekenen, zowel voor de Condorcet methode van minste omkeringen en de methode van Dodgson.

Hoofdstuk 7 zal twee onderwerpen behandelen. Vooreerst bespreken we het Onmogelijkheidstheorema van Arrow, welke stelt dat er geen rangschikking regel bestaat die een aantal intuïtieve en aantrekkelijke eigenschappen simultaan vertoont. Welke rangschikking regels dan wel welke eigenschappen vertonen, is dan ook een dankbaar onderwerp voor onderzoek geweest. In dit hoofdstuk zullen we dan ook een aantal eigenschappen bespreken van de rangschikking regels gebaseerd op monotoniteit, al zullen we geen volledige axiomatische karakterisatie doorvoeren. Vervolgens bespreken we of de zoektochten naar elk van de verschillende monotoniteit-gebaseerde consensus toestanden, onafhankelijk zijn van elkaar.

In Hoofdstuk 8 bekijken we de meer algemene setting waar een stemmer een rangschikking uitdrukt waarin sommige kandidaten als evenwaardig beschouwd worden, een niet-strikte rangschikking. Met andere woorden, voor elke twee kandidaten $a$ en $b$, zal elke stemmer uitdrukken ' $a$ is beter dan $b$ ', ' $b$ is beter dan $a$ ' of ' $a$ en $b$ zijn even goed'. We kunnen dit beschouwen als een representatie op een bipolaire kwalitatieve schaal met drie verschillende labels. Aangezien de stemmers nog steeds een rangschikking uitdrukken, veronderstellen we dat de beter dan en even goed relaties transitief zijn.

In Hoofdstuk 9 bespreken we verschillende types winnende kandidaten. Er zijn omstandigheden waarin het bepalen van de winnende kandidaat eenvoudig is; bijvoorbeeld wanneer elke stemmer dezelfde kandidaat boven alle andere verkiest. Een dergelijke 'unanieme winnaar' zal in de realiteit jammer genoeg slechts zelden aanwezig zijn. Wanneer (meer dan) de helft van alle stemmers dezelfde kandidaat boven alle anderen verkiest hebben we een (strikte) meerderheid kandidaat. Het spreekt vanzelf dat ook de aanwezigheid van een meerderheid kandidaat niet verzekerd is. Condorcet, die enkel de paarsgewijze preferentie relatie beschouwde, suggereerde een zwakkere voorwaarde voor een meerderheid kandidaat: de Condorcet winnaar is een kandidaat die door meer dan de helft van de stemmers boven elke andere kandidaat verkozen wordt. Jammer genoeg is ook het bestaan van een dergelijke kandidaat niet verzekerd, aangezien er een cykel kan optreden, waarbij de Condorcet meerderheid relatie niet transitief blijkt te zijn. Aangezien dit kan 
optreden zelfs wanneer elke stemmer zelf transitiviteit respecteert, wordt dit al een paradox beschouwd. Een Borda winnaar, een kandidaat die de grootste Borda score behaald, is wel steeds aanwezig. In dit hoofdstuk introduceren we twee nieuwe types winnaars: De (quasi)positionele winnaar en de paarsgewijze winnaar. De aanwezigheid van deze twee winnaars zal volstaan om Borda en Condorcet eindelijk met elkaar te verzoenen: Wanneer deze twee tegelijkertijd aanwezig zijn, zullen de Borda en de Condorcet winnaar een en dezelfde zijn.

In Hoofdstuk 10 passen we de methodologiën uit de vorige hoofdstukken toe op enkele problemen uit de praktijk. Presidentsverkiezingen (social choice), rangschikking van beheersplannen (meervoudige criteria analyse) and zoektocht naar consensus in een groep experts (groepsbeslissing technieken) zijn enkele van de mogelijke grootschalige toepassingen. Eigenlijk is elke dagdagelijkse beslissing waar meer dan een criterium of oordeel in rekening gebracht wordt, een potentiële toepassing. In deze thesis behandelen we voorbeelden uit een ecosysteem beheersprobleem in Iran, een milieugerelateerd beslissingsprobleem in Argentinië en een consumenten preferentie analyse toepassing in België.

Hoofdstuk 11 ten slotte bevat de conclusies en een blik vooruit naar potentieel verder onderzoek. 


\title{
Curriculum vitae
}

\section{Personal data}

\author{
Name: $\quad$ Raúl Pérez Fernández \\ Date of birth: 28/12/1990 \\ Place of birth: Oviedo, Asturias, Spain \\ e-mail: $\quad$ raul.perezfernandez@ugent.be \\ raulpf90@gmail.com
}

\section{Education}

1993-2002. Primary school. Colegio Montevil, Gijón, Asturias, Spain.

2002-2008. Secondary school. I.E.S. Montevil and I.E.S. Doña Jimena, Gijón, Asturias, Spain.

2008-2012. Degree in Mathematics. Speciality: Statistics. University of Oviedo, Oviedo, Asturias, Spain.

2011-2012. ERASMUS student. Université Joseph Fourier, Grenoble, France.

2012-2013. Master in Mathematical Modelling, Statistics and Computing. University of Oviedo, Oviedo, Asturias, Spain. 
2013-present. PhD student in Mathematics and Statistics. University of Oviedo, Oviedo, Asturias, Spain.

2015-present. PhD student in Applied Biological Sciences. Ghent University, Ghent, Belgium.

\section{Publications in international journals (WoS)}

R. Pérez-Fernández, P. Alonso, I. Díaz, S. Montes, Multi-factorial risk assessment: An approach based on fuzzy preference relations, Fuzzy Sets and Systems 278 (2015) 67-80.

R. Pérez-Fernández, P. Alonso, H. Bustince, I. Díaz, A. Jurio, S. Montes, Ordering finitely generated sets and finite-interval valued hesitant fuzzy sets, Information Sciences 325 (2015) 375-392.

R. Pérez-Fernández, P. Alonso, H. Bustince, I. Díaz, S. Montes, Applications of finite interval-valued hesitant fuzzy preference relations in group decision making, Information Sciences 326 (2016) 89-101.

R. Pérez-Fernández, M. Rademaker, P. Alonso, I. Díaz, S. Montes, B. De Baets, Representations of votes facilitating monotonicity-based ranking rules: From votrix to votex, International Journal of Approximate Reasoning 73 (2016) 87-107.

R. Pérez-Fernández, M. Rademaker, B. De Baets, The scorix: A popular representation of votes revisited, International Journal of Approximate Reasoning 78 (2016) 241-251.

R. Pérez-Fernández, M. Rademaker, B. De Baets, Monometrics and their role in the rationalisation of ranking rules, Information Fusion 34 (2017) 16-27.

H. Bouremel, R. Pérez-Fernández, L. Zedam, B. De Baets, The clone relation of a binary relation, Information Sciences 382-382 (2017) 308-325.

R. Pérez-Fernández, P. Alonso, I. Díaz, S. Montes, B. De Baets, Monotonicity as a tool for differentiating between truth and optimality in the aggregation of rankings, Journal of Mathematical Psychology 77 (2017) 1-9. 
R. Pérez-Fernández, M. Rademaker, P. Alonso, I. Díaz, S. Montes, B. De Baets, Monotonicity-based ranking on the basis of multiple partially specified reciprocal relations, Fuzzy Sets and Systems, in press, http://dx.doi.org/10.1016/j.fss.2016.12.008.

R. Pérez-Fernández, B. De Baets, Recursive monotonicity of the scorix: Borda meets Condorcet, Group Decision and Negotiation, in press, http://dx.doi.org/10.1007/s10726017-9525-y.

R. Pérez-Fernández, B. De Baets, Representations of votes based on pairwise information: Monotonicity versus consistency, Information Sciences, pending minor revision.

\section{Conference proceedings}

R. Pérez-Fernández, P. Alonso, I. Díaz, S. Montes, Aggregation of risk-assessment matrices for human reliability in transportation systems, Proceedings of the EUROFUSE 2013 Workshop on Uncertainty and Imprecision Modelling in Decision Making in Oviedo, Spain, 2013, pp. 225-232.

R. Pérez-Fernández, M. Rademaker, P. Alonso, I. Díaz, B. De Baets, Adding feasibility constraints to a ranking rule under a monotonicity constraint, Proceedings of the joint 16th World Congress of the International Fuzzy Systems Association (IFSA) and the 9th Conference of the European Society for Fuzzy Logic and Technology (EUSFLAT) in Gijón, Spain, 2015, pp. 1302-1309. (Best student paper award nomination)

R. Pérez-Fernández, B. De Baets, The role of betweenness relations, monometrics and penalty functions in data aggregation, Proceedings of the joint 17th World Congress of the International Fuzzy Systems Association (IFSA) and the 9th International Conference on Soft Computing and Intelligent Systems (SCIS) in Otsu, Japan, 2017.

\section{Conference abstracts}

B. De Baets, R. Pérez-Fernández, Ranking rules characterized by means of monometrics and consensus states, Book of Abstracts of the International Symposium on Aggregation and Structures (ISAS) in Luxembourg city, Luxembourg, 2016, pp. 30-32. 
R. Pérez-Fernández, B. De Baets, Monotonicity: A cornerstone of social choice theory, Book of Abstracts of the International Symposium on Aggregation and Structures (ISAS) in Luxembourg city, Luxembourg, 2016, pp. 60-62.

\section{Organizing committee memberships}

EUROFUSE 2013 Workshop on Uncertainty and Imprecision Modelling in Decision Making in Oviedo, Spain, 2013. 